\title{
N-glycoproteomics of human seminal plasma glycoproteins
}

Mayank Saraswat ${ }^{1,2}$, Sakari Joenväära ${ }^{2}$, Anil Kumar Tomar ${ }^{3}$, Sarman Singh $^{4}$, Savita Yadav ${ }^{3}$, Risto Renkonen ${ }^{1,2, *}$

${ }^{1}$ Haartman Institute, Transplantation laboratory, Haartmaninkatu 3, PO Box 21, FI-00014 University of Helsinki, Finland.

${ }^{2}$ HUSLAB, Helsinki University Hospital, Helsinki, Finland.

${ }^{3}$ Department of Biophysics, All India Institute of Medical Sciences, New Delhi - 110029.

${ }^{4}$ Division of Clinical Microbiology \& Molecular Medicine, Department of Laboratory Medicine, All India Institute of Medical Sciences, New Delhi-110029, India.

Table of contents

Page 1: $\quad$ Supplementary Table 1 and supplementary Figure 1.

Page 2-244: Annotated spectrum of all the glycopeptides. 


\section{$\mathrm{N}$-glycoproteomics of human seminal plasma glycoproteins}

Table S-1: Presents the clinical semen parameters of the healthy individuals which were used for the study. Sperm count and sperm motility is provided for the persons whose seminal plasma was used for the study.

\begin{tabular}{|l|l|l|l|}
\hline Serial Number & Code & $\begin{array}{l}\text { Sperm count } \\
\text { (million/mL) }\end{array}$ & $\begin{array}{l}\text { Sperm Motility (\% of } \\
\text { total sperm) }\end{array}$ \\
\hline 1 & $\mathrm{~N} 1$ & 185 & 60 \\
\hline 2 & $\mathrm{~N} 2$ & 160 & 60 \\
\hline 3 & $\mathrm{~N} 3$ & 116 & 50 \\
\hline 4 & $\mathrm{~N} 11$ & 102 & 55 \\
\hline 5 & $\mathrm{~N} 12$ & 78 & 50 \\
\hline 6 & $\mathrm{~N} 13$ & 60 & 55 \\
\hline 7 & $\mathrm{~N} 21$ & 86 & 50 \\
\hline
\end{tabular}

Figure S-1: Molecular function GoOntology analysis of 50 human seminal plasma glycoproteins. The GoRetriever tool from AgBase was used for mapping Go Ontology terms to the glycoproteins which was subsequently visualized using GoSlim and MS Excel. The number of proteins mapped to a given category is shown with the name of the category.

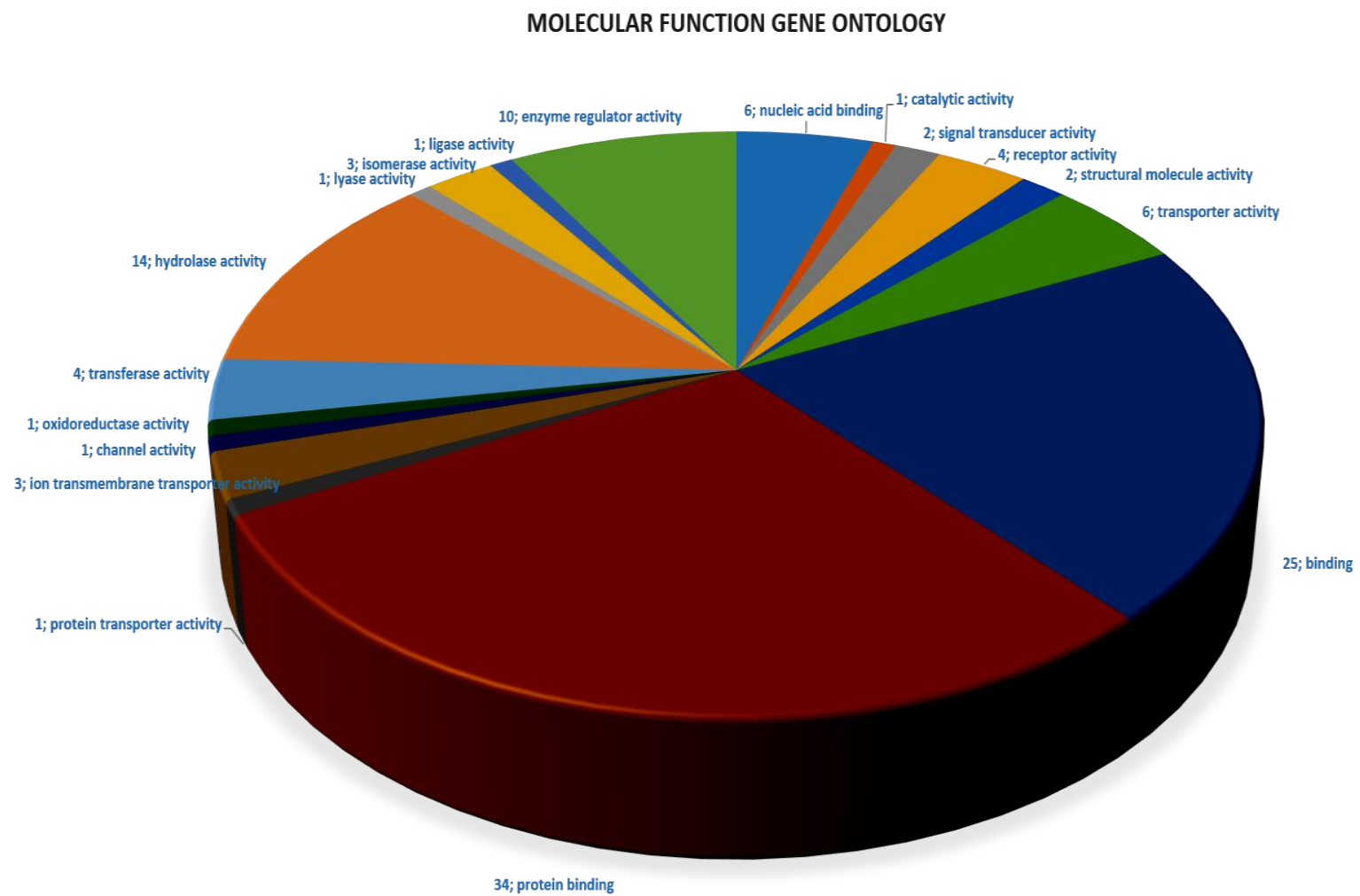


$\mathrm{m} / \mathrm{z} 1000.4222$ charge 4 scan $0-0$

Score $=61.67$, Hits $=44$, Explained Intensity $=0.26$

Peptide: RNT2_HUMAN[206,223]:QDQQLQNCTEPGEQPSPK

Glycan: $\mathrm{FHNH}(\mathrm{FHNH}) \mathrm{HNN}$, H5N4F2

Charge: $4 \mathrm{H}$
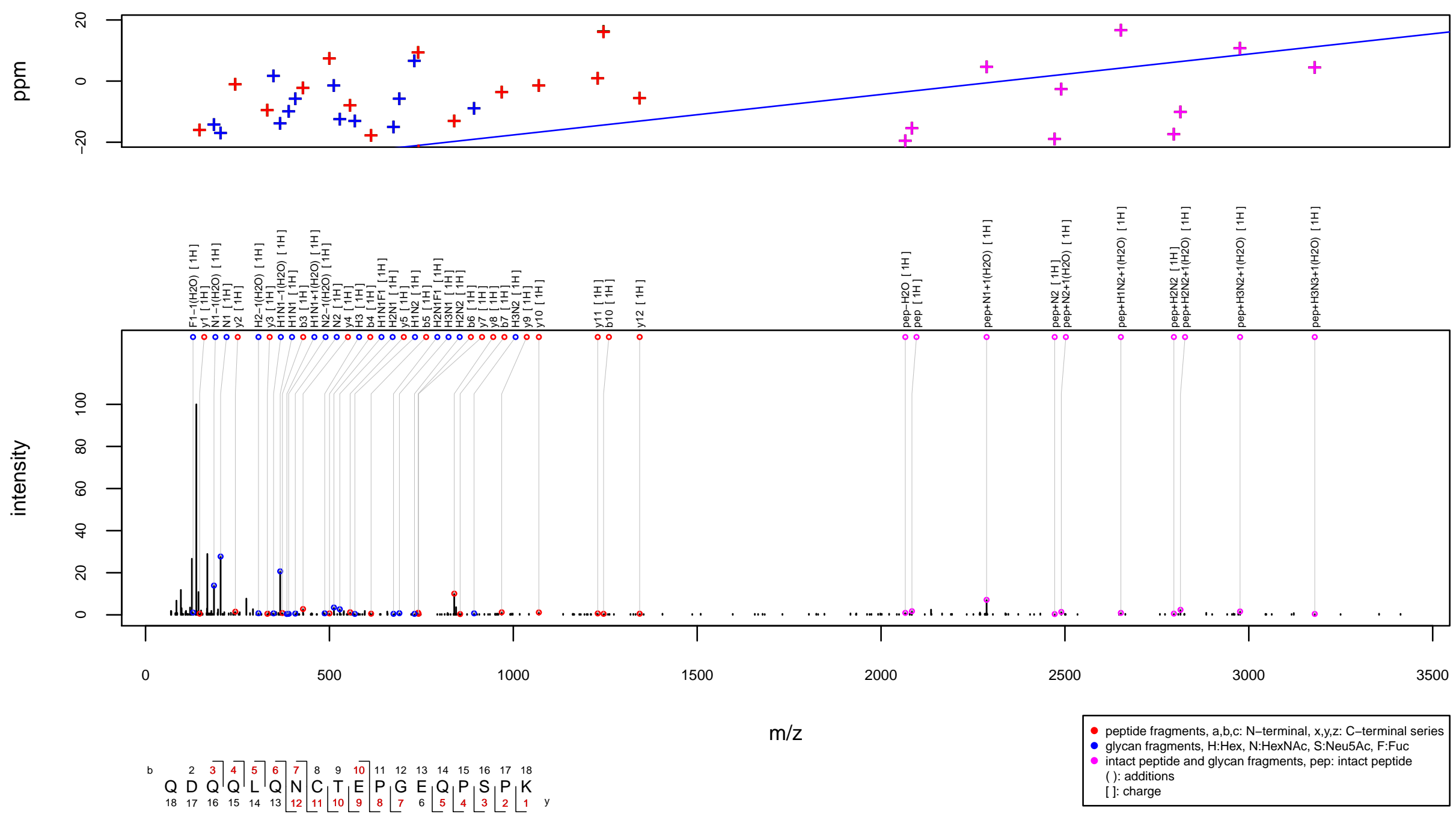


\section{$\mathrm{m} / \mathrm{z} 1000.9734$ charge 4 scan $0-0$}

Score $=66.85$, Hits $=44$, Explained Intensity $=0.38$

Peptide: FINC_HUMAN[997,1016]:LDAPTNLQFVNETDSTVLVR

Glycan: $\mathrm{HNH}(\mathrm{HNH}) \mathrm{HN}(\mathrm{F}) \mathrm{N}, \mathrm{H} 5 \mathrm{~N} 4 \mathrm{~F} 1$

Charge: $4 \mathrm{H}$
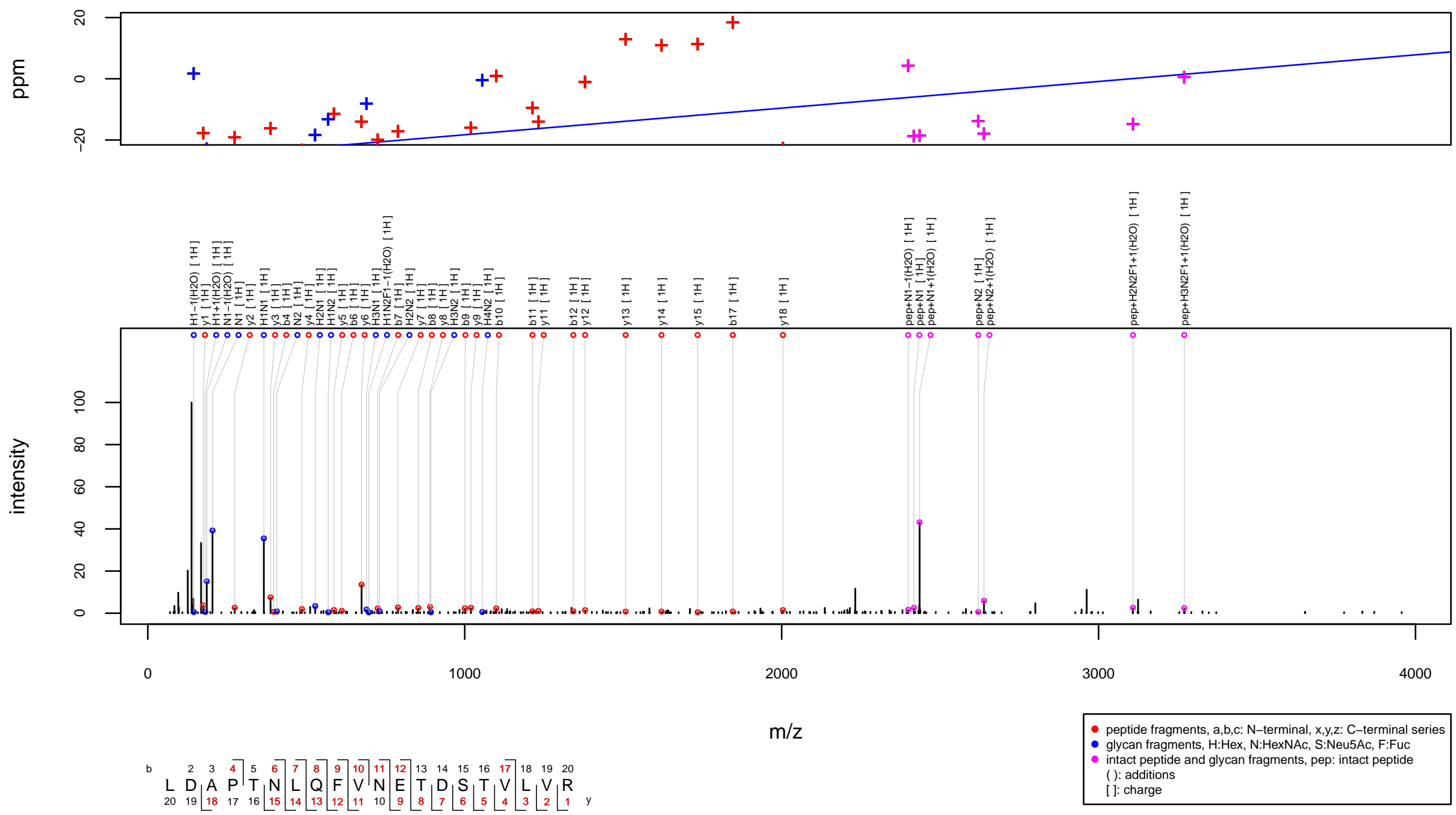
$\mathrm{m} / \mathrm{z} 1009.4437$ charge 4 scan $0-0$

Score $=46.13$, Hits $=29$, Explained Intensity $=0.37$

Peptide: CLUS_HUMAN[372,385]:LANLTQGEDQYYLR

Glycan: $\mathrm{SHNH}(\mathrm{SHNH}) \mathrm{HN}(\mathrm{F}) \mathrm{N}, \mathrm{S} 2 \mathrm{H} 5 \mathrm{~N} 4 \mathrm{~F} 1$

Charge: $4 \mathrm{H}$
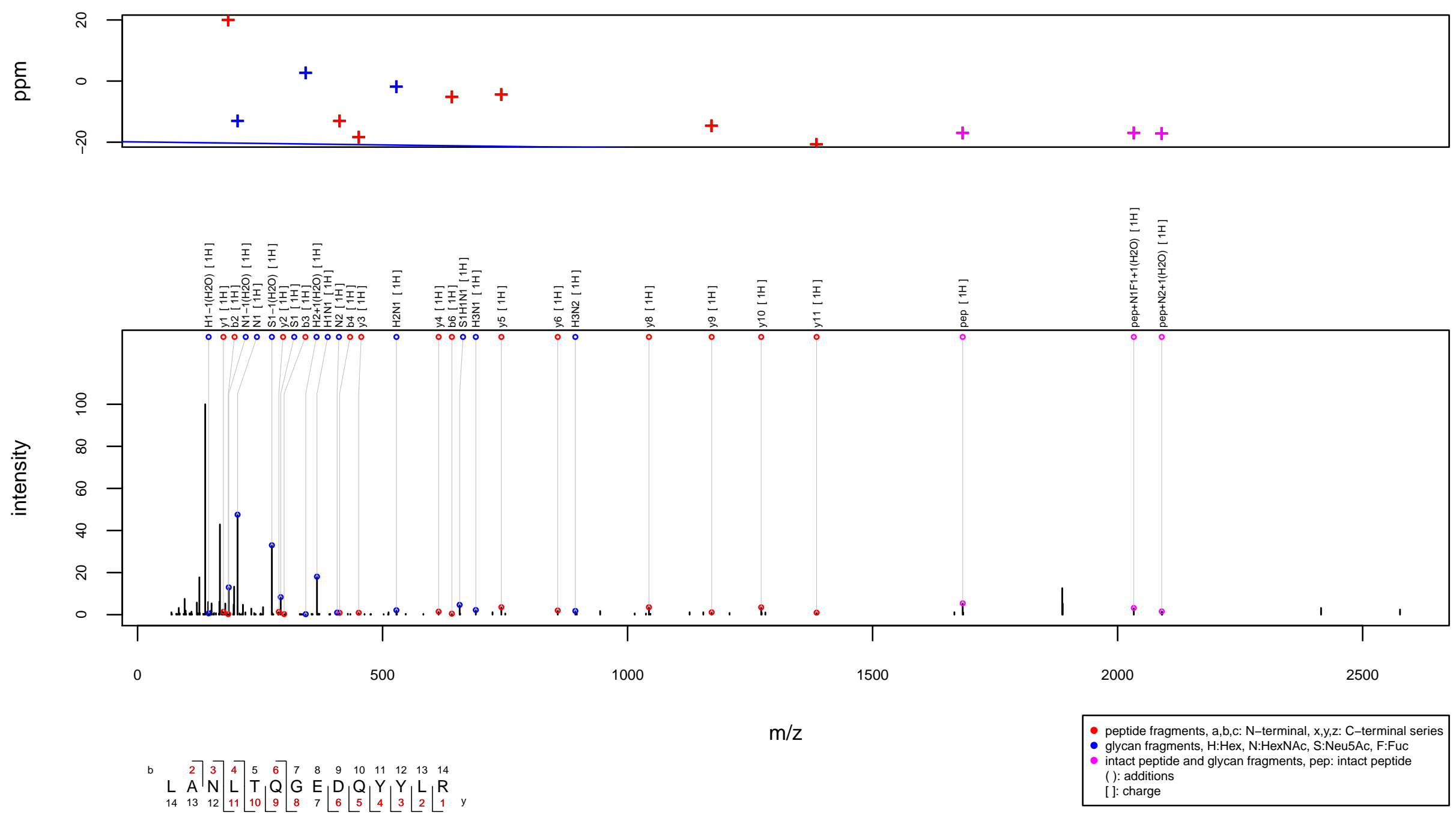
$\mathrm{m} / \mathrm{z} 1009.6426$ charge 4 scan $0-0$

Score $=93.4$, Hits $=73$, Explained Intensity $=0.41$ Peptide: PPAP_HUMAN[91,98]:KFLNESYK

Glycan: $\mathrm{SHNH}(\mathrm{SHN}(\mathrm{SHN}) \mathrm{H}) \mathrm{HN}(\mathrm{F}) \mathrm{N}, \mathrm{S} 3 \mathrm{H} 6 \mathrm{~N} 5 \mathrm{~F} 1$

Charge: $4 \mathrm{H}$
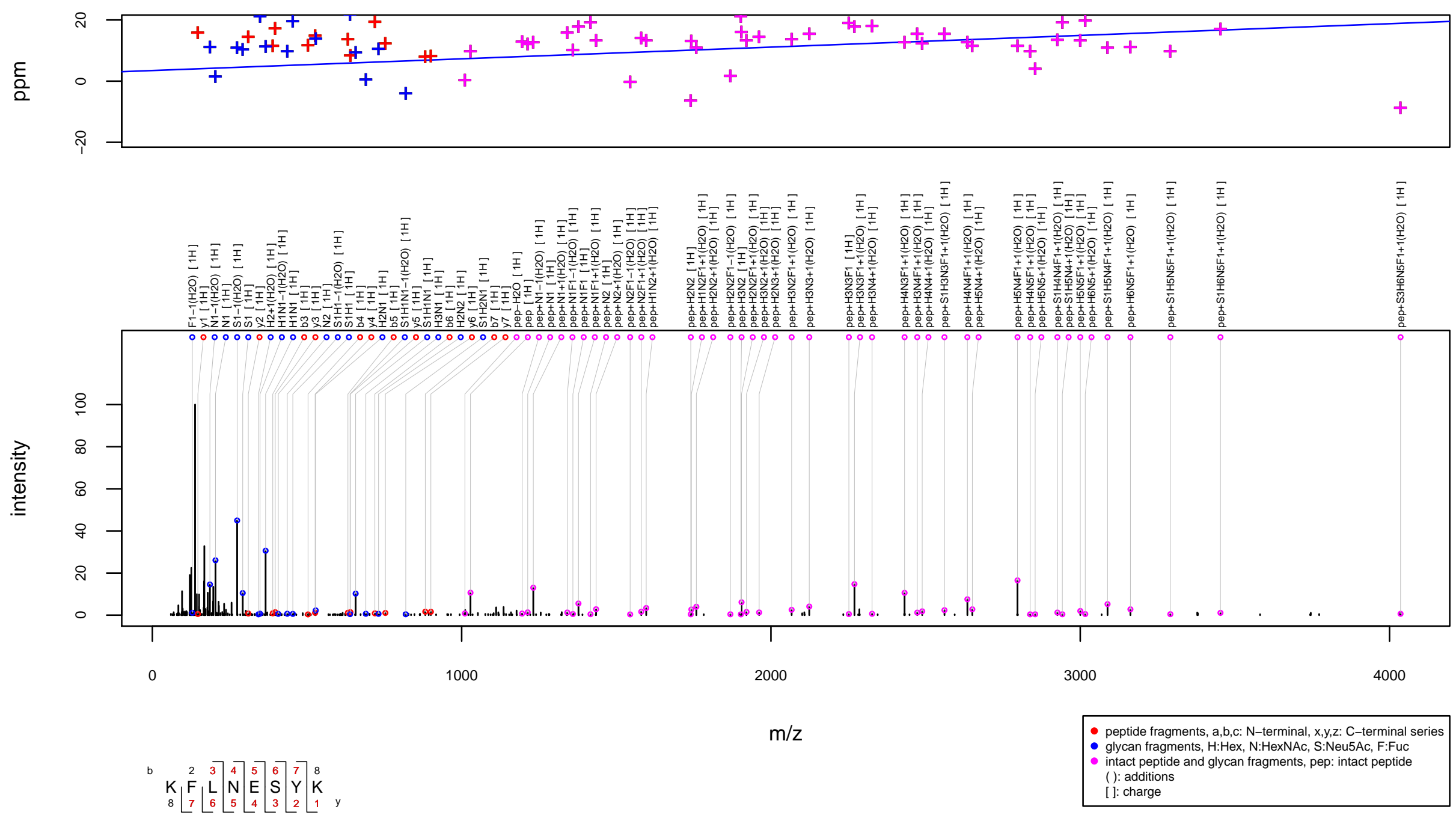
$\mathrm{m} / \mathrm{z} 1016.64$ charge 4 scan $0-0$

Score $=48.42$, Hits $=58$, Explained Intensity $=0.23$ Peptide: PPAP_HUMAN[91,98]:KFLNESYK

Glycan: H6N8F3

Charge: $4 \mathrm{H}$
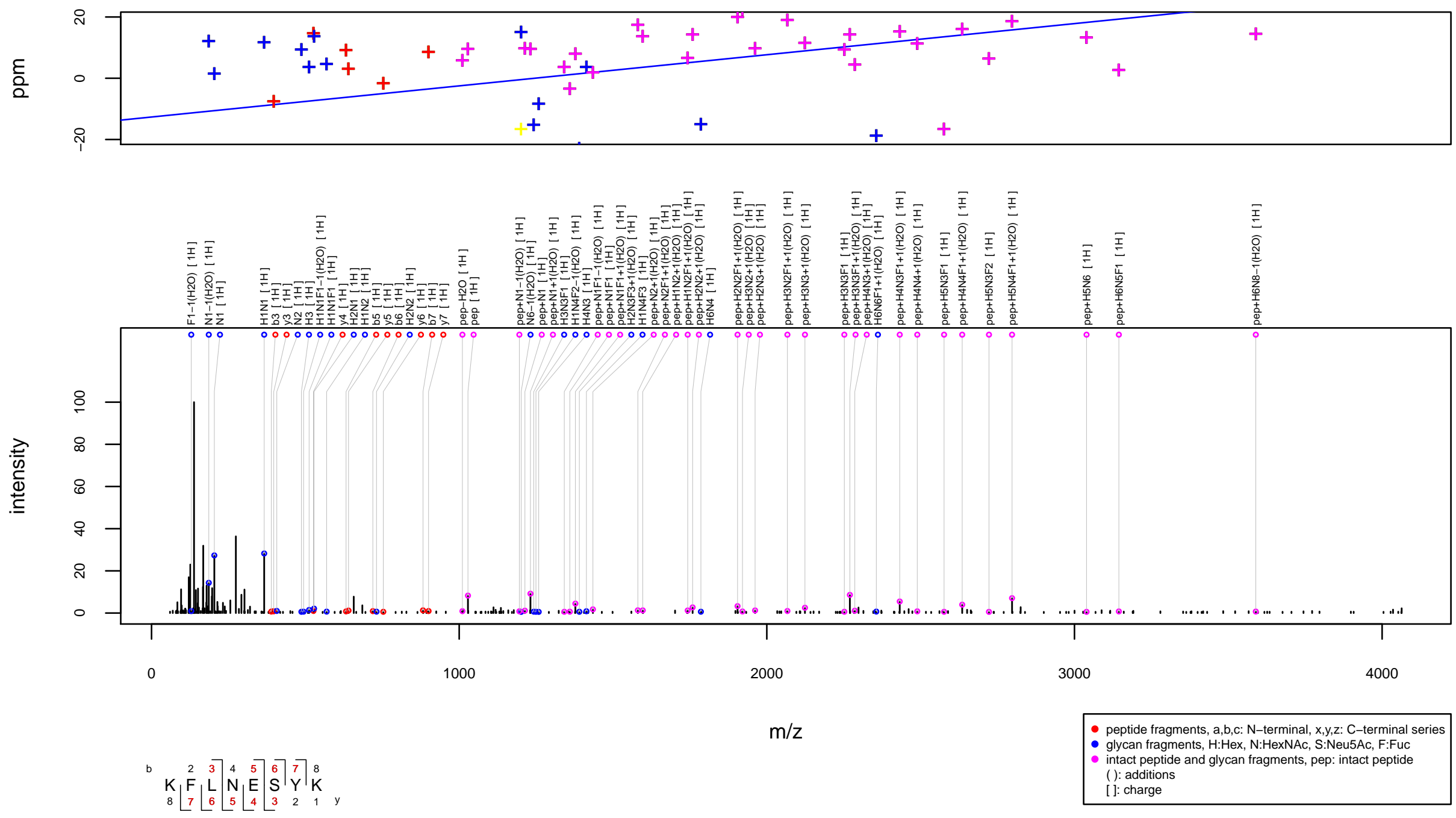
$\mathrm{m} / \mathrm{z} 1016.8979$ charge 4 scan 0-0

Score $=92.73$, Hits $=65$, Explained Intensity $=0.43$

Peptide: CLUS_HUMAN[80,89]:KKEDALNETR

Glycan: $\mathrm{SHNH}(\mathrm{SHN}(\mathrm{SHN}) \mathrm{H}) \mathrm{HNN}, \mathrm{S} 3 \mathrm{H} 6 \mathrm{~N} 5$

Charge: $4 \mathrm{H}$
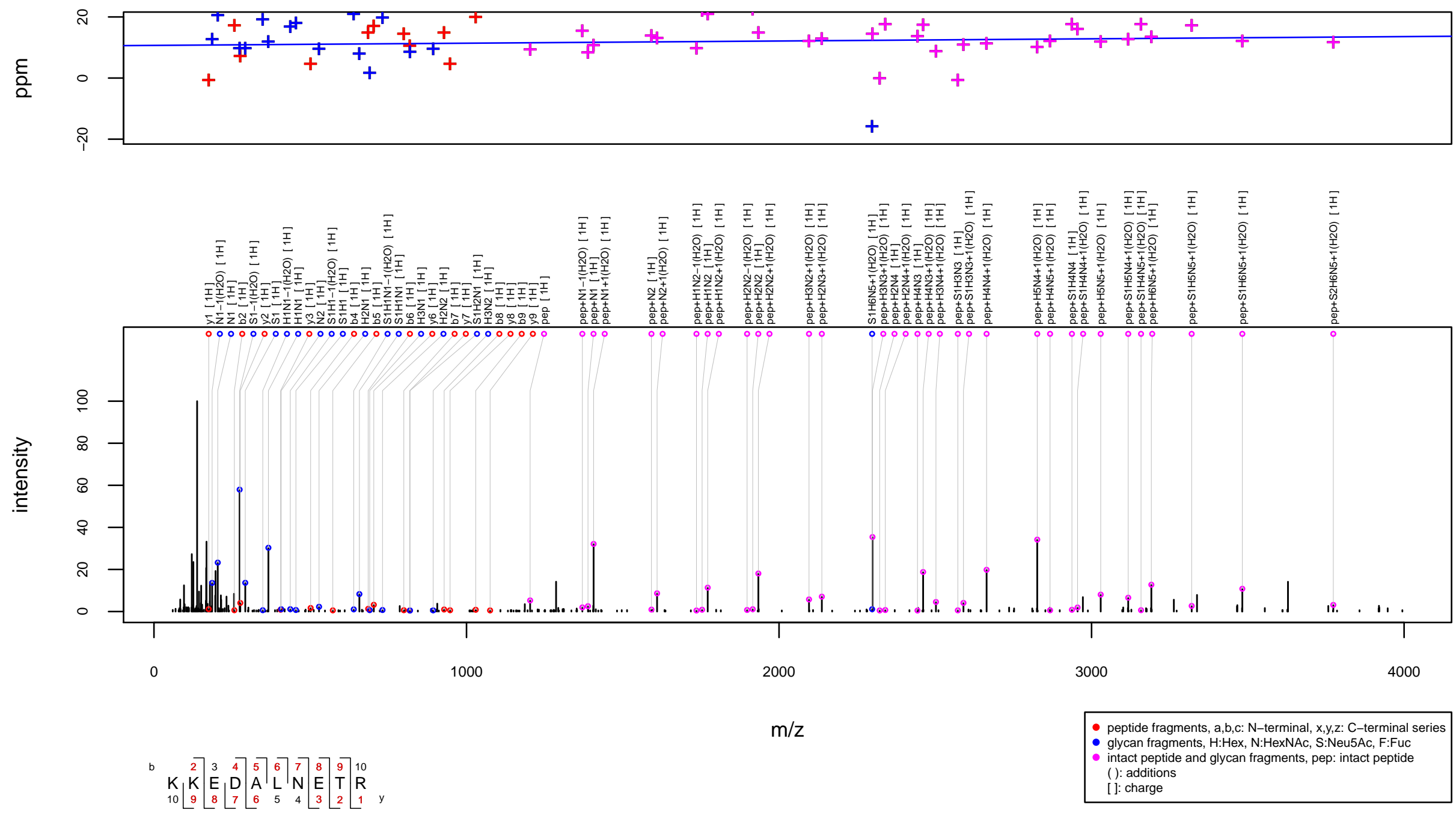
$\mathrm{m} / \mathrm{z} 1017.4301$ charge 4 scan $0-0$

Score $=64.89$, Hits $=87$, Explained Intensity $=0.36$ Peptide: CLUS_HUMAN[80,89]:KKEDALNETR Glycan: S1H6N5F4

Charge: $4 \mathrm{H}$
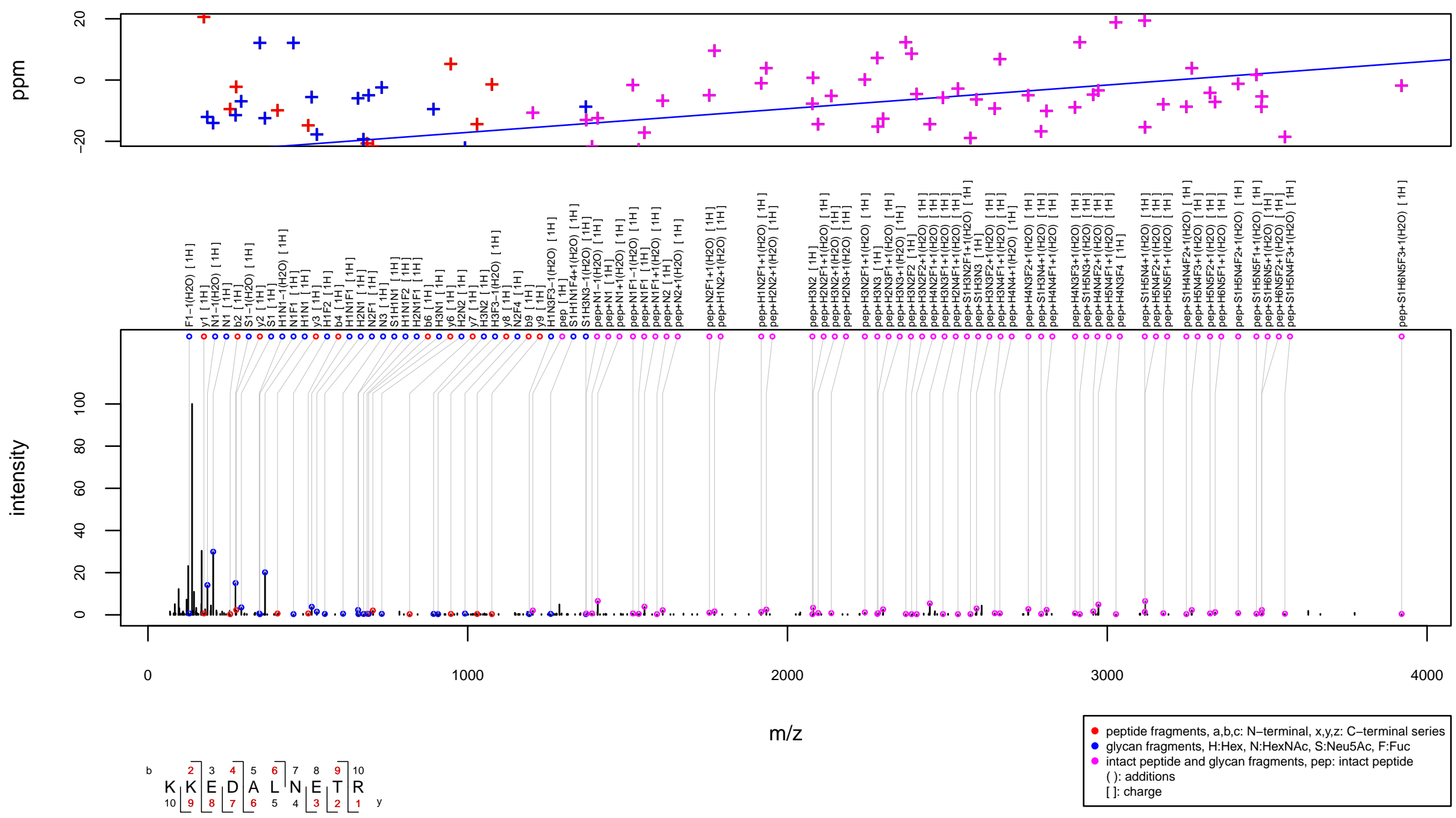
$\mathrm{m} / \mathrm{z} 1019.4911$ charge 4 scan $0-0$

Score $=50.73$, Hits $=39$, Explained Intensity $=0.32$

Peptide: IPSP_HUMAN[254,274]:VVGVPYQGNATALFILPSEGK

Glycan: $\mathrm{FHNH}(\mathrm{HNH}) \mathrm{HN}(\mathrm{F}) \mathrm{N}, \mathrm{H} 5 \mathrm{~N} 4 \mathrm{~F} 2$

Charge: $4 \mathrm{H}$
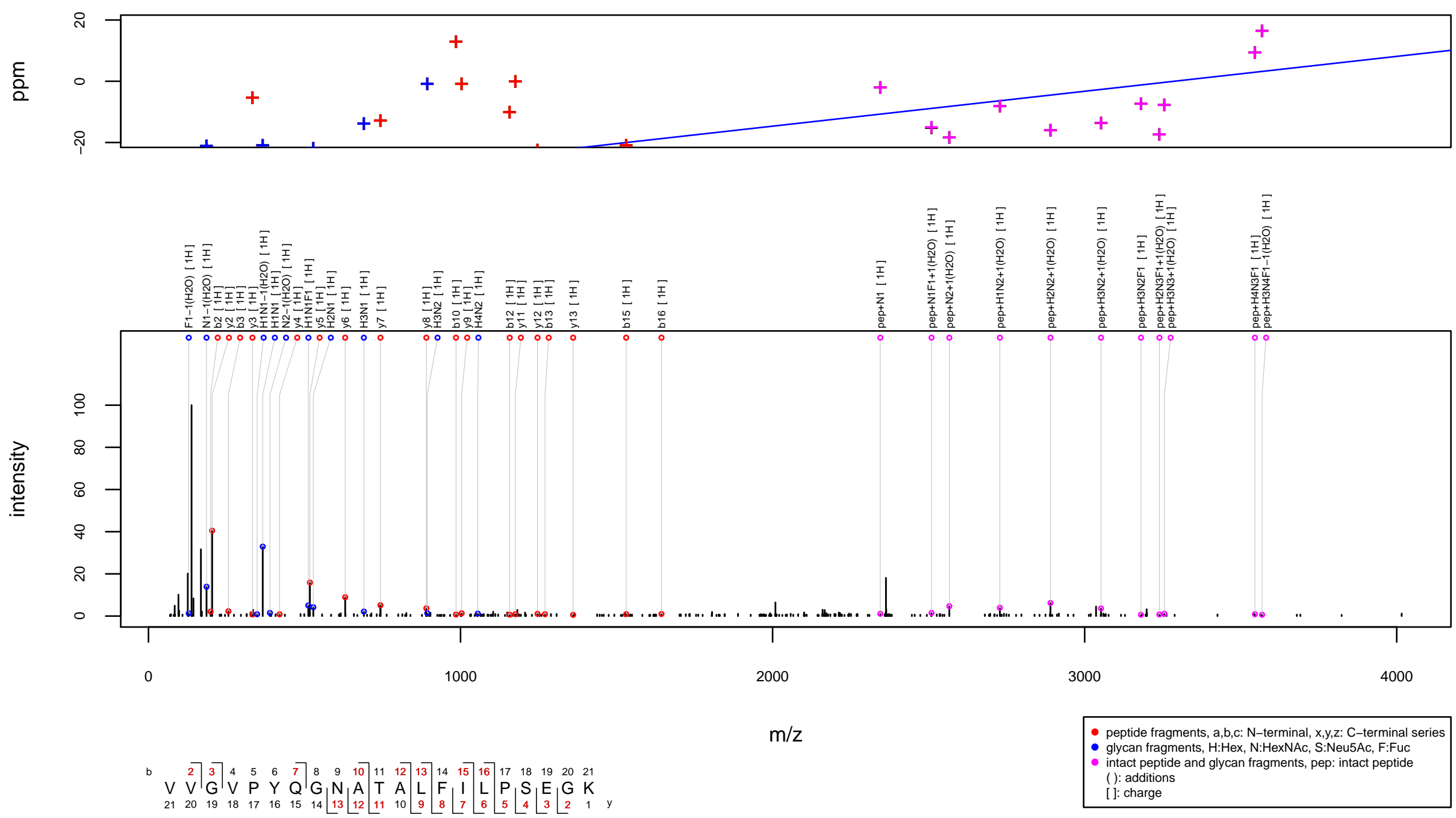
$\mathrm{m} / \mathrm{z} 1022.4171$ charge 3 scan $0-0$

Score $=76.4$, Hits $=65$, Explained Intensity $=0.36$

Peptide: SEMG2_HUMAN[272,281]:NLSQDQEHGR

Glycan: S1H3N4F2

Charge: $3 \mathrm{H}$
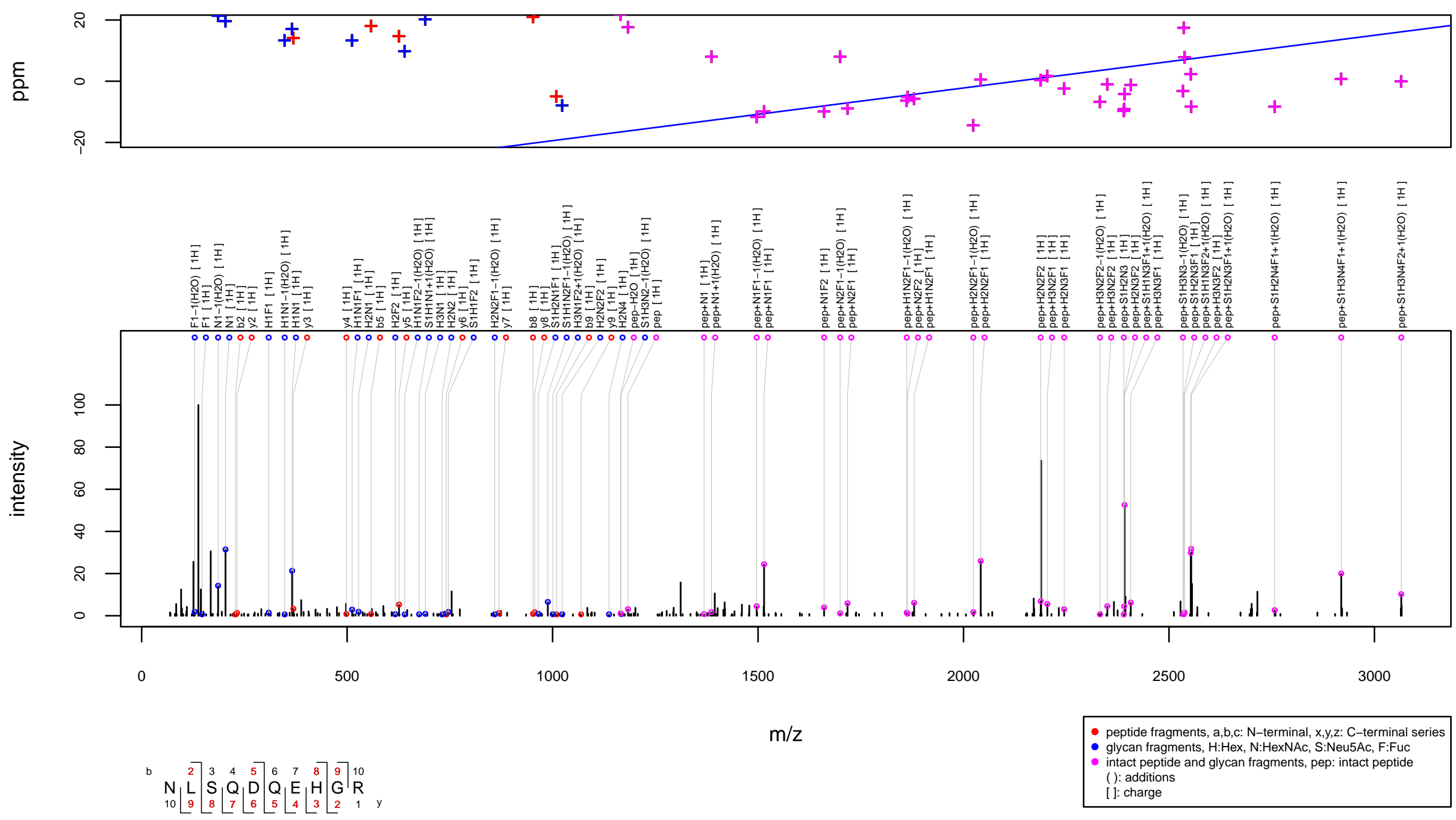
m/z 1025.972 charge 4 scan $0-0$

Score $=37.28$, Hits $=28$, Explained Intensity $=0.4$

Peptide: PPAP_HUMAN[333,355]:NETQHEPYPLMLPGCSPSCPLER

Glycan: $\mathrm{NH}(\mathrm{H}) \mathrm{HN}(\mathrm{F}) \mathrm{N}, \mathrm{H} 3 \mathrm{~N} 3 \mathrm{~F} 1$

Charge: $4 \mathrm{H}$
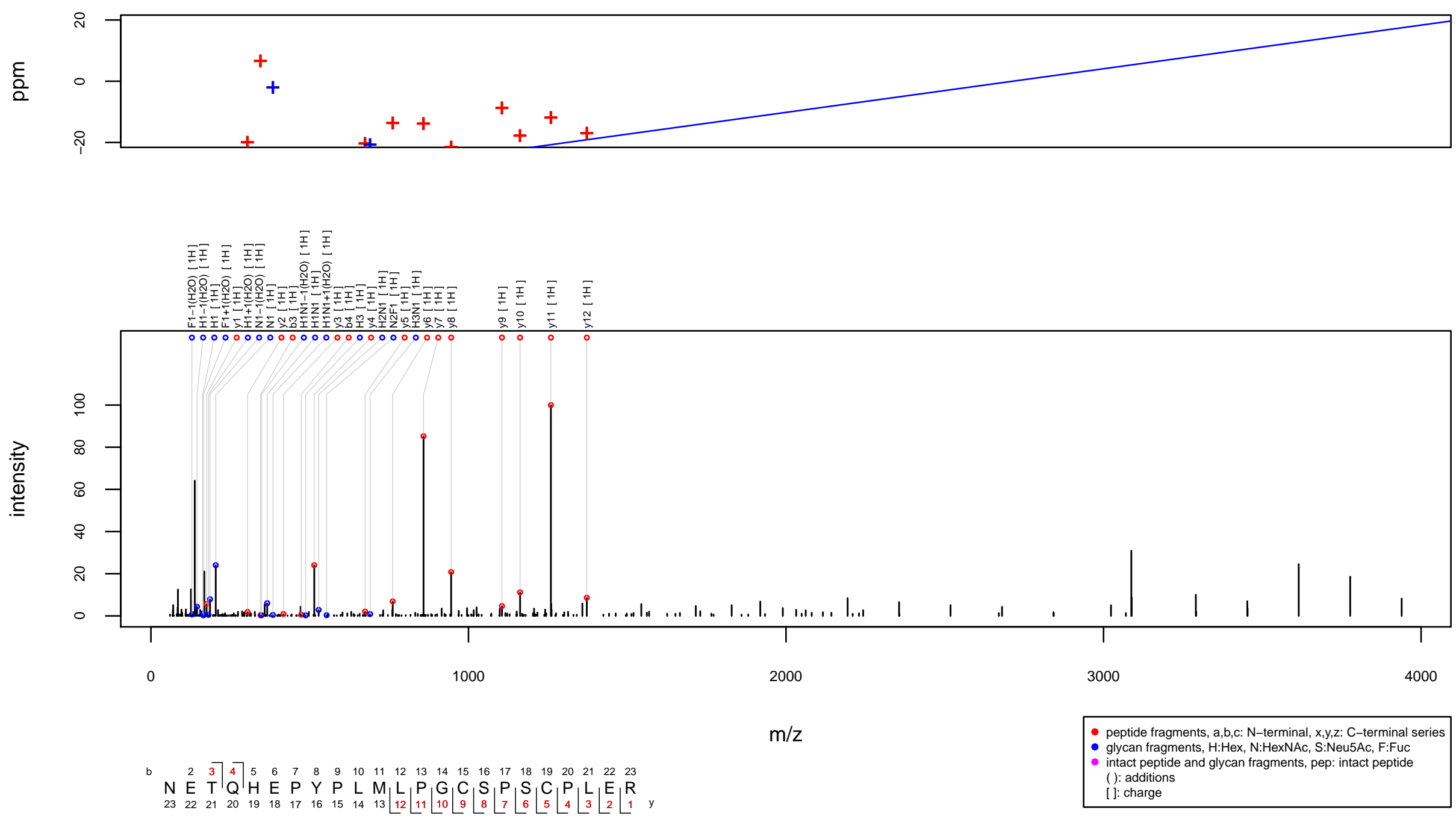
$\mathrm{m} / \mathrm{z} 1026.7052$ charge 4 scan $0-0$

Score $=48.4$, Hits $=39$, Explained Intensity $=0.32$

Peptide: TIMP1_HUMAN[46,60]:FVGTPEVNQTTLYQR

Glycan: $\mathrm{SHNH}(\mathrm{SHNH}) \mathrm{HN}(\mathrm{F}) \mathrm{N}, \mathrm{S} 2 \mathrm{H} 5 \mathrm{~N} 4 \mathrm{~F} 1$

Charge: $4 \mathrm{H}$
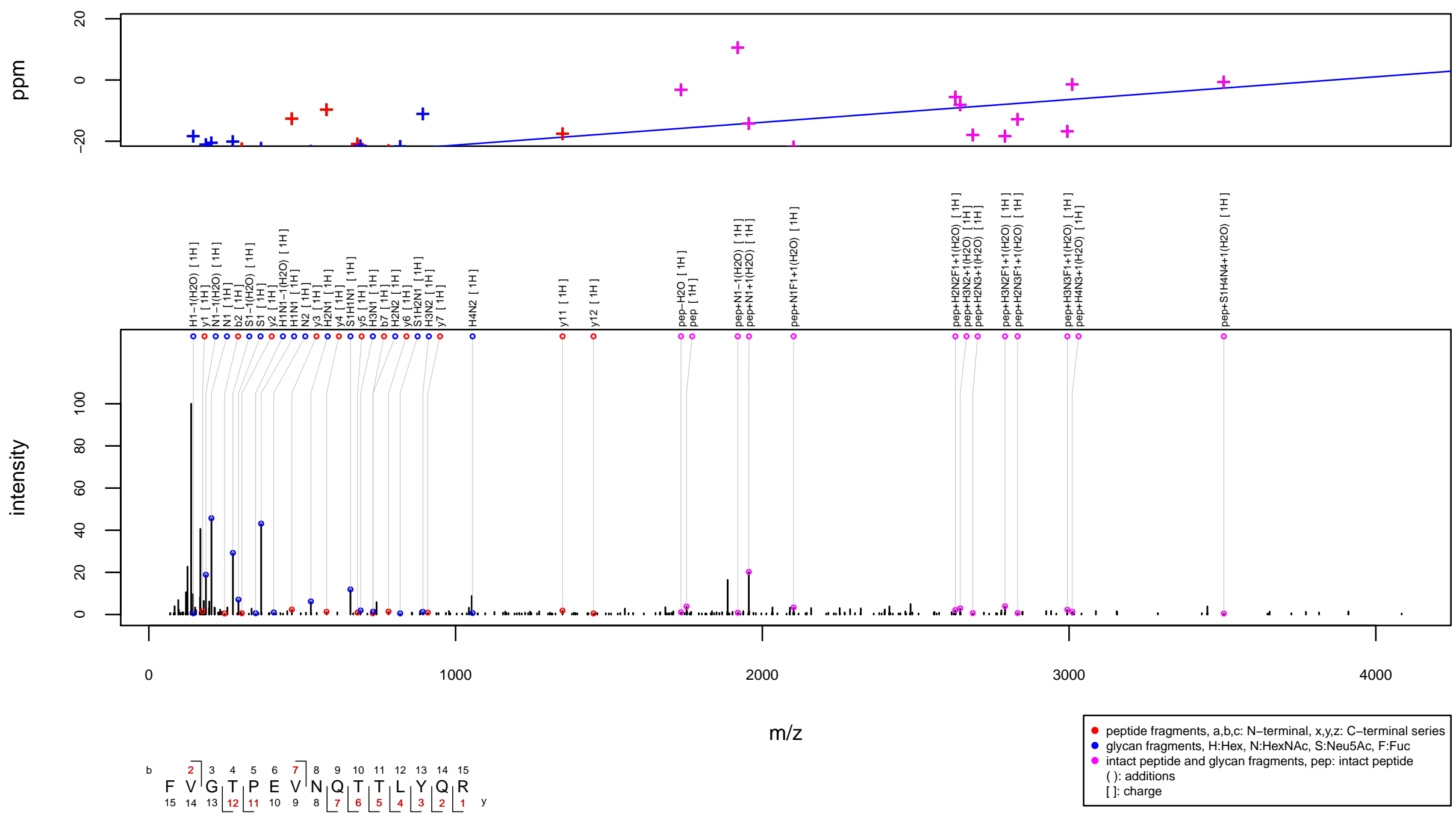
$\mathrm{m} / \mathrm{z} 1027.1925$ charge 4 scan $0-0$

Score $=25.7$, Hits $=33$, Explained Intensity $=0.2$

Peptide: RBP2_HUMAN[830,840]:LNSSNSASPHR

Glycan: S1H7N6F2

Charge: $4 \mathrm{H}$
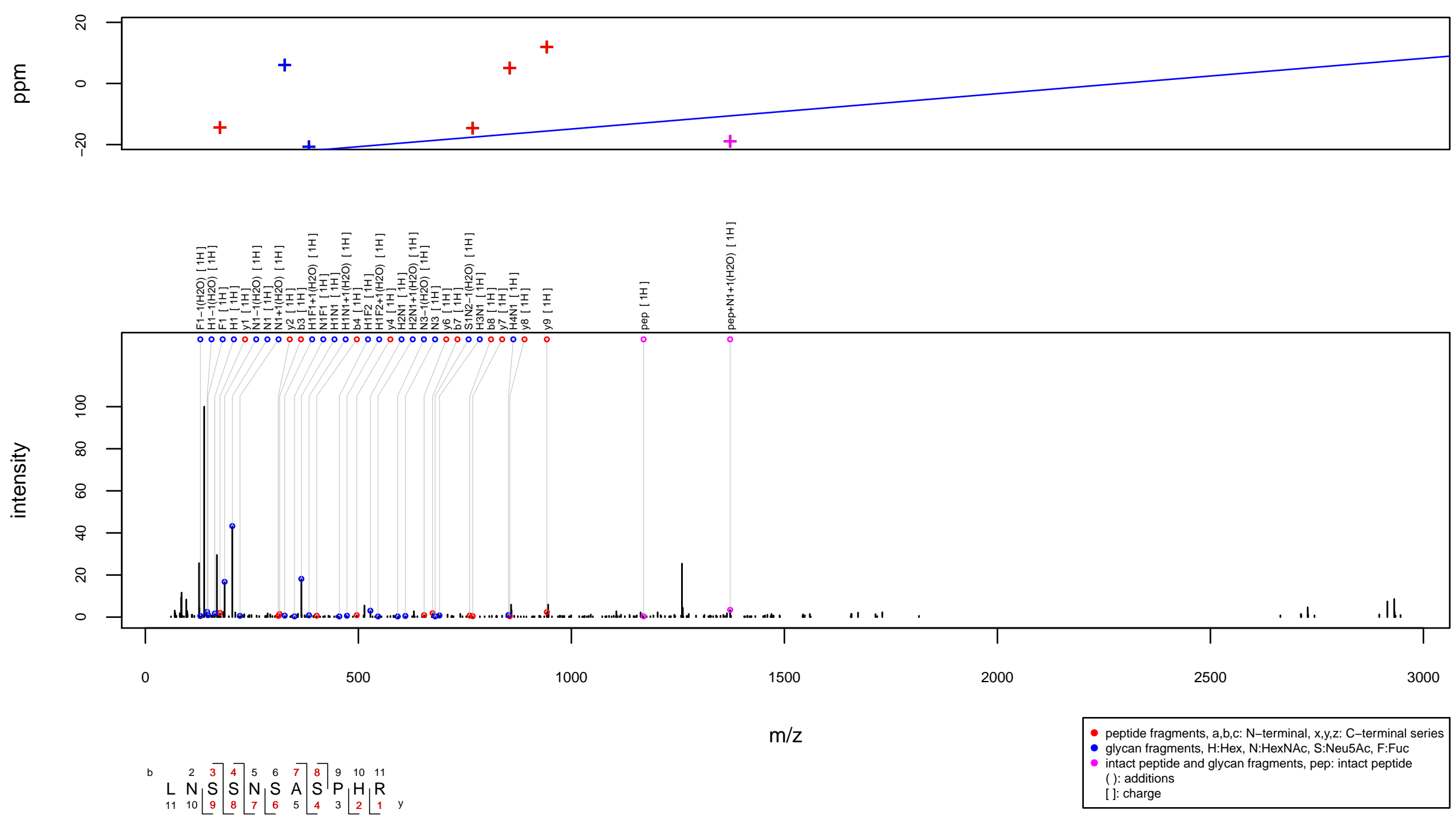
$\mathrm{m} / \mathrm{z} 1027.3876$ charge 3 scan $0-0$

Score $=57.52$, Hits $=43$, Explained Intensity $=0.29$

Peptide: PAEP_HUMAN[79,87]:WENNSCVEK

Glycan: $\mathrm{FHNH}(\mathrm{HNH}) \mathrm{HN}(\mathrm{F}) \mathrm{N}, \mathrm{H} 5 \mathrm{~N} 4 \mathrm{~F} 2$

Charge: $3 \mathrm{H}$
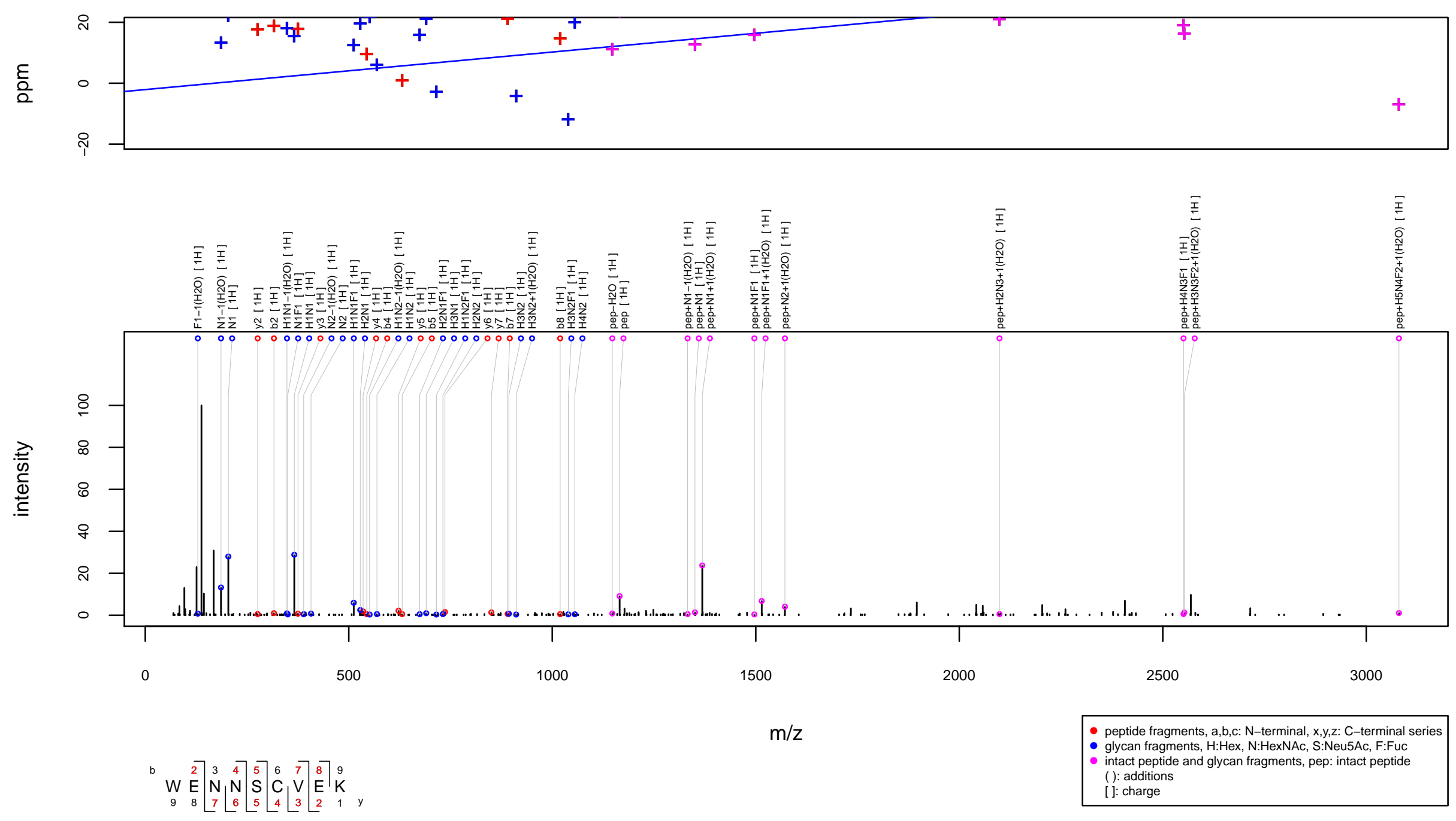
$\mathrm{m} / \mathrm{z} 1027.4122$ charge 3 scan $0-0$

Score $=94.7$, Hits $=61$, Explained Intensity $=0.51$

Peptide: SEMG2_HUMAN[272,282]:NLSQDQEHGRK

Glycan: $\mathrm{HNH}(\mathrm{HNH}) \mathrm{HN}(\mathrm{F}) \mathrm{N}, \mathrm{H} 5 \mathrm{~N} 4 \mathrm{~F} 1$

Charge: $3 \mathrm{H}$
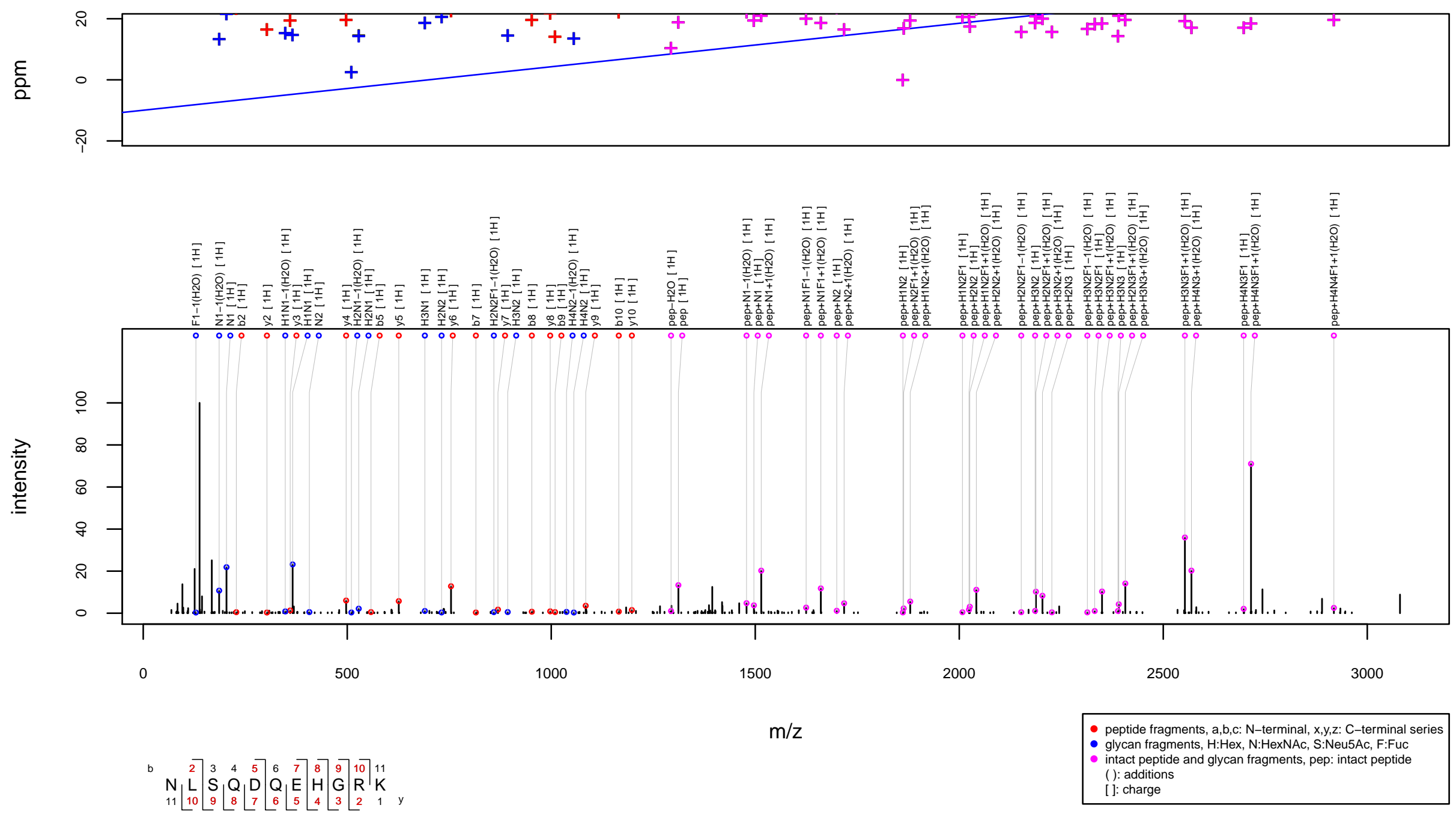
$\mathrm{m} / \mathrm{z} 1028.2142$ charge 4 scan $0-0$

Score $=66.46$, Hits $=54$, Explained Intensity $=0.4$

Peptide: CLUS_HUMAN[372,385]:LANLTQGEDQYYLR

Glycan: $\mathrm{HN}(\mathrm{F}(\mathrm{H}) \mathrm{N}) \mathrm{H}(\mathrm{F}(\mathrm{H}) \mathrm{NH}) \mathrm{HN}(\mathrm{F}) \mathrm{N}, \mathrm{H} 6 \mathrm{~N} 5 \mathrm{~F} 3$

Charge: $4 \mathrm{H}$
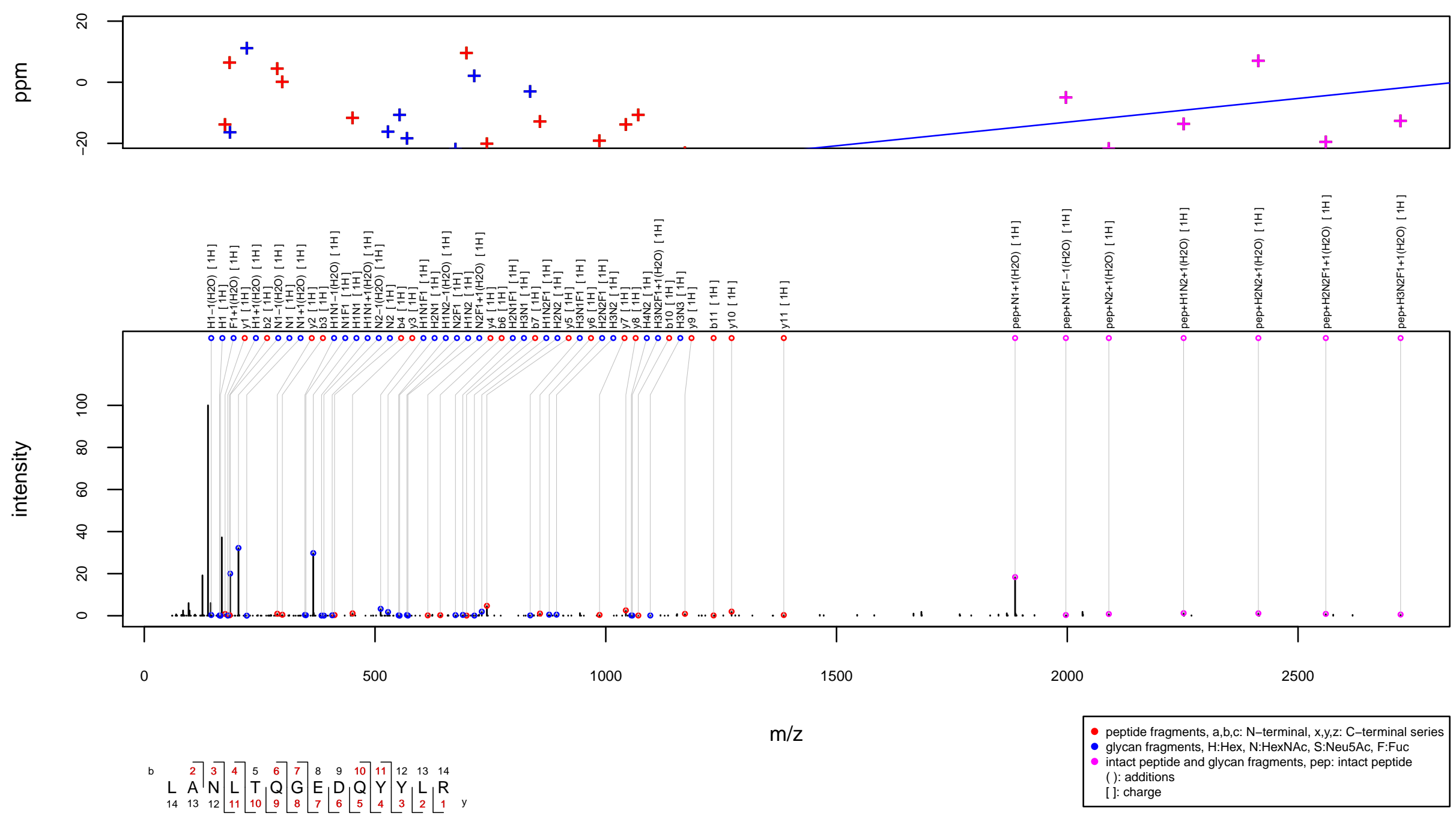
$\mathrm{m} / \mathrm{z} 1028.6589$ charge 4 scan $0-0$

Score $=86.41$, Hits $=62$, Explained Intensity $=0.38$

Peptide: FINC_HUMAN[516,533]:DQCIVDDITYNVNDTFHK

Glycan: H5N4F2

Charge: $4 \mathrm{H}$
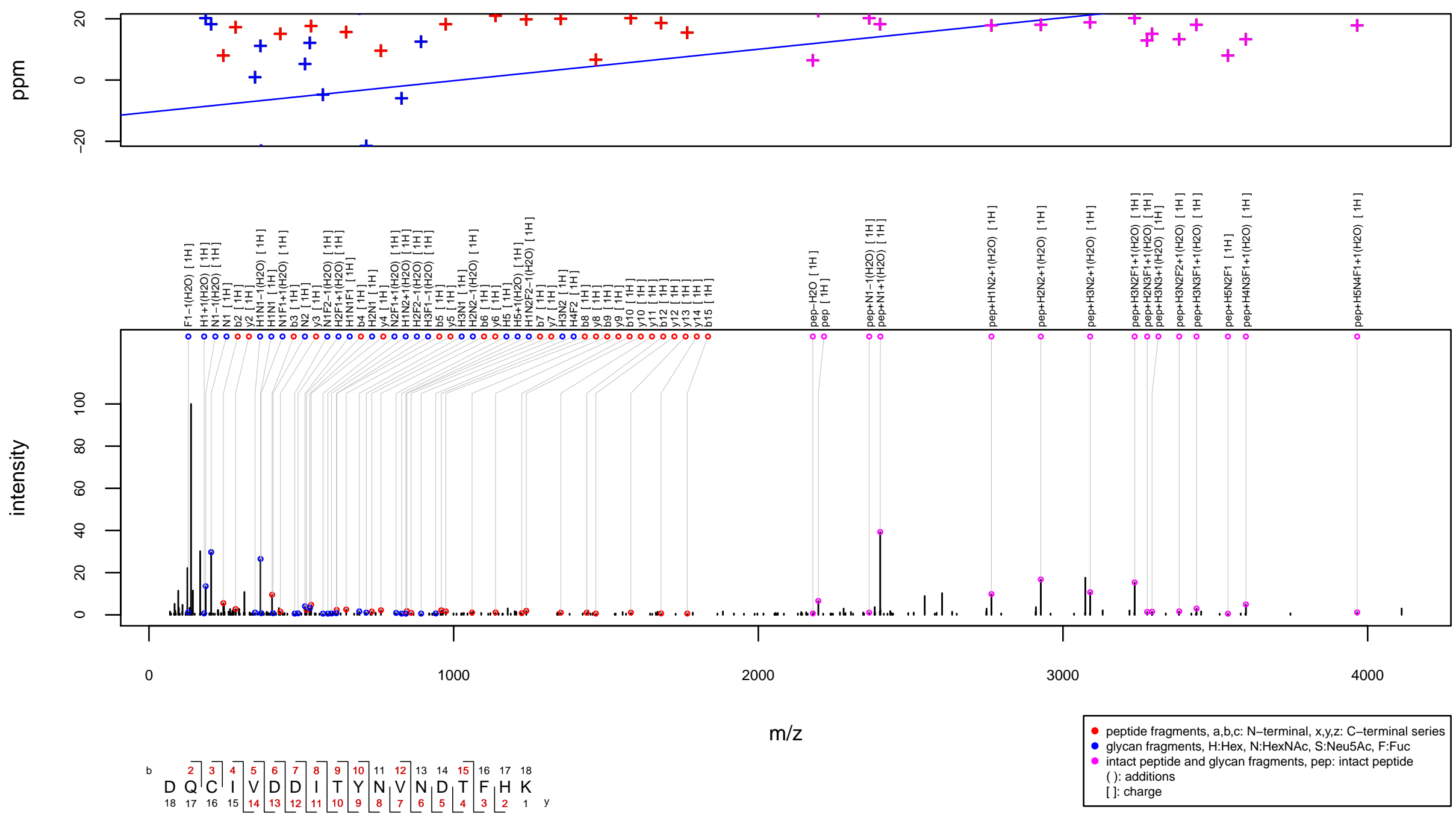
$\mathrm{m} / \mathrm{z} 1030.1078$ charge 3 scan $0-0$

Score $=86.92$, Hits $=63$, Explained Intensity $=0.35$ Peptide: PPAP_HUMAN[91,98]:KFLNESYK

Glycan: $\mathrm{SHNH}(\mathrm{HNH}) \mathrm{HN}(\mathrm{F}) \mathrm{N}, \mathrm{S} 1 \mathrm{H} 5 \mathrm{~N} 4 \mathrm{~F} 1$

Charge: $3 \mathrm{H}$
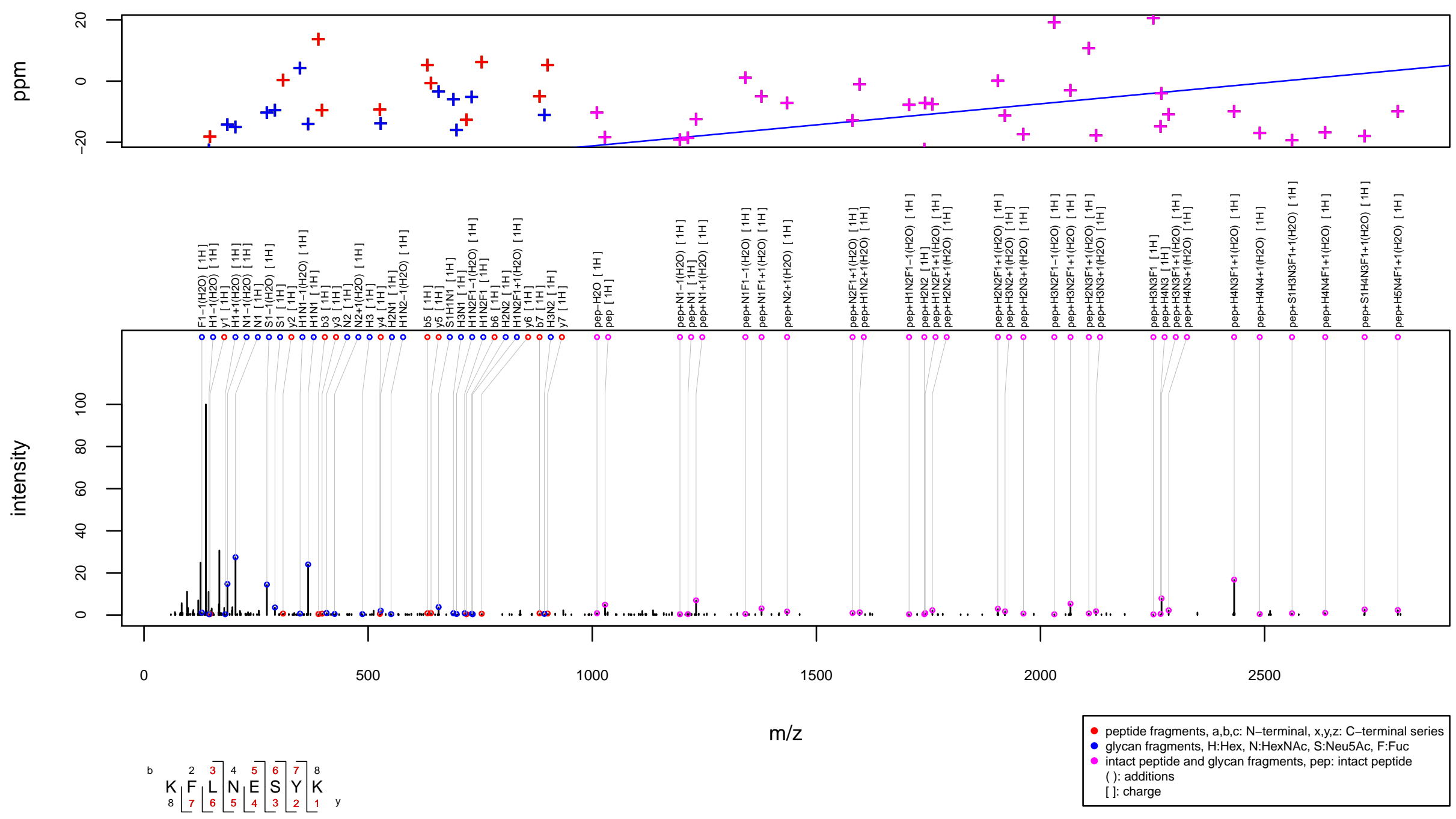
$\mathrm{m} / \mathrm{z} 1030.441$ charge 3 scan $0-0$

Score $=55.97$, Hits $=35$, Explained Intensity $=0.36$ Peptide: ACE_HUMAN[706,718]:KFDVNQLQNTTIK Glycan: $\mathrm{HHH}(\mathrm{H}(\mathrm{H}) \mathrm{H}) \mathrm{HNN}, \mathrm{H} 7 \mathrm{~N} 2$

Charge: $3 \mathrm{H}$
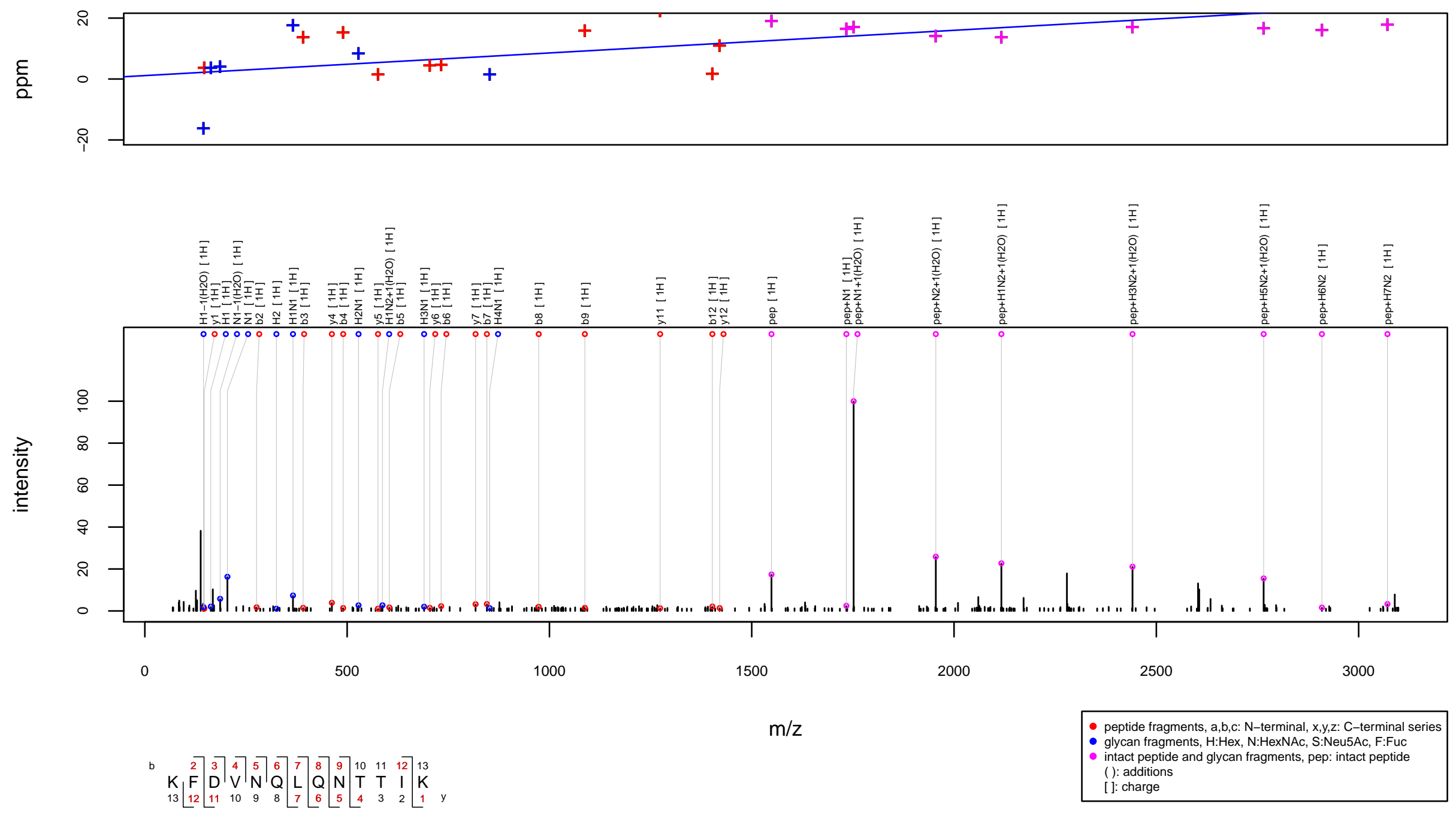
$\mathrm{m} / \mathrm{z} 1032.2092$ charge 4 scan $0-0$

Score $=48.19$, Hits $=36$, Explained Intensity $=0.31$

Peptide: ZA2G_HUMAN[103,120]:DIVEYYNDSNGSHVLQGR

Glycan: $\mathrm{SHNH}(\mathrm{HNH}) \mathrm{HN}(\mathrm{F}) \mathrm{N}$, S1H5N4F1

Charge: $4 \mathrm{H}$
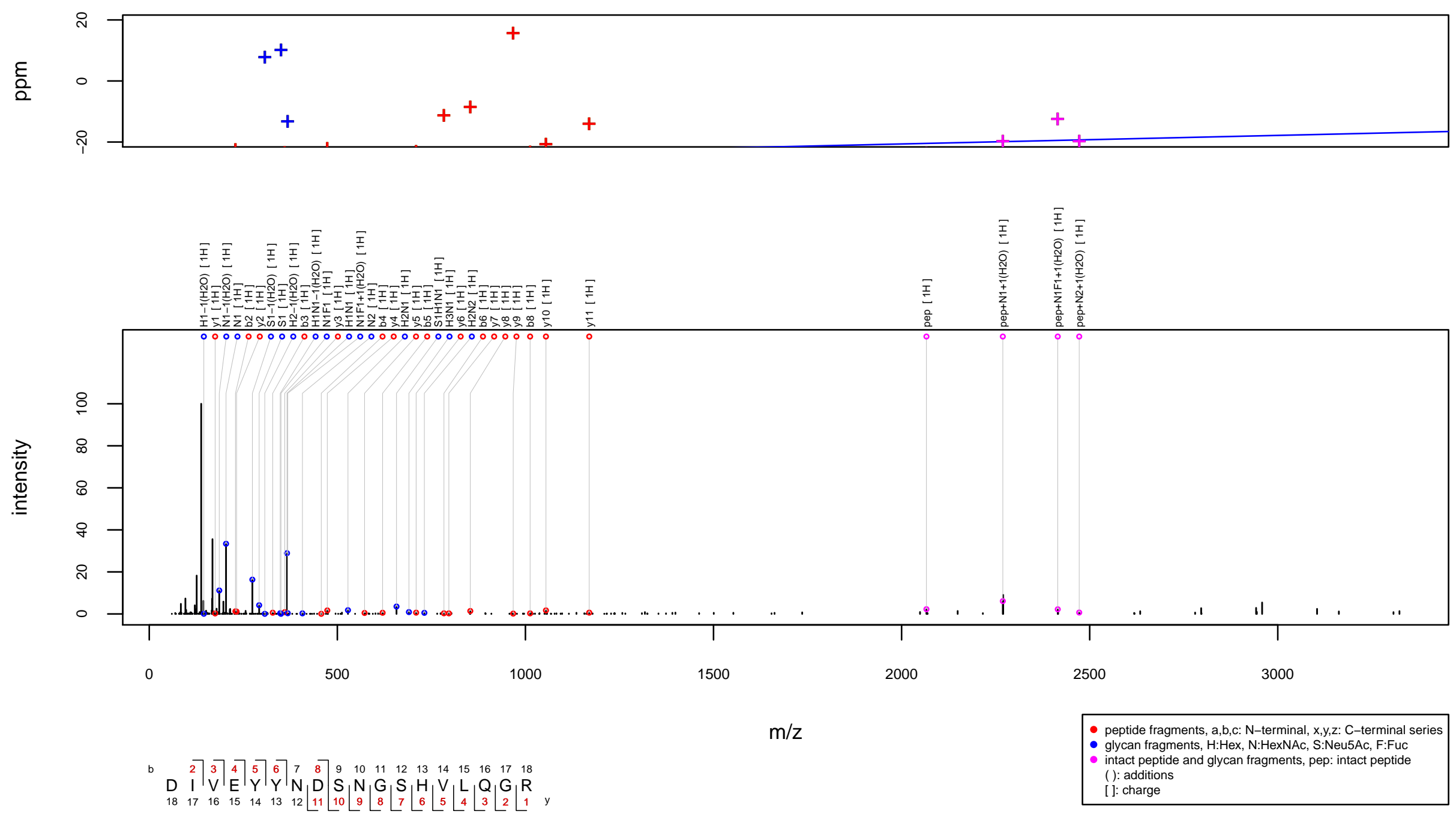
$\mathrm{m} / \mathrm{z} 1033.4011$ charge 3 scan $0-0$

Score $=130.24$, Hits $=84$, Explained Intensity $=0.62$ Peptide: SEMG2_HUMAN[272,281]:NLSQDQEHGR Glycan: $\mathrm{FHNH}(\mathrm{HNH}) \mathrm{HN}(\mathrm{F}) \mathrm{N}, \mathrm{H} 5 \mathrm{~N} 4 \mathrm{~F} 2$

Charge: $3 \mathrm{H}$
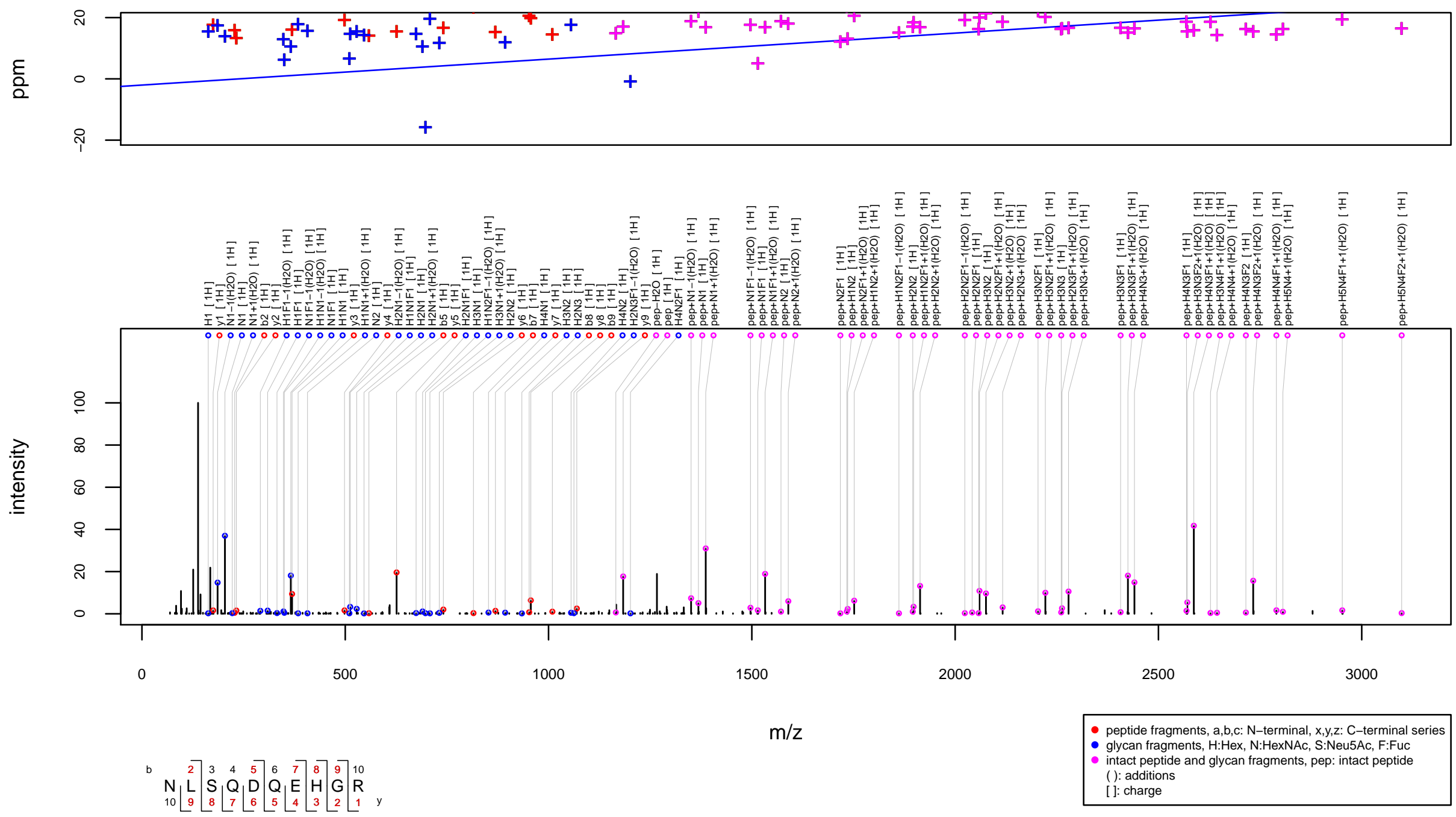
$\mathrm{m} / \mathrm{z} 1035.7443$ charge 3 scan $0-0$

Score $=77.92$, Hits $=50$, Explained Intensity $=0.46$ Peptide: PPAP_HUMAN[92,98]:FLNESYK

Glycan: $\mathrm{SHNH}(\mathrm{SHNH}) \mathrm{HNN}, \mathrm{S} 2 \mathrm{H} 5 \mathrm{~N} 4$

Charge: $3 \mathrm{H}$
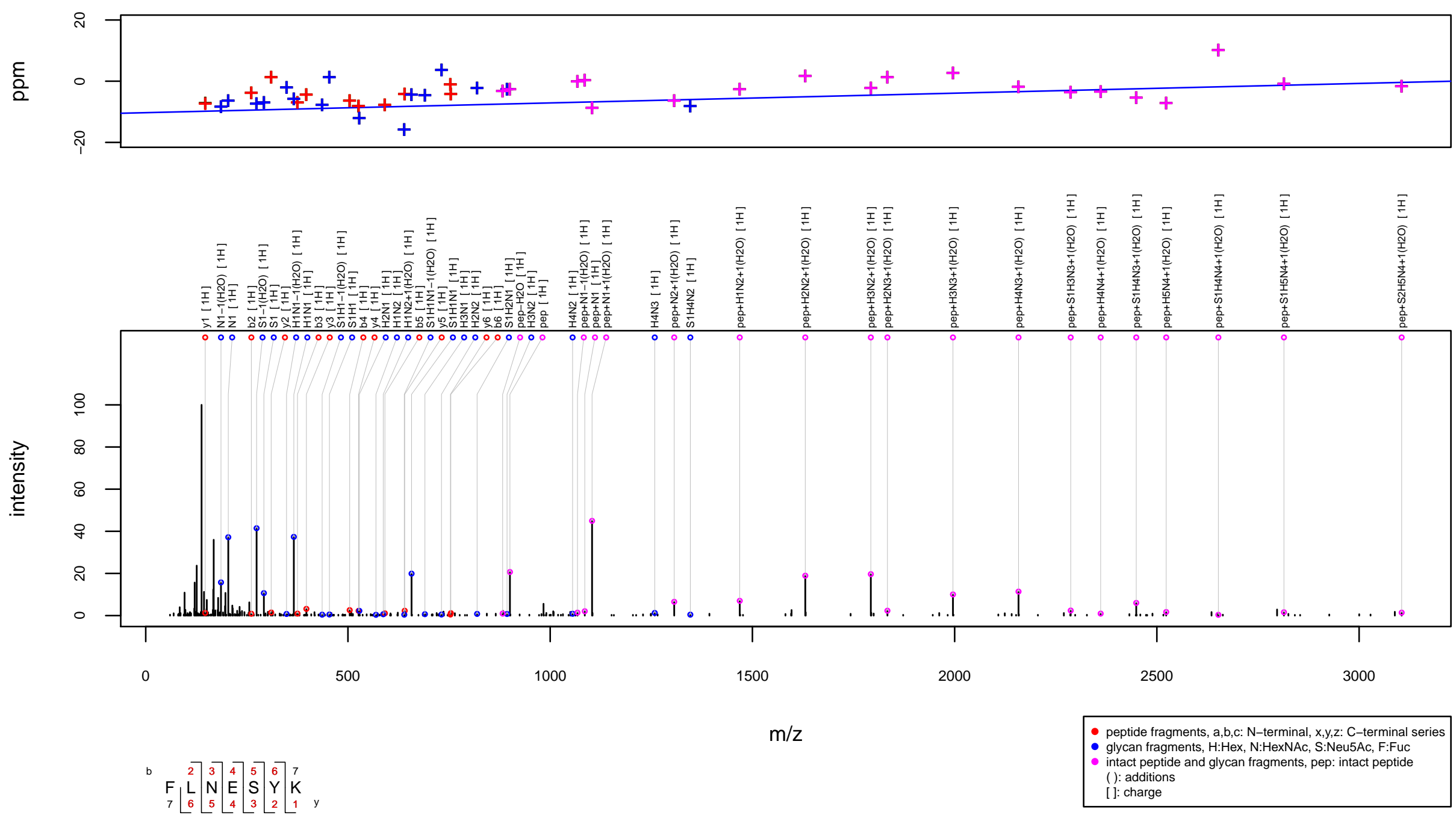
$\mathrm{m} / \mathrm{z} 1036.9288$ charge 4 scan $0-0$

Score $=117.31$, Hits $=78$, Explained Intensity $=0.51$

Peptide: RNT2_HUMAN[206,223]:QDQQLQNCTEPGEQPSPK

Glycan: $\mathrm{FH}(\mathrm{F}) \mathrm{NH}(\mathrm{HNH}) \mathrm{HN}(\mathrm{F}) \mathrm{N}, \mathrm{H} 5 \mathrm{~N} 4 \mathrm{~F} 3$

Charge: $4 \mathrm{H}$
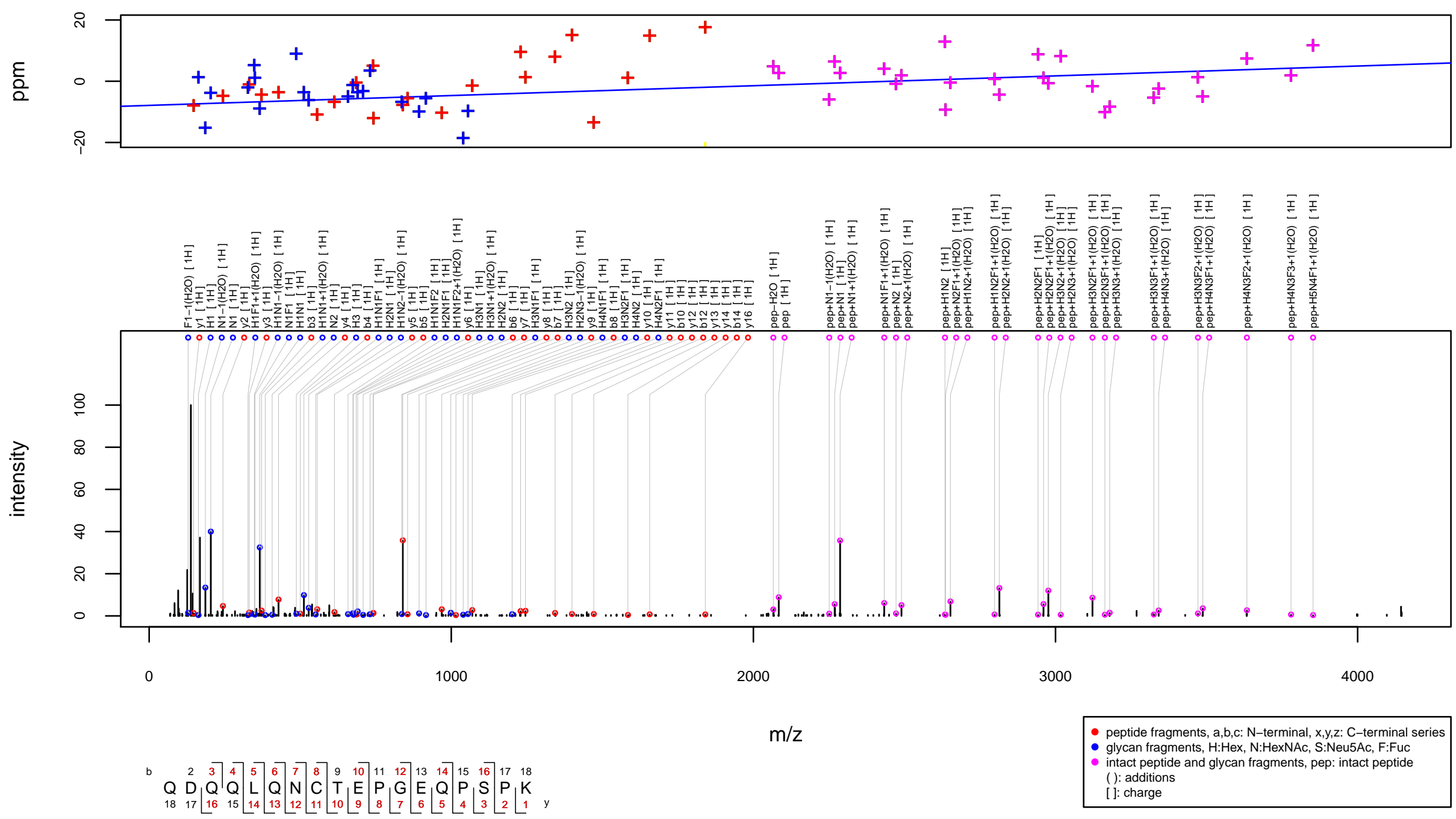
$\mathrm{m} / \mathrm{z} 1036.986$ charge 4 scan $0-0$

Score $=50.76$, Hits $=44$, Explained Intensity $=0.33$ Peptide: TIMP1_HUMAN[46,60]:FVGTPEVNQTTLYQR Glycan: $\mathrm{SNNH}(\mathrm{SHNH}) \mathrm{HN}(\mathrm{F}) \mathrm{N}, \mathrm{S} 2 \mathrm{H} 4 \mathrm{~N} 5 \mathrm{~F} 1$

Charge: $4 \mathrm{H}$
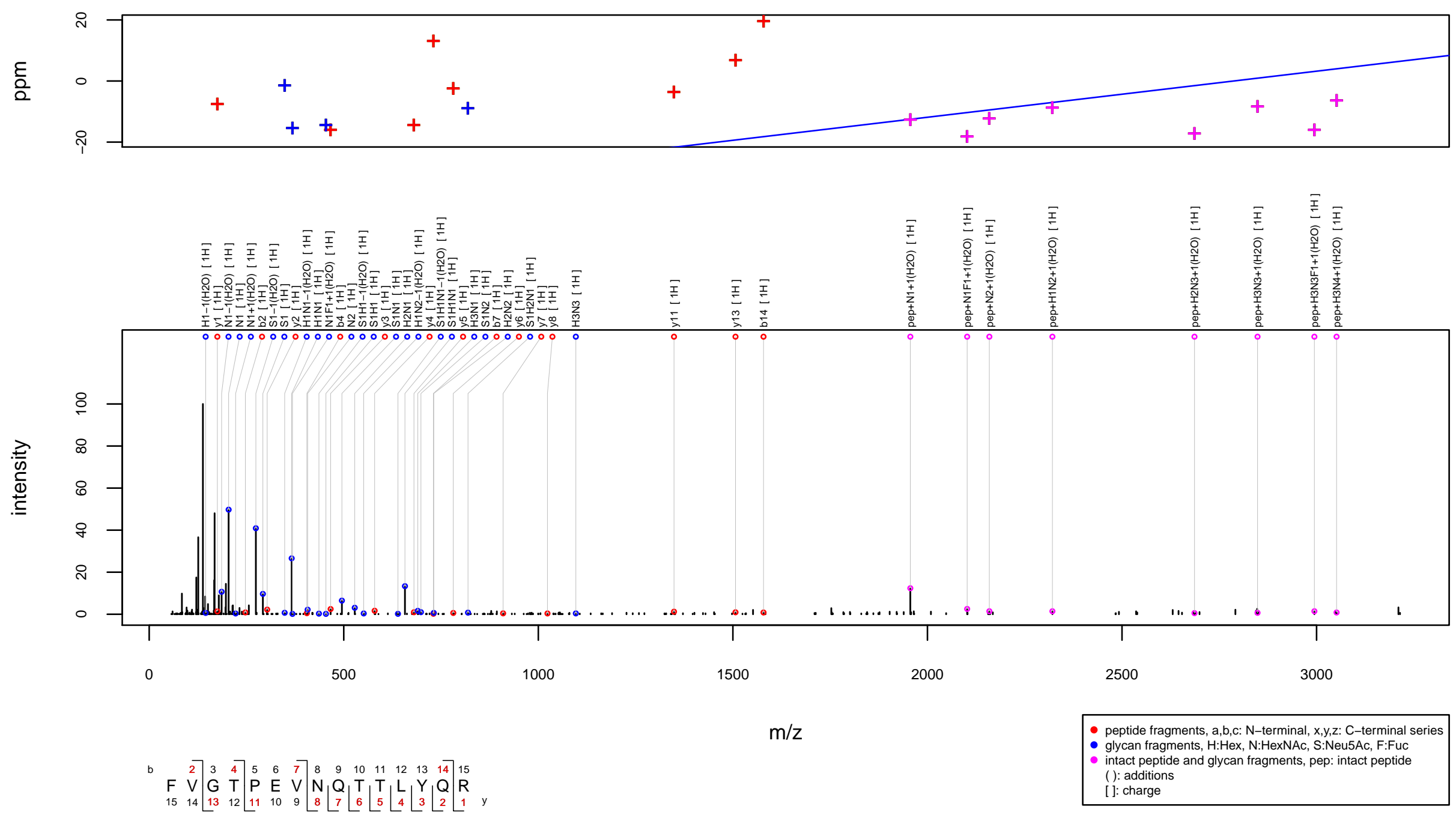
$\mathrm{m} / \mathrm{z} 1037.4908$ charge 4 scan $0-0$

Score $=70.48$, Hits $=49$, Explained Intensity $=0.36$

Peptide: FINC_HUMAN[997,1016]:LDAPTNLQFVNETDSTVLVR

Glycan: $\mathrm{FHNH}(\mathrm{HNH}) \mathrm{HN}(\mathrm{F}) \mathrm{N}, \mathrm{H} 5 \mathrm{~N} 4 \mathrm{~F} 2$

Charge: $4 \mathrm{H}$
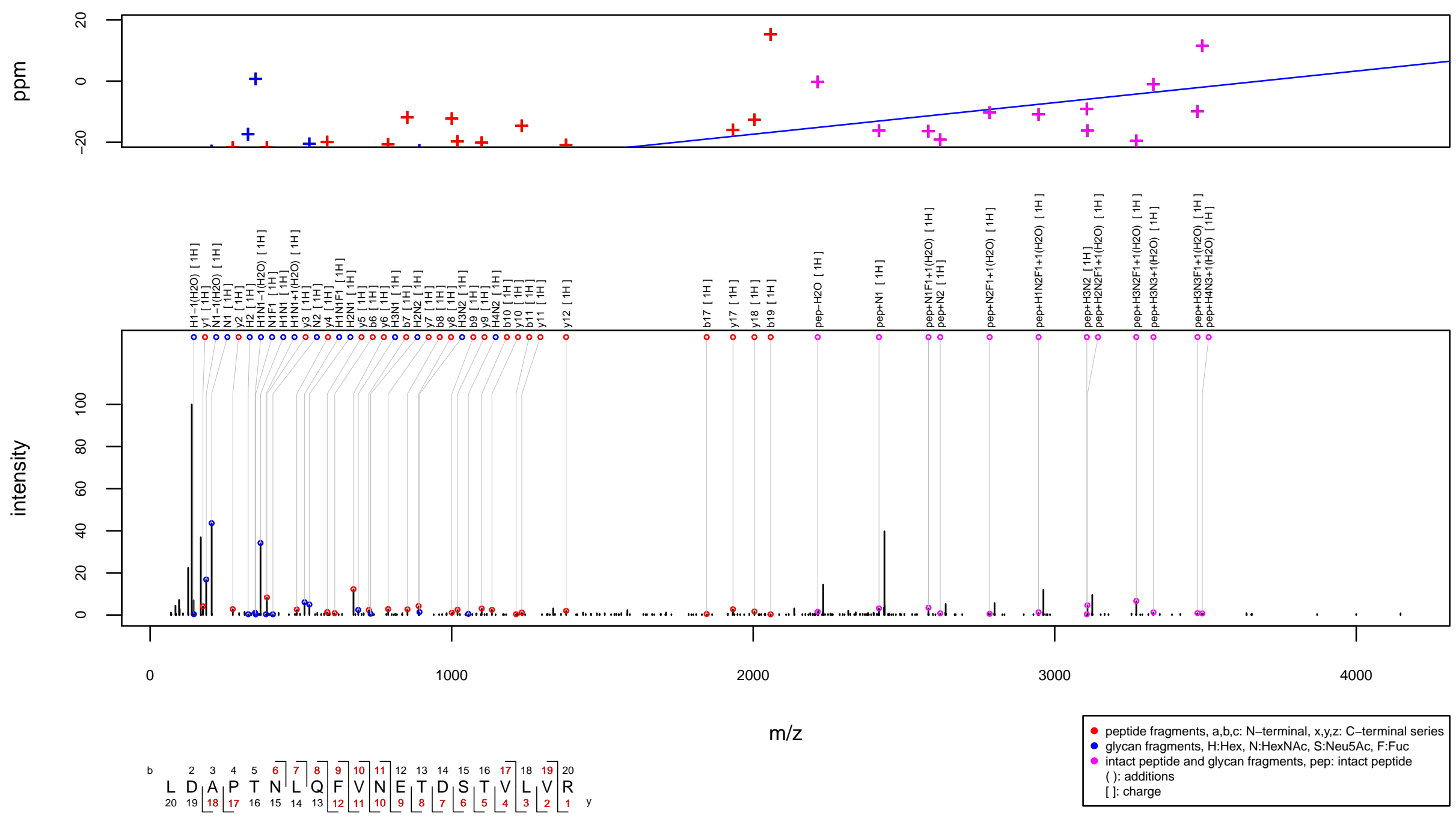


\section{$\mathrm{m} / \mathrm{z} 1037.5056$ charge 4 scan 0-0}

Score $=59.49$, Hits $=38$, Explained Intensity $=0.3$

Peptide: AMPN_HUMAN[674,690]:VPVTLALNNTLFLIEER

Glycan: $\mathrm{SHNH}(\mathrm{SHNH}) \mathrm{HNN}, \mathrm{S} 2 \mathrm{H} 5 \mathrm{~N} 4$

Charge: $4 \mathrm{H}$
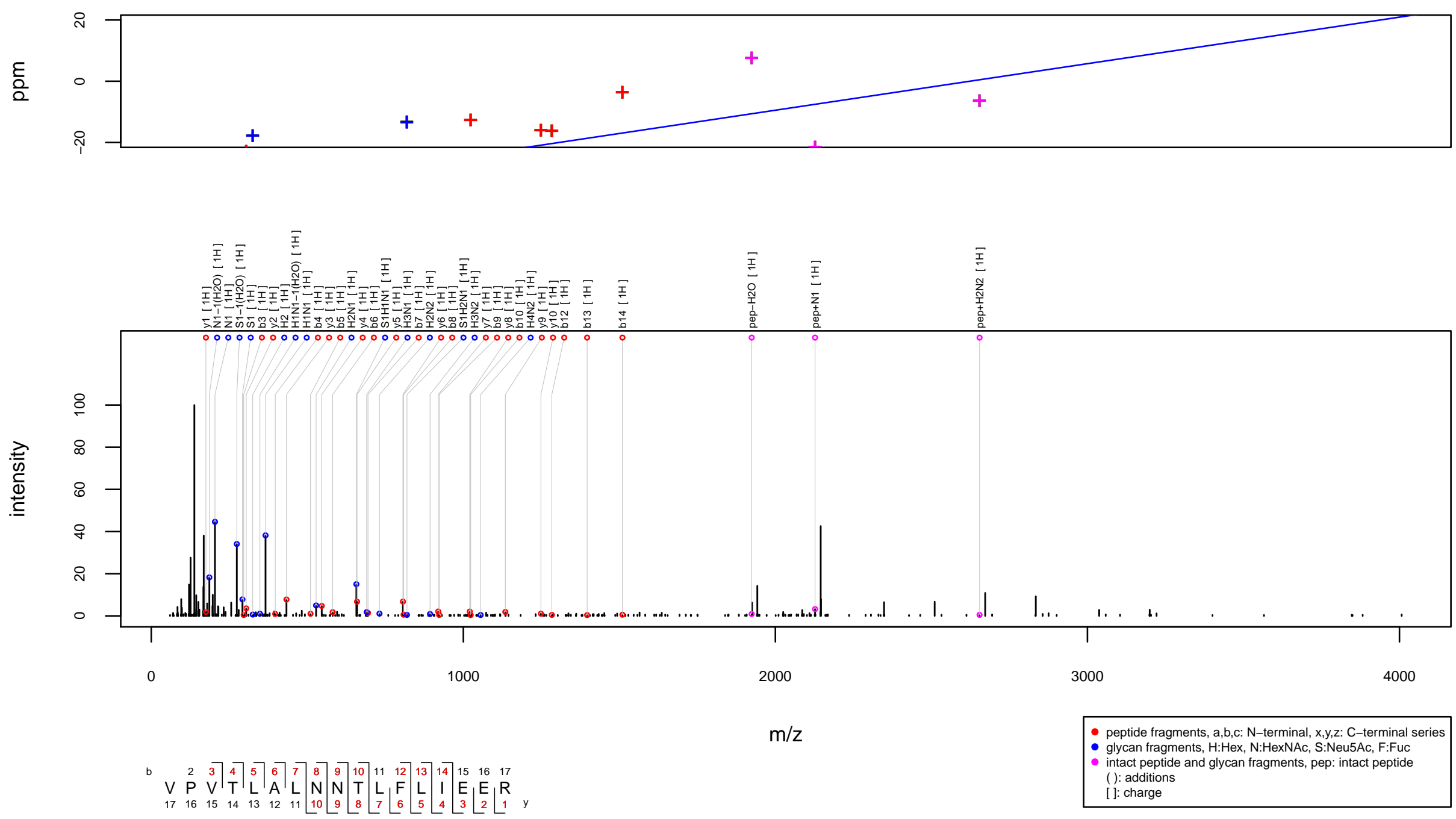
$\mathrm{m} / \mathrm{z} 1038.9476$ charge 4 scan $0-0$

Score $=81.34$, Hits $=55$, Explained Intensity $=0.42$ Peptide: CD59_HUMAN[40,55]:TAVNCSSDFDACLITK Glycan: $\mathrm{SHNH}(\mathrm{SHNH}) \mathrm{HN}(\mathrm{F}) \mathrm{N}, \mathrm{S} 2 \mathrm{H} 5 \mathrm{~N} 4 \mathrm{~F} 1$

Charge: $4 \mathrm{H}$
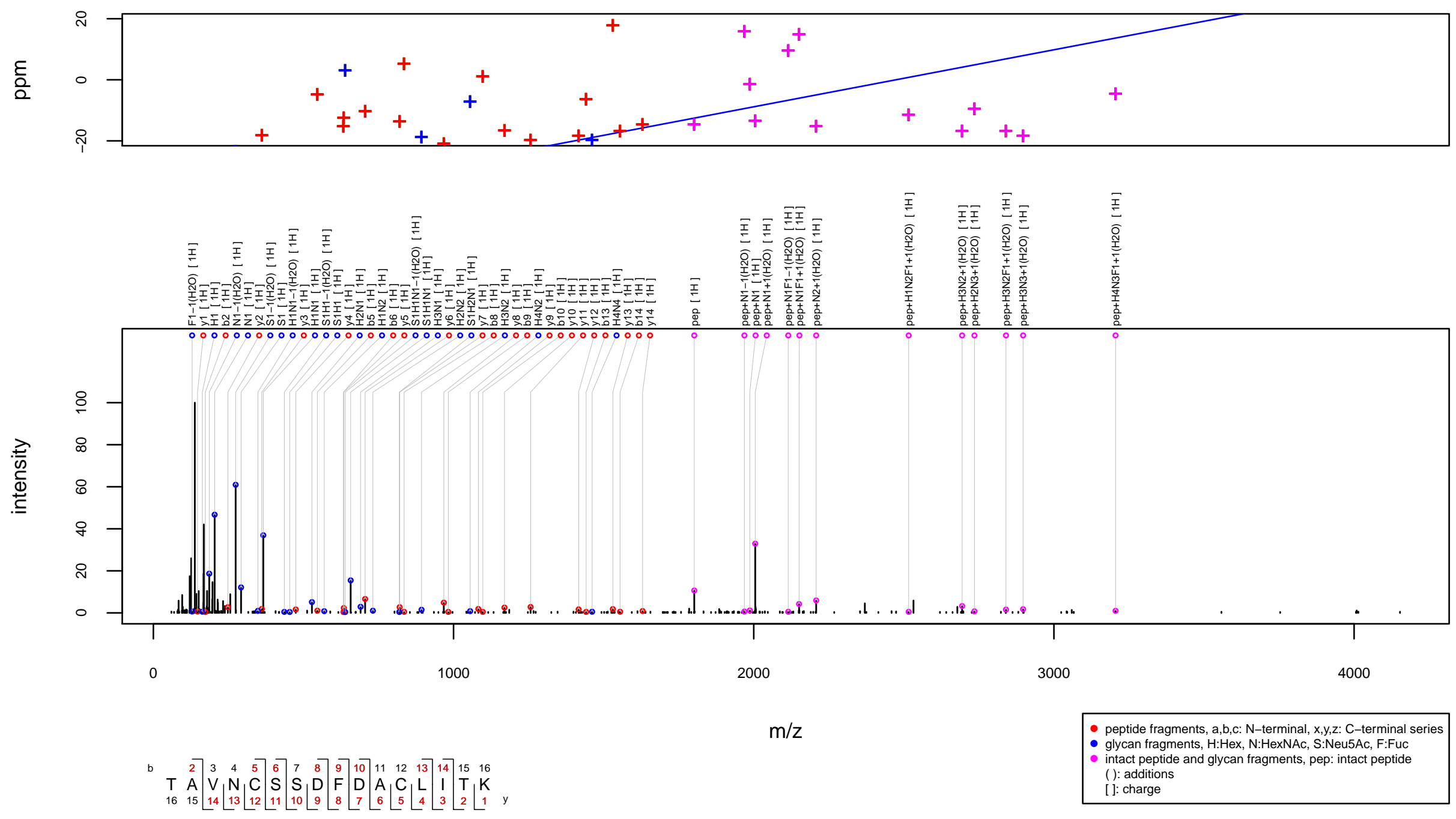
$\mathrm{m} / \mathrm{z} 1039.6895$ charge 4 scan $0-0$

Score $=38.8$, Hits $=37$, Explained Intensity $=0.22$

Peptide: TIMP1_HUMAN[46,60]:FVGTPEVNQTTLYQR

Glycan: H3N8F2

Charge: $4 \mathrm{H}$
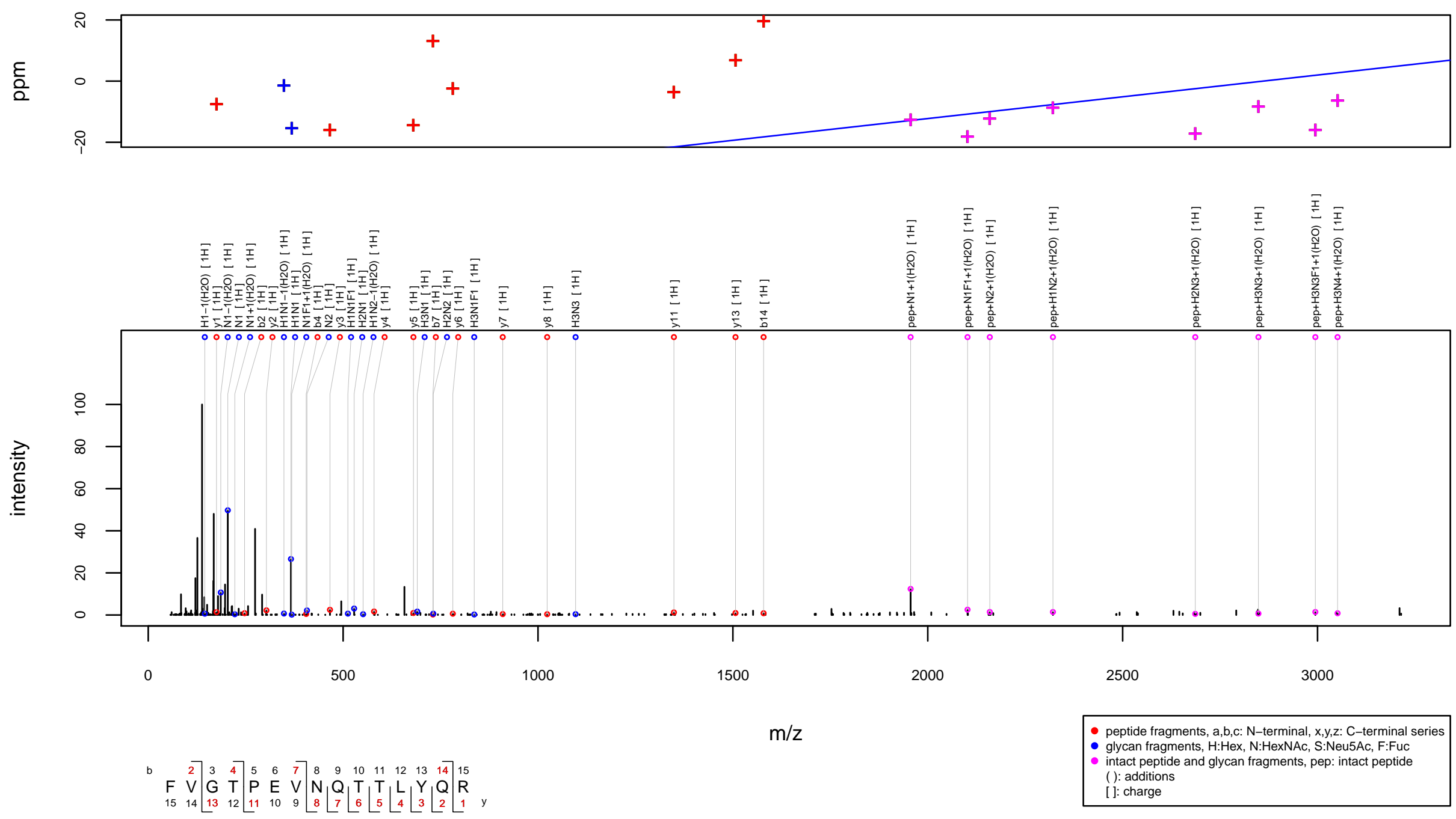
$\mathrm{m} / \mathrm{z} 1039.7212$ charge 4 scan $0-0$

Score $=39.4$, Hits $=30$, Explained Intensity $=0.25$

Peptide: TRFL_HUMAN[485,503]:TAGWNIPMGLLFNQTGSCK

Glycan: $\mathrm{SHNH}(\mathrm{HNH}) \mathrm{HNN}$, S1H5N4

Charge: $4 \mathrm{H}$
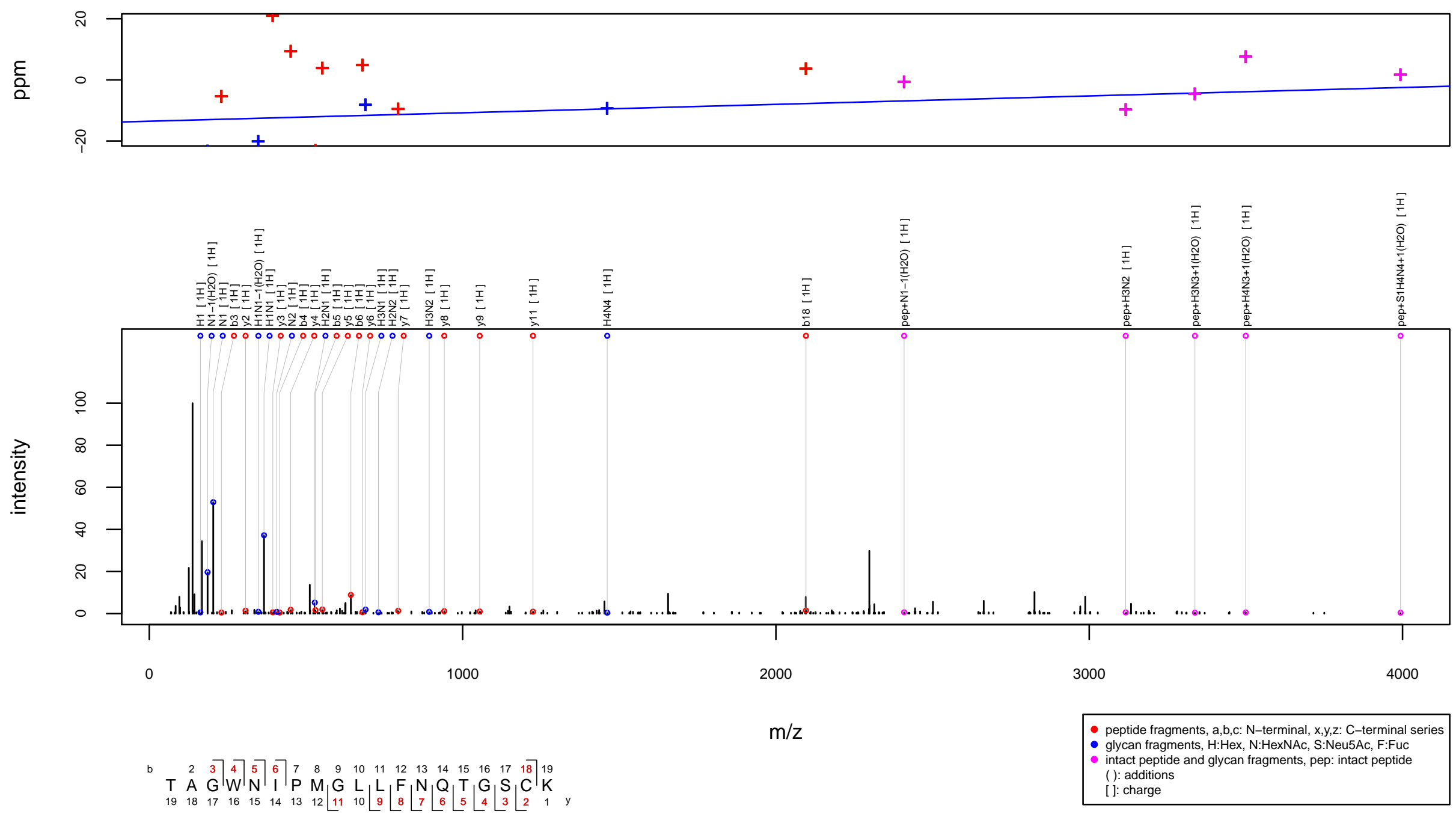
$\mathrm{m} / \mathrm{z} 1043.1052$ charge 3 scan $0-0$

Score $=51.46$, Hits $=33$, Explained Intensity $=0.41$

Peptide: CATL1_HUMAN[217,233]:YSVANDTGFVDIPKQEK

Glycan: $\mathrm{H}(\mathrm{H}) \mathrm{H}(\mathrm{H}) \mathrm{HNN}, \mathrm{H} 5 \mathrm{~N} 2$

Charge: $3 \mathrm{H}$
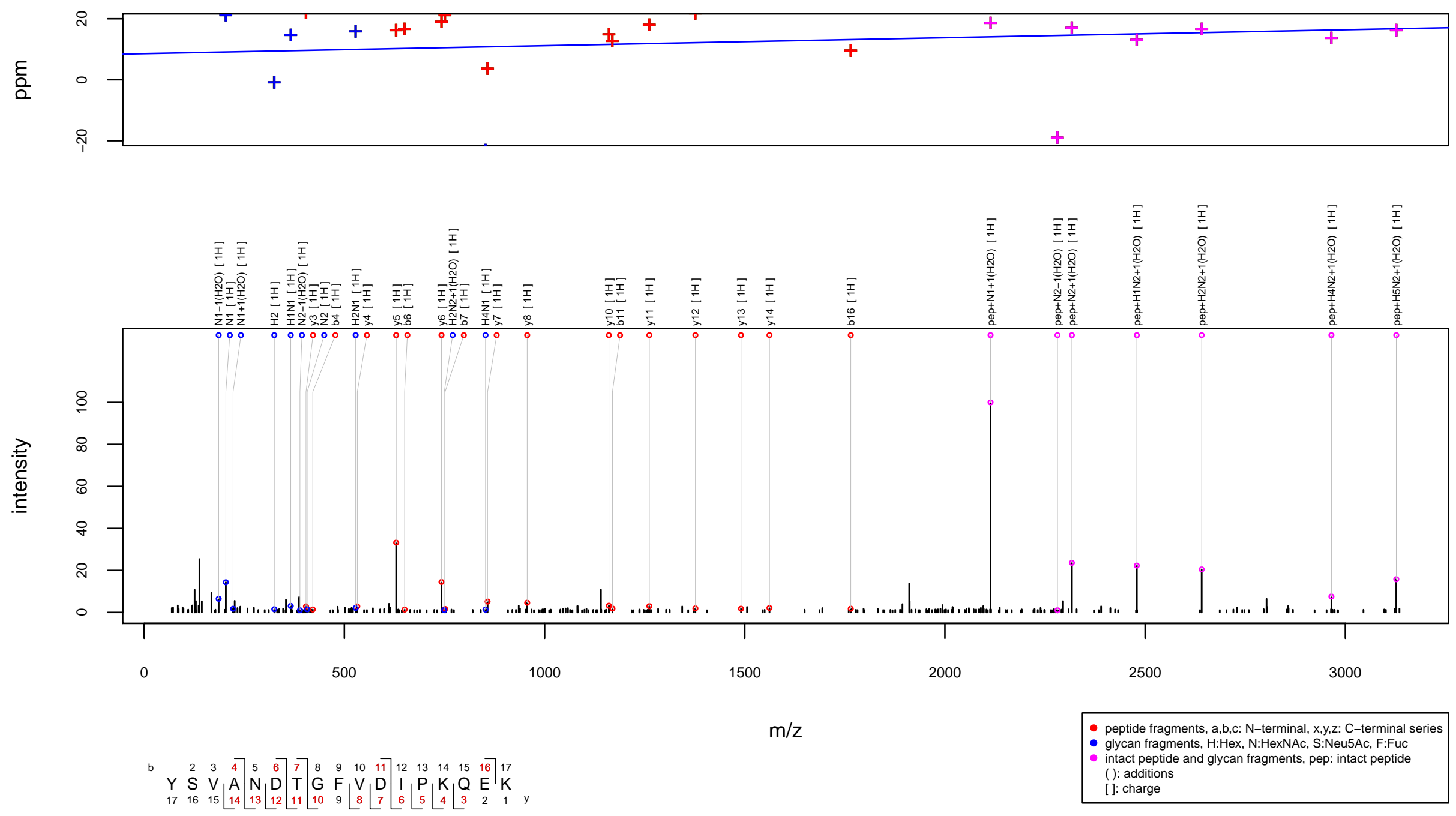
$\mathrm{m} / \mathrm{z} 1045.4728$ charge 4 scan $0-0$

Score $=70.45$, Hits $=55$, Explained Intensity $=0.45$

Peptide: TIMP1_HUMAN[46,60]:FVGTPEVNQTTLYQR

Glycan: $\mathrm{HN}(\mathrm{F}(\mathrm{H}) \mathrm{N}) \mathrm{H}(\mathrm{F}(\mathrm{H}) \mathrm{NH}) \mathrm{HN}(\mathrm{F}) \mathrm{N}, \mathrm{H} 6 \mathrm{~N} 5 \mathrm{~F} 3$

Charge: $4 \mathrm{H}$
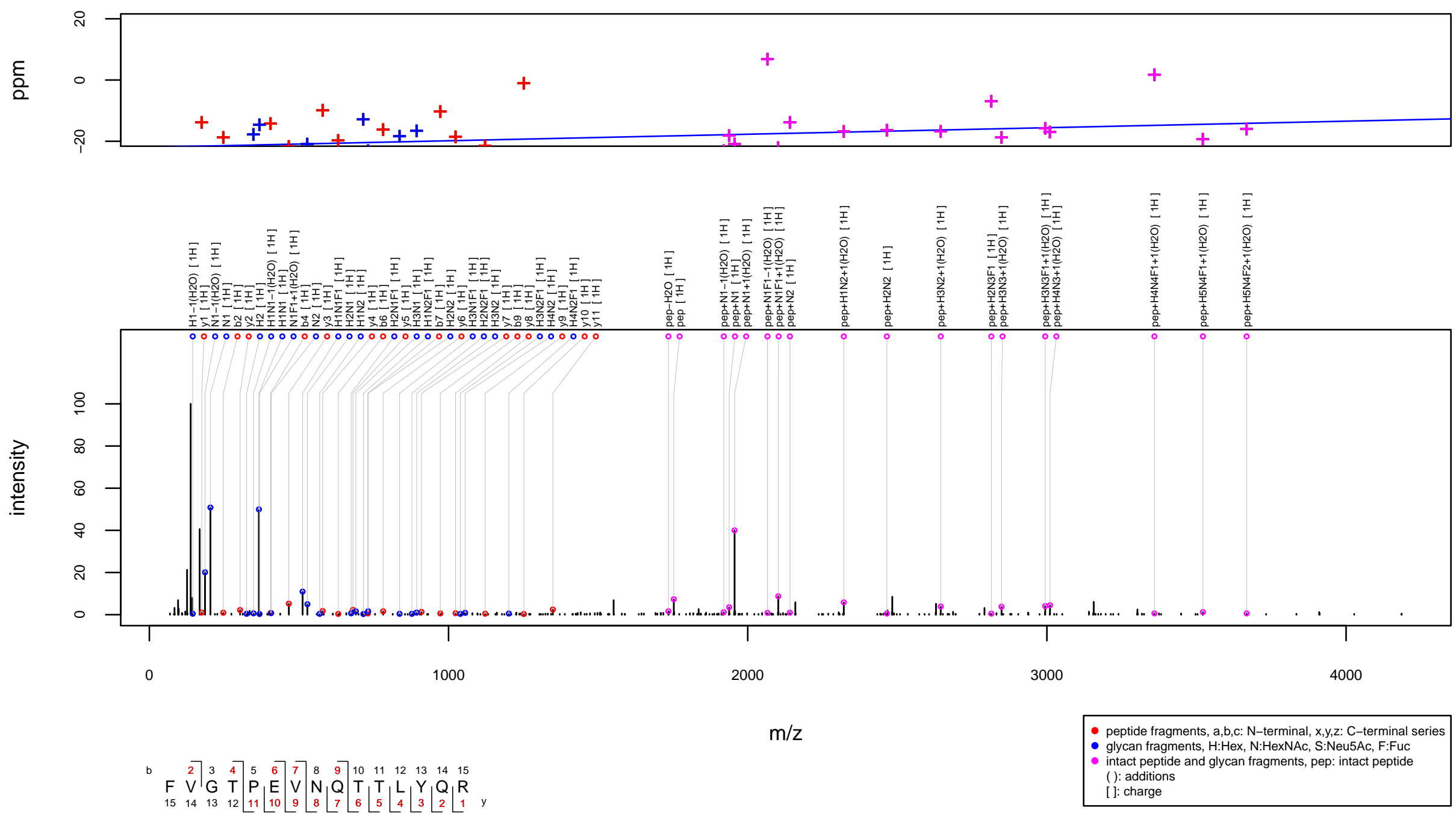
m/z 1052.4897 charge 3 scan $0-0$

Score $=43.34$, Hits $=32$, Explained Intensity $=0.31$ Peptide: AMPN_HUMAN[231,241]:AEFNITLIHPK

Glycan: $\mathrm{SHNH}(\mathrm{H}(\mathrm{H}) \mathrm{H}) \mathrm{HNN}, \mathrm{S} 1 \mathrm{H} 6 \mathrm{~N} 3$

Charge: $3 \mathrm{H}$
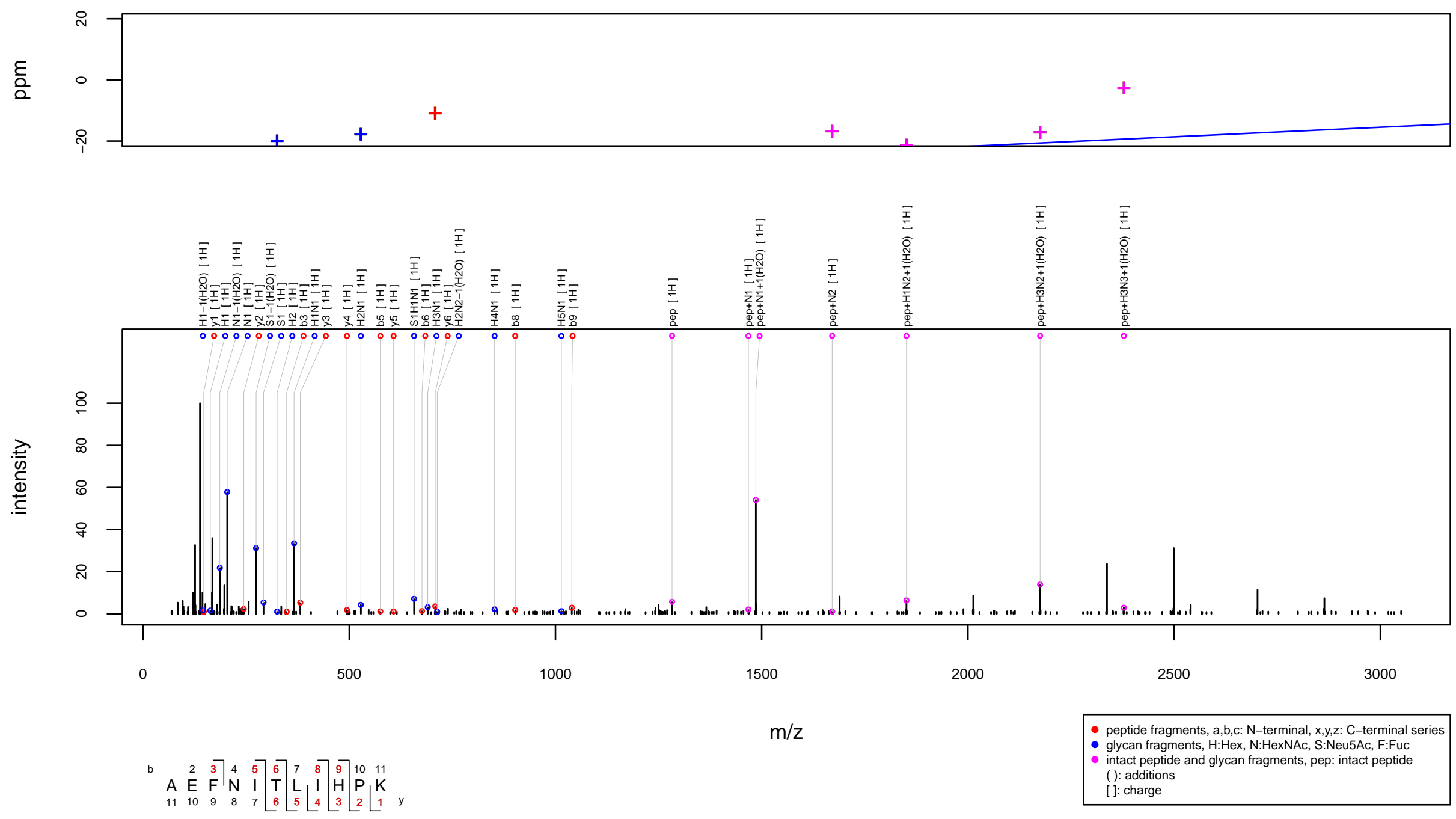
$\mathrm{m} / \mathrm{z} 1052.8308$ charge 3 scan $0-0$

Score $=40.26$, Hits $=31$, Explained Intensity $=0.31$

Peptide: TIMP1_HUMAN[46,60]:FVGTPEVNQTTLYQR

Glycan: H4N3F1

Charge: $3 \mathrm{H}$
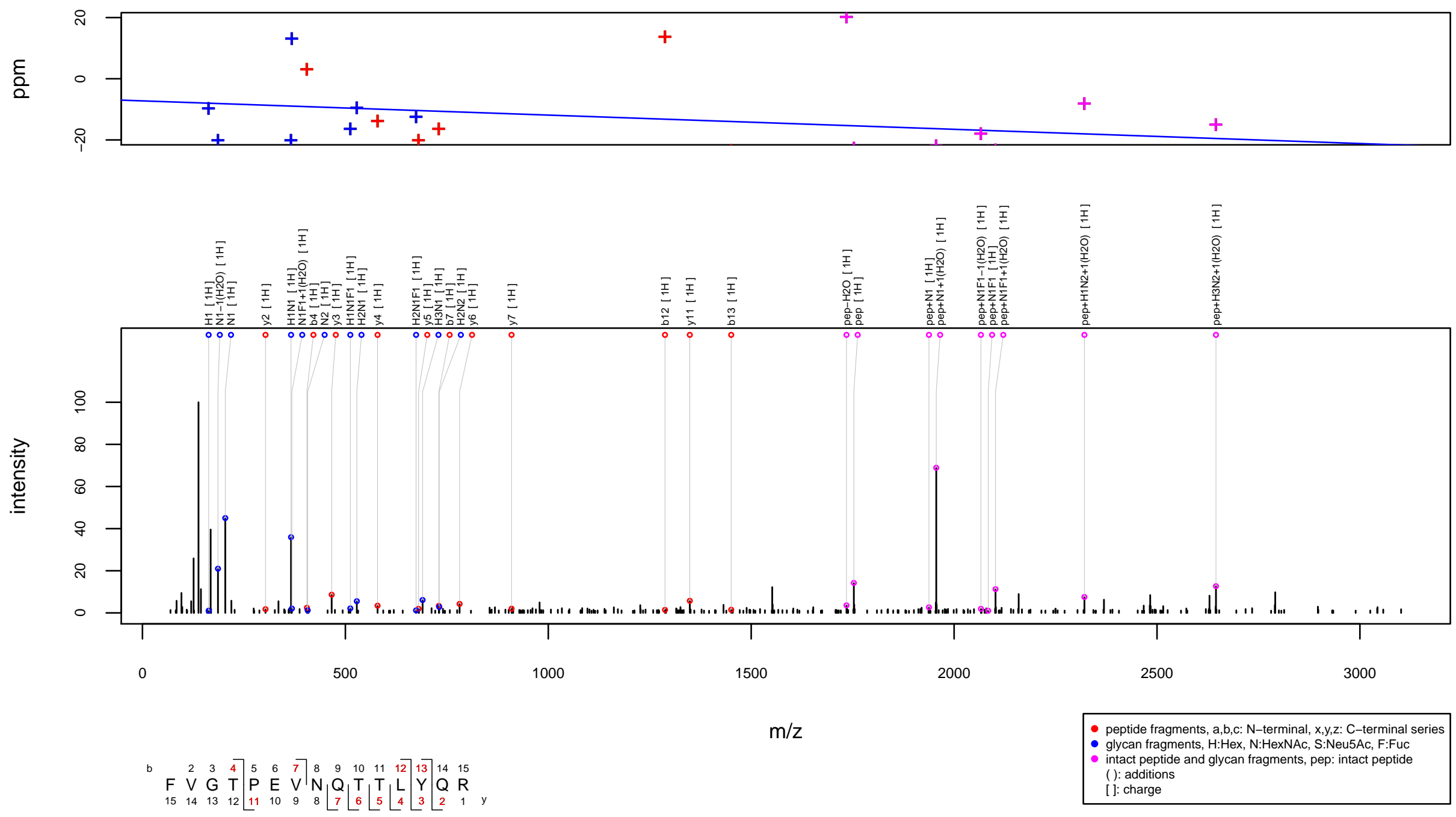
$\mathrm{m} / \mathrm{z} 1053.4102$ charge 4 scan $0-0$

Score $=44.3$, Hits $=41$, Explained Intensity $=0.19$

Peptide: ECM1_HUMAN[519,538]:GQGEQGSTGGTNISSTSEPK

Glycan: H3N6F4

Charge: $4 \mathrm{H}$
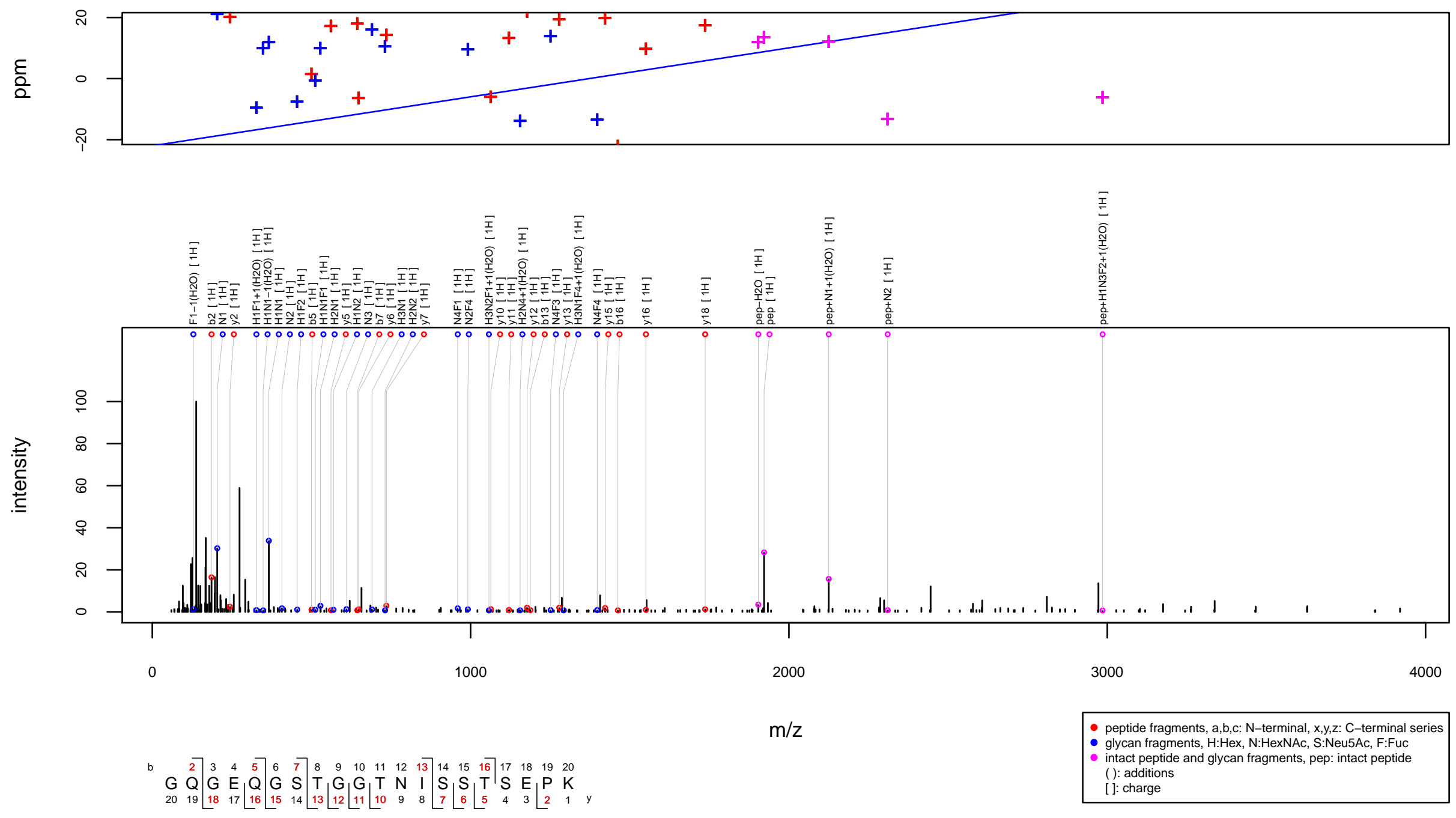
$\mathrm{m} / \mathrm{z} 1053.4135$ charge 4 scan $0-0$

Score $=115.21$, Hits $=88$, Explained Intensity $=0.5$

Peptide: CLUS_HUMAN[80,89]:KKEDALNETR

Glycan: $\mathrm{SHNH}(\mathrm{SHN}(\mathrm{SHN}) \mathrm{H}) \mathrm{HN}(\mathrm{F}) \mathrm{N}, \mathrm{S} 3 \mathrm{H} 6 \mathrm{~N} 5 \mathrm{~F} 1$

Charge: $4 \mathrm{H}$
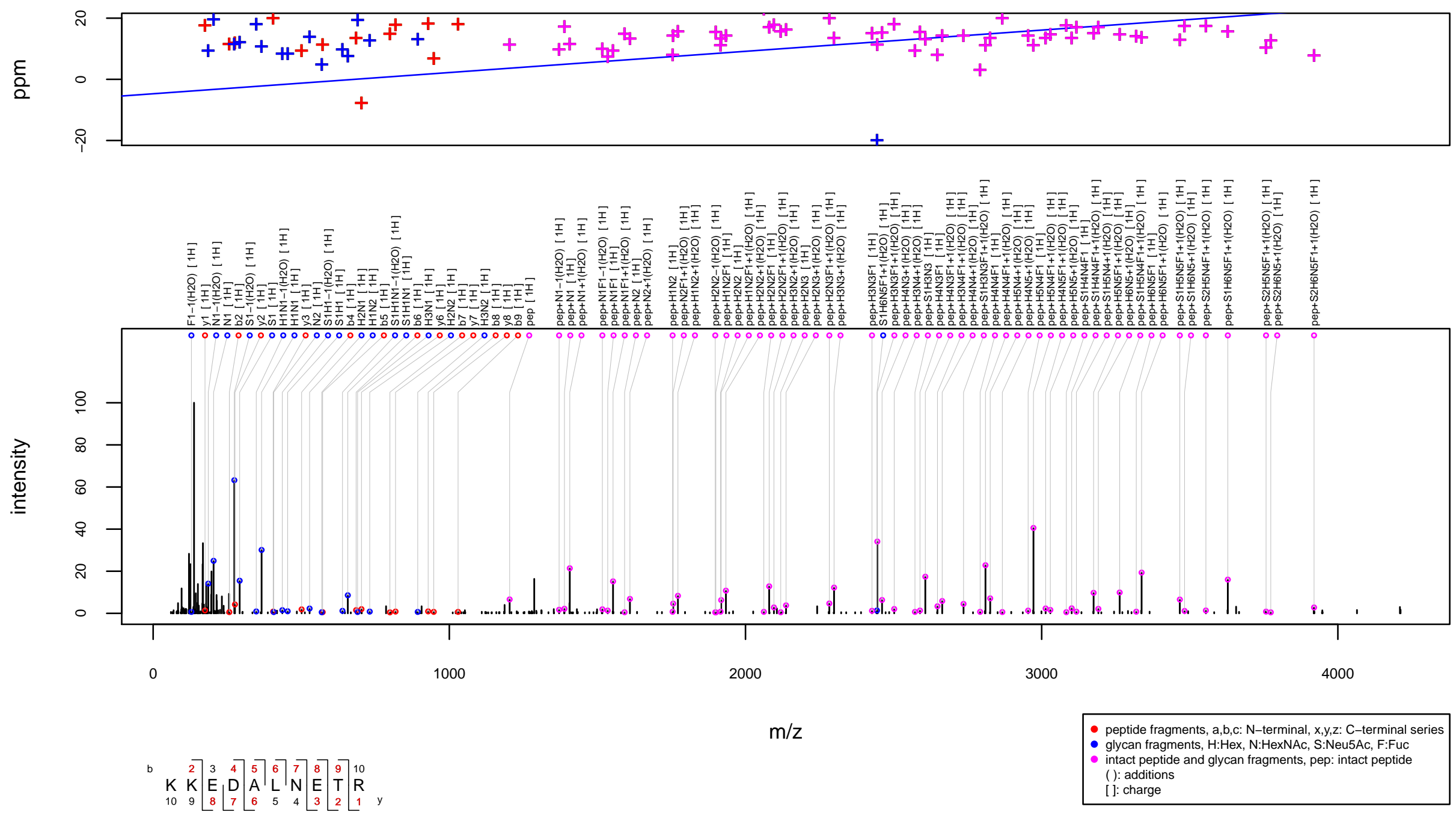
$\mathrm{m} / \mathrm{z} 1055.4965$ charge 3 scan $0-0$

Score $=67.11$, Hits $=43$, Explained Intensity $=0.33$ Peptide: CBPE_HUMAN[138,150]:GNETIVNLIHSTR Glycan: $\mathrm{SHNH}(\mathrm{HH}) \mathrm{HNN}$, S1H5N3

Charge: $3 \mathrm{H}$
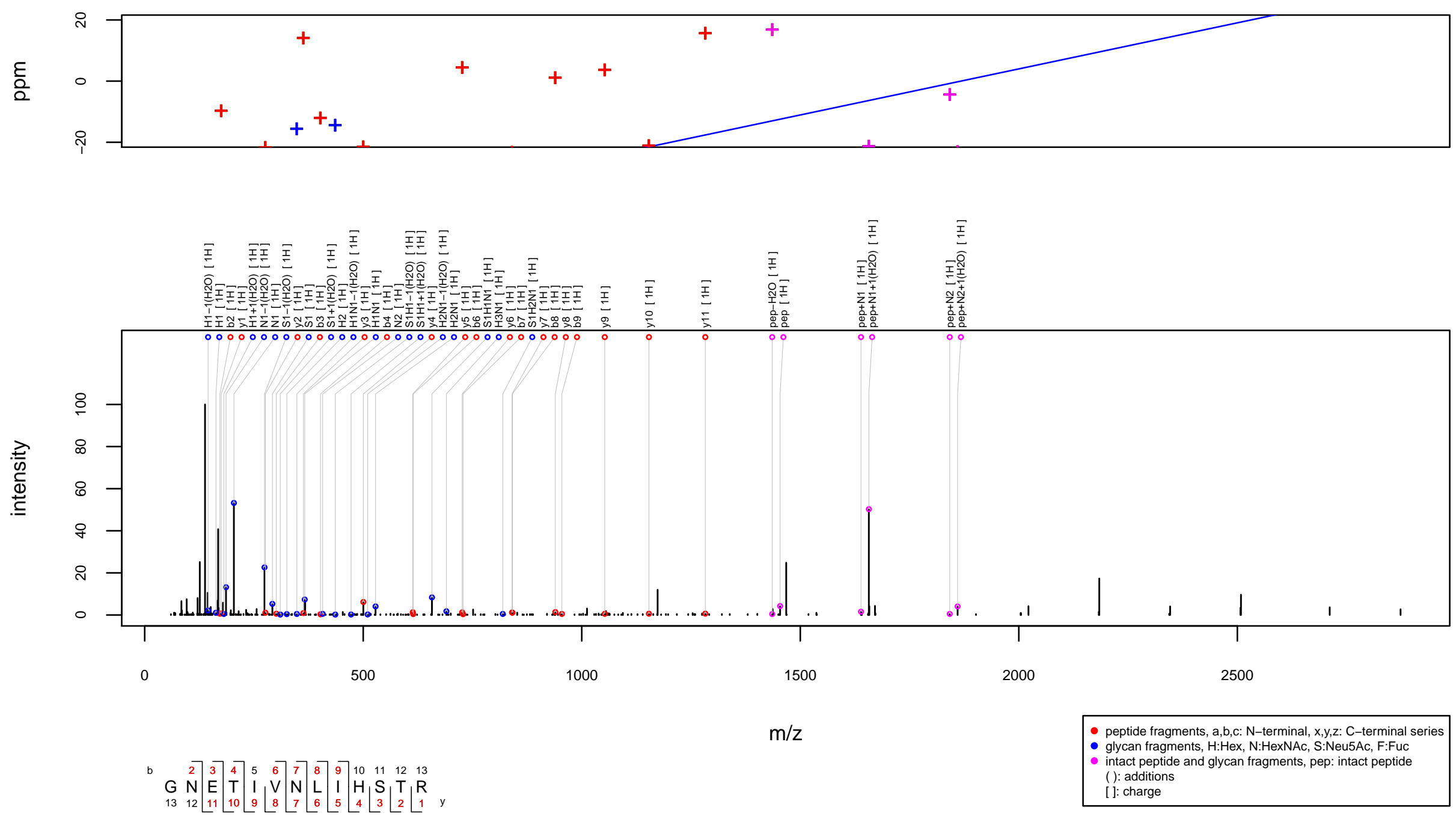
$\mathrm{m} / \mathrm{z} 1056.0089$ charge 4 scan $0-0$

Score $=65.11$, Hits $=48$, Explained Intensity $=0.41$

Peptide: IPSP_HUMAN[254,274]:VVGVPYQGNATALFILPSEGK

Glycan: $\mathrm{FHNH}(\mathrm{FHNH}) \mathrm{HN}(\mathrm{F}) \mathrm{N}, \mathrm{H} 5 \mathrm{~N} 4 \mathrm{F3}$

Charge: $4 \mathrm{H}$
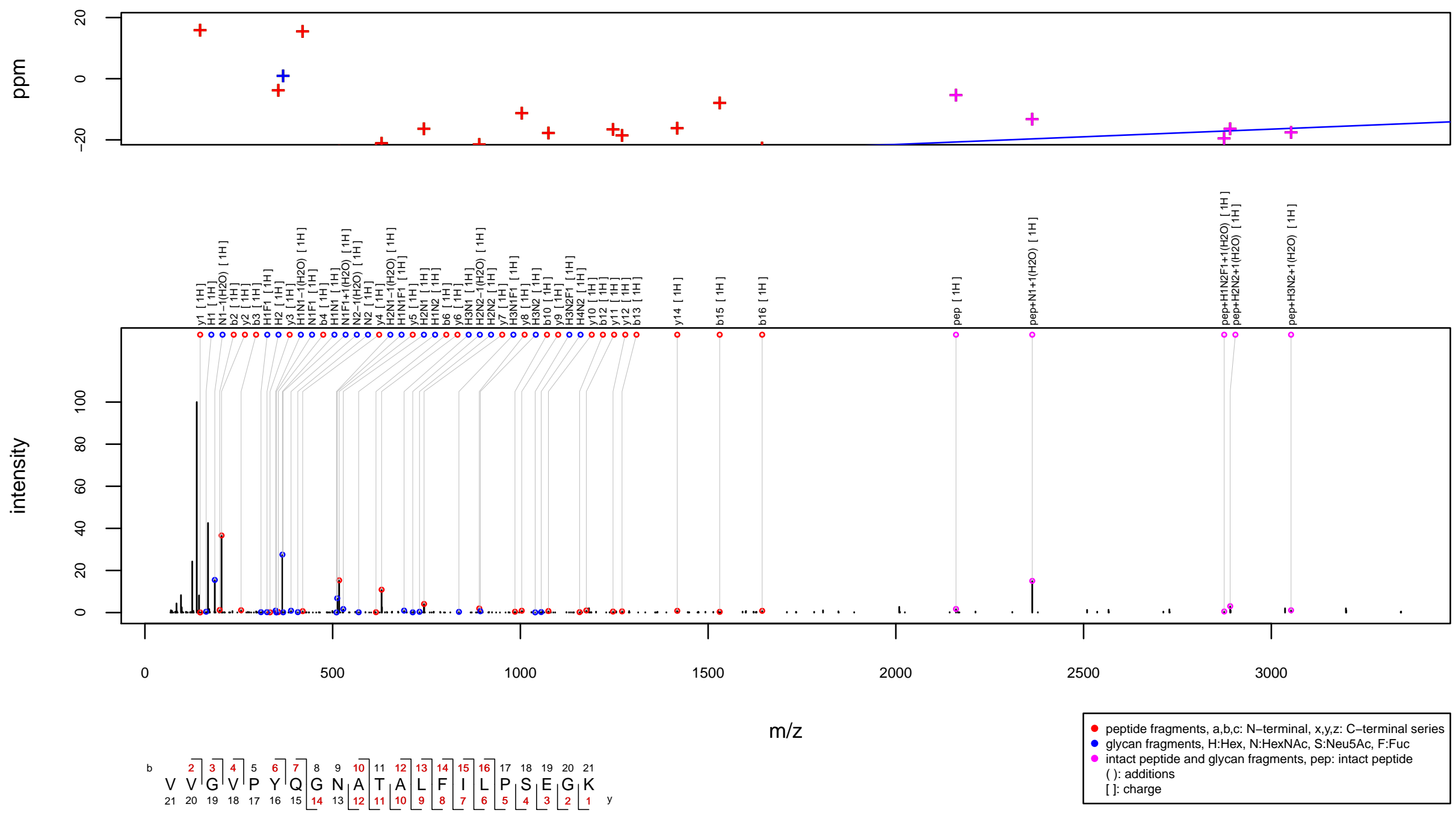


\section{$\mathrm{m} / \mathrm{z} 1059.1198$ charge 5 scan 0-0}

Score $=53.68$, Hits $=37$, Explained Intensity $=0.36$

Peptide: TRFL_HUMAN[141,170]:TAGWNVPIGTLRPFLNWTGPPEPIEAAVAR

Glycan: $\mathrm{FHNH}(\mathrm{FHNH}) \mathrm{HN}(\mathrm{F}) \mathrm{N}, \mathrm{H} 5 \mathrm{~N} 4 \mathrm{~F} 3$

Charge: $5 \mathrm{H}$
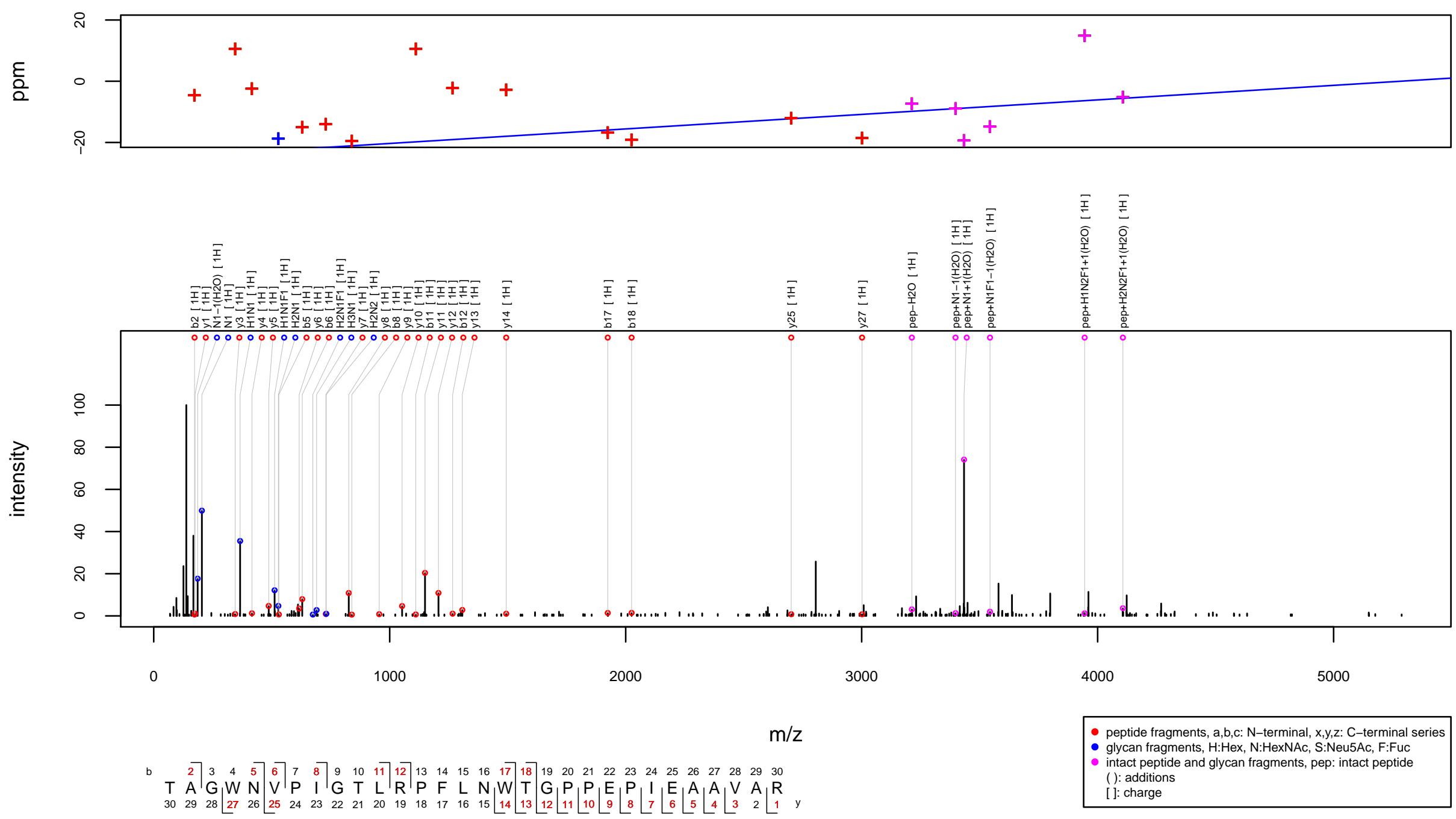
m/z 1060.4094 charge 4 scan 0-0

Score $=59.51$, Hits $=67$, Explained Intensity $=0.31$

Peptide: CLUS_HUMAN[80,89]:KKEDALNETR

Glycan: H6N8F3

Charge: $4 \mathrm{H}$
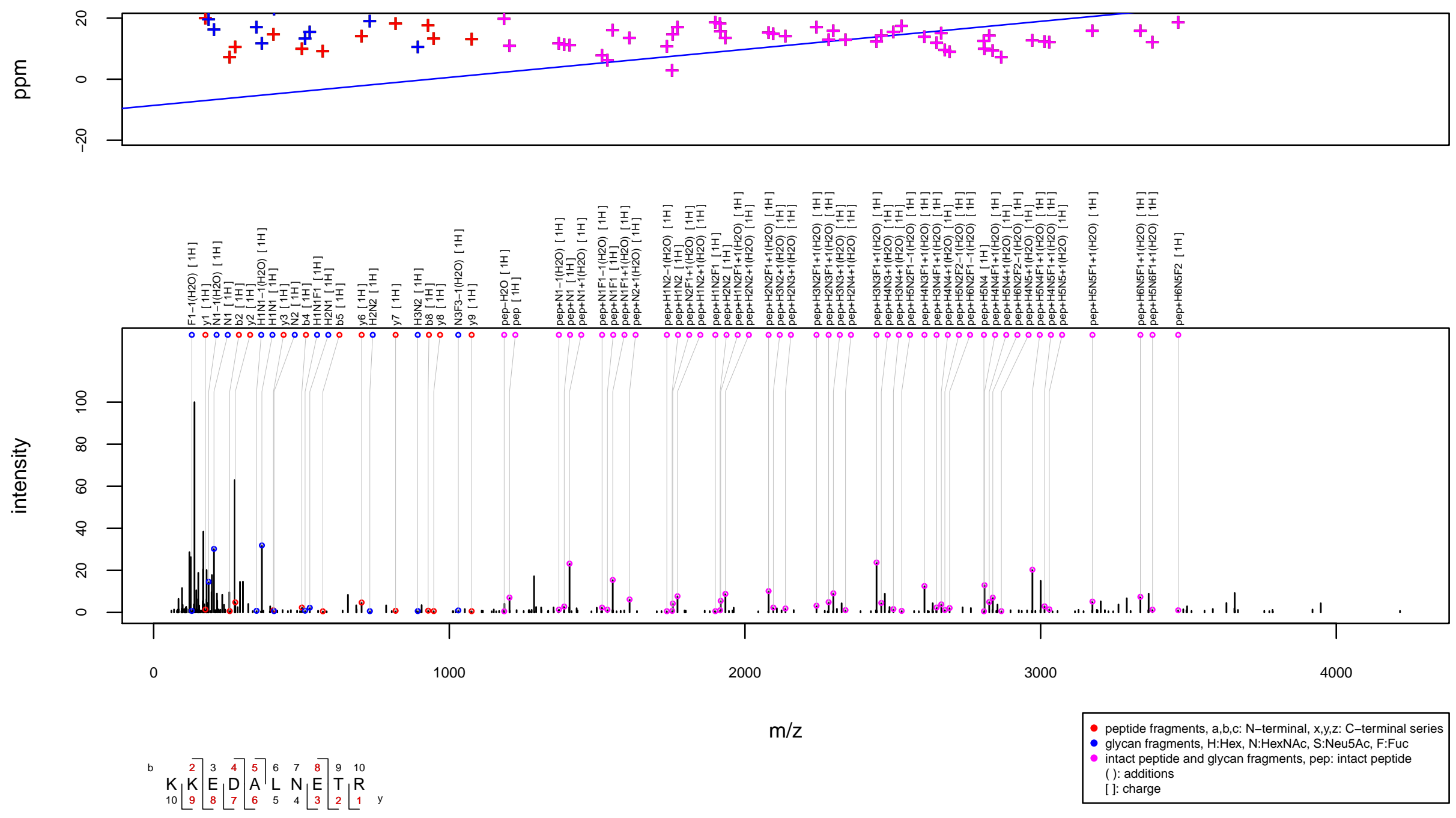


\section{$\mathrm{m} / \mathrm{z} 1063.5142$ charge 4 scan 0-0}

Score $=60.5$, Hits $=41$, Explained Intensity $=0.32$

Peptide: ASAH1_HUMAN[275,296]:ILAPAYFILGGNQSGEGCVITR

Glycan: $\mathrm{FHNH}(\mathrm{HNH}) \mathrm{HN}(\mathrm{F}) \mathrm{N}, \mathrm{H} 5 \mathrm{~N} 4 \mathrm{~F} 2$

Charge: $4 \mathrm{H}$
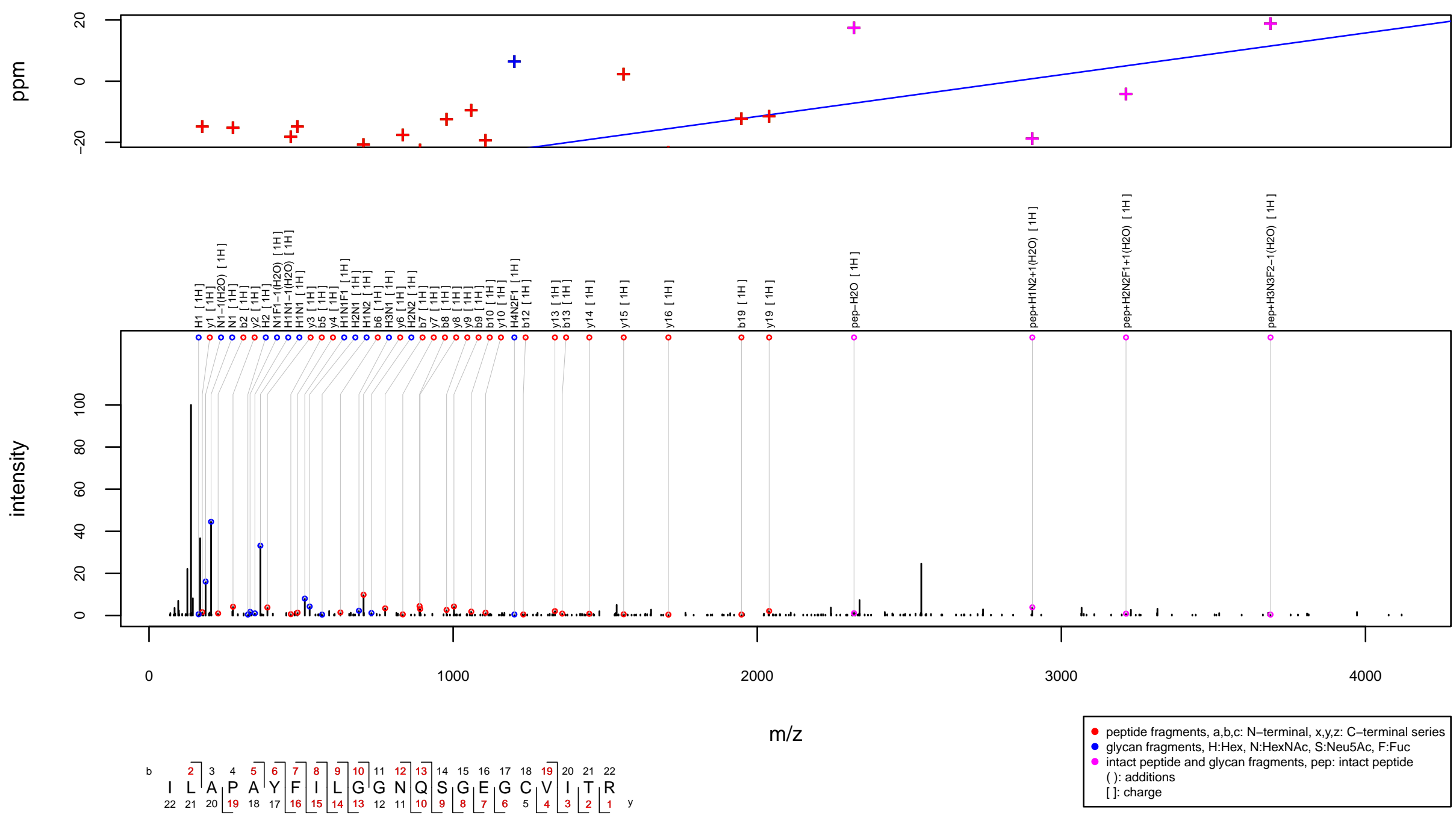
m/z 1064.7299 charge 4 scan 0-0

Score $=85.55$, Hits $=65$, Explained Intensity $=0.5$

Peptide: CLUS_HUMAN[372,385]:LANLTQGEDQYYLR

Glycan: $\mathrm{F}(\mathrm{H}) \mathrm{NH}(\mathrm{F}(\mathrm{H}) \mathrm{N}(\mathrm{F}(\mathrm{H}) \mathrm{N}) \mathrm{H}) \mathrm{HN}(\mathrm{F}) \mathrm{N}, \mathrm{H} 6 \mathrm{~N} 5 \mathrm{~F} 4$

Charge: $4 \mathrm{H}$
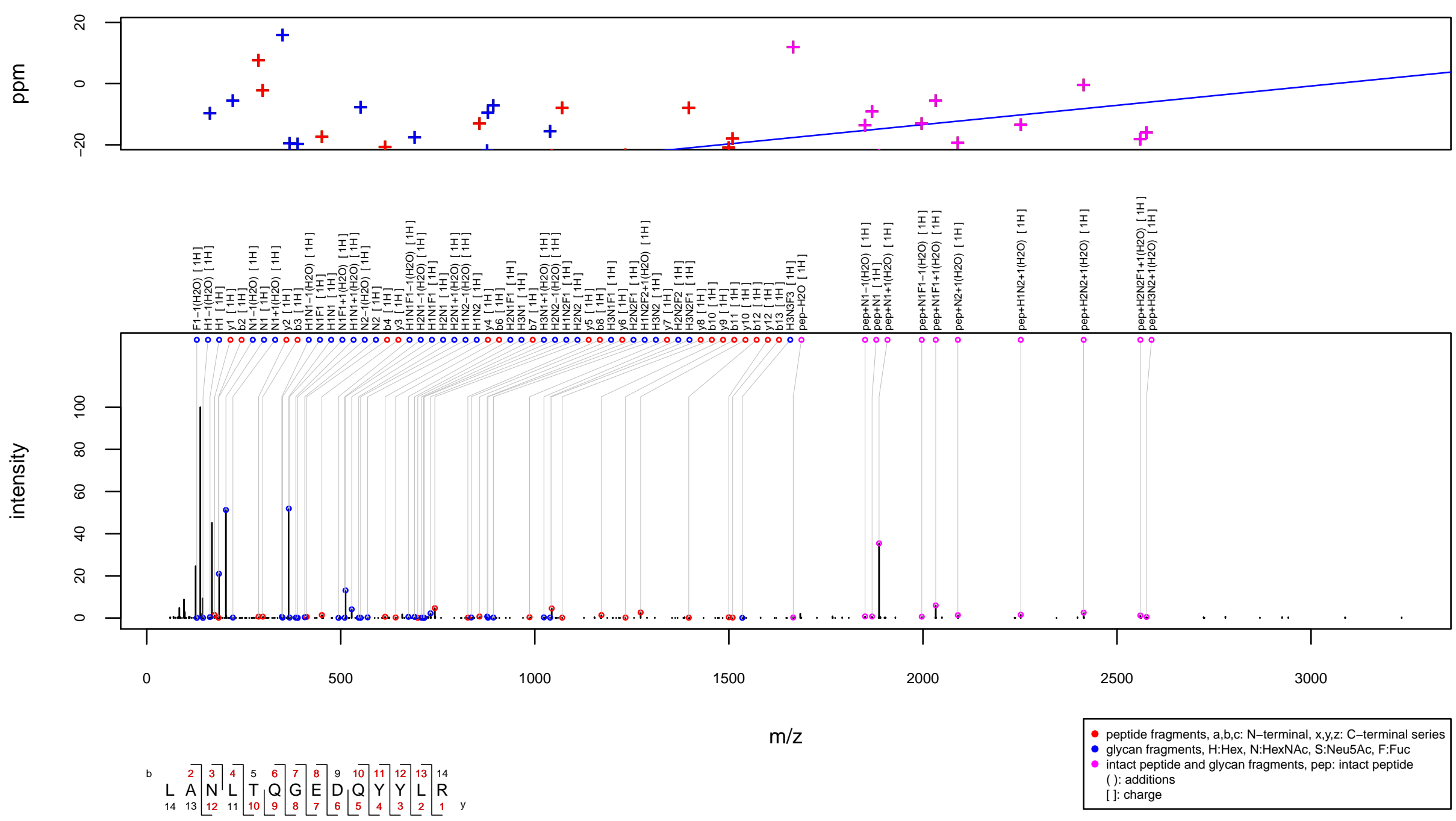
$\mathrm{m} / \mathrm{z} 1065.2054$ charge 4 scan $0-0$

Score $=110.05$, Hits $=71$, Explained Intensity $=0.42$

Peptide: FINC_HUMAN[516,533]:DQCIVDDITYNVNDTFHK

Glycan: $\mathrm{FHNH}(\mathrm{FHNH}) \mathrm{HN}(\mathrm{F}) \mathrm{N}, \mathrm{H} 5 \mathrm{~N} 4 \mathrm{F3}$

Charge: $4 \mathrm{H}$
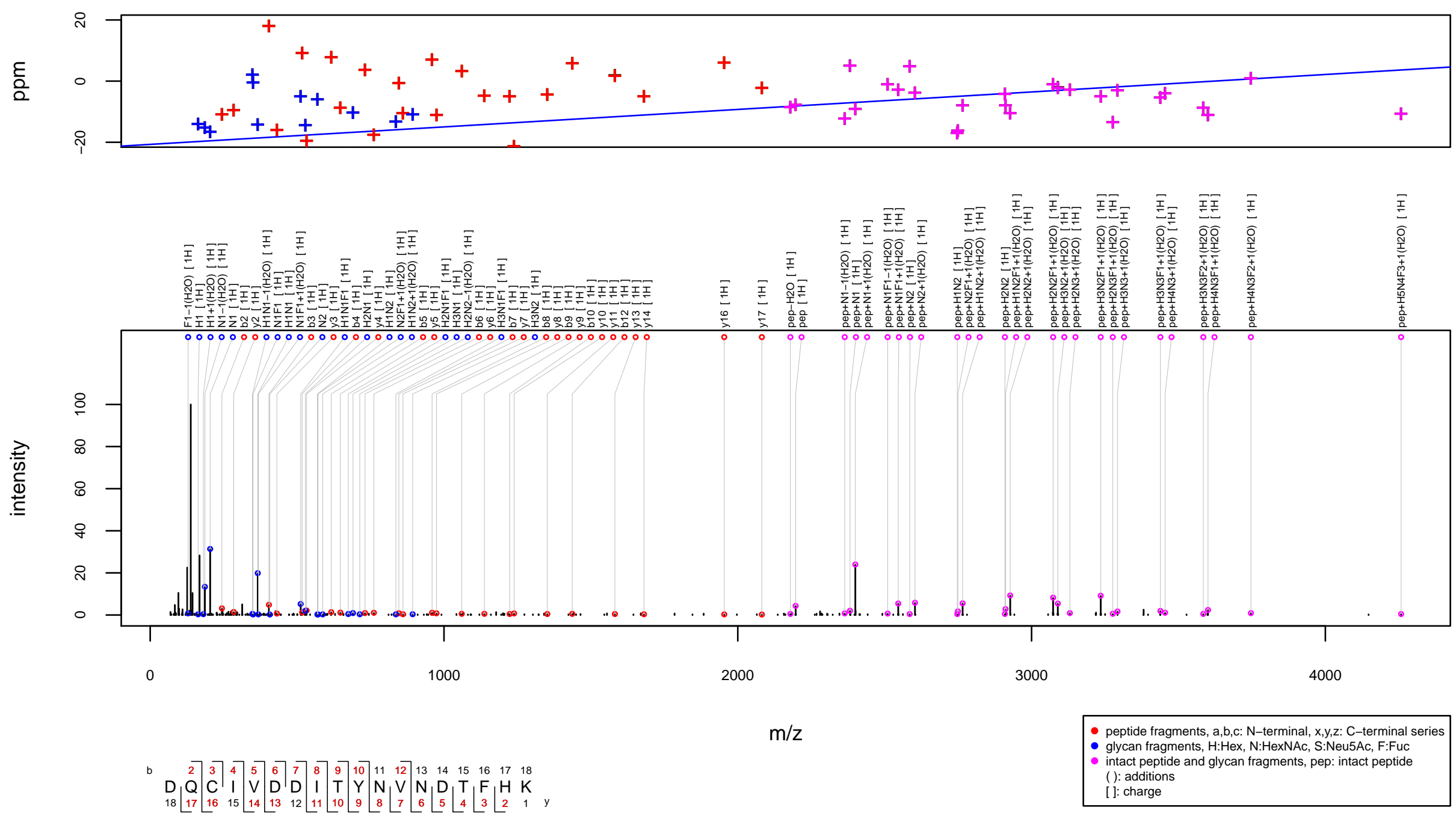
$\mathrm{m} / \mathrm{z} 1066.1676$ charge 3 scan $0-0$

Score $=52.76$, Hits $=38$, Explained Intensity $=0.33$ Peptide: AMPN_HUMAN[231,241]:AEFNITLIHPK

Glycan: $\mathrm{SHNH}(\mathrm{HNH}) \mathrm{HNN}, \mathrm{S} 1 \mathrm{H} 5 \mathrm{~N} 4$

Charge: $3 \mathrm{H}$
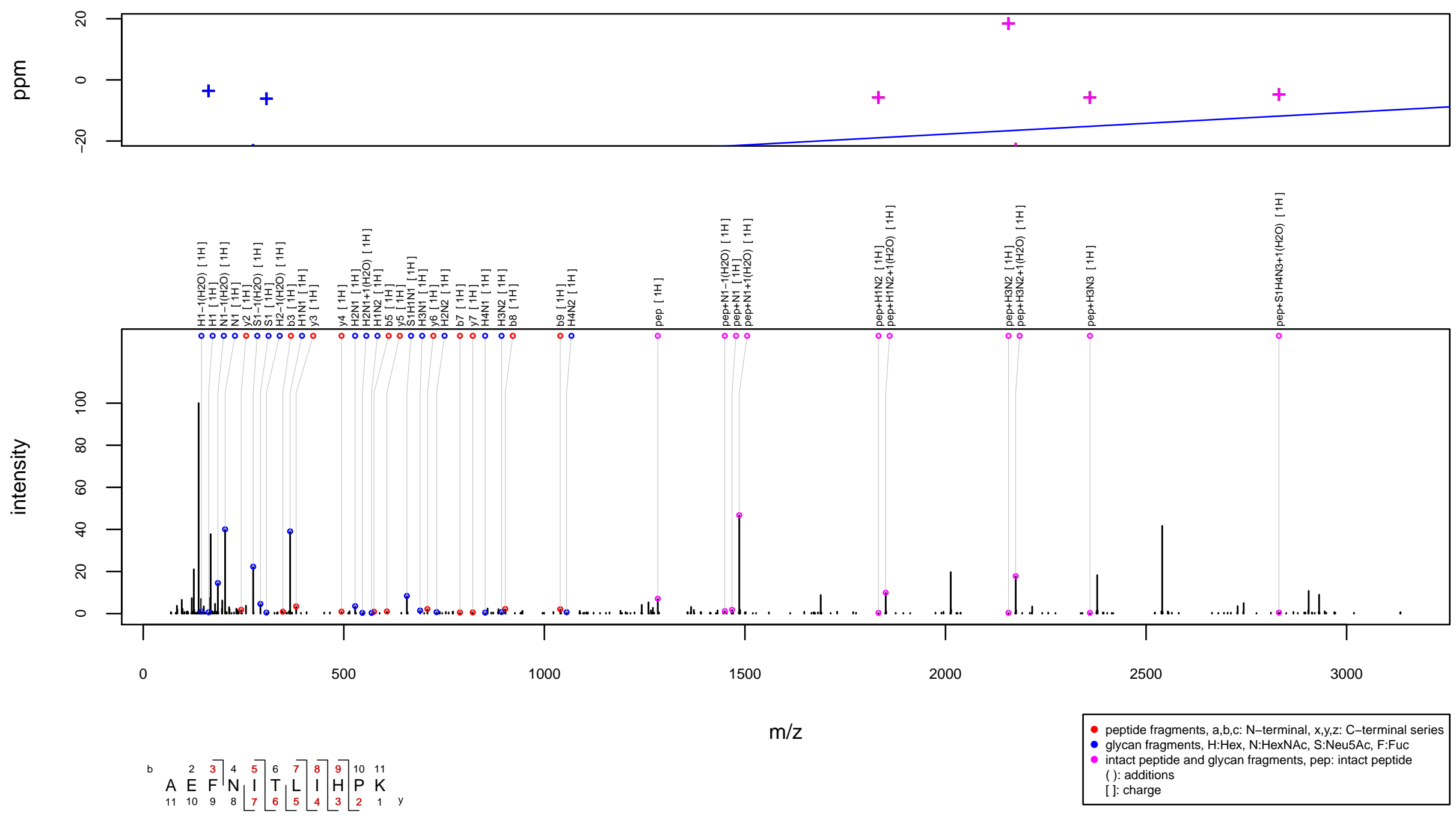
m/z 1066.7593 charge 3 scan 0-0

Score $=74.2$, Hits $=55$, Explained Intensity $=0.36$ Peptide: ZA2G_HUMAN[121,129]:FGCEIENNR

Glycan: $\mathrm{SHNH}(\mathrm{HNH}) \mathrm{HN}(\mathrm{F}) \mathrm{N}, \mathrm{S} 1 \mathrm{H} 5 \mathrm{~N} 4 \mathrm{~F} 1$

Charge: $3 \mathrm{H}$
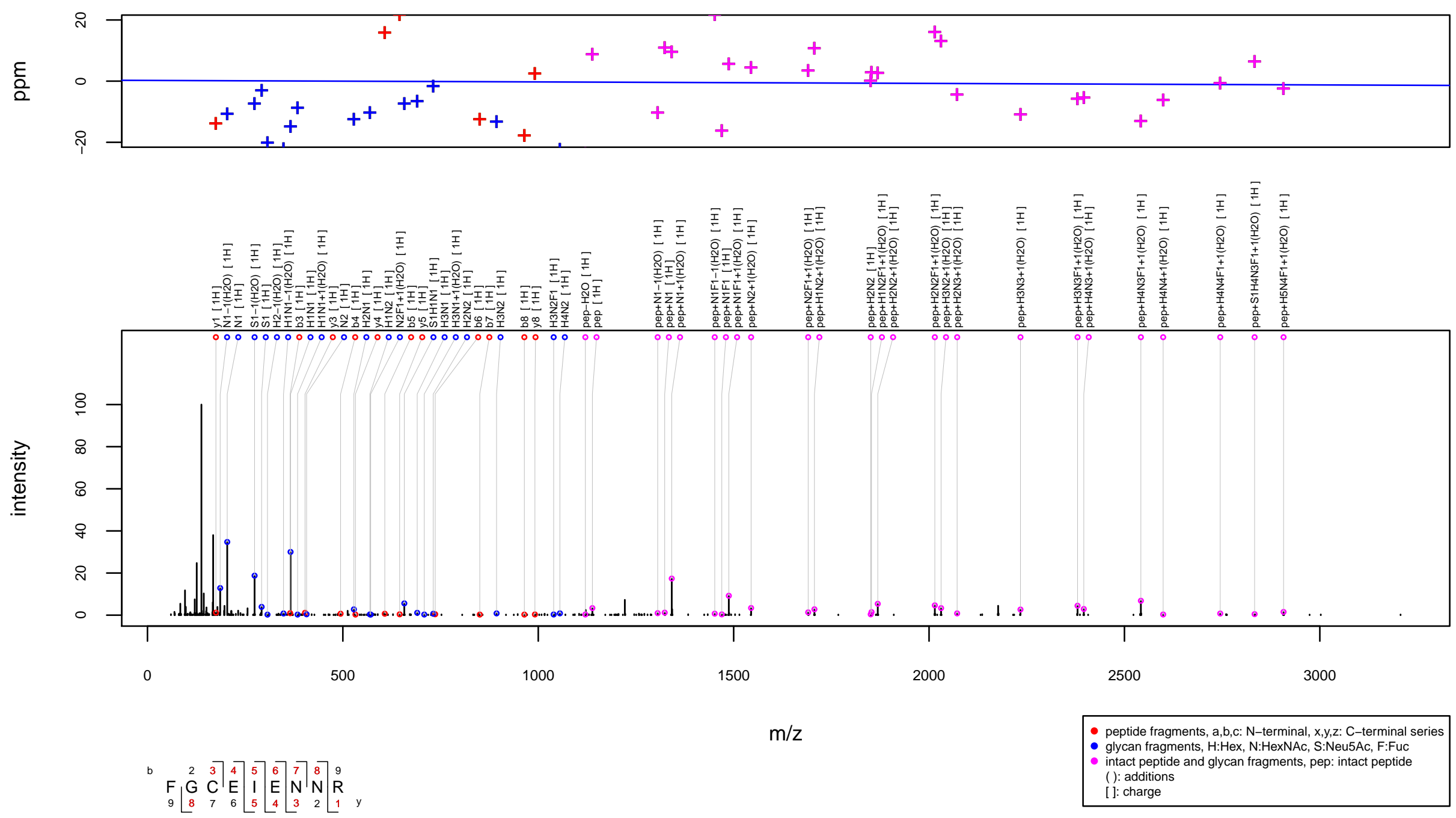
$\mathrm{m} / \mathrm{z} 1067.2461$ charge 4 scan 0-0

Score $=28.15$, Hits $=25$, Explained Intensity $=0.21$

Peptide: EHMT1_HUMAN[232,246]:EPKEEINKNISDFGR

Glycan: $\mathrm{S} 2 \mathrm{H} 3 \mathrm{~N} 7$

Charge: $4 \mathrm{H}$
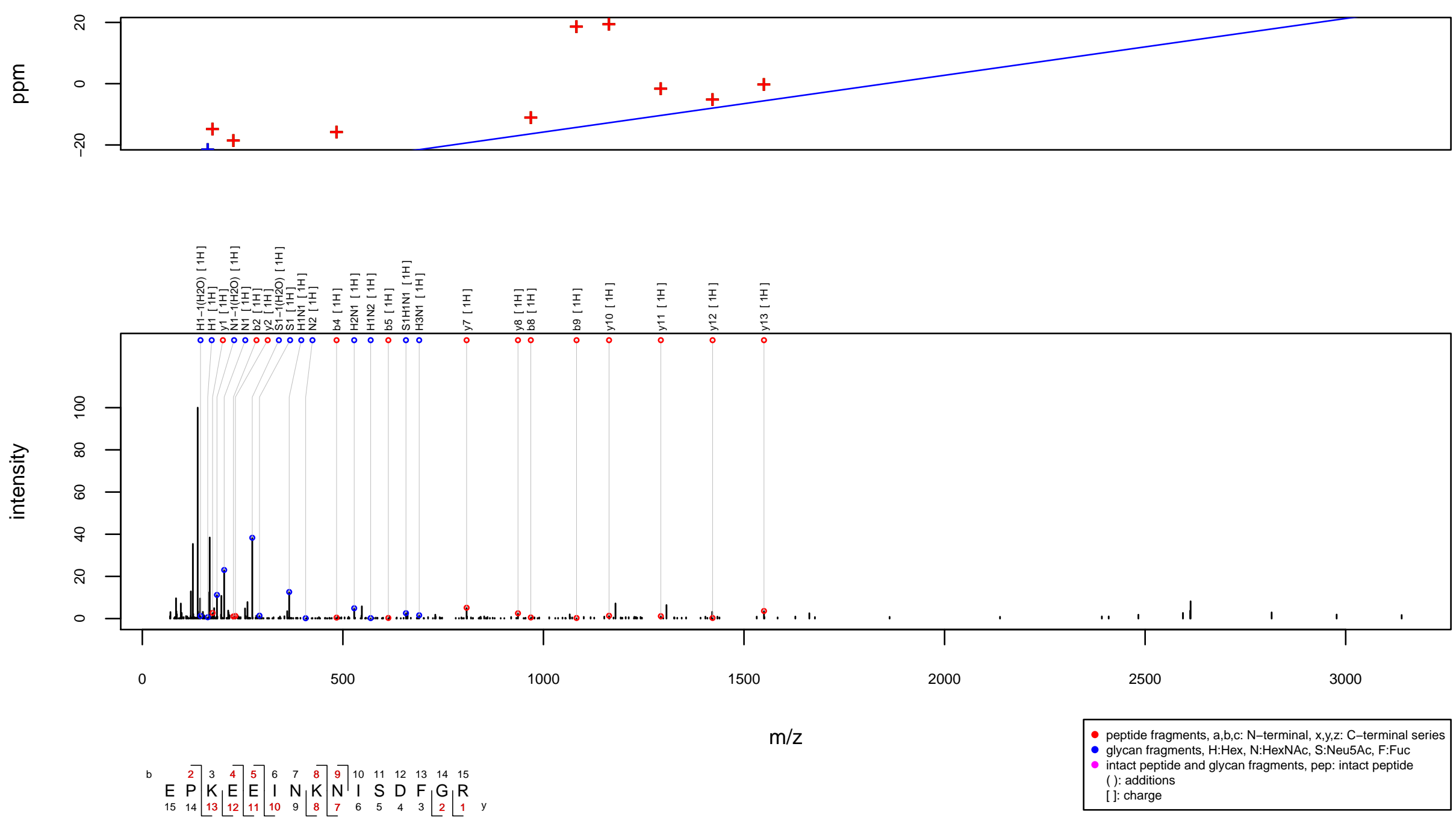
m/z 1067.503 charge 4 scan 0-0

Score $=28.48$, Hits $=31$, Explained Intensity $=0.22$

Peptide: EHMT1_HUMAN[232,246]:EPKEEINKNISDFGR

Glycan: S1H3N7F2

Charge: $4 \mathrm{H}$
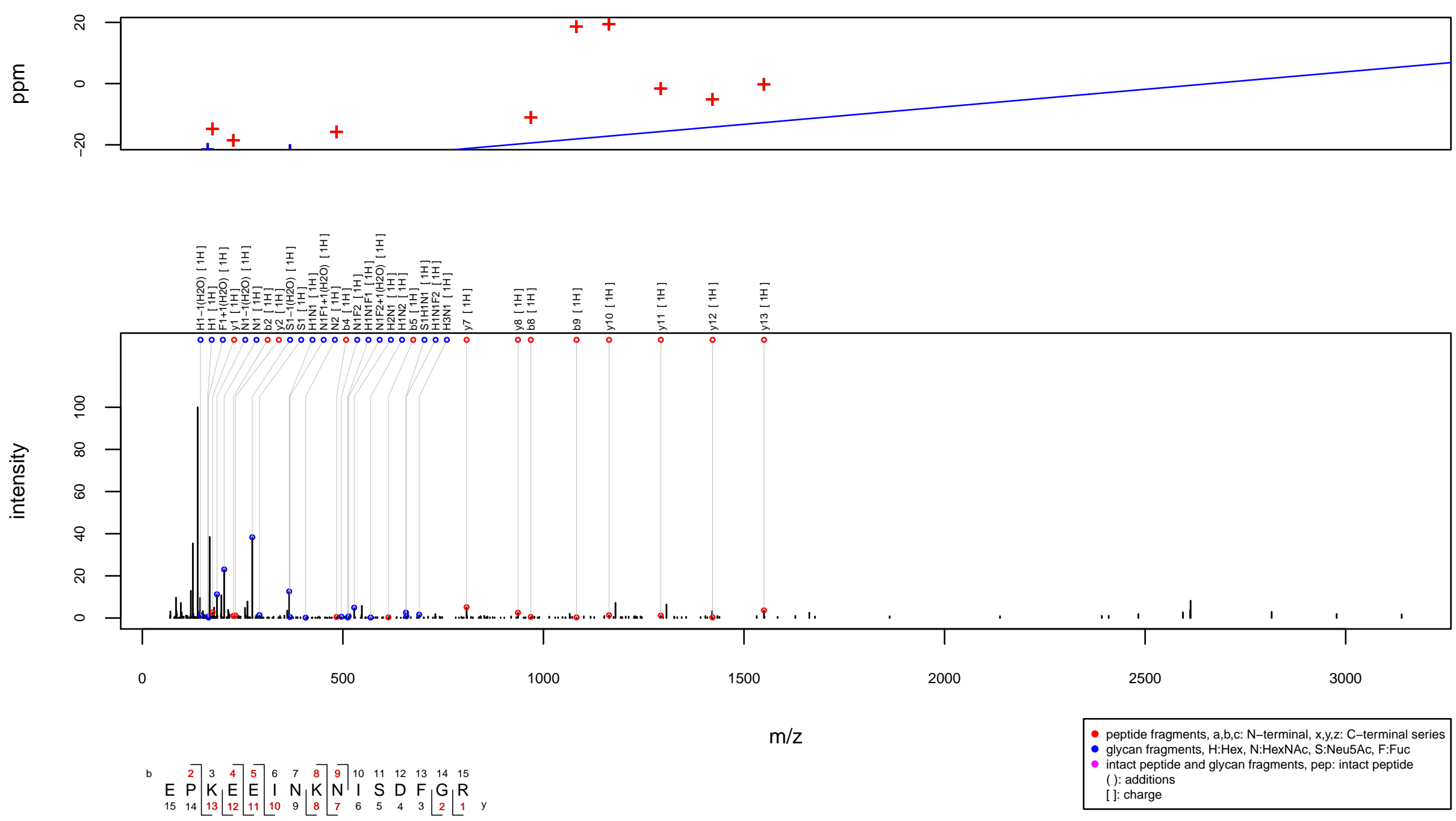
m/z 1067.9755 charge 4 scan 0-0

Score $=34.07$, Hits $=30$, Explained Intensity $=0.17$ Peptide: ZN821_HUMAN[283,293]:SRRDNETPEER Glycan: H9N7

Charge: $4 \mathrm{H}$
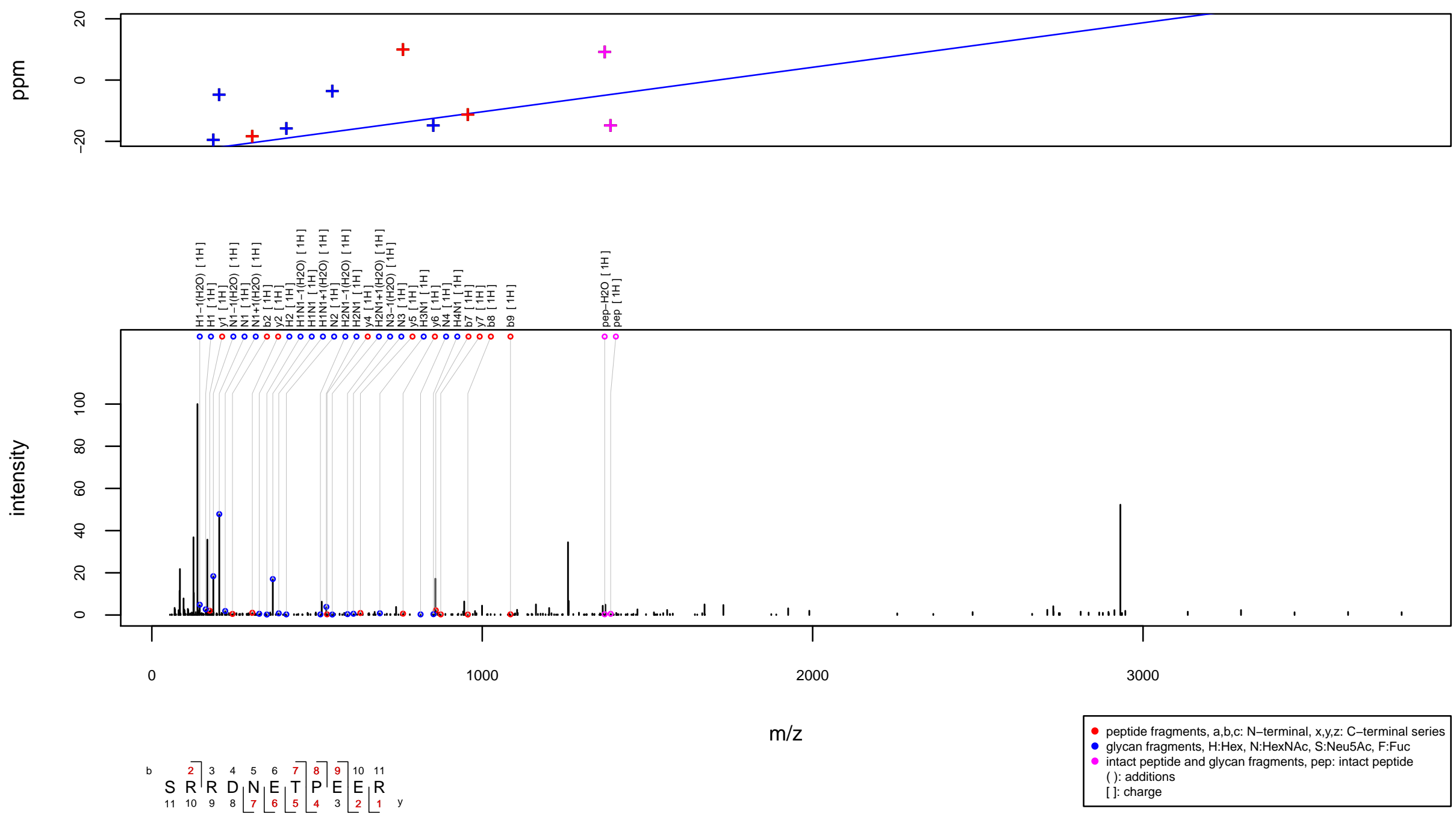
$\mathrm{m} / \mathrm{z} 1068.2511$ charge 4 scan $0-0$

Score $=73.17$, Hits $=47$, Explained Intensity $=0.33$

Peptide: PTGDS_HUMAN[67,85]:SVVAPATDGGLNLTSTFLR

Glycan: $\mathrm{SHNH}(\mathrm{SHNH}) \mathrm{HN}(\mathrm{F}) \mathrm{N}, \mathrm{S} 2 \mathrm{H} 5 \mathrm{~N} 4 \mathrm{~F} 1$

Charge: $4 \mathrm{H}$
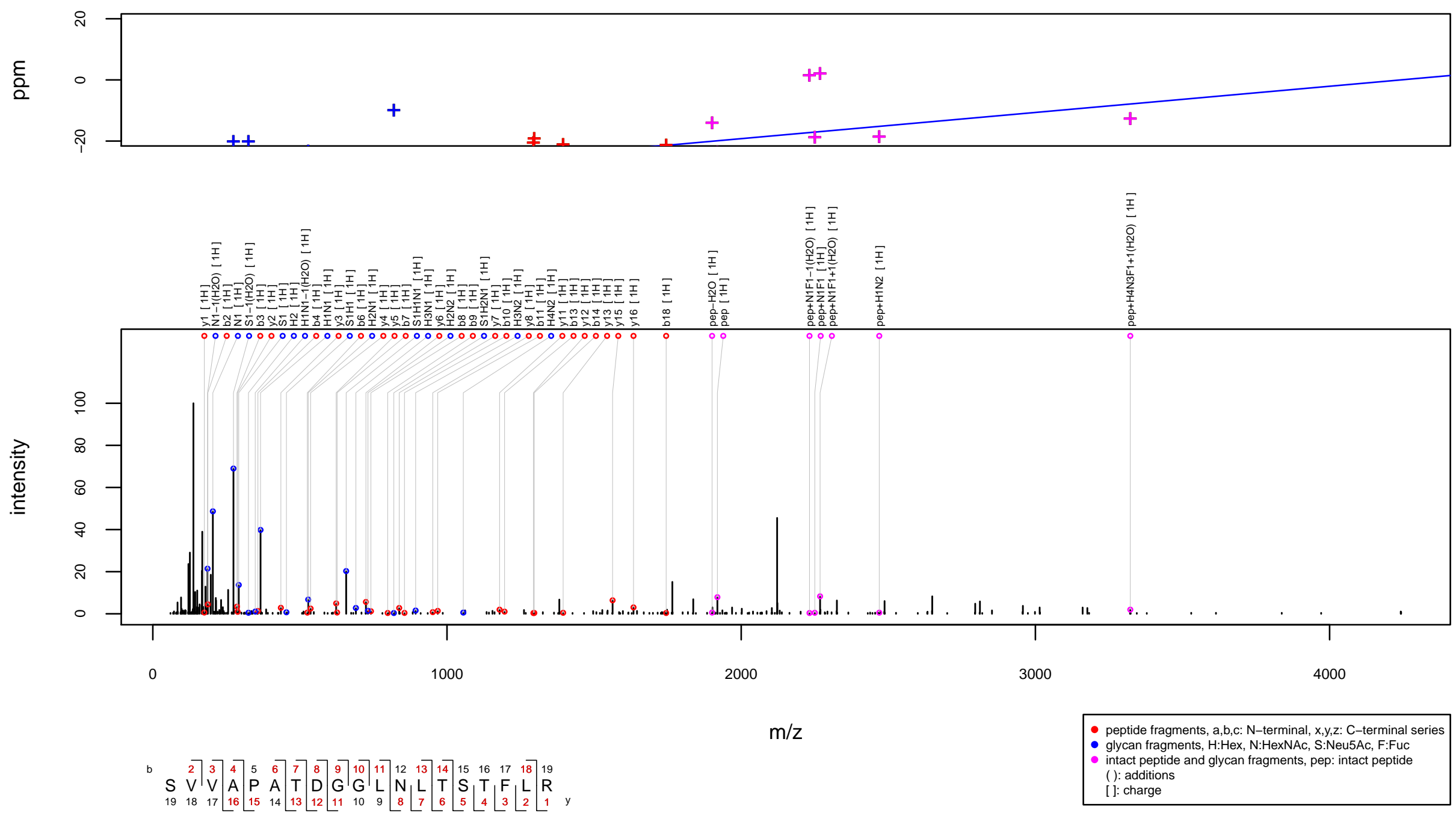
$\mathrm{m} / \mathrm{z} 1068.5211$ charge 2 scan 0-0

Score $=25.29$, Hits $=16$, Explained Intensity $=0.09$ Peptide: BCD1_HUMAN[331,340]:KENSTFFDKK Glycan: $\mathrm{H}(\mathrm{H}) \mathrm{HNN}, \mathrm{H} 3 \mathrm{~N} 2$

Charge: $2 \mathrm{H}$
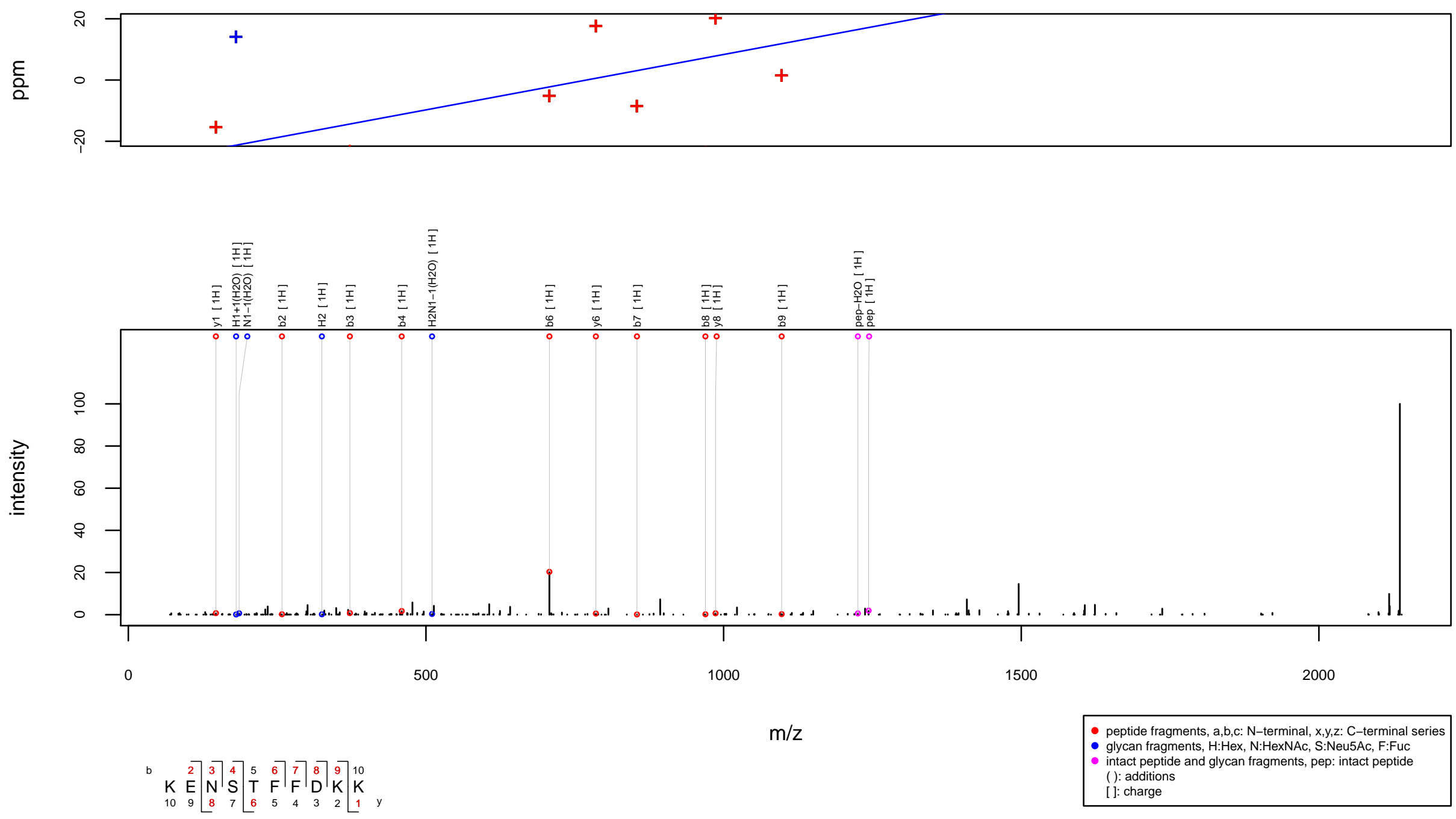
$\mathrm{m} / \mathrm{z} 1072.4548$ charge 4 scan $0-0$

Score $=95.75$, Hits $=78$, Explained Intensity $=0.38$

Peptide: CLUS_HUMAN[80,89]:KKEDALNETR

Glycan: $\mathrm{F}(\mathrm{H}) \mathrm{NH}(\mathrm{F}) \mathrm{NH}(\mathrm{F}(\mathrm{H}) \mathrm{NH}(\mathrm{F}) \mathrm{NH}) \mathrm{HN}(\mathrm{F}) \mathrm{N}, \mathrm{H} 7 \mathrm{N6F5}$

Charge: $4 \mathrm{H}$
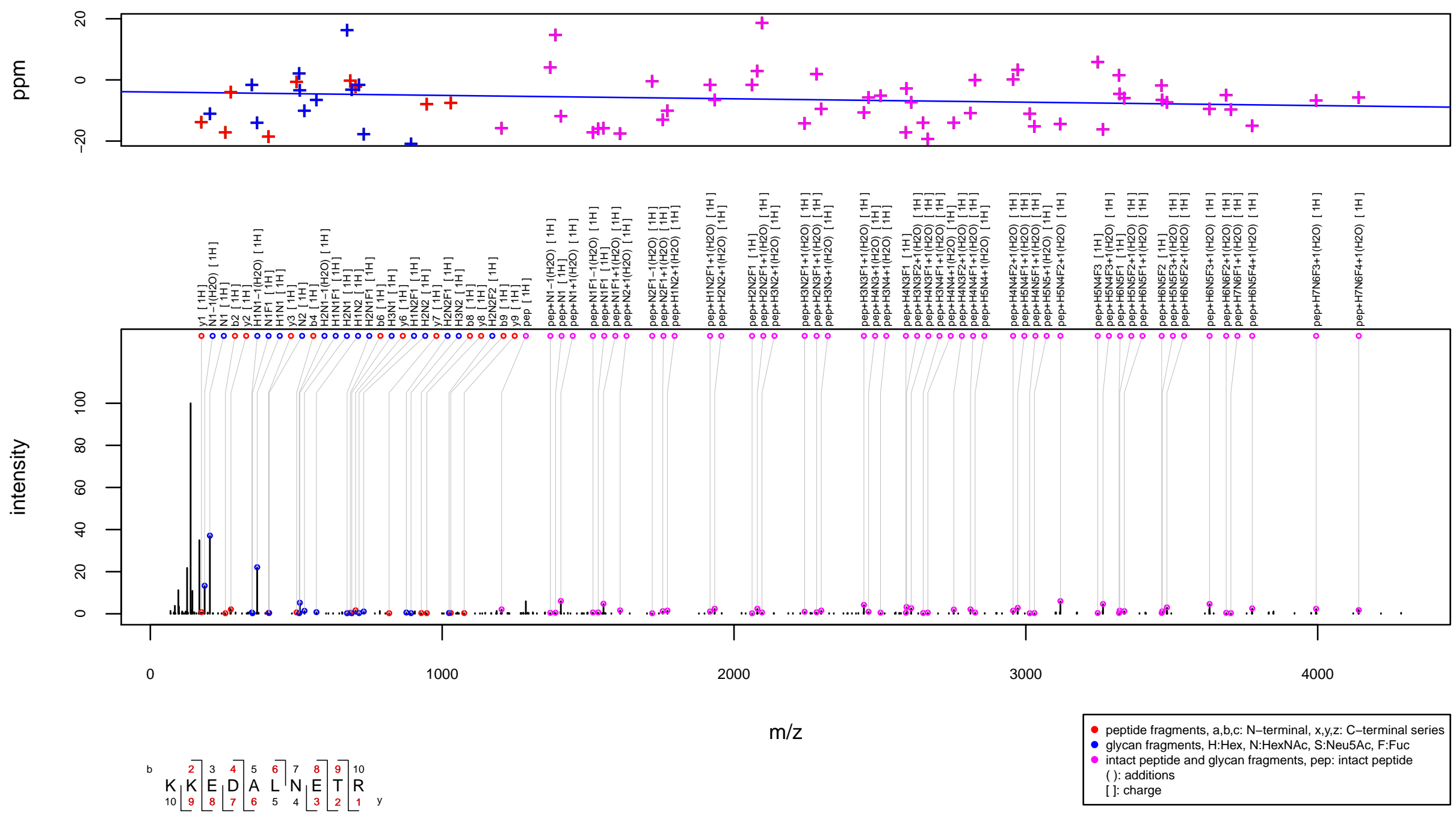
$\mathrm{m} / \mathrm{z} 1073.7472$ charge 4 scan $0-0$

Score $=37.41$, Hits $=30$, Explained Intensity $=0.28$

Peptide: FINC_HUMAN[997,1016]:LDAPTNLQFVNETDSTVLVR

Glycan: $\mathrm{SHNH}(\mathrm{HNH}) \mathrm{HN}(\mathrm{F}) \mathrm{N}$, S1H5N4F1

Charge: $4 \mathrm{H}$
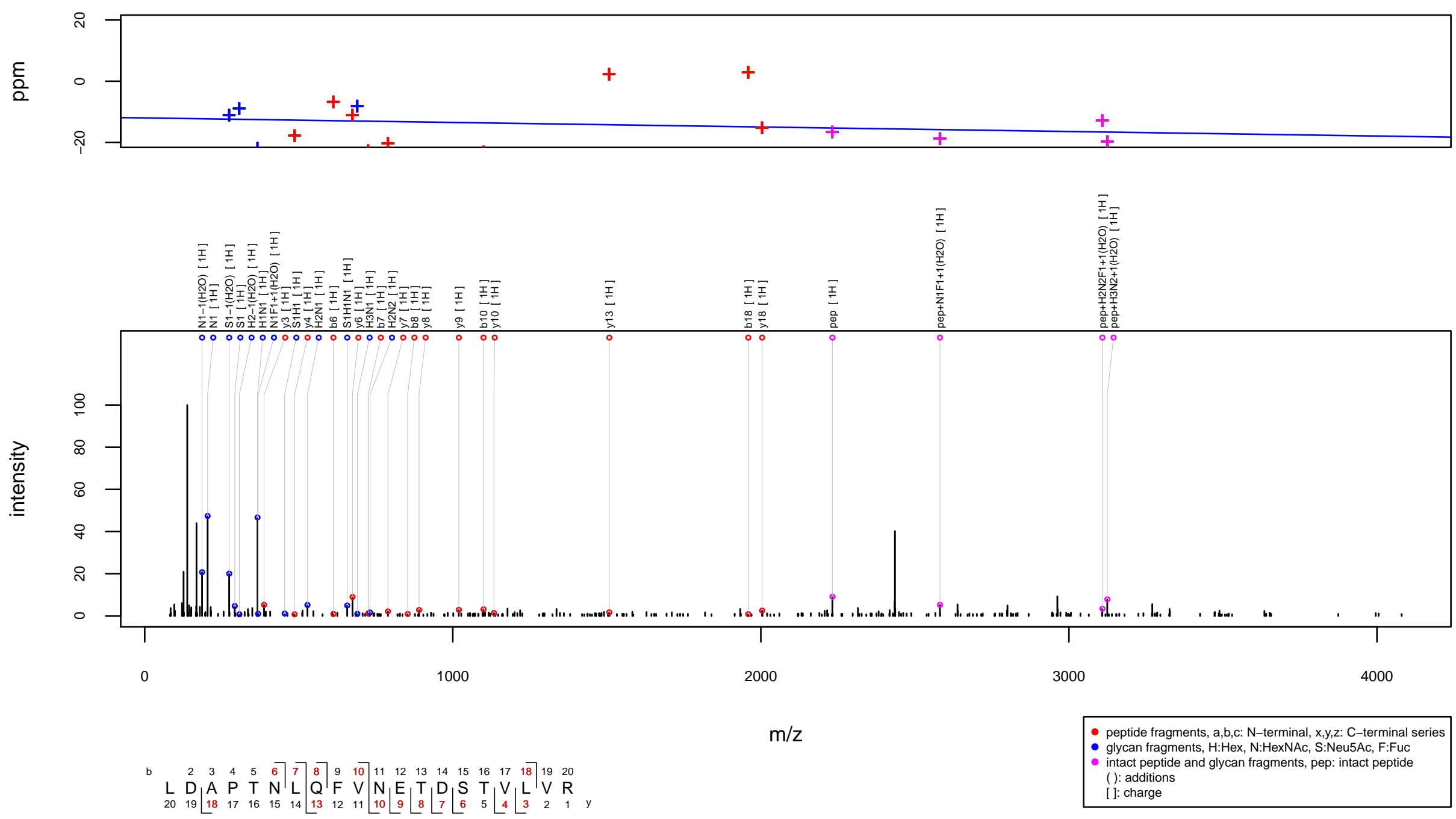
$\mathrm{m} / \mathrm{z} 1074.0127$ charge 4 scan $0-0$

Score $=87.19$, Hits $=59$, Explained Intensity $=0.42$

Peptide: FINC_HUMAN[997,1016]:LDAPTNLQFVNETDSTVLVR

Glycan: $\mathrm{FHNH}(\mathrm{FHNH}) \mathrm{HN}(\mathrm{F}) \mathrm{N}, \mathrm{H} 5 \mathrm{~N} 4 \mathrm{~F} 3$

Charge: $4 \mathrm{H}$
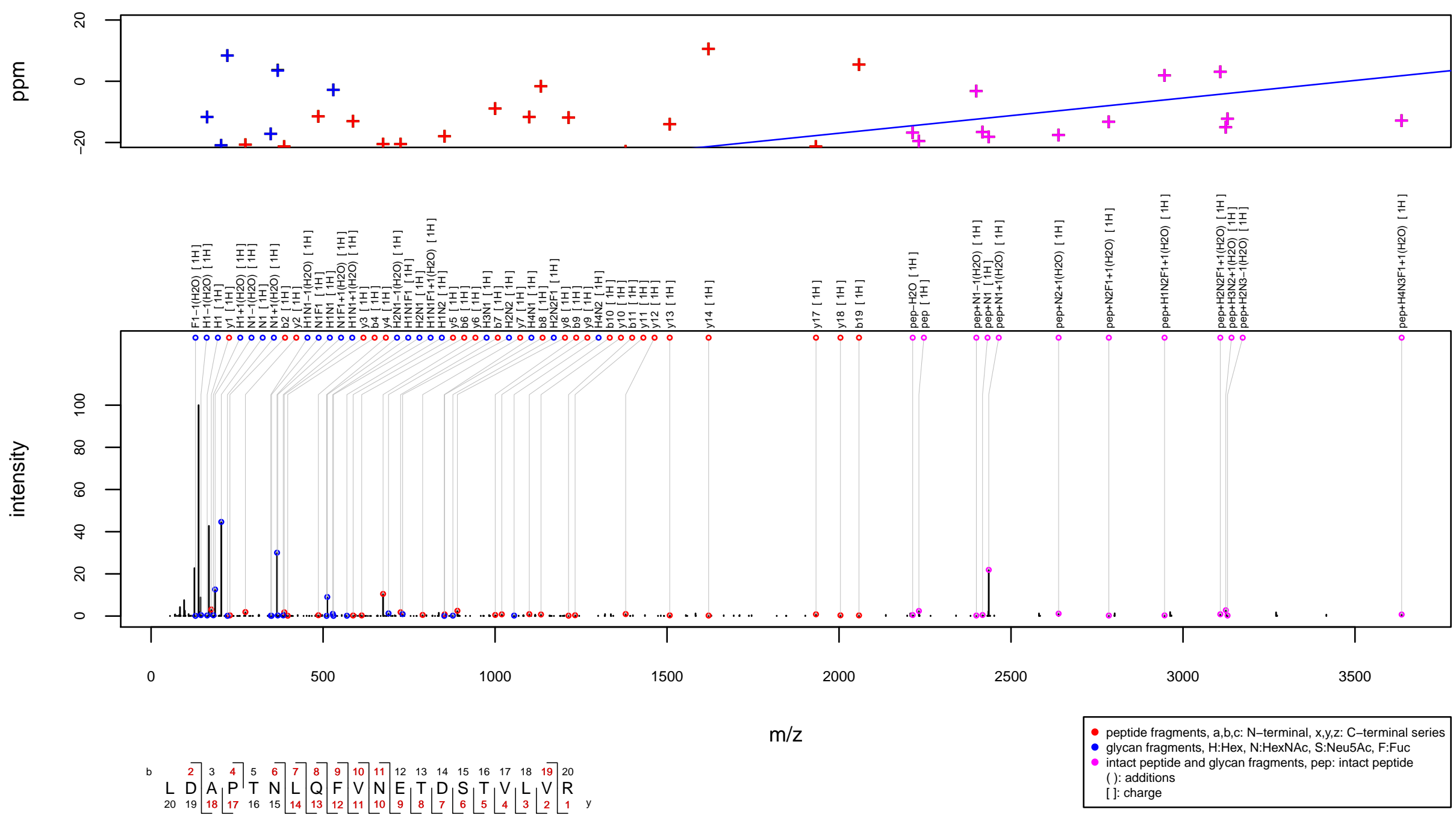
$\mathrm{m} / \mathrm{z} 1076.0712$ charge 3 scan $0-0$

Score $=87.94$, Hits $=73$, Explained Intensity $=0.43$ Peptide: PAEP_HUMAN[79,87]:WENNSCVEK

Glycan: H5N4F3

Charge: $3 \mathrm{H}$
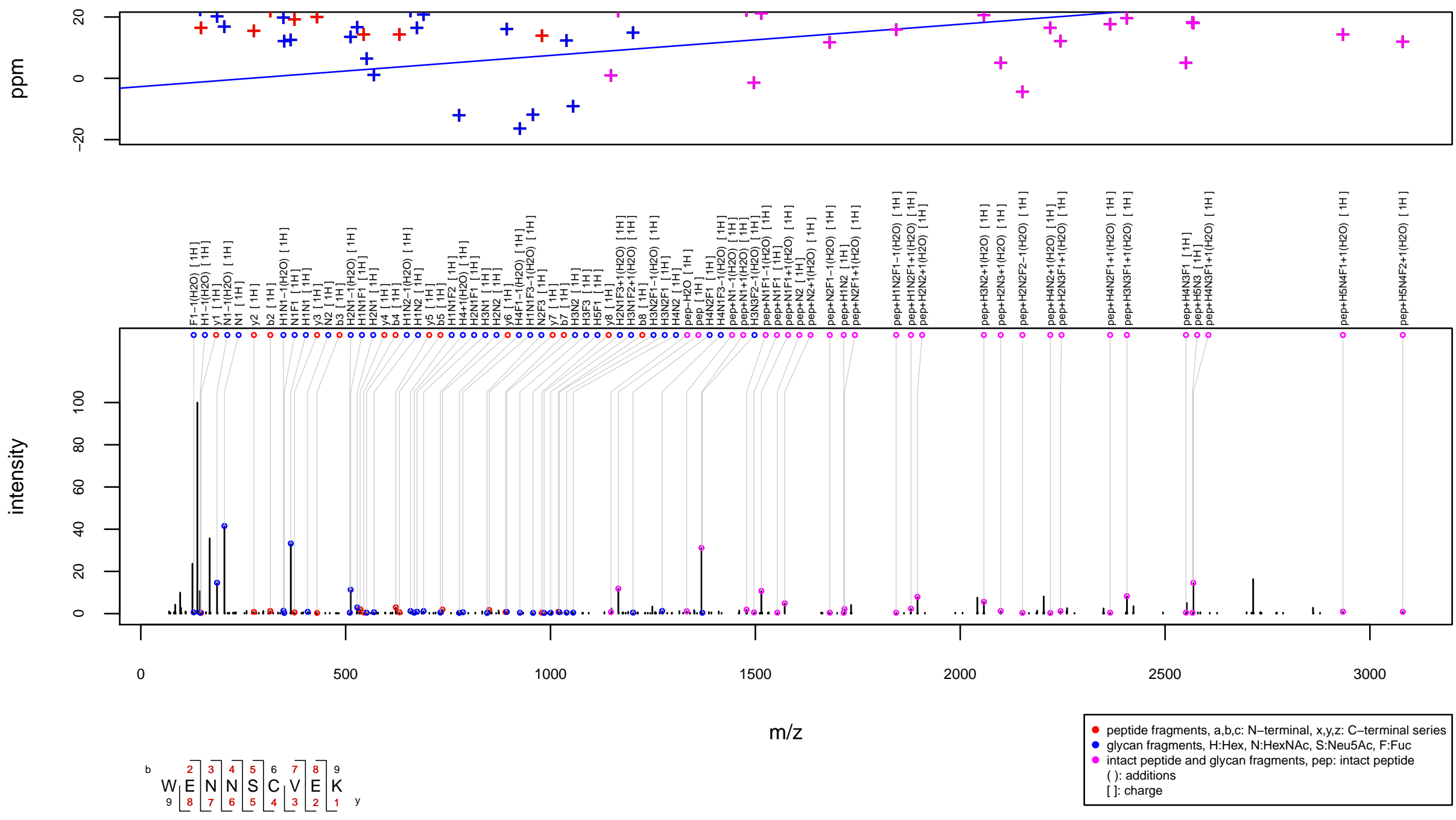
m/z 1076.0978 charge 3 scan $0-0$

Score $=113.56$, Hits $=76$, Explained Intensity $=0.61$ Peptide: SEMG2_HUMAN[272,282]:NLSQDQEHGRK Glycan: $\mathrm{F}(\mathrm{H}) \mathrm{HNH}(\mathrm{NH}) \mathrm{HN}(\mathrm{F}) \mathrm{N}, \mathrm{H} 5 \mathrm{~N} 4 \mathrm{~F} 2$

Charge: $3 \mathrm{H}$
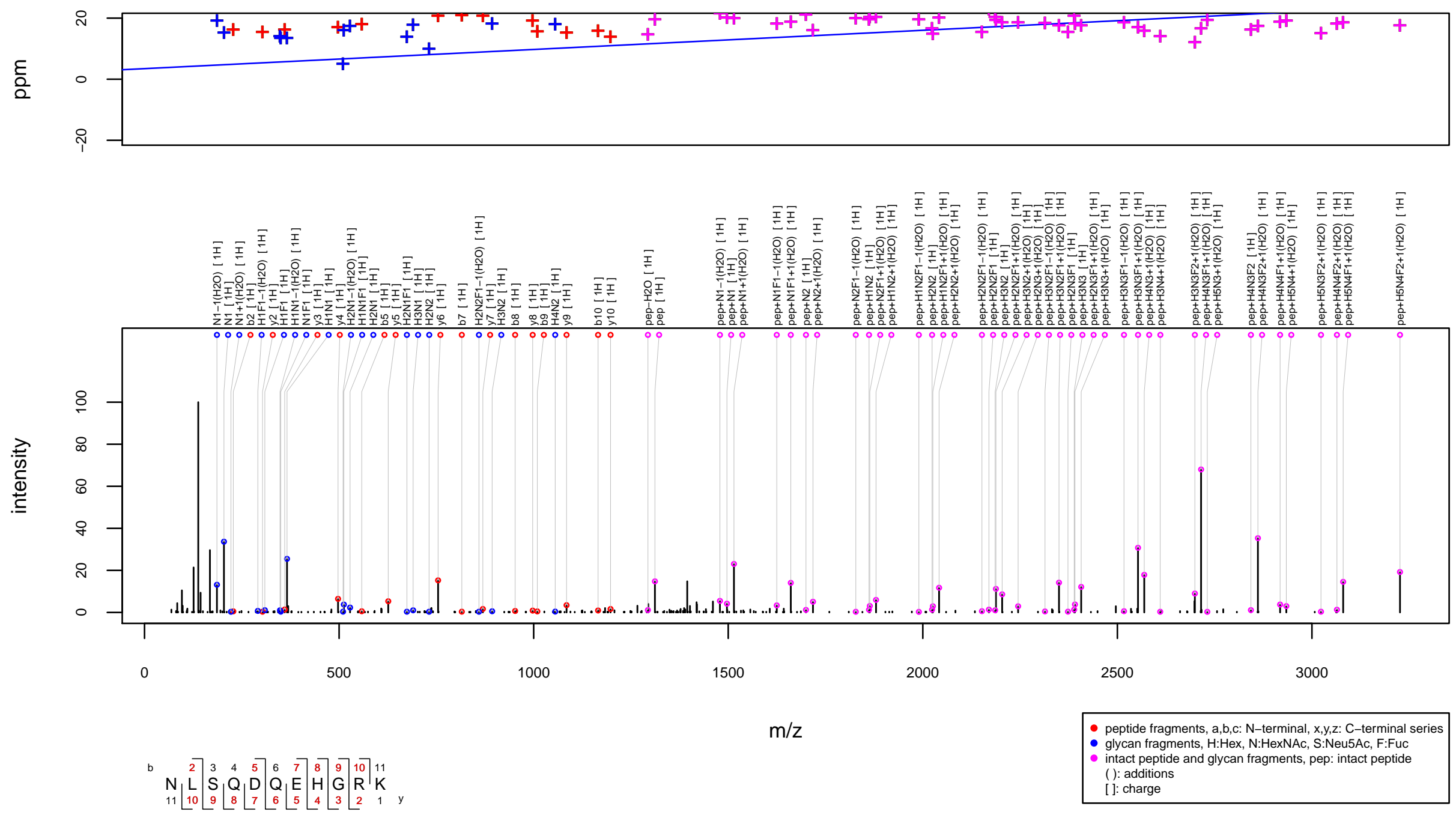
m/z 1077.932 charge 4 scan $0-0$

Score $=84.76$, Hits $=64$, Explained Intensity $=0.39$

Peptide: KLK11_HUMAN[120,137]:TATESFPHPGFNNSLPNK

Glycan: $\mathrm{SHNH}(\mathrm{SHNH}) \mathrm{HN}(\mathrm{F}) \mathrm{N}, \mathrm{S} 2 \mathrm{H} 5 \mathrm{~N} 4 \mathrm{~F} 1$

Charge: $4 \mathrm{H}$
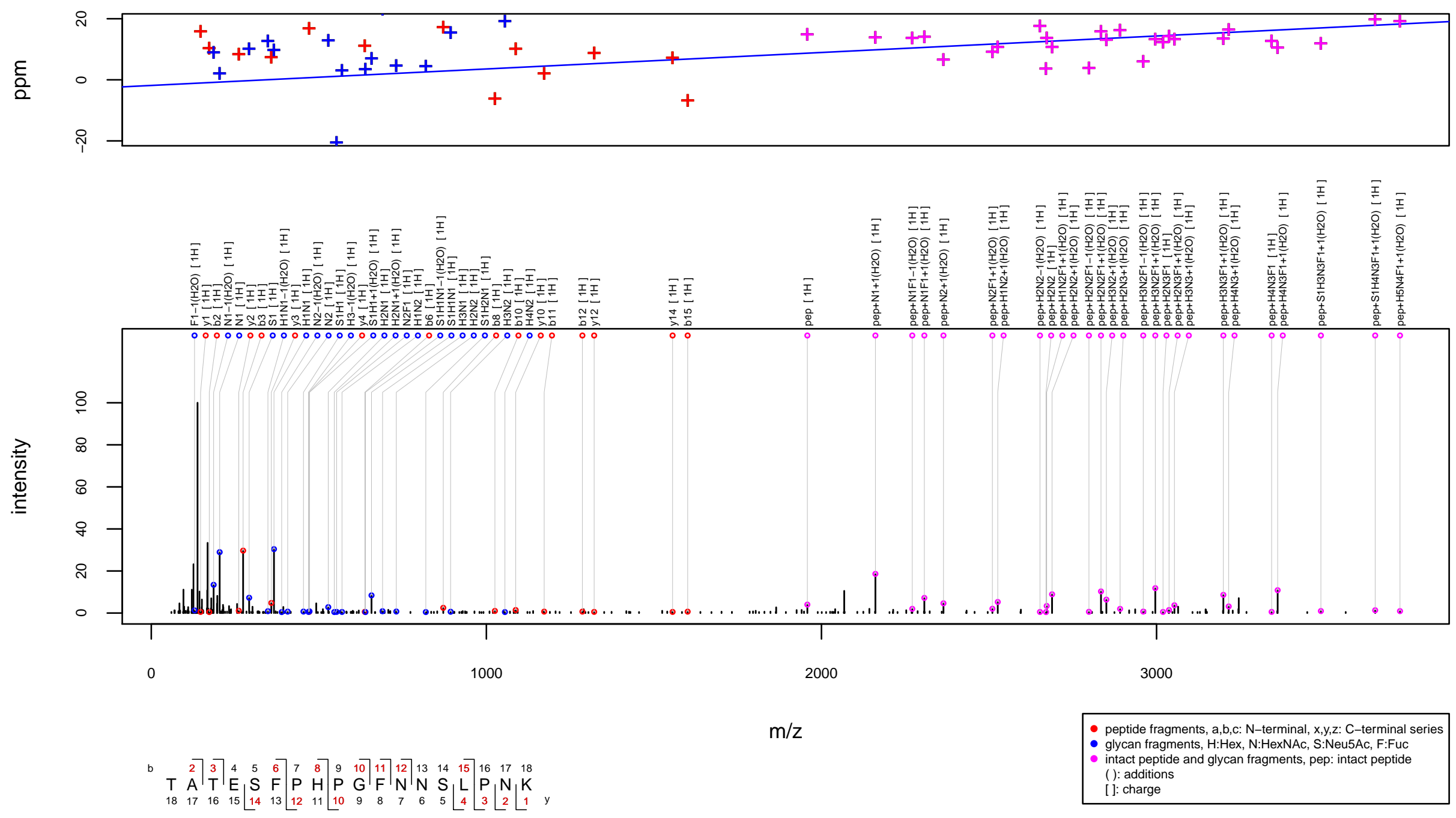
m/z 1077.9517 charge 4 scan 0-0

Score $=54.08$, Hits $=44$, Explained Intensity $=0.37$

Peptide: CLUS_HUMAN[372,385]:LANLTQGEDQYYLR

Glycan: S4H4N4

Charge: $4 \mathrm{H}$
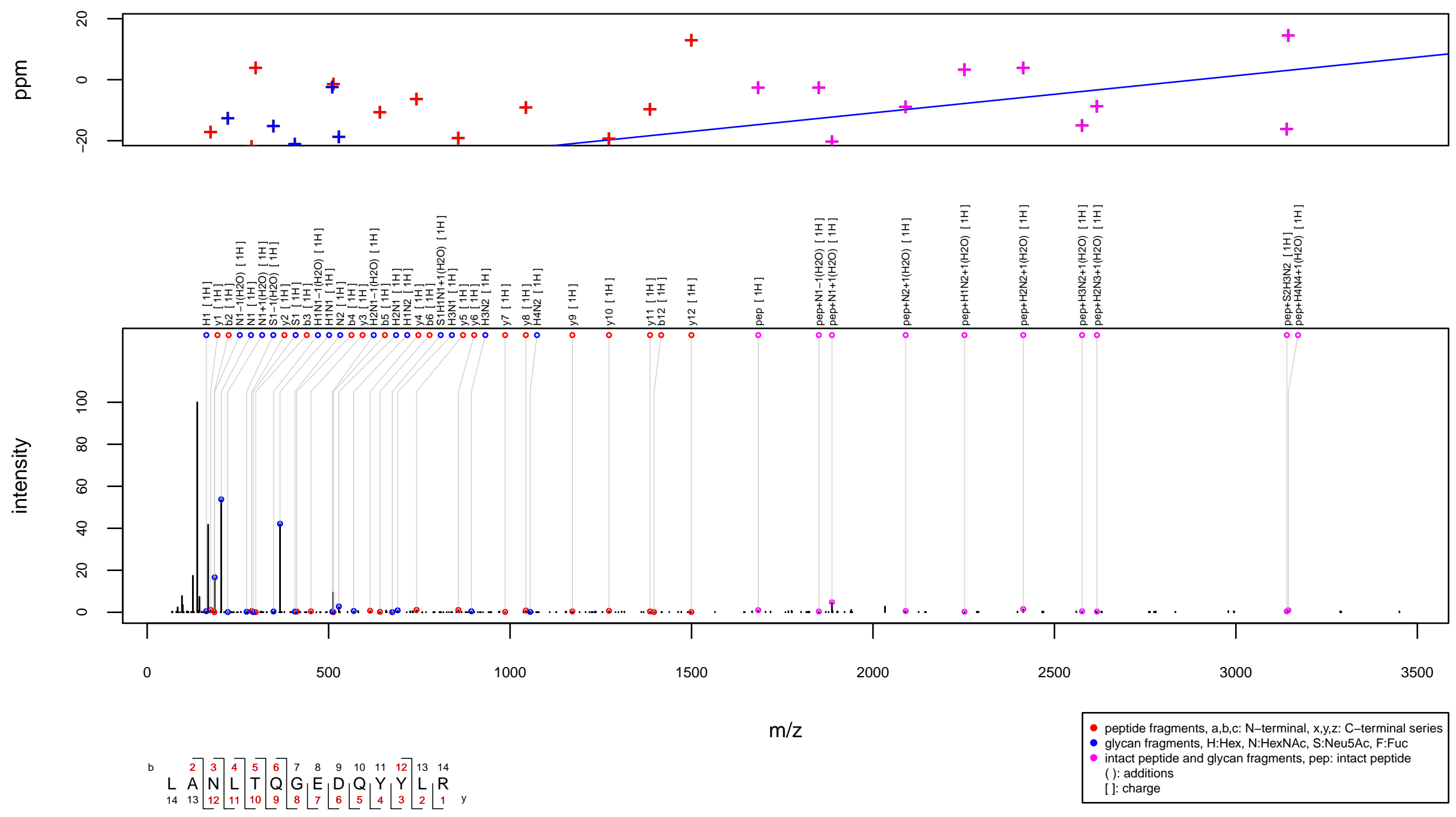
$\mathrm{m} / \mathrm{z} 1078.2074$ charge 4 scan $0-0$

Score $=61$, Hits $=64$, Explained Intensity $=0.42$

Peptide: CLUS_HUMAN[372,385]:LANLTQGEDQYYLR

Glycan: S3H4N4F2

Charge: $4 \mathrm{H}$
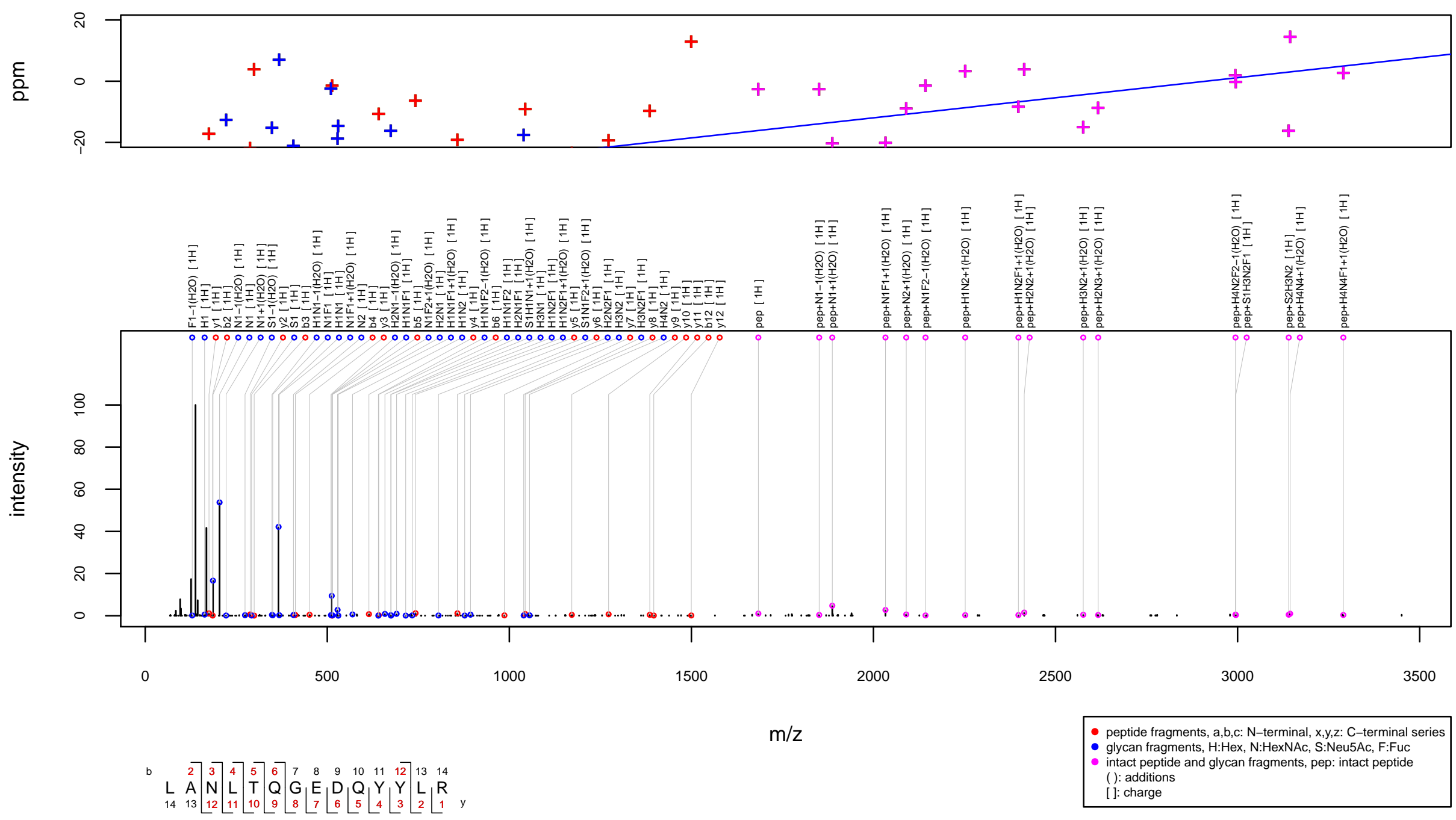
$\mathrm{m} / \mathrm{z} 1078.4546$ charge 3 scan $0-0$

Score $=85.96$, Hits $=55$, Explained Intensity $=0.46$ Peptide: PPAP_HUMAN[91,98]:KFLNESYK

Glycan: $\mathrm{SHNH}(\mathrm{SHNH}) \mathrm{HNN}, \mathrm{S} 2 \mathrm{H} 5 \mathrm{~N} 4$

Charge: $3 \mathrm{H}$
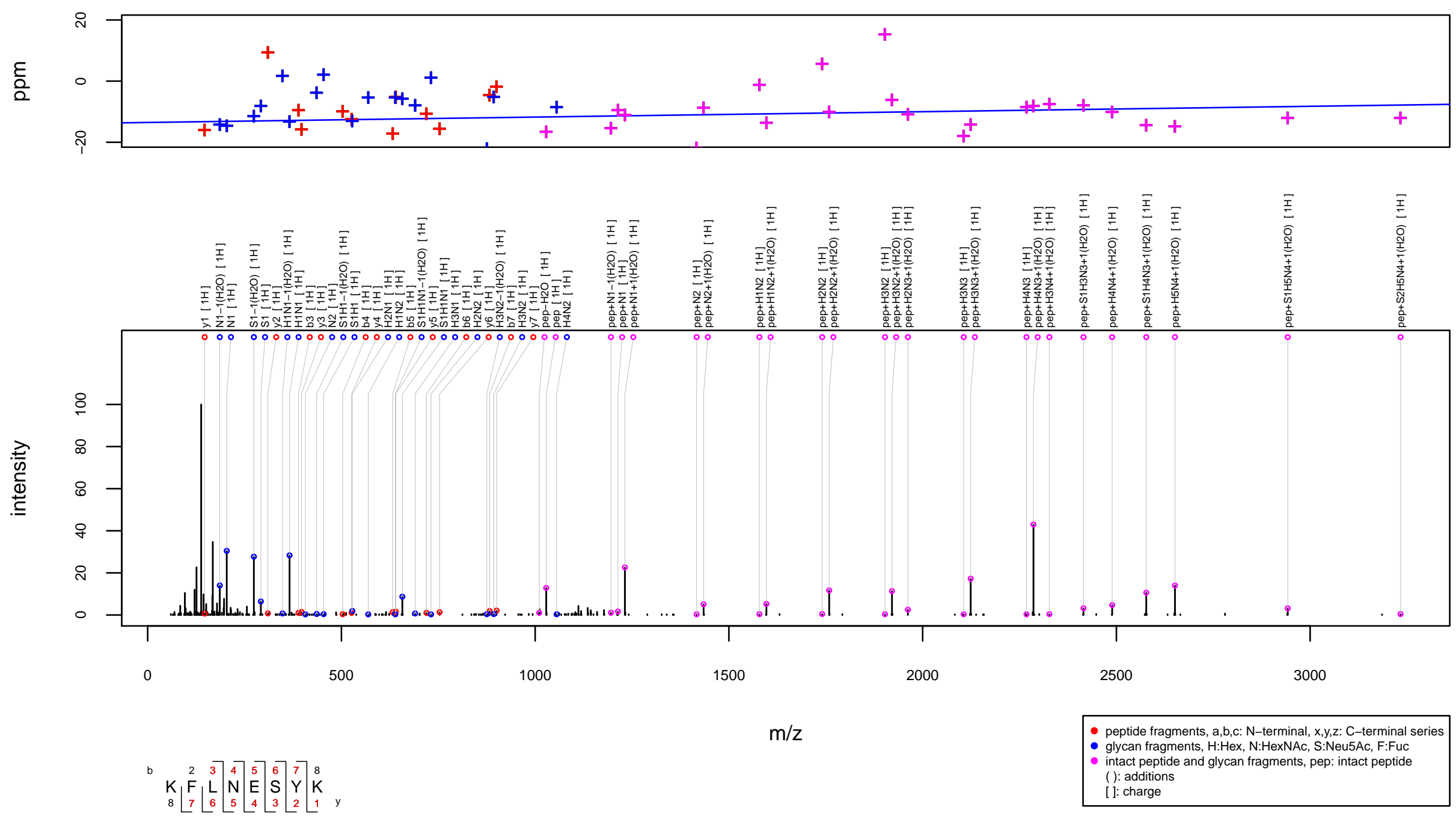
$\mathrm{m} / \mathrm{z} 1078.4568$ charge 3 scan $0-0$

Score $=65.91$, Hits $=48$, Explained Intensity $=0.45$ Peptide: CLUS_HUMAN[372,385]:LANLTQGEDQYYLR Glycan: H4N3F2

Charge: $3 \mathrm{H}$
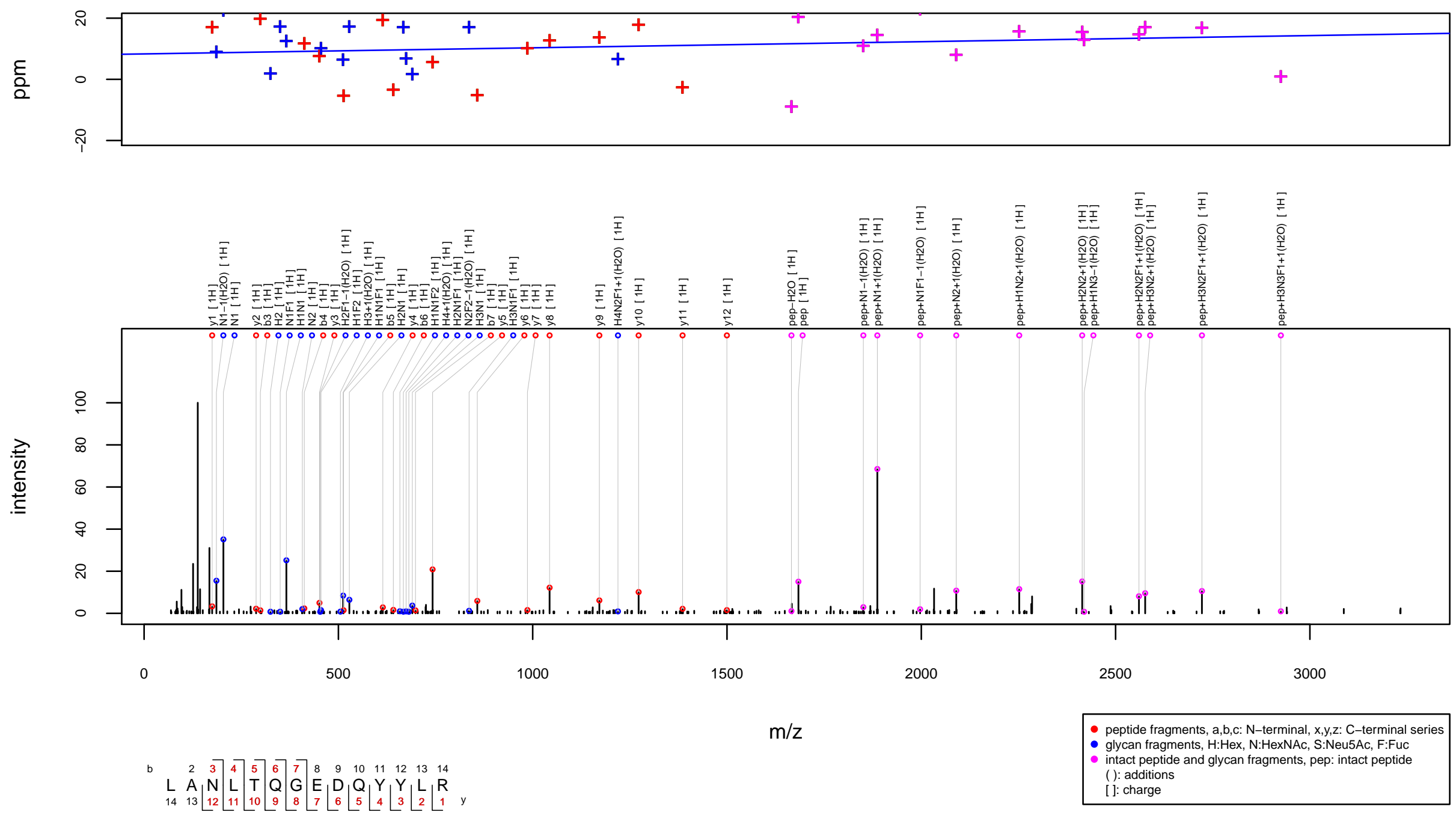
$\mathrm{m} / \mathrm{z} 1078.4623$ charge 4 scan $0-0$

Score $=60.51$, Hits $=68$, Explained Intensity $=0.42$

Peptide: CLUS_HUMAN[372,385]:LANLTQGEDQYYLR

Glycan: S2H4N4F4

Charge: $4 \mathrm{H}$
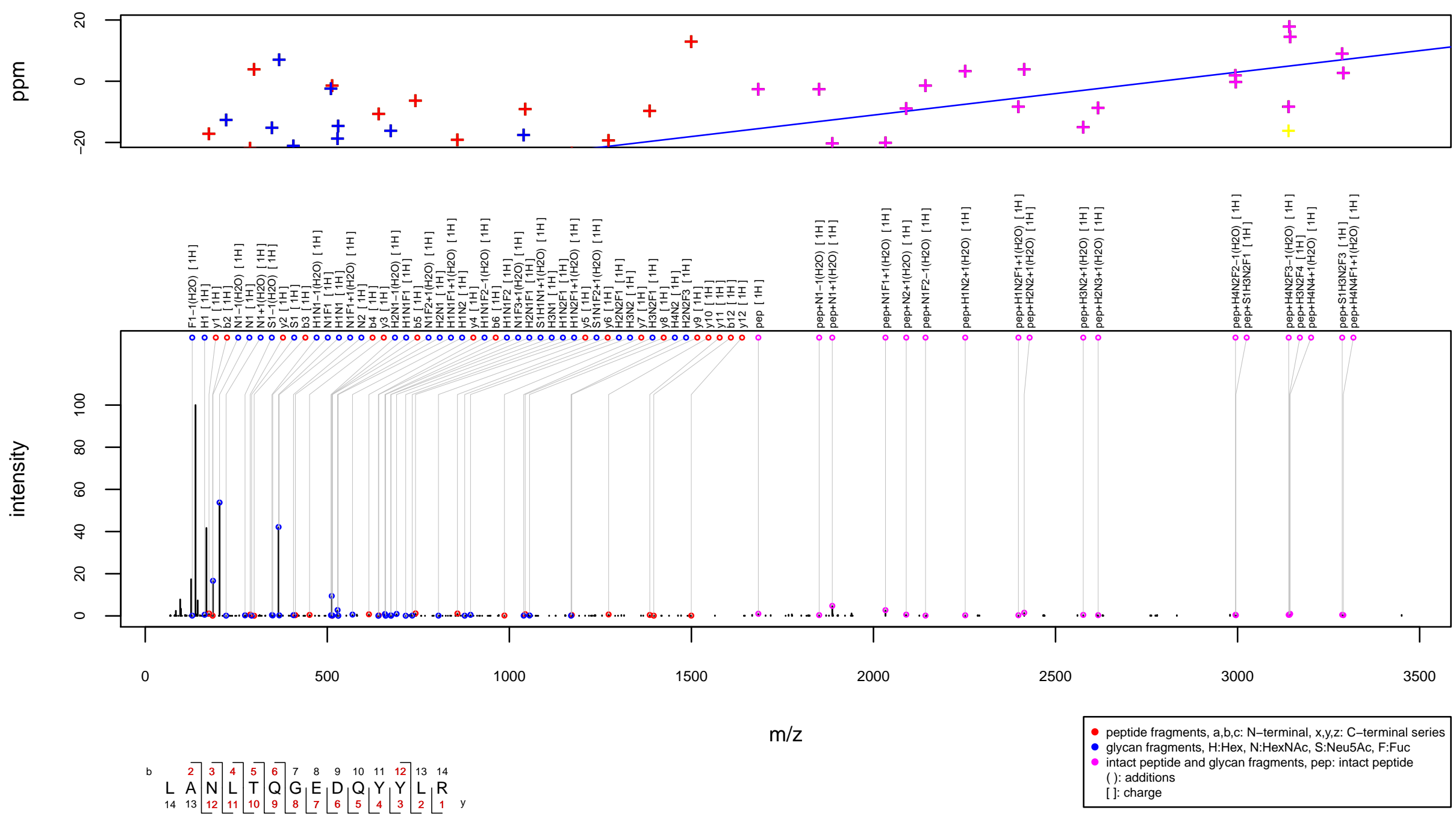
$\mathrm{m} / \mathrm{z} 1078.4963$ charge 3 scan $0-0$

Score $=65.32$, Hits $=43$, Explained Intensity $=0.35$ Peptide: CLUS_HUMAN[372,385]:LANLTQGEDQYYLR Glycan: $\mathrm{FHNH}(\mathrm{H}) \mathrm{HN}(\mathrm{F}) \mathrm{N}, \mathrm{H} 4 \mathrm{~N} 3 \mathrm{~F} 2$

Charge: $3 \mathrm{H}$
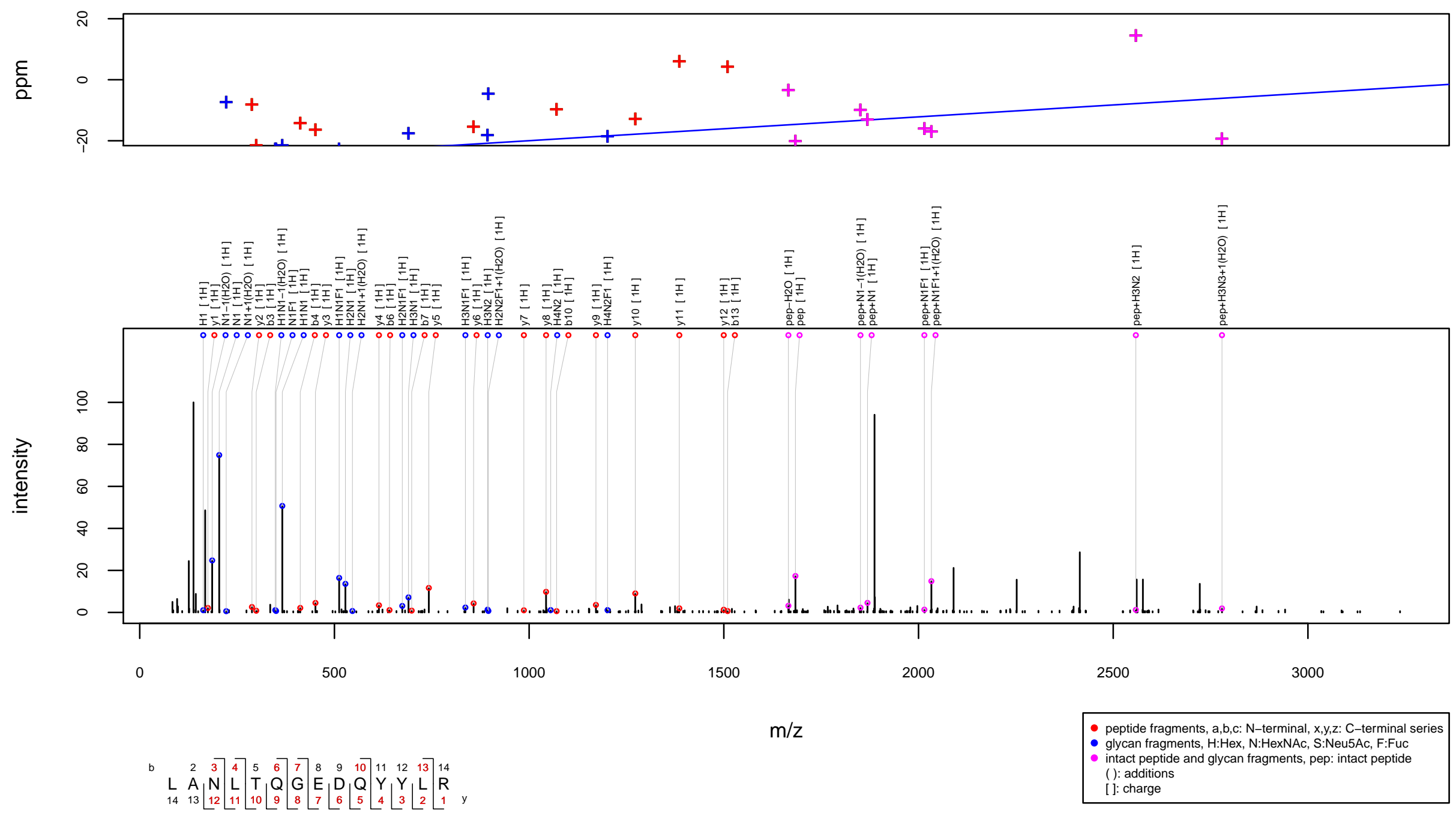
$\mathrm{m} / \mathrm{z} 1081.4478$ charge 4 scan $0-0$

Score $=104.73$, Hits $=70$, Explained Intensity $=0.51$

Peptide: SAP_HUMAN[214,232]:TNSTFVQALVEHVKEECDR

Glycan: $\mathrm{FH}(\mathrm{F}) \mathrm{NH}(\mathrm{HNH}) \mathrm{HN}(\mathrm{F}) \mathrm{N}, \mathrm{H} 5 \mathrm{~N} 4 \mathrm{~F} 3$

Charge: $4 \mathrm{H}$
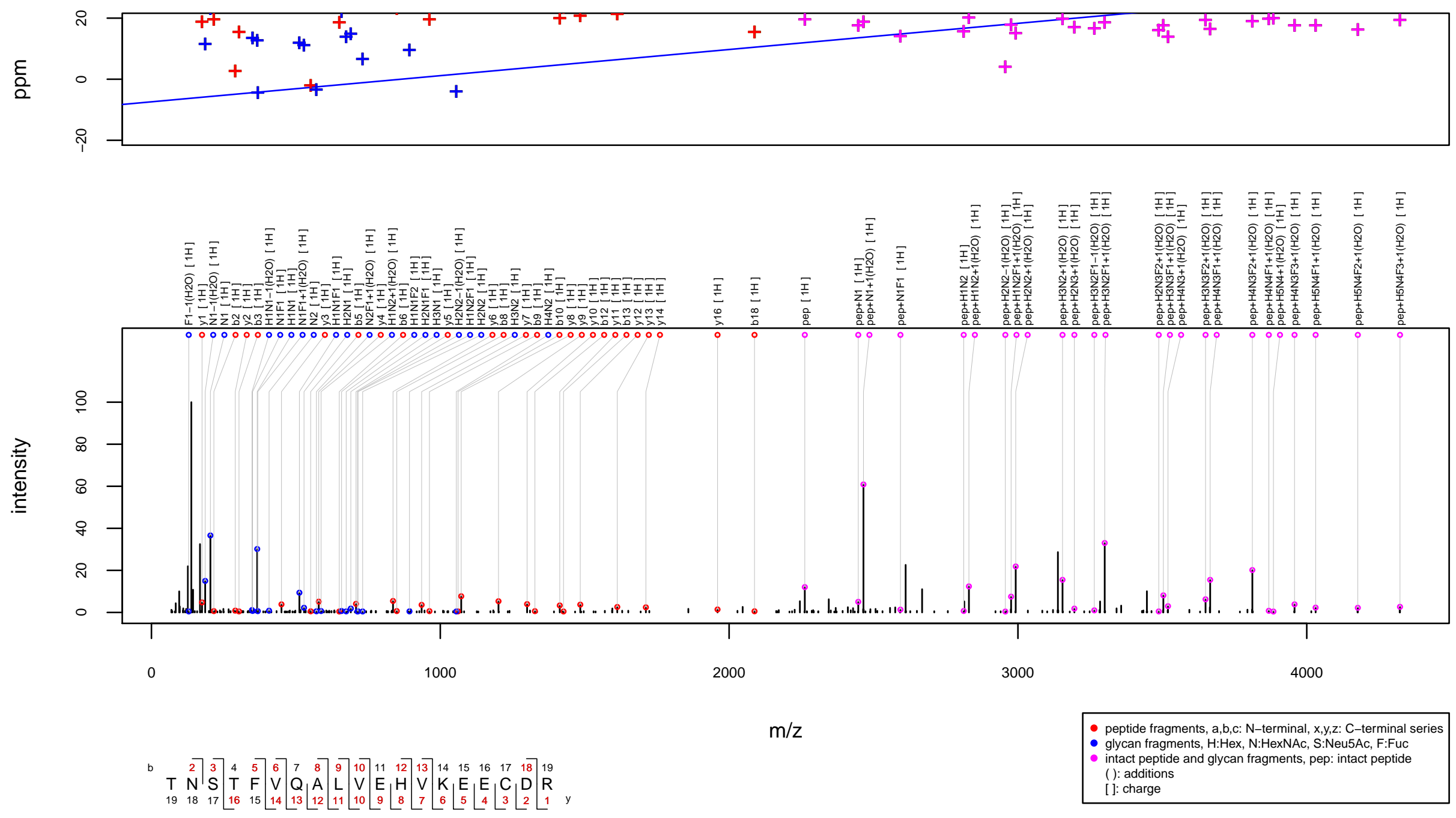
m/z 1081.989 charge 4 scan 0-0

Score $=79.62$, Hits $=62$, Explained Intensity $=0.48$ Peptide: TIMP1_HUMAN[46,60]:FVGTPEVNQTTLYQR Glycan: $\mathrm{F}(\mathrm{H}) \mathrm{NH}(\mathrm{F}(\mathrm{H}) \mathrm{N}(\mathrm{F}(\mathrm{H}) \mathrm{N}) \mathrm{H}) \mathrm{HN}(\mathrm{F}) \mathrm{N}, \mathrm{H} 6 \mathrm{~N} 5 \mathrm{~F} 4$

Charge: $4 \mathrm{H}$
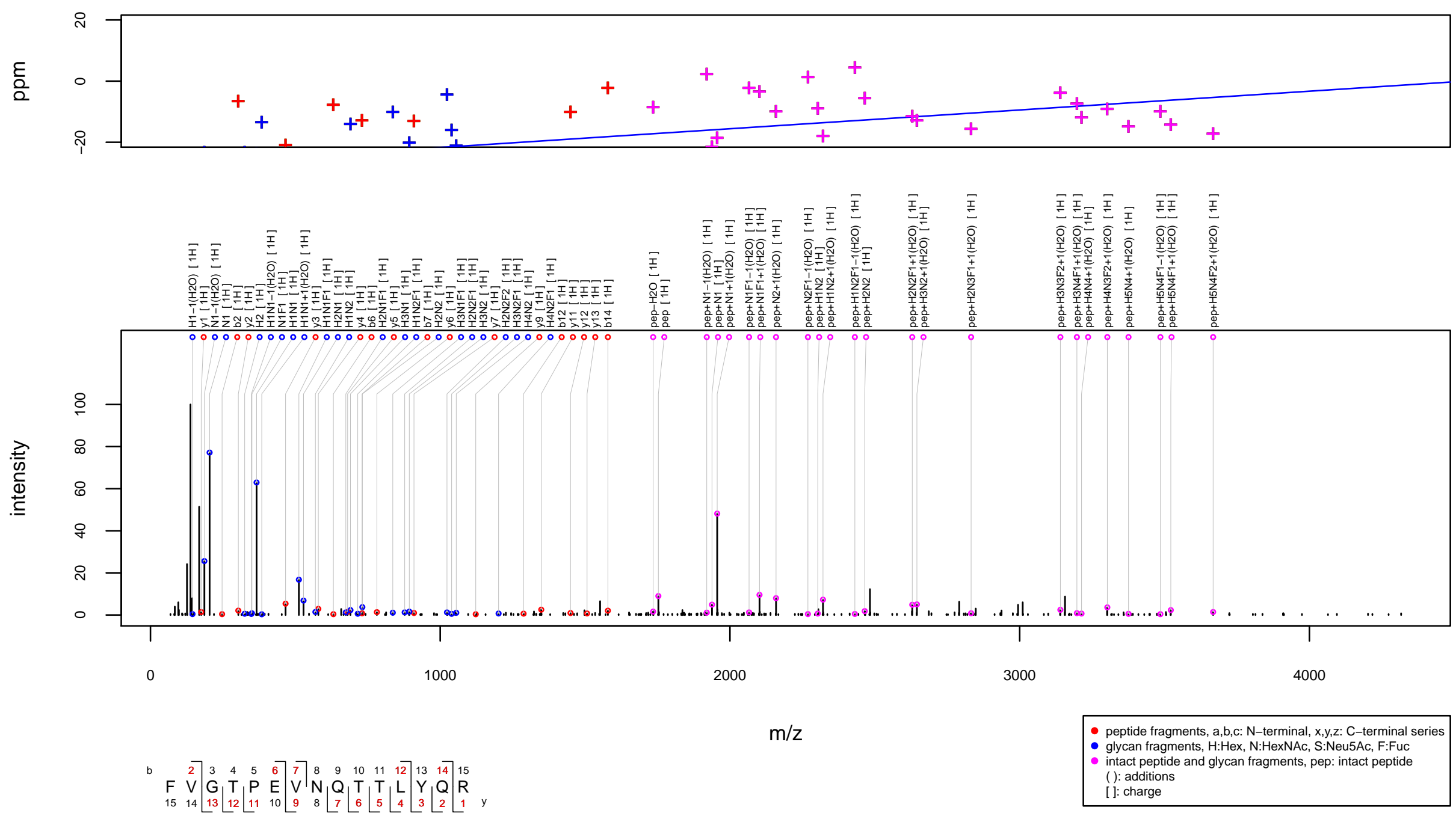
$\mathrm{m} / \mathrm{z} 1082.0856$ charge 3 scan $0-0$

Score $=127.15$, Hits $=87$, Explained Intensity $=0.62$ Peptide: SEMG2_HUMAN[272,281]:NLSQDQEHGR Glycan: $\mathrm{FH}(\mathrm{F}) \mathrm{NH}(\mathrm{HNH}) \mathrm{HN}(\mathrm{F}) \mathrm{N}, \mathrm{H} 5 \mathrm{~N} 4 \mathrm{~F} 3$

Charge: $3 \mathrm{H}$
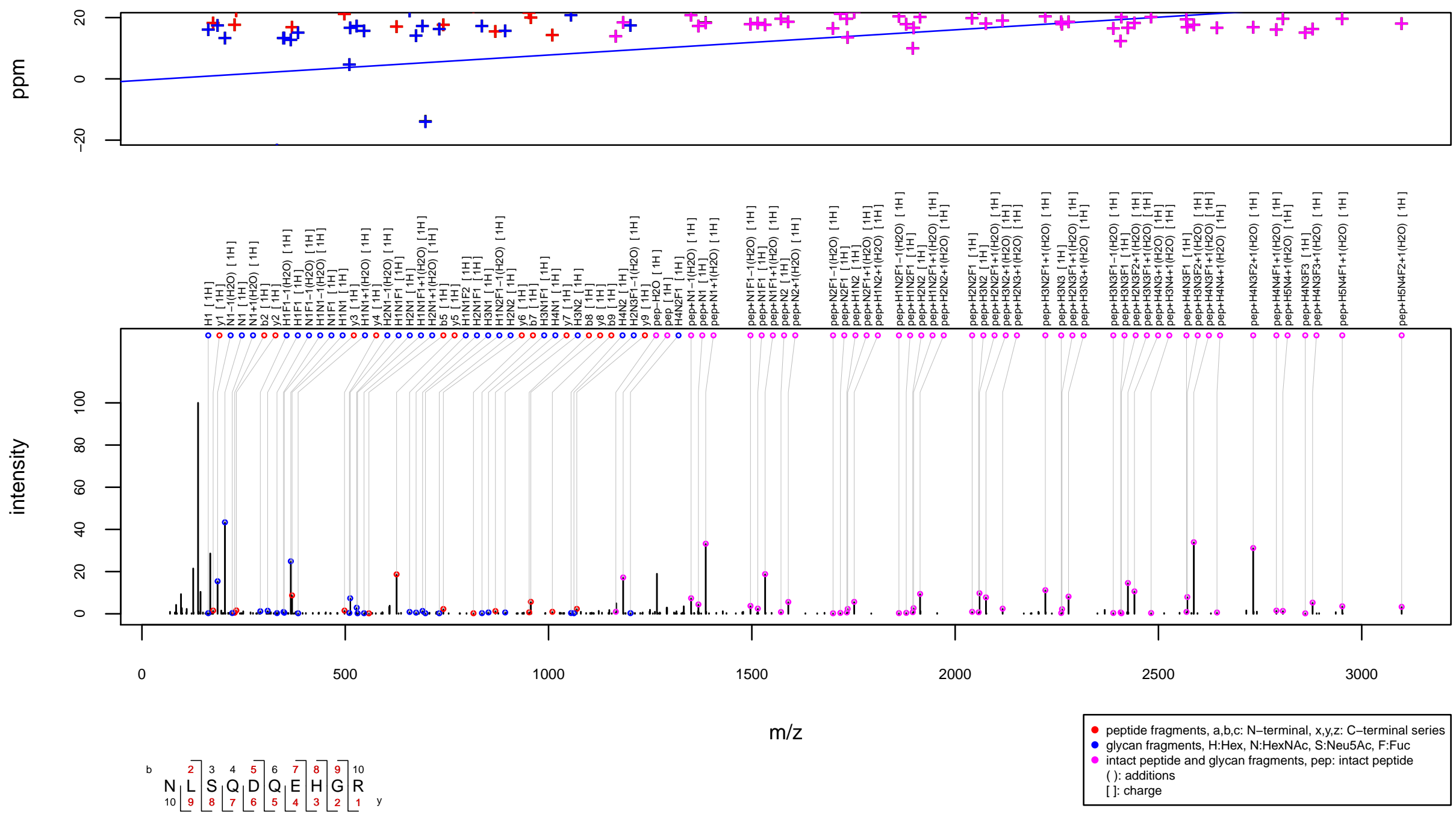
$\mathrm{m} / \mathrm{z} 1084.4346$ charge 3 scan $0-0$

Score $=97.95$, Hits $=68$, Explained Intensity $=0.49$

Peptide: PPAP_HUMAN[92,98]:FLNESYK

Glycan: $\mathrm{SHNH}(\mathrm{SHNH}) \mathrm{HN}(\mathrm{F}) \mathrm{N}, \mathrm{S} 2 \mathrm{H} 5 \mathrm{~N} 4 \mathrm{~F} 1$

Charge: $3 \mathrm{H}$
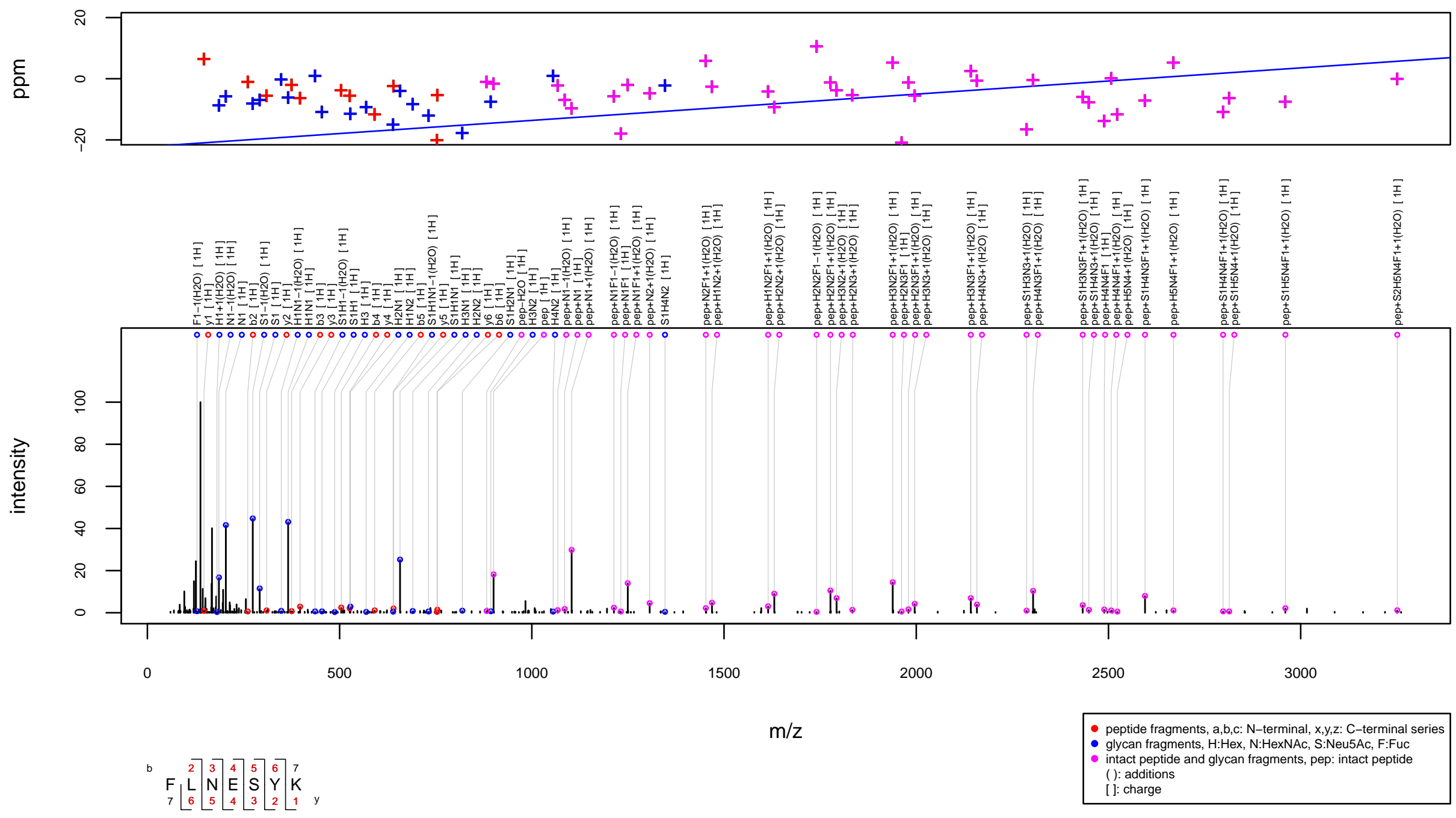
$\mathrm{m} / \mathrm{z} 1085.751$ charge 4 scan $0-0$

Score $=29.97$, Hits $=27$, Explained Intensity $=0.26$

Peptide: EHMT1_HUMAN[232,246]:EPKEEINKNISDFGR

Glycan: S1H4N8

Charge: $4 \mathrm{H}$
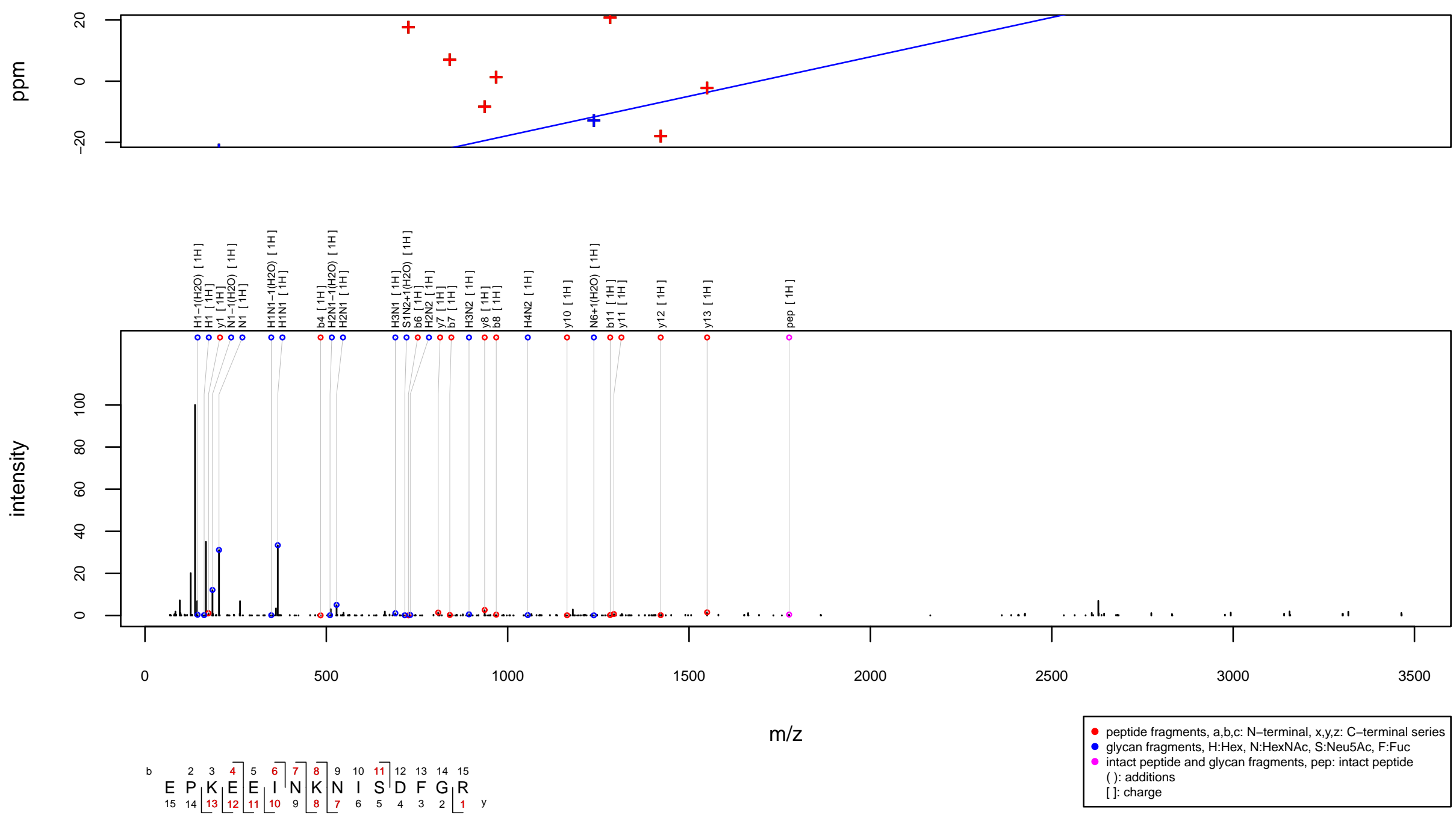
$\mathrm{m} / \mathrm{z} 1094.8289$ charge 3 scan 0-0

Score $=56.38$, Hits $=35$, Explained Intensity $=0.2$

Peptide: ZA2G_HUMAN[103,120]:DIVEYYNDSNGSHVLQGR

Glycan: $\mathrm{H}(\mathrm{H}) \mathrm{H}(\mathrm{H}) \mathrm{HNN}, \mathrm{H} 5 \mathrm{~N} 2$

Charge: $3 \mathrm{H}$
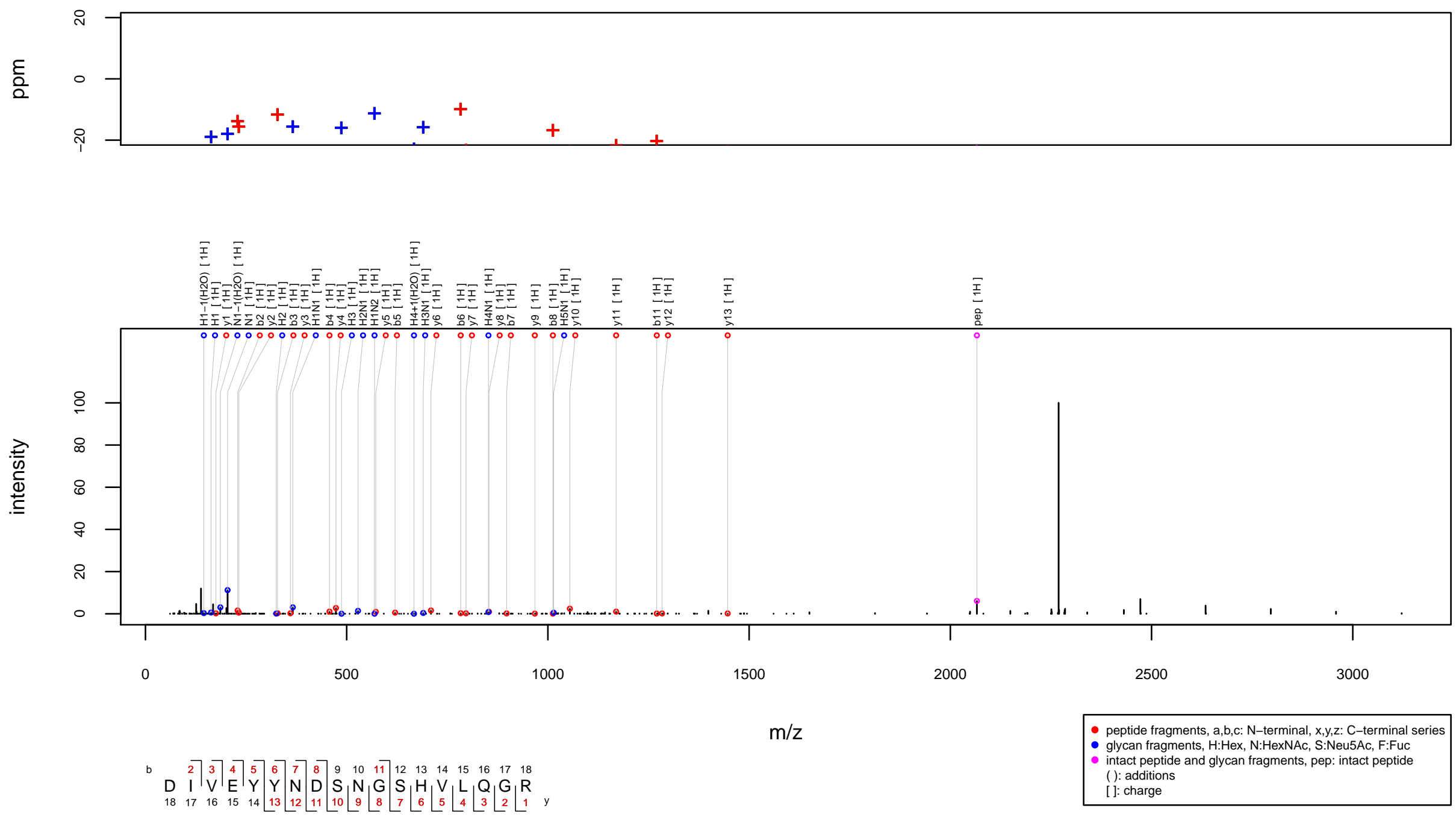
$\mathrm{m} / \mathrm{z} 1095.2506$ charge 4 scan $0-0$

Score $=40.22$, Hits $=30$, Explained Intensity $=0.27$

Peptide: AMPN_HUMAN[253,274]:GPSTPLPEDPNWNVTEFHTTPK

Glycan: $\mathrm{SHNH}(\mathrm{HNH}) \mathrm{HNN}, \mathrm{S} 1 \mathrm{H} 5 \mathrm{~N} 4$

Charge: $4 \mathrm{H}$
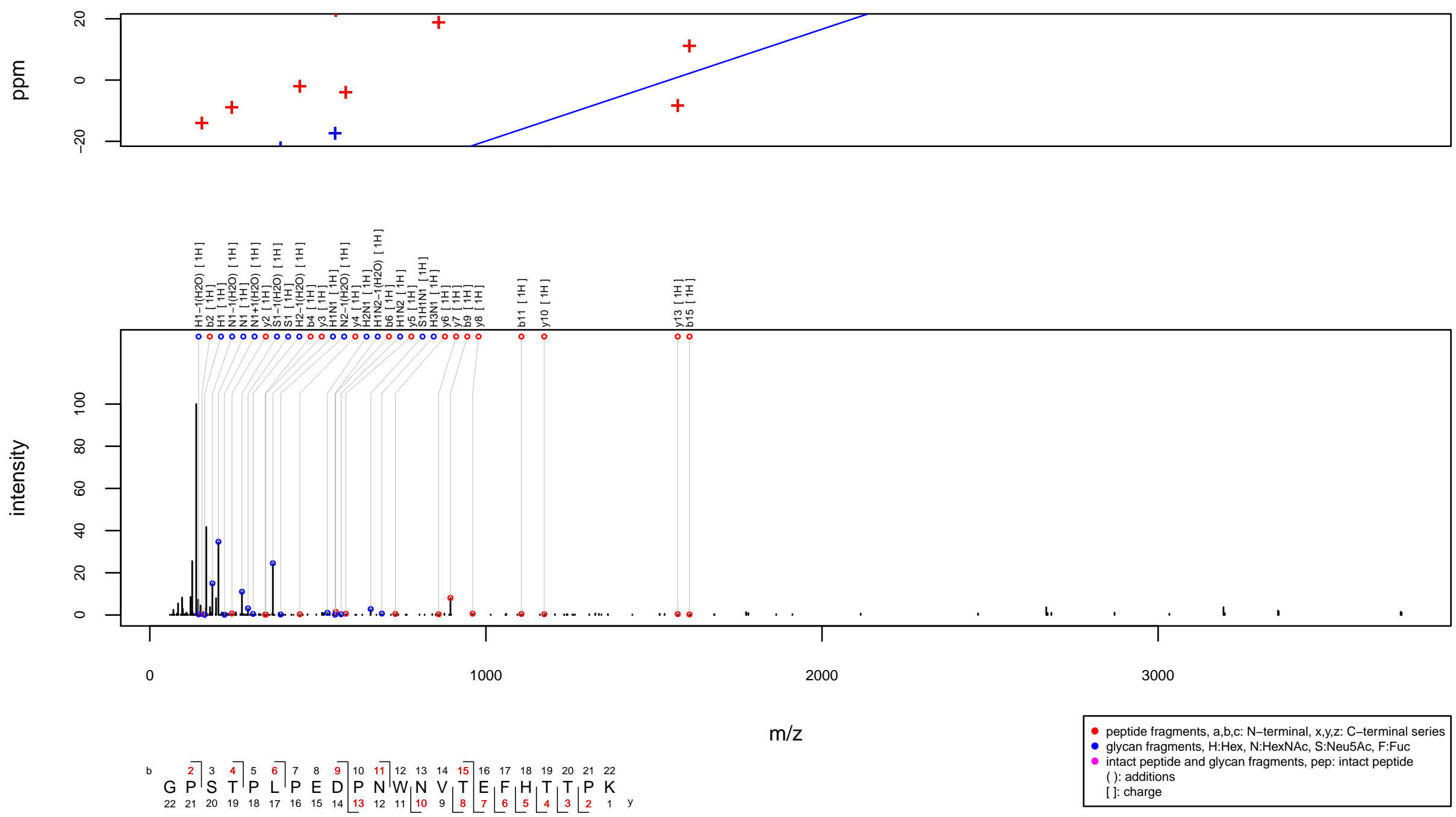
$\mathrm{m} / \mathrm{z} 1095.5071$ charge 4 scan $0-0$

Score $=41$, Hits $=31$, Explained Intensity $=0.23$

Peptide: AMPN_HUMAN[253,274]:GPSTPLPEDPNWNVTEFHTTPK

Glycan: $\mathrm{FHNH}(\mathrm{FHNH}) \mathrm{HNN}, \mathrm{H} 5 \mathrm{~N} 4 \mathrm{~F} 2$

Charge: $4 \mathrm{H}$
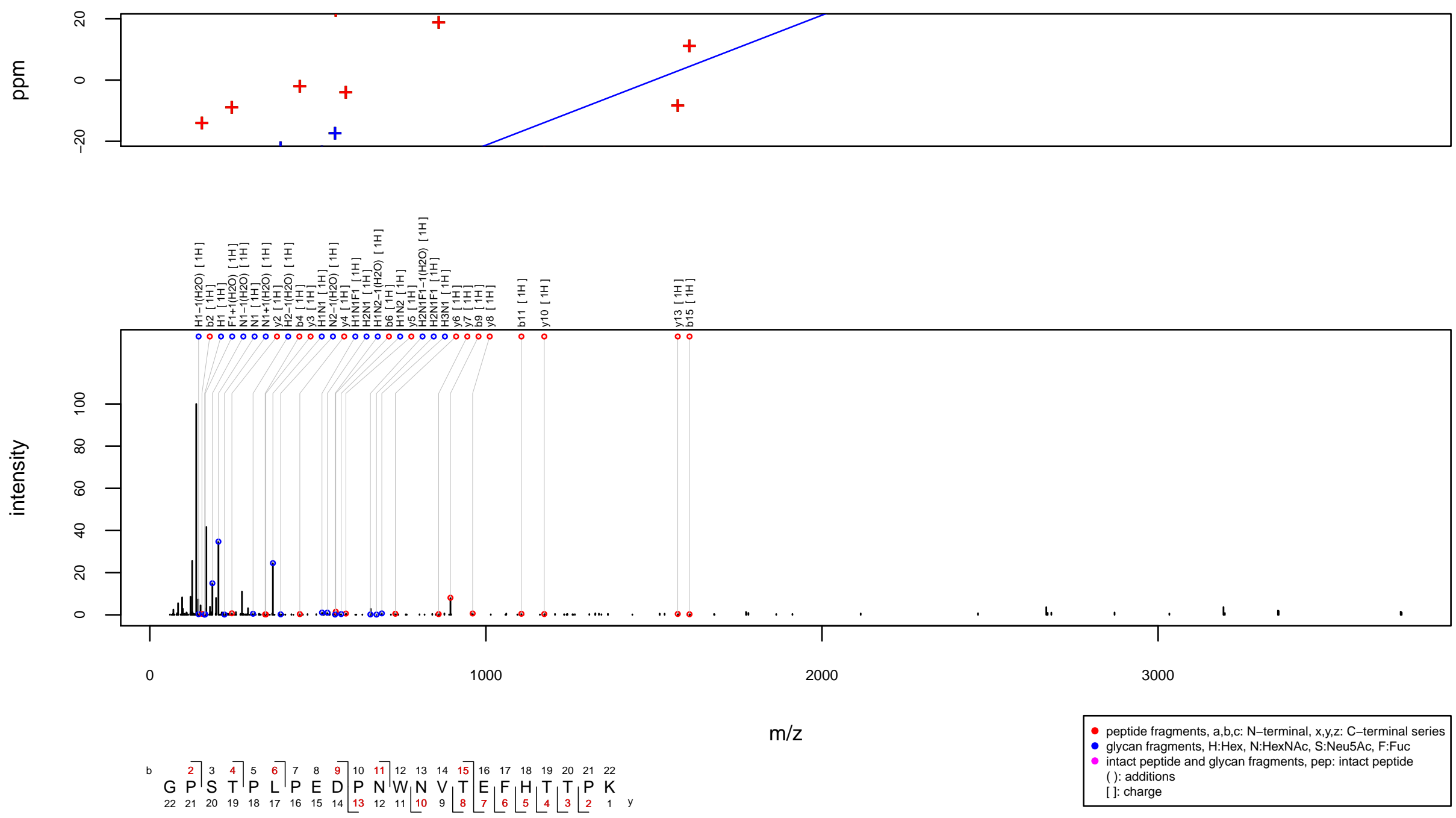
$\mathrm{m} / \mathrm{z} 1096.2025$ charge 3 scan 0-0

Score $=63.04$, Hits $=34$, Explained Intensity $=0.34$

Peptide: FINC_HUMAN[997,1016]:LDAPTNLQFVNETDSTVLVR

Glycan: $\mathrm{HH}(\mathrm{H}) \mathrm{HNN}, \mathrm{H} 4 \mathrm{~N} 2$

Charge: $3 \mathrm{H}$
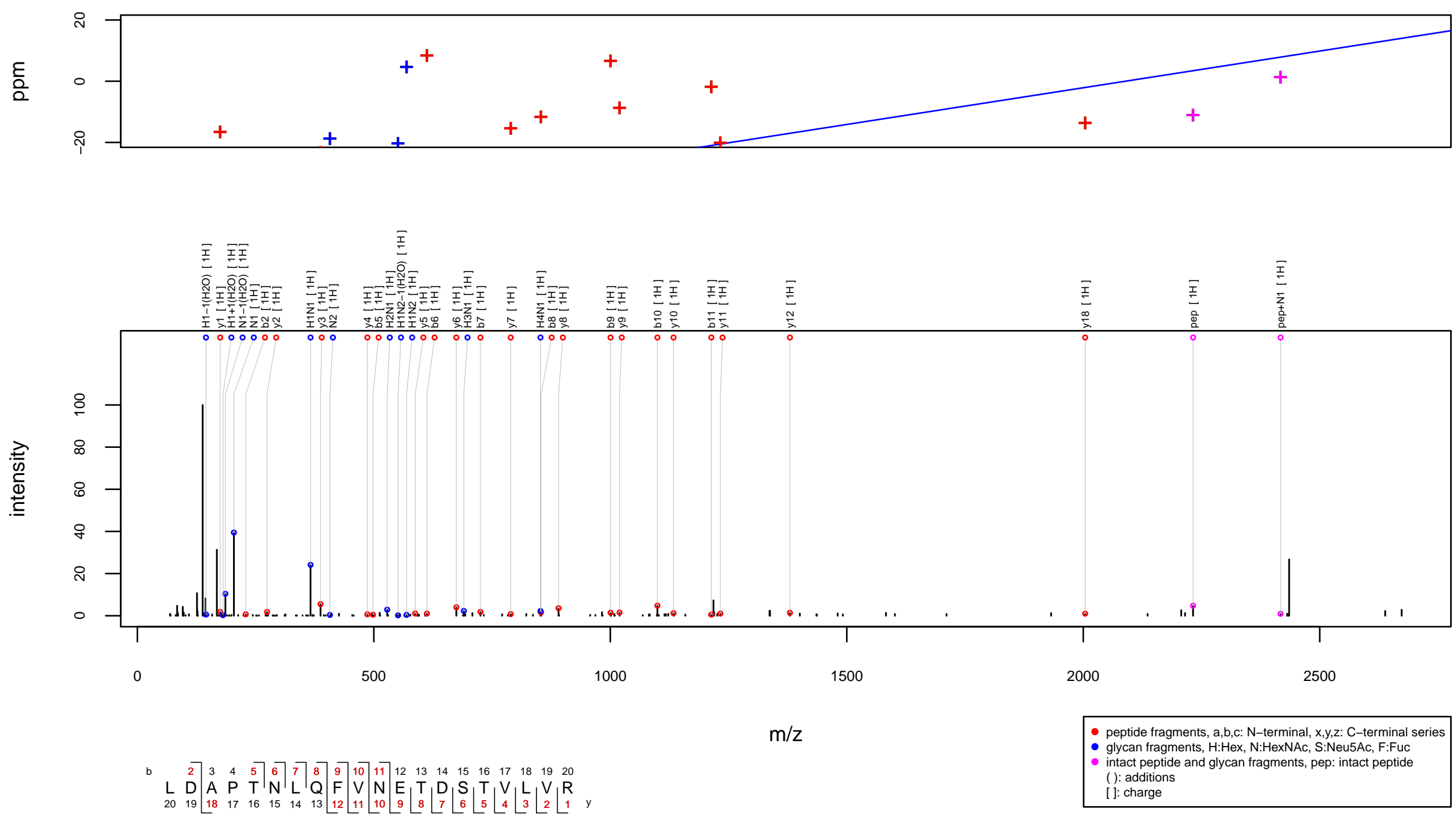
$\mathrm{m} / \mathrm{z} 1100.9901$ charge 4 scan 0-0

Score $=51.59$, Hits $=47$, Explained Intensity $=0.41$ Peptide: CLUS_HUMAN[372,385]:LANLTQGEDQYYLR Glycan: $\mathrm{F}(\mathrm{H}) \mathrm{NH}(\mathrm{F}) \mathrm{NH}(\mathrm{SHNH}) \mathrm{HN}(\mathrm{F}) \mathrm{N}, \mathrm{S} 1 \mathrm{H} 6 \mathrm{~N} 5 \mathrm{~F} 3$

Charge: $4 \mathrm{H}$
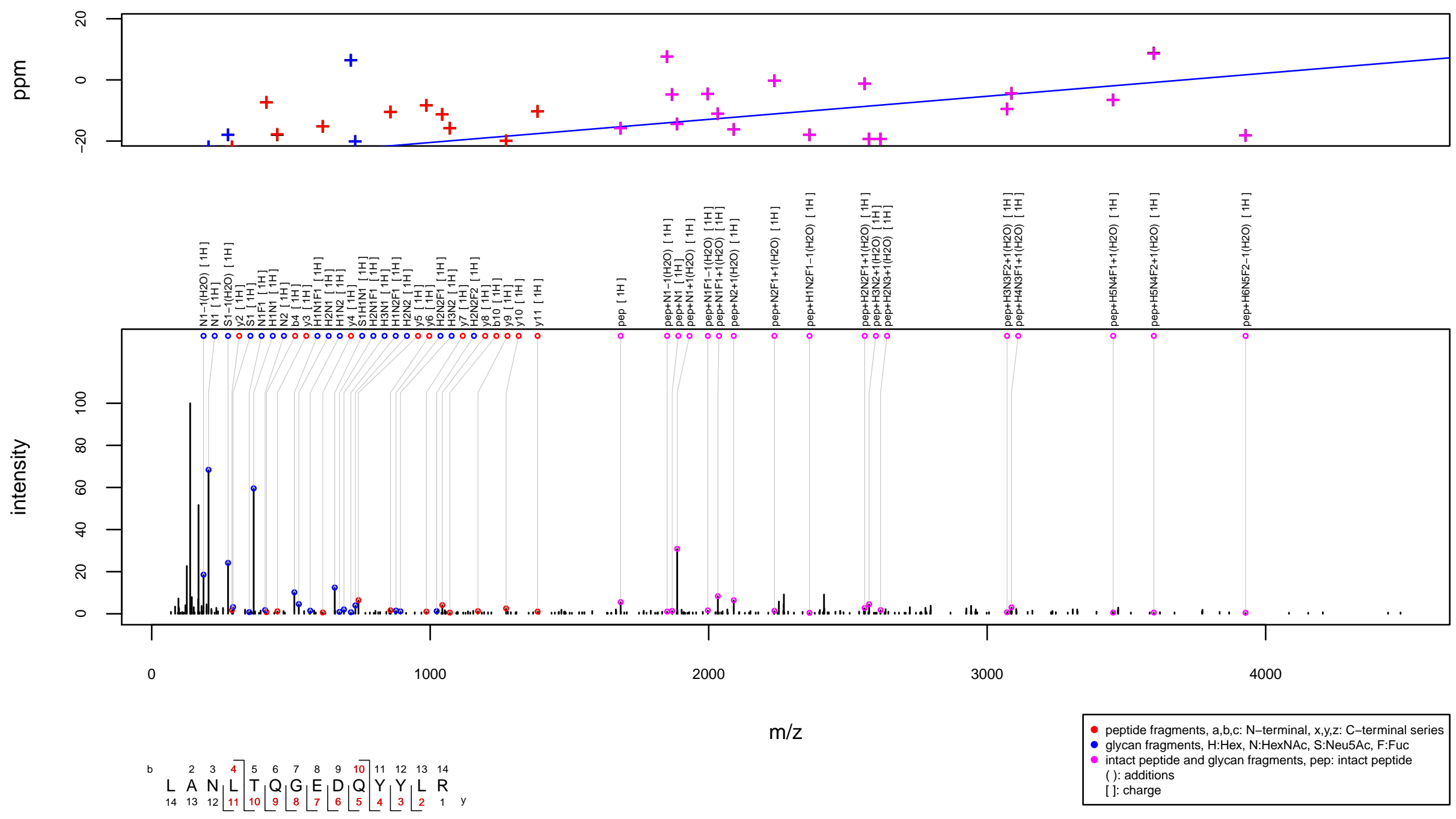
$\mathrm{m} / \mathrm{z} 1104.9371$ charge 4 scan $0-0$

Score $=97.31$, Hits $=65$, Explained Intensity $=0.5$

Peptide: ZA2G_HUMAN[103,120]:DIVEYYNDSNGSHVLQGR

Glycan: $\mathrm{SHNH}(\mathrm{SHNH}) \mathrm{HN}(\mathrm{F}) \mathrm{N}, \mathrm{S} 2 \mathrm{H} 5 \mathrm{~N} 4 \mathrm{~F} 1$

Charge: $4 \mathrm{H}$
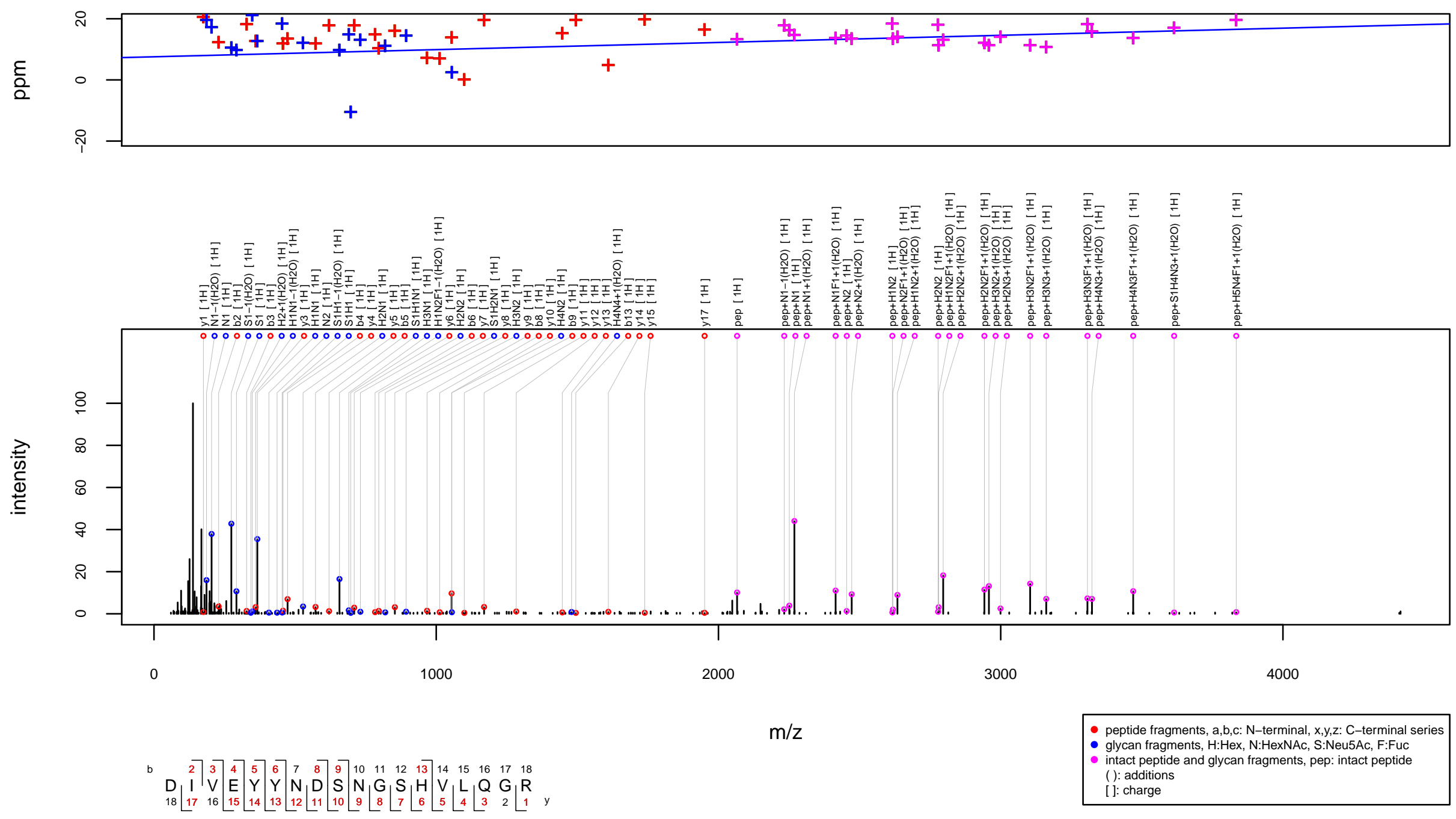
$\mathrm{m} / \mathrm{z} 1105.2312$ charge 4 scan $0-0$

Score $=43.52$, Hits $=47$, Explained Intensity $=0.29$

Peptide: ZA2G_HUMAN[103,120]:DIVEYYNDSNGSHVLQGR

Glycan: S1H5N4F3

Charge: $4 \mathrm{H}$
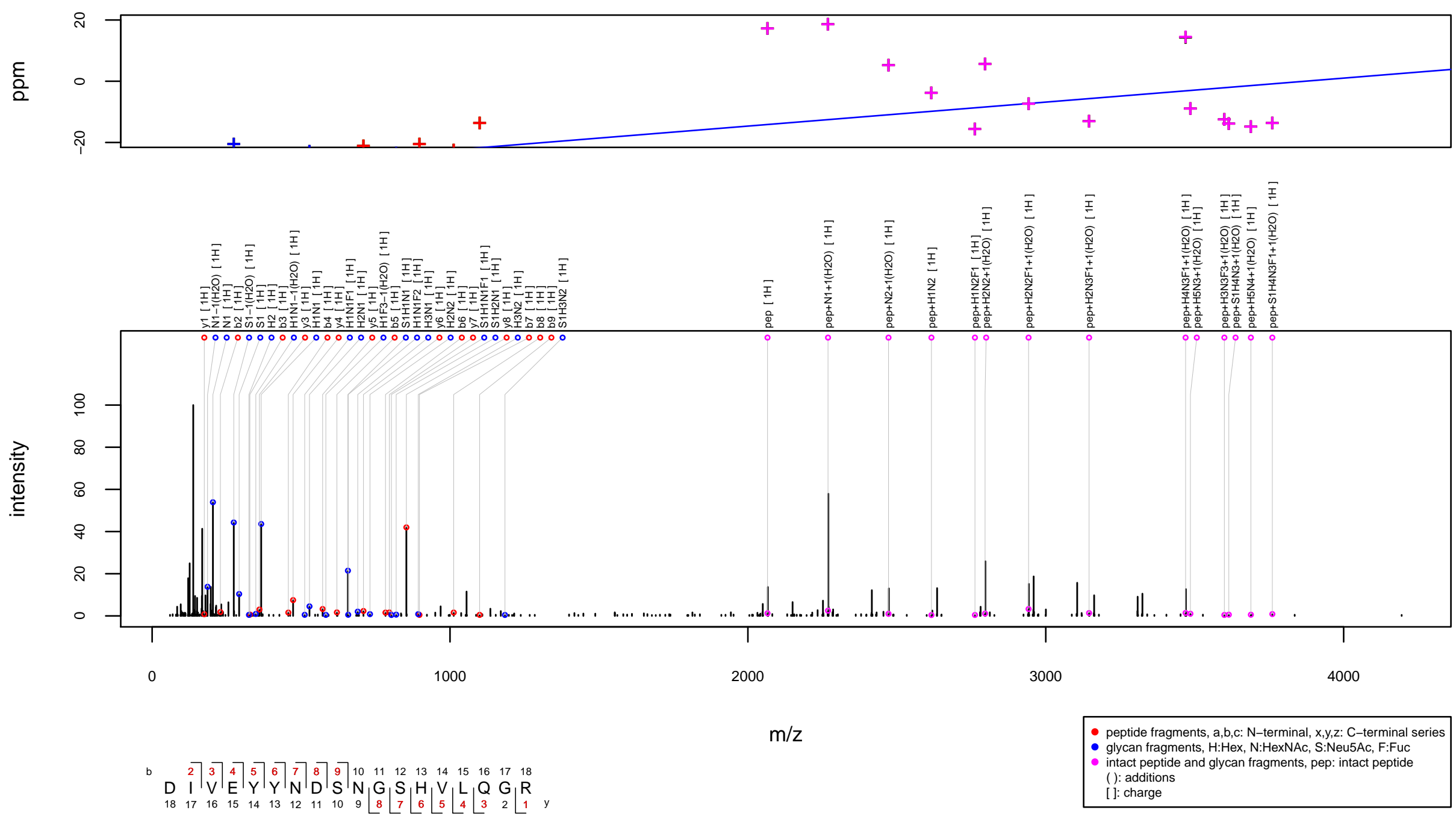


\section{$\mathrm{m} / \mathrm{z} 1107.0155$ charge 4 scan $0-0$}

Score $=30.27$, Hits $=25$, Explained Intensity $=0.32$

Peptide: PPAP_HUMAN[333,355]:NETQHEPYPLMLPGCSPSCPLER

Glycan: H5N3F1

Charge: $4 \mathrm{H}$
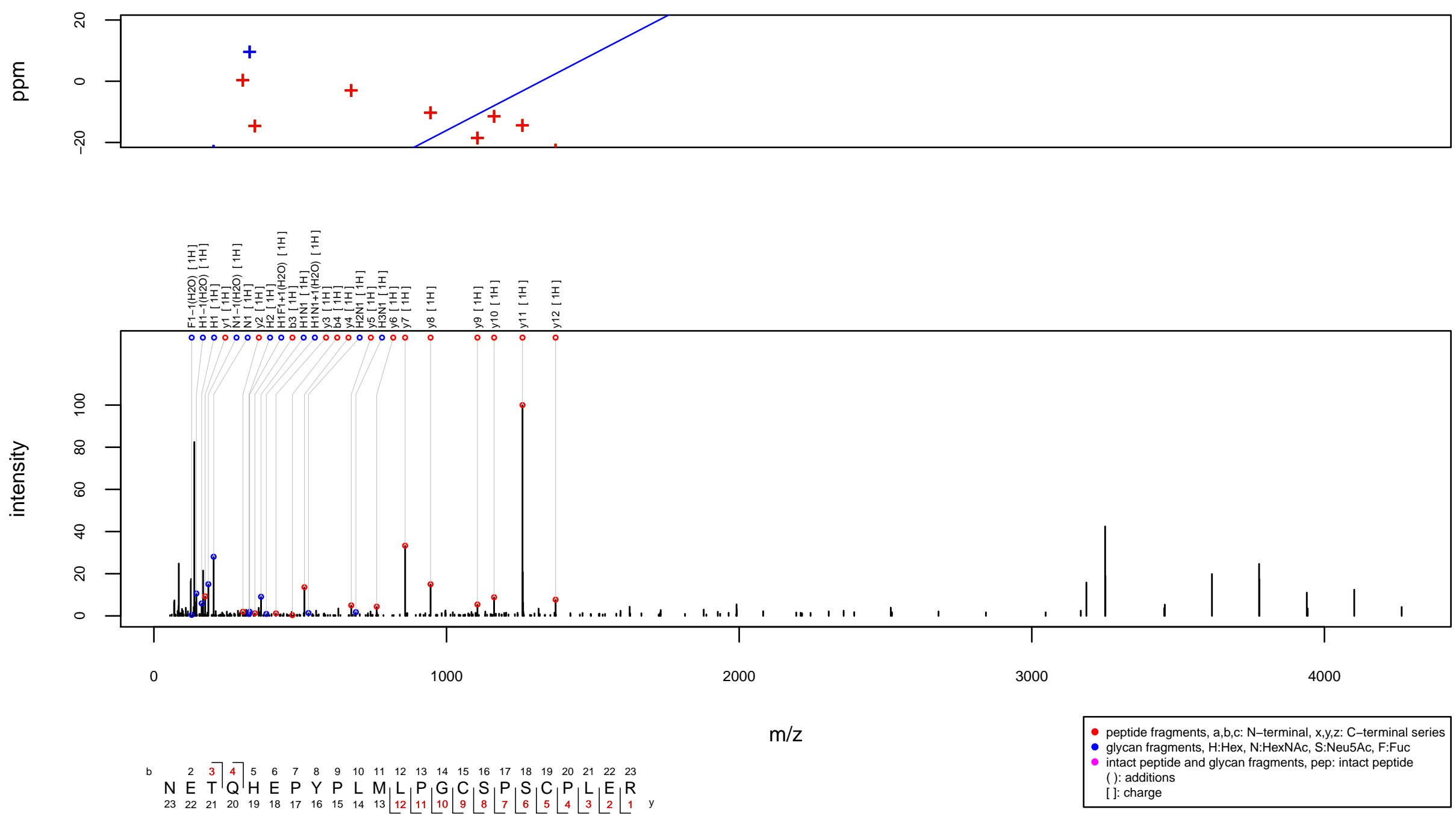
$\mathrm{m} / \mathrm{z} 1109.1223$ charge 3 scan 0-0

Score $=67.4$, Hits $=52$, Explained Intensity $=0.34$

Peptide: PPAP_HUMAN[92,98]:FLNESYK

Glycan: SHNHNH(F(H)NH)HNN, S1H6N5F1

Charge: $3 \mathrm{H}$
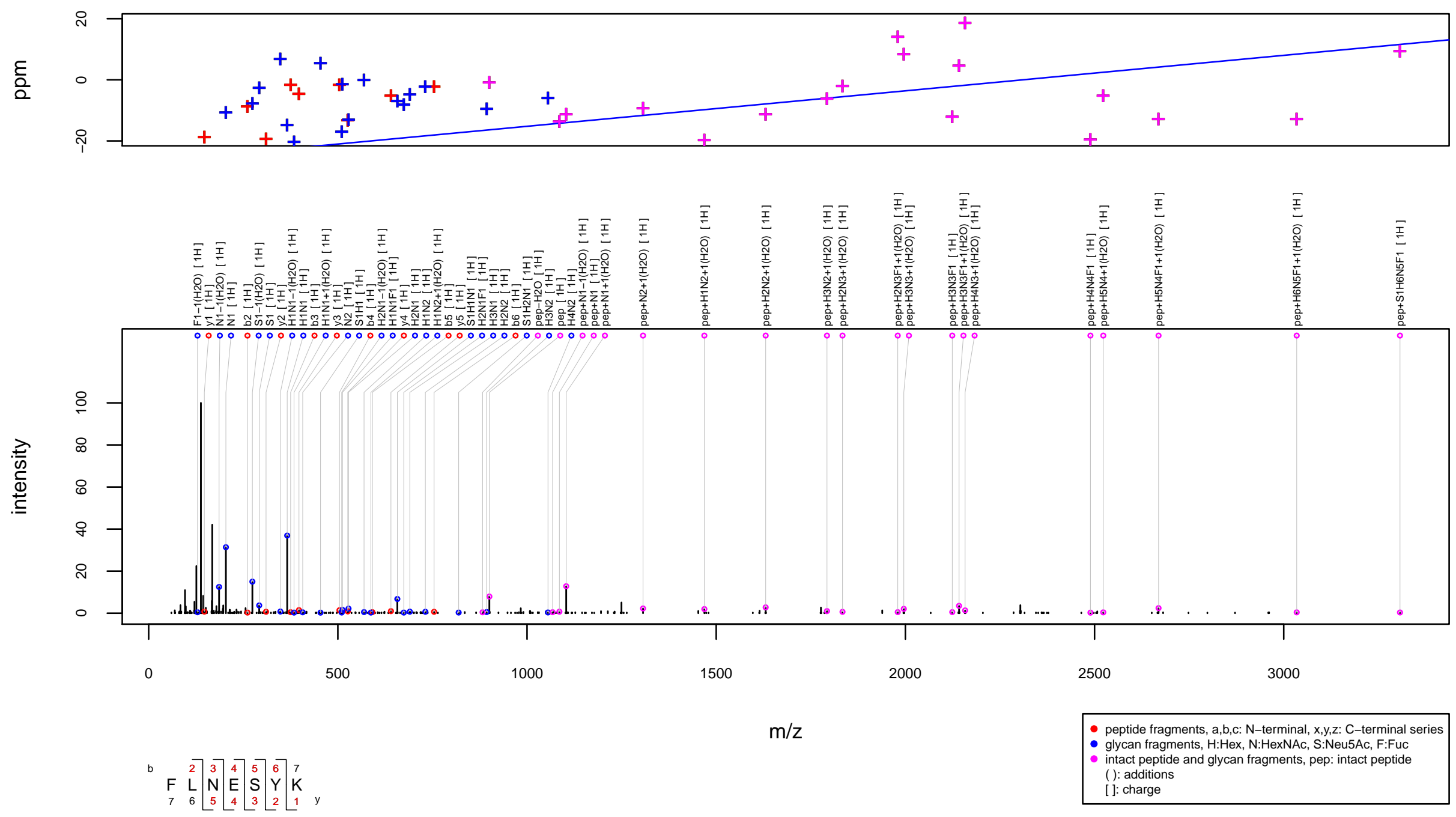
$\mathrm{m} / \mathrm{z} 1109.4868$ charge 3 scan 0-0

Score $=77.43$, Hits $=57$, Explained Intensity $=0.41$ Peptide: CBPE_HUMAN[138,150]:GNETIVNLIHSTR Glycan: S1H6N3

Charge: $3 \mathrm{H}$
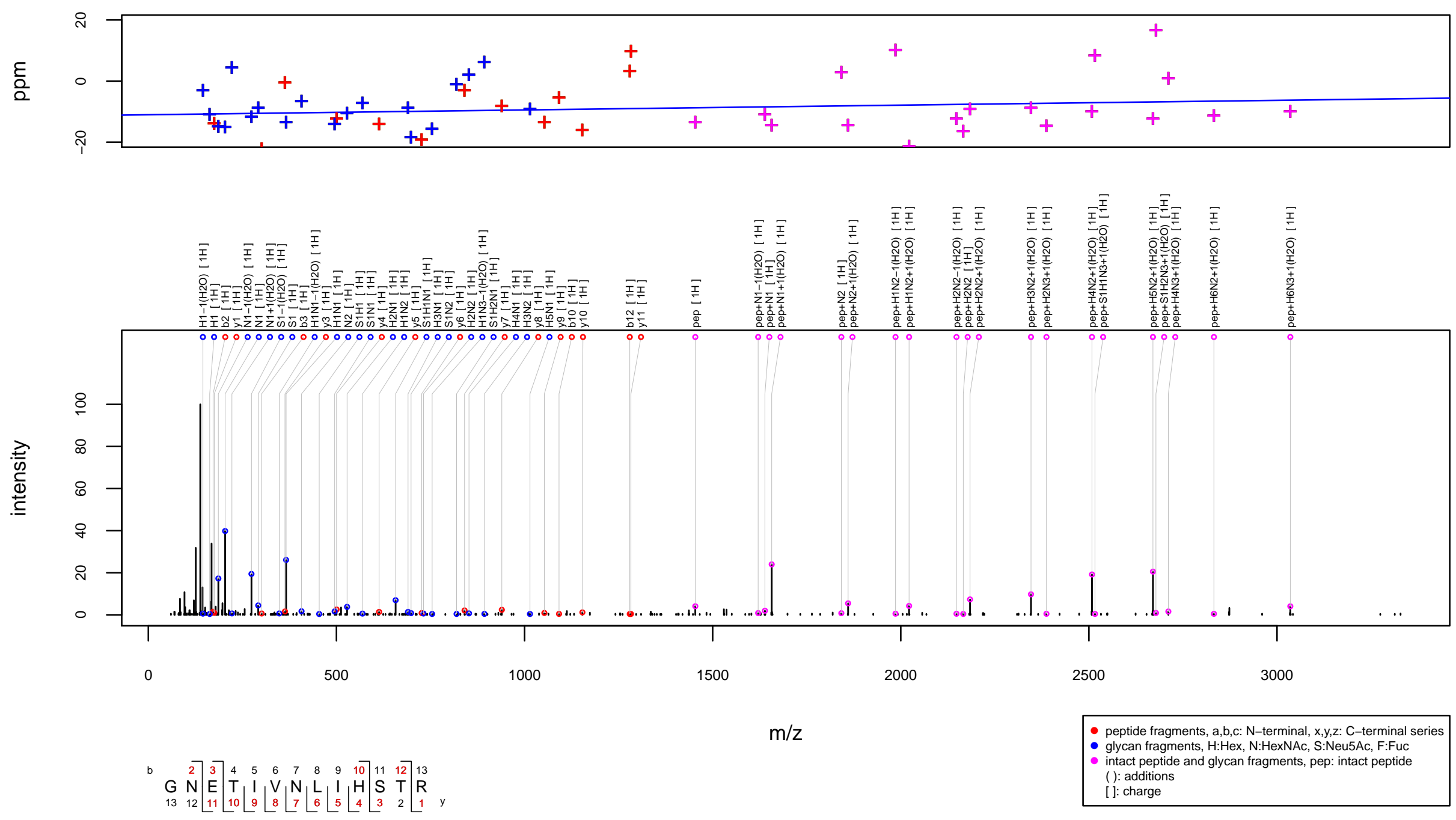
$\mathrm{m} / \mathrm{z} 1110.2711$ charge 4 scan $0-0$

Score $=65.31$, Hits $=46$, Explained Intensity $=0.3$

Peptide: CBPE_HUMAN[382,406]:DLQGNPIANATISVEGIDHDVTSAK

Glycan: $\mathrm{SHNH}(\mathrm{H}(\mathrm{H}) \mathrm{H}) \mathrm{HNN}$, S1H6N3

Charge: $4 \mathrm{H}$
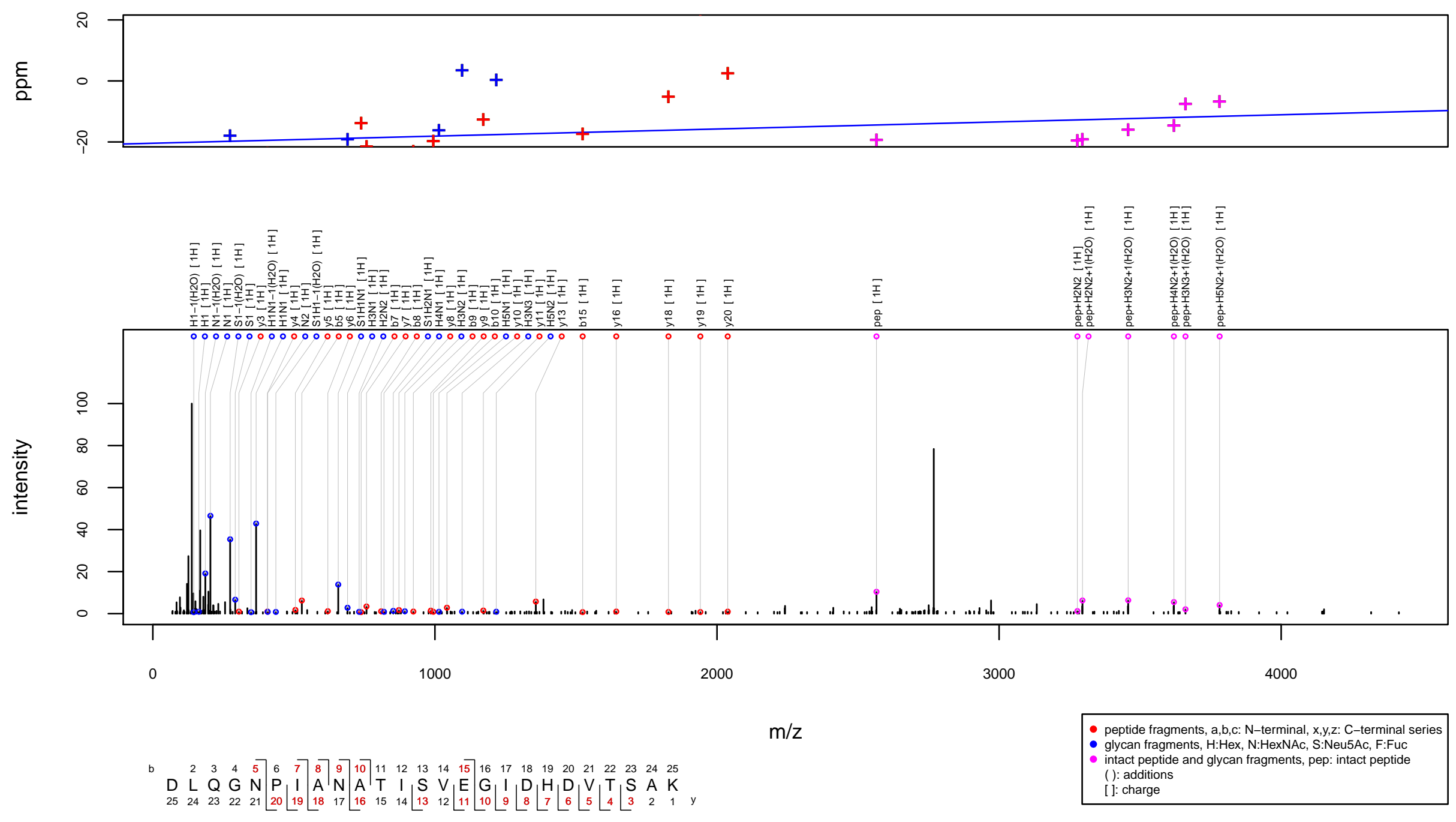
$\mathrm{m} / \mathrm{z} 1112.7742$ charge 4 scan $0-0$

Score $=47.77$, Hits $=36$, Explained Intensity $=0.36$

Peptide: FCGBP_HUMAN[1821,1845]:NPNNDQVFPNGTLAPSIPIWGGSWR

Glycan: $\mathrm{SHNH}(\mathrm{HH}) \mathrm{HNN}$, S1H5N3

Charge: $4 \mathrm{H}$
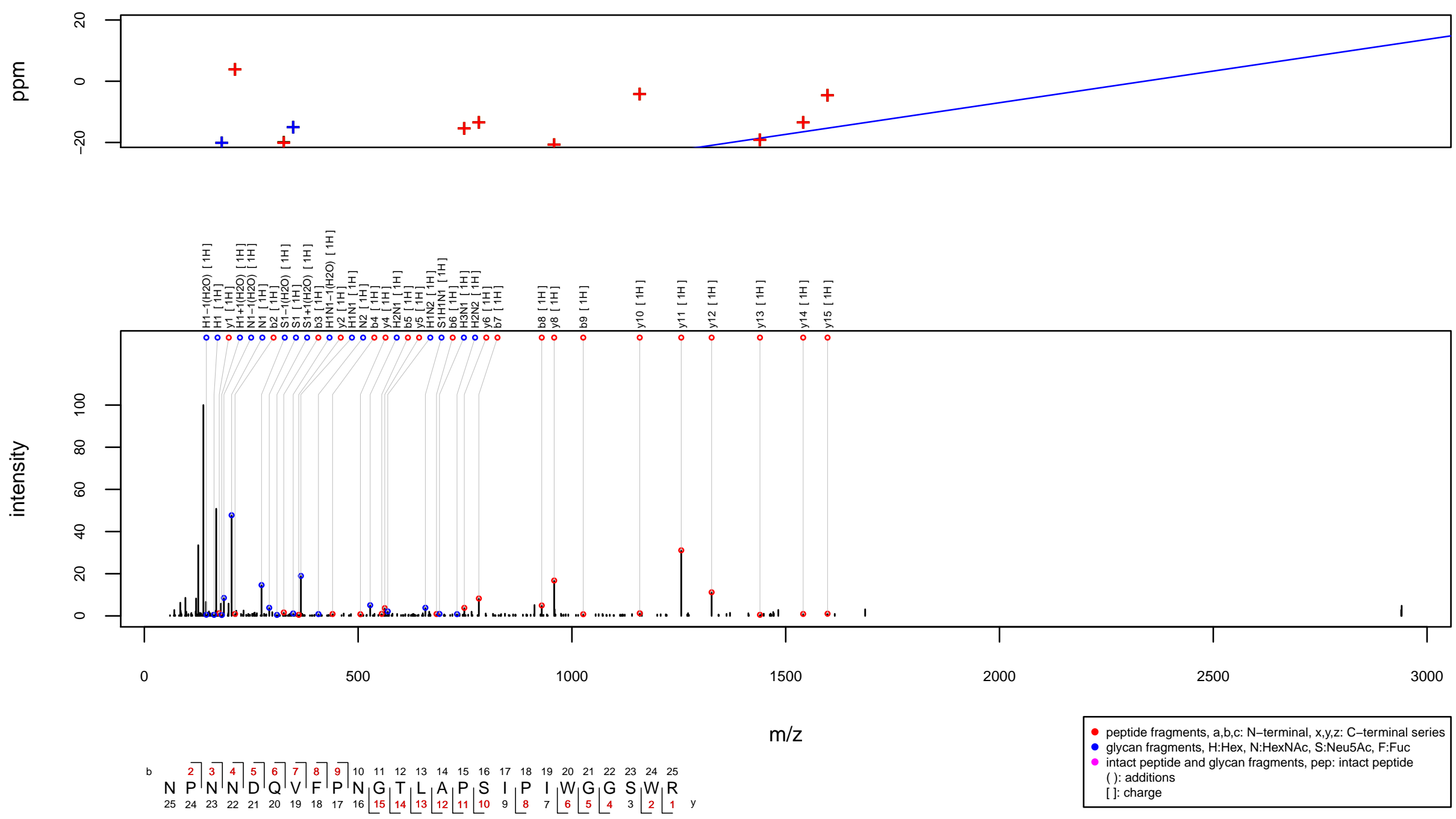
$\mathrm{m} / \mathrm{z} 1115.7412$ charge 4 scan $0-0$

Score $=35.47$, Hits $=28$, Explained Intensity $=0.23$

Peptide: ZA2G_HUMAN[103,120]:DIVEYYNDSNGSHVLQGR

Glycan: $\mathrm{NN}(\mathrm{HN}) \mathrm{H}(\mathrm{HN}(\mathrm{HN}) \mathrm{H}) \mathrm{HNN}, \mathrm{H} 6 \mathrm{~N} 7$

Charge: $4 \mathrm{H}$
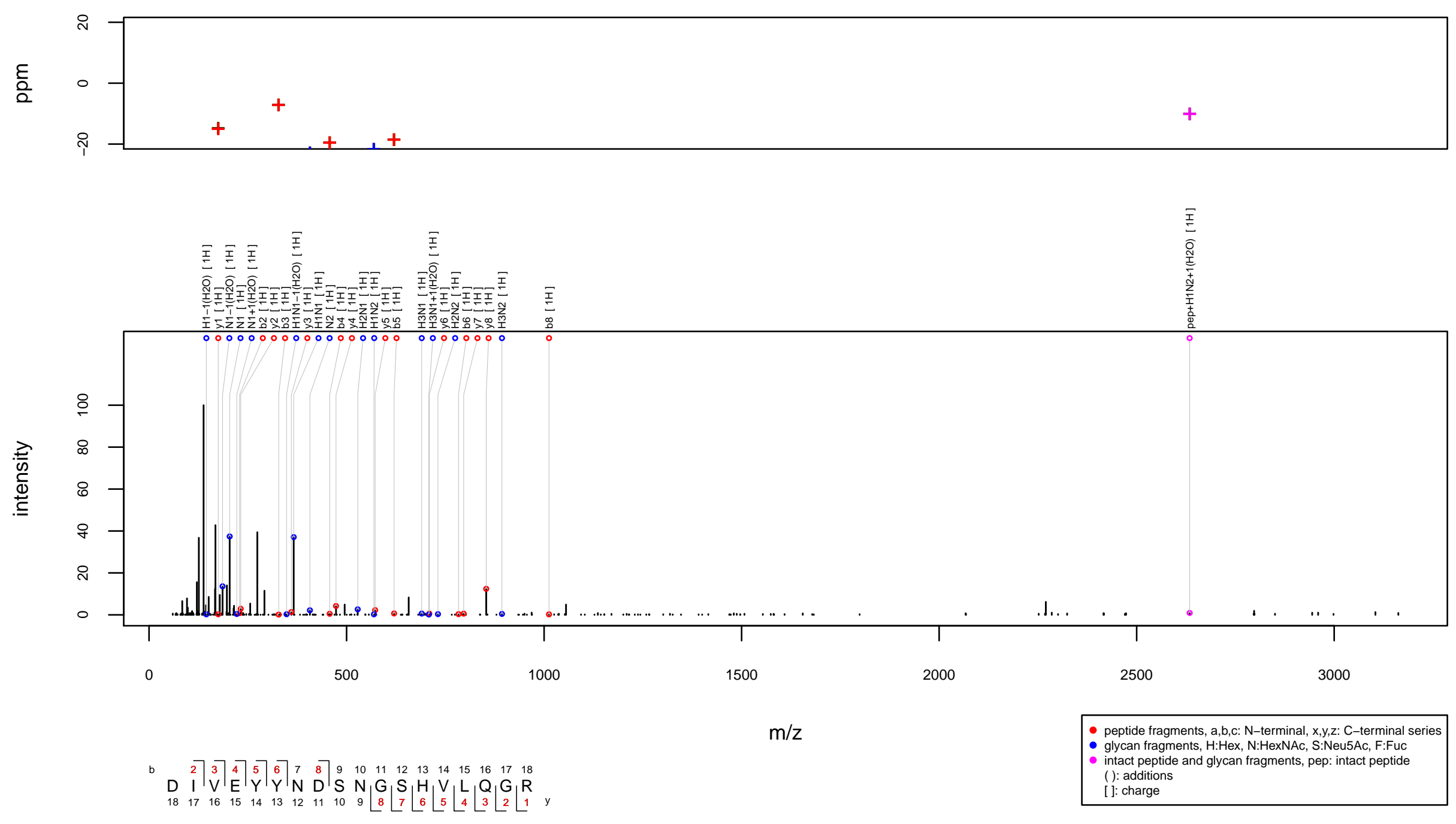
$\mathrm{m} / \mathrm{z} 1116.678$ charge 5 scan $0-0$

Score $=86.3$, Hits $=55$, Explained Intensity $=0.44$

Peptide: WFDC2_HUMAN[33,60]:TGVCPELQADQNCTQECVSDSECADNLK

Glycan: $\mathrm{SHNH}(\mathrm{SHNH}) \mathrm{HN}(\mathrm{F}) \mathrm{N}$, S2H5N4F1

Charge: $5 \mathrm{H}$
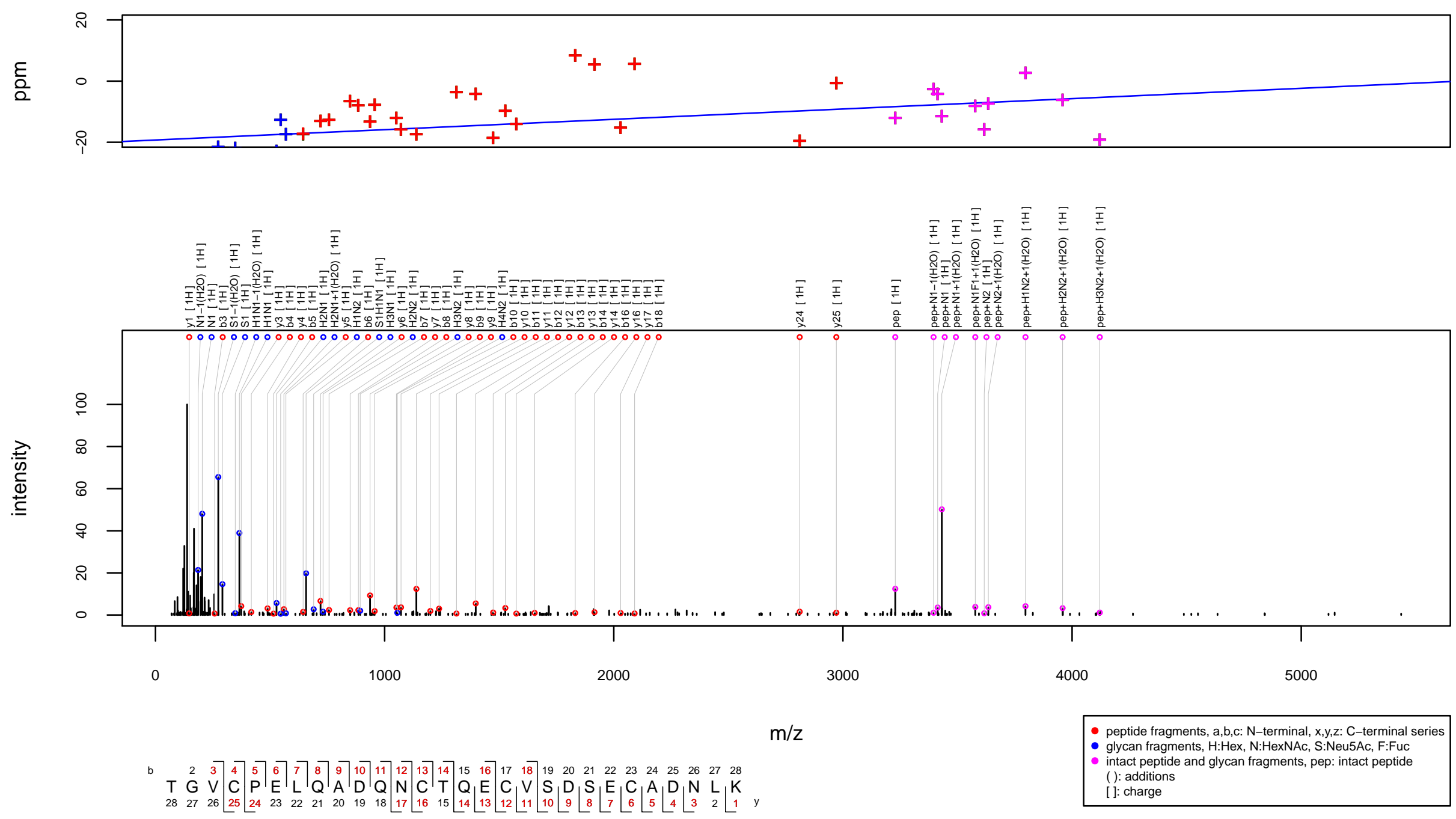
$\mathrm{m} / \mathrm{z} 1116.8694$ charge 5 scan $0-0$

Score $=51.3$, Hits $=47$, Explained Intensity $=0.34$

Peptide: WFDC2_HUMAN[33,60]:TGVCPELQADQNCTQECVSDSECADNLK

Glycan: S1H5N4F3

Charge: $5 \mathrm{H}$
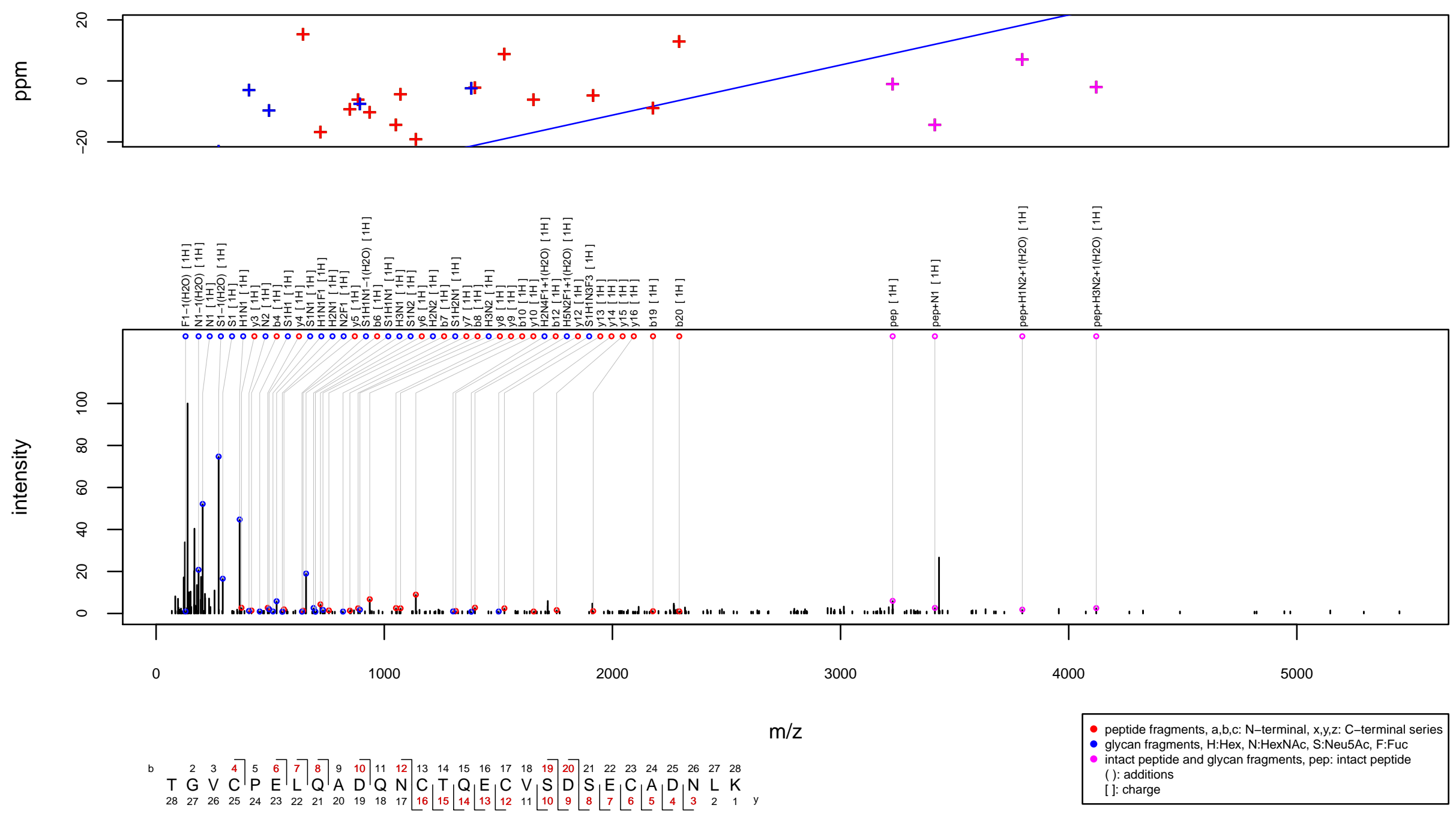


\section{$\mathrm{m} / \mathrm{z} 1117.1411$ charge 5 scan $0-0$}

Score $=50.14$, Hits $=38$, Explained Intensity $=0.28$

Peptide: TRFL_HUMAN[141,170]:TAGWNVPIGTLRPFLNWTGPPEPIEAAVAR

Glycan: $\mathrm{SHNH}(\mathrm{SHNH}) \mathrm{HN}(\mathrm{F}) \mathrm{N}, \mathrm{S} 2 \mathrm{H} 5 \mathrm{~N} 4 \mathrm{~F} 1$

Charge: $5 \mathrm{H}$
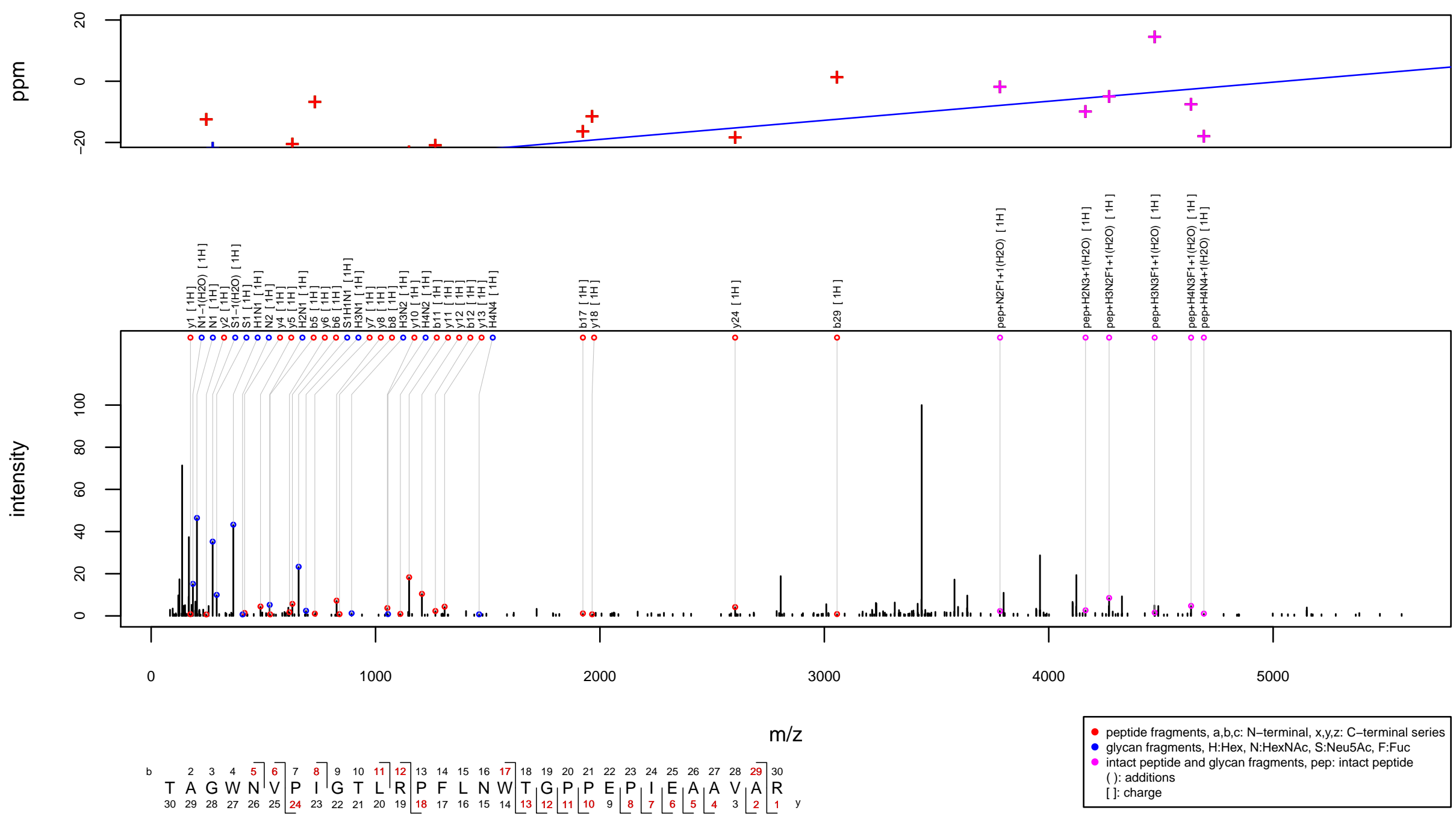


\section{$\mathrm{m} / \mathrm{z} 1118.2666$ charge 4 scan $0-0$}

Score $=43.08$, Hits $=33$, Explained Intensity $=0.38$

Peptide: CLUS_HUMAN[352,371]:MLNTSSLLEQLNEQFNWVSR

Glycan: $\mathrm{SHNH}(\mathrm{HNH}) \mathrm{HNN}, \mathrm{S} 1 \mathrm{H} 5 \mathrm{~N} 4$

Charge: $4 \mathrm{H}$
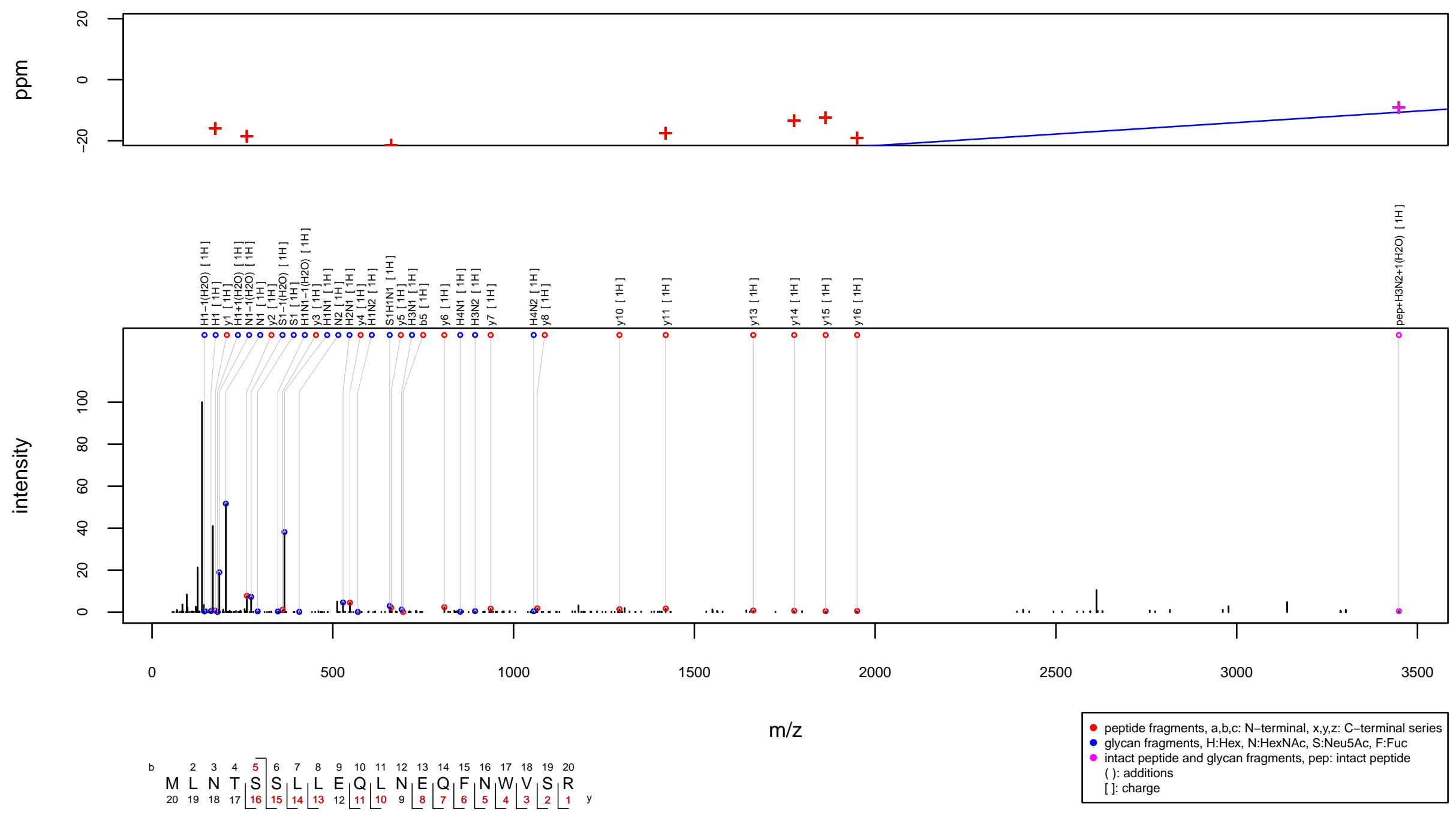
$\mathrm{m} / \mathrm{z} 1122.4734$ charge 4 scan $0-0$

Score $=64.58$, Hits $=48$, Explained Intensity $=0.37$

Peptide: FCGBP_HUMAN[1051,1072]:LDSLVAQQLQSKNECGILADPK

Glycan: $\mathrm{SHNH}(\mathrm{HNH}) \mathrm{HN}(\mathrm{F}) \mathrm{N}, \mathrm{S} 1 \mathrm{H} 5 \mathrm{~N} 4 \mathrm{~F} 1$

Charge: $4 \mathrm{H}$
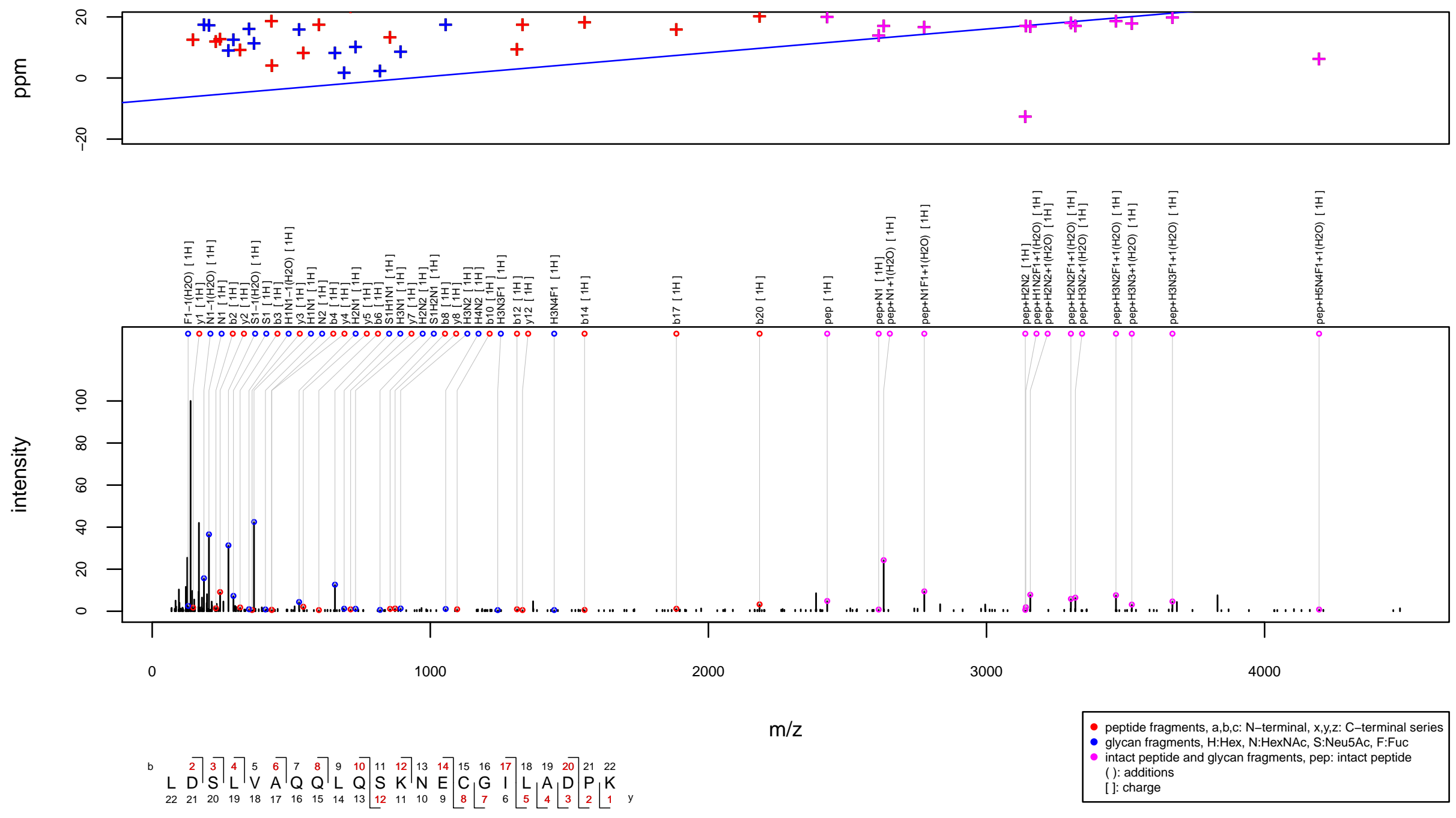
$\mathrm{m} / \mathrm{z} 1124.5089$ charge 4 scan $0-0$

Score $=61.41$, Hits $=48$, Explained Intensity $=0.42$ Peptide: GGT2_HUMAN[504,517]:LHNKLLPNVTTVER Glycan: $\mathrm{SHNH}(\mathrm{SHN}(\mathrm{SHN}) \mathrm{H}) \mathrm{HNN}, \mathrm{S} 3 \mathrm{H} 6 \mathrm{~N} 5$

Charge: $4 \mathrm{H}$
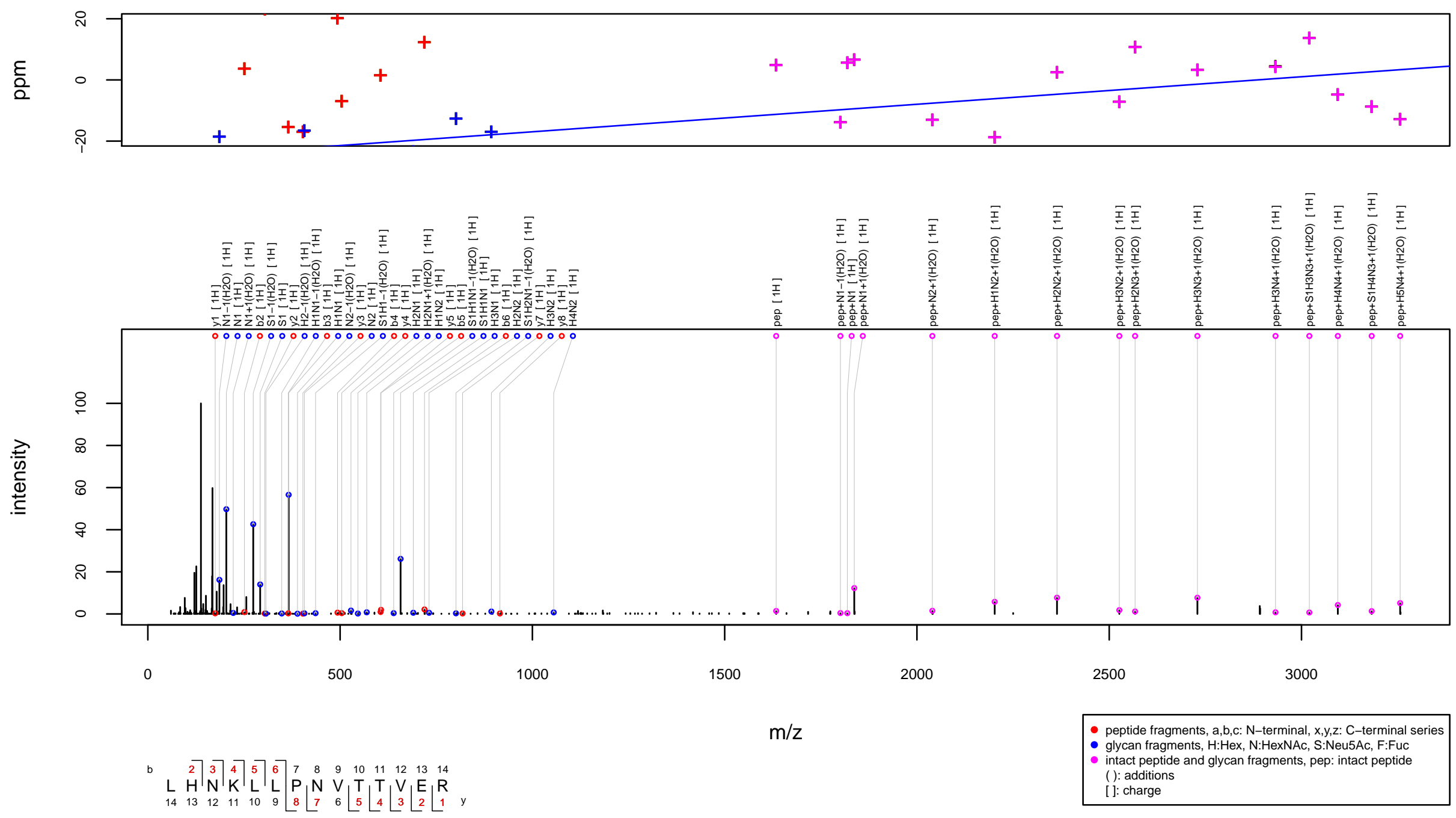
$\mathrm{m} / \mathrm{z} 1124.7834$ charge 3 scan $0-0$

Score $=110.81$, Hits $=74$, Explained Intensity $=0.63$ Peptide: SEMG2_HUMAN[272,282]:NLSQDQEHGRK Glycan: $\mathrm{FHNH}(\mathrm{FHNH}) \mathrm{HN}(\mathrm{F}) \mathrm{N}, \mathrm{H} 5 \mathrm{~N} 4 \mathrm{~F} 3$

Charge: $3 \mathrm{H}$
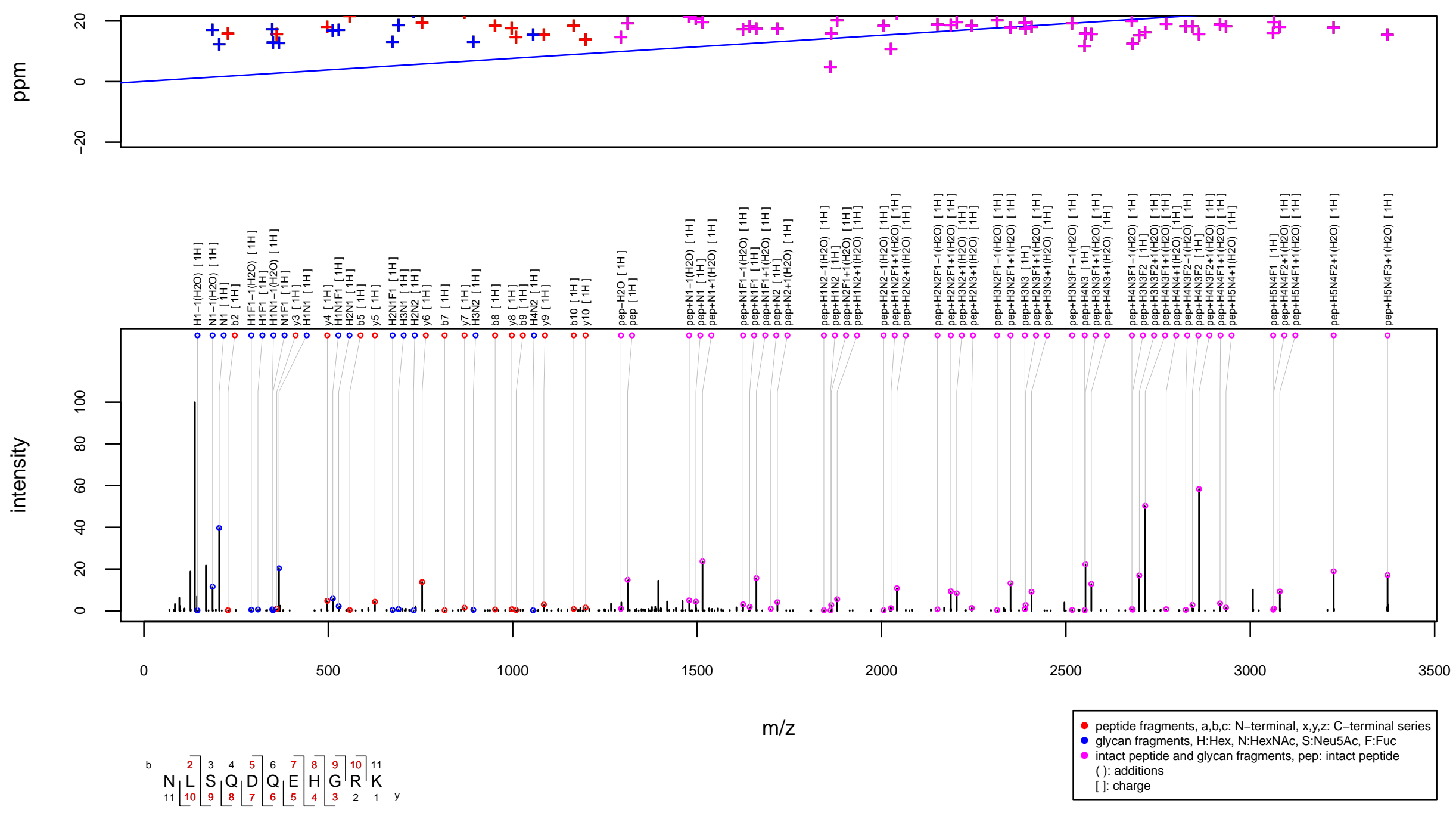
$\mathrm{m} / \mathrm{z} 1127.1409$ charge 3 scan $0-0$

Score $=107.88$, Hits $=73$, Explained Intensity $=0.49$

Peptide: PPAP_HUMAN[91,98]:KFLNESYK

Glycan: $\mathrm{SHNH}(\mathrm{SHNH}) \mathrm{HN}(\mathrm{F}) \mathrm{N}, \mathrm{S} 2 \mathrm{H} 5 \mathrm{~N} 4 \mathrm{~F} 1$

Charge: $3 \mathrm{H}$
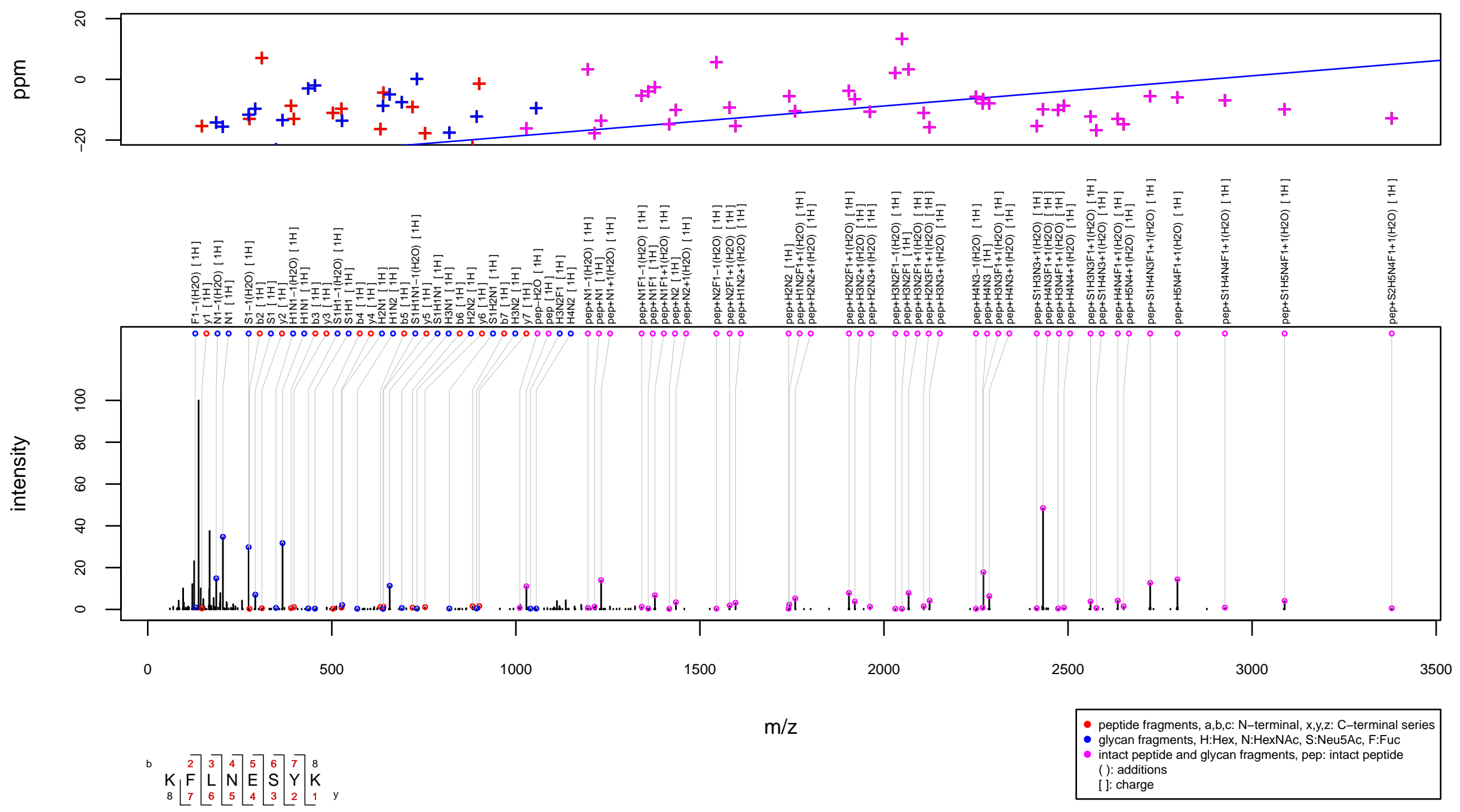
$\mathrm{m} / \mathrm{z} 1128.8018$ charge 4 scan $0-0$

Score $=45.79$, Hits $=35$, Explained Intensity $=0.33$

Peptide: AMPN_HUMAN[674,690]:VPVTLALNNTLFLIEER

Glycan: $\mathrm{SHNH}(\mathrm{SHN}(\mathrm{HN}) \mathrm{H}) \mathrm{HNN}$, S2H6N5

Charge: $4 \mathrm{H}$
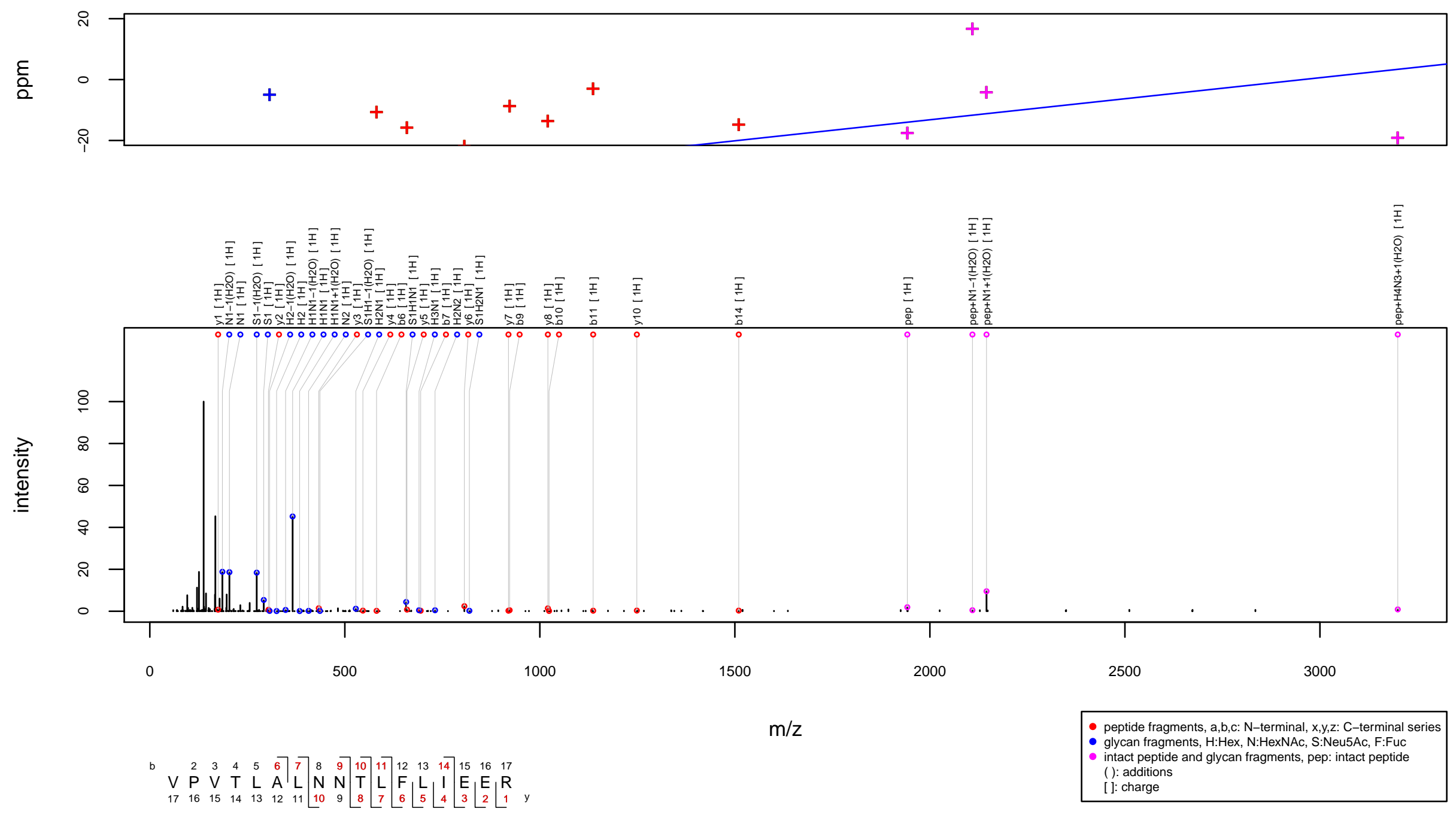
$\mathrm{m} / \mathrm{z} 1130.7727$ charge 3 scan $0-0$

Score $=118.29$, Hits $=83$, Explained Intensity $=0.63$ Peptide: SEMG2_HUMAN[272,281]:NLSQDQEHGR Glycan: $\mathrm{FHNH}(\mathrm{FH}(\mathrm{F}) \mathrm{NH}) \mathrm{HN}(\mathrm{F}) \mathrm{N}, \mathrm{H} 5 \mathrm{~N} 4 \mathrm{~F} 4$

Charge: $3 \mathrm{H}$
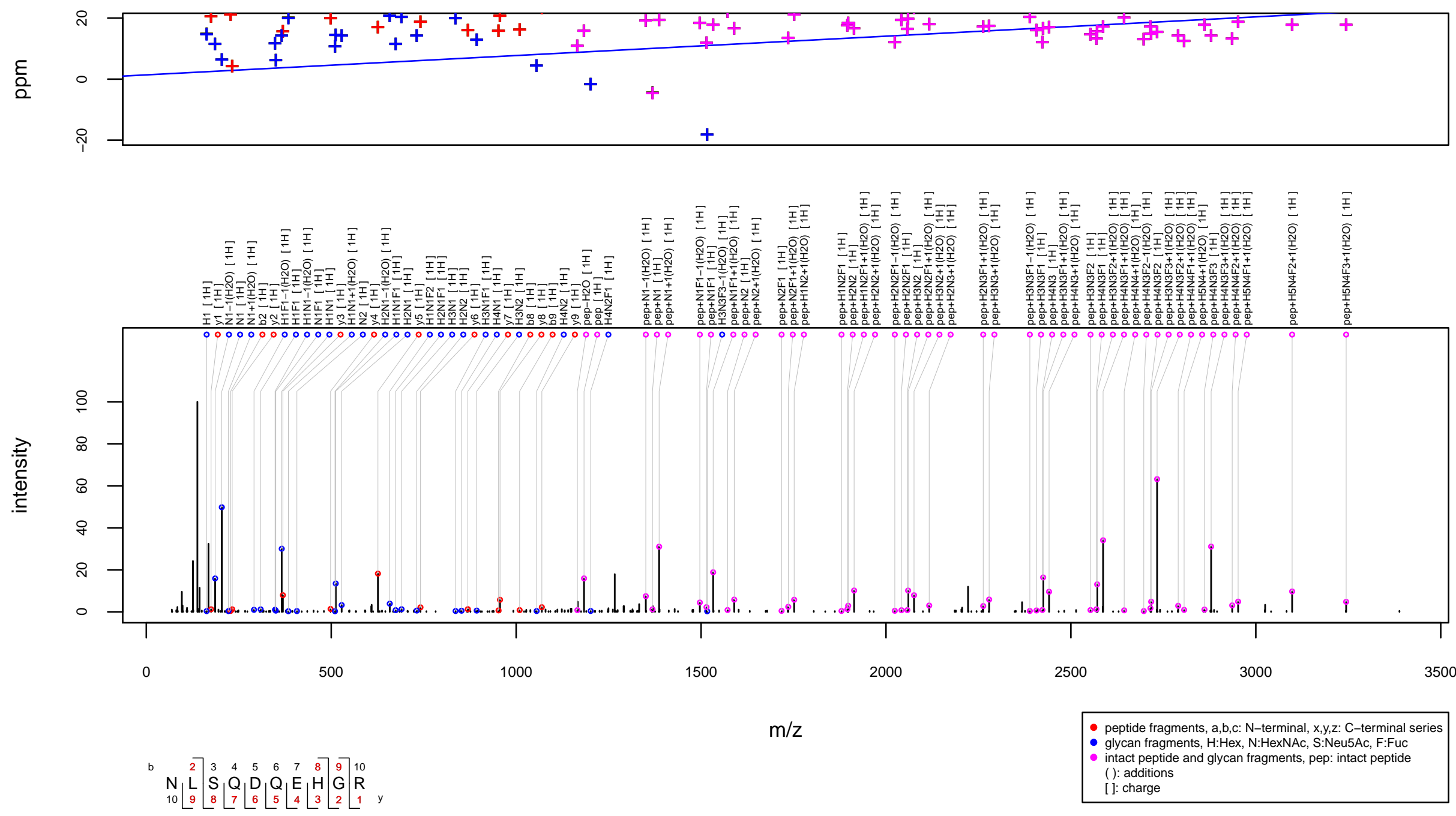
$\mathrm{m} / \mathrm{z} 1137.0074$ charge 4 scan $0-0$

Score $=52.18$, Hits $=39$, Explained Intensity $=0.42$

Peptide: CLUS_HUMAN[372,385]:LANLTQGEDQYYLR

Glycan: $\mathrm{SHNH}(\mathrm{SHN}(\mathrm{SHN}) \mathrm{H}) \mathrm{HNN}, \mathrm{S} 3 \mathrm{H} 6 \mathrm{~N} 5$

Charge: $4 \mathrm{H}$
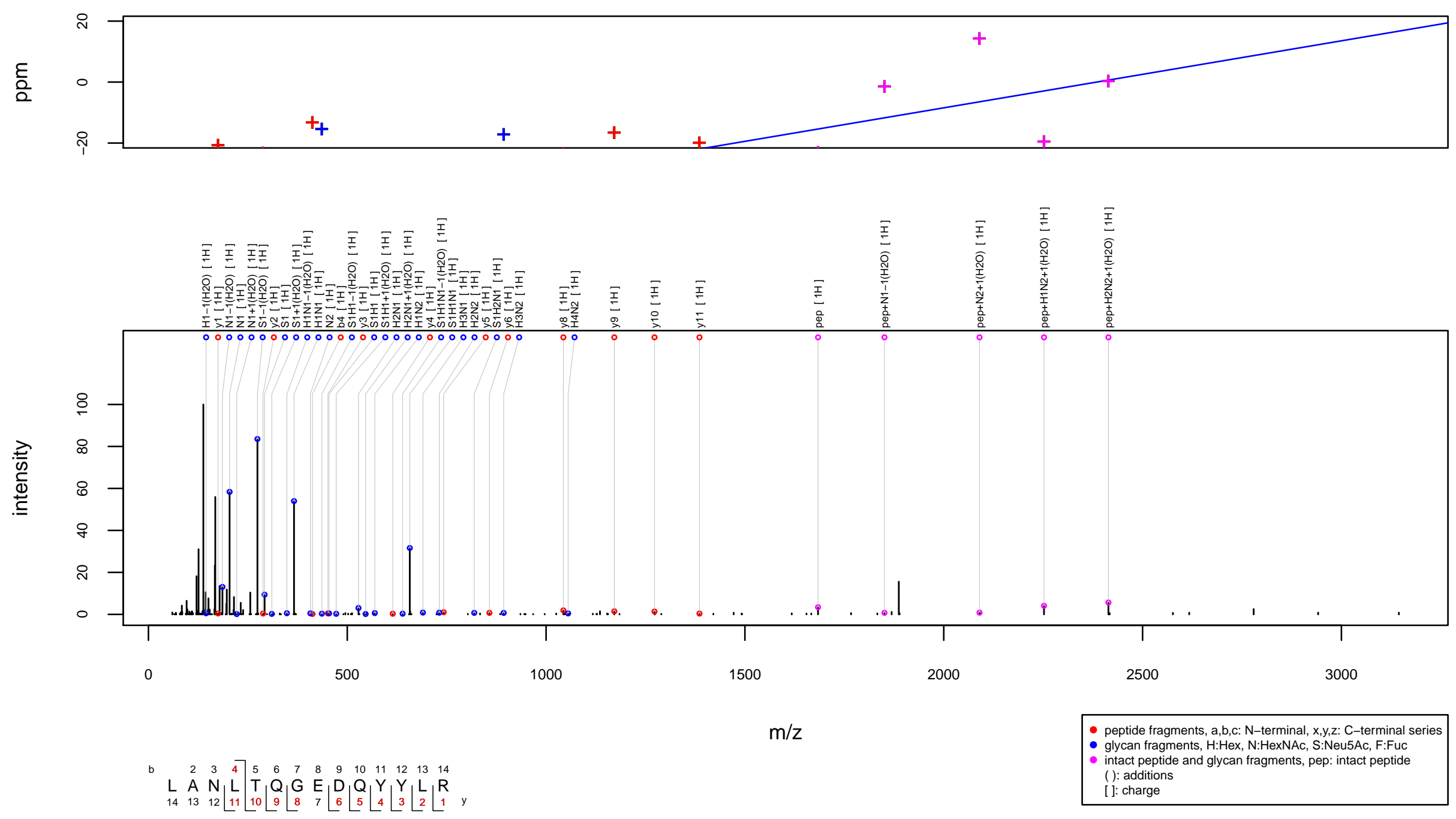
$\mathrm{m} / \mathrm{z} 1137.4991$ charge 4 scan $0-0$

Score $=56.47$, Hits $=72$, Explained Intensity $=0.49$ Peptide: CLUS_HUMAN[372,385]:LANLTQGEDQYYLR Glycan: S1H6N5F4

Charge: $4 \mathrm{H}$
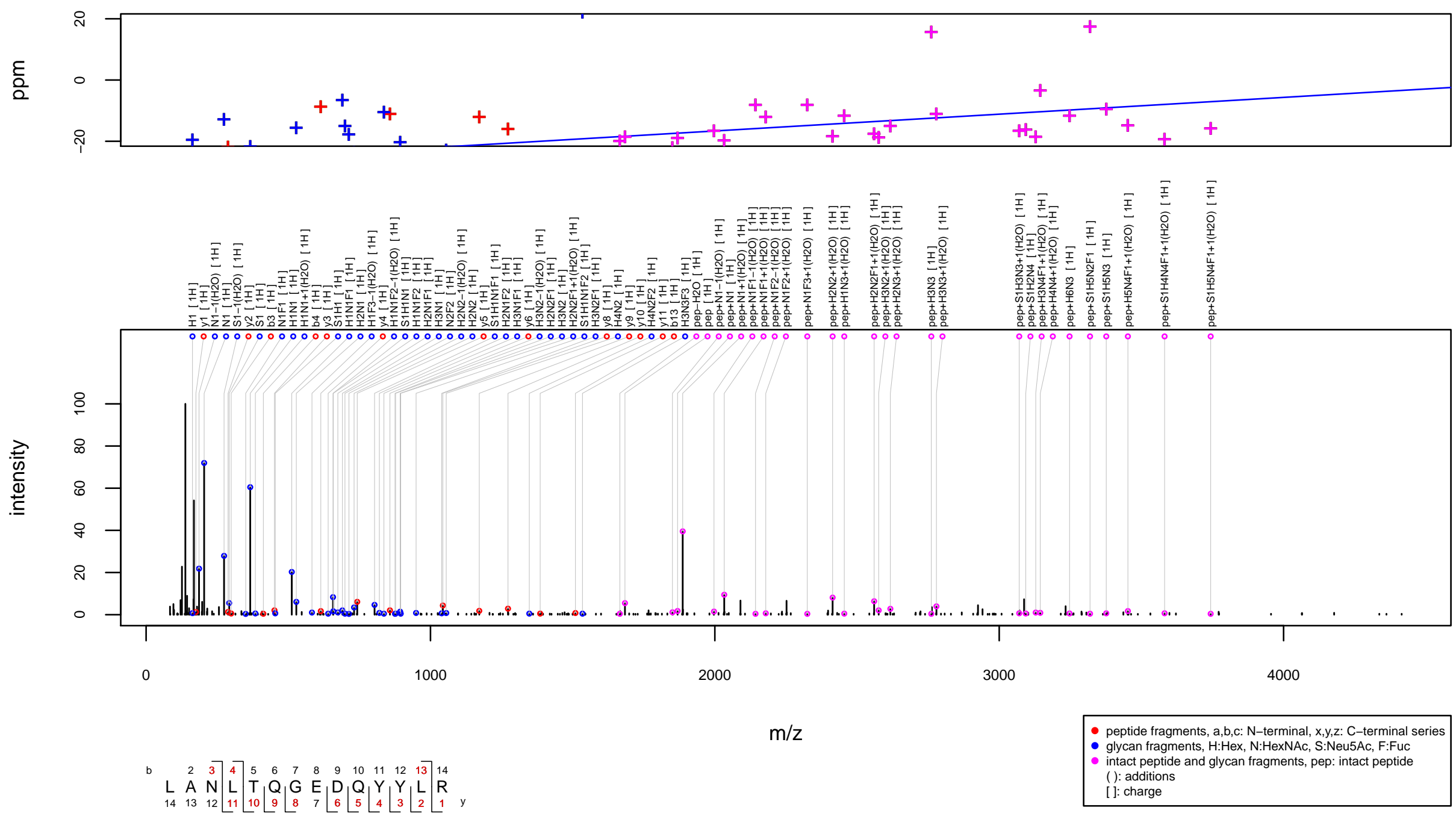
$\mathrm{m} / \mathrm{z} 1138.4982$ charge 3 scan $0-0$

Score $=65.87$, Hits $=40$, Explained Intensity $=0.51$

Peptide: FINC_HUMAN[516,533]:DQCIVDDITYNVNDTFHK

Glycan: $\mathrm{H}(\mathrm{H}) \mathrm{H}(\mathrm{H}) \mathrm{HNN}, \mathrm{H} 5 \mathrm{~N} 2$

Charge: $3 \mathrm{H}$
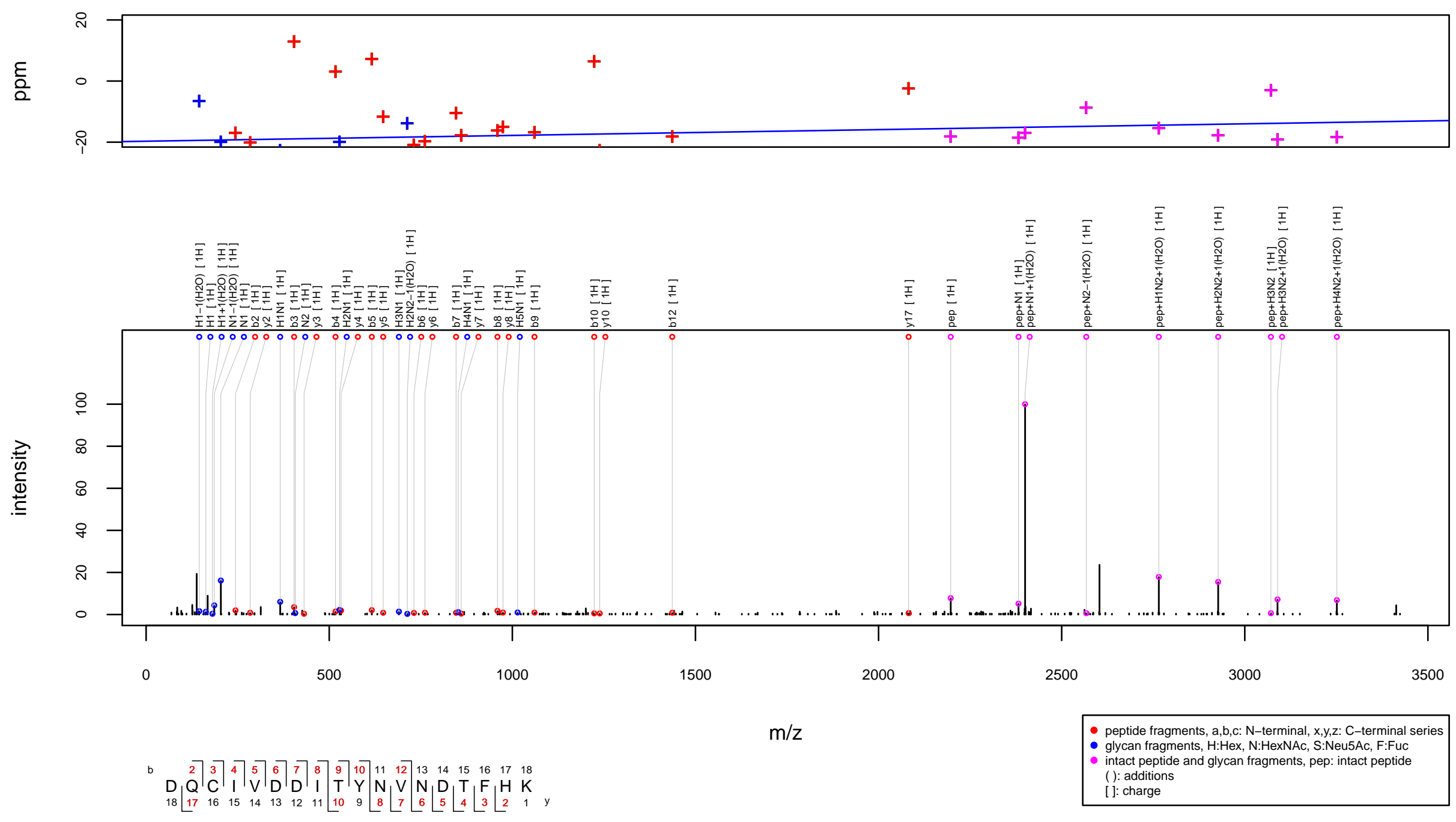


\section{$\mathrm{m} / \mathrm{z} 1140.5179$ charge 4 scan $0-0$}

Score $=33.76$, Hits $=33$, Explained Intensity $=0.3$

Peptide: CLUS_HUMAN[352,371]:MLNTSSLLEQLNEQFNWVSR

Glycan: S1H5N3F2

Charge: $4 \mathrm{H}$
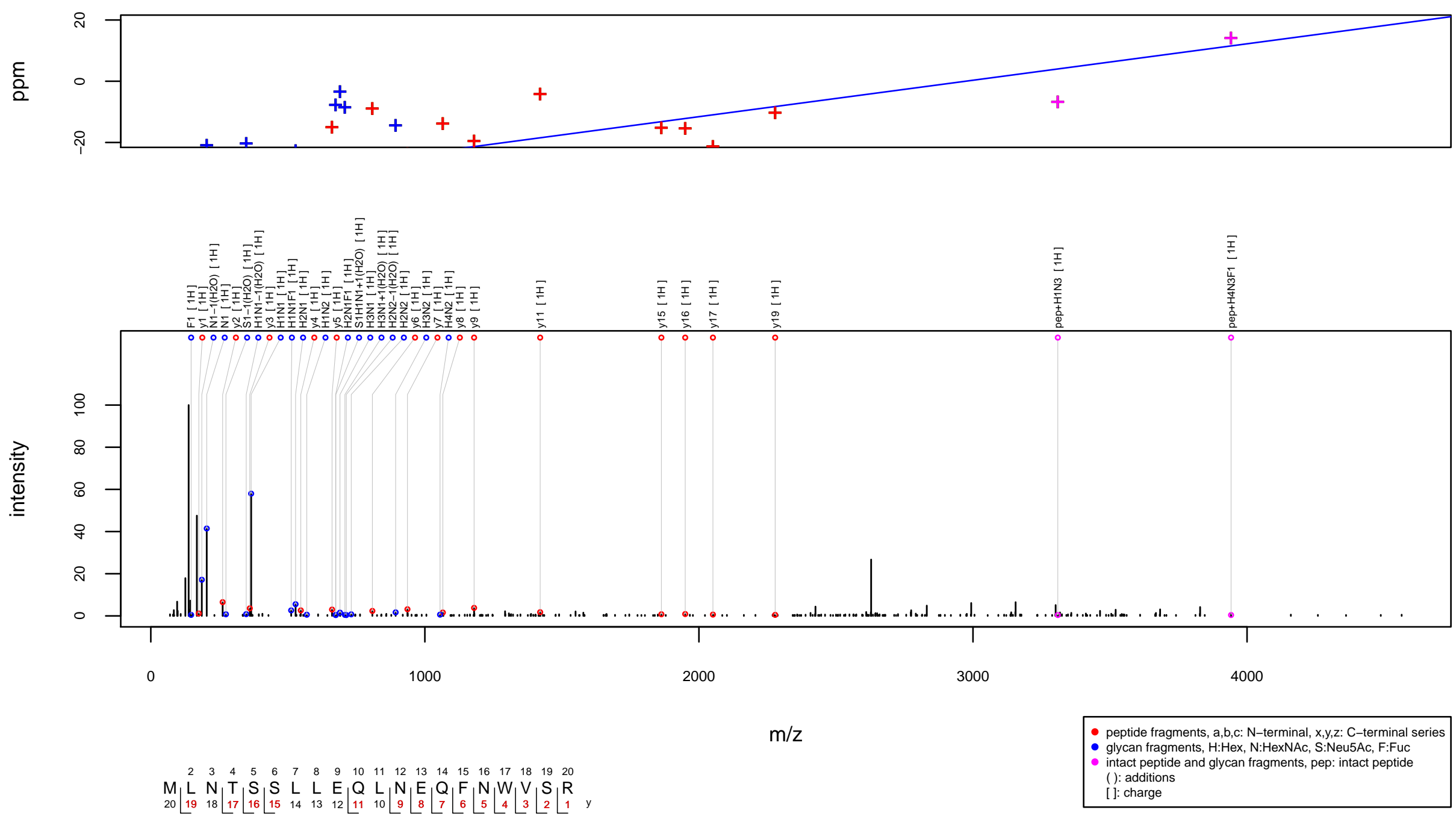


\section{$\mathrm{m} / \mathrm{z} 1143.2539$ charge 4 scan $0-0$}

Score $=48.31$, Hits $=39$, Explained Intensity $=0.33$

Peptide: CLUS_HUMAN[139,158]:QLEEFLNQSSPFYFWMNGDR

Glycan: $\mathrm{F}(\mathrm{H}) \mathrm{NH}(\mathrm{F}(\mathrm{H}) \mathrm{NH}) \mathrm{HNN}, \mathrm{H} 5 \mathrm{~N} 4 \mathrm{~F} 2$

Charge: $4 \mathrm{H}$
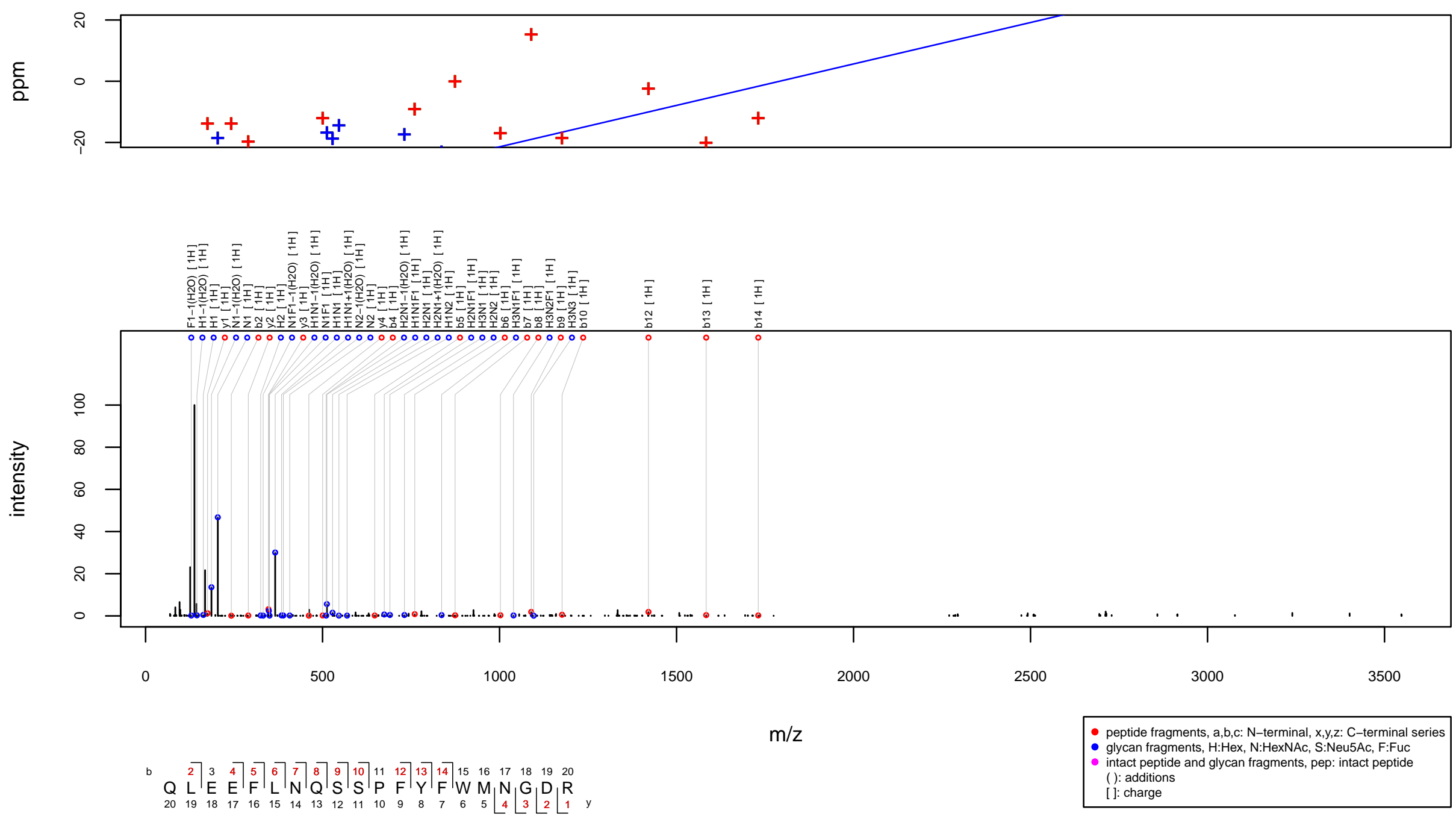


\section{$\mathrm{m} / \mathrm{z} 1147.2877$ charge 4 scan $0-0$}

Score $=43.99$, Hits $=33$, Explained Intensity $=0.49$

Peptide: IPSP_HUMAN[254,274]:VVGVPYQGNATALFILPSEGK

Glycan: $\mathrm{HN}(\mathrm{F}(\mathrm{H}) \mathrm{N}) \mathrm{H}(\mathrm{F}(\mathrm{H}) \mathrm{NH}) \mathrm{HN}(\mathrm{F}) \mathrm{N}, \mathrm{H} 6 \mathrm{~N} 5 \mathrm{~F} 3$

Charge: $4 \mathrm{H}$
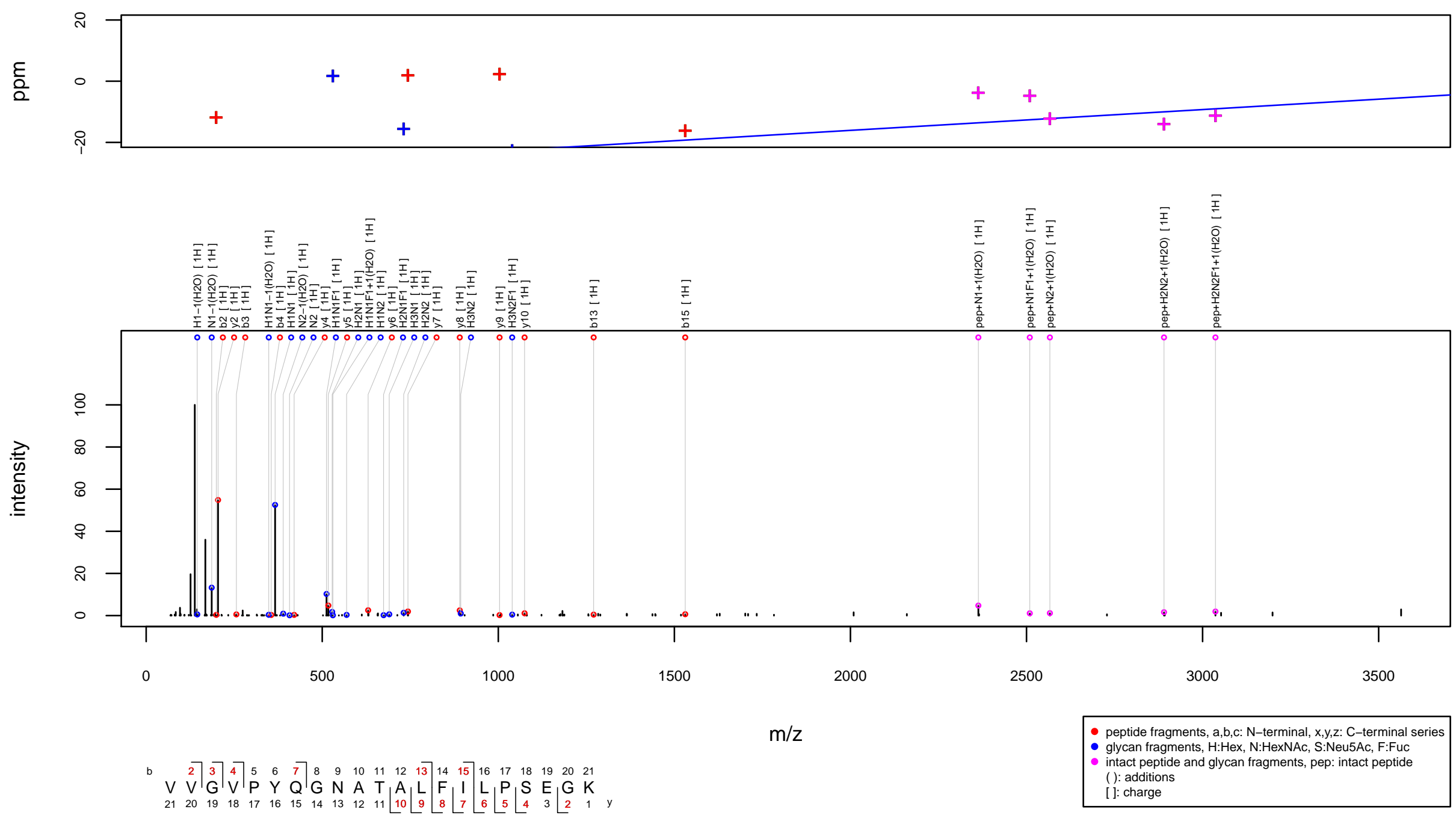
$\mathrm{m} / \mathrm{z} 1150.2253$ charge 3 scan $0-0$

Score $=92.02$, Hits $=52$, Explained Intensity $=0.39$

Peptide: FINC_HUMAN[997,1016]:LDAPTNLQFVNETDSTVLVR

Glycan: $\mathrm{H}(\mathrm{H}) \mathrm{H}(\mathrm{H}) \mathrm{HNN}$, H5N2

Charge: $3 \mathrm{H}$
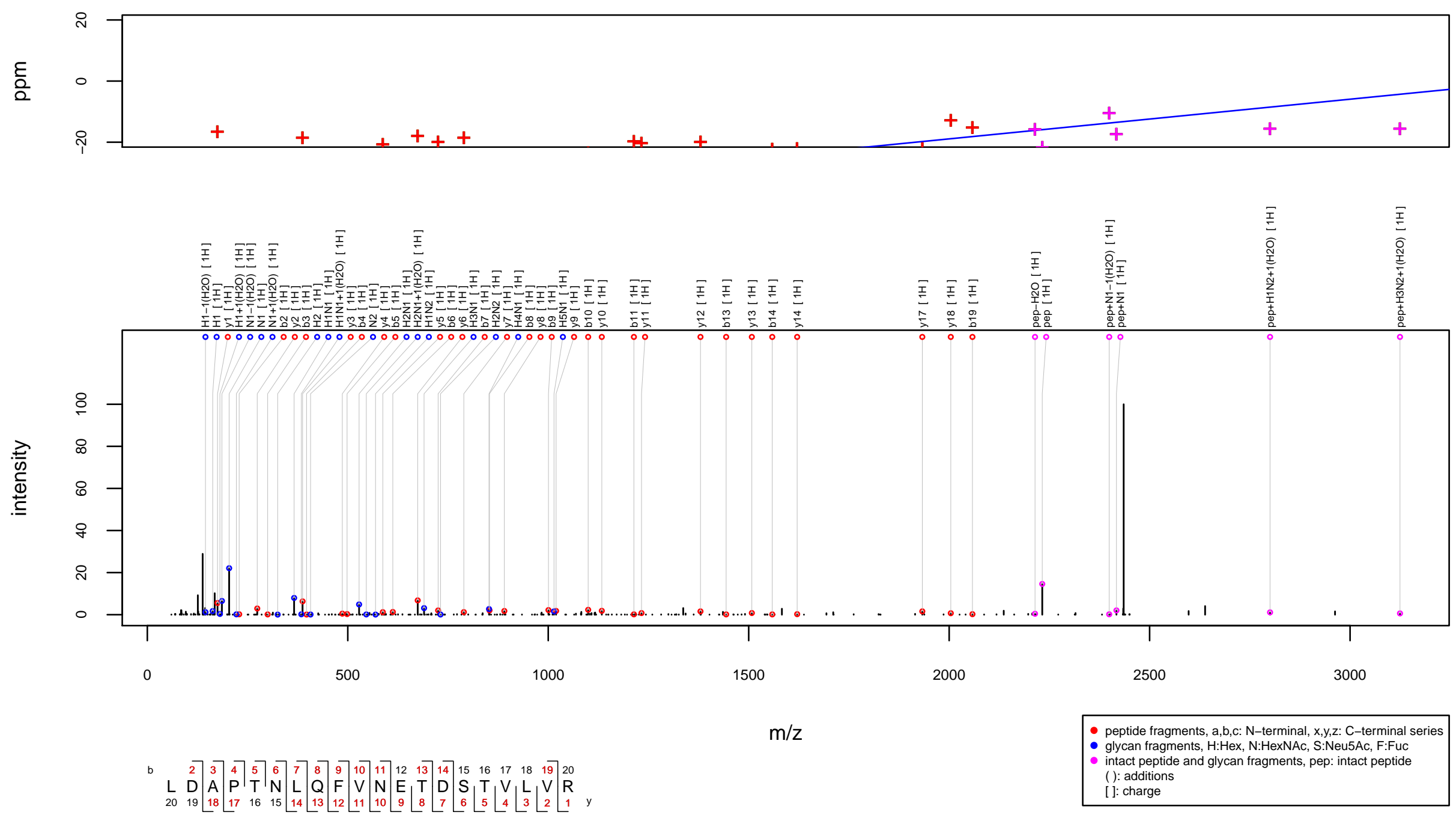


\section{$\mathrm{m} / \mathrm{z} 1151.5204$ charge 3 scan 0-0}

Score $=65.1$, Hits $=41$, Explained Intensity $=0.3$

Peptide: CLUS_HUMAN[372,385]:LANLTQGEDQYYLR

Glycan: $\mathrm{HNH}(\mathrm{HNH}) \mathrm{HN}(\mathrm{F}) \mathrm{N}, \mathrm{H} 5 \mathrm{~N} 4 \mathrm{~F} 1$

Charge: $3 \mathrm{H}$
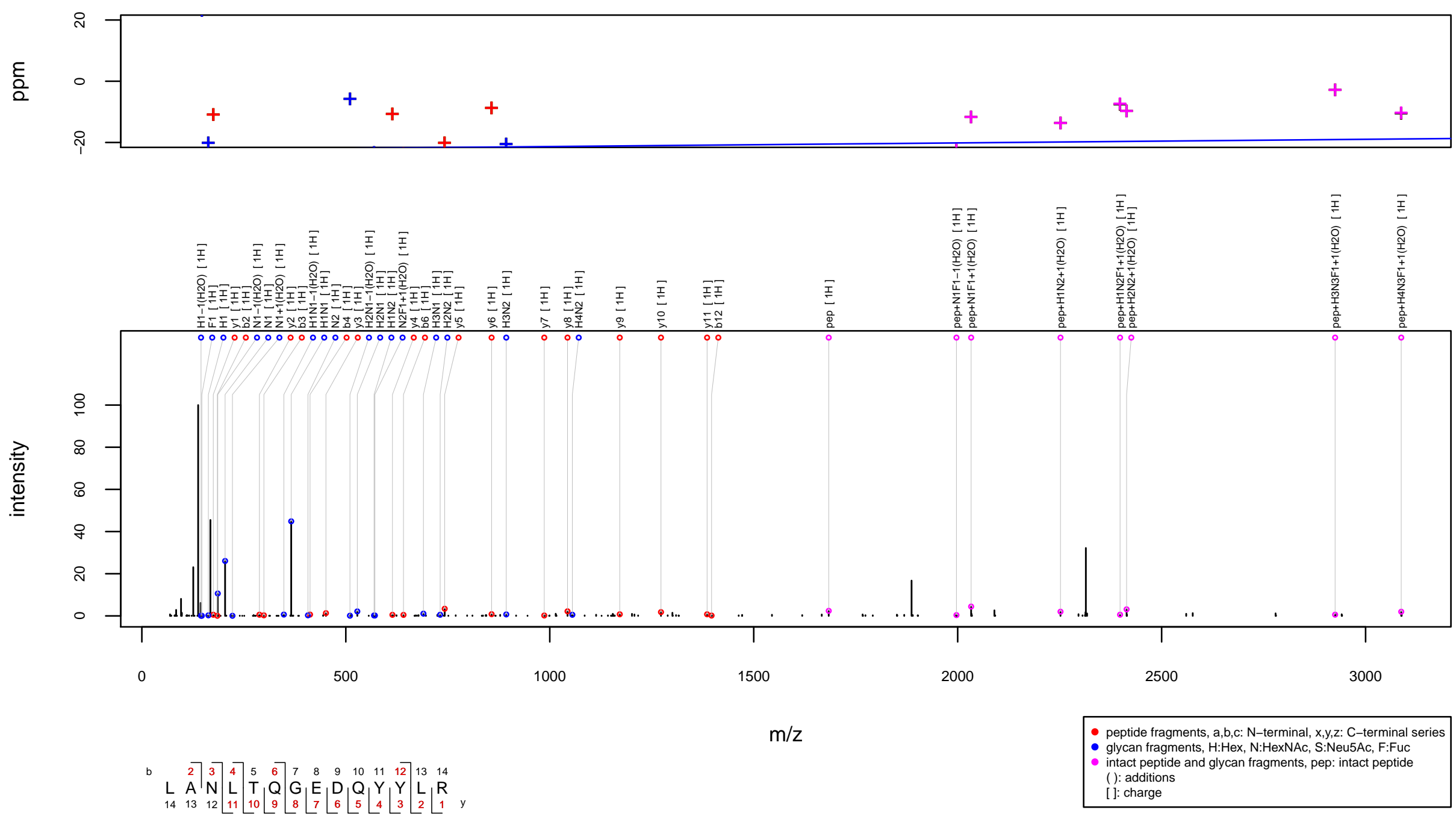
$\mathrm{m} / \mathrm{z} 1153.2892$ charge 4 scan $0-0$

Score $=53.11$, Hits $=39$, Explained Intensity $=0.39$

Peptide: FCGBP_HUMAN[1821,1845]:NPNNDQVFPNGTLAPSIPIWGGSWR

Glycan: $\mathrm{SHNH}(\mathrm{H}(\mathrm{H}) \mathrm{H}) \mathrm{HNN}, \mathrm{S} 1 \mathrm{H} 6 \mathrm{~N} 3$

Charge: $4 \mathrm{H}$
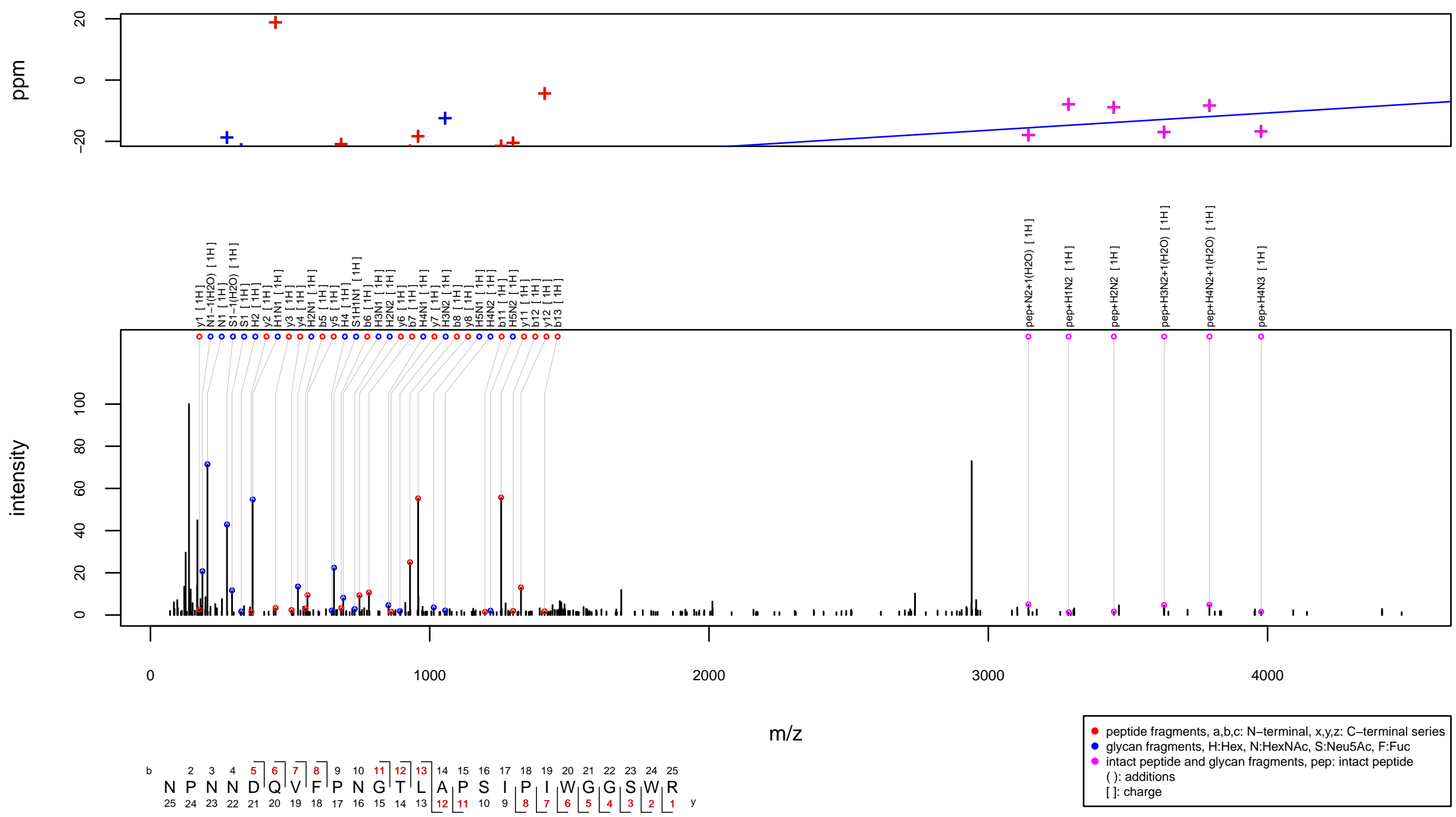
$\mathrm{m} / \mathrm{z} 1155.2144$ charge 3 scan $0-0$

Score $=42.56$, Hits $=30$, Explained Intensity $=0.28$ Peptide: APC_HUMAN[1814,1826]:QNLKNNSKVFNDK Glycan: $\mathrm{FHNH}(\mathrm{FHNH}) \mathrm{HNN}, \mathrm{H} 5 \mathrm{~N} 4 \mathrm{~F} 2$

Charge: $3 \mathrm{H}$
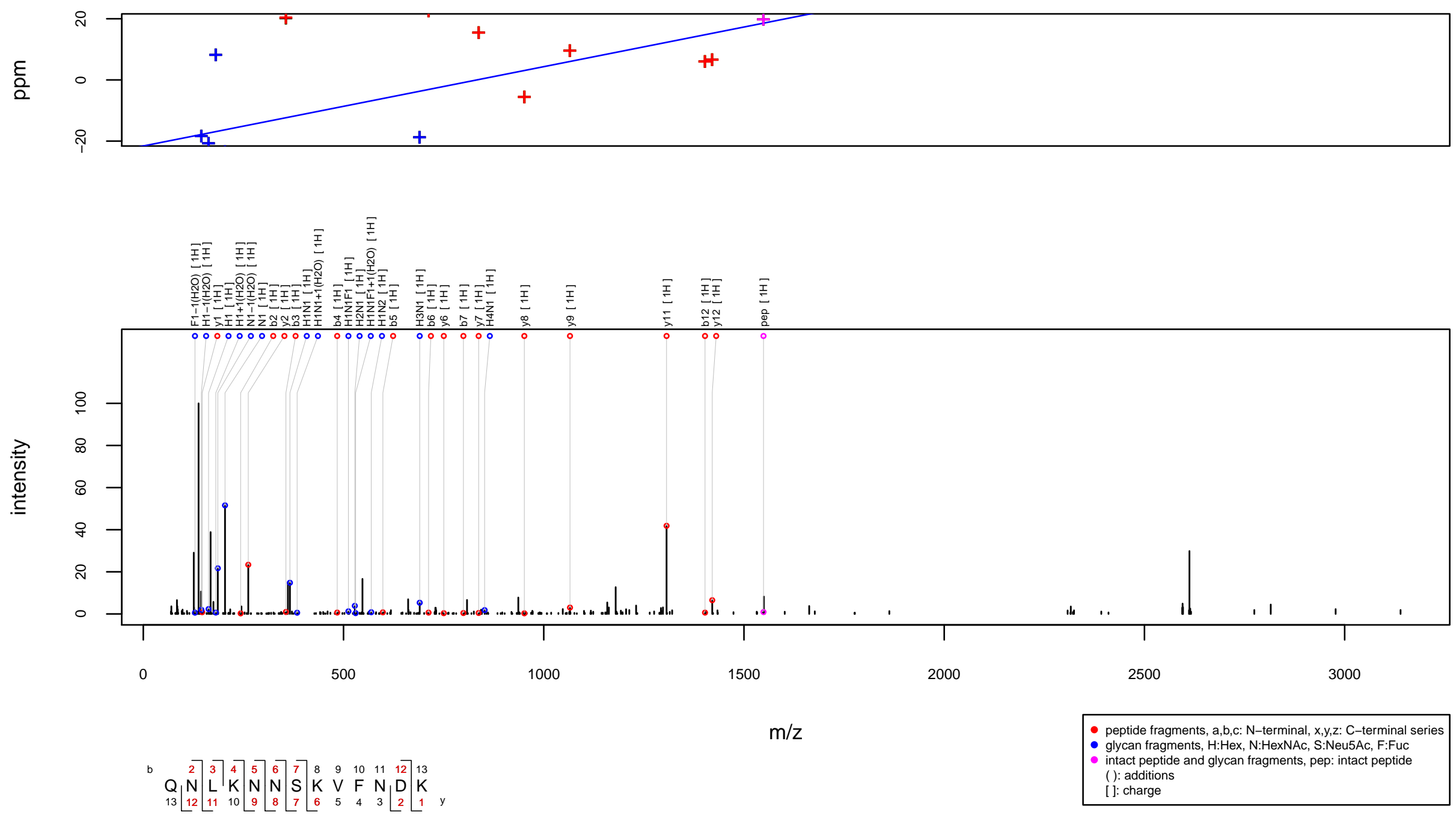
$\mathrm{m} / \mathrm{z} 1156.0087$ charge 4 scan $0-0$

Score $=85.49$, Hits $=67$, Explained Intensity $=0.54$ Peptide: CLUS_HUMAN[372,385]:LANLTQGEDQYYLR Glycan: $\mathrm{HNH}(\mathrm{F}) \mathrm{NH}(\mathrm{F}(\mathrm{H}) \mathrm{NH}(\mathrm{F}) \mathrm{NH}) \mathrm{HN}(\mathrm{F}) \mathrm{N}, \mathrm{H} 7 \mathrm{N6F} 4$

Charge: $4 \mathrm{H}$
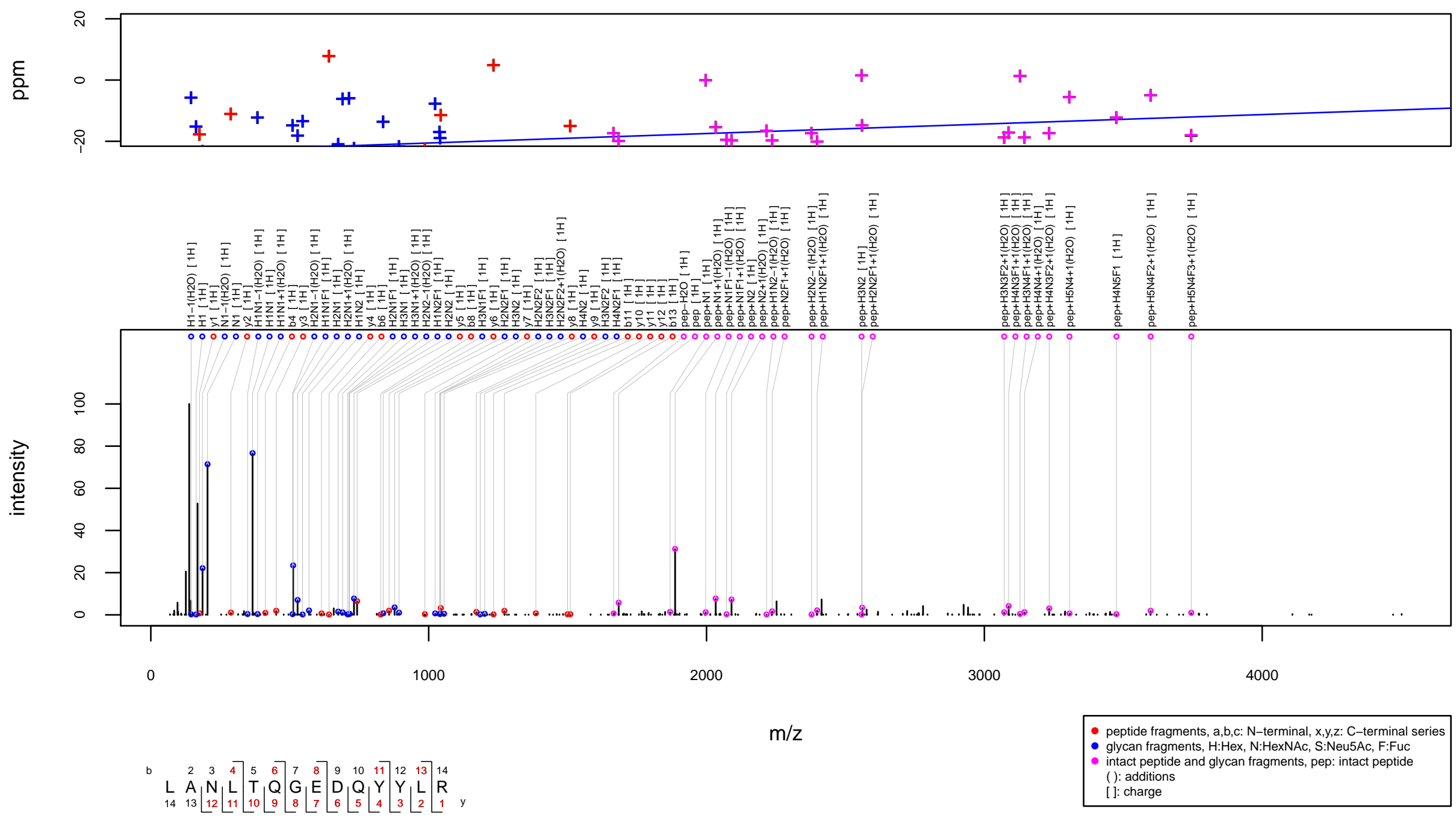
$\mathrm{m} / \mathrm{z} 1156.499$ charge 4 scan $0-0$

Score $=71.51$, Hits $=56$, Explained Intensity $=0.44$

Peptide: FINC_HUMAN[516,533]:DQCIVDDITYNVNDTFHK

Glycan: $\mathrm{HN}(\mathrm{F}(\mathrm{H}) \mathrm{N}) \mathrm{H}(\mathrm{F}(\mathrm{H}) \mathrm{NH}) \mathrm{HN}(\mathrm{F}) \mathrm{N}, \mathrm{H} 6 \mathrm{~N} 5 \mathrm{~F} 3$

Charge: $4 \mathrm{H}$
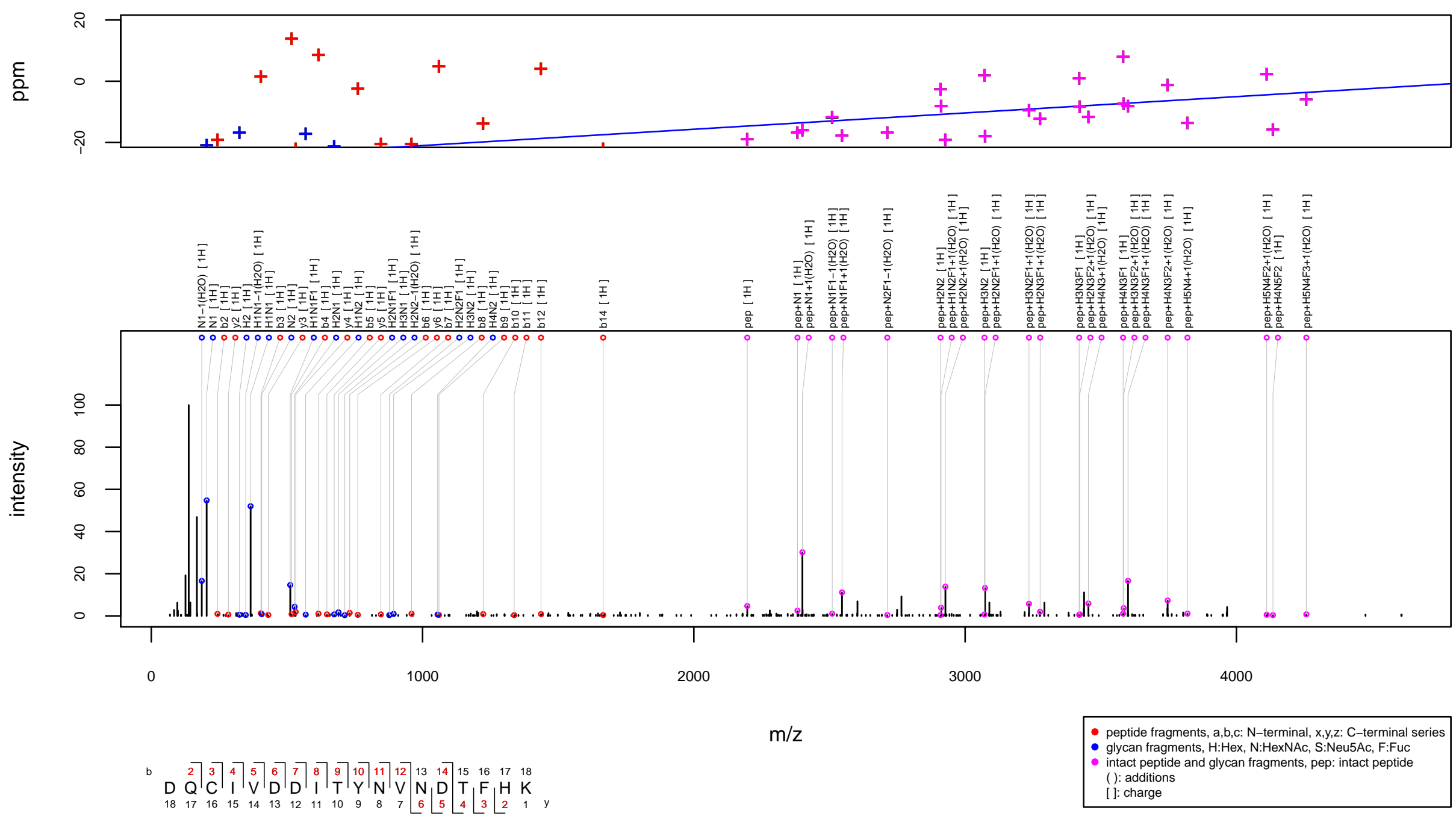
$\mathrm{m} / \mathrm{z} 1157.4614$ charge 3 scan $0-0$

Score $=88.44$, Hits $=61$, Explained Intensity $=0.5$

Peptide: PPAP_HUMAN[92,98]:FLNESYK

Glycan: $\mathrm{SHNH}(\mathrm{SHN}(\mathrm{HN}) \mathrm{H}) \mathrm{HNN}$, S2H6N5

Charge: $3 \mathrm{H}$
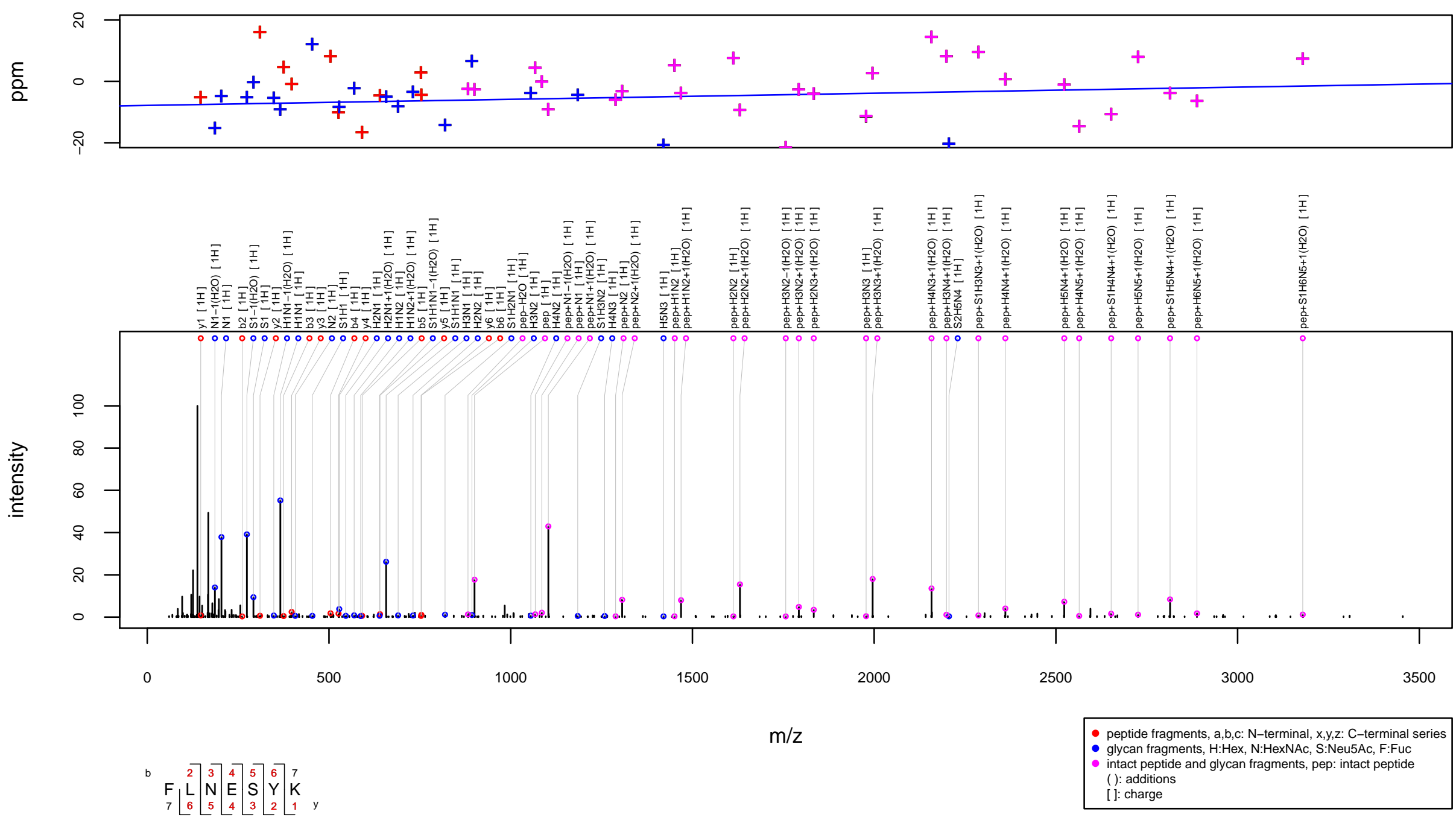
$\mathrm{m} / \mathrm{z} 1159.4873$ charge 3 scan $0-0$

Score $=37.12$, Hits $=31$, Explained Intensity $=0.22$

Peptide: KCNU1_HUMAN[657,670]:VSASTSSISNFTTR

Glycan: $\mathrm{SHNH}(\mathrm{H}(\mathrm{H}) \mathrm{H}) \mathrm{HN}(\mathrm{F}) \mathrm{N}, \mathrm{S} 1 \mathrm{H} 6 \mathrm{~N} 3 \mathrm{~F} 1$

Charge: $3 \mathrm{H}$
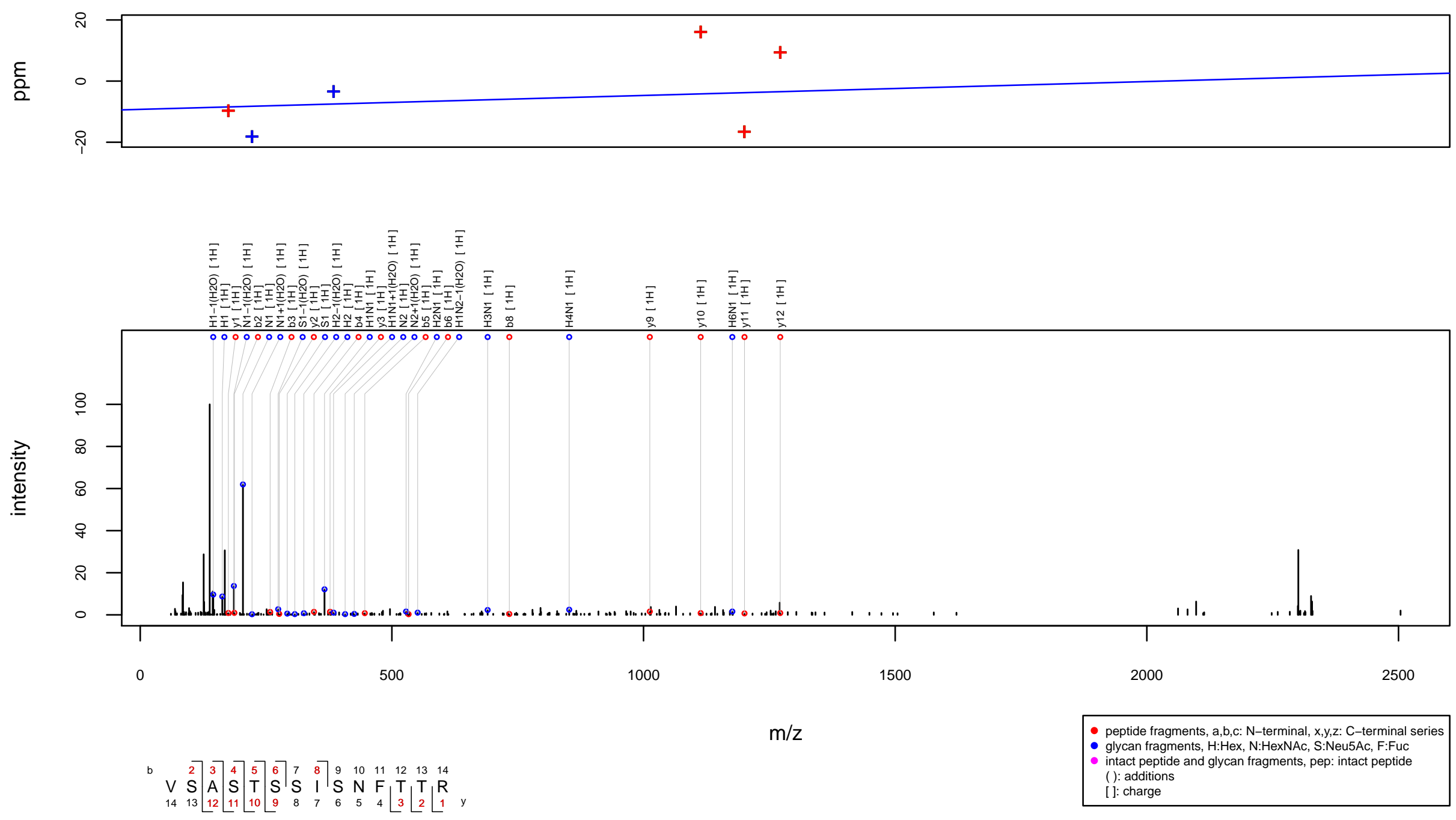
$\mathrm{m} / \mathrm{z} 1163.1996$ charge 3 scan 0-0

Score $=50.29$, Hits $=37$, Explained Intensity $=0.29$ Peptide: AMPN_HUMAN[231,241]:AEFNITLIHPK

Glycan: $\mathrm{SHNH}(\mathrm{SHNH}) \mathrm{HNN}, \mathrm{S} 2 \mathrm{H} 5 \mathrm{~N} 4$

Charge: $3 \mathrm{H}$
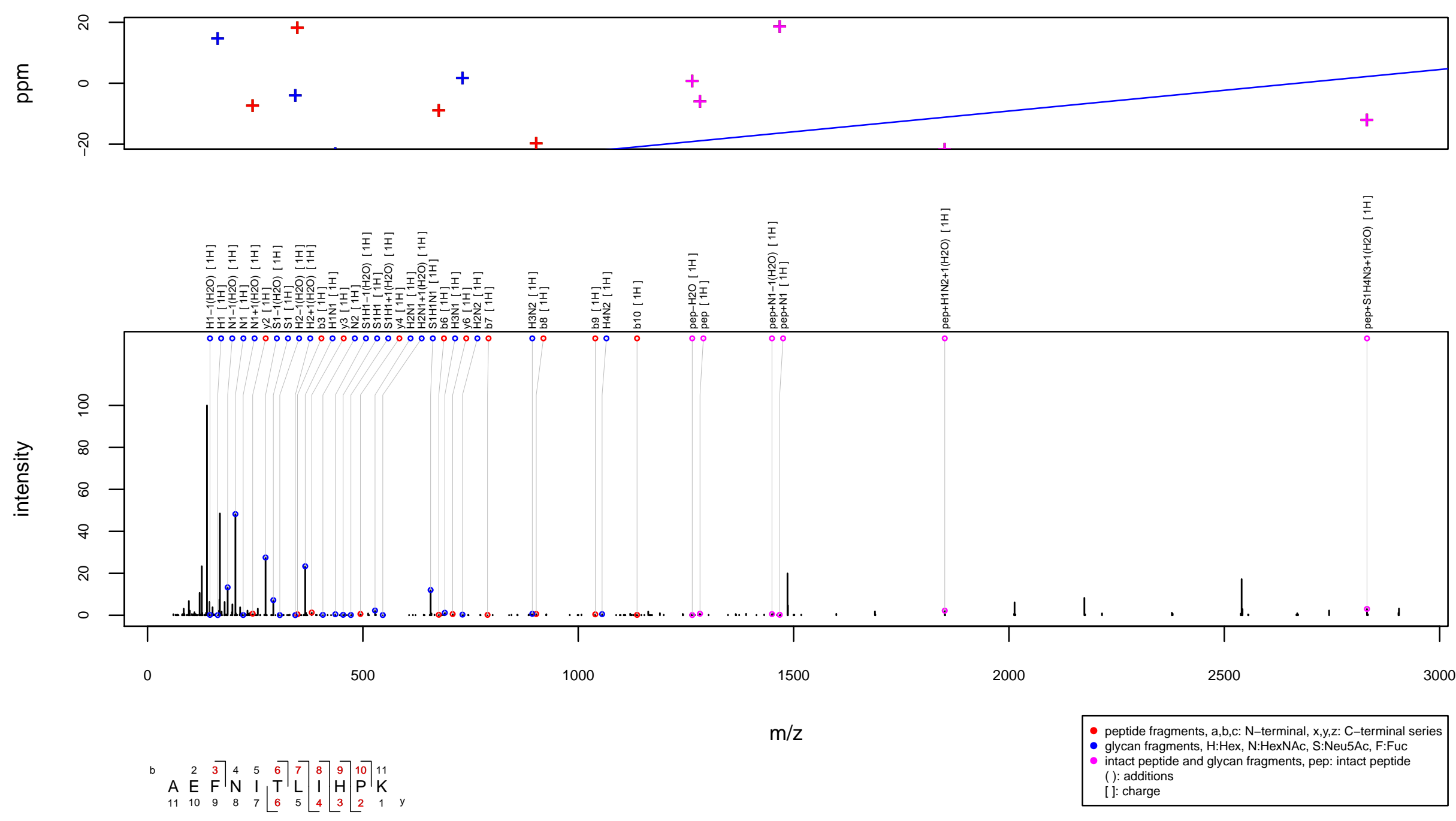
$\mathrm{m} / \mathrm{z} 1163.5461$ charge 4 scan $0-0$

Score $=63.21$, Hits $=45$, Explained Intensity $=0.37$

Peptide: FCGBP_HUMAN[1821,1845]:NPNNDQVFPNGTLAPSIPIWGGSWR

Glycan: $\mathrm{SHNH}(\mathrm{HNH}) \mathrm{HNN}, \mathrm{S} 1 \mathrm{H} 5 \mathrm{~N} 4$

Charge: $4 \mathrm{H}$
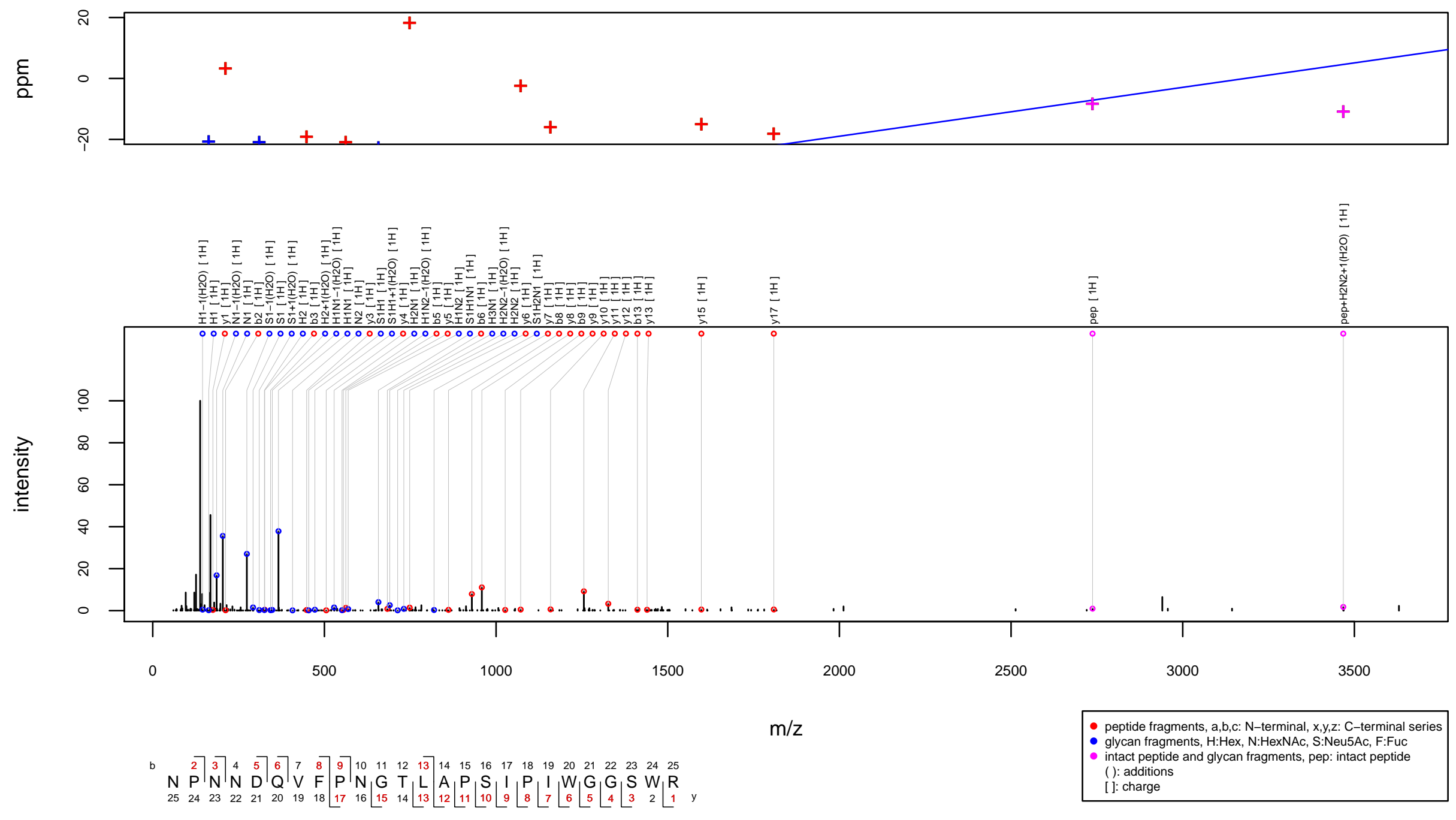
$\mathrm{m} / \mathrm{z} 1163.7979$ charge 4 scan $0-0$

Score $=47.34$, Hits $=37$, Explained Intensity $=0.31$

Peptide: FCGBP_HUMAN[1821,1845]:NPNNDQVFPNGTLAPSIPIWGGSWR

Glycan: $\mathrm{FHNH}(\mathrm{FHNH}) \mathrm{HNN}$, H5N4F2

Charge: $4 \mathrm{H}$
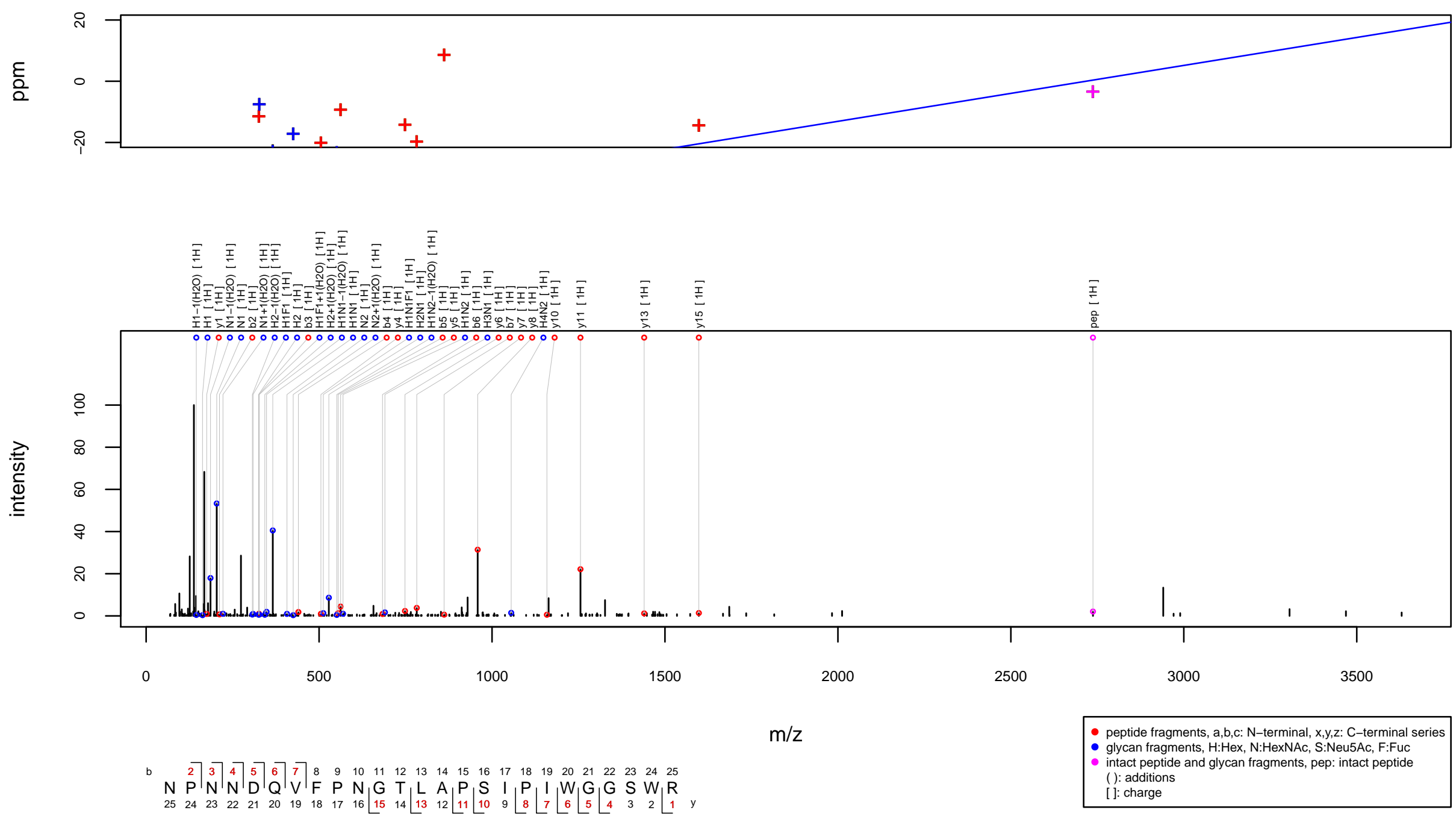


\section{$\mathrm{m} / \mathrm{z} 1165.0388$ charge 4 scan $0-0$}

Score $=84.27$, Hits $=60$, Explained Intensity $=0.48$

Peptide: FINC_HUMAN[997,1016]:LDAPTNLQFVNETDSTVLVR

Glycan: $\mathrm{SHNHNH}(\mathrm{F}(\mathrm{H}) \mathrm{NH}) \mathrm{HNN}, \mathrm{S} 1 \mathrm{H} 6 \mathrm{~N} 5 \mathrm{~F} 1$

Charge: $4 \mathrm{H}$
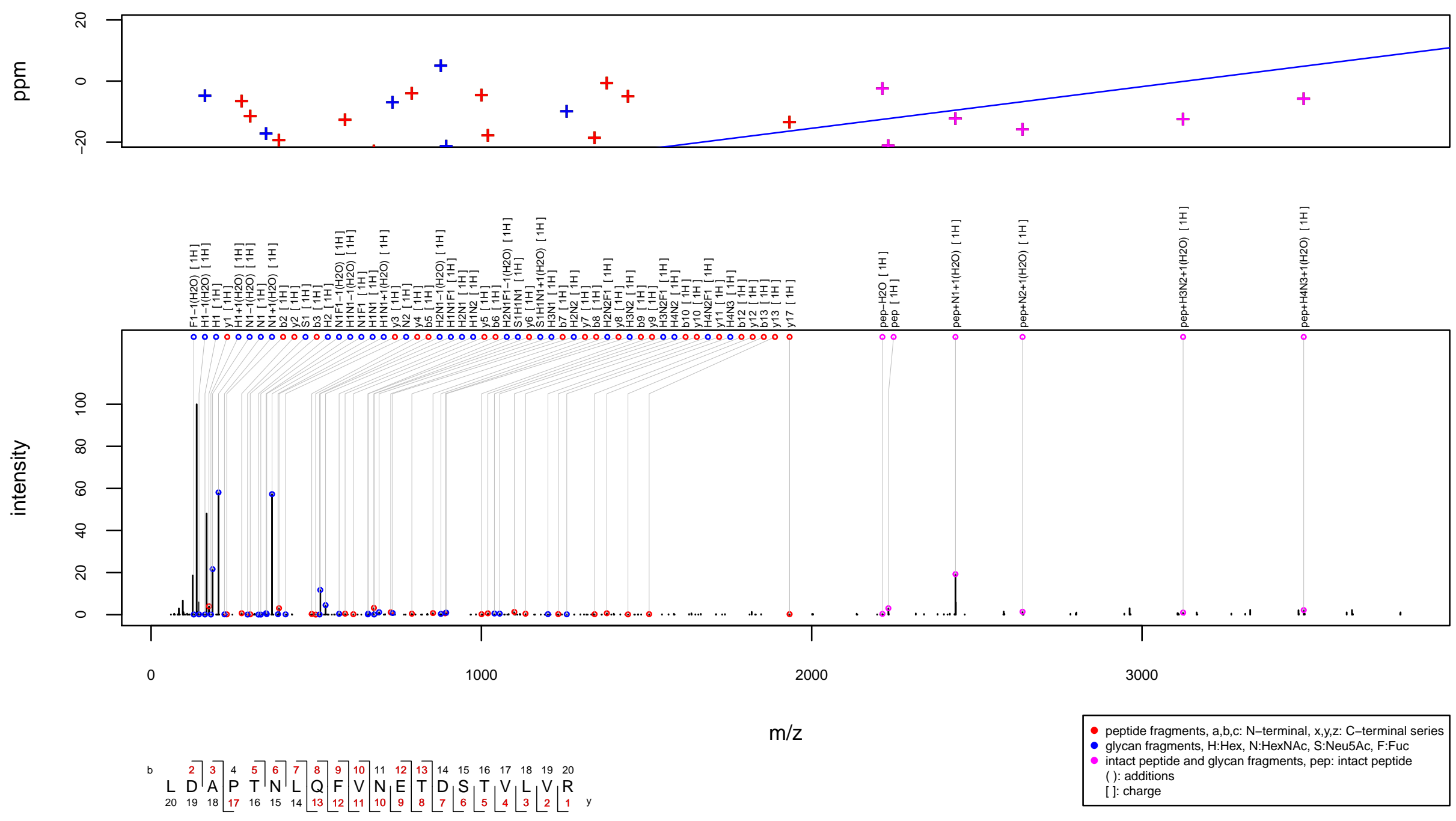
$\mathrm{m} / \mathrm{z} 1173.5101$ charge 4 scan $0-0$

Score $=82.24$, Hits $=61$, Explained Intensity $=0.44$ Peptide: CLUS_HUMAN[372,385]:LANLTQGEDQYYLR Glycan: $\mathrm{SHNH}(\mathrm{SHN}(\mathrm{SHN}) \mathrm{H}) \mathrm{HN}(\mathrm{F}) \mathrm{N}, \mathrm{S} 3 \mathrm{H} 6 \mathrm{~N} 5 \mathrm{~F} 1$

Charge: $4 \mathrm{H}$
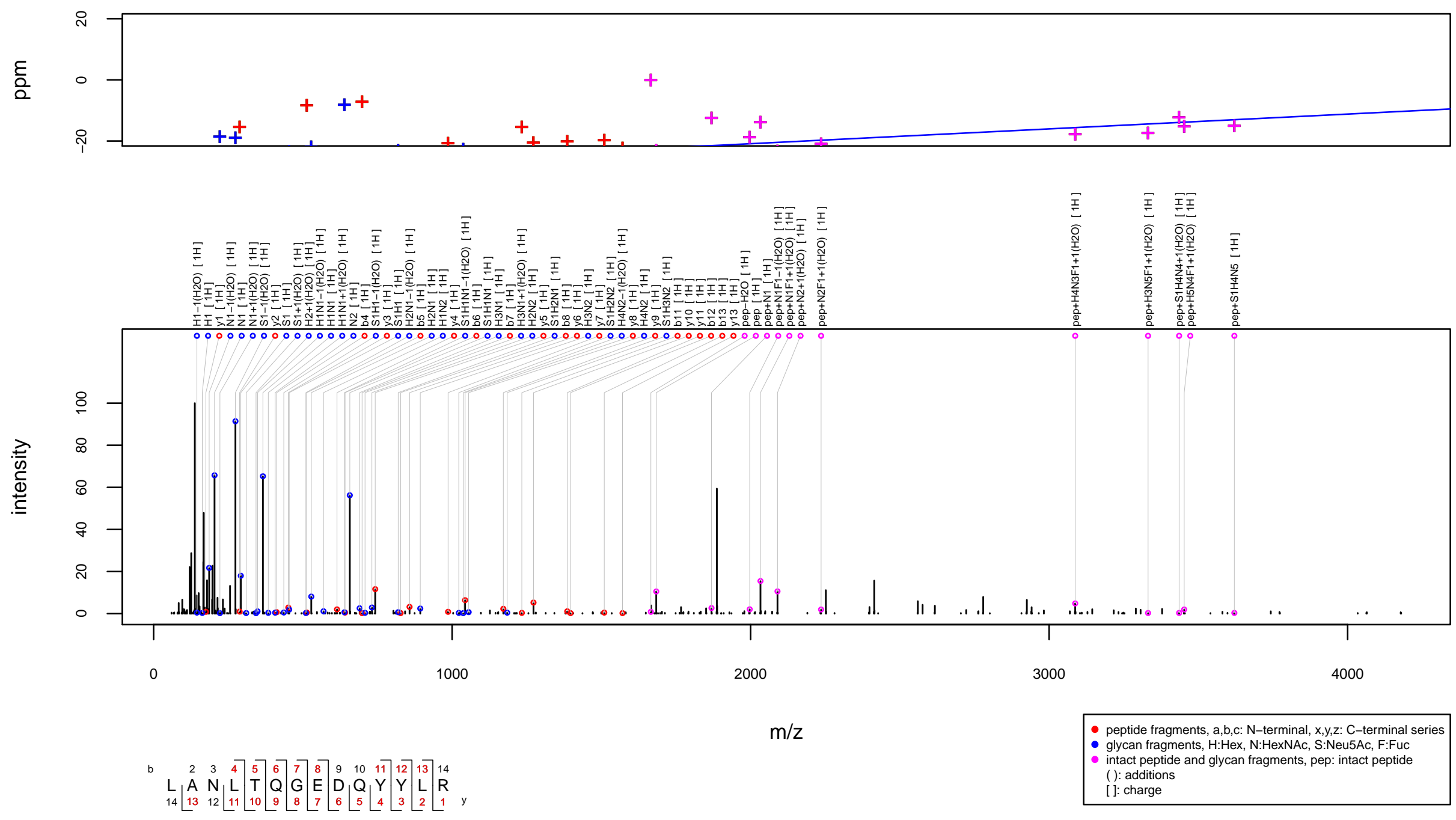
$\mathrm{m} / \mathrm{z} 1173.8123$ charge 3 scan $0-0$

Score $=103.79$, Hits $=82$, Explained Intensity $=0.45$ Peptide: CLUS_HUMAN[82,89]:EDALNETR

Glycan: $\mathrm{F}(\mathrm{H}) \mathrm{NH}(\mathrm{F}(\mathrm{H}) \mathrm{N}(\mathrm{F}(\mathrm{H}) \mathrm{N}) \mathrm{H}) \mathrm{HN}(\mathrm{F}) \mathrm{N}, \mathrm{H} 6 \mathrm{~N} 5 \mathrm{~F} 4$

Charge: $3 \mathrm{H}$
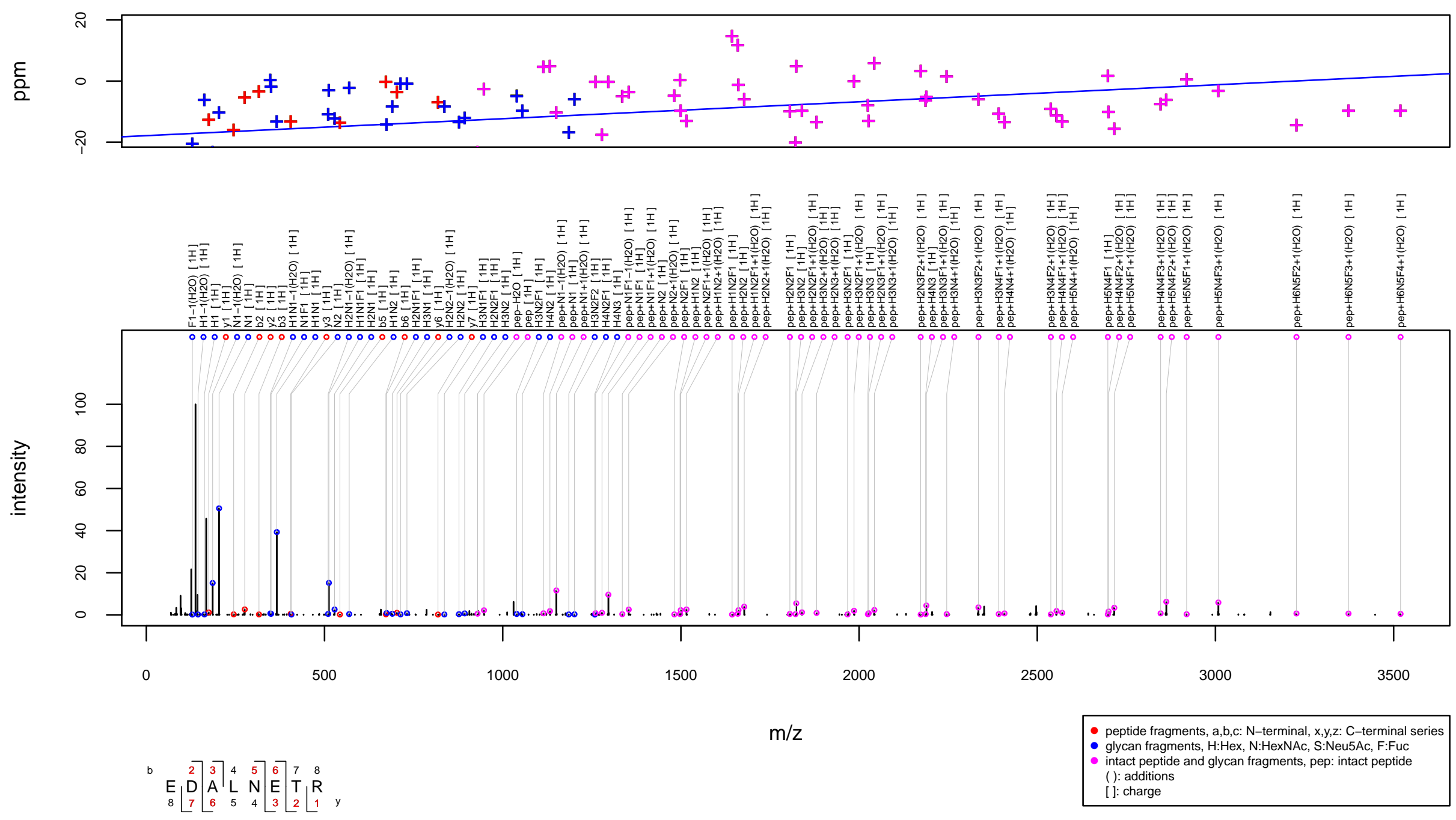
$\mathrm{m} / \mathrm{z} 1174.5398$ charge 3 scan $0-0$

Score $=78.36$, Hits $=58$, Explained Intensity $=0.45$ Peptide: TIMP1_HUMAN[46,60]:FVGTPEVNQTTLYQR Glycan: H5N4F1

Charge: $3 \mathrm{H}$
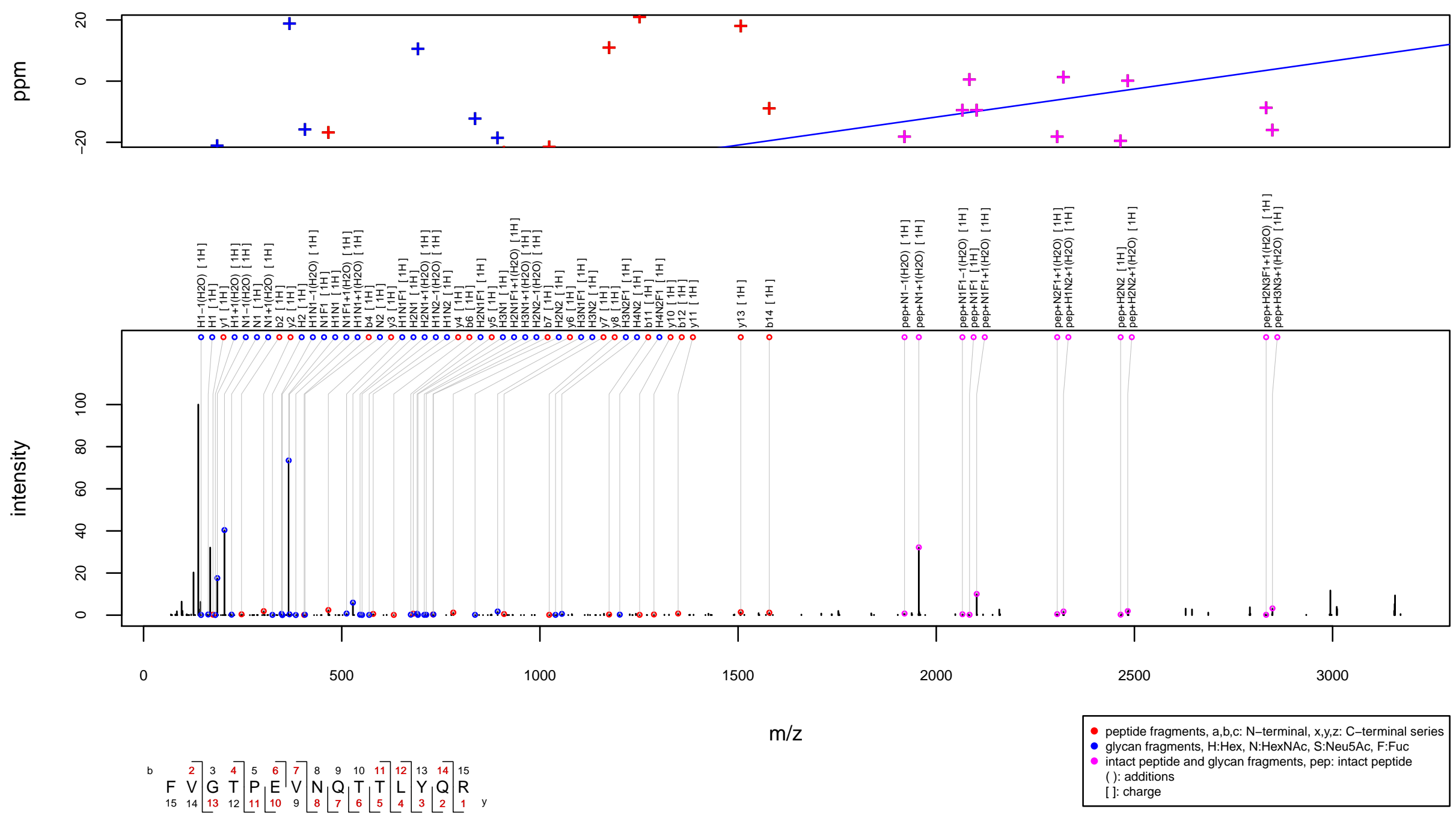
$\mathrm{m} / \mathrm{z} 1177.0314$ charge 4 scan $0-0$

Score $=37.85$, Hits $=43$, Explained Intensity $=0.37$

Peptide: CLUS_HUMAN[352,371]:MLNTSSLLEQLNEQFNWVSR

Glycan: S1H5N3F3

Charge: $4 \mathrm{H}$
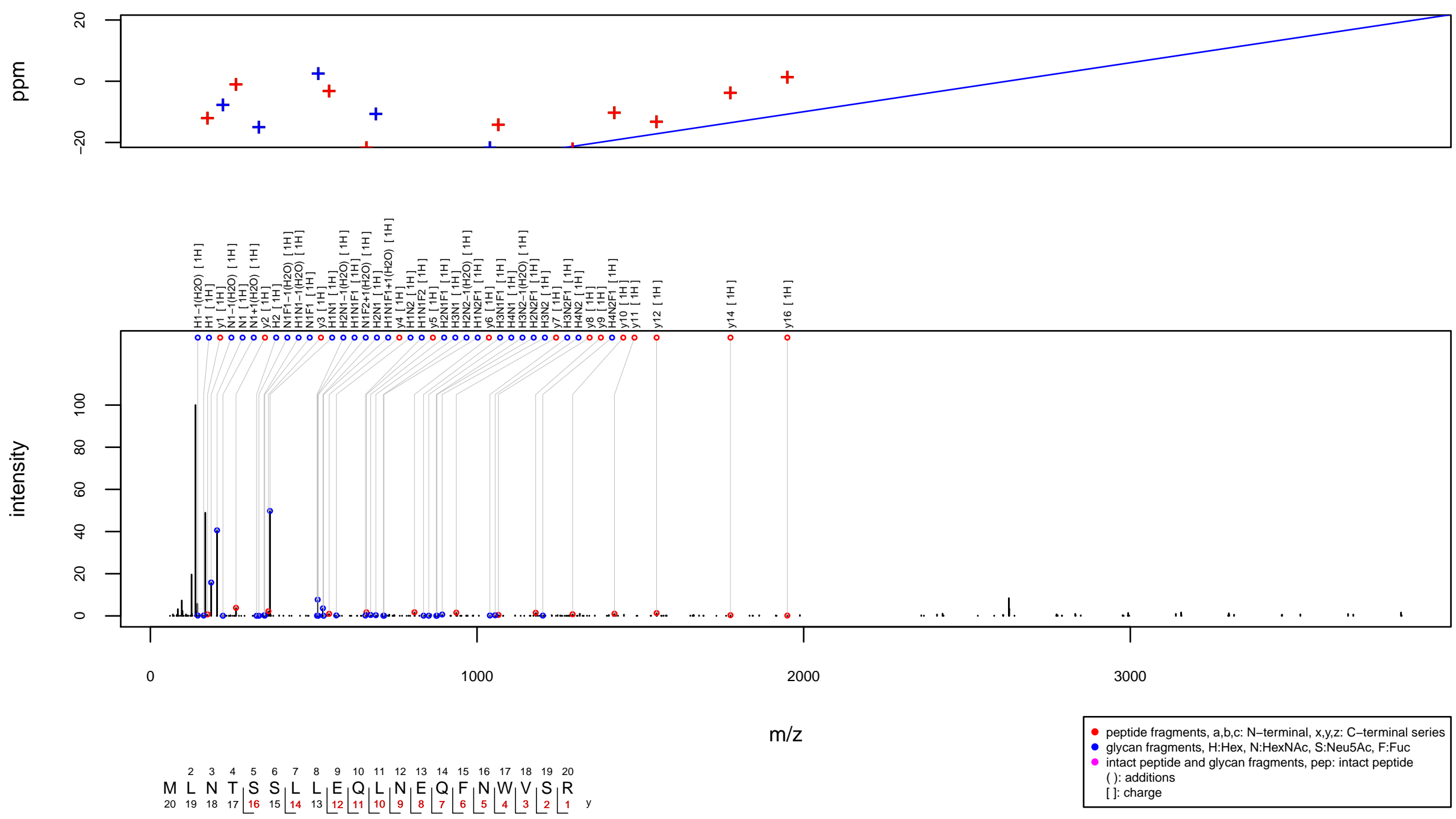
$\mathrm{m} / \mathrm{z} 1180.258$ charge 3 scan $0-0$

Score $=30.23$, Hits $=24$, Explained Intensity $=0.11$ Peptide: WDR43_HUMAN[309,324]:KPLTSNCTIQIATPGK Glycan: $\mathrm{HNH}(\mathrm{NH})(\mathrm{N}) \mathrm{HN}(\mathrm{F}) \mathrm{N}, \mathrm{H} 4 \mathrm{~N} 5 \mathrm{~F} 1$

Charge: $3 \mathrm{H}$
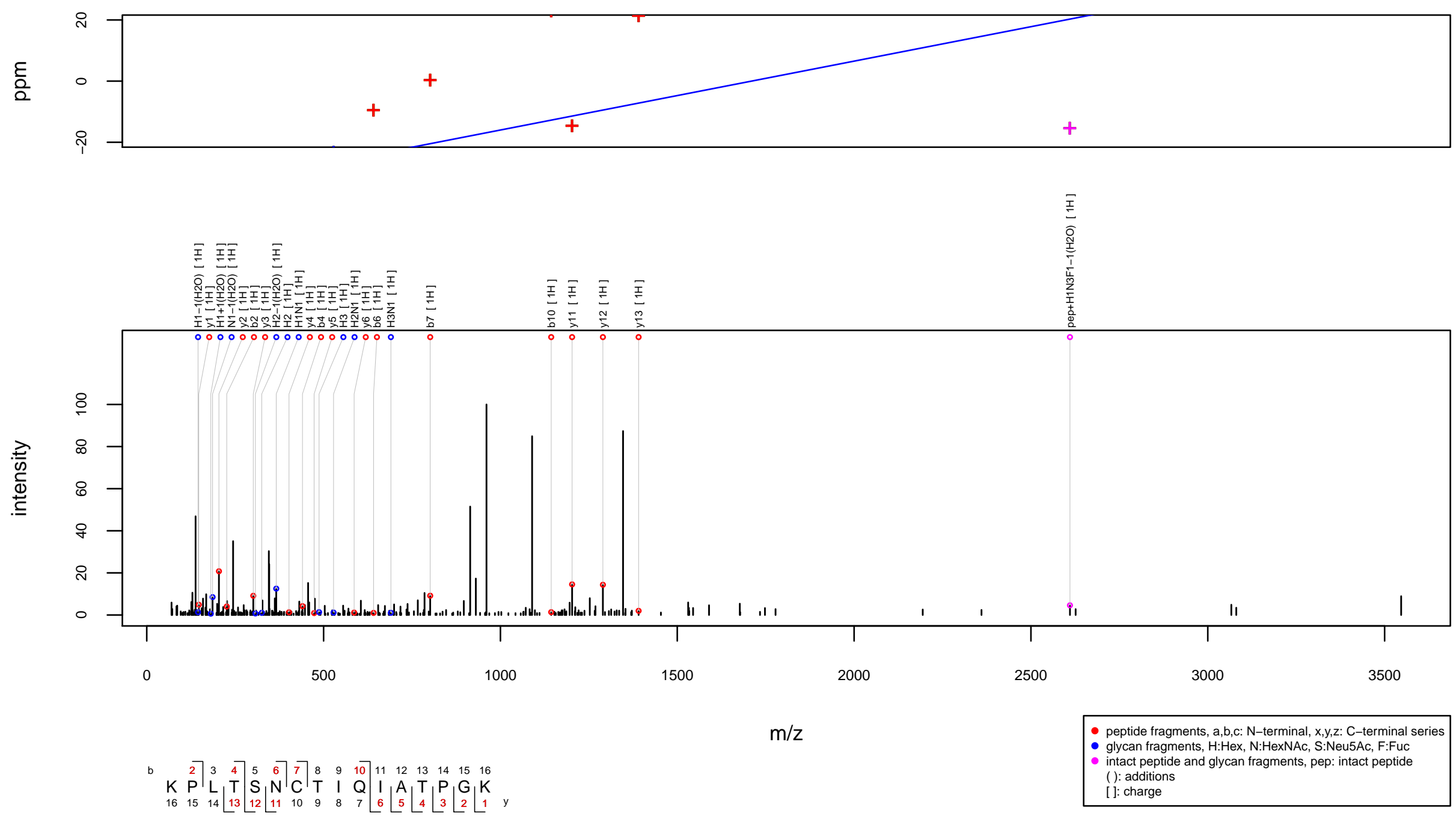
$\mathrm{m} / \mathrm{z} 1180.5099$ charge 4 scan 0-0

Score $=43.03$, Hits $=55$, Explained Intensity $=0.29$

Peptide: CLUS_HUMAN[372,385]:LANLTQGEDQYYLR

Glycan: H6N8F3

Charge: $4 \mathrm{H}$
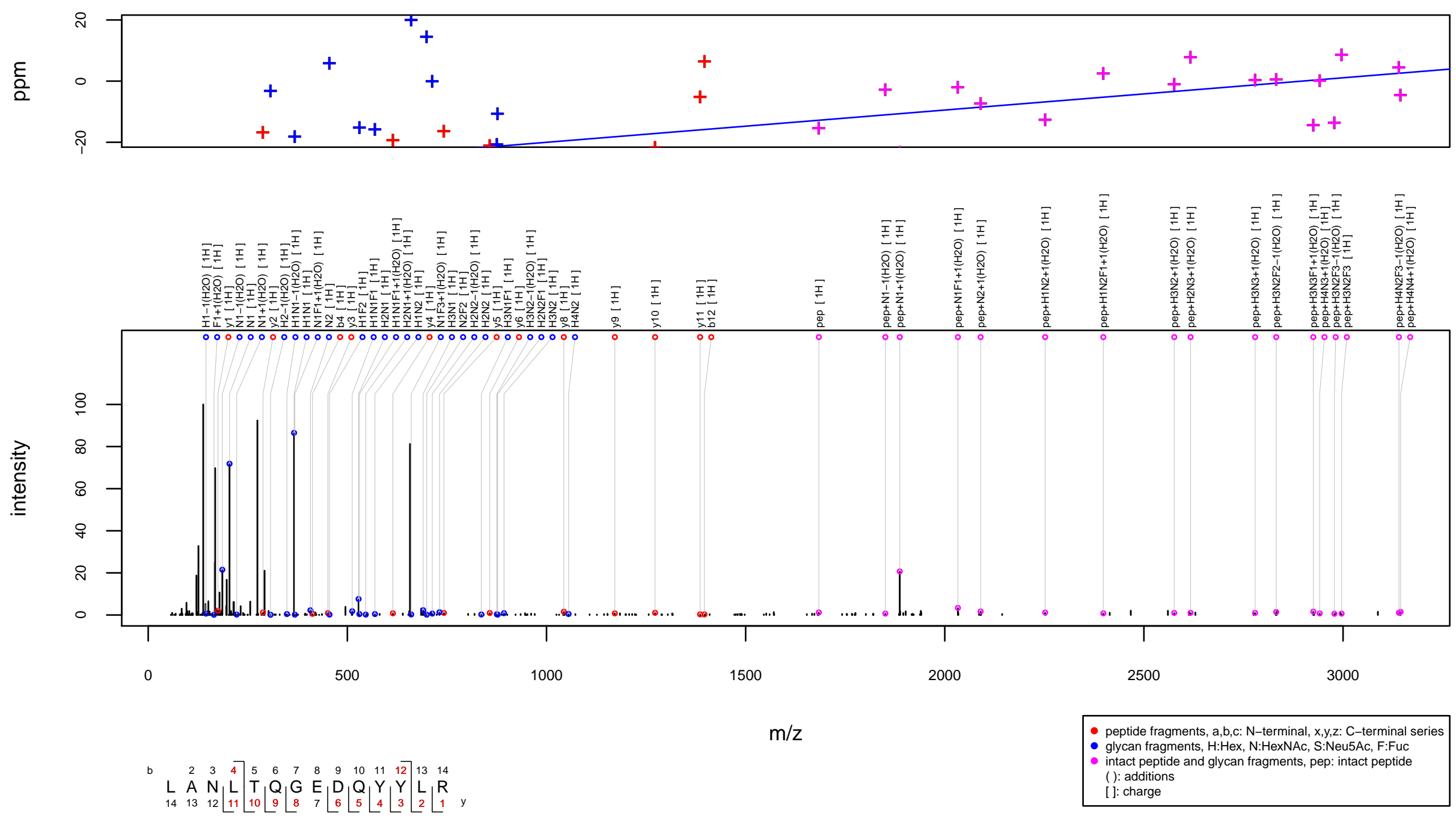
$\mathrm{m} / \mathrm{z} 1180.7645$ charge 4 scan $0-0$

Score $=68.44$, Hits $=46$, Explained Intensity $=0.33$

Peptide: TRFE_HUMAN[622,642]:QQQHLFGSNVTDCSGNFCLFR

Glycan: $\mathrm{SHNH}(\mathrm{SHNH}) \mathrm{HNN}, \mathrm{S} 2 \mathrm{H} 5 \mathrm{~N} 4$

Charge: $4 \mathrm{H}$
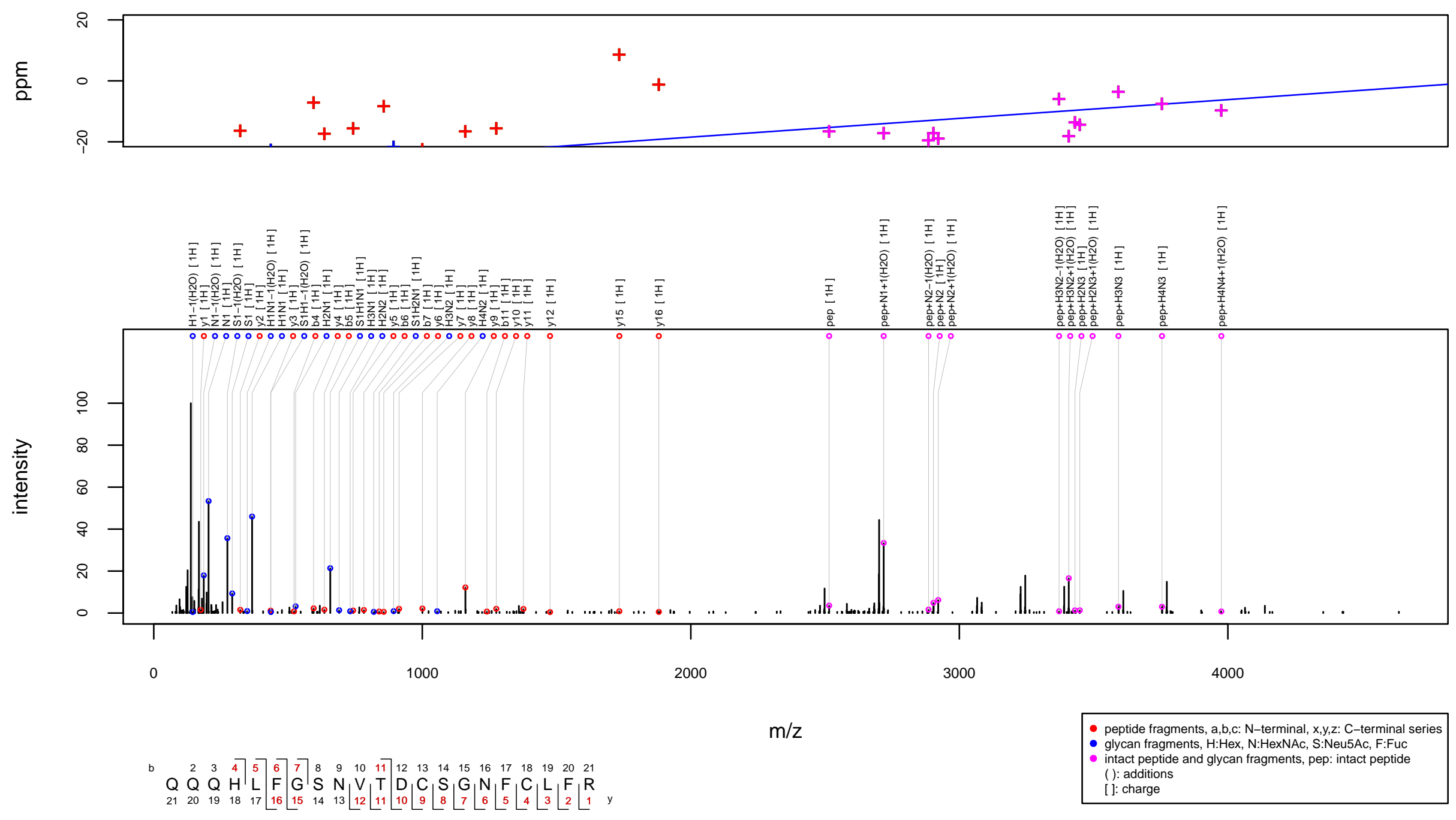
$\mathrm{m} / \mathrm{z} 1182.7714$ charge 4 scan $0-0$

Score $=22.24$, Hits $=19$, Explained Intensity $=0.03$

Peptide: STRCL_HUMAN[957,972]:GLLECAANGTLSPEGR

Glycan: $\mathrm{F}(\mathrm{H}) \mathrm{NH}(\mathrm{F}) \mathrm{NH}(\mathrm{F}(\mathrm{H}) \mathrm{NH}(\mathrm{F}) \mathrm{NH}) \mathrm{HN}(\mathrm{F}) \mathrm{N}, \mathrm{H} 7 \mathrm{N6F5}$

Charge: $4 \mathrm{H}$
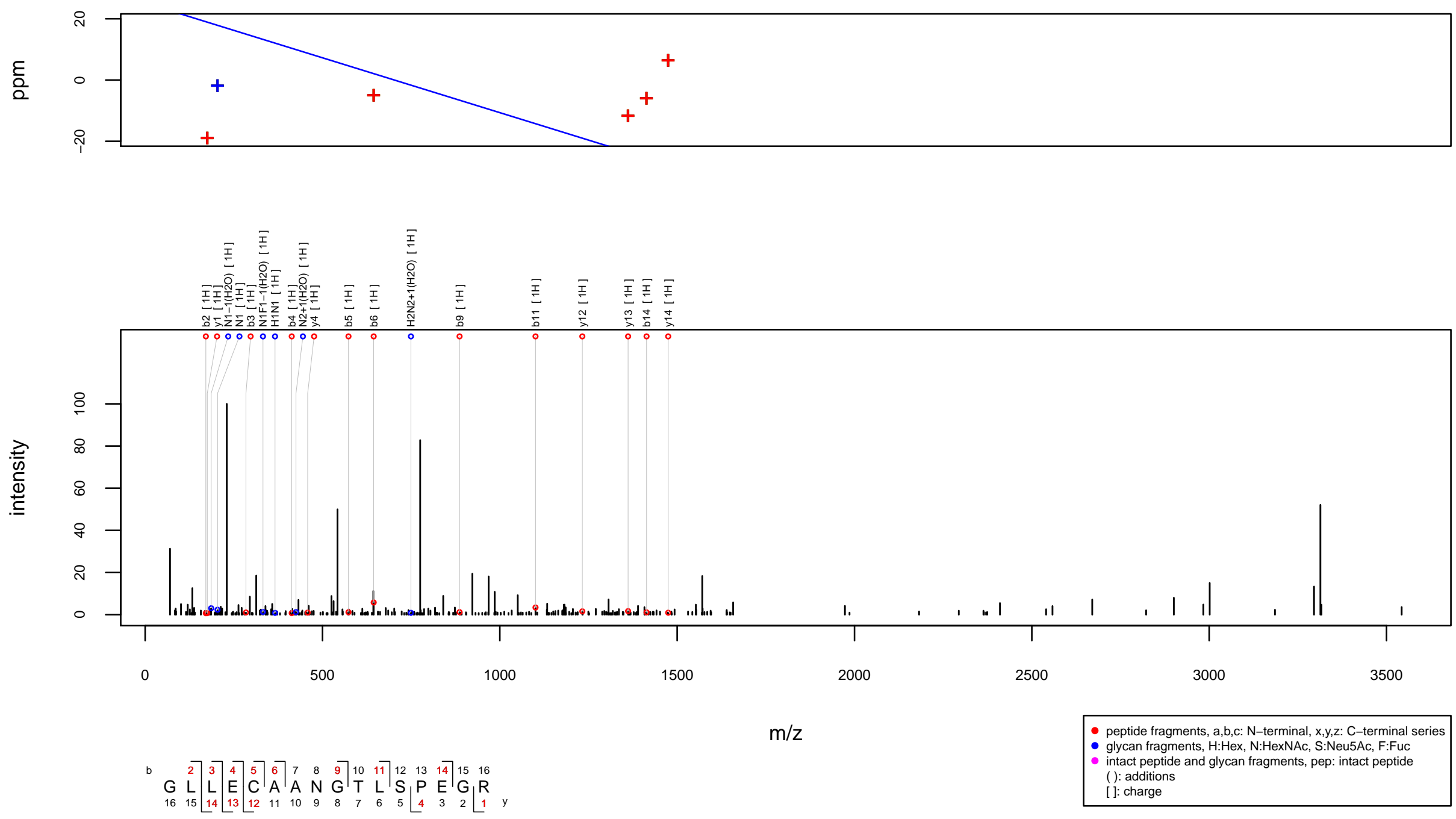
$\mathrm{m} / \mathrm{z} 1183.3201$ charge 4 scan $0-0$

Score $=56.2$, Hits $=39$, Explained Intensity $=0.37$

Peptide: PIGR_HUMAN[168,190]:QIGLYPVLVIDSSGYVNPNYTGR

Glycan: $\mathrm{SHNH}(\mathrm{SHNH}) \mathrm{HNN}$, S2H5N4

Charge: $4 \mathrm{H}$
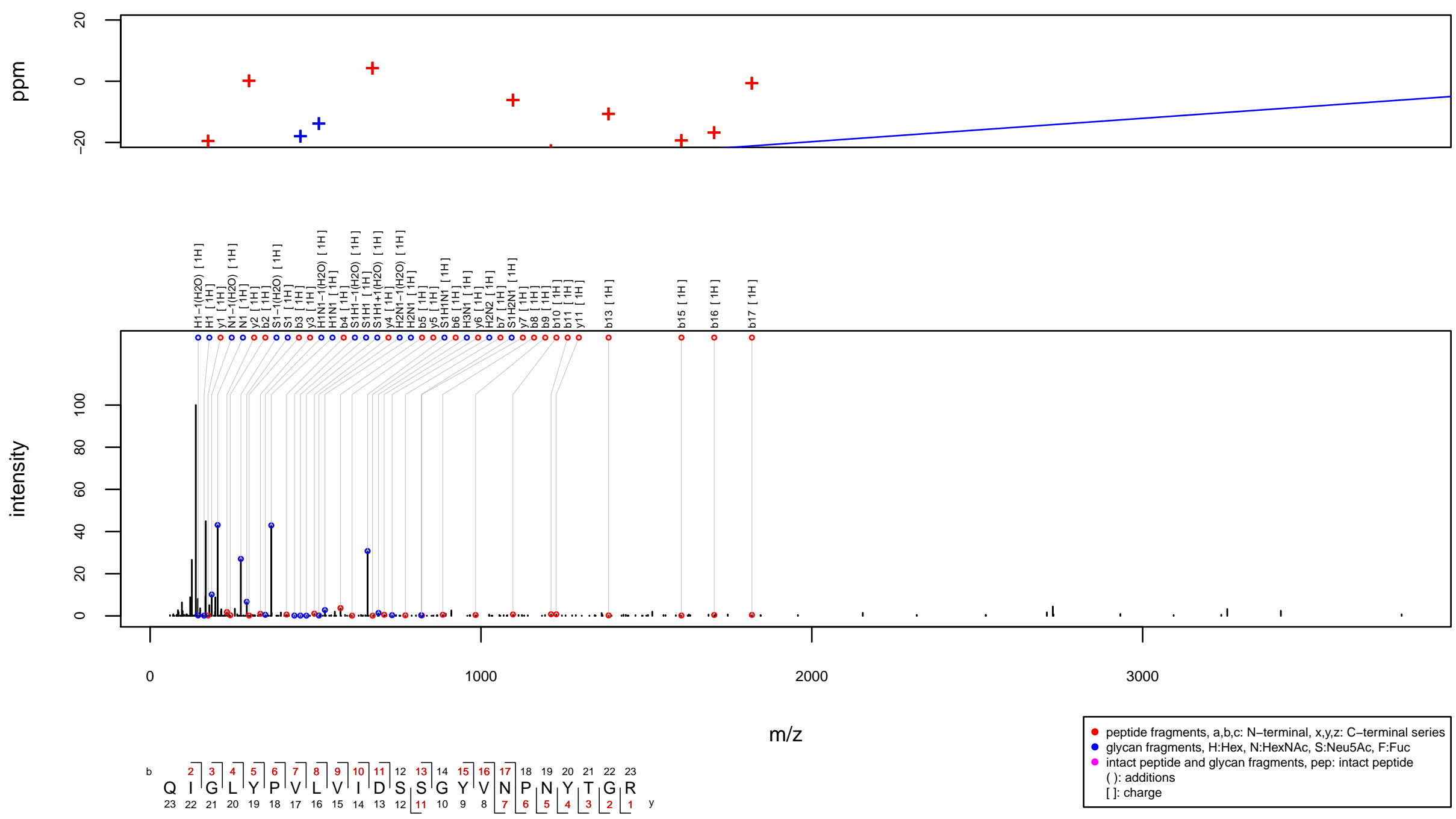
$\mathrm{m} / \mathrm{z} 1183.8076$ charge 4 scan $0-0$

Score $=69.41$, Hits $=66$, Explained Intensity $=0.54$

Peptide: IPSP_HUMAN[254,274]:VVGVPYQGNATALFILPSEGK

Glycan: H6N5F4

Charge: $4 \mathrm{H}$
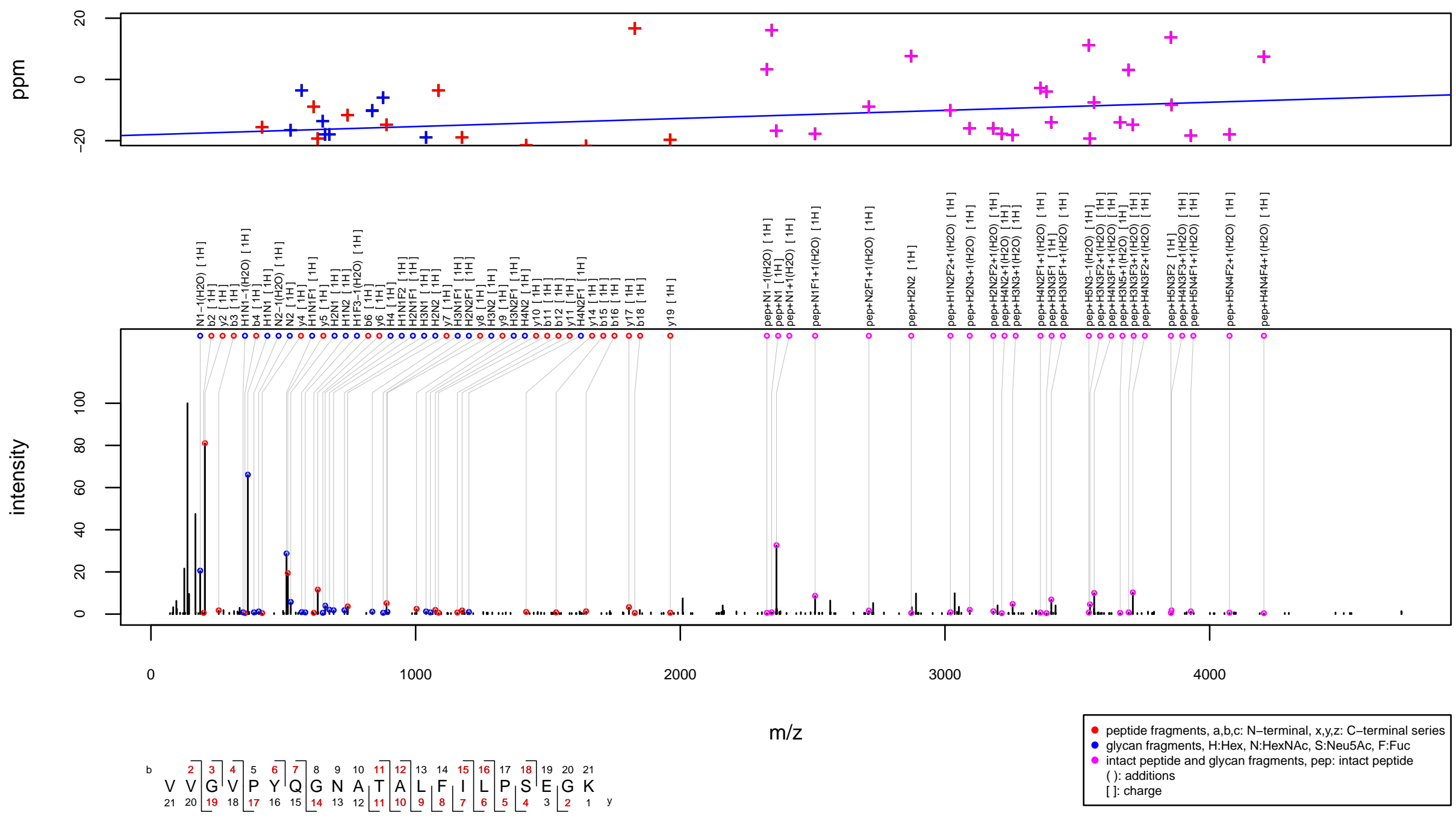
$\mathrm{m} / \mathrm{z} 1189.7004$ charge 5 scan $0-0$

Score $=84.94$, Hits $=60$, Explained Intensity $=0.48$

Peptide: WFDC2_HUMAN[33,60]:TGVCPELQADQNCTQECVSDSECADNLK

Glycan: $\mathrm{SHNH}(\mathrm{SHN}(\mathrm{HN}) \mathrm{H}) \mathrm{HN}(\mathrm{F}) \mathrm{N}, \mathrm{S} 2 \mathrm{H} 6 \mathrm{~N} 5 \mathrm{~F} 1$

Charge: $5 \mathrm{H}$
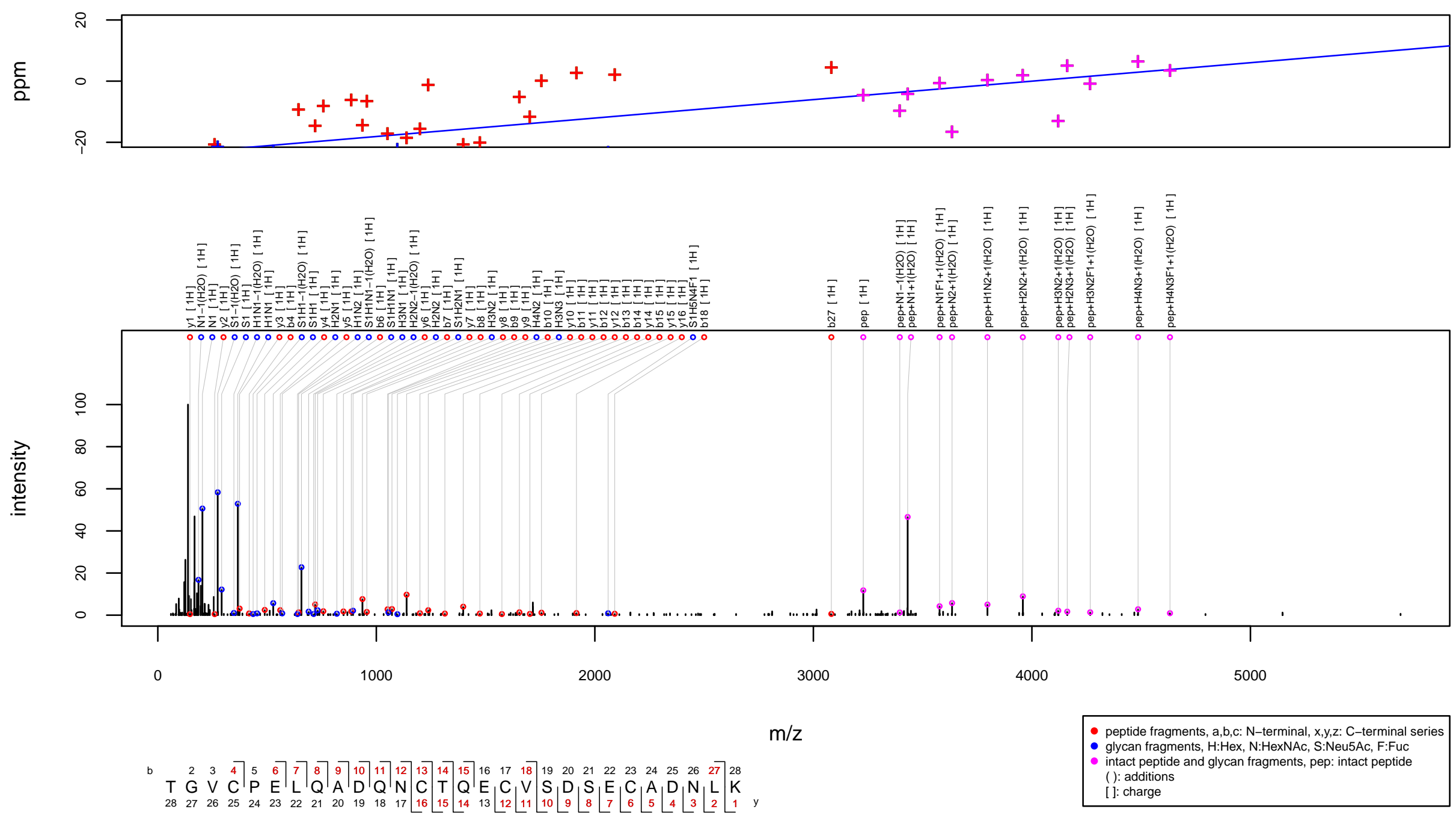
$\mathrm{m} / \mathrm{z} 1192.2521$ charge 4 scan $0-0$

Score $=64.59$, Hits $=83$, Explained Intensity $=0.52$

Peptide: CLUS_HUMAN[372,385]:LANLTQGEDQYYLR

Glycan: S1H7N6F3

Charge: $4 \mathrm{H}$
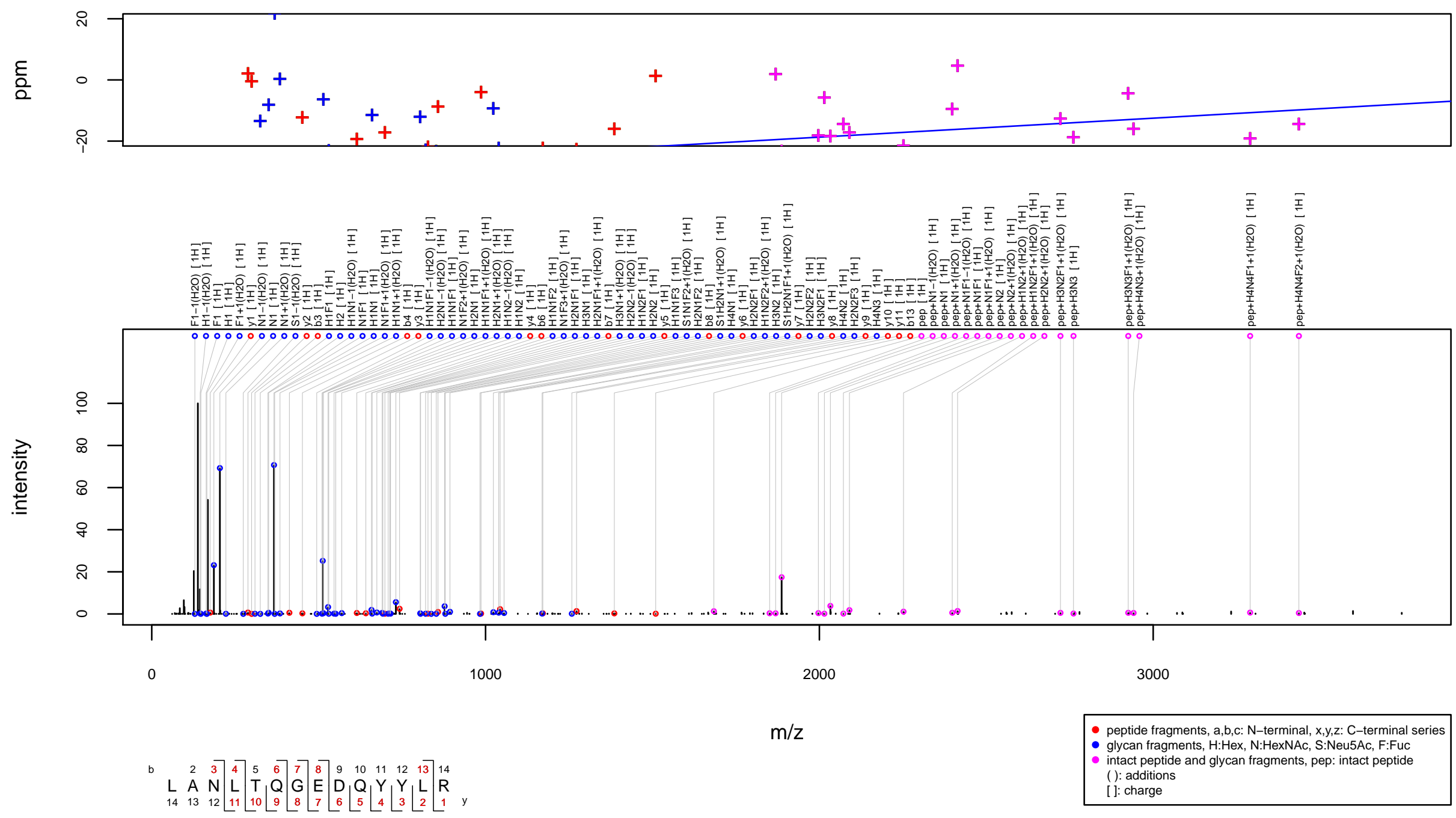
$\mathrm{m} / \mathrm{z} 1192.5098$ charge 4 scan 0-0

Score $=113.64$, Hits $=85$, Explained Intensity $=0.48$ Peptide: CLUS_HUMAN[372,385]:LANLTQGEDQYYLR Glycan: $\mathrm{F}(\mathrm{H}) \mathrm{NH}(\mathrm{F}) \mathrm{NH}(\mathrm{F}(\mathrm{H}) \mathrm{NH}(\mathrm{F}) \mathrm{NH}) \mathrm{HN}(\mathrm{F}) \mathrm{N}, \mathrm{H} 7 \mathrm{N6F5}$

Charge: $4 \mathrm{H}$
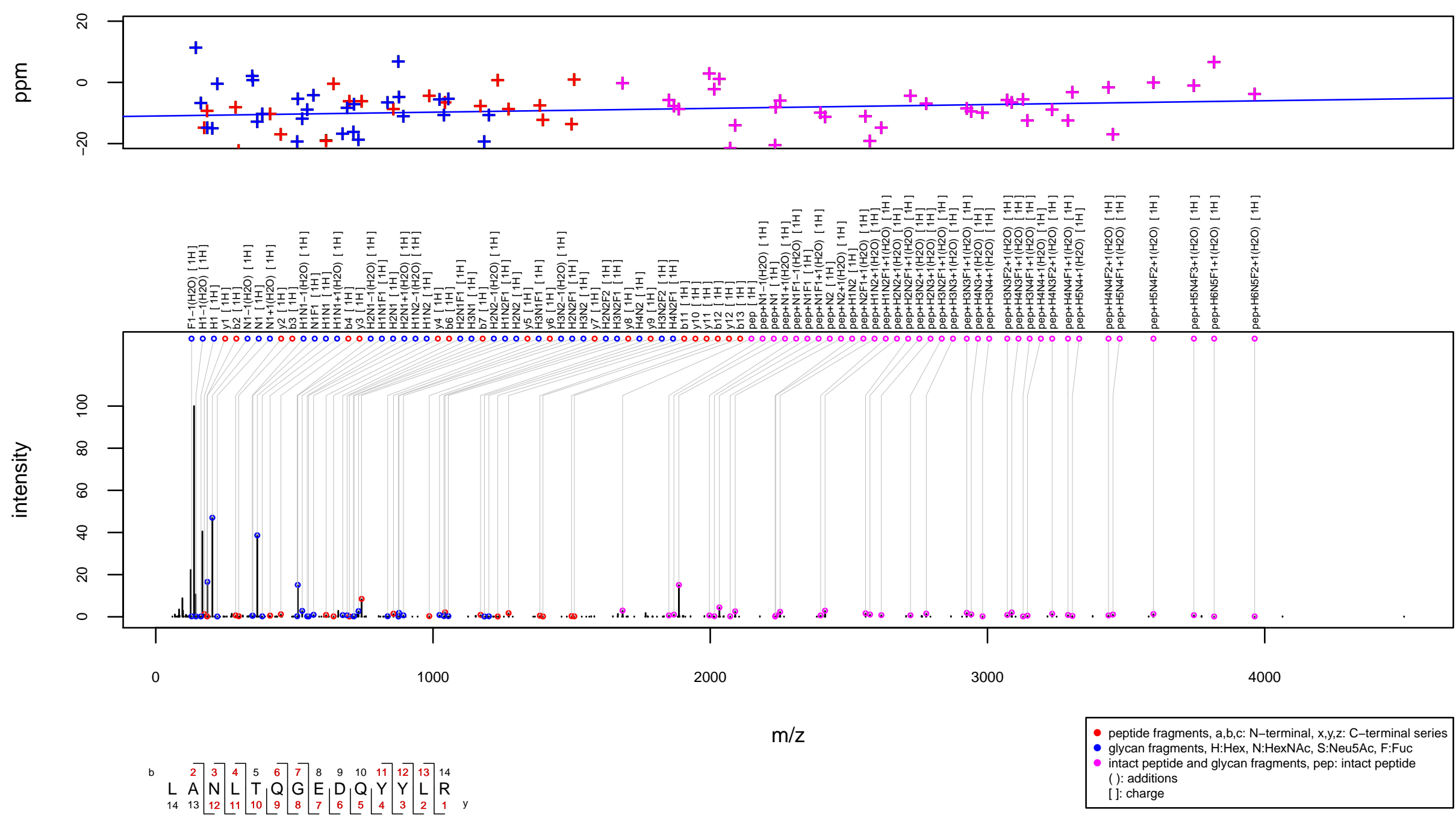
$\mathrm{m} / \mathrm{z} 1193.0112$ charge 4 scan $0-0$

Score $=57.86$, Hits $=48$, Explained Intensity $=0.44$ Peptide: FINC_HUMAN[516,533]:DQCIVDDITYNVNDTFHK Glycan: $\mathrm{F}(\mathrm{H}) \mathrm{NH}(\mathrm{F}(\mathrm{H}) \mathrm{N}(\mathrm{F}(\mathrm{H}) \mathrm{N}) \mathrm{H}) \mathrm{HN}(\mathrm{F}) \mathrm{N}, \mathrm{H} 6 \mathrm{~N} 5 \mathrm{~F} 4$

Charge: $4 \mathrm{H}$
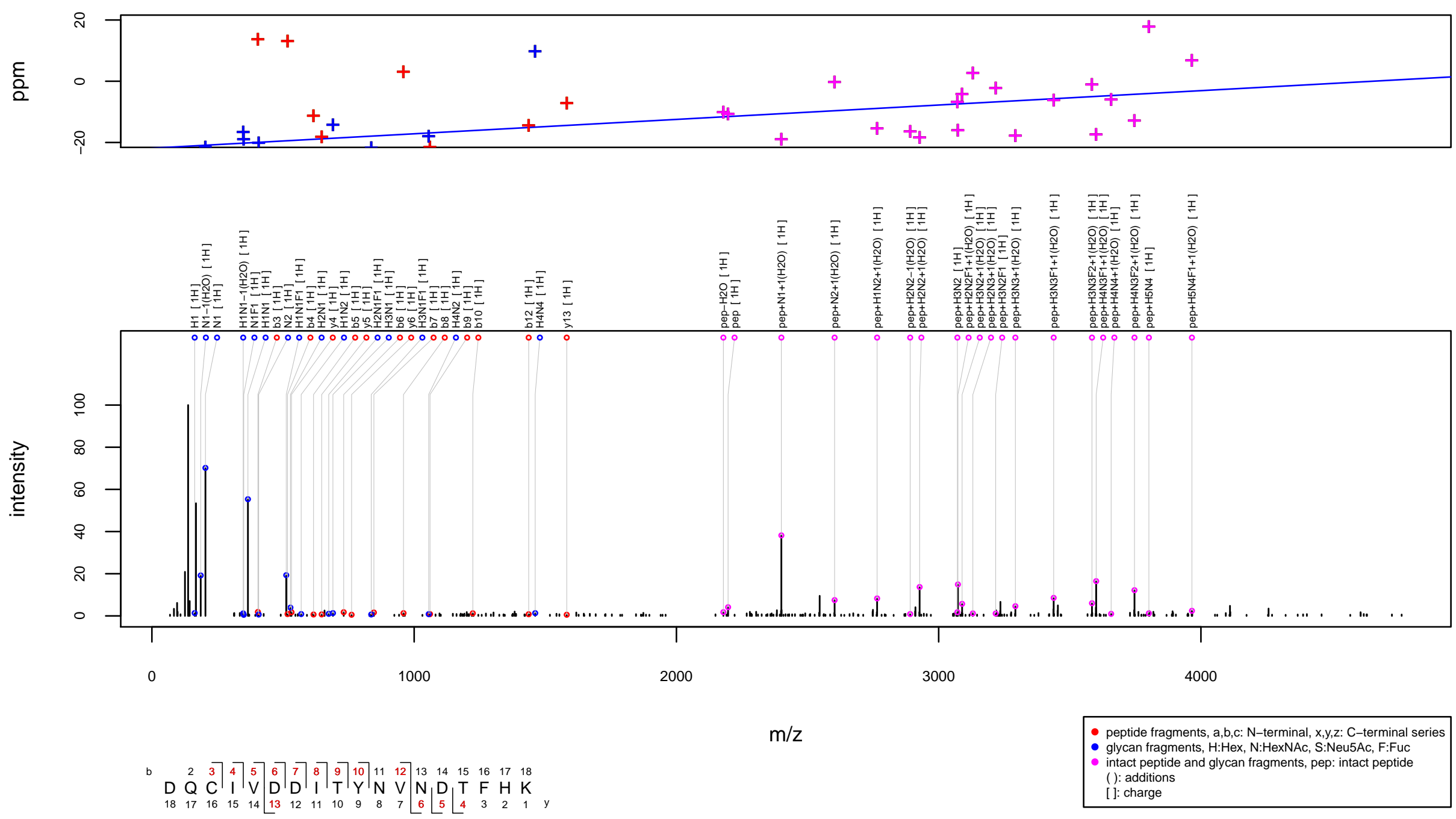
$\mathrm{m} / \mathrm{z} 1193.2502$ charge 4 scan $0-0$

Score $=116.61$, Hits $=69$, Explained Intensity $=0.53$

Peptide: CBPE_HUMAN[382,406]:DLQGNPIANATISVEGIDHDVTSAK

Glycan: $\mathrm{SHNH}(\mathrm{SHNH}) \mathrm{HNN}$, S2H5N4

Charge: $4 \mathrm{H}$
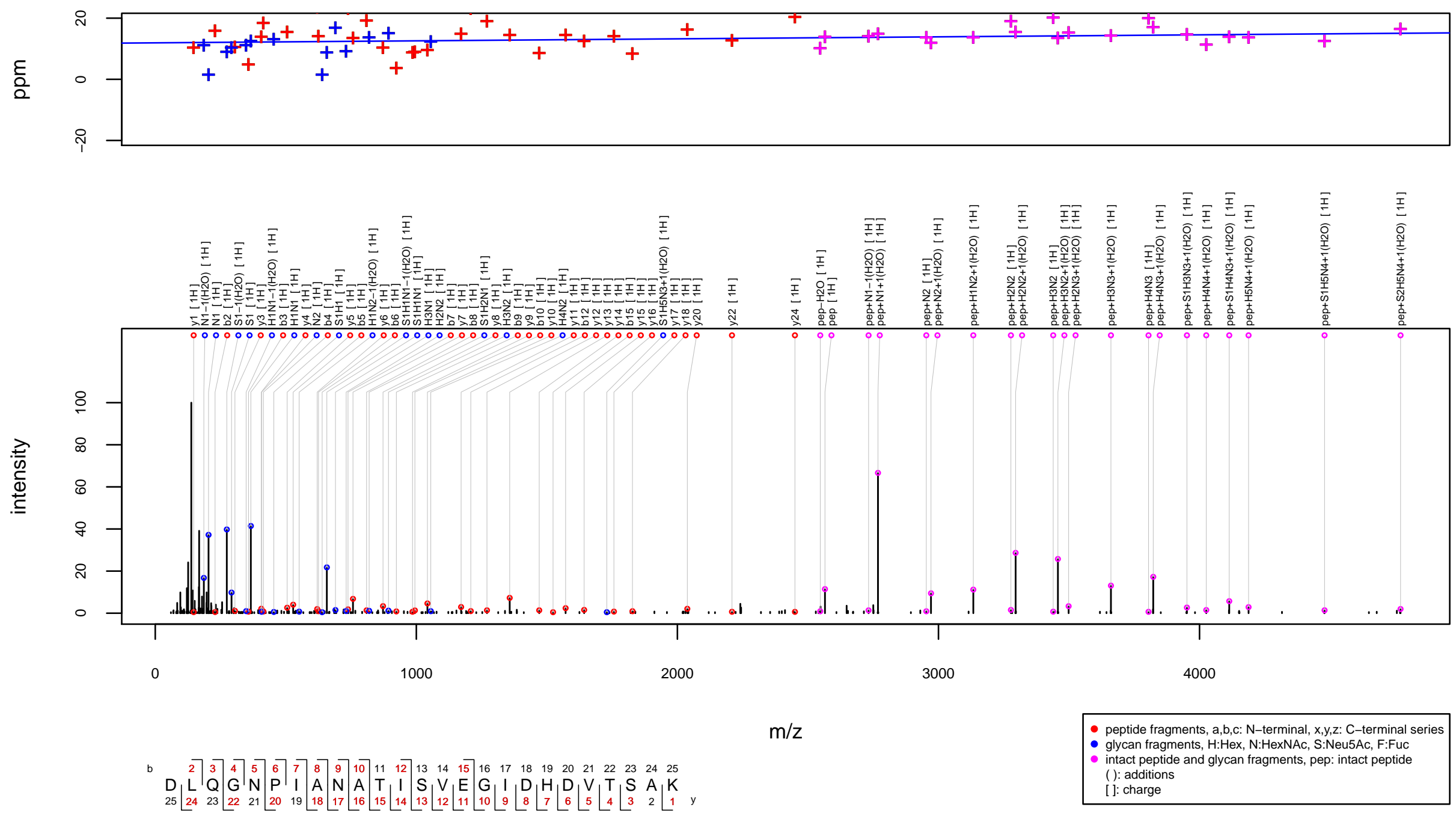
$\mathrm{m} / \mathrm{z} 1195.0117$ charge 4 scan $0-0$

Score $=68.02$, Hits $=53$, Explained Intensity $=0.45$

Peptide: A1AG1_HUMAN[87,101]:QDQCIYNTTYLNVQR

Glycan: $\mathrm{SHNH}(\mathrm{SHN}(\mathrm{SHN}) \mathrm{H}) \mathrm{HNN}, \mathrm{S} 3 \mathrm{H} 6 \mathrm{~N} 5$

Charge: $4 \mathrm{H}$
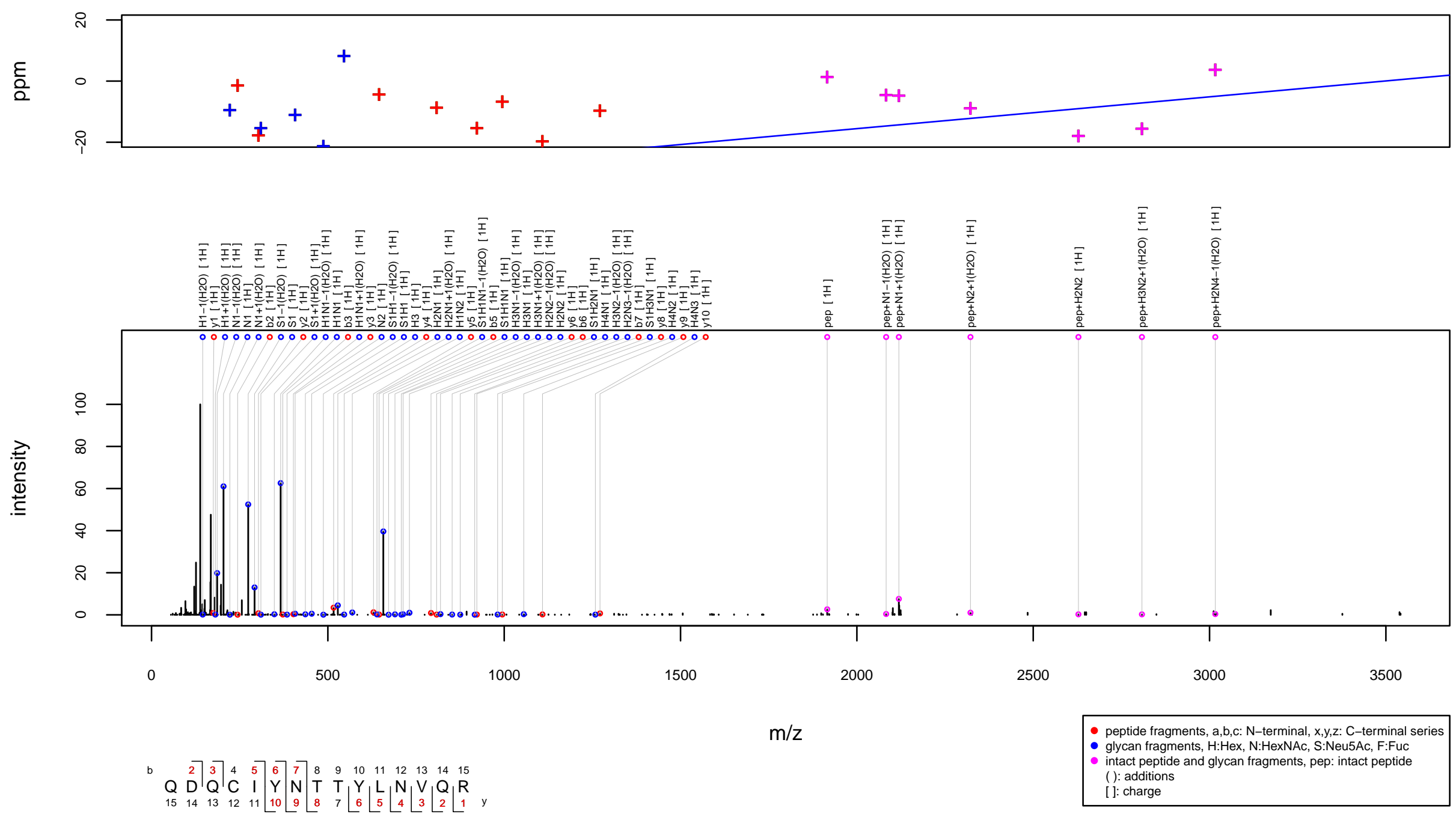
$\mathrm{m} / \mathrm{z} 1198.9141$ charge 3 scan $0-0$

Score $=72.19$, Hits $=45$, Explained Intensity $=0.37$

Peptide: FINC_HUMAN[997,1016]:LDAPTNLQFVNETDSTVLVR

Glycan: $\mathrm{H}(\mathrm{H}) \mathrm{H}(\mathrm{H}) \mathrm{HN}(\mathrm{F}) \mathrm{N}, \mathrm{H} 5 \mathrm{~N} 2 \mathrm{~F} 1$

Charge: $3 \mathrm{H}$
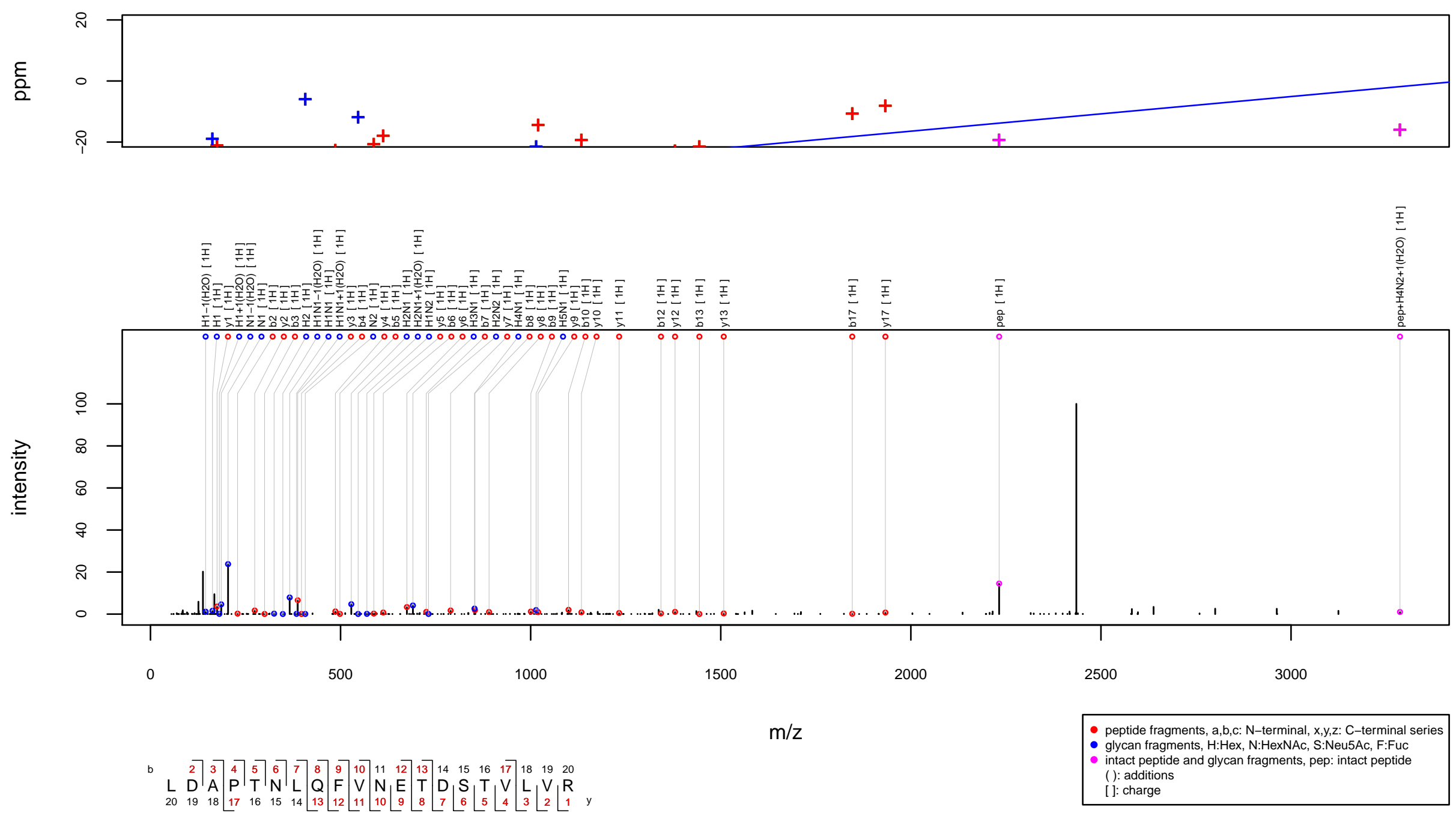
$\mathrm{m} / \mathrm{z} 1200.2087$ charge 3 scan $0-0$

Score $=79.99$, Hits $=54$, Explained Intensity $=0.49$

Peptide: CLUS_HUMAN[372,385]:LANLTQGEDQYYLR

Glycan: $\mathrm{FHNH}(\mathrm{HNH}) \mathrm{HN}(\mathrm{F}) \mathrm{N}, \mathrm{H} 5 \mathrm{~N} 4 \mathrm{~F} 2$

Charge: $3 \mathrm{H}$
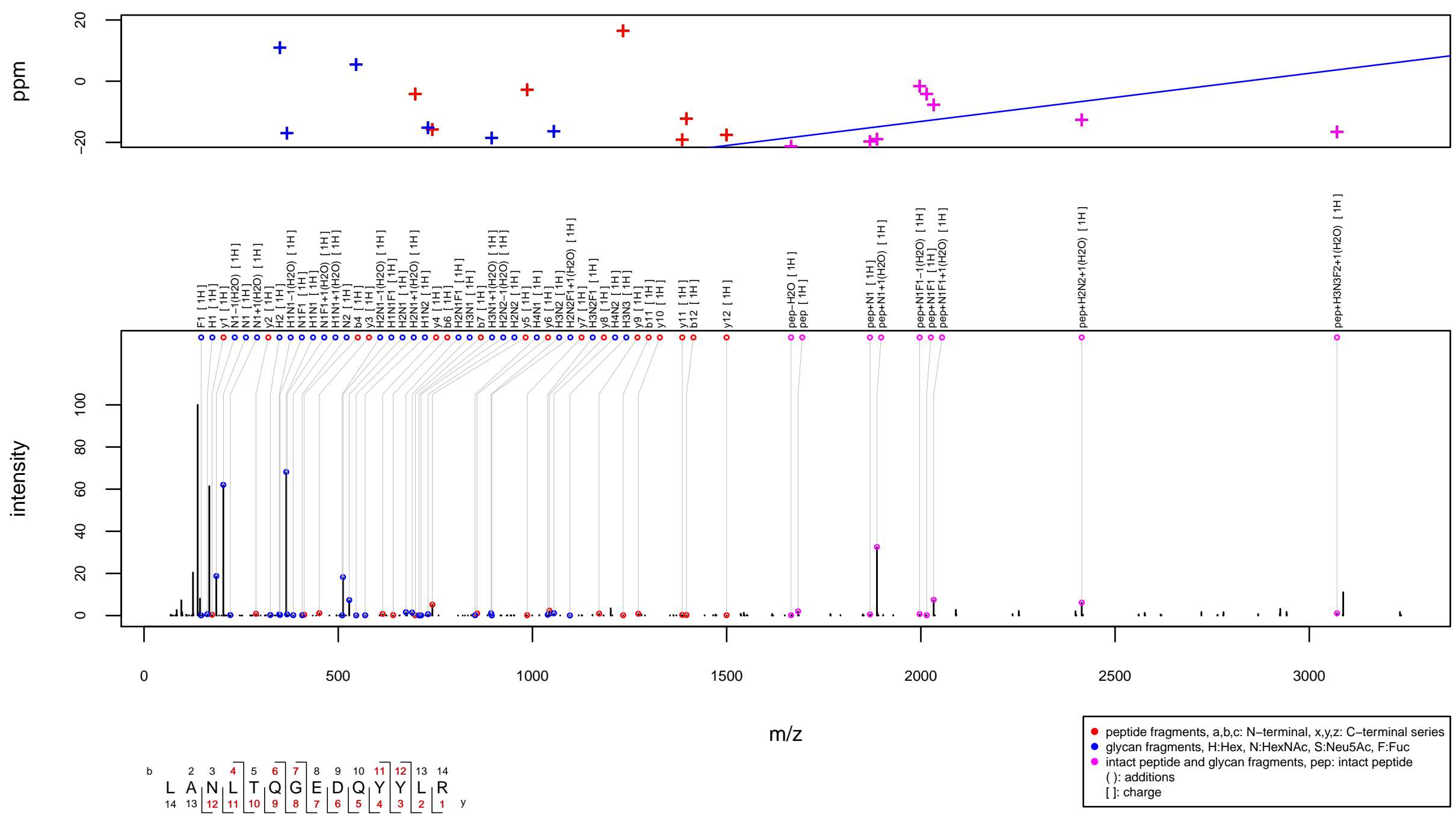


\section{$\mathrm{m} / \mathrm{z} 1200.8611$ charge 3 scan $0-0$}

Score $=40.7$, Hits $=26$, Explained Intensity $=0.26$

Peptide: FINC_HUMAN[516,533]:DQCIVDDITYNVNDTFHK

Glycan: $\mathrm{HNH}(\mathrm{H}) \mathrm{HN}(\mathrm{F}) \mathrm{N}, \mathrm{H} 4 \mathrm{~N} 3 \mathrm{~F} 1$

Charge: $3 \mathrm{H}$
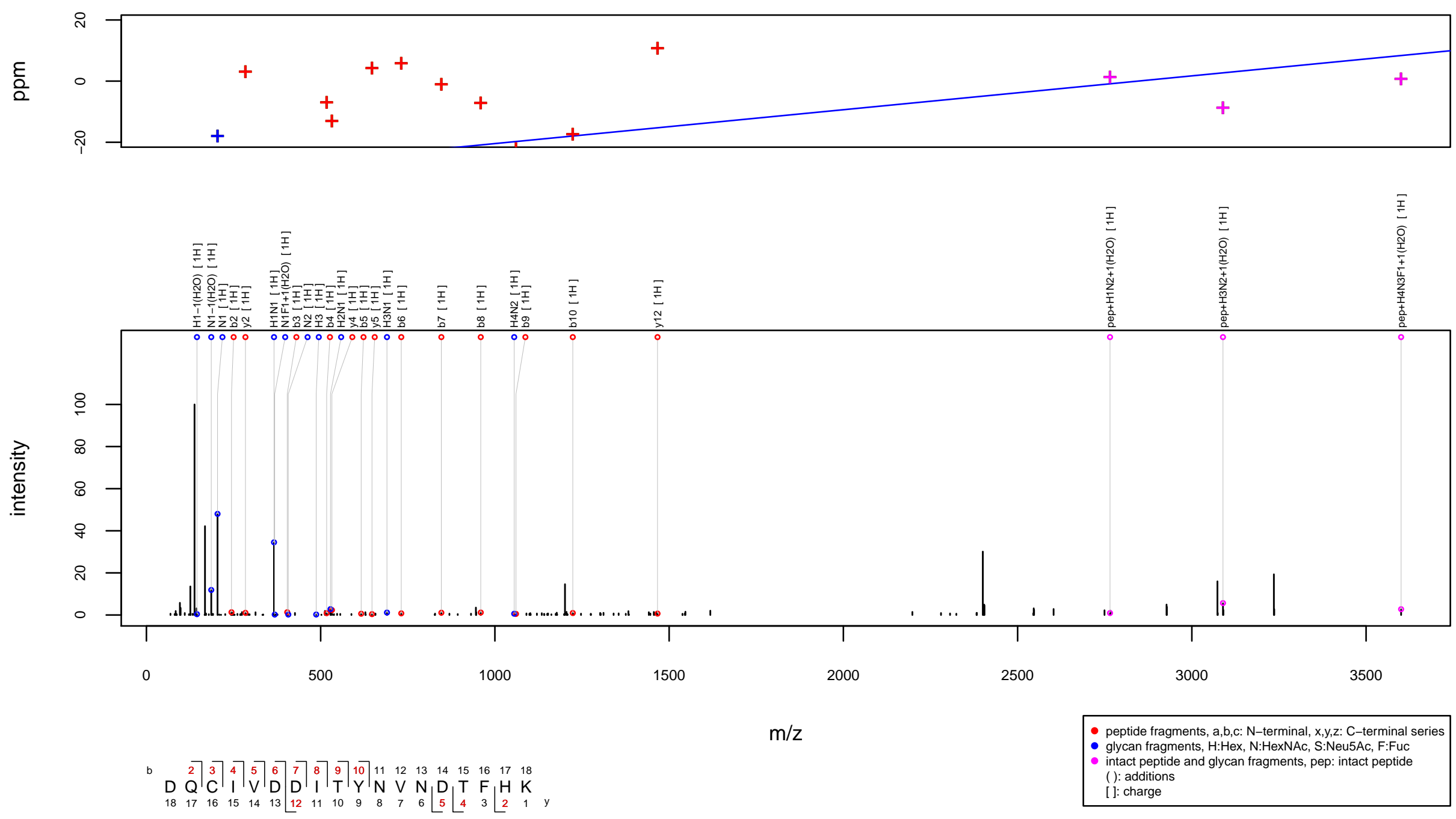
$\mathrm{m} / \mathrm{z} 1201.8079$ charge 4 scan $0-0$

Score $=82.58$, Hits $=66$, Explained Intensity $=0.47$

Peptide: FINC_HUMAN[997,1016]:LDAPTNLQFVNETDSTVLVR

Glycan: $\mathrm{F}(\mathrm{H}) \mathrm{NH}(\mathrm{F}(\mathrm{H}) \mathrm{N}(\mathrm{F}(\mathrm{H}) \mathrm{N}) \mathrm{H}) \mathrm{HN}(\mathrm{F}) \mathrm{N}, \mathrm{H} 6 \mathrm{~N} 5 \mathrm{~F} 4$

Charge: $4 \mathrm{H}$
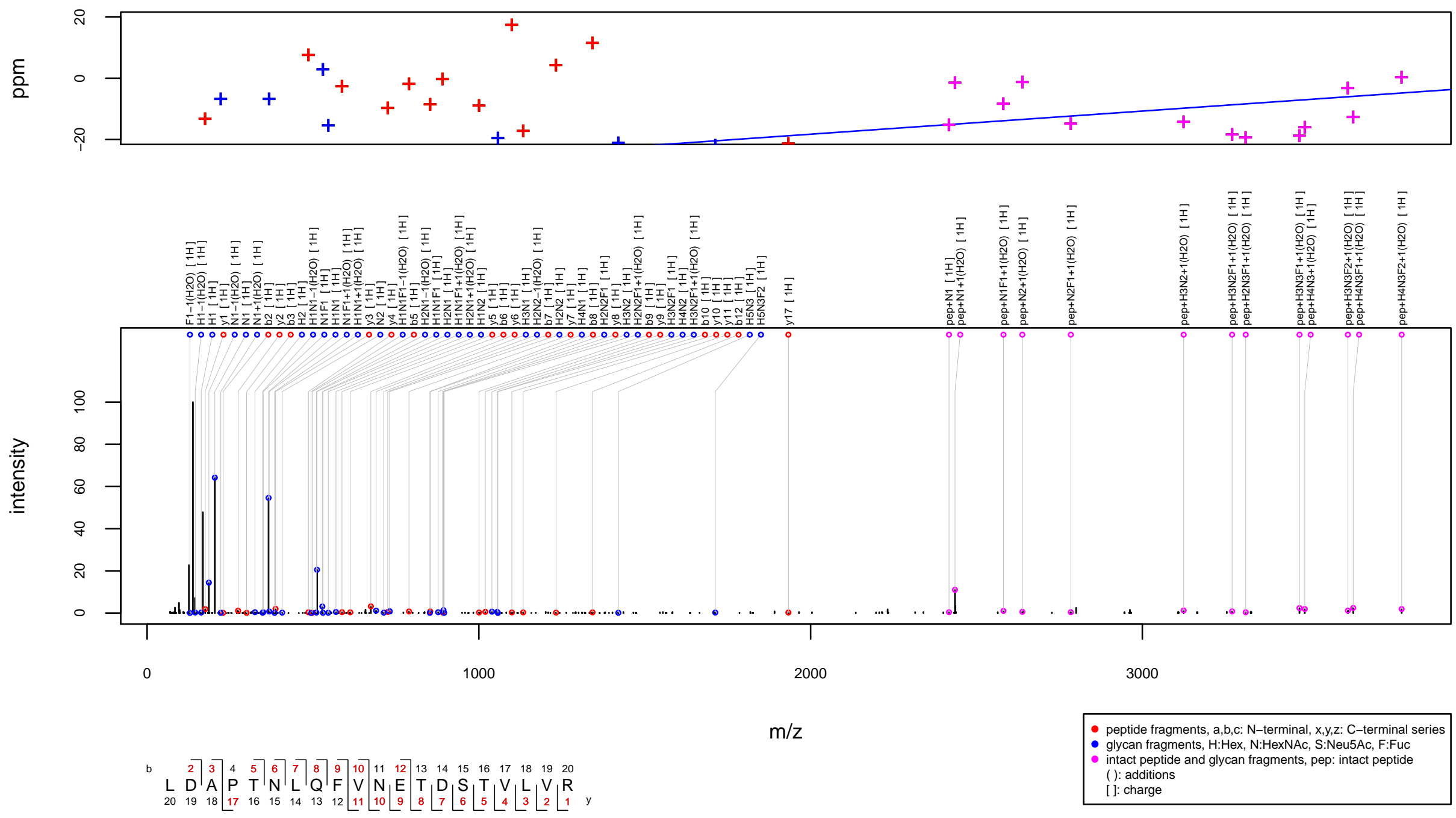
$\mathrm{m} / \mathrm{z} 1204.2415$ charge 3 scan $0-0$

Score $=86.37$, Hits $=50$, Explained Intensity $=0.35$

Peptide: FINC_HUMAN[997,1016]:LDAPTNLQFVNETDSTVLVR

Glycan: $\mathrm{HH}(\mathrm{H}(\mathrm{H}) \mathrm{H}) \mathrm{HNN}$, H6N2

Charge: $3 \mathrm{H}$
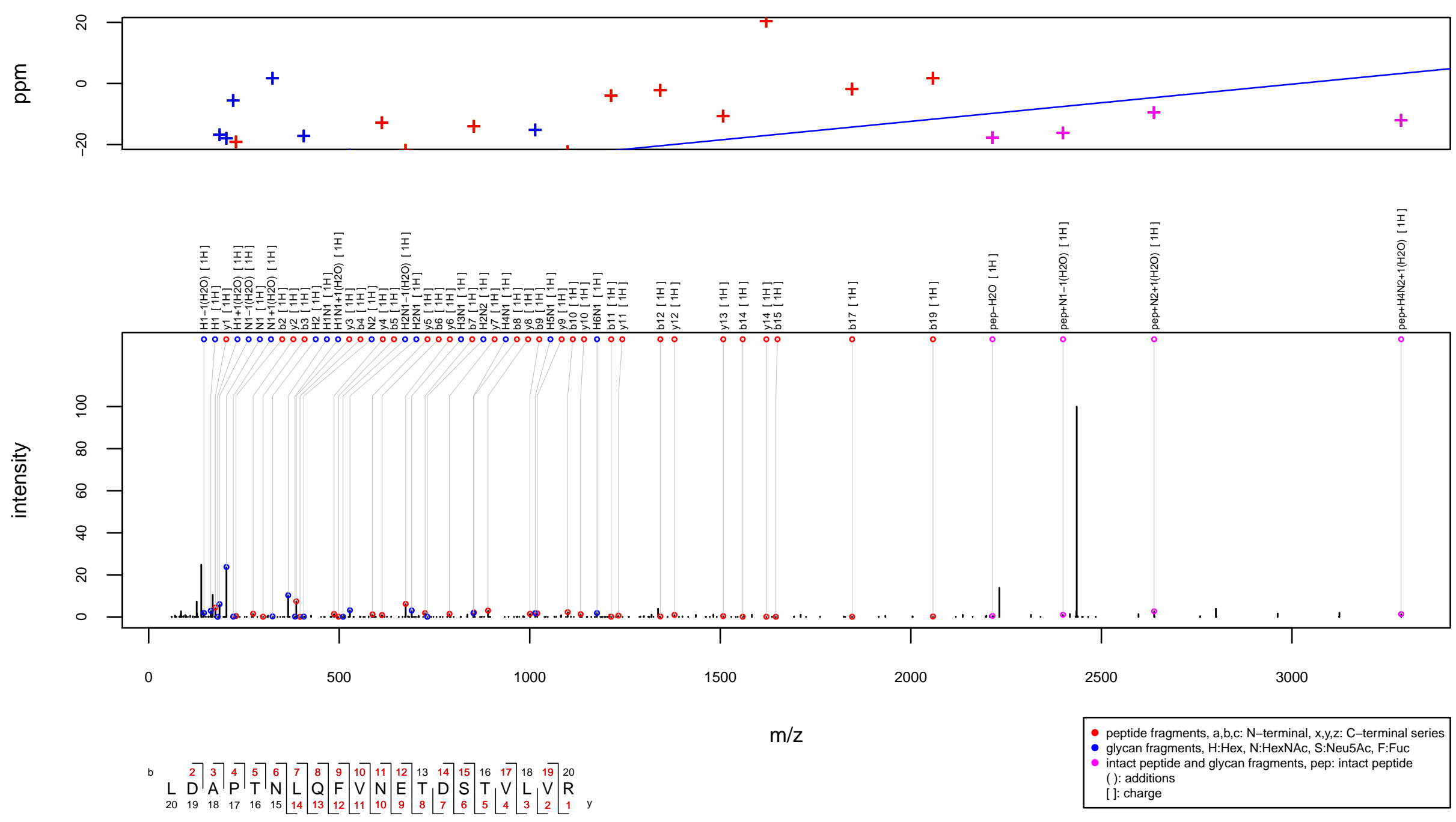


\section{m/z 1205.2576 charge 3 scan 0-0}

Score $=55.62$, Hits $=43$, Explained Intensity $=0.34$

Peptide: PTGDS_HUMAN[67,85]:SVVAPATDGGLNLTSTFLR

Glycan: $\mathrm{SHNH}(\mathrm{H}) \mathrm{HN}(\mathrm{F}) \mathrm{N}$, S1H4N3F1

Charge: $3 \mathrm{H}$
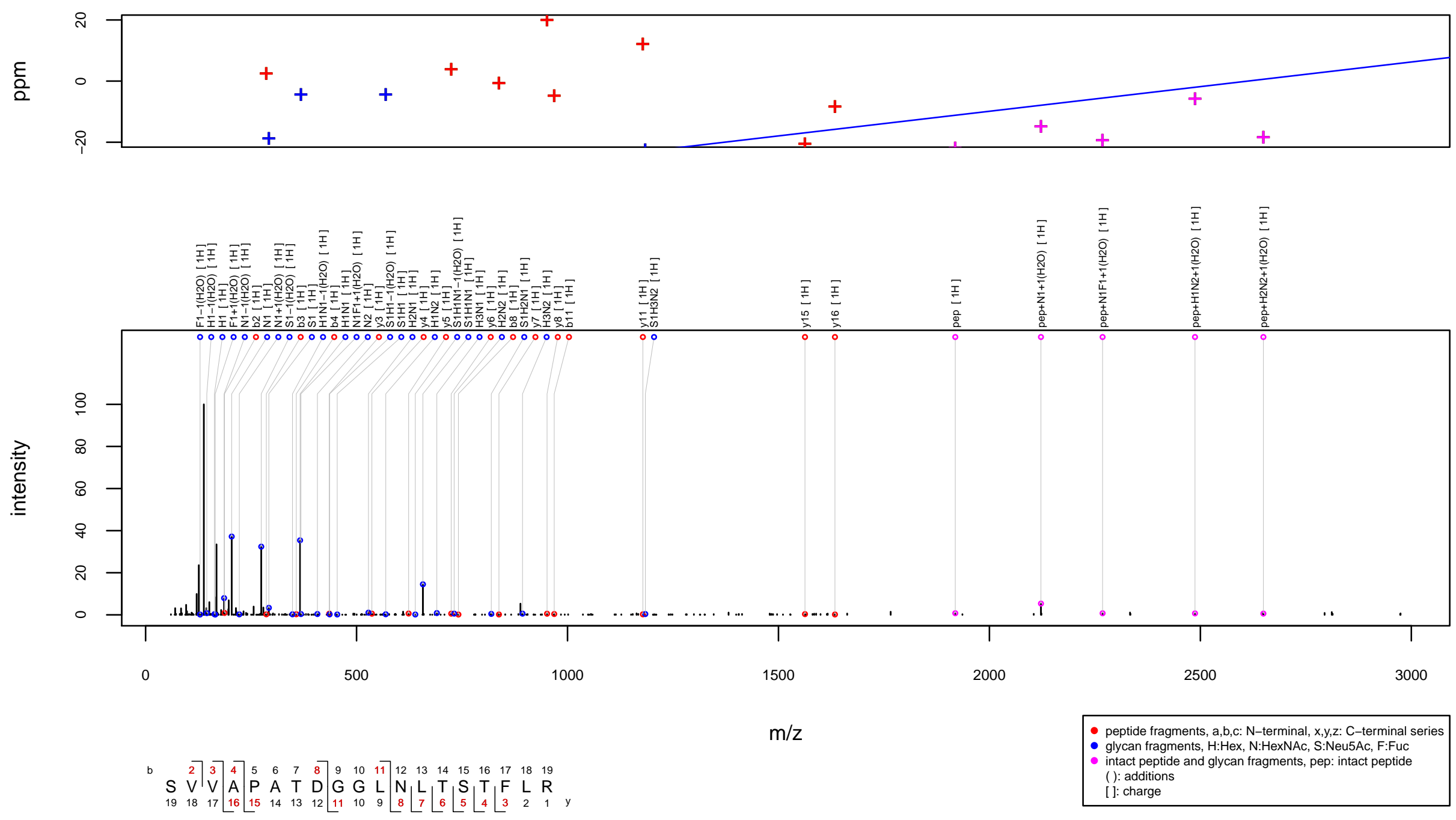
$\mathrm{m} / \mathrm{z} 1206.1254$ charge 3 scan $0-0$

Score $=106.8$, Hits $=81$, Explained Intensity $=0.5$

Peptide: PPAP_HUMAN[92,98]:FLNESYK

Glycan: $\mathrm{SHNH}(\mathrm{SHN}(\mathrm{HN}) \mathrm{H}) \mathrm{HN}(\mathrm{F}) \mathrm{N}, \mathrm{S} 2 \mathrm{H} 6 \mathrm{~N} 5 \mathrm{~F} 1$

Charge: $3 \mathrm{H}$
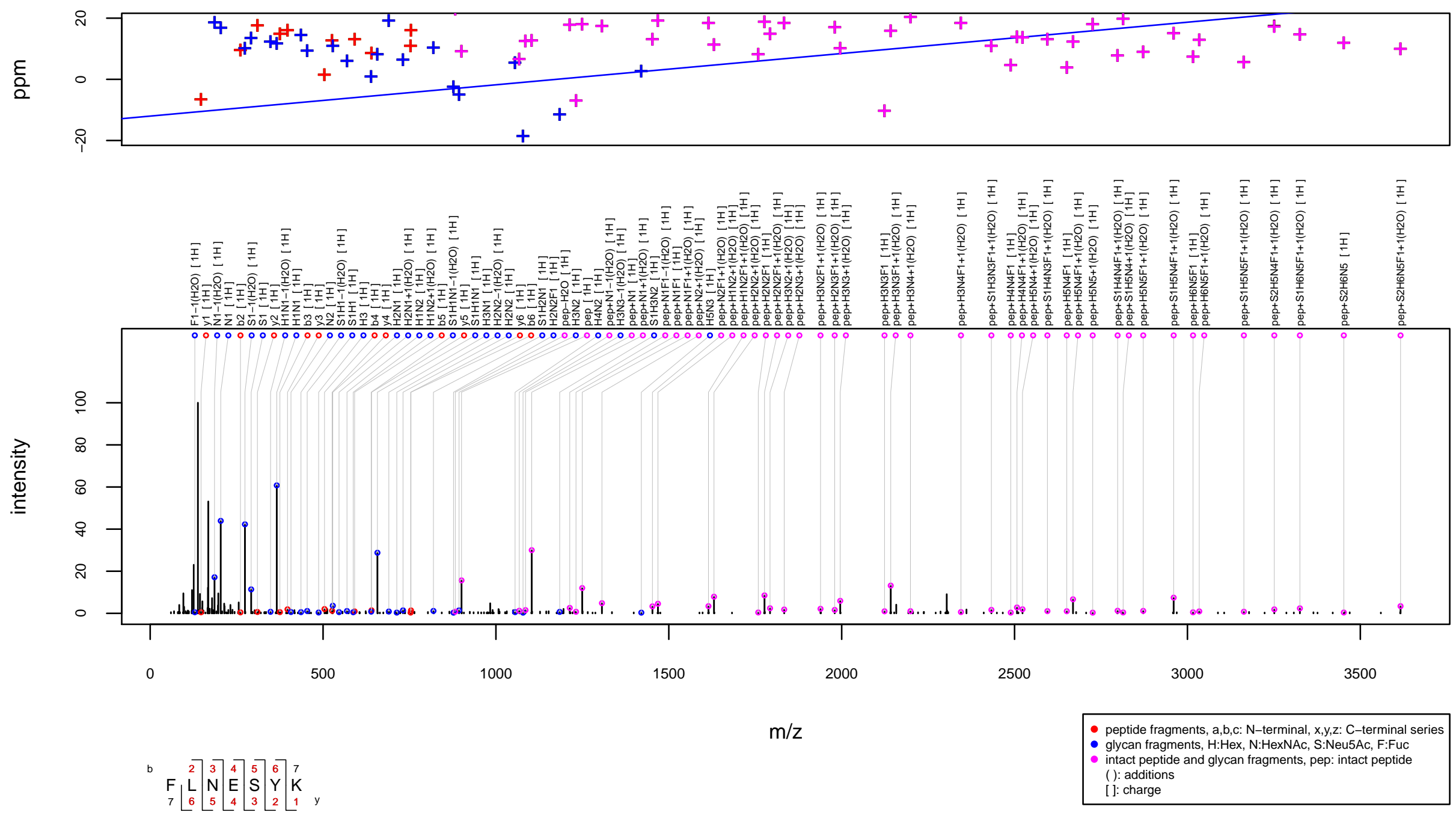
m/z 1206.7893 charge 3 scan 0-0

Score $=78.2$, Hits $=61$, Explained Intensity $=0.41$

Peptide: PIP_HUMAN[97,106]:TFYWDFYTNR

Glycan: $\mathrm{SHNH}(\mathrm{F}(\mathrm{H}) \mathrm{NH}) \mathrm{HN}(\mathrm{F}) \mathrm{N}, \mathrm{S} 1 \mathrm{H} 5 \mathrm{~N} 4 \mathrm{~F} 2$

Charge: $3 \mathrm{H}$
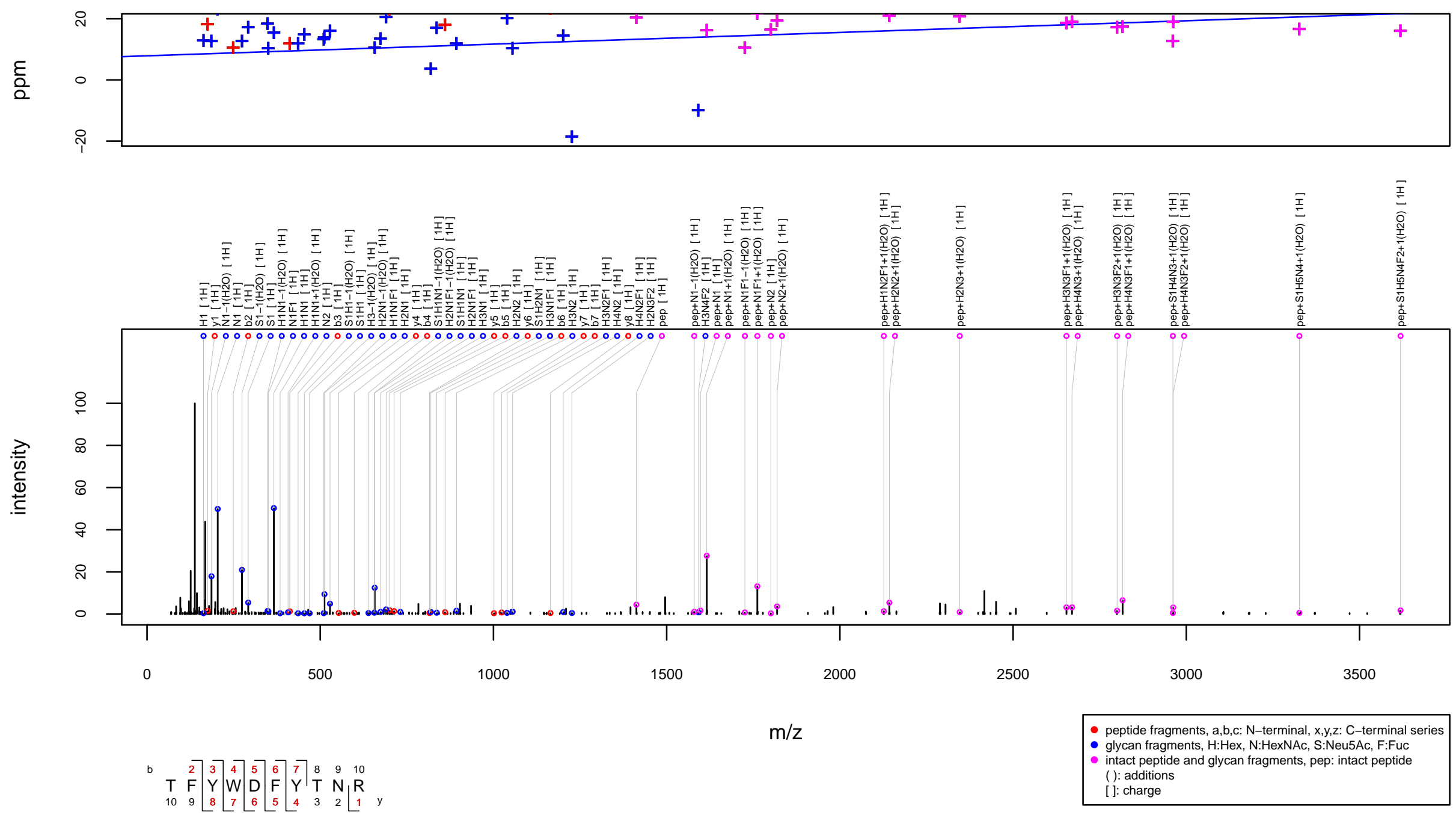
$\mathrm{m} / \mathrm{z} 1211.8479$ charge 3 scan $0-0$

Score $=100.52$, Hits $=69$, Explained Intensity $=0.56$ Peptide: SAP_HUMAN[214,227]:TNSTFVQALVEHVK Glycan: $\mathrm{FHNH}(\mathrm{FHNH}) \mathrm{HN}(\mathrm{F}) \mathrm{N}, \mathrm{H} 5 \mathrm{~N} 4 \mathrm{~F} 3$

Charge: $3 \mathrm{H}$
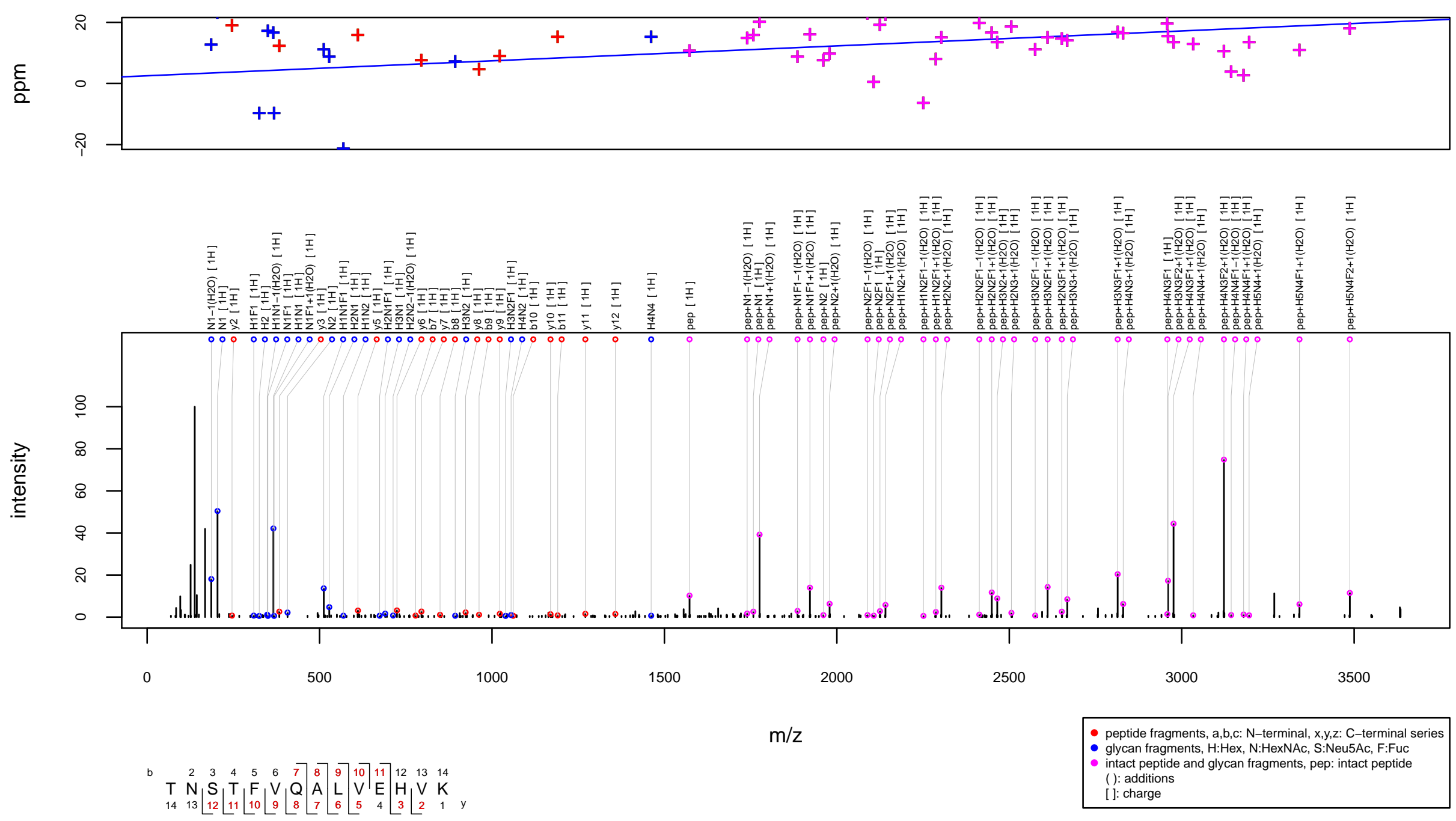
$\mathrm{m} / \mathrm{z} 1212.5862$ charge 3 scan $0-0$

Score $=59.7$, Hits $=36$, Explained Intensity $=0.32$

Peptide: FINC_HUMAN[997,1016]:LDAPTNLQFVNETDSTVLVR

Glycan: H4N3F1

Charge: $3 \mathrm{H}$
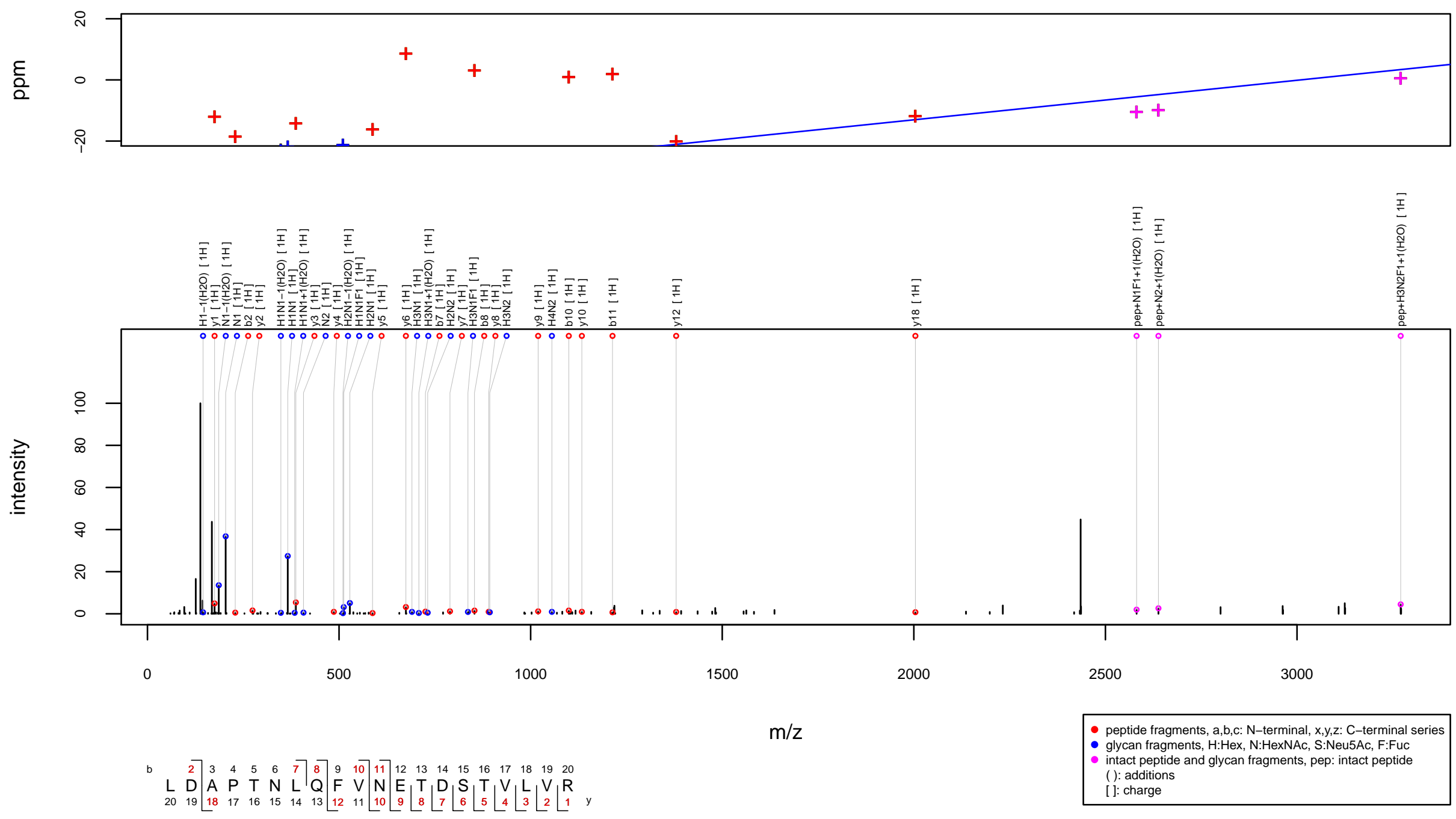
$\mathrm{m} / \mathrm{z} 1219.123$ charge 5 scan $0-0$

Score $=39.38$, Hits $=43$, Explained Intensity $=0.34$

Peptide: WFDC2_HUMAN[33,60]:TGVCPELQADQNCTQECVSDSECADNLK

Glycan: S1H6N5F4

Charge: $5 \mathrm{H}$
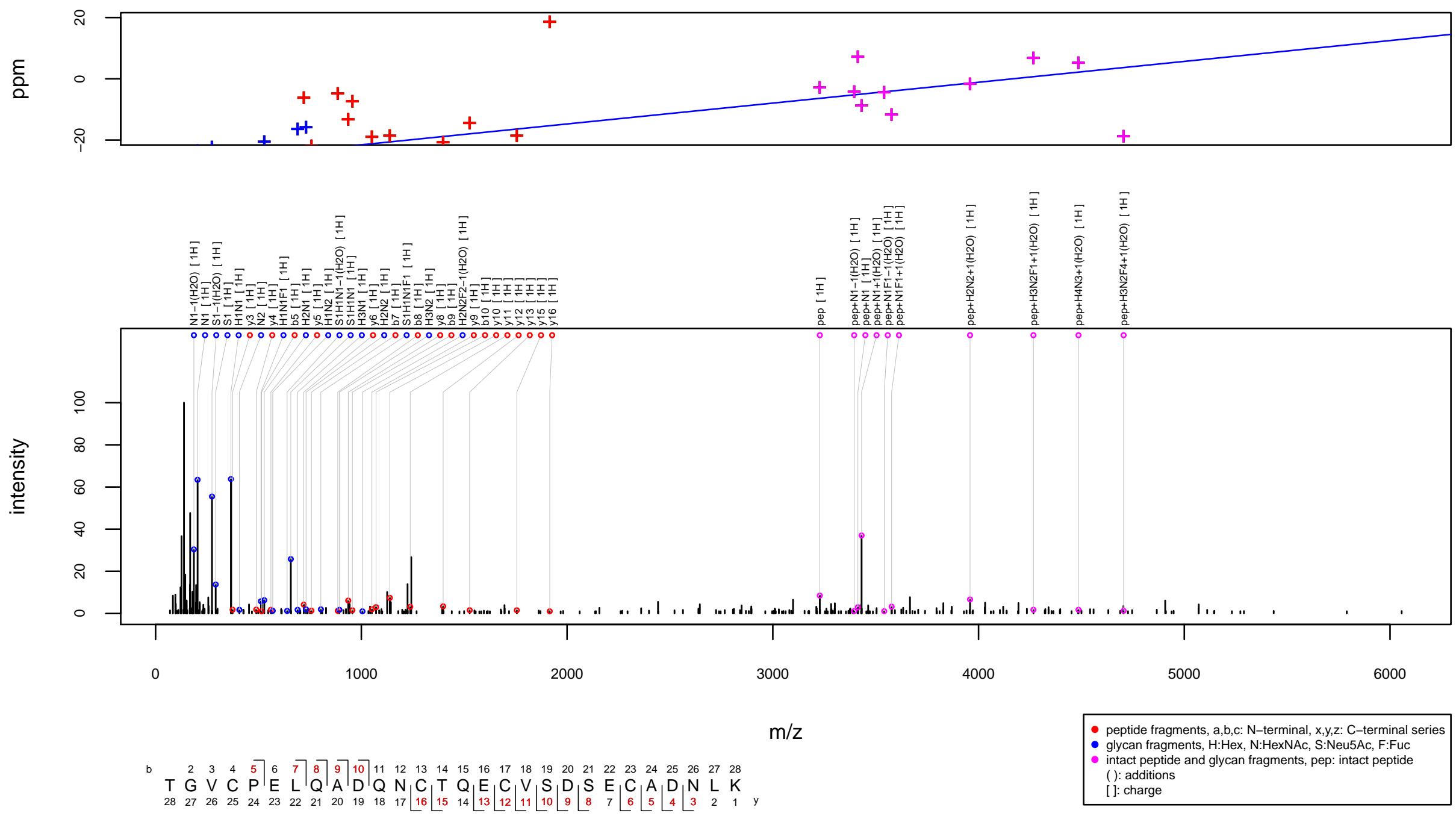
$\mathrm{m} / \mathrm{z} 1223.231$ charge 3 scan $0-0$

Score $=87.14$, Hits $=59$, Explained Intensity $=0.46$ Peptide: TIMP1_HUMAN[46,60]:FVGTPEVNQTTLYQR Glycan: $\mathrm{FHNH}(\mathrm{HNH}) \mathrm{HN}(\mathrm{F}) \mathrm{N}, \mathrm{H} 5 \mathrm{~N} 4 \mathrm{~F} 2$

Charge: $3 \mathrm{H}$
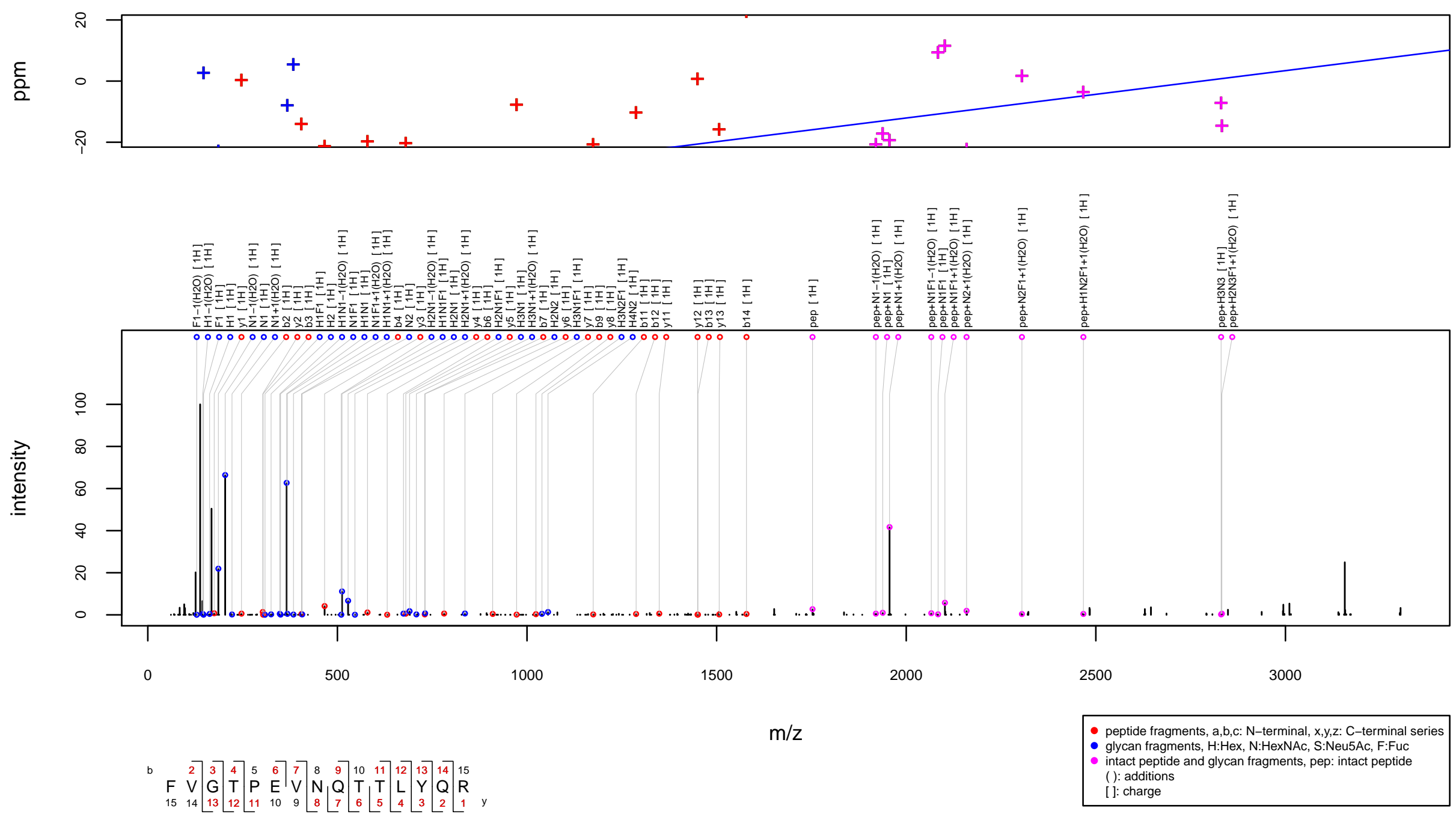
$\mathrm{m} / \mathrm{z} 1225.2358$ charge 3 scan $0-0$

Score $=55.04$, Hits $=43$, Explained Intensity $=0.41$

Peptide: QSOX1_HUMAN[130,147]:NGSGAVFPVAGADVQTLR

Glycan: $\mathrm{F}(\mathrm{H}) \mathrm{HNH}(\mathrm{NH}) \mathrm{HN}(\mathrm{F}) \mathrm{N}, \mathrm{H} 5 \mathrm{~N} 4 \mathrm{~F} 2$

Charge: $3 \mathrm{H}$
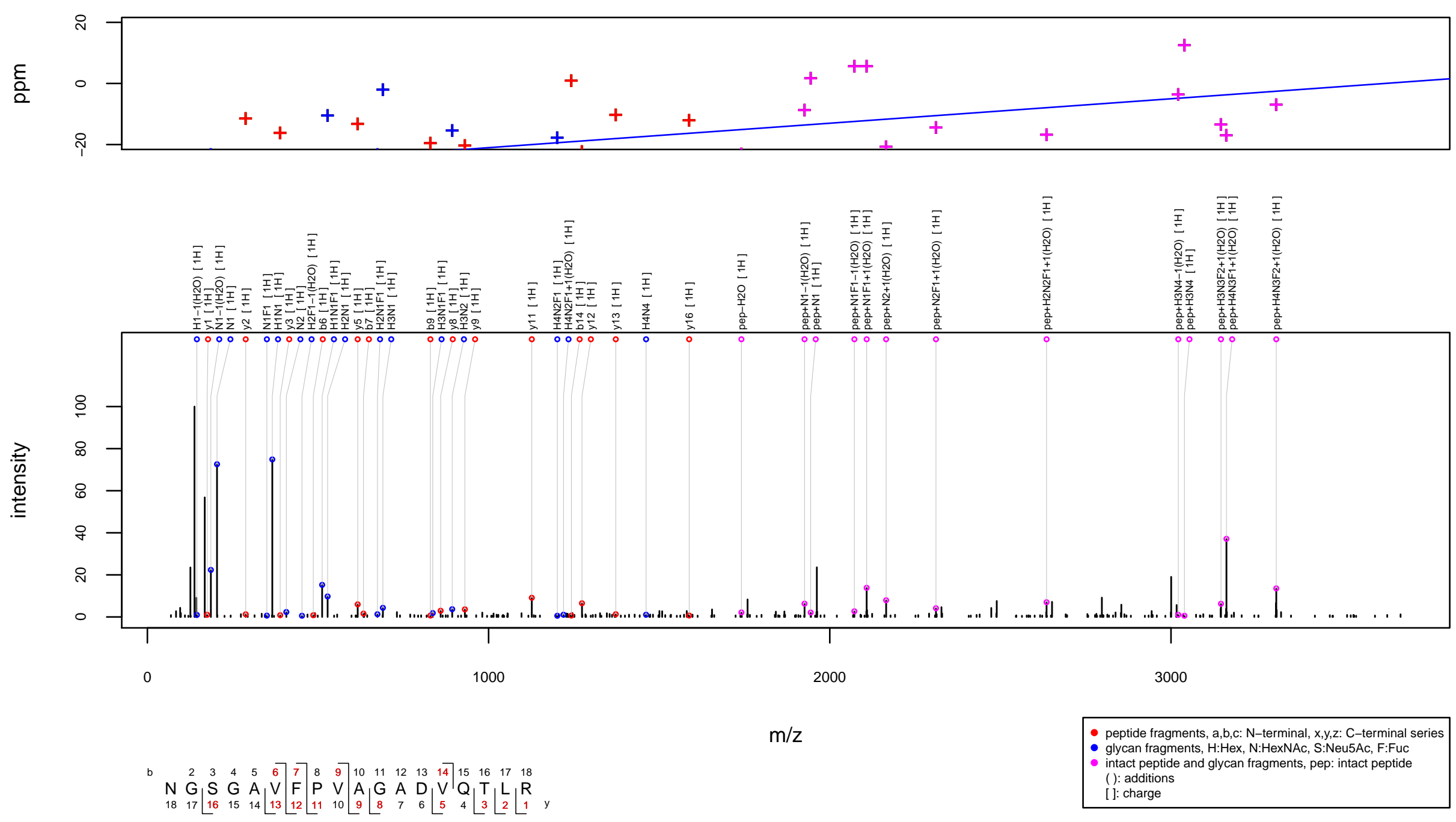
$\mathrm{m} / \mathrm{z} 1227.8845$ charge 3 scan $0-0$

Score $=73.45$, Hits $=51$, Explained Intensity $=0.4$

Peptide: TRFE_HUMAN[421,433]:CGLVPVLAENYNK

Glycan: $\mathrm{SHNH}(\mathrm{SHNH}) \mathrm{HNN}, \mathrm{S} 2 \mathrm{H} 5 \mathrm{~N} 4$

Charge: $3 \mathrm{H}$
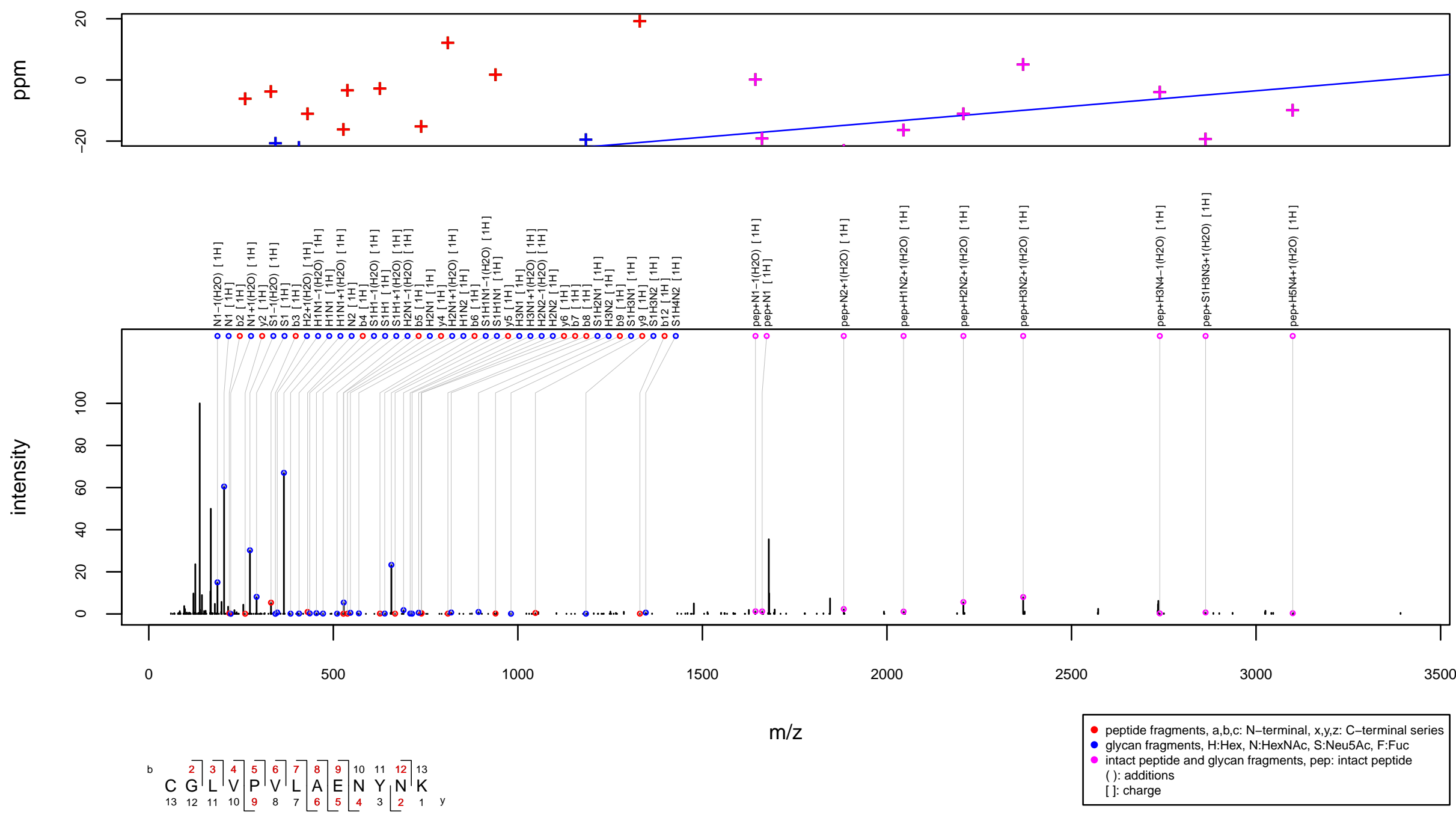
$\mathrm{m} / \mathrm{z} 1128.7745$ charge 4 scan $0-0$

Score $=72.91$, Hits $=50$, Explained Intensity $=0.4$

Peptide: FINC_HUMAN[997,1016]:LDAPTNLQFVNETDSTVLVR

Glycan: $\mathrm{F}(\mathrm{H}) \mathrm{NHNH}(\mathrm{HNH}) \mathrm{HN}(\mathrm{F}) \mathrm{N}, \mathrm{H} 6 \mathrm{~N} 5 \mathrm{~F} 2$

Charge: $4 \mathrm{H}$
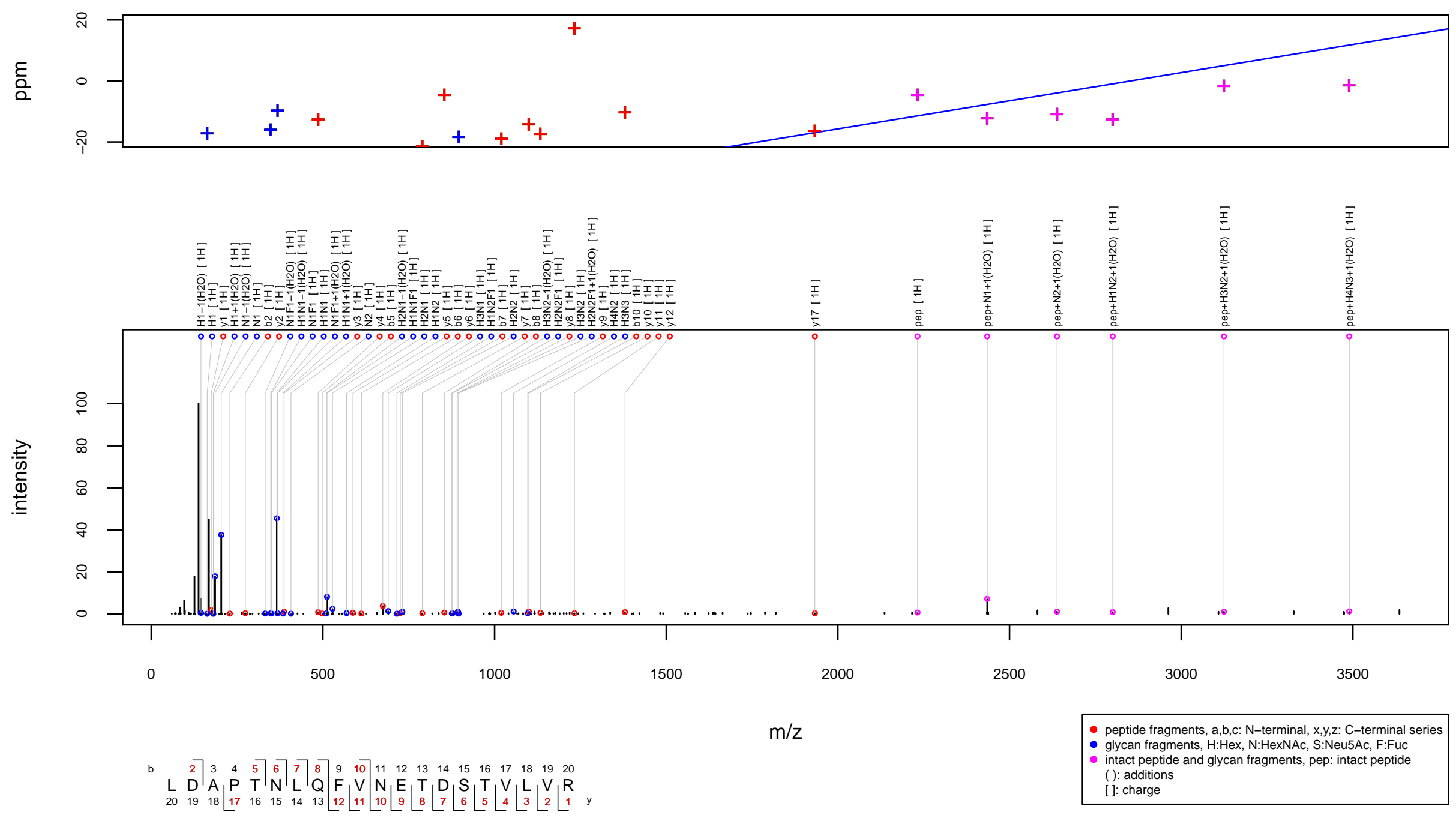
$\mathrm{m} / \mathrm{z} 1228.7853$ charge 4 scan $0-0$

Score $=64.16$, Hits $=61$, Explained Intensity $=0.53$

Peptide: CLUS_HUMAN[372,385]:LANLTQGEDQYYLR Glycan: $\mathrm{SHNH}(\mathrm{F}) \mathrm{NH}(\mathrm{F}(\mathrm{H}) \mathrm{NH}(\mathrm{F}) \mathrm{NH}) \mathrm{HN}(\mathrm{F}) \mathrm{N}, \mathrm{S} 1 \mathrm{H} 7 \mathrm{~N} 6 \mathrm{~F} 4$

Charge: $4 \mathrm{H}$
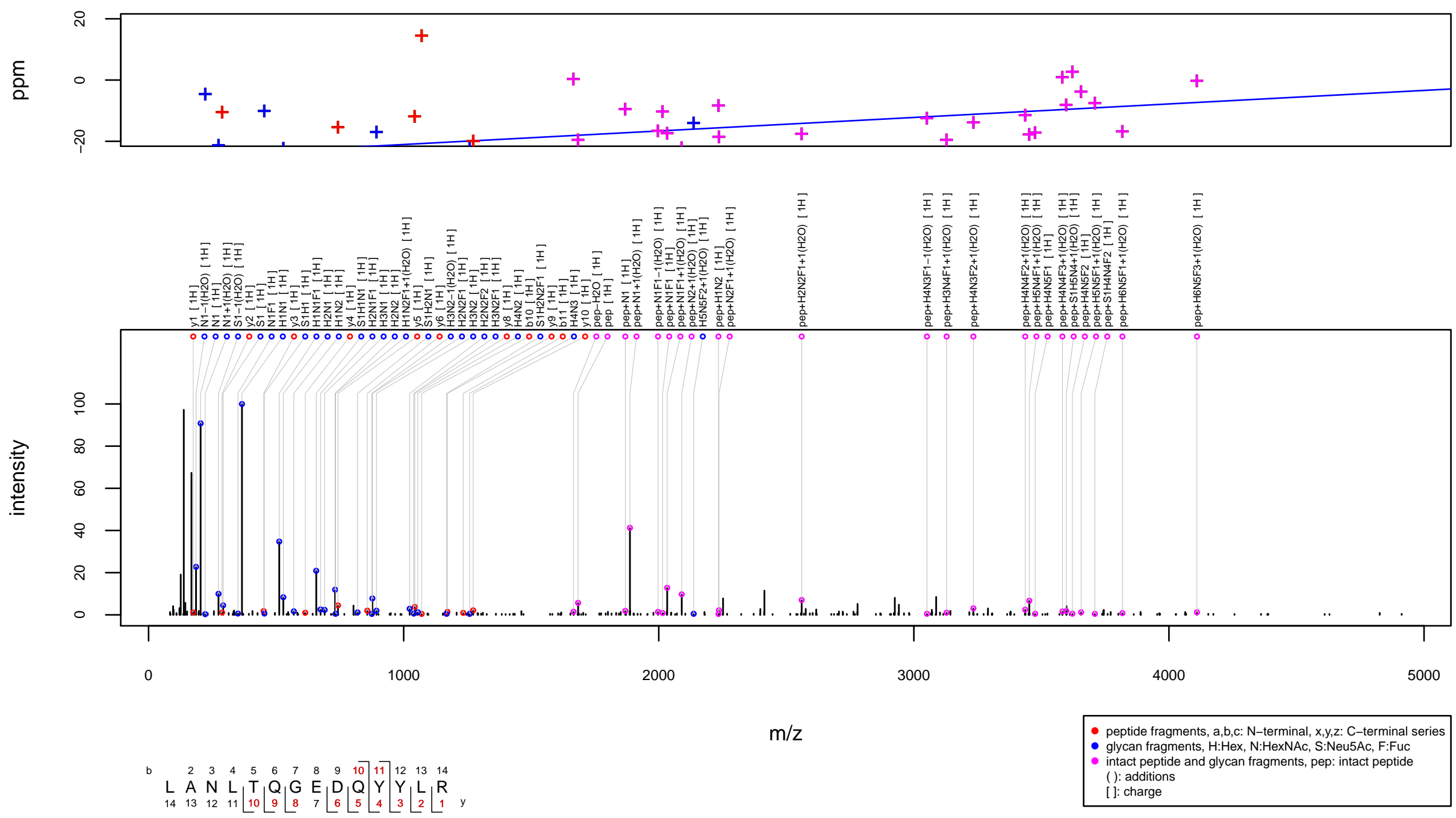
$\mathrm{m} / \mathrm{z} 1231.4723$ charge 4 scan $0-0$

Score $=79.97$, Hits $=59$, Explained Intensity $=0.35$

Peptide: WFDC2_HUMAN[33,60]:TGVCPELQADQNCTQECVSDSECADNLK

Glycan: S1H4N3F1

Charge: $4 \mathrm{H}$
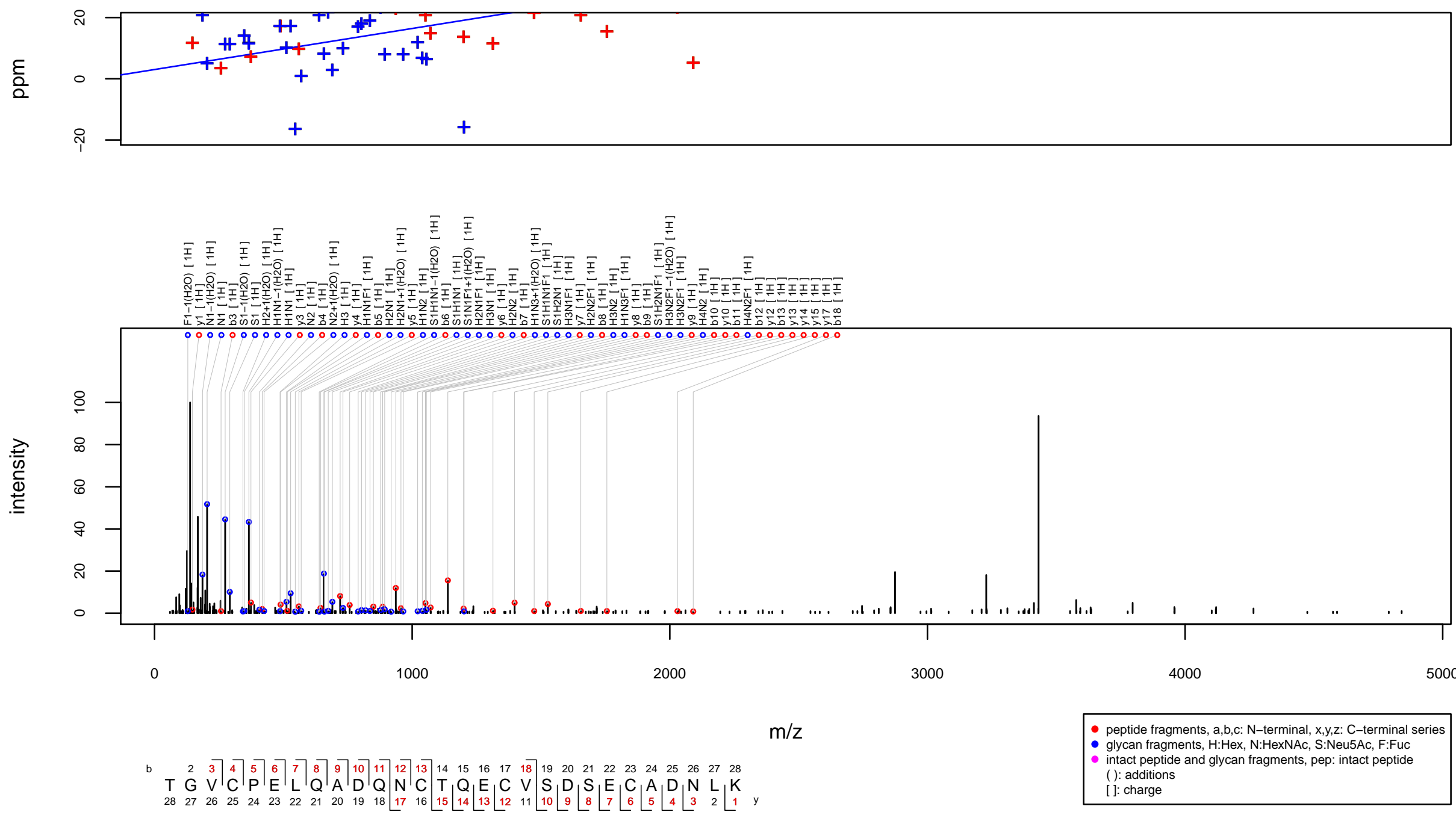
$\mathrm{m} / \mathrm{z} 1246.0673$ charge 4 scan $0-0$

Score $=51.93$, Hits $=41$, Explained Intensity $=0.41$

Peptide: CLUS_HUMAN[352,371]:MLNTSSLLEQLNEQFNWVSR

Glycan: $\operatorname{SHNHNH}(\mathrm{F}(\mathrm{H}) \mathrm{NH}) \mathrm{HNN}, \mathrm{S} 1 \mathrm{H} 6 \mathrm{~N} 5 \mathrm{~F} 1$

Charge: $4 \mathrm{H}$
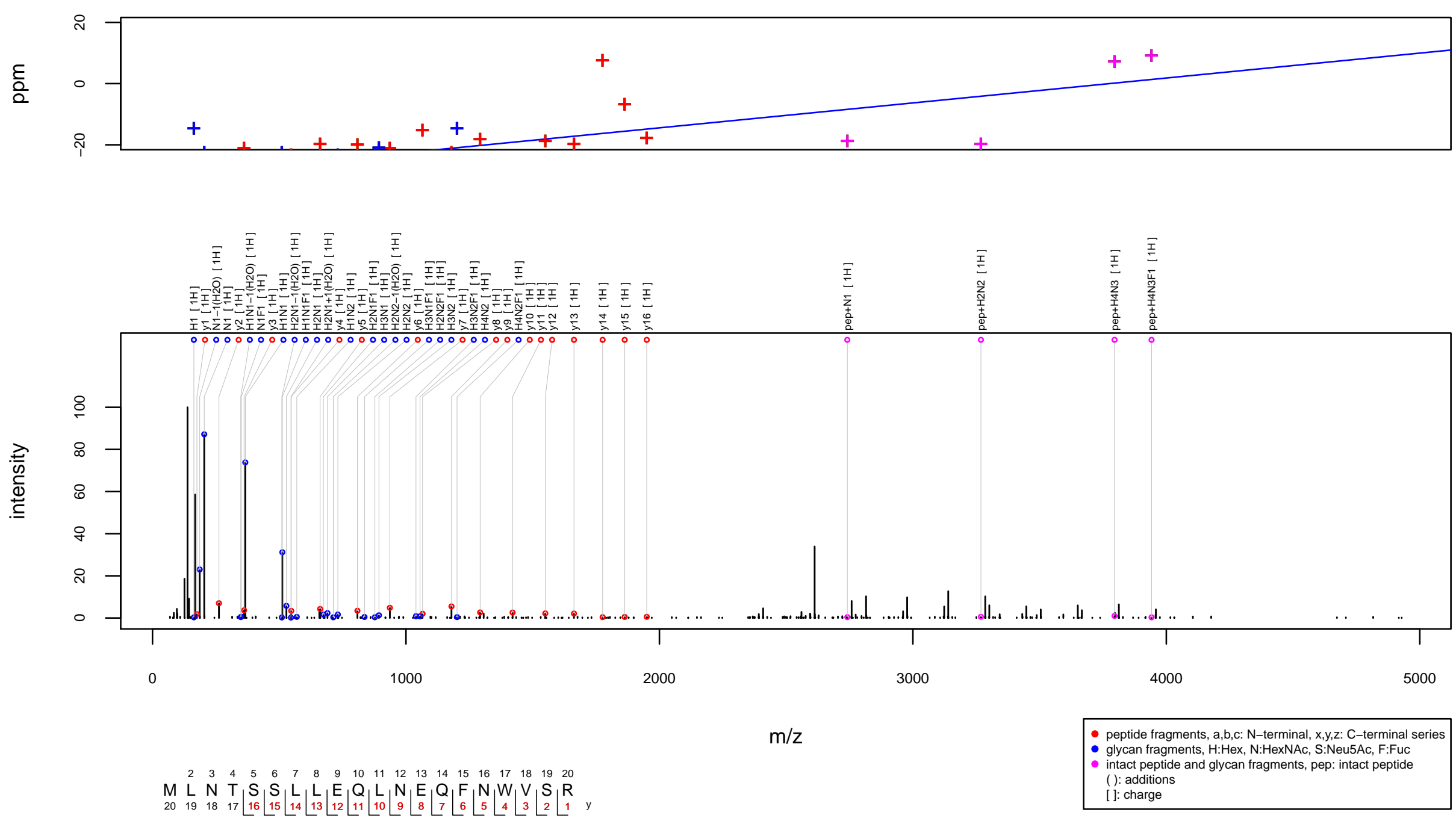
$\mathrm{m} / \mathrm{z} 1247.9152$ charge 5 scan $0-0$

Score $=105.18$, Hits $=71$, Explained Intensity $=0.54$

Peptide: WFDC2_HUMAN[33,60]:TGVCPELQADQNCTQECVSDSECADNLK

Glycan: $\mathrm{SHNH}(\mathrm{SHN}(\mathrm{SHN}) \mathrm{H}) \mathrm{HN}(\mathrm{F}) \mathrm{N}, \mathrm{S} 3 \mathrm{H} 6 \mathrm{~N} 5 \mathrm{~F} 1$

Charge: $5 \mathrm{H}$
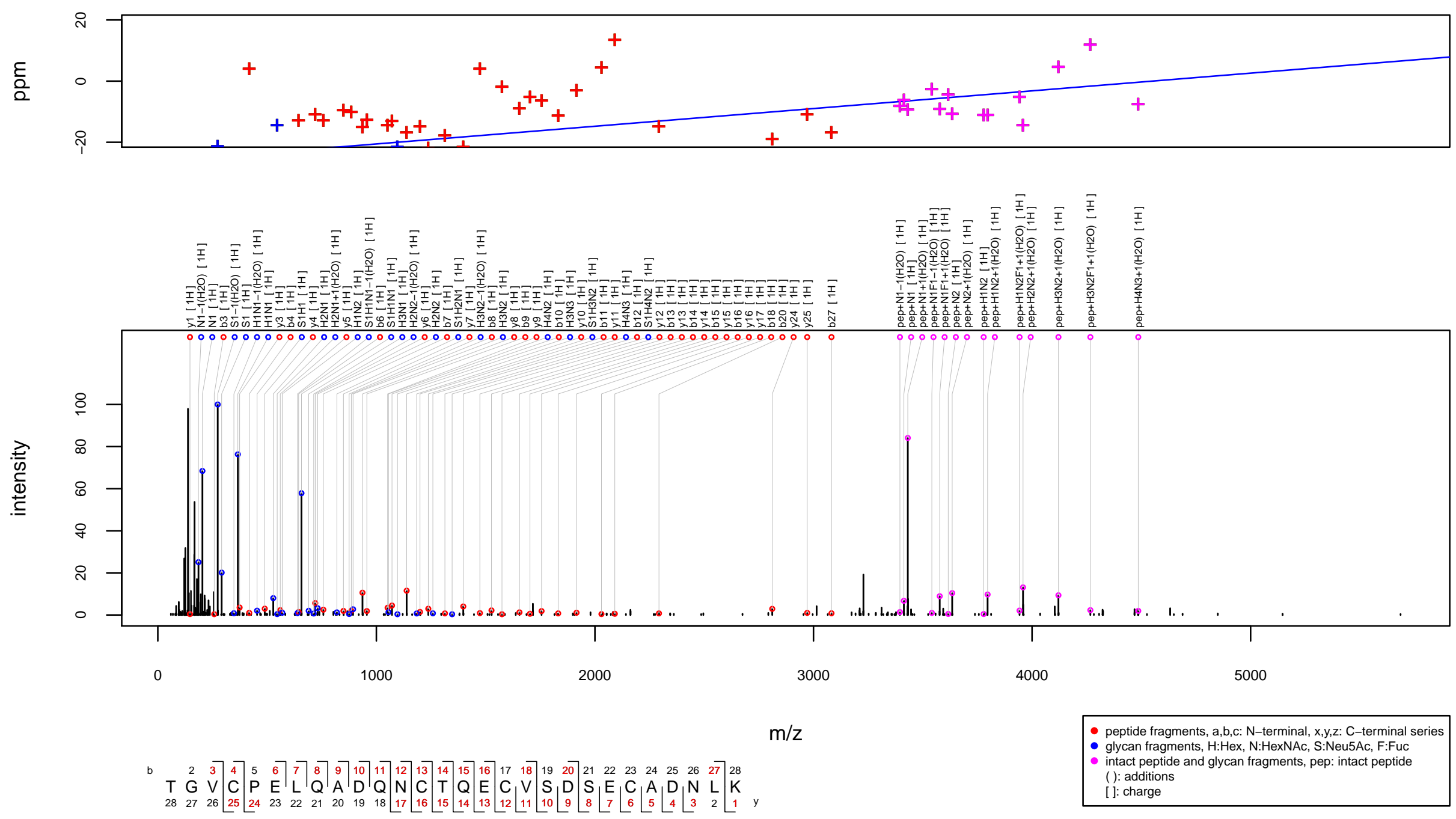
$\mathrm{m} / \mathrm{z} 1250.6161$ charge 4 scan $0-0$

Score $=60.09$, Hits $=47$, Explained Intensity $=0.4$

Peptide: TRFL_HUMAN[141,170]:TAGWNVPIGTLRPFLNWTGPPEPIEAAVAR

Glycan: H5N4F1

Charge: $4 \mathrm{H}$
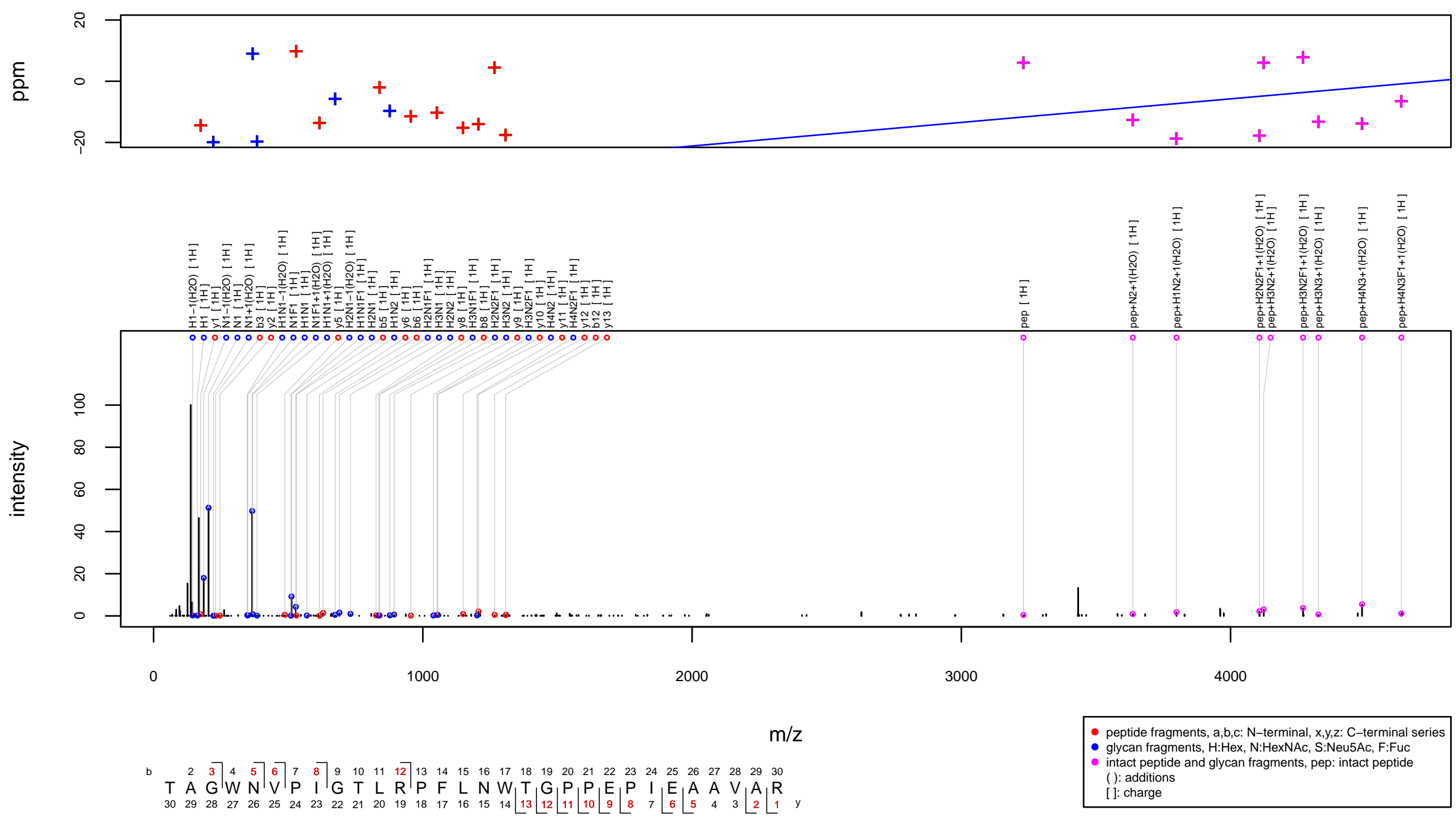
$\mathrm{m} / \mathrm{z} 1254.227$ charge 3 scan $0-0$

Score $=49.64$, Hits $=38$, Explained Intensity $=0.29$

Peptide: ZA2G_HUMAN[103,120]:DIVEYYNDSNGSHVLQGR

Glycan: $\mathrm{SHNH}(\mathrm{H}) \mathrm{HN}(\mathrm{F}) \mathrm{N}, \mathrm{S} 1 \mathrm{H} 4 \mathrm{~N} 3 \mathrm{~F} 1$

Charge: $3 \mathrm{H}$
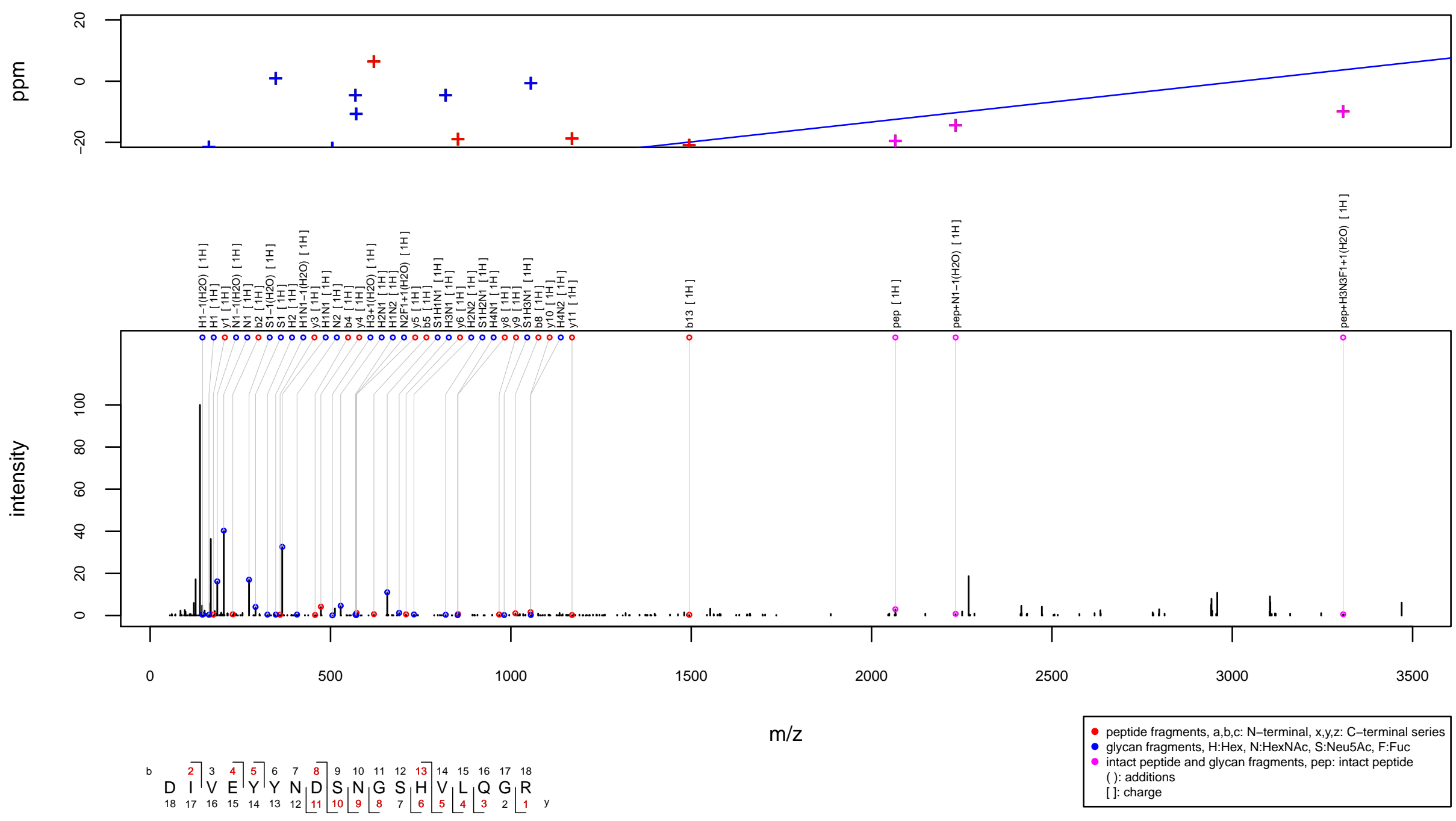
$\mathrm{m} / \mathrm{z} 1261.2524$ charge 3 scan 0-0

Score $=92.73$, Hits $=53$, Explained Intensity $=0.56$

Peptide: CBPE_HUMAN[382,406]:DLQGNPIANATISVEGIDHDVTSAK

Glycan: $\mathrm{H}(\mathrm{H}) \mathrm{H}(\mathrm{H}) \mathrm{HNN}, \mathrm{H} 5 \mathrm{~N} 2$

Charge: $3 \mathrm{H}$
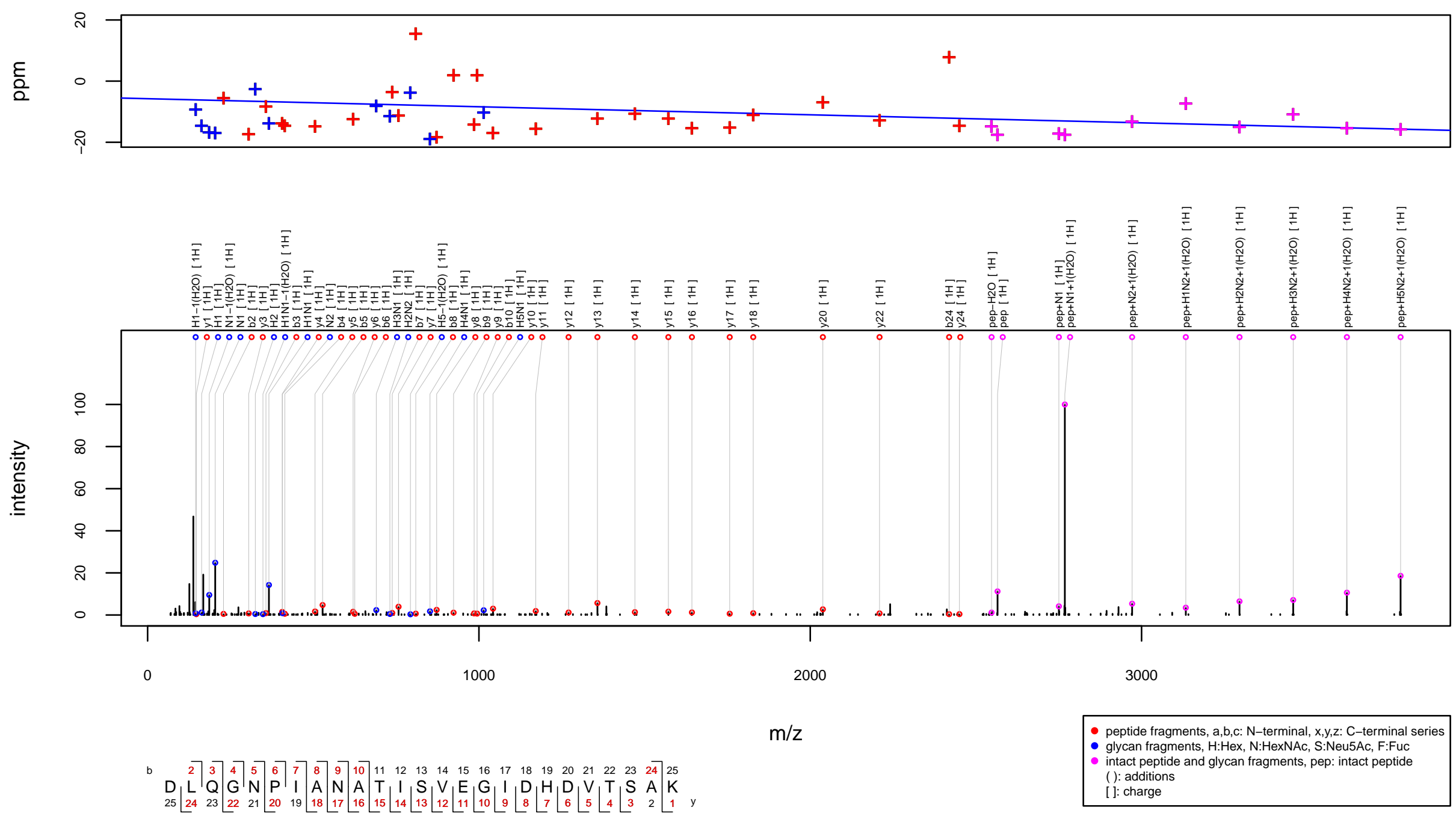
$\mathrm{m} / \mathrm{z} 1261.2699$ charge 3 scan $0-0$

Score $=62.34$, Hits $=38$, Explained Intensity $=0.4$

Peptide: FINC_HUMAN[997,1016]:LDAPTNLQFVNETDSTVLVR

Glycan: $\mathrm{FHNH}(\mathrm{H}) \mathrm{HN}(\mathrm{F}) \mathrm{N}, \mathrm{H} 4 \mathrm{~N} 3 \mathrm{~F} 2$

Charge: $3 \mathrm{H}$
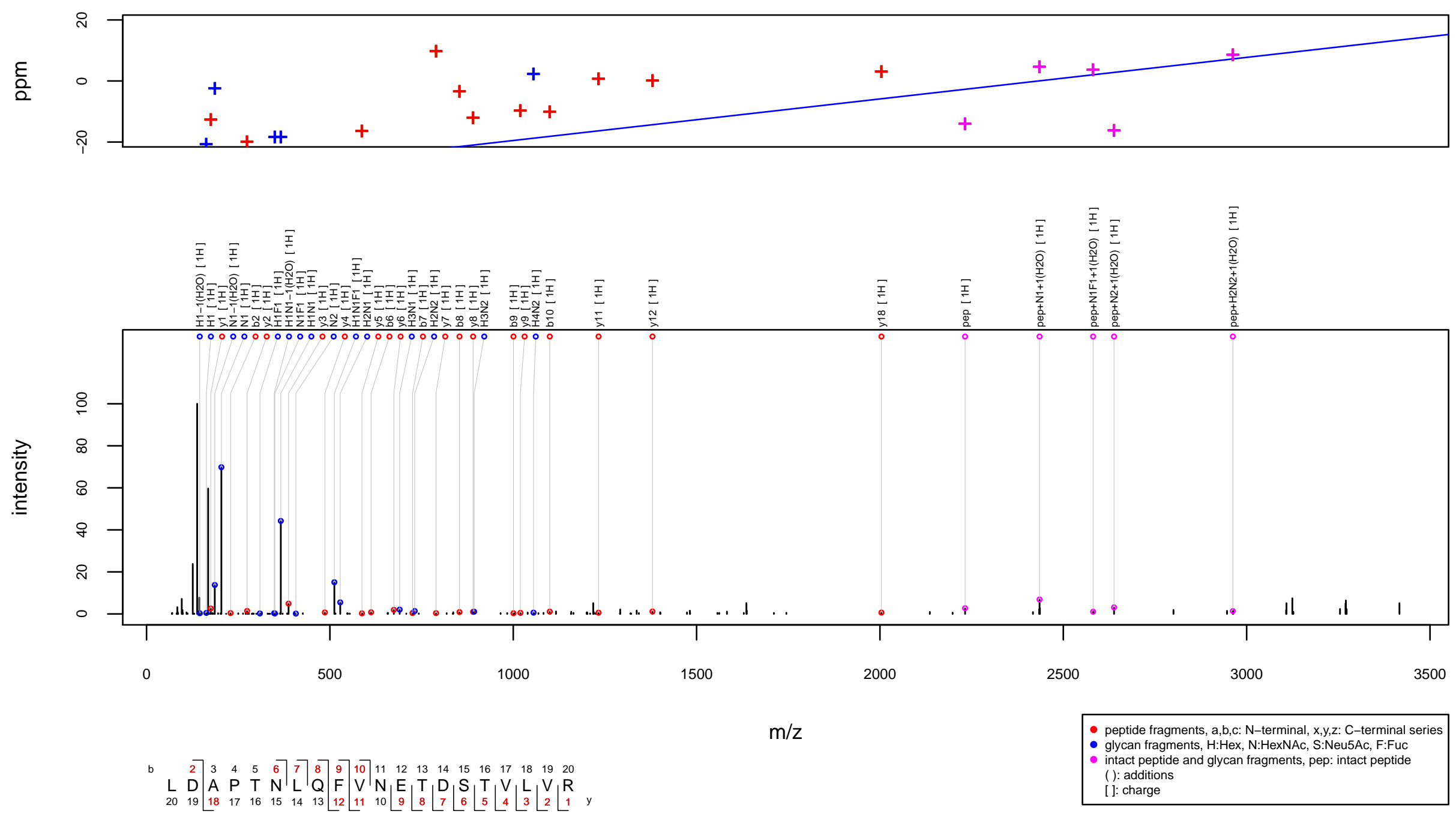
$\mathrm{m} / \mathrm{z} 1271.9167$ charge 3 scan $0-0$

Score $=85.94$, Hits $=63$, Explained Intensity $=0.46$ Peptide: TIMP1_HUMAN[46,60]:FVGTPEVNQTTLYQR Glycan: $\mathrm{FH}(\mathrm{F}) \mathrm{NH}(\mathrm{HNH}) \mathrm{HN}(\mathrm{F}) \mathrm{N}, \mathrm{H} 5 \mathrm{~N} 4 \mathrm{~F} 3$

Charge: $3 \mathrm{H}$
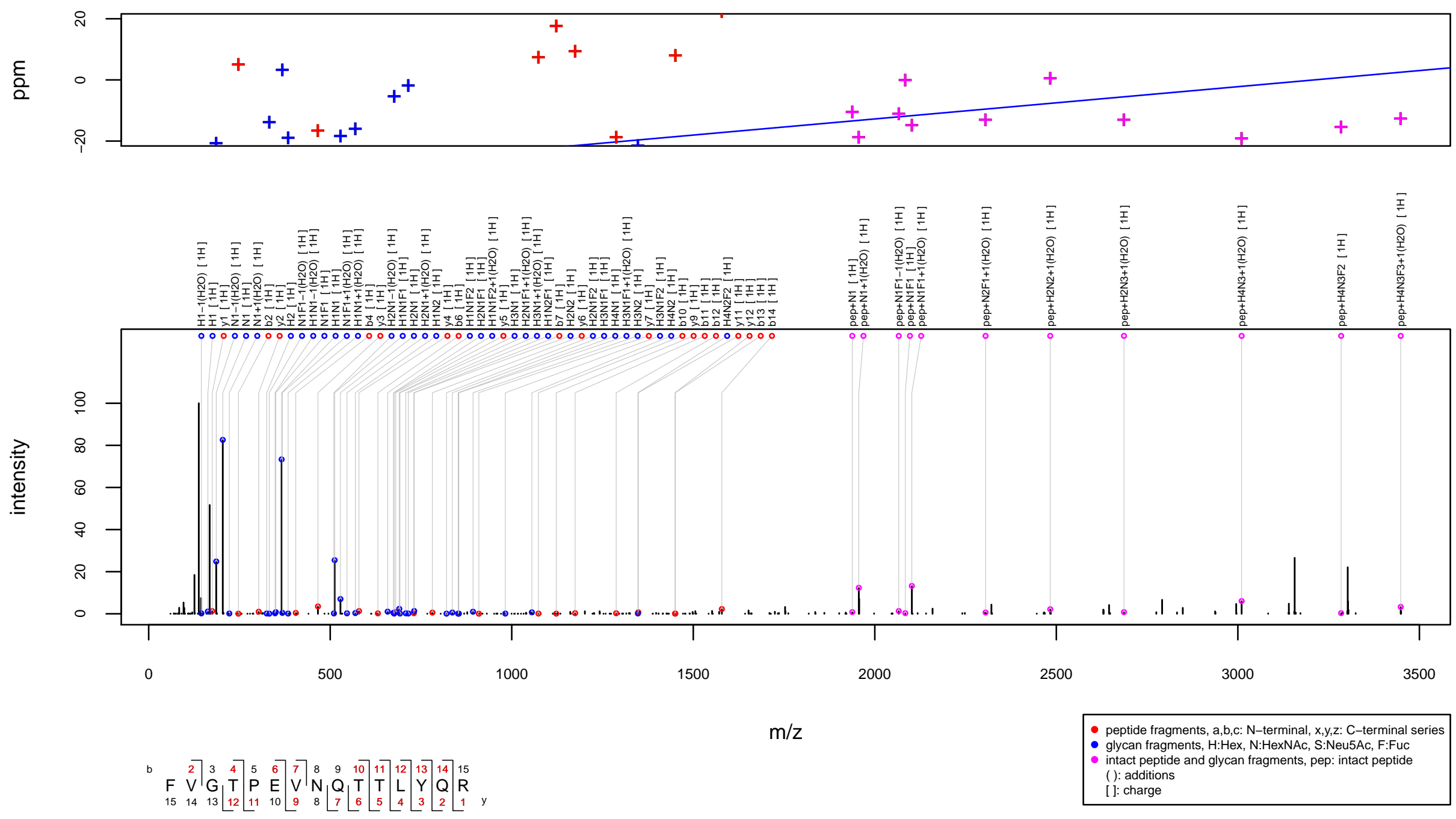
$\mathrm{m} / \mathrm{z} 1273.2375$ charge 3 scan $0-0$

Score $=64.39$, Hits $=44$, Explained Intensity $=0.42$

Peptide: CLUS_HUMAN[372,385]:LANLTQGEDQYYLR

Glycan: $\mathrm{HNHNH}(\mathrm{F}(\mathrm{H}) \mathrm{NH}) \mathrm{HNN}, \mathrm{H} 6 \mathrm{~N} 5 \mathrm{~F} 1$

Charge: $3 \mathrm{H}$
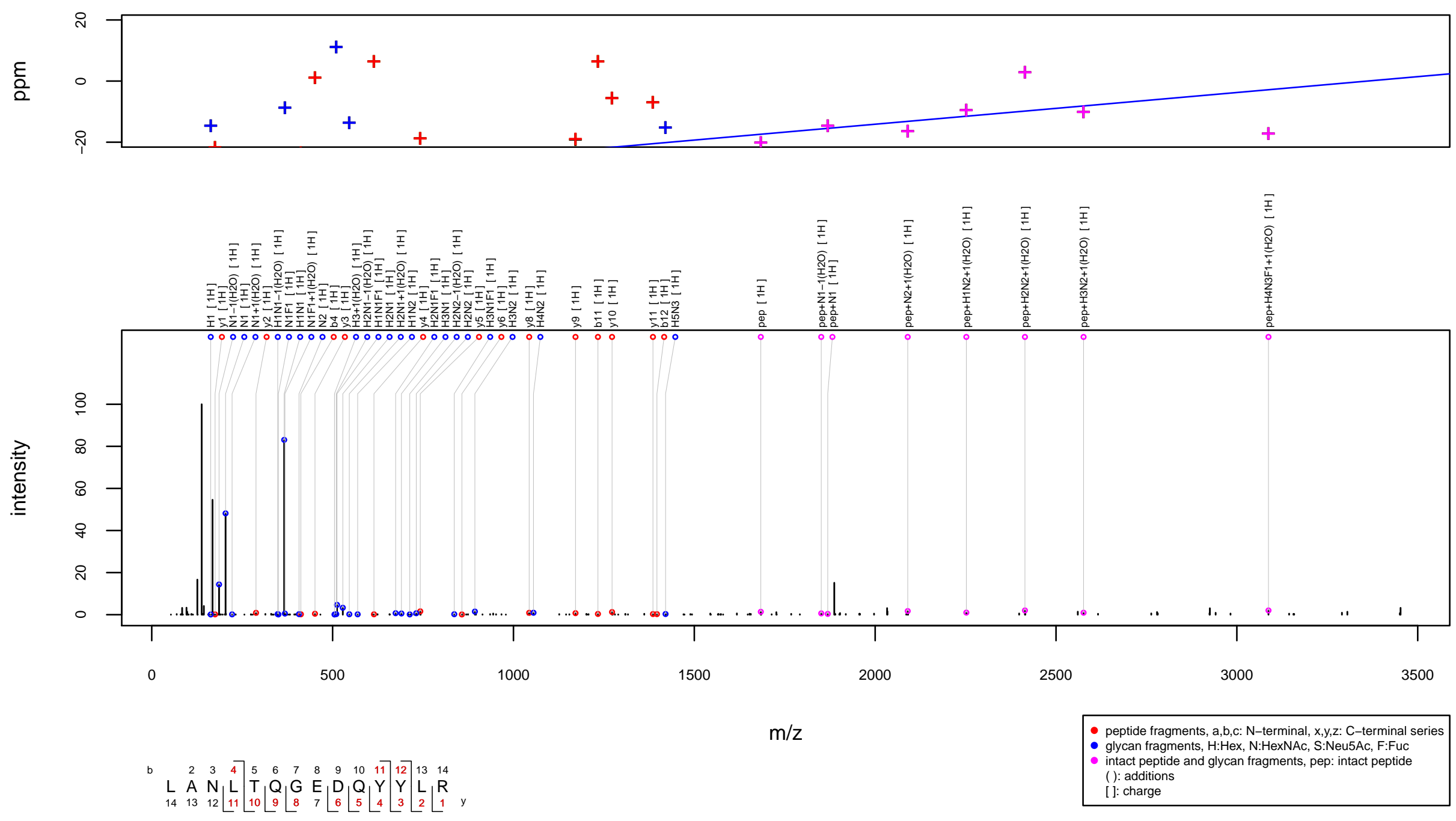
$\mathrm{m} / \mathrm{z} 1307.2351$ charge 3 scan $0-0$

Score $=82.32$, Hits $=68$, Explained Intensity $=0.46$ Peptide: LG3BP_HUMAN[64,76]:ALGFENATQALGR Glycan: $\mathrm{F}(\mathrm{H}) \mathrm{NH}(\mathrm{F}(\mathrm{H}) \mathrm{N}(\mathrm{F}(\mathrm{H}) \mathrm{N}) \mathrm{H}) \mathrm{HN}(\mathrm{F}) \mathrm{N}, \mathrm{H} 6 \mathrm{~N} 5 \mathrm{~F} 4$

Charge: $3 \mathrm{H}$
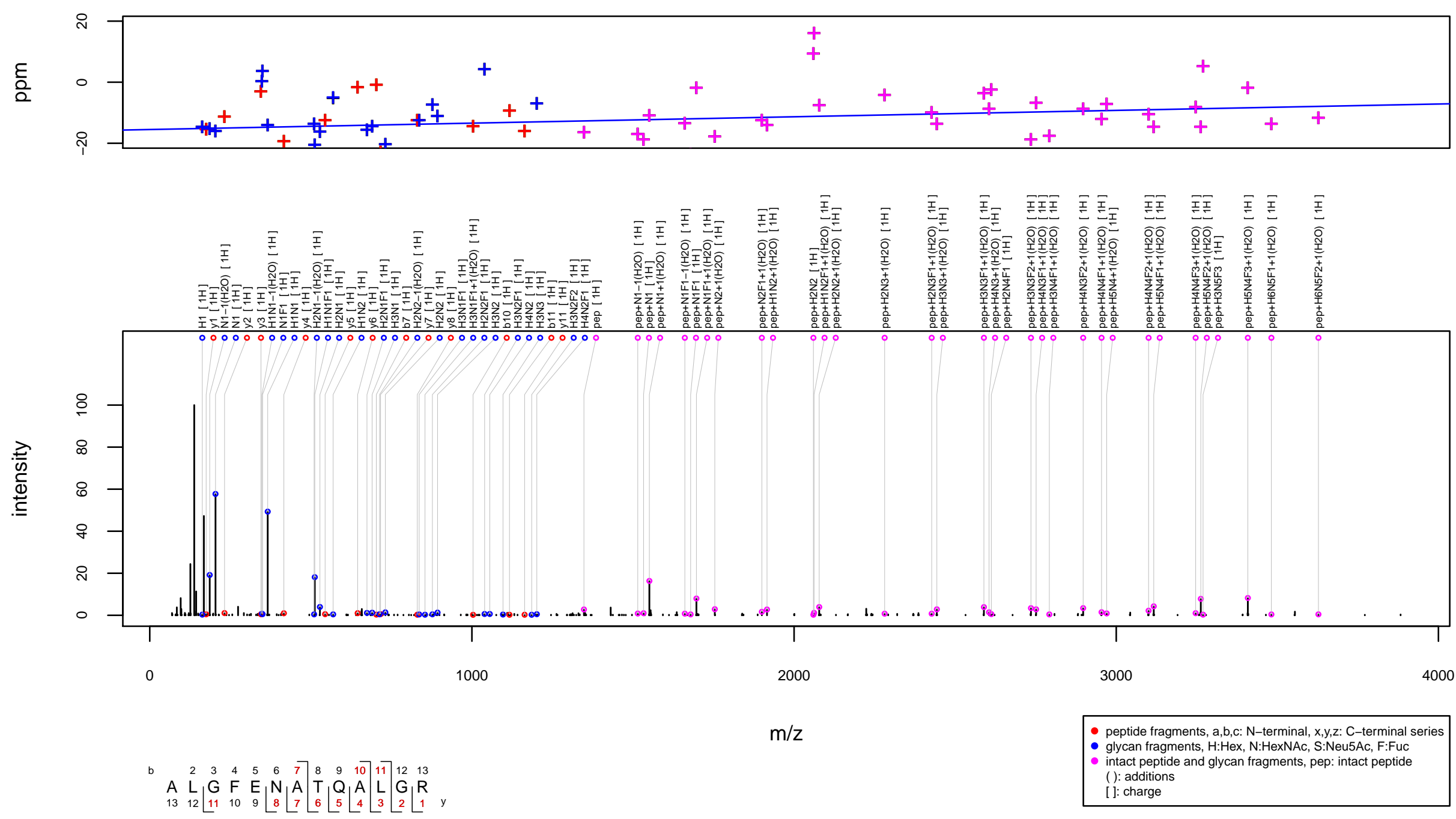


\section{$\mathrm{m} / \mathrm{z} 1314.2761$ charge 3 scan $0-0$}

Score $=80.54$, Hits $=54$, Explained Intensity $=0.45$

Peptide: HEMO_HUMAN[447,462]:ALPQPQNVTSLLGCTH

Glycan: $\mathrm{SHNH}(\mathrm{SHNH}) \mathrm{HNN}, \mathrm{S} 2 \mathrm{H} 5 \mathrm{~N} 4$

Charge: $3 \mathrm{H}$
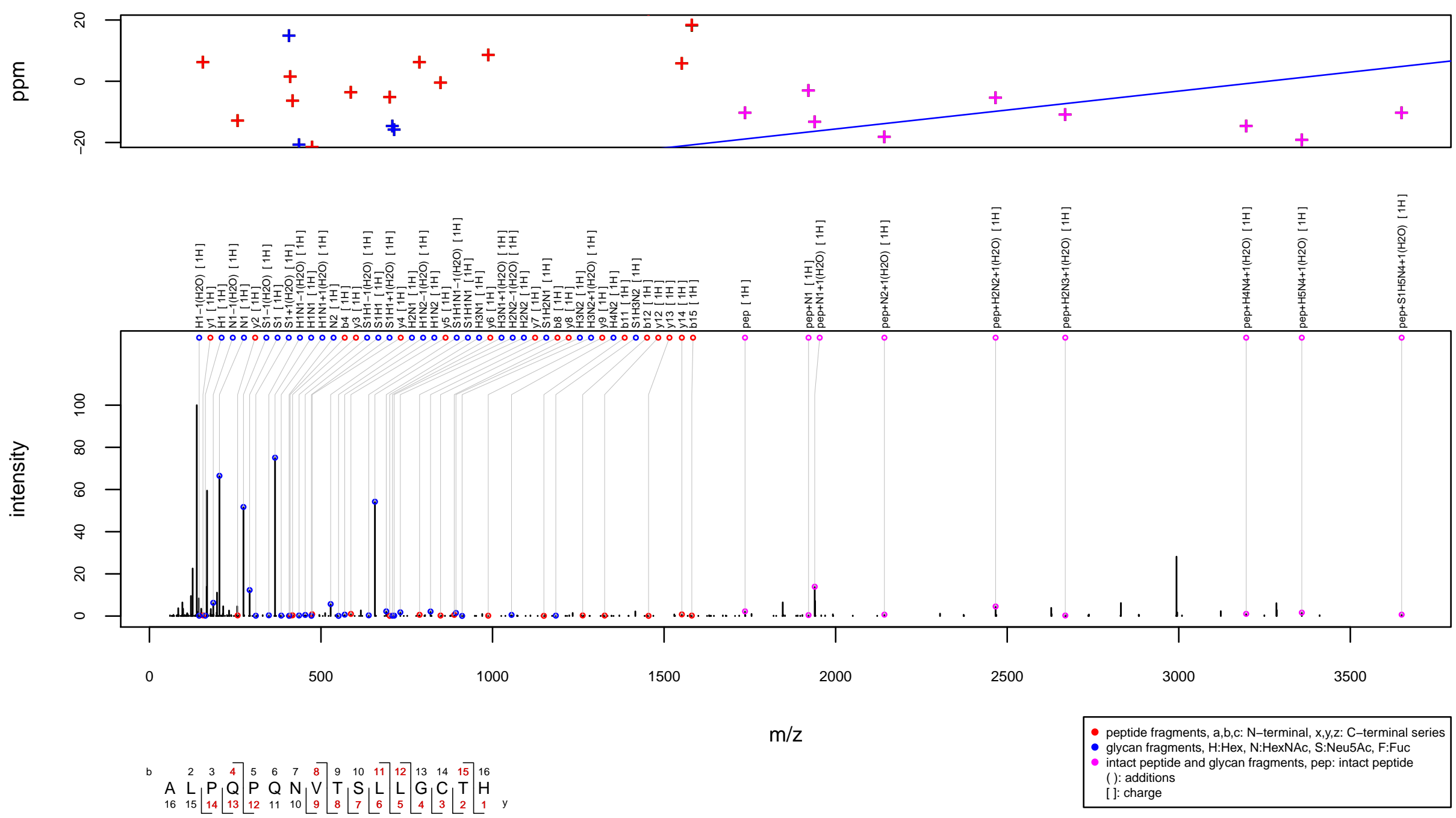
$\mathrm{m} / \mathrm{z} 1319.8384$ charge 4 scan $0-0$

Score $=84.56$, Hits $=62$, Explained Intensity $=0.49$

Peptide: MUC6_HUMAN[884,909]:FVFDGNCEYILATDVCGVNDSQPTFK

Glycan: $\mathrm{HNHNH}(\mathrm{F}(\mathrm{H}) \mathrm{NH}) \mathrm{HN}(\mathrm{F}) \mathrm{N}, \mathrm{H} 6 \mathrm{~N} 5 \mathrm{~F} 2$

Charge: $4 \mathrm{H}$
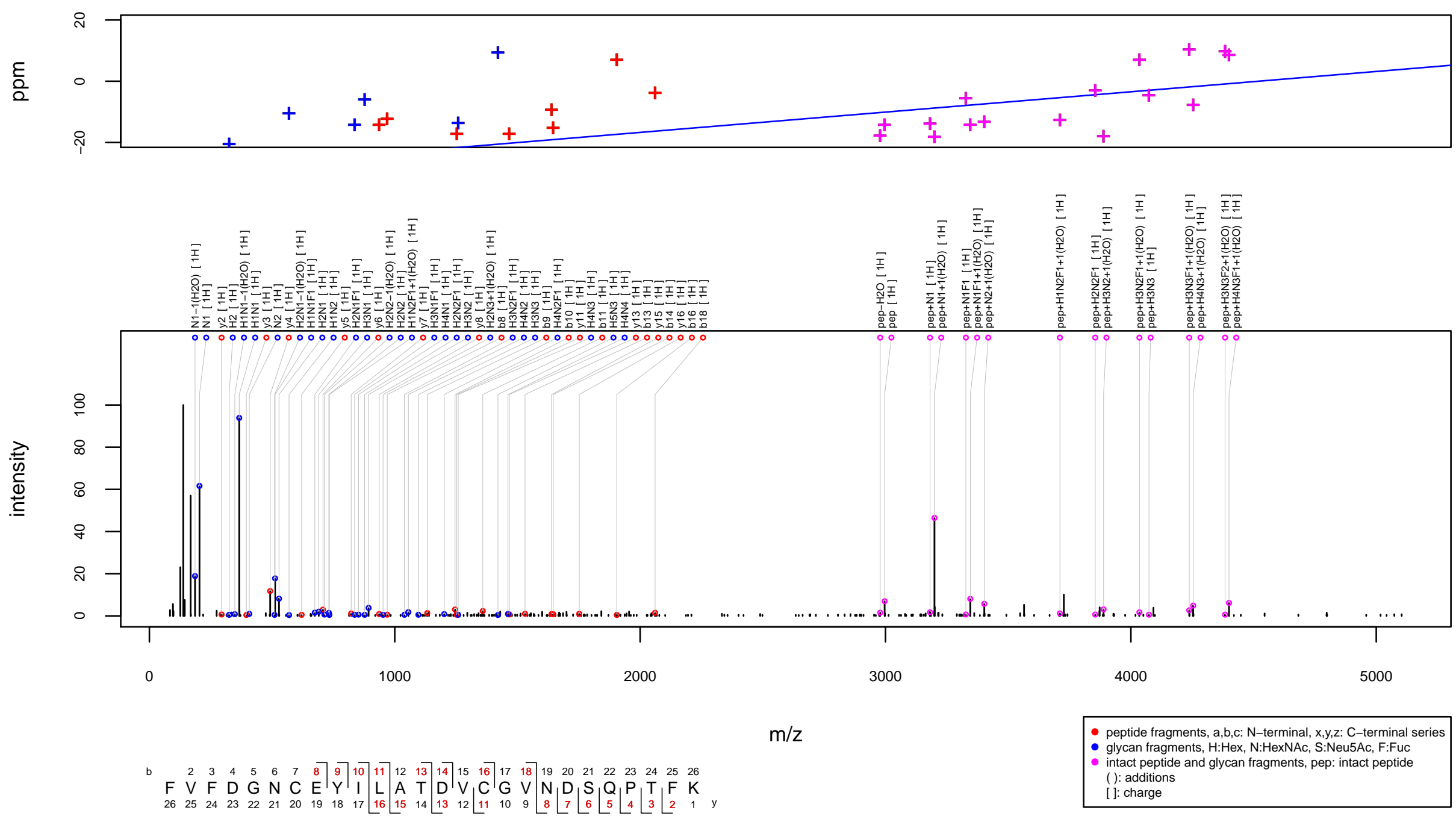
$\mathrm{m} / \mathrm{z} 1319.9214$ charge 3 scan $0-0$

Score $=59.1$, Hits $=41$, Explained Intensity $=0.41$

Peptide: TIMP1_HUMAN[46,60]:FVGTPEVNQTTLYQR

Glycan: $\mathrm{SHNH}(\mathrm{SHNH}) \mathrm{HNN}, \mathrm{S} 2 \mathrm{H} 5 \mathrm{~N} 4$

Charge: $3 \mathrm{H}$
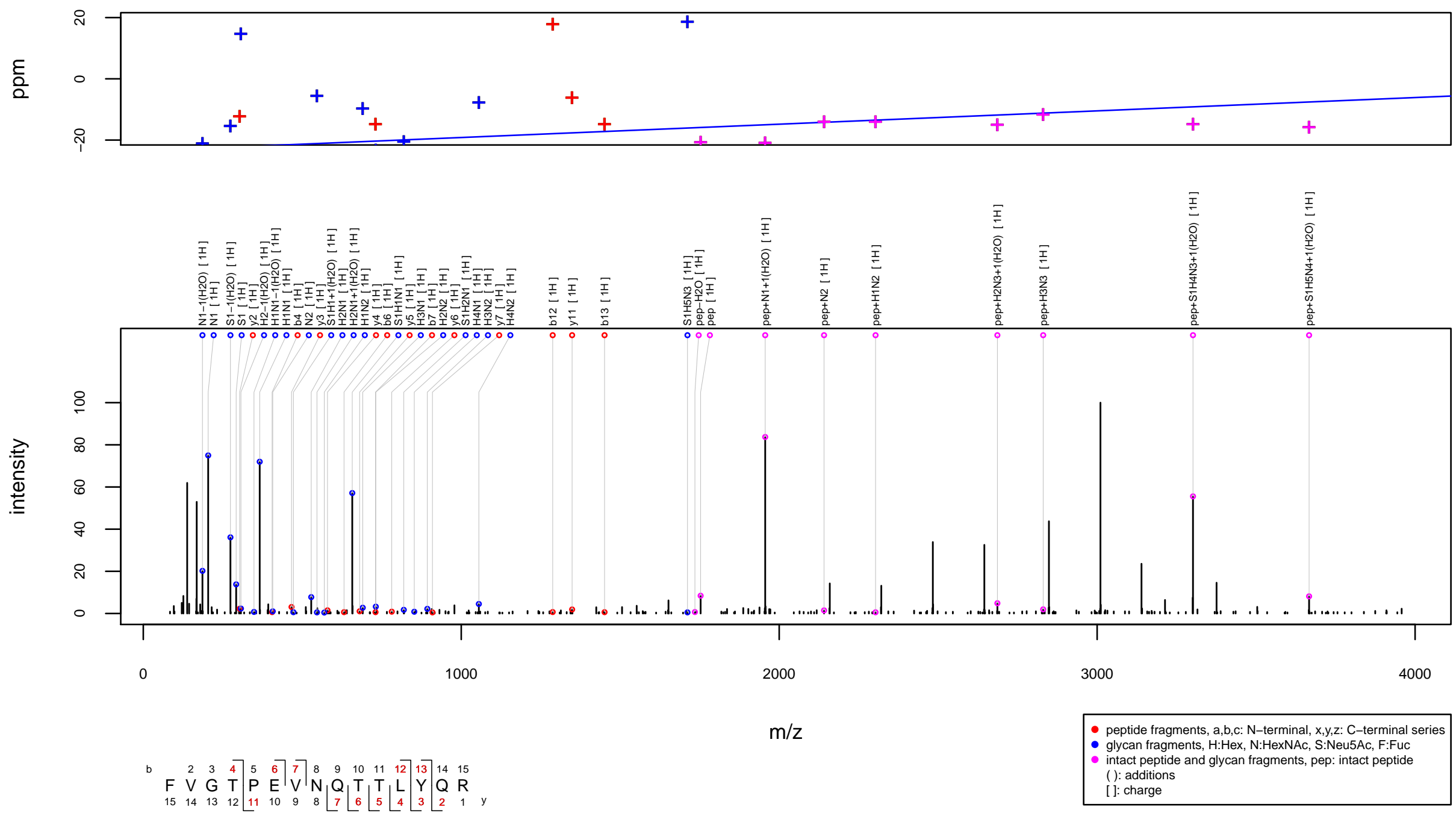
$\mathrm{m} / \mathrm{z} 1320.2701$ charge 4 scan $0-0$

Score $=65.47$, Hits $=56$, Explained Intensity $=0.39$

Peptide: CLUS_HUMAN[372,385]:LANLTQGEDQYYLR

Glycan: $\mathrm{HNHN}(\mathrm{HN}) \mathrm{H}(\mathrm{HNHN}(\mathrm{HNHN}) \mathrm{H}) \mathrm{HN}(\mathrm{F}) \mathrm{N}, \mathrm{H} 10 \mathrm{~N} 9 \mathrm{~F} 1$

Charge: $4 \mathrm{H}$
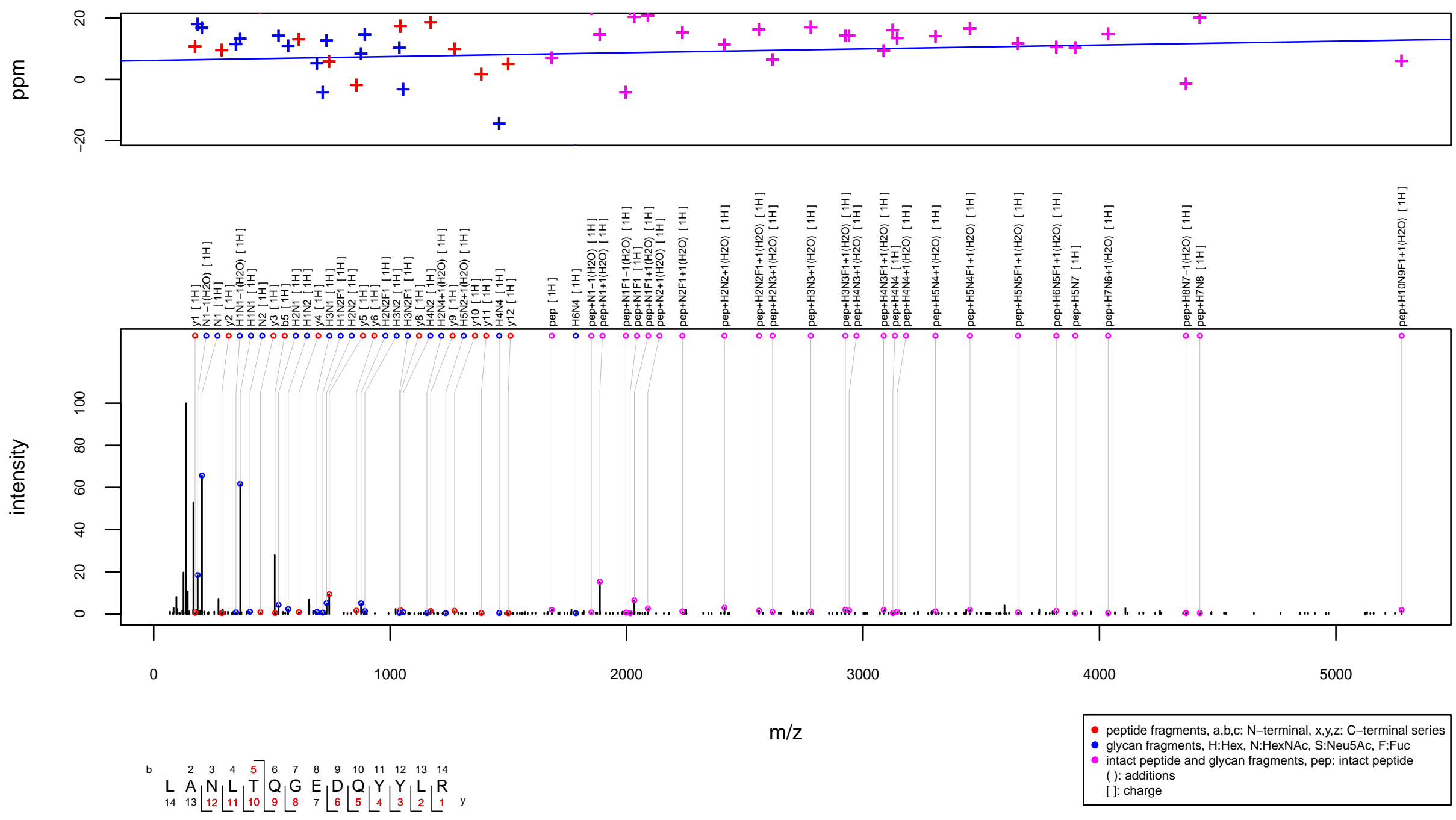
$\mathrm{m} / \mathrm{z} 1320.2963$ charge 4 scan $0-0$

Score $=101.78$, Hits $=114$, Explained Intensity $=0.6$ Peptide: CLUS_HUMAN[372,385]:LANLTQGEDQYYLR Glycan: H8N7F6

Charge: $4 \mathrm{H}$
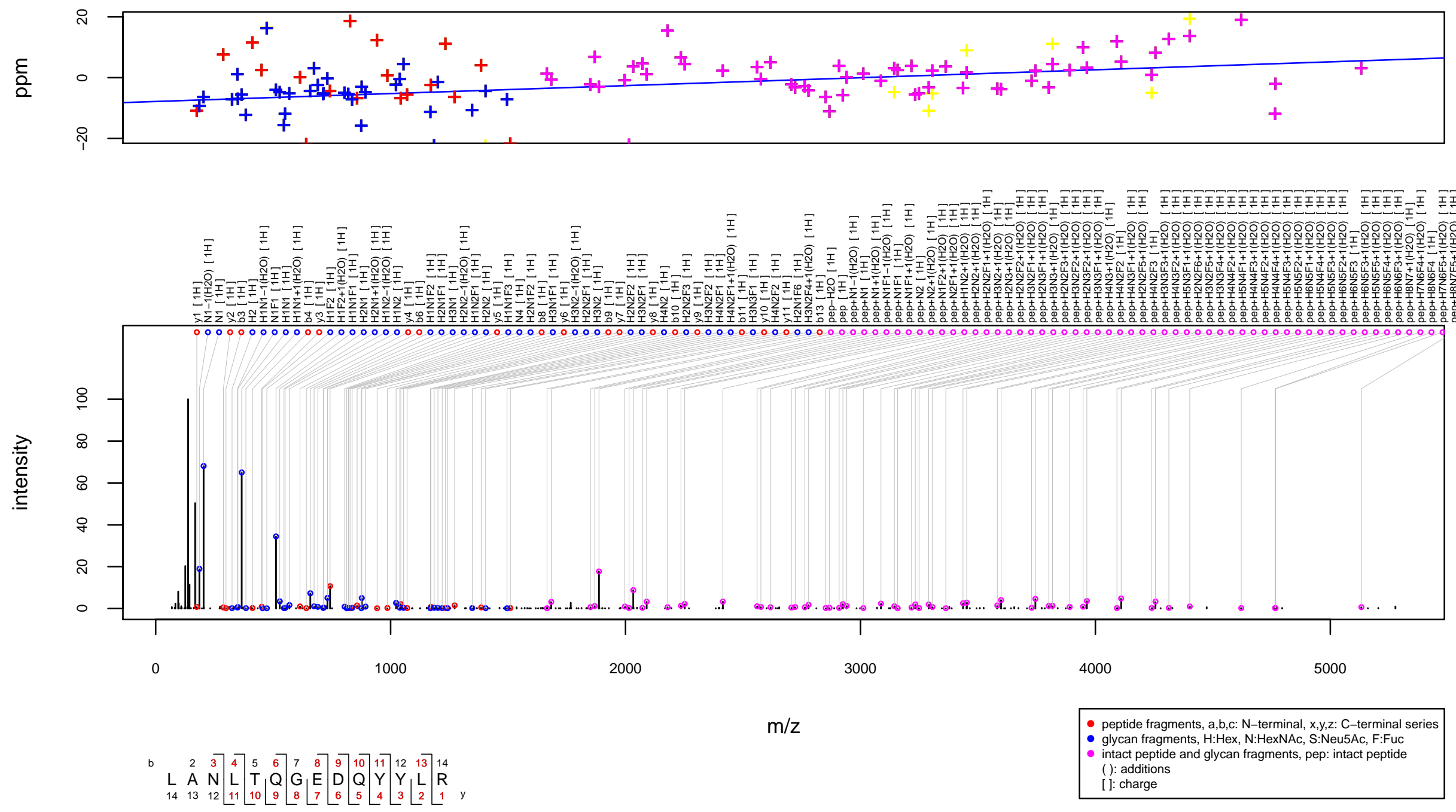


\section{$\mathrm{m} / \mathrm{z} 1320.6165$ charge 3 scan 0-0}

Score $=61.54$, Hits $=48$, Explained Intensity $=0.35$

Peptide: FINC_HUMAN[997,1016]:LDAPTNLQFVNETDSTVLVR

Glycan: H6N3F1

Charge: $3 \mathrm{H}$
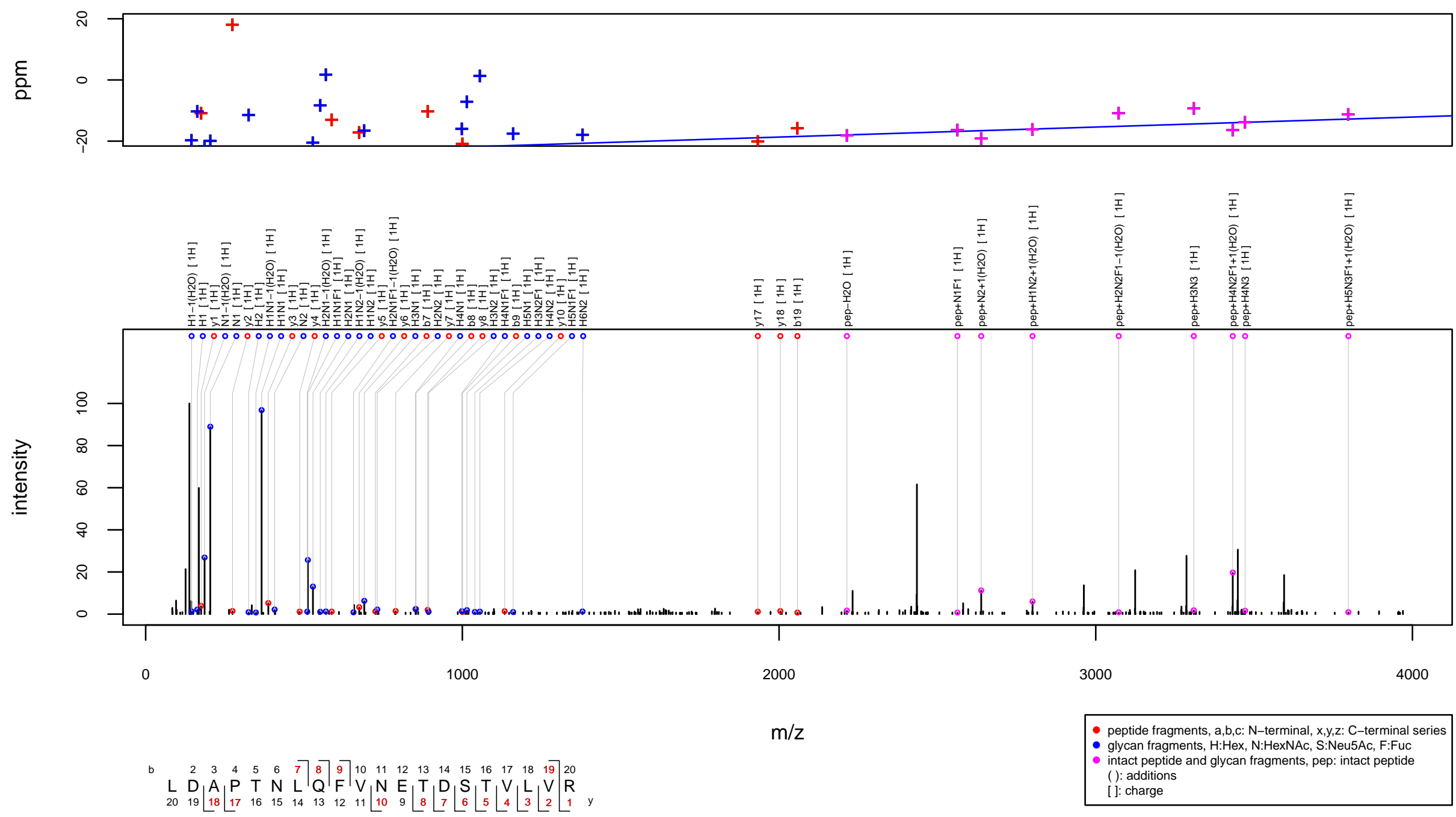
$\mathrm{m} / \mathrm{z} 1320.9302$ charge 3 scan $0-0$

Score $=70.3$, Hits $=48$, Explained Intensity $=0.43$

Peptide: A1AT_HUMAN[268,283]:YLGNATAIFFLPDEGK

Glycan: $\mathrm{SHNH}(\mathrm{SHNH}) \mathrm{HNN}, \mathrm{S} 2 \mathrm{H} 5 \mathrm{~N} 4$

Charge: $3 \mathrm{H}$
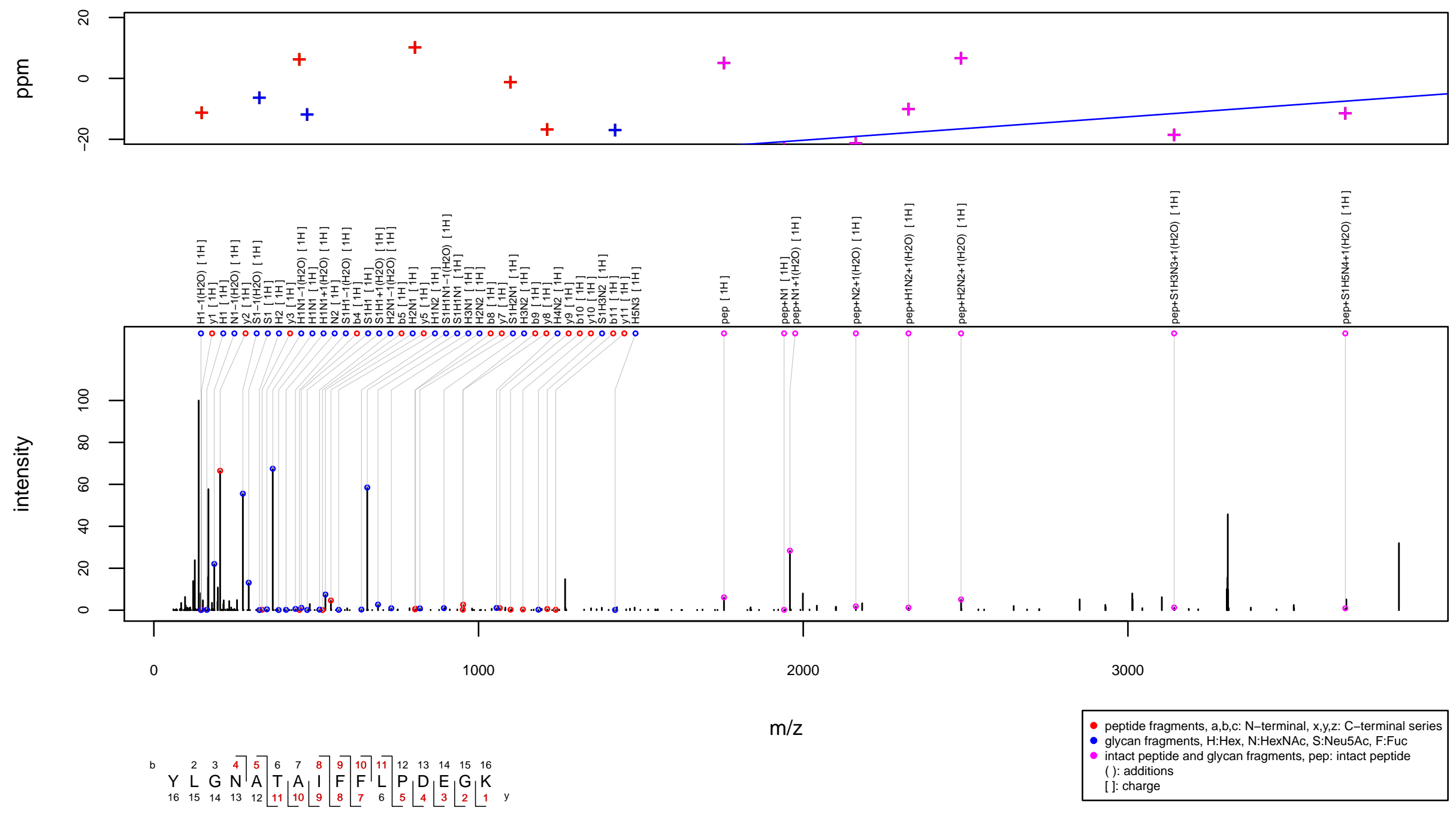
$\mathrm{m} / \mathrm{z} 1321.924$ charge 3 scan $0-0$

Score $=73.69$, Hits $=57$, Explained Intensity $=0.44$

Peptide: CLUS_HUMAN[372,385]:LANLTQGEDQYYLR

Glycan: $\mathrm{HNHNH}(\mathrm{F}(\mathrm{H}) \mathrm{NH}) \mathrm{HN}(\mathrm{F}) \mathrm{N}, \mathrm{H} 6 \mathrm{~N} 5 \mathrm{~F} 2$

Charge: $3 \mathrm{H}$
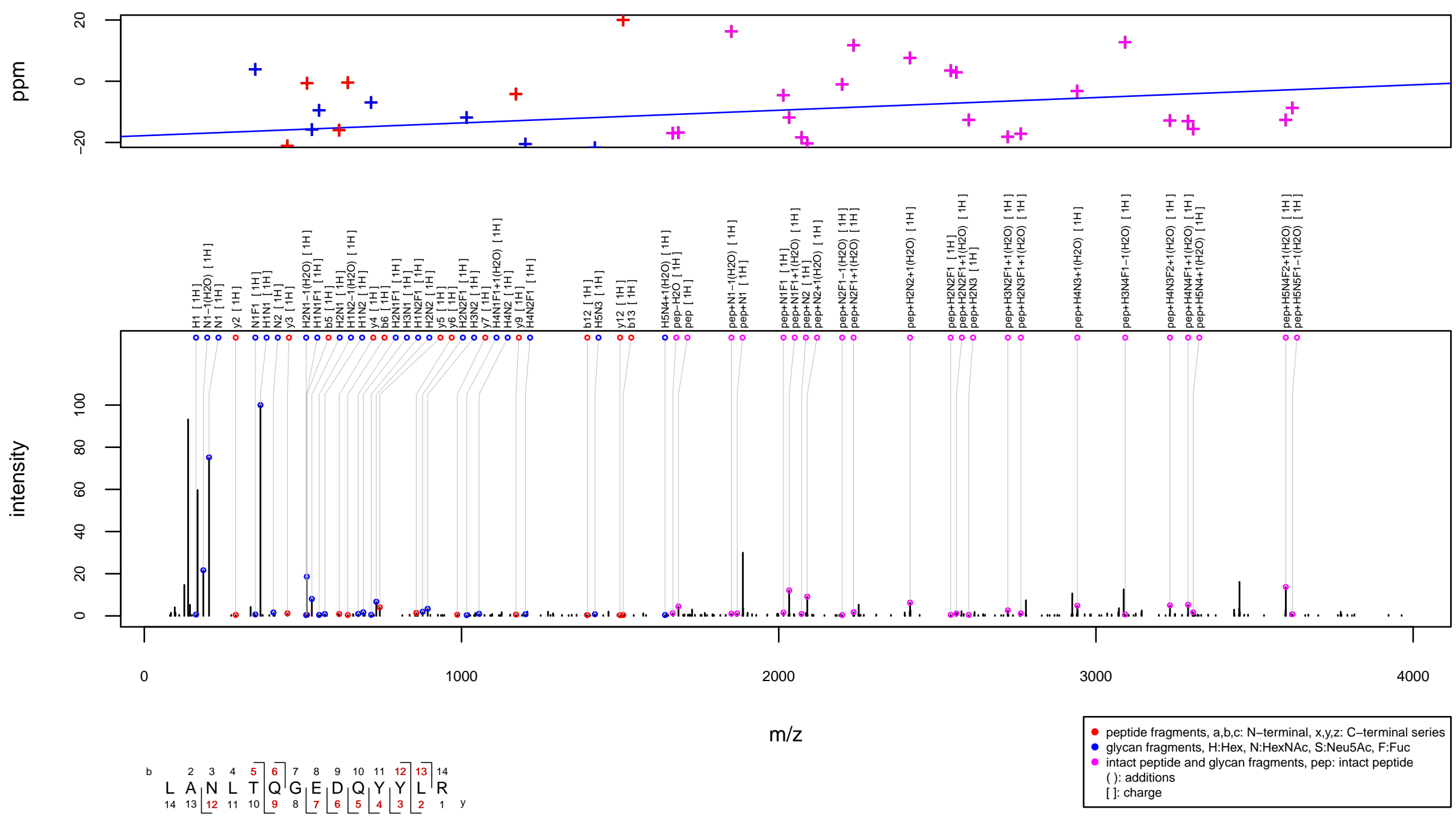
$\mathrm{m} / \mathrm{z} 1322.5773$ charge 3 scan $0-0$

Score $=78.7$, Hits $=57$, Explained Intensity $=0.46$

Peptide: FINC_HUMAN[516,533]:DQCIVDDITYNVNDTFHK

Glycan: H5N4F1

Charge: $3 \mathrm{H}$
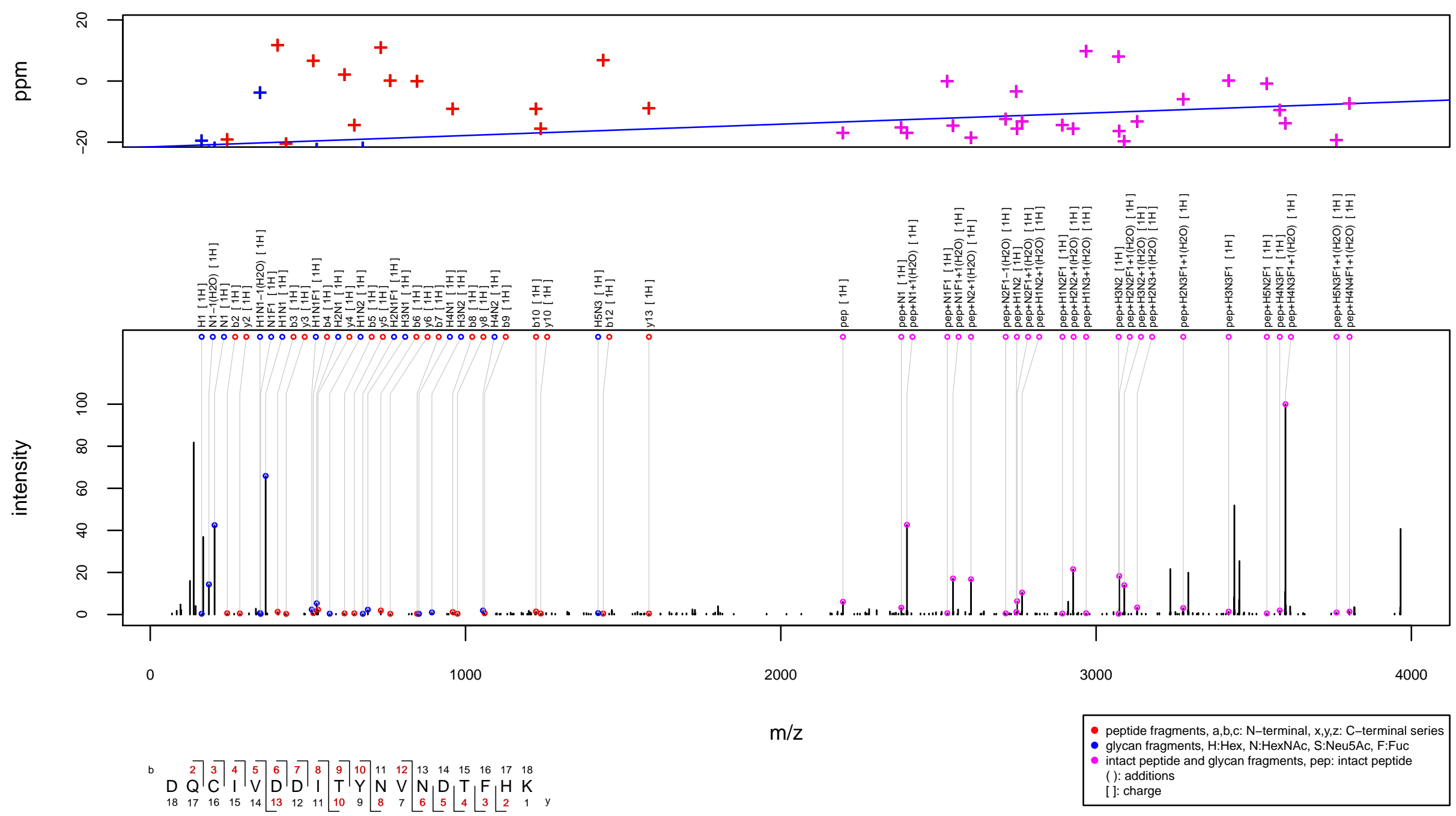


\section{$\mathrm{m} / \mathrm{z} 1323.6493$ charge 4 scan 0-0}

Score $=58.79$, Hits $=44$, Explained Intensity $=0.46$

Peptide: TRFL_HUMAN[141,170]:TAGWNVPIGTLRPFLNWTGPPEPIEAAVAR

Glycan: $\mathrm{FHNH}(\mathrm{FHNH}) \mathrm{HN}(\mathrm{F}) \mathrm{N}, \mathrm{H} 5 \mathrm{~N} 4 \mathrm{~F} 3$

Charge: $4 \mathrm{H}$
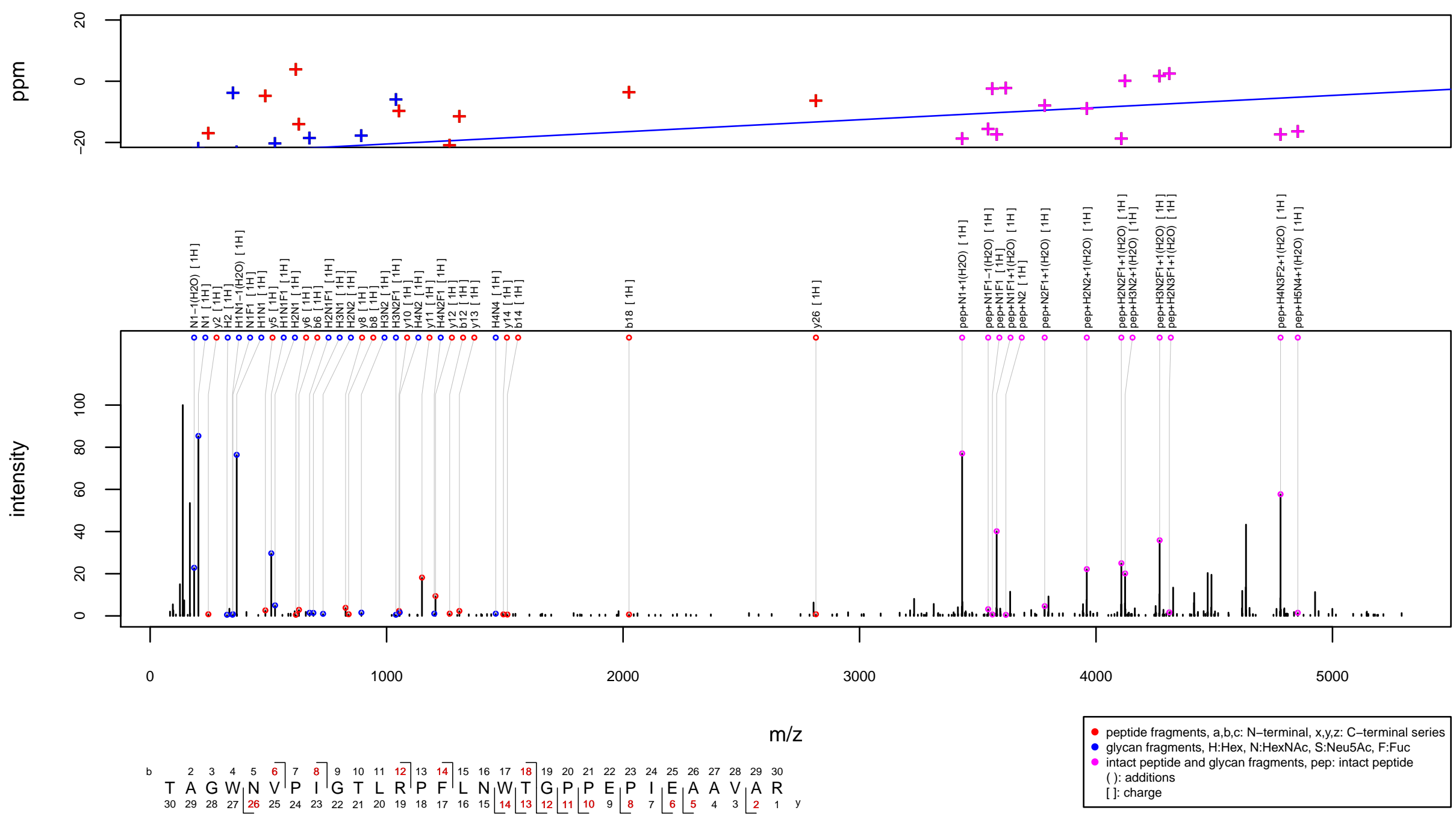
$\mathrm{m} / \mathrm{z} 1328.8339$ charge 3 scan $0-0$

Score $=93.67$, Hits $=91$, Explained Intensity $=0.51$

Peptide: PIP_HUMAN[97,106]:TFYWDFYTNR

Glycan: H6N5F4

Charge: $3 \mathrm{H}$
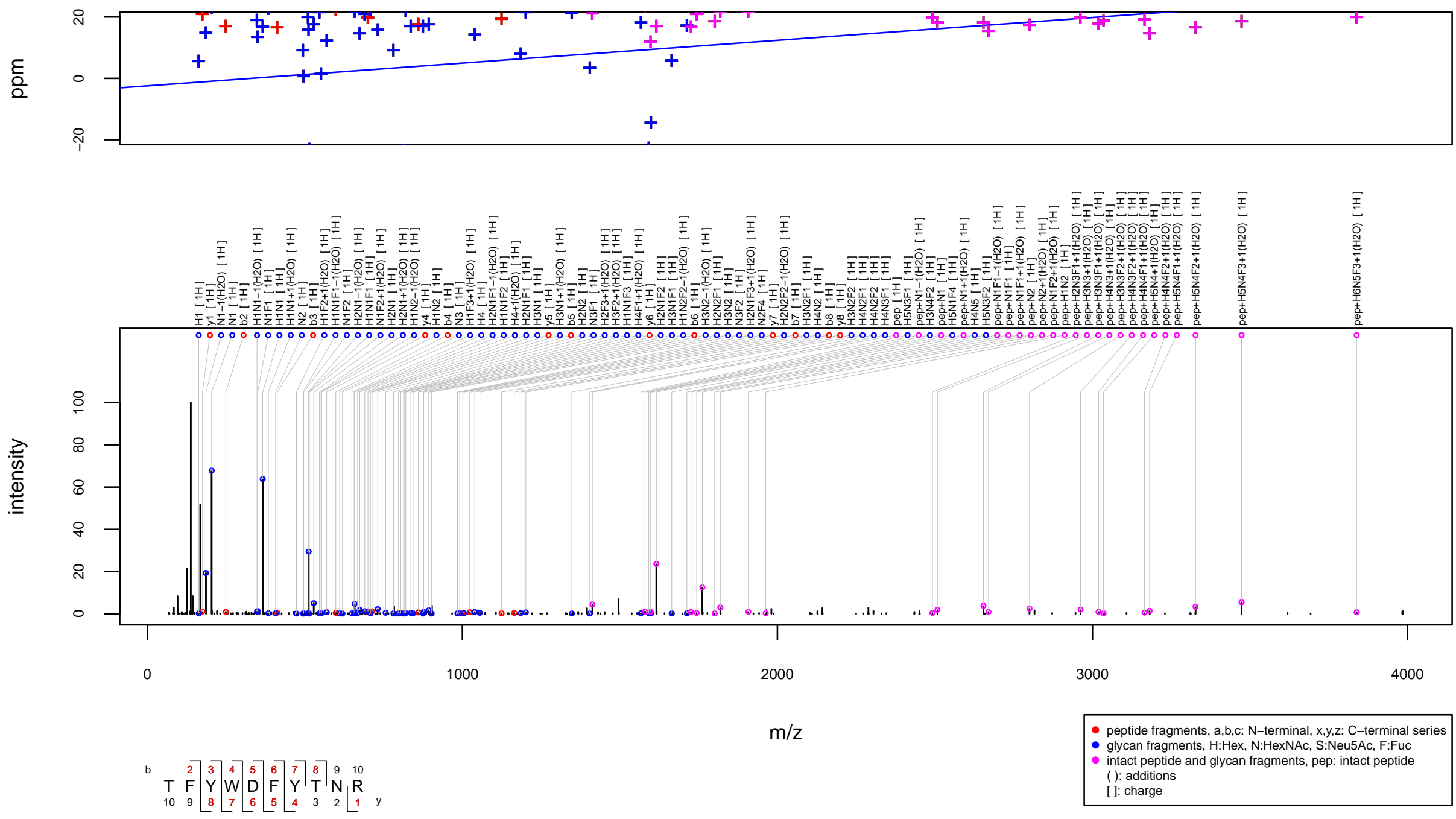
$\mathrm{m} / \mathrm{z} 1331.8923$ charge 3 scan $0-0$

Score $=45.32$, Hits $=64$, Explained Intensity $=0.45$ Peptide: SO1B3_HUMAN[306,316]:NQTANLTNQGK Glycan: S2H6N4F3

Charge: $3 \mathrm{H}$
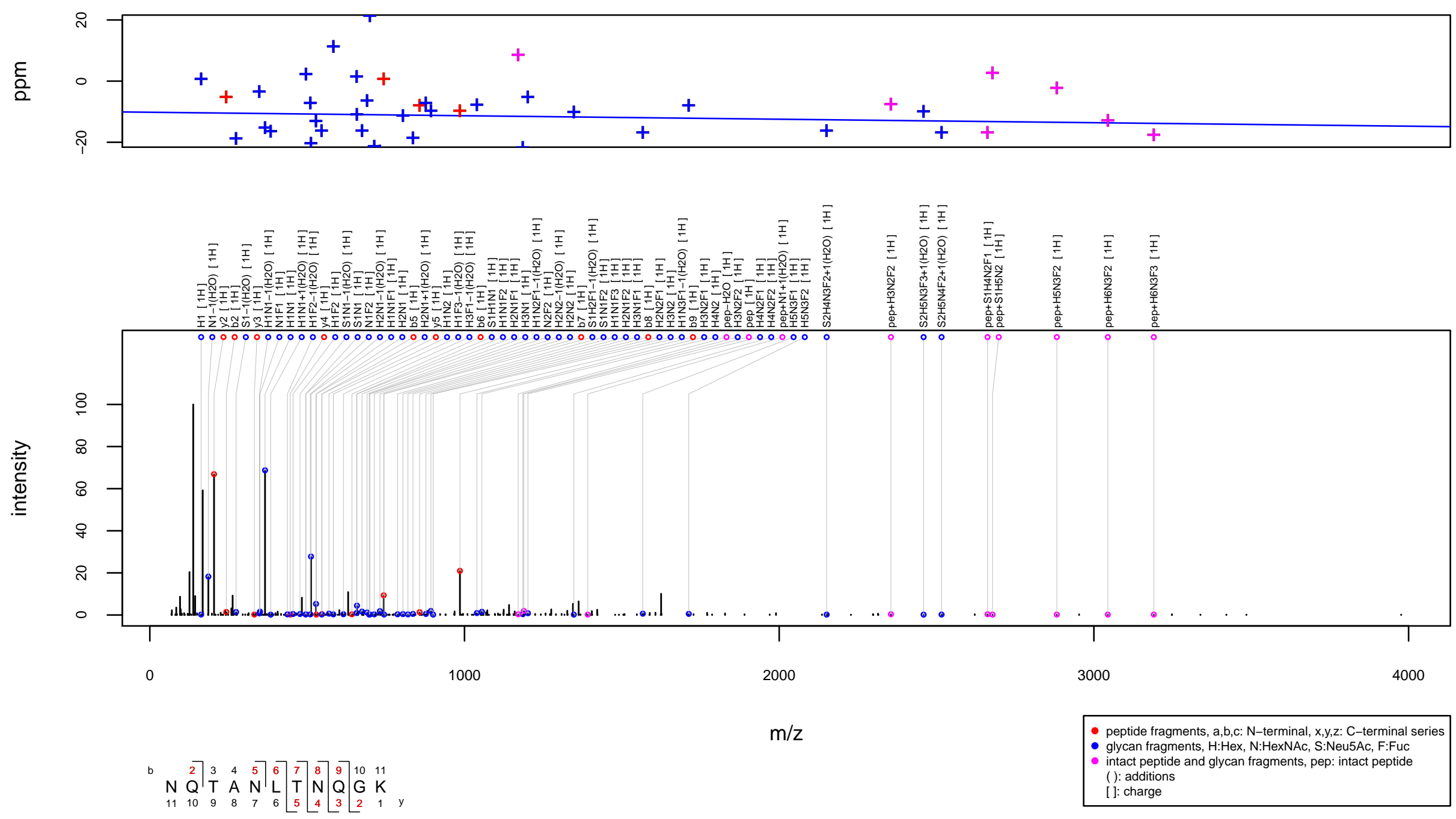


\section{m/z 1334.2977 charge 3 scan 0-0}

Score $=76.44$, Hits $=50$, Explained Intensity $=0.36$

Peptide: FINC_HUMAN[997,1016]:LDAPTNLQFVNETDSTVLVR

Glycan: $\mathrm{HNH}(\mathrm{HNH}) \mathrm{HN}(\mathrm{F}) \mathrm{N}, \mathrm{H} 5 \mathrm{~N} 4 \mathrm{~F} 1$

Charge: $3 \mathrm{H}$
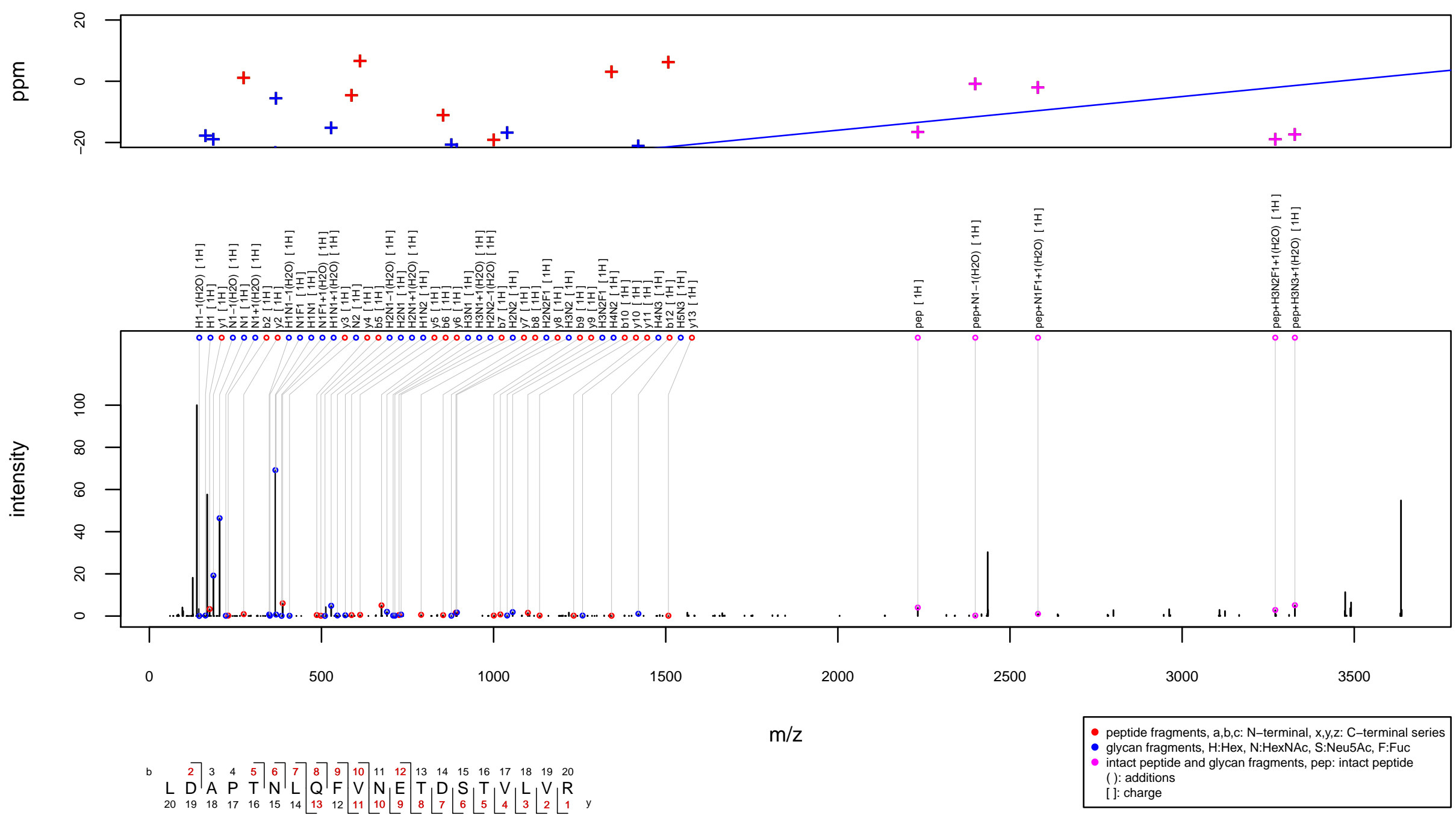


\section{$\mathrm{m} / \mathrm{z} 1334.52$ charge 3 scan $0-0$}

Score $=55.73$, Hits $=44$, Explained Intensity $=0.25$

Peptide: SAP_HUMAN[93,107]:TCDWLPKPNMSASCK

Glycan: $\mathrm{SHNH}(\mathrm{HNH}) \mathrm{HN}(\mathrm{F}) \mathrm{N}, \mathrm{S} 1 \mathrm{H} 5 \mathrm{~N} 4 \mathrm{~F} 1$

Charge: $3 \mathrm{H}$
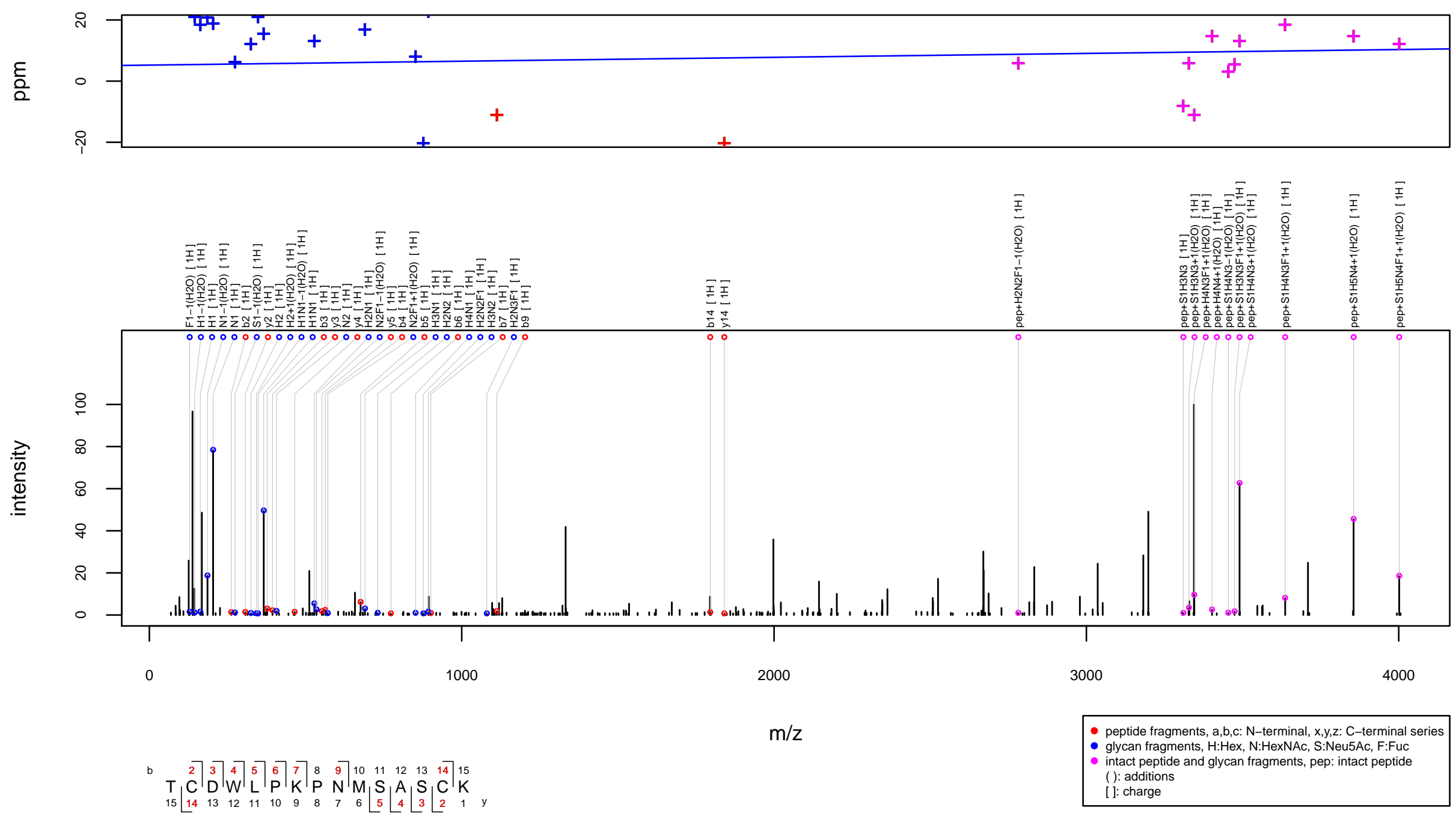


\section{$\mathrm{m} / \mathrm{z} 1341.8815$ charge 3 scan $0-0$}

Score $=66.91$, Hits $=46$, Explained Intensity $=0.52$

Peptide: TSN1_HUMAN[149,165]:CCGFTNYTDFEDSPYFK

Glycan: $\mathrm{SHNH}(\mathrm{H}(\mathrm{H}) \mathrm{H}) \mathrm{HNN}, \mathrm{S} 1 \mathrm{H} 6 \mathrm{~N} 3$

Charge: $3 \mathrm{H}$
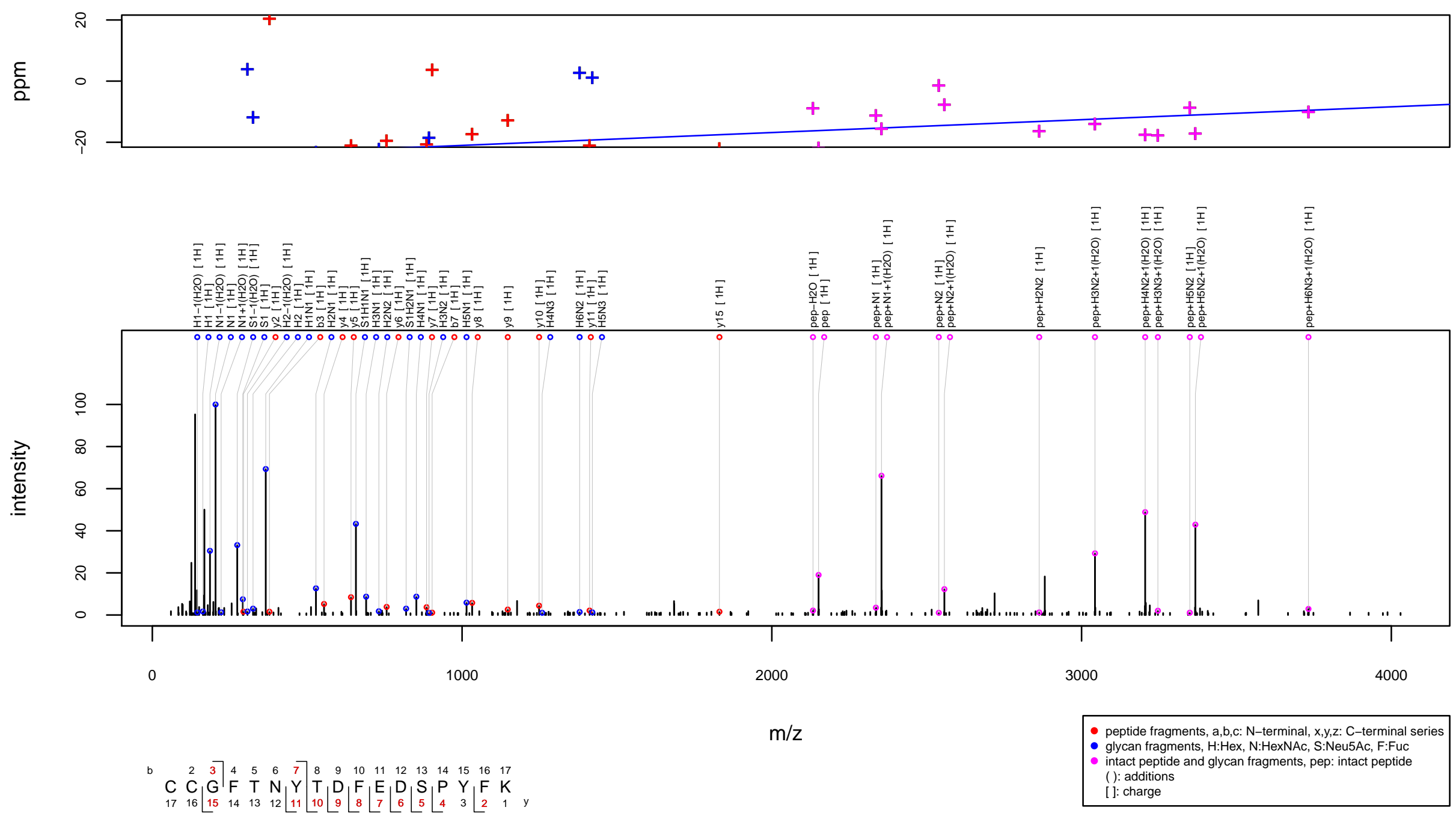
$\mathrm{m} / \mathrm{z} 1358.985$ charge 3 scan $0-0$

Score $=68.95$, Hits $=45$, Explained Intensity $=0.5$

Peptide: IPSP_HUMAN[254,274]:VVGVPYQGNATALFILPSEGK

Glycan: $\mathrm{FHNH}(\mathrm{HNH}) \mathrm{HN}(\mathrm{F}) \mathrm{N}, \mathrm{H} 5 \mathrm{~N} 4 \mathrm{~F} 2$

Charge: $3 \mathrm{H}$
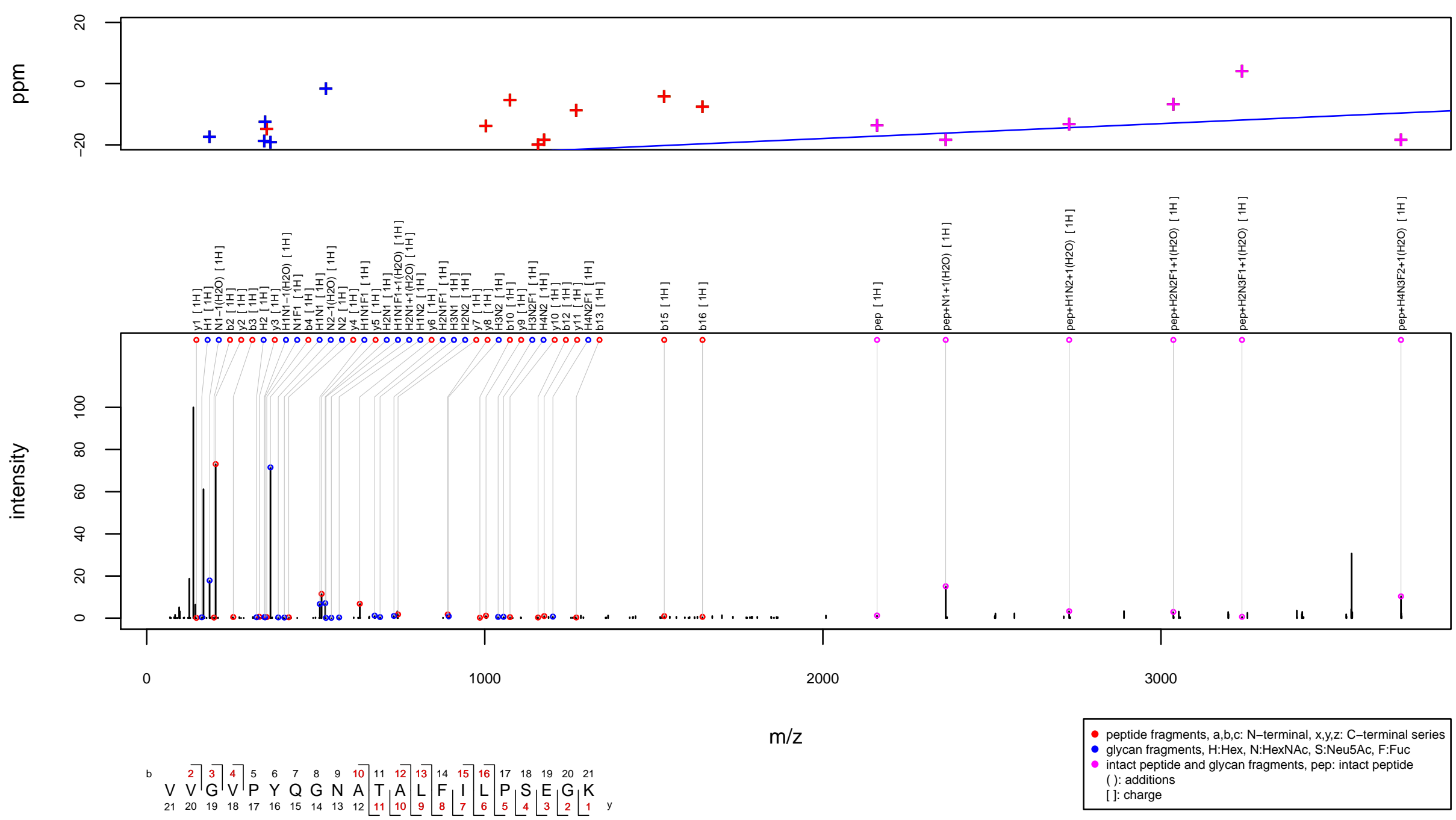
$\mathrm{m} / \mathrm{z} 1368.612$ charge 3 scan $0-0$

Score $=71.45$, Hits $=51$, Explained Intensity $=0.53$

Peptide: TIMP1_HUMAN[46,60]:FVGTPEVNQTTLYQR

Glycan: $\mathrm{SHNH}(\mathrm{SHNH}) \mathrm{HN}(\mathrm{F}) \mathrm{N}, \mathrm{S} 2 \mathrm{H} 5 \mathrm{~N} 4 \mathrm{~F} 1$

Charge: $3 \mathrm{H}$
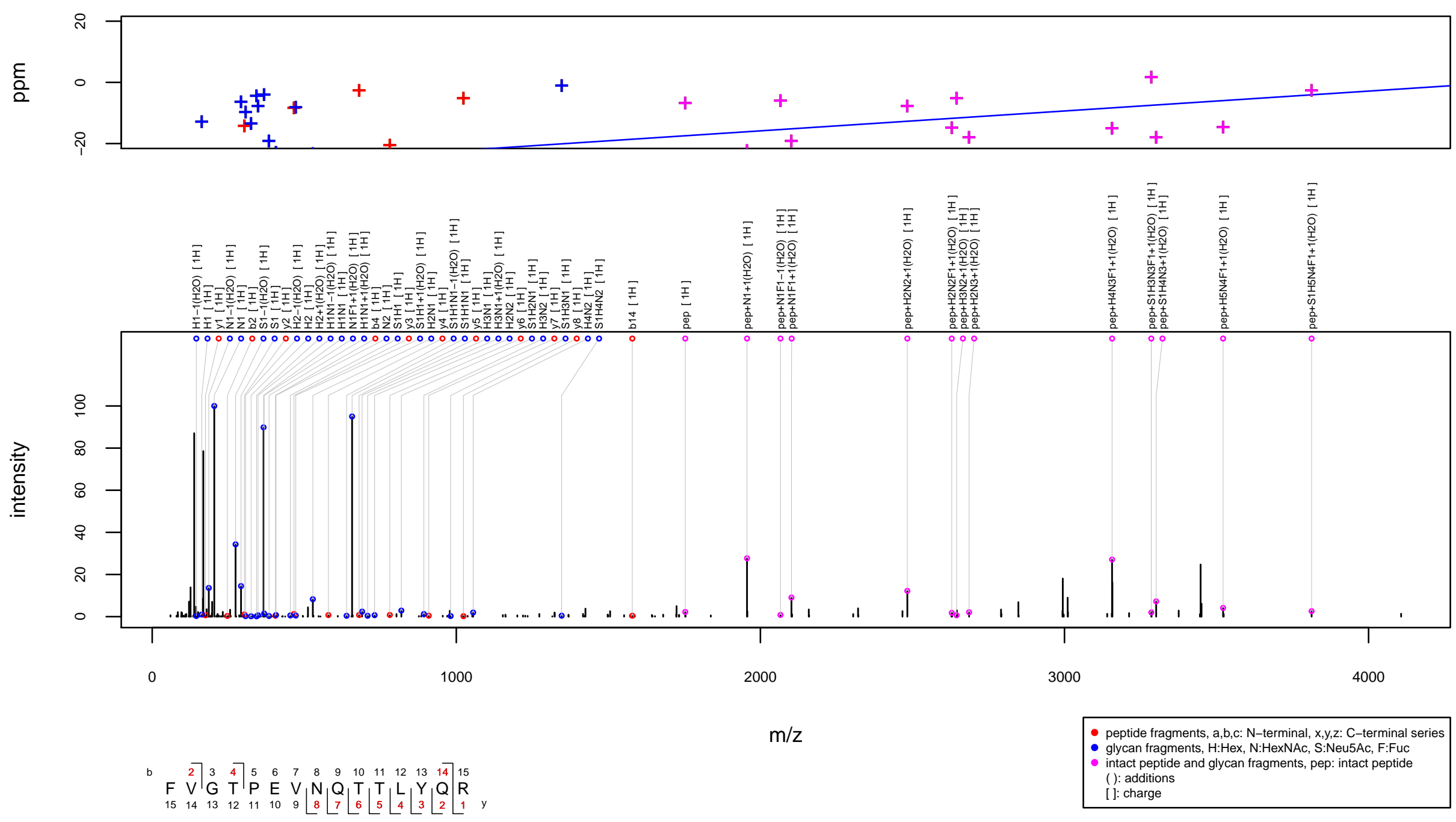
$\mathrm{m} / \mathrm{z} 1369.3003$ charge 3 scan $0-0$

Score $=73.14$, Hits $=51$, Explained Intensity $=0.47$

Peptide: FINC_HUMAN[997,1016]:LDAPTNLQFVNETDSTVLVR

Glycan: $\mathrm{F}(\mathrm{H}) \mathrm{NH}(\mathrm{H}(\mathrm{H}) \mathrm{H}) \mathrm{HN}(\mathrm{F}) \mathrm{N}, \mathrm{H} 6 \mathrm{~N} 3 \mathrm{~F} 2$

Charge: $3 \mathrm{H}$
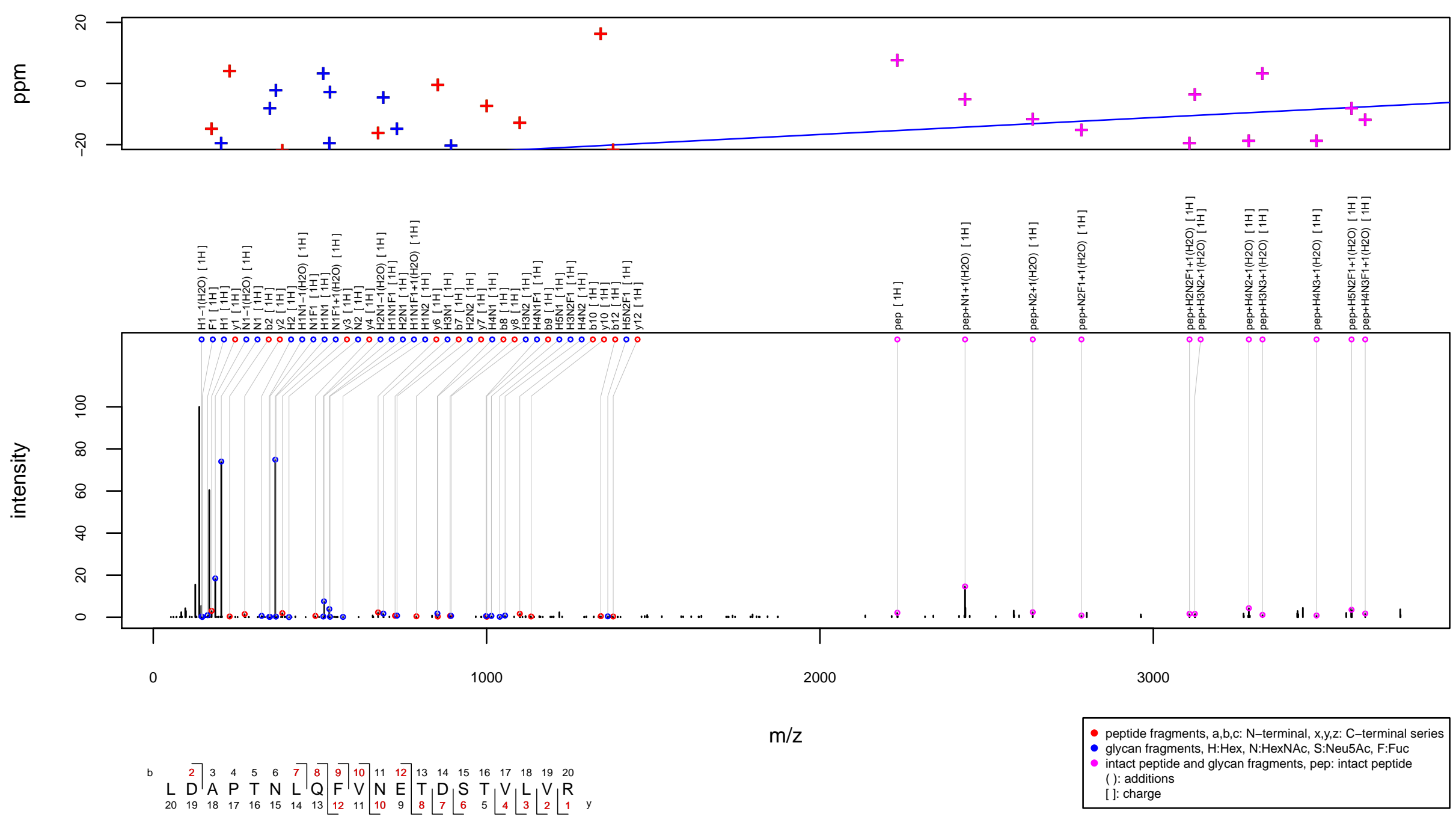
$\mathrm{m} / \mathrm{z} 1371.264$ charge 3 scan $0-0$

Score $=63.8$, Hits $=43$, Explained Intensity $=0.44$

Peptide: FINC_HUMAN[516,533]:DQCIVDDITYNVNDTFHK

Glycan: $\mathrm{FHNH}(\mathrm{HNH}) \mathrm{HN}(\mathrm{F}) \mathrm{N}, \mathrm{H} 5 \mathrm{~N} 4 \mathrm{~F} 2$

Charge: $3 \mathrm{H}$
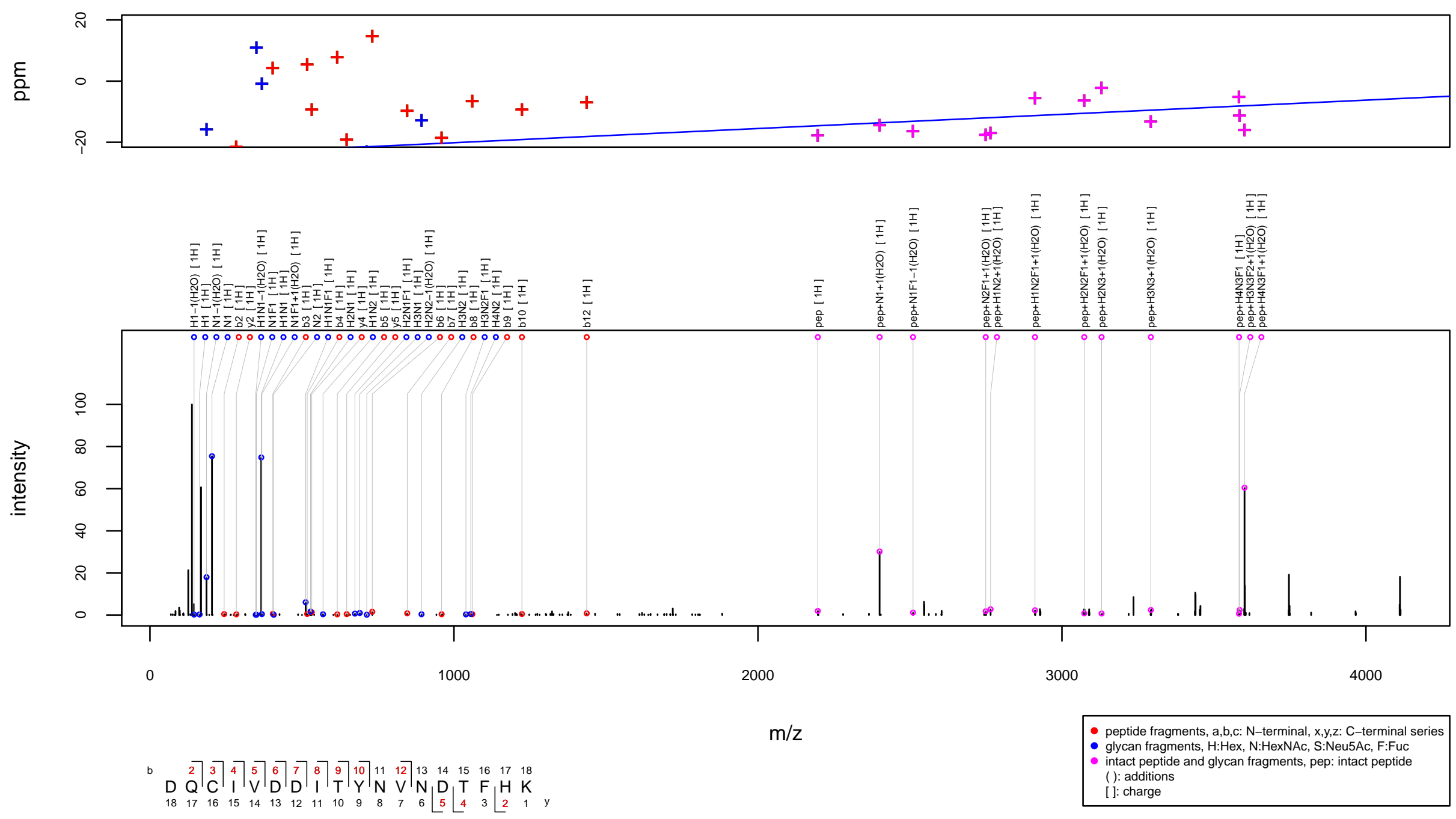
$\mathrm{m} / \mathrm{z} 1379.1503$ charge 5 scan $0-0$

Score $=79.97$, Hits $=61$, Explained Intensity $=0.52$

Peptide: WFDC2_HUMAN[33,60]:TGVCPELQADQNCTQECVSDSECADNLK

Glycan: SHNSHNH(SHNSHNH)HN(F)N, S4H7N6F1

Charge: $5 \mathrm{H}$
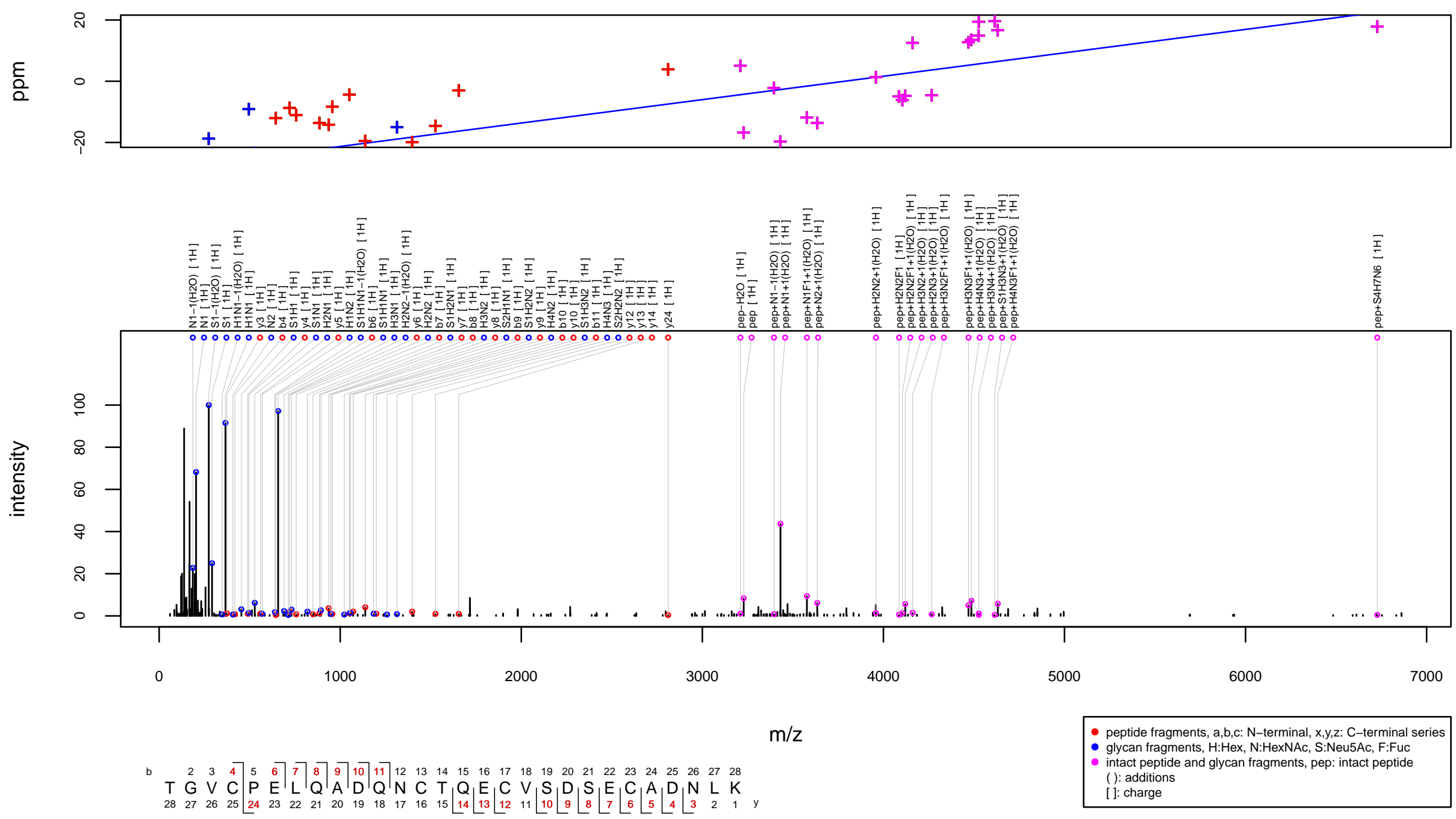
$\mathrm{m} / \mathrm{z} 1382.2478$ charge 3 scan $0-0$

Score $=125.97$, Hits $=85$, Explained Intensity $=0.56$

Peptide: RNT2_HUMAN[206,223]:QDQQLQNCTEPGEQPSPK

Glycan: $\mathrm{FH}(\mathrm{F}) \mathrm{NH}(\mathrm{HNH}) \mathrm{HN}(\mathrm{F}) \mathrm{N}, \mathrm{H} 5 \mathrm{~N} 4 \mathrm{~F} 3$

Charge: $3 \mathrm{H}$
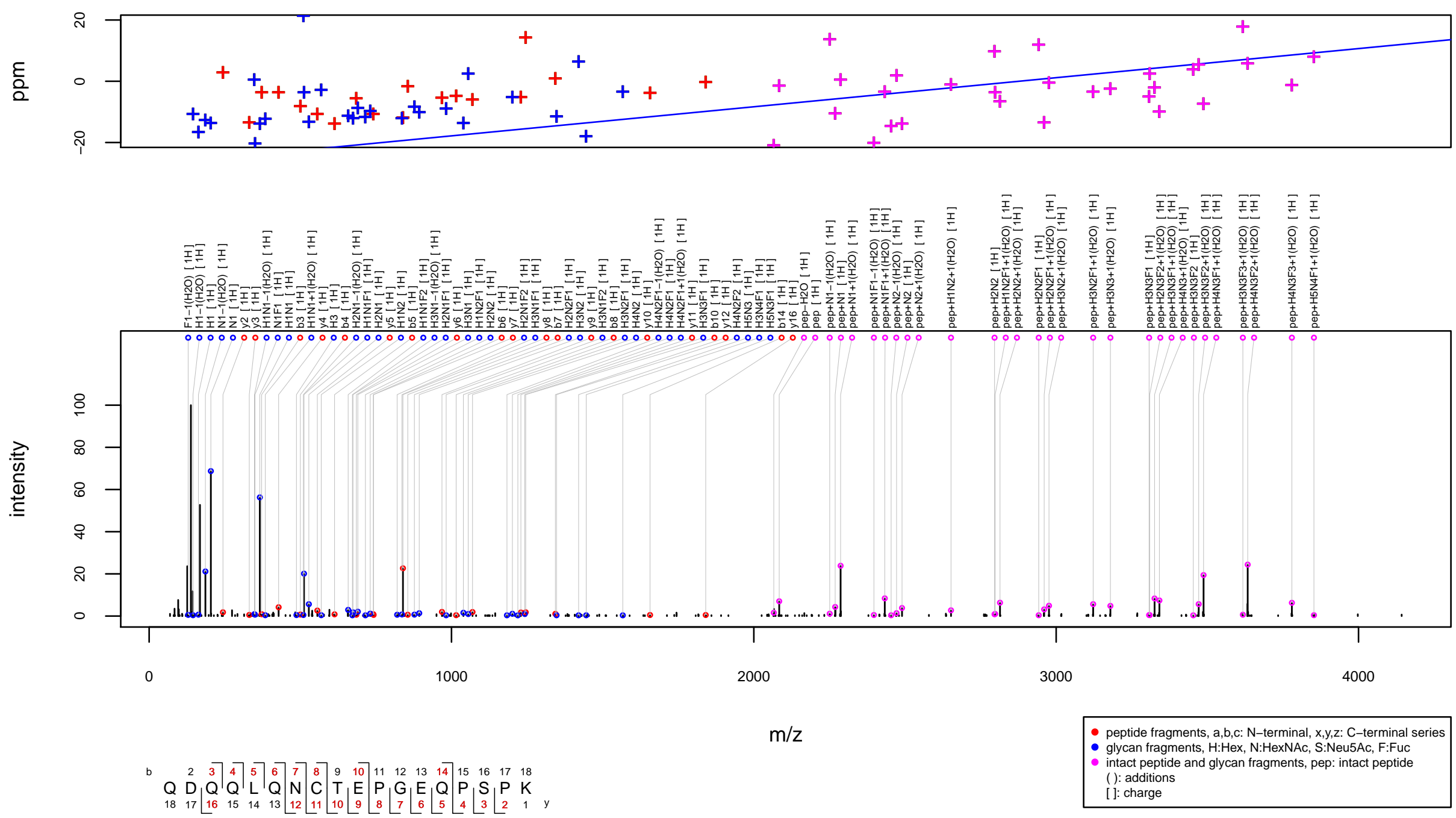
$\mathrm{m} / \mathrm{z} 1382.9832$ charge 3 scan $0-0$

Score $=84.47$, Hits $=57$, Explained Intensity $=0.5$

Peptide: FINC_HUMAN[997,1016]:LDAPTNLQFVNETDSTVLVR

Glycan: $\mathrm{FHNH}(\mathrm{HNH}) \mathrm{HN}(\mathrm{F}) \mathrm{N}, \mathrm{H} 5 \mathrm{~N} 4 \mathrm{~F} 2$

Charge: $3 \mathrm{H}$
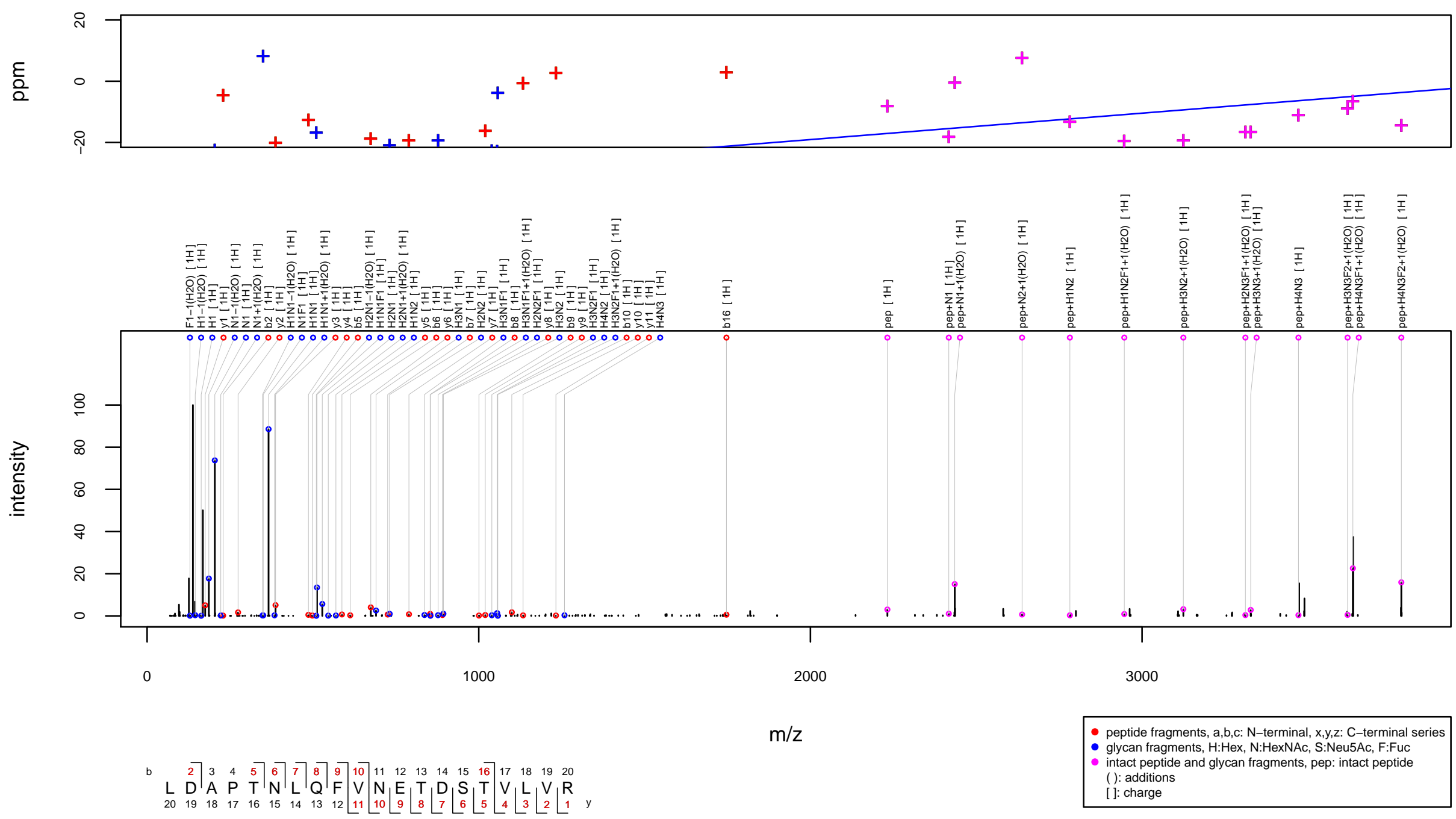
$\mathrm{m} / \mathrm{z} 1395.5841$ charge 4 scan $0-0$

Score $=103.53$, Hits $=69$, Explained Intensity $=0.5$

Peptide: WFDC2_HUMAN[33,60]:TGVCPELQADQNCTQECVSDSECADNLK

Glycan: $\mathrm{SHNH}(\mathrm{SHNH}) \mathrm{HN}(\mathrm{F}) \mathrm{N}, \mathrm{S} 2 \mathrm{H} 5 \mathrm{~N} 4 \mathrm{~F} 1$

Charge: $4 \mathrm{H}$
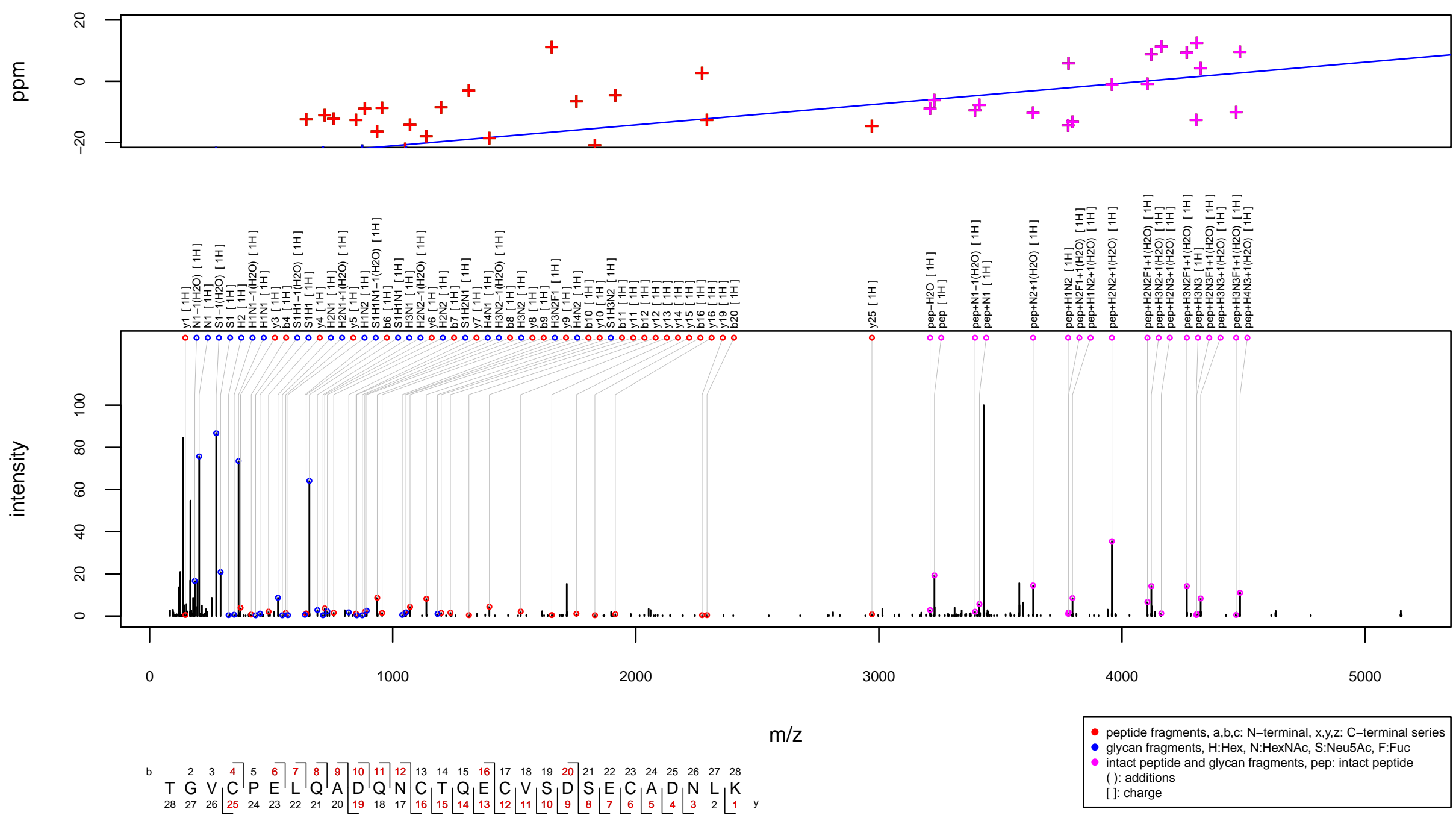


\section{$\mathrm{m} / \mathrm{z} 1407.6755$ charge 3 scan $0-0$}

Score $=73.86$, Hits $=52$, Explained Intensity $=0.51$

Peptide: IPSP_HUMAN[254,274]:VVGVPYQGNATALFILPSEGK

Glycan: $\mathrm{FH}(\mathrm{F}) \mathrm{NH}(\mathrm{HNH}) \mathrm{HN}(\mathrm{F}) \mathrm{N}, \mathrm{H} 5 \mathrm{~N} 4 \mathrm{~F} 3$

Charge: $3 \mathrm{H}$
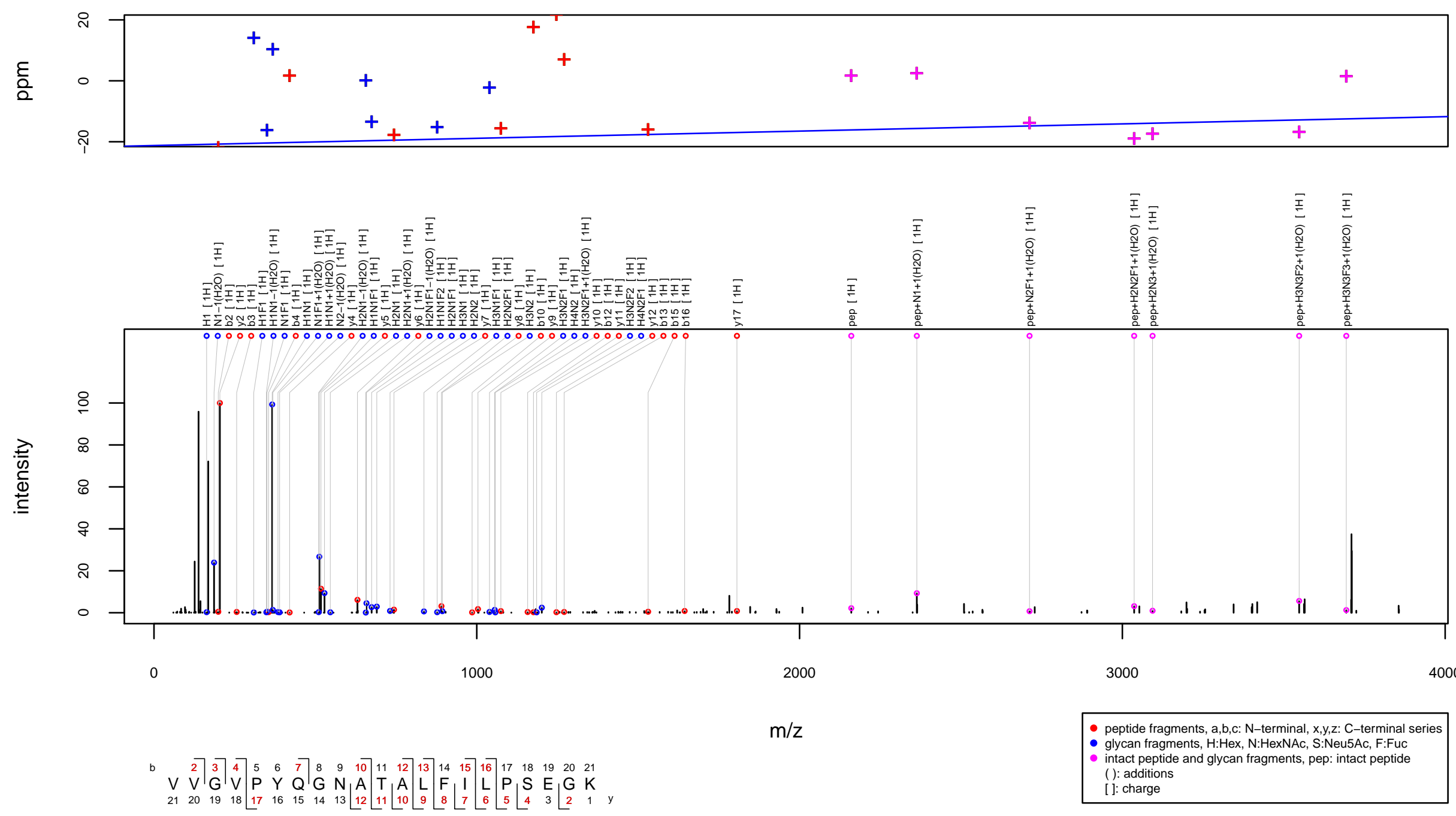
$\mathrm{m} / \mathrm{z} 1414.3613$ charge 4 scan $0-0$

Score $=75.85$, Hits $=57$, Explained Intensity $=0.47$

Peptide: WFDC2_HUMAN[33,60]:TGVCPELQADQNCTQECVSDSECADNLK

Glycan: $\mathrm{HN}(\mathrm{F}(\mathrm{H}) \mathrm{N}) \mathrm{H}(\mathrm{F}(\mathrm{H}) \mathrm{NH}) \mathrm{HN}(\mathrm{F}) \mathrm{N}, \mathrm{H} 6 \mathrm{~N} 5 \mathrm{~F} 3$

Charge: $4 \mathrm{H}$
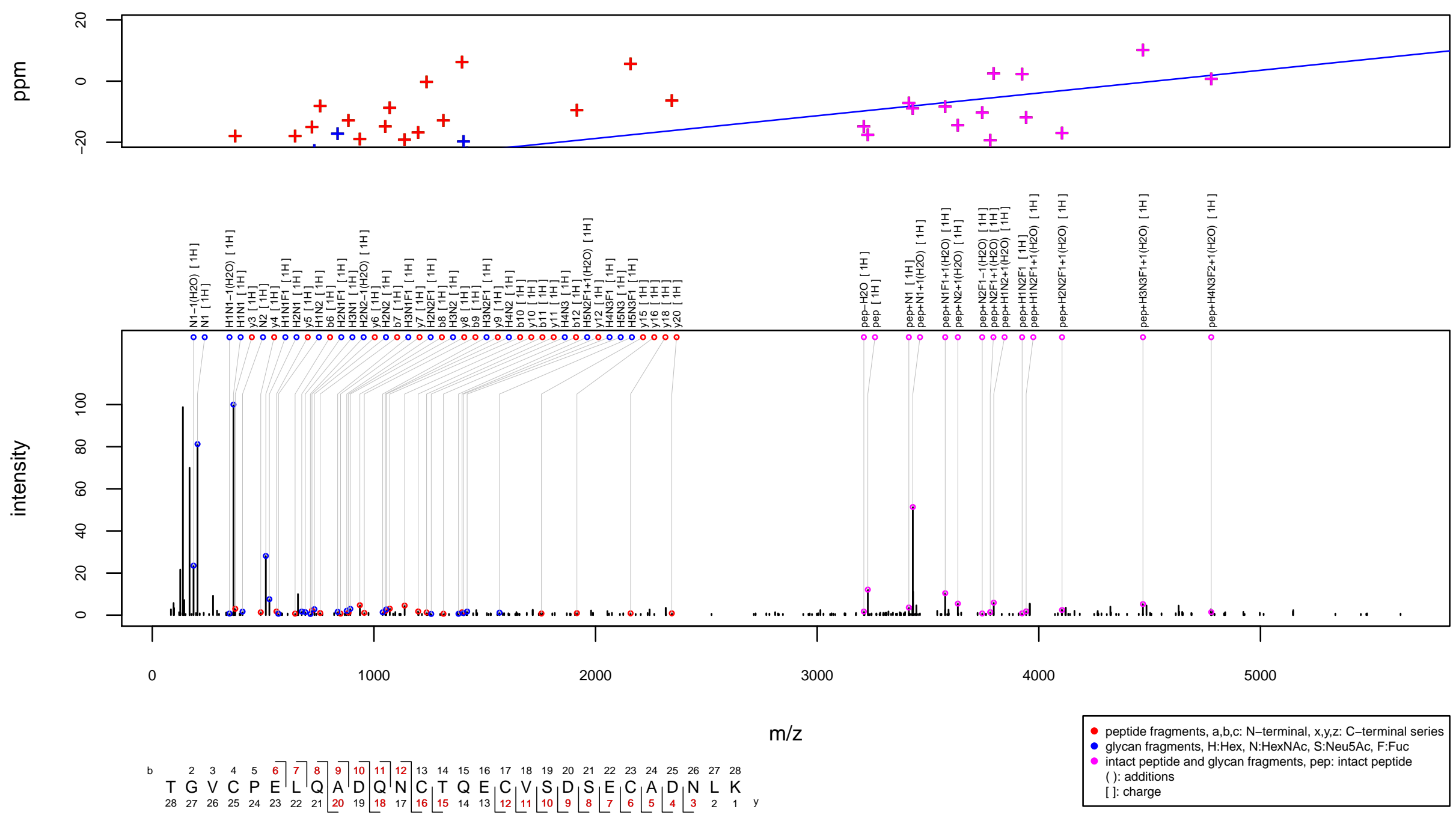
$\mathrm{m} / \mathrm{z} 1418.9684$ charge 3 scan $0-0$

Score $=80.24$, Hits $=66$, Explained Intensity $=0.52$

Peptide: CLUS_HUMAN[372,385]:LANLTQGEDQYYLR

Glycan: SHNHNH(F(H)NH)HN(F)N, S1H6N5F2

Charge: $3 \mathrm{H}$
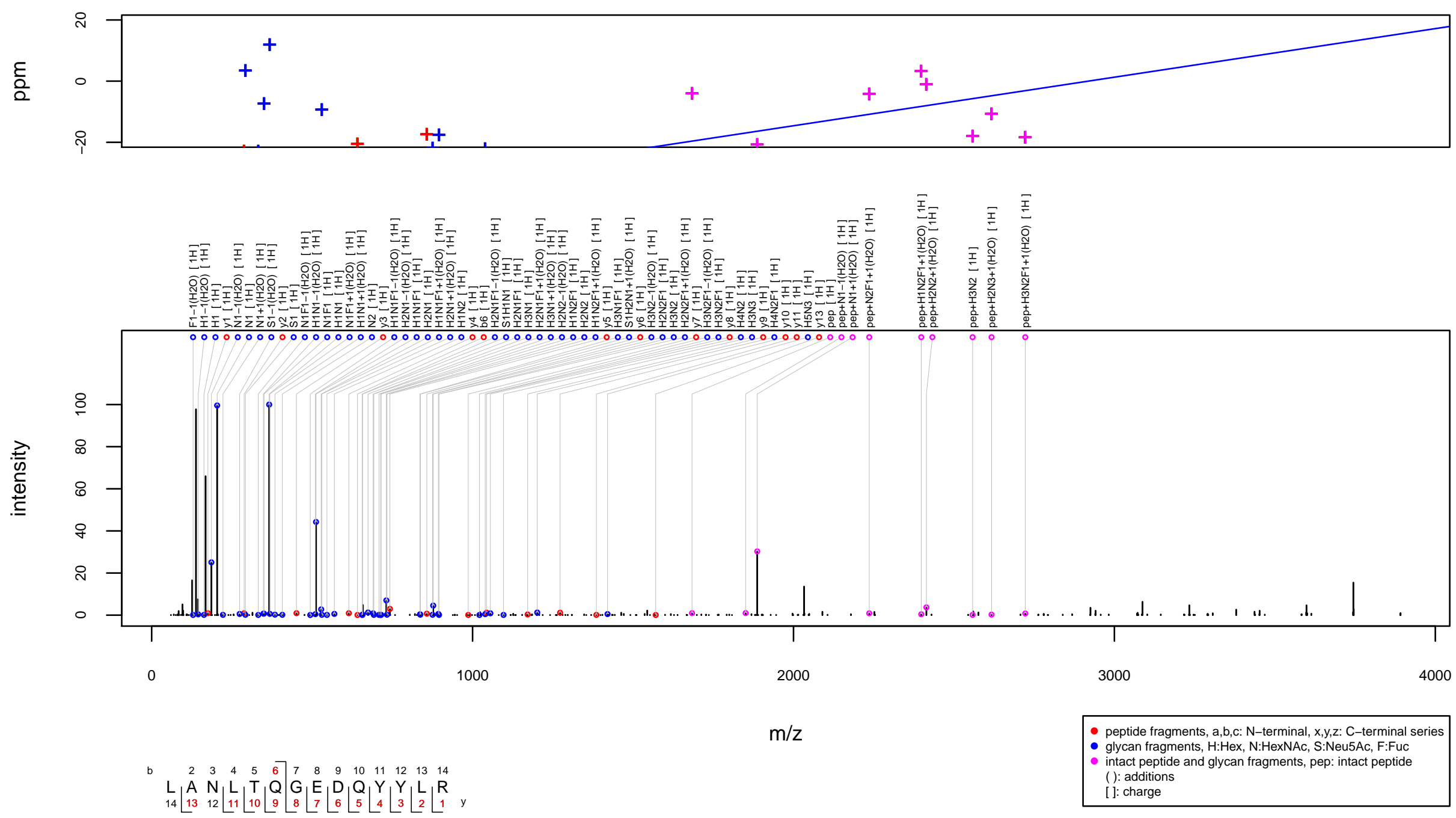
$\mathrm{m} / \mathrm{z} 1419.2809$ charge 3 scan $0-0$

Score $=111.13$, Hits $=82$, Explained Intensity $=0.56$ Peptide: CLUS_HUMAN[372,385]:LANLTQGEDQYYLR Glycan: $\mathrm{F}(\mathrm{H}) \mathrm{NH}(\mathrm{F}(\mathrm{H}) \mathrm{N}(\mathrm{F}(\mathrm{H}) \mathrm{N}) \mathrm{H}) \mathrm{HN}(\mathrm{F}) \mathrm{N}, \mathrm{H} 6 \mathrm{~N} 5 \mathrm{~F} 4$

Charge: $3 \mathrm{H}$
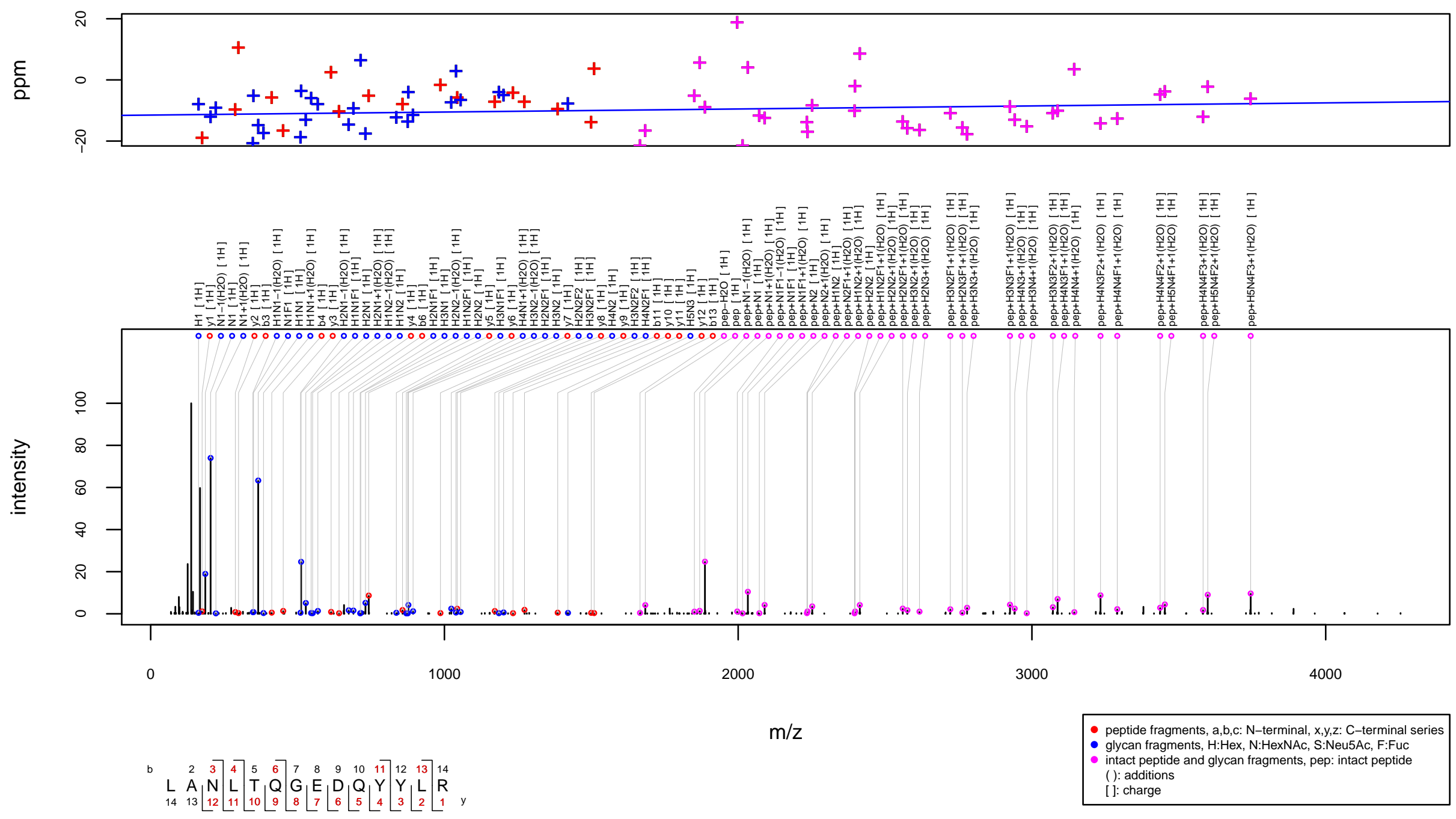
$\mathrm{m} / \mathrm{z} 1419.9388$ charge 3 scan $0-0$

Score $=121.41$, Hits $=81$, Explained Intensity $=0.59$

Peptide: FINC_HUMAN[516,533]:DQCIVDDITYNVNDTFHK

Glycan: $\mathrm{FH}(\mathrm{F}) \mathrm{NH}(\mathrm{HNH}) \mathrm{HN}(\mathrm{F}) \mathrm{N}, \mathrm{H} 5 \mathrm{~N} 4 \mathrm{~F} 3$

Charge: $3 \mathrm{H}$
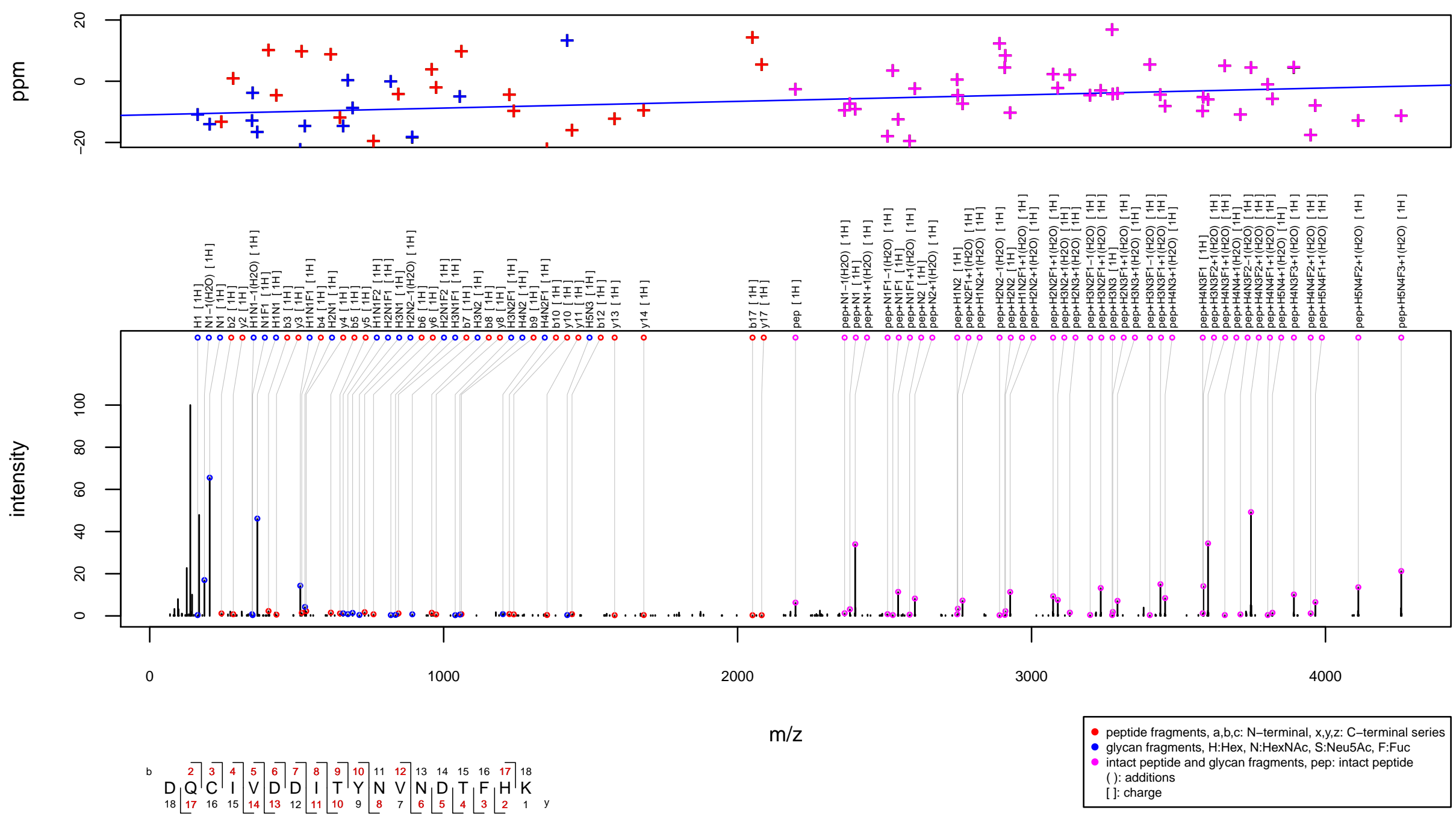
$\mathrm{m} / \mathrm{z} 1423.9995$ charge 3 scan $0-0$

Score $=70.57$, Hits $=49$, Explained Intensity $=0.55$

Peptide: PTGDS_HUMAN[67,85]:SVVAPATDGGLNLTSTFLR

Glycan: $\mathrm{SHNH}(\mathrm{SHNH}) \mathrm{HN}(\mathrm{F}) \mathrm{N}, \mathrm{S} 2 \mathrm{H} 5 \mathrm{~N} 4 \mathrm{~F} 1$

Charge: $3 \mathrm{H}$
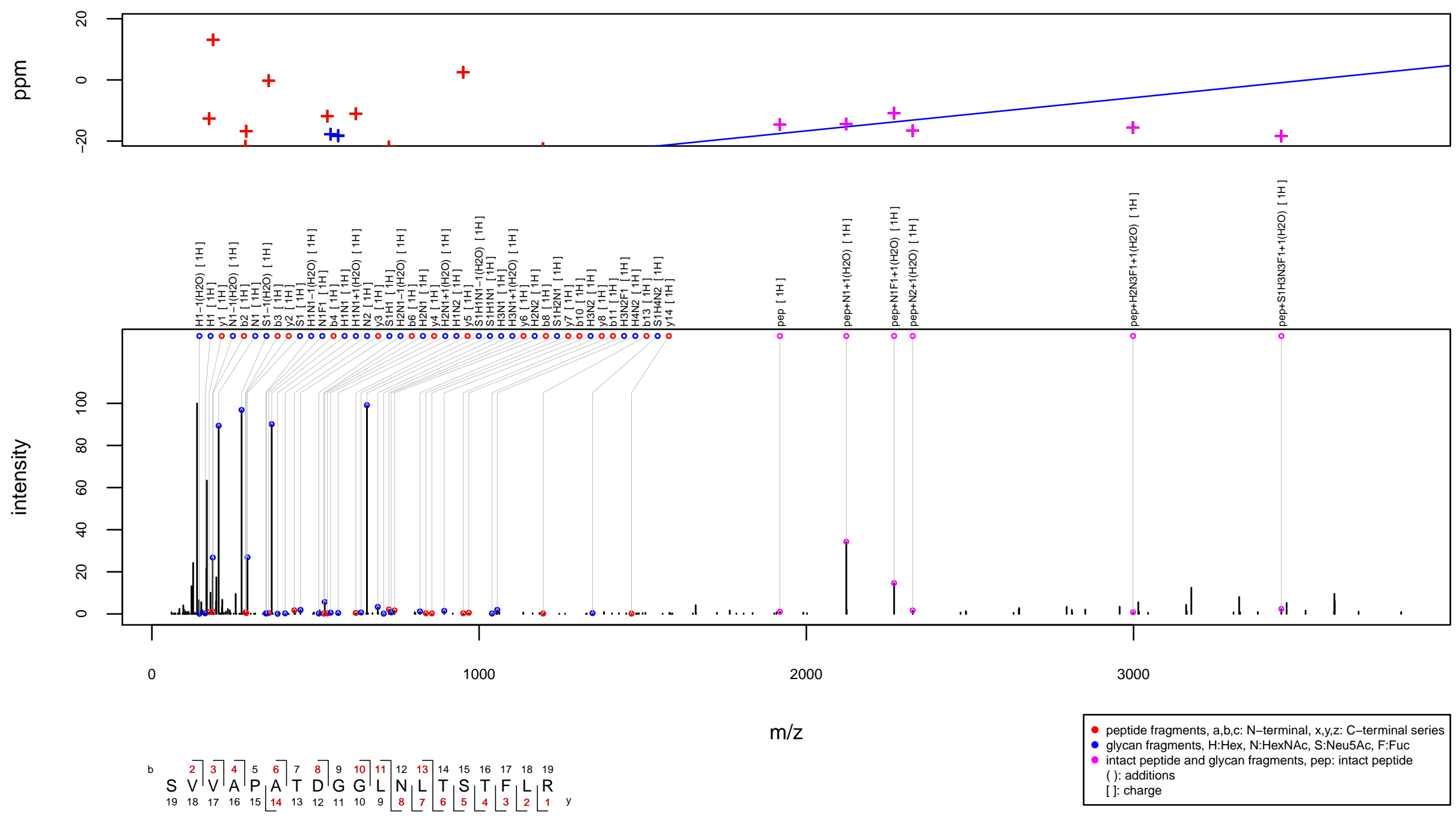
$\mathrm{m} / \mathrm{z} 1431.672$ charge 3 scan $0-0$

Score $=86.54$, Hits $=63$, Explained Intensity $=0.57$

Peptide: FINC_HUMAN[997,1016]:LDAPTNLQFVNETDSTVLVR

Glycan: $\mathrm{FH}(\mathrm{F}) \mathrm{NH}(\mathrm{HNH}) \mathrm{HN}(\mathrm{F}) \mathrm{N}, \mathrm{H} 5 \mathrm{~N} 4 \mathrm{~F} 3$

Charge: $3 \mathrm{H}$
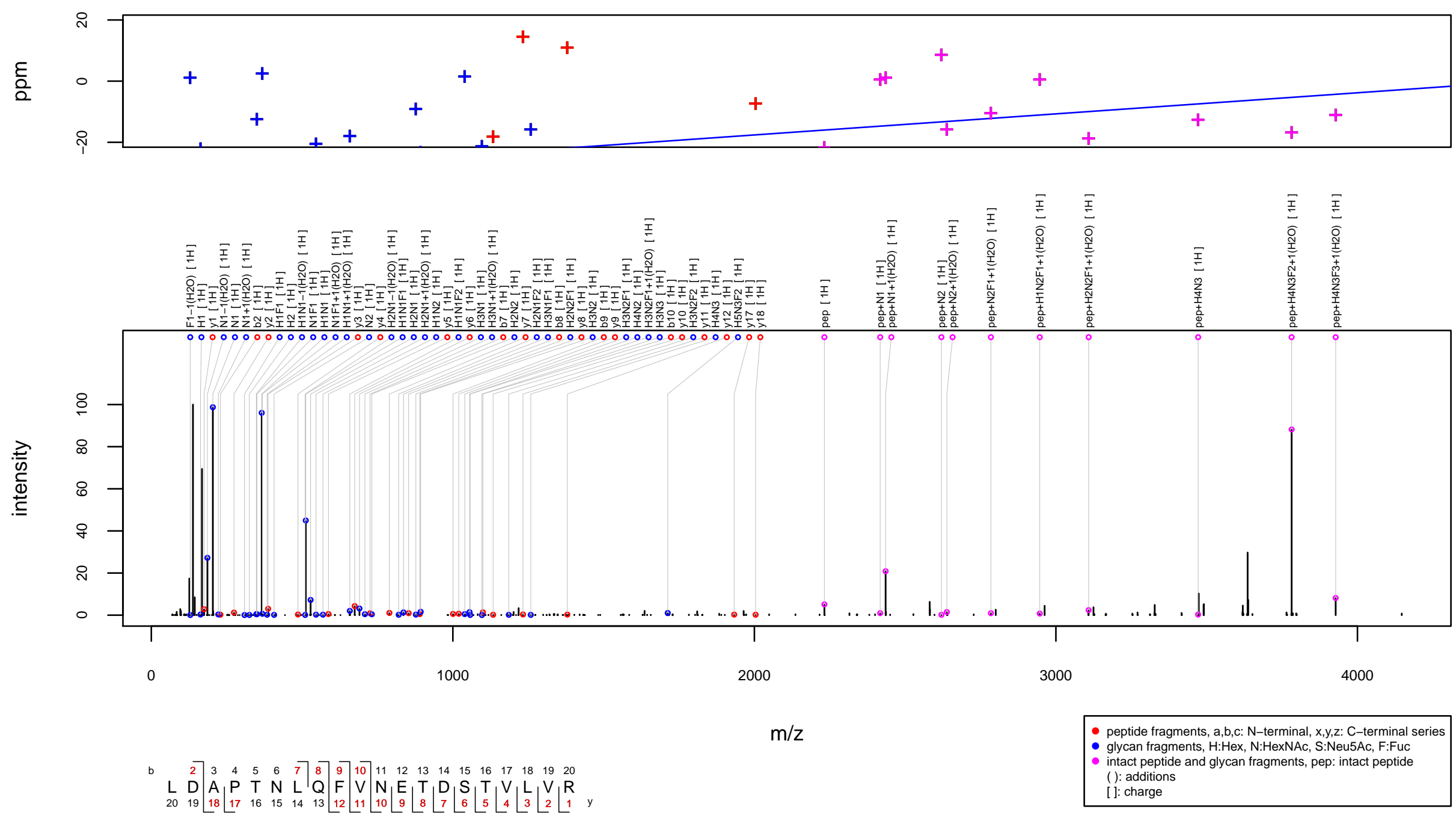
$\mathrm{m} / \mathrm{z} 1450.8647$ charge 4 scan $0-0$

Score $=89.13$, Hits $=68$, Explained Intensity $=0.54$

Peptide: WFDC2_HUMAN[33,60]:TGVCPELQADQNCTQECVSDSECADNLK

Glycan: $\mathrm{F}(\mathrm{H}) \mathrm{NH}(\mathrm{F}(\mathrm{H}) \mathrm{N}(\mathrm{F}(\mathrm{H}) \mathrm{N}) \mathrm{H}) \mathrm{HN}(\mathrm{F}) \mathrm{N}, \mathrm{H} 6 \mathrm{~N} 5 \mathrm{~F} 4$

Charge: $4 \mathrm{H}$
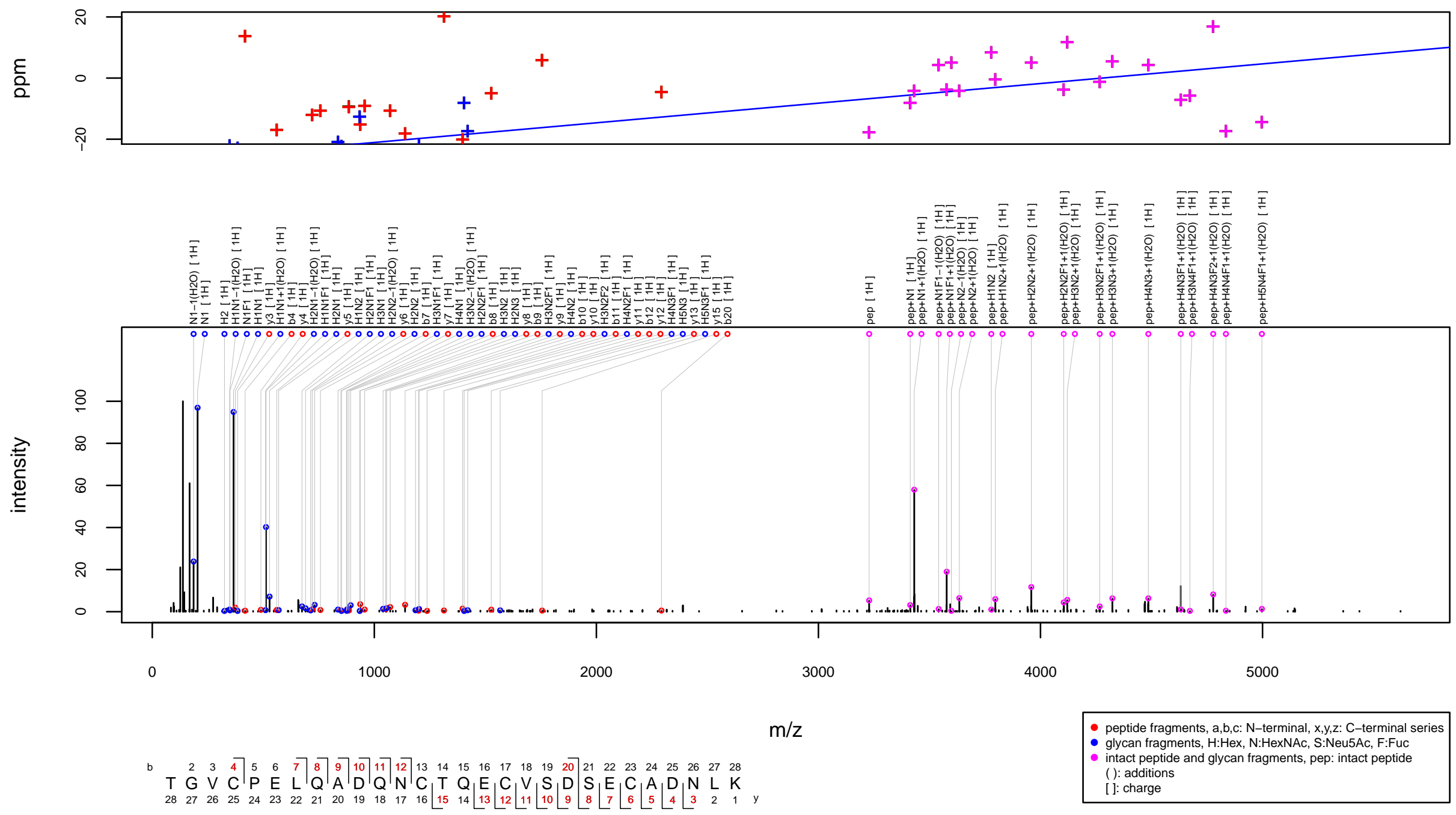
$\mathrm{m} / \mathrm{z} 1457.6029$ charge 3 scan $0-0$

Score $=63.59$, Hits $=40$, Explained Intensity $=0.42$

Peptide: MUC6_HUMAN[655,679]:SSVDNCTIPCTGNTTFSYNSQACER

Glycan: $\mathrm{NH}(\mathrm{NH})(\mathrm{N}) \mathrm{HNN}, \mathrm{H} 3 \mathrm{~N} 5$

Charge: $3 \mathrm{H}$
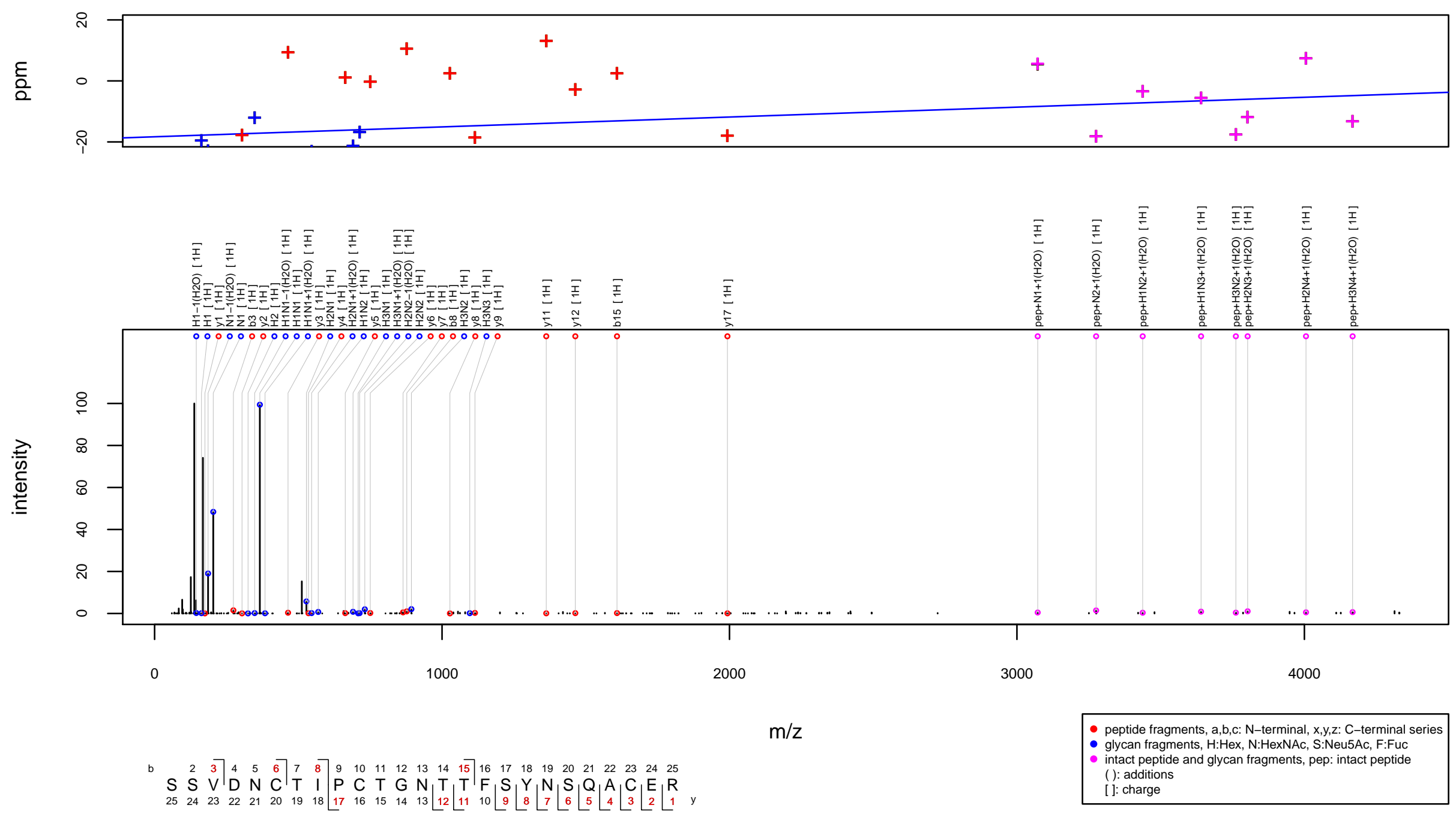
$\mathrm{m} / \mathrm{z} 1486.8715$ charge 4 scan $0-0$

Score $=91.29$, Hits $=68$, Explained Intensity $=0.6$

Peptide: WFDC2_HUMAN[33,60]:TGVCPELQADQNCTQECVSDSECADNLK

Glycan: $\mathrm{SHNH}(\mathrm{SHN}(\mathrm{HN}) \mathrm{H}) \mathrm{HN}(\mathrm{F}) \mathrm{N}, \mathrm{S} 2 \mathrm{H} 6 \mathrm{~N} 5 \mathrm{~F} 1$

Charge: $4 \mathrm{H}$
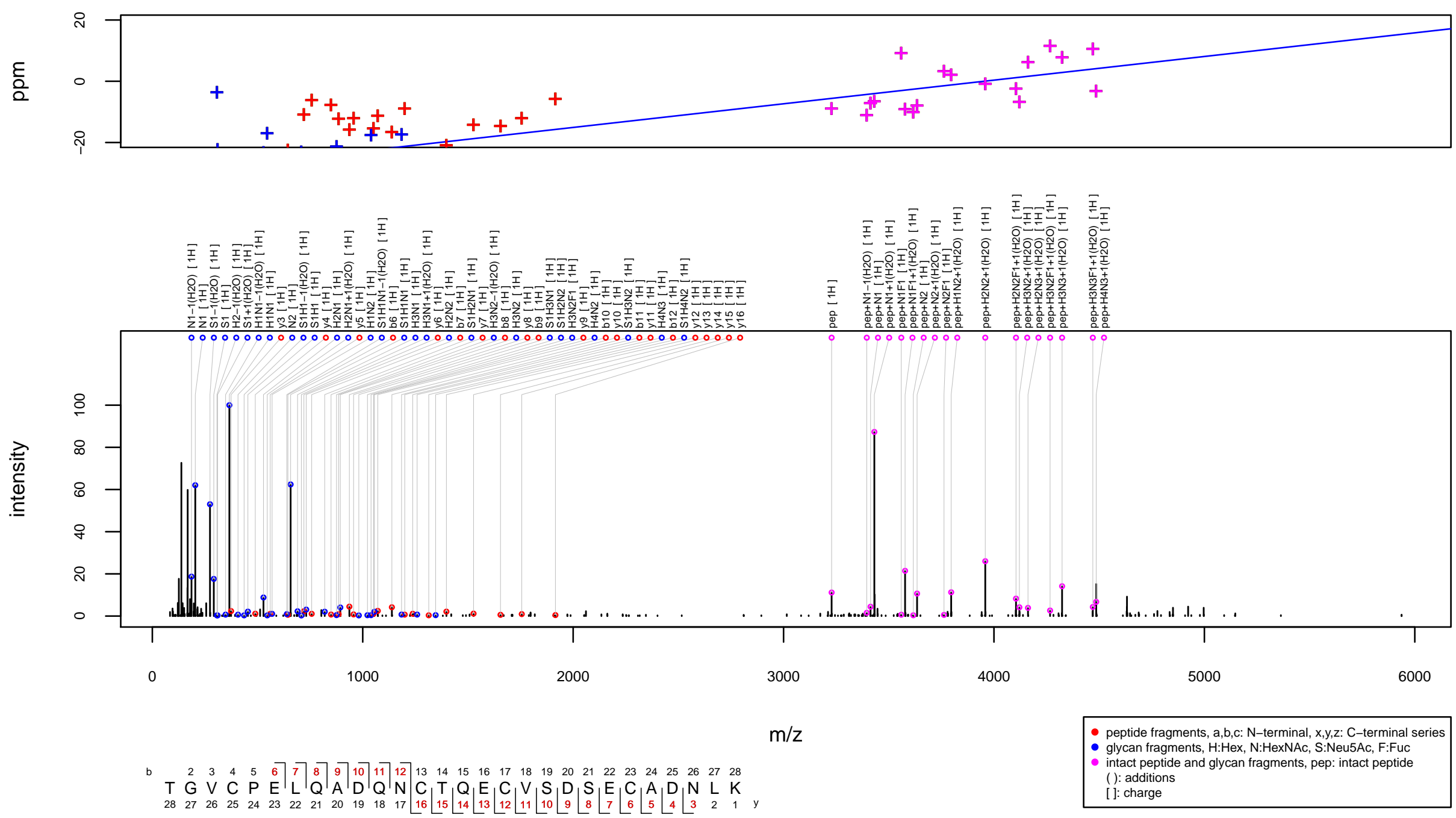
$\mathrm{m} / \mathrm{z} 1559.6439$ charge 4 scan $0-0$

Score $=107.66$, Hits $=78$, Explained Intensity $=0.65$

Peptide: WFDC2_HUMAN[33,60]:TGVCPELQADQNCTQECVSDSECADNLK

Glycan: $\mathrm{SHNH}(\mathrm{SHN}(\mathrm{SHN}) \mathrm{H}) \mathrm{HN}(\mathrm{F}) \mathrm{N}, \mathrm{S} 3 \mathrm{H} 6 \mathrm{~N} 5 \mathrm{~F} 1$

Charge: $4 \mathrm{H}$
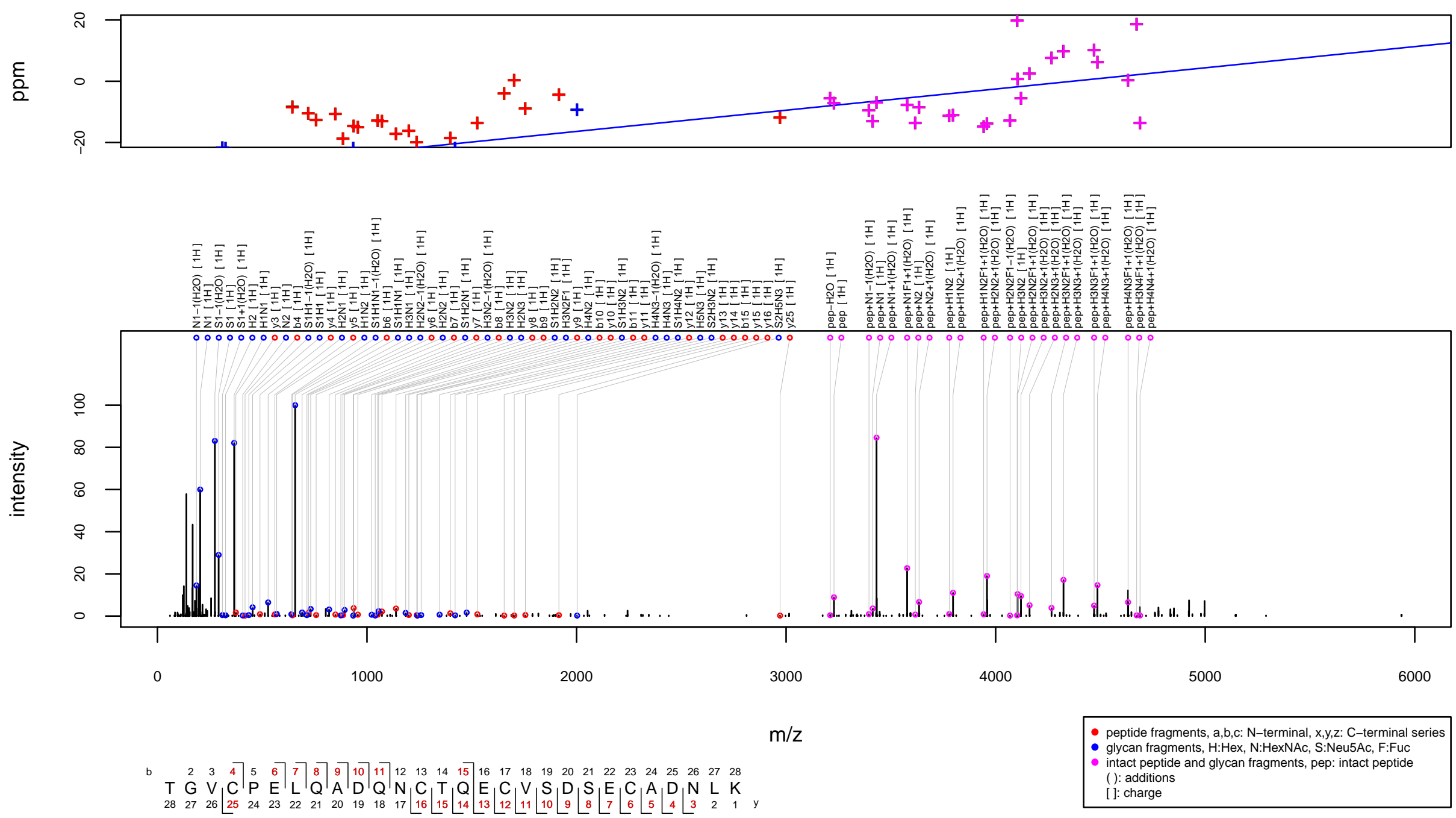


\section{$\mathrm{m} / \mathrm{z} 1590.7316$ charge 3 scan 0-0}

Score $=53.42$, Hits $=38$, Explained Intensity $=0.41$

Peptide: CBPE_HUMAN[382,406]:DLQGNPIANATISVEGIDHDVTSAK

Glycan: $\mathrm{SHNH}(\mathrm{SHNH}) \mathrm{HNN}$, S2H5N4

Charge: $3 \mathrm{H}$
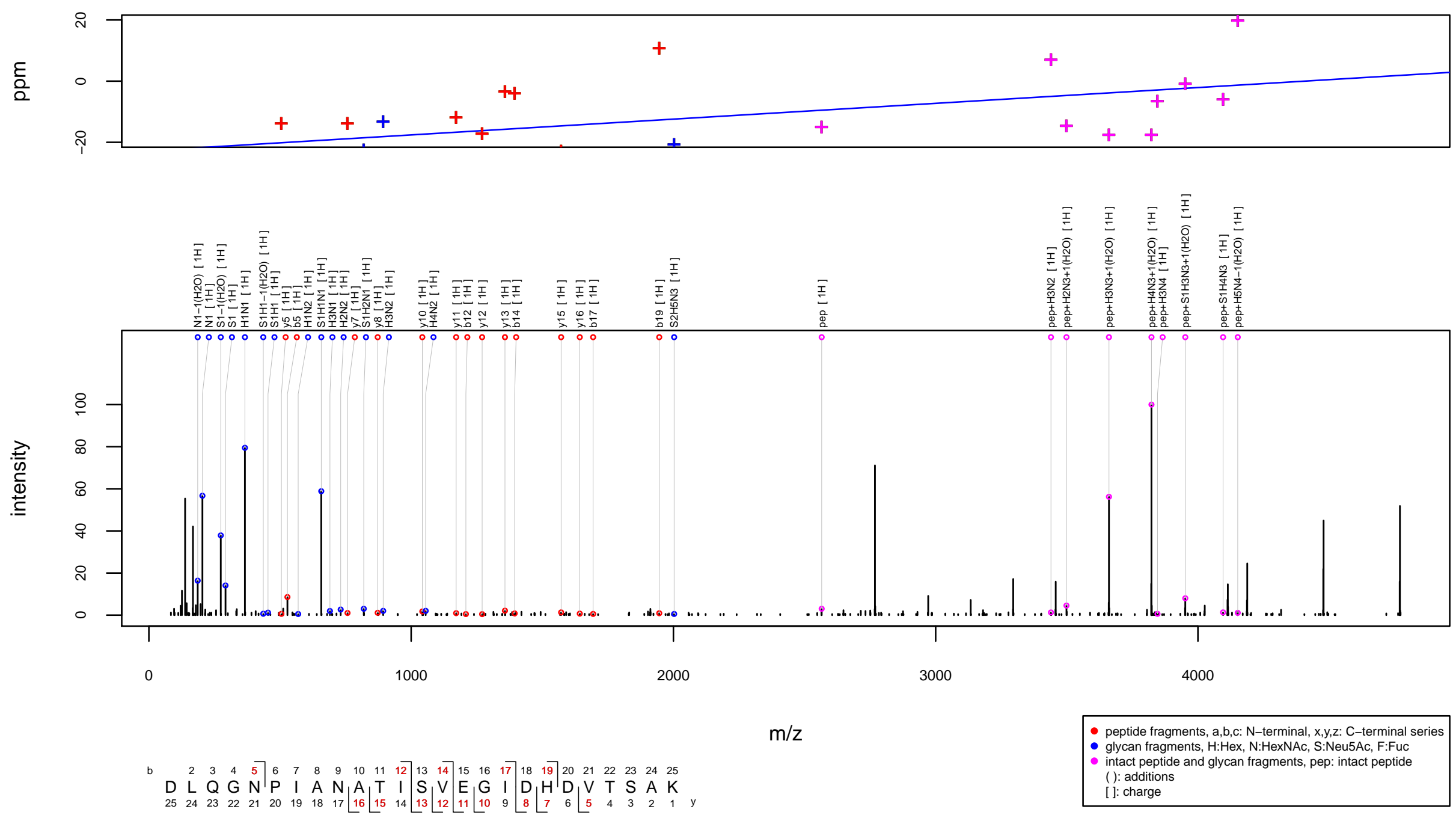
$\mathrm{m} / \mathrm{z} 1724.822$ charge 2 scan $0-0$

Score $=50.77$, Hits $=26$, Explained Intensity $=0.81$

Peptide: FINC_HUMAN[997,1016]:LDAPTNLQFVNETDSTVLVR

Glycan: $\mathrm{H}(\mathrm{H}) \mathrm{H}(\mathrm{H}) \mathrm{HNN}, \mathrm{H} 5 \mathrm{~N} 2$

Charge: $2 \mathrm{H}$
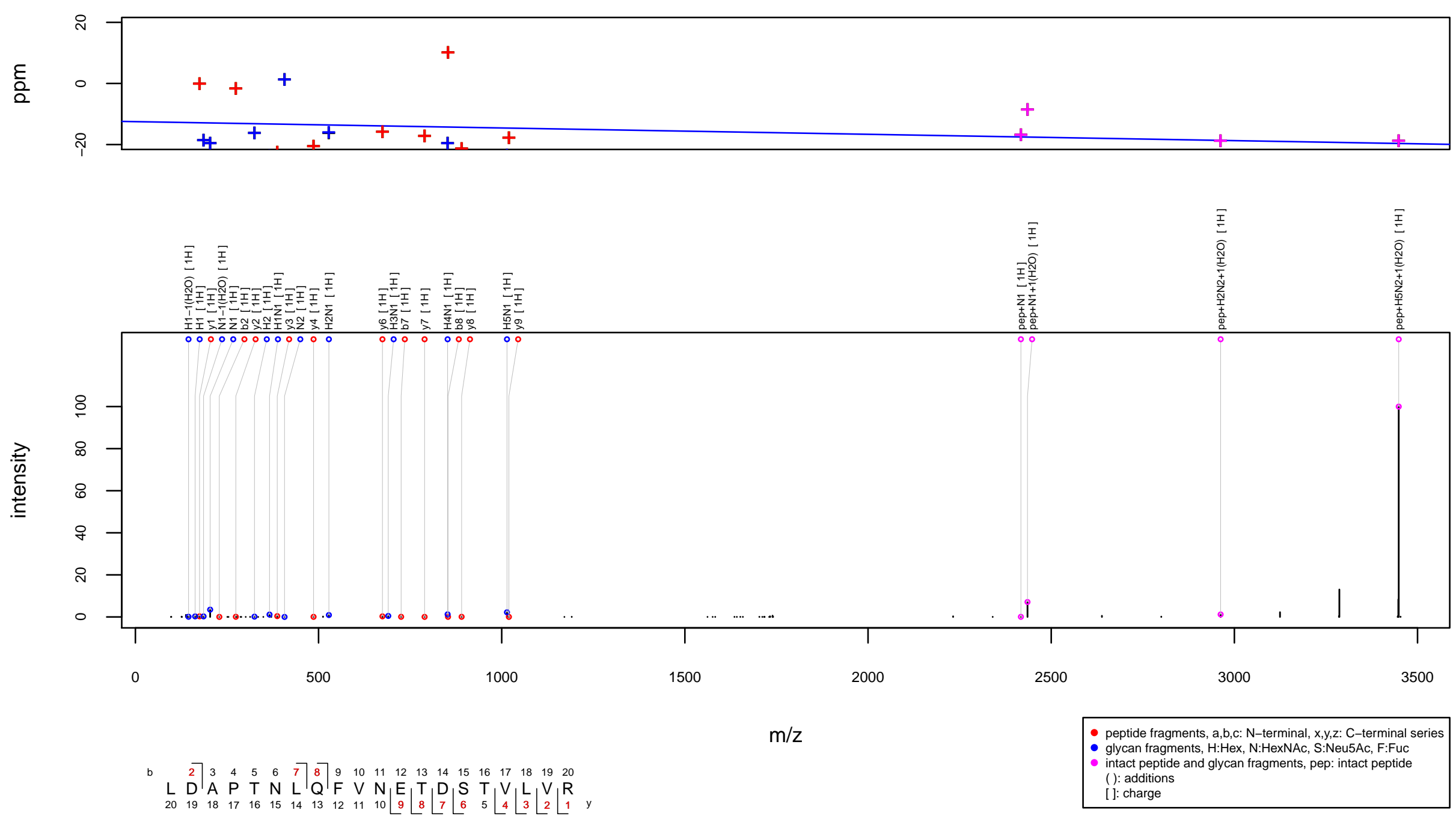
$\mathrm{m} / \mathrm{z} 660.8302$ charge 4 scan $0-0$

Score $=27.41$, Hits $=18$, Explained Intensity $=0.05$

Peptide: CDS2_HUMAN[58,65]:ALSNLSSR

Glycan: S1H3N5

Charge: $4 \mathrm{H}$
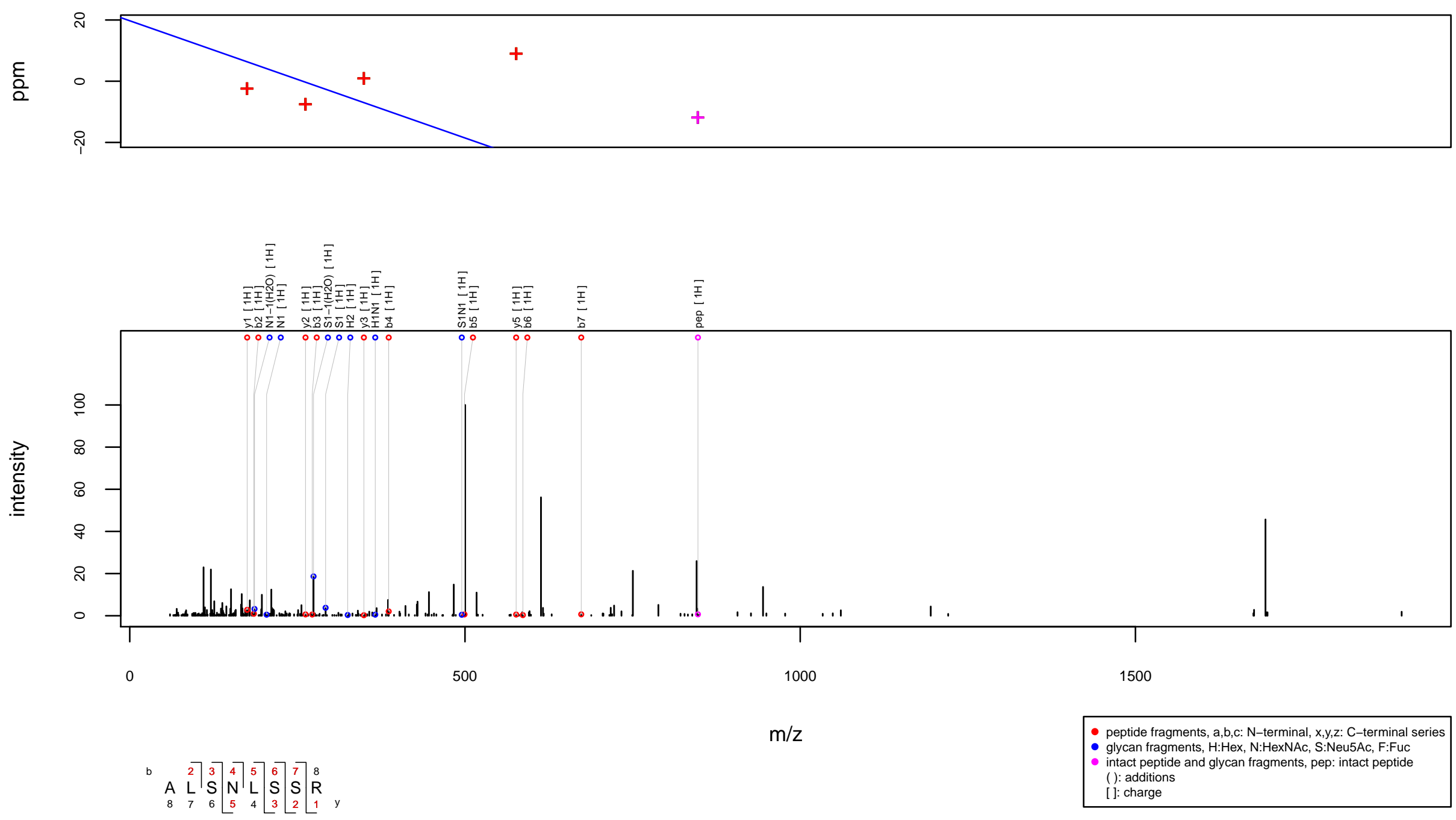
$\mathrm{m} / \mathrm{z} 661.086$ charge 4 scan $0-0$

Score $=24.2$, Hits $=16$, Explained Intensity $=0.02$

Peptide: CDS2_HUMAN[58,65]:ALSNLSSR

Glycan: H3N5F2

Charge: $4 \mathrm{H}$
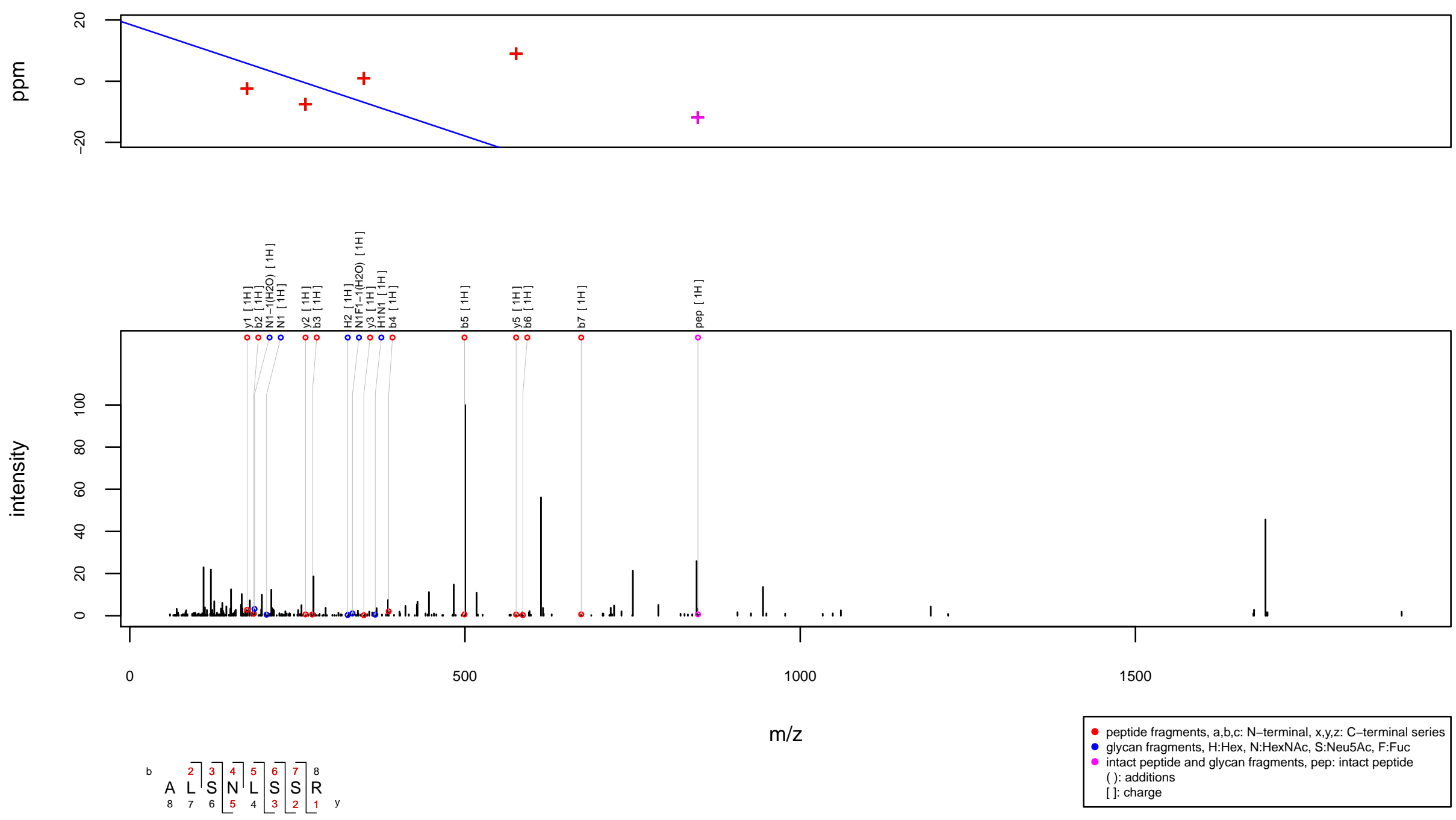
$\mathrm{m} / \mathrm{z} 791.8721$ charge 4 scan $0-0$

Score $=40.97$, Hits $=27$, Explained Intensity $=0.21$ Peptide: CBPE_HUMAN[138,150]:GNETIVNLIHSTR Glycan: $\mathrm{SHNH}(\mathrm{HH}) \mathrm{HNN}, \mathrm{S} 1 \mathrm{H} 5 \mathrm{~N} 3$

Charge: $4 \mathrm{H}$
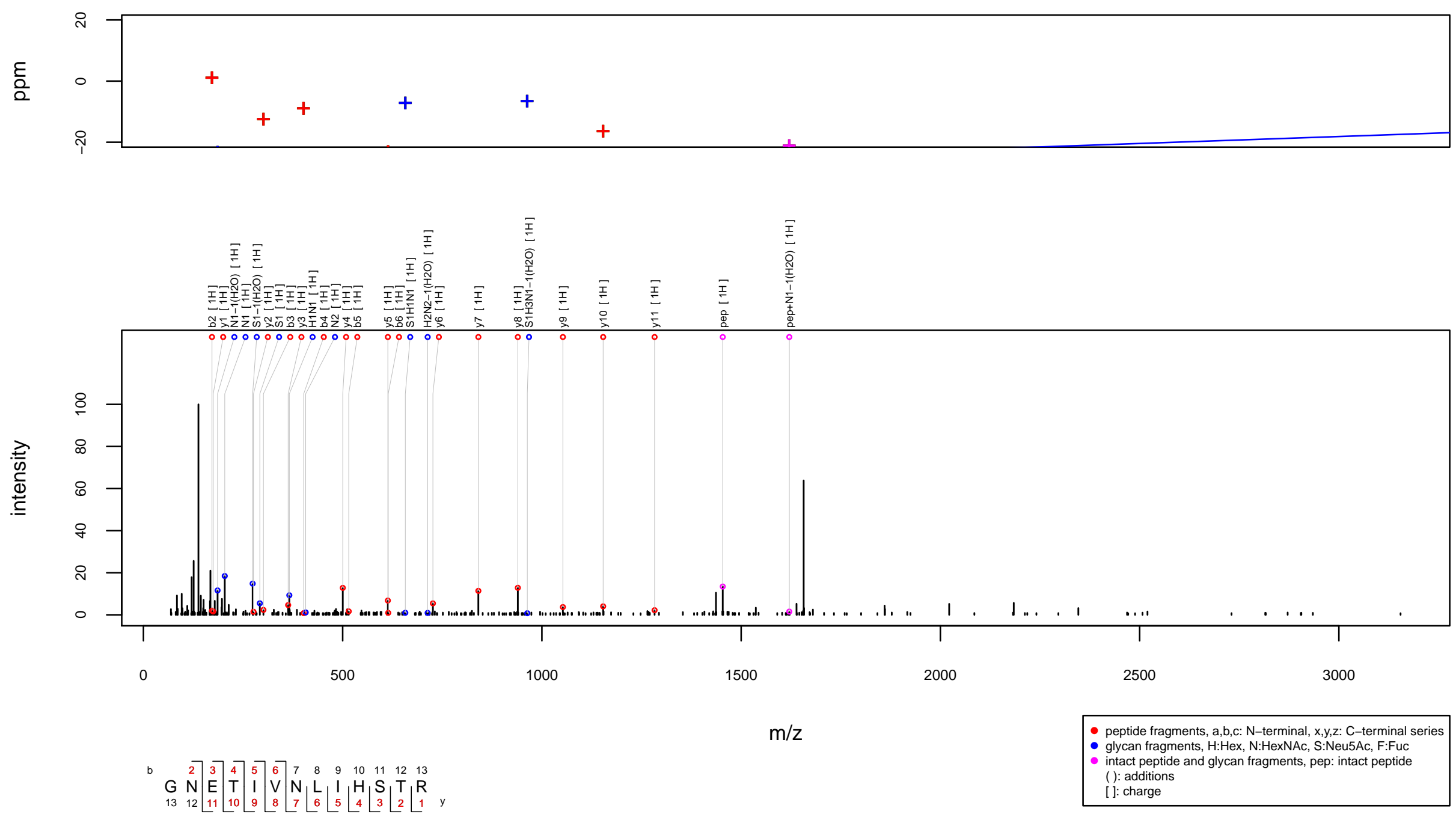
m/z 813.5967 charge 4 scan 0-0

Score $=34.03$, Hits $=26$, Explained Intensity $=0.06$ Peptide: CUBN_HUMAN[2082,2090]:AGFNASFHK Glycan: H4N8

Charge: $4 \mathrm{H}$
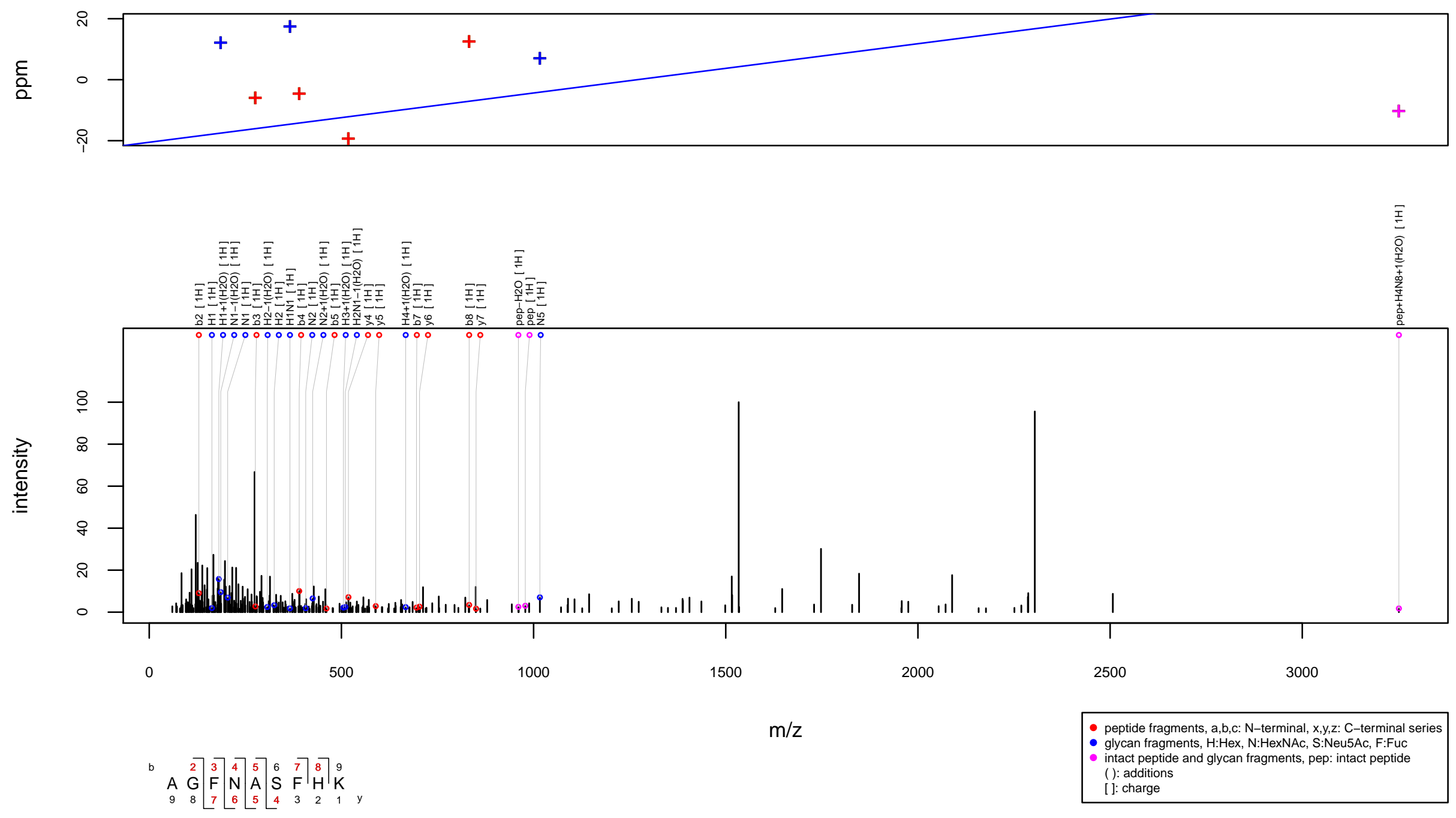
m/z 825.7395 charge 5 scan $0-0$

Score $=38.46$, Hits $=32$, Explained Intensity $=0.13$

Peptide: SEMG2_HUMAN[266,281]:NQHQTKNLSQDQEHGR

Glycan: S2H5N4

Charge: $5 \mathrm{H}$
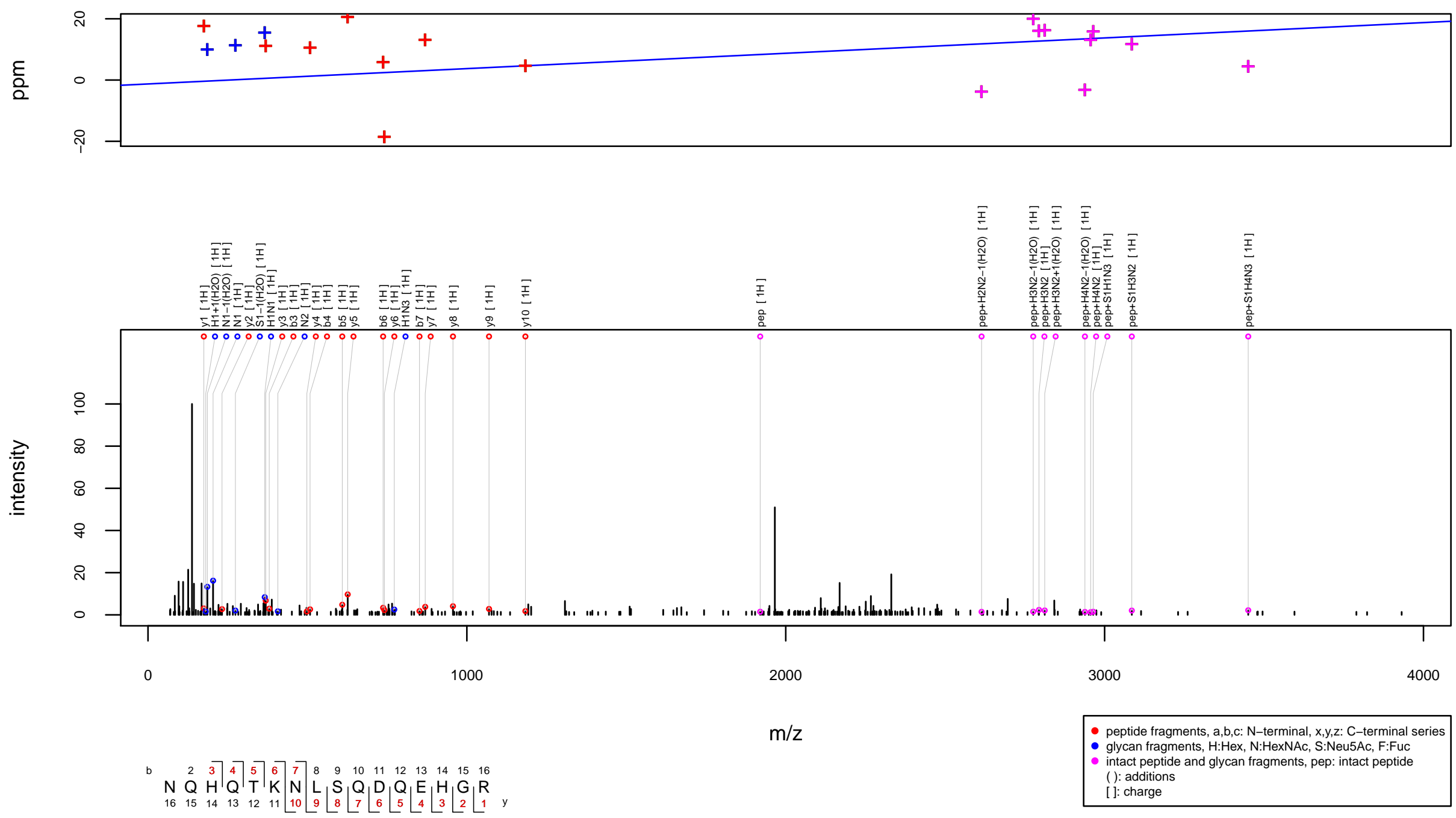
$\mathrm{m} / \mathrm{z} 832.3874$ charge 4 scan $0-0$

Score $=63.64$, Hits $=41$, Explained Intensity $=0.31$ Peptide: CBPE_HUMAN[138,150]:GNETIVNLIHSTR Glycan: $\mathrm{SHNH}(\mathrm{H}(\mathrm{H}) \mathrm{H}) \mathrm{HNN}$, S1H6N3

Charge: $4 \mathrm{H}$
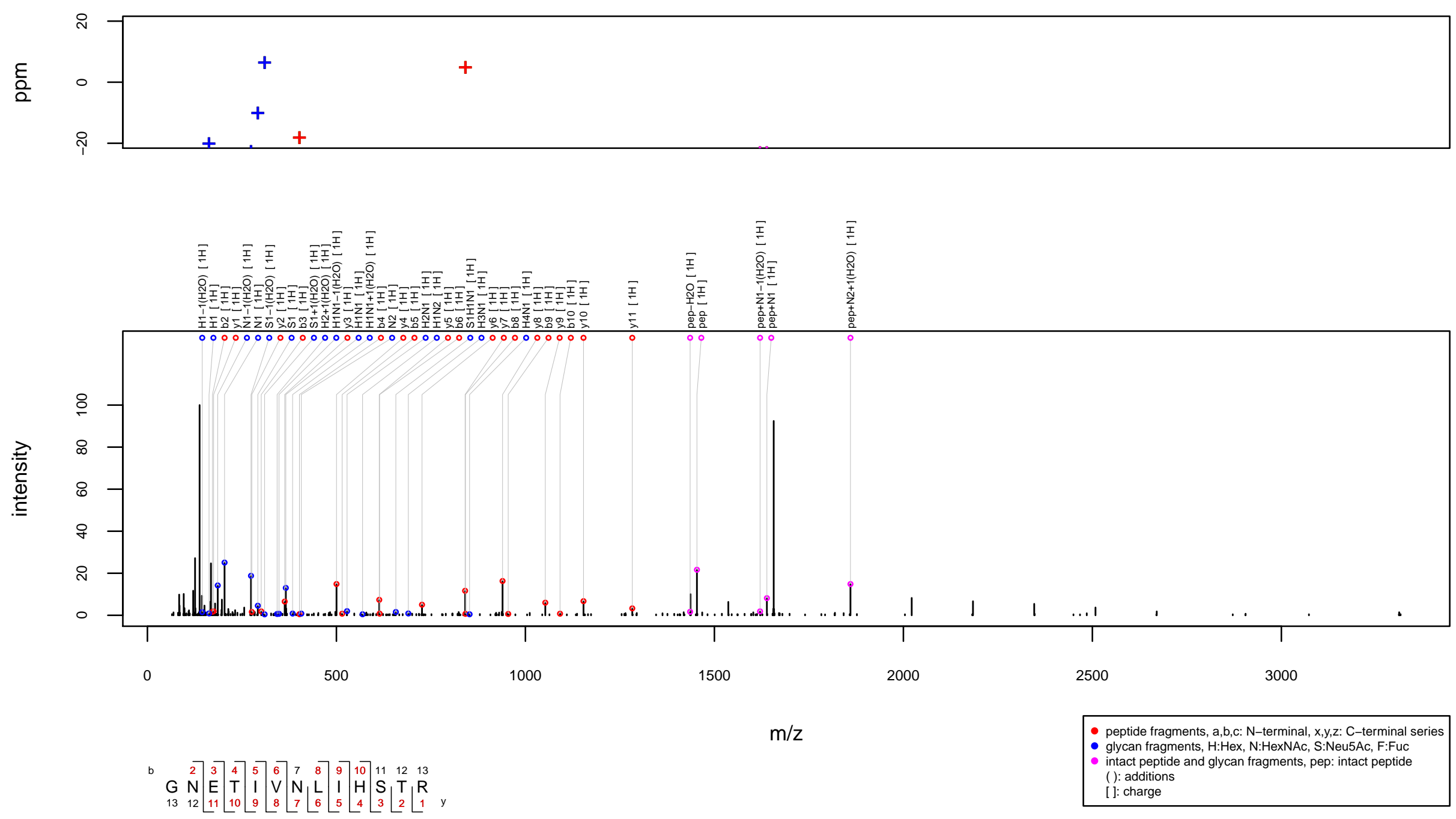
m/z 833.7398 charge 3 scan $0-0$

Score $=52.04$, Hits $=30$, Explained Intensity $=0.16$ Peptide: AMPN_HUMAN[231,241]:AEFNITLIHPK

Glycan: $\mathrm{H}(\mathrm{H}) \mathrm{H}(\mathrm{H}) \mathrm{HNN}, \mathrm{H} 5 \mathrm{~N} 2$

Charge: $3 \mathrm{H}$
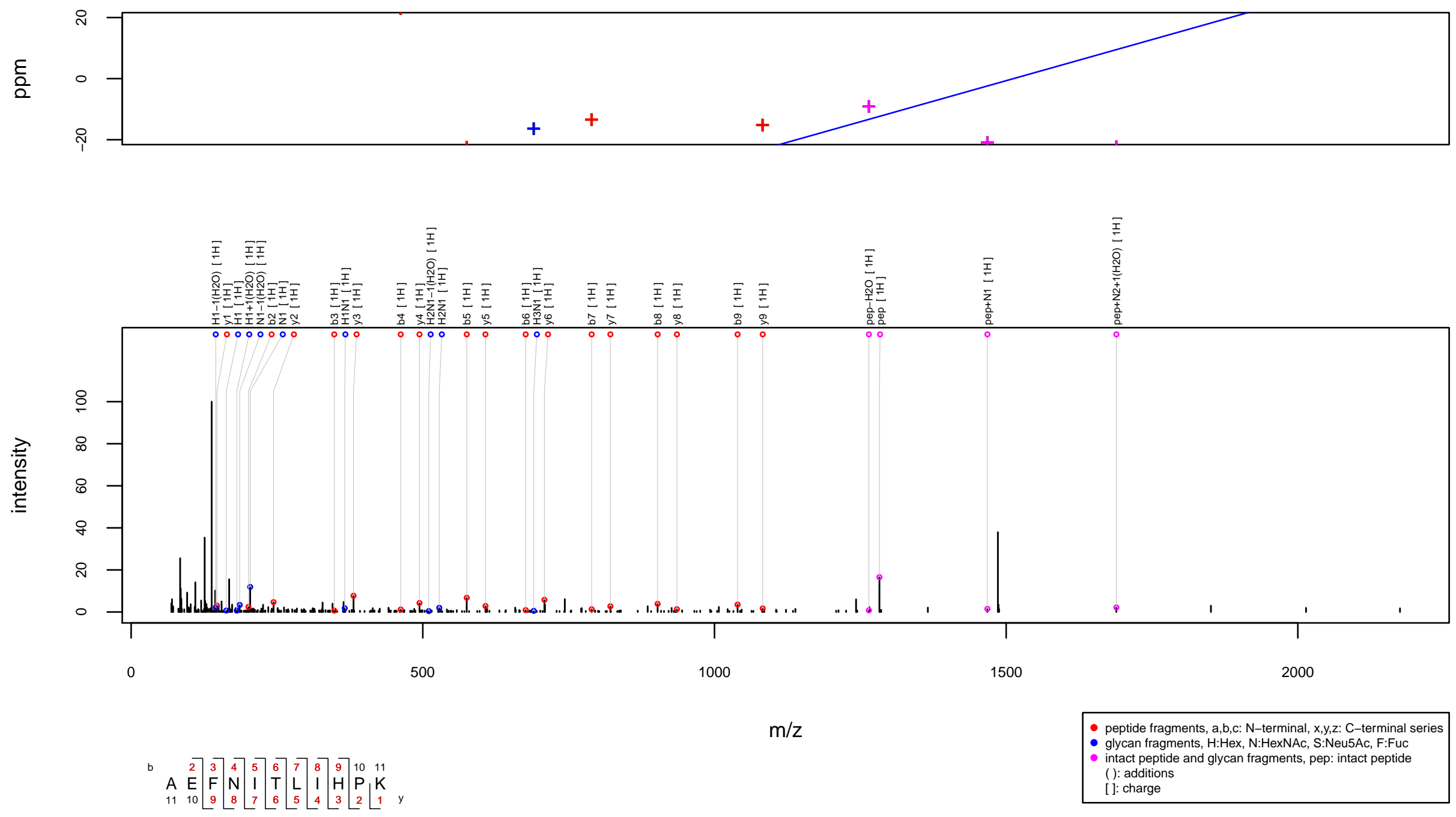
$\mathrm{m} / \mathrm{z} 840.0128$ charge 3 scan $0-0$

Score $=56.44$, Hits $=40$, Explained Intensity $=0.32$ Peptide: MUC6_HUMAN[486,491]:NITVFR

Glycan: $\mathrm{HNH}(\mathrm{HNH}) \mathrm{HN}(\mathrm{F}) \mathrm{N}, \mathrm{H} 5 \mathrm{~N} 4 \mathrm{~F} 1$

Charge: $3 \mathrm{H}$
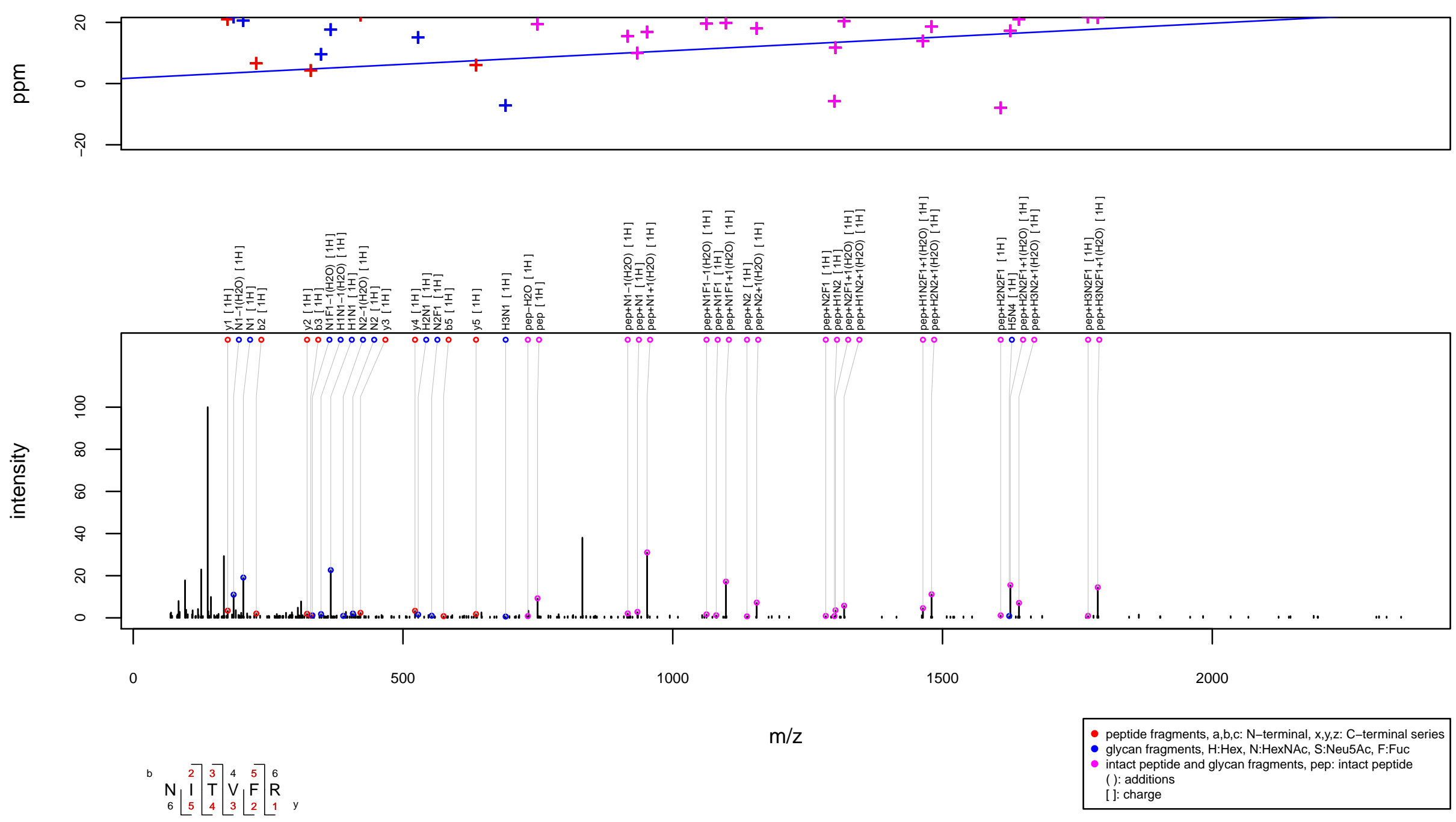
$\mathrm{m} / \mathbf{z} 841.7088$ charge 3 scan $0-0$

Score $=39.68$, Hits $=24$, Explained Intensity $=0.31$

Peptide: PPAP_HUMAN[92,98]:FLNESYK

Glycan: $\mathrm{HNH}(\mathrm{HNH}) \mathrm{HNN}, \mathrm{H} 5 \mathrm{~N} 4$

Charge: $3 \mathrm{H}$
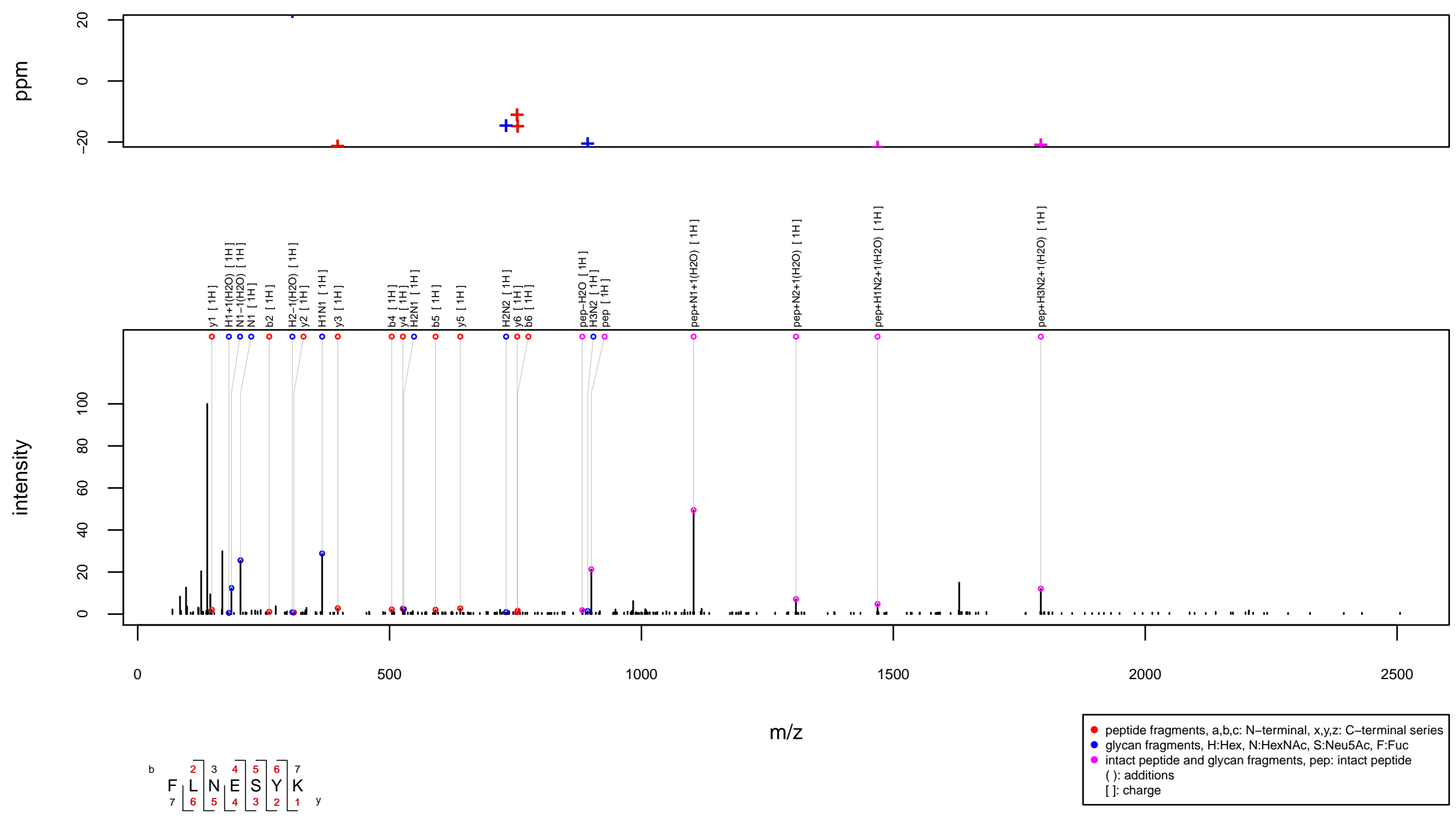
$\mathrm{m} / \mathbf{z} 843.3423$ charge 3 scan $0-0$

Score $=65.89$, Hits $=39$, Explained Intensity $=0.43$

Peptide: SEMG2_HUMAN[272,282]:NLSQDQEHGRK

Glycan: $\mathrm{H}(\mathrm{H}) \mathrm{H}(\mathrm{H}) \mathrm{HNN}, \mathrm{H} 5 \mathrm{~N} 2$

Charge: $3 \mathrm{H}$
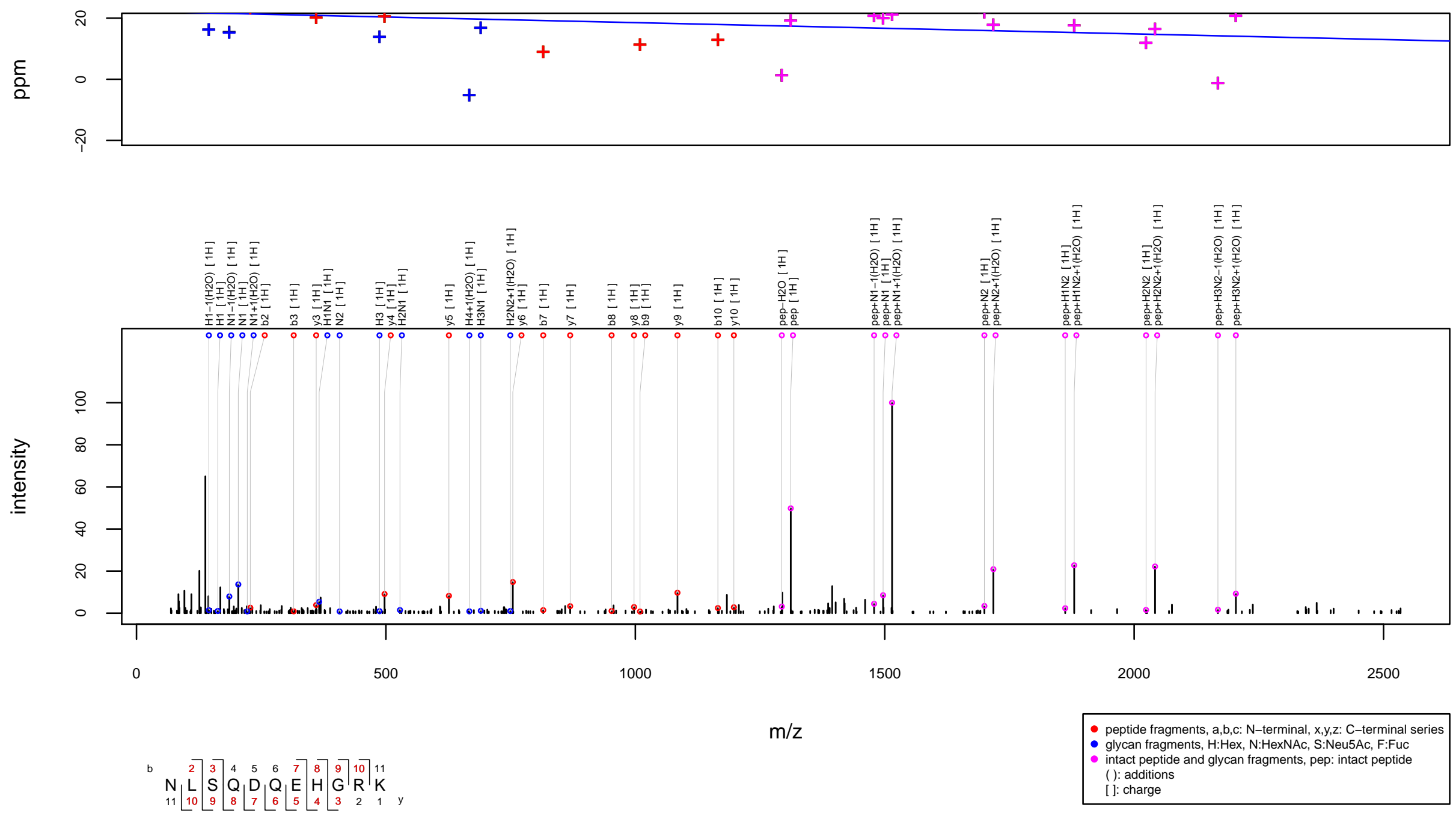
$\mathrm{m} / \mathrm{z} 845.6109$ charge 4 scan $0-0$

Score $=76.18$, Hits $=55$, Explained Intensity $=0.28$

Peptide: PPAP_HUMAN[91,98]:KFLNESYK

Glycan: $\mathrm{SHNH}(\mathrm{SHNH}) \mathrm{HN}(\mathrm{F}) \mathrm{N}, \mathrm{S} 2 \mathrm{H} 5 \mathrm{~N} 4 \mathrm{~F} 1$

Charge: $4 \mathrm{H}$
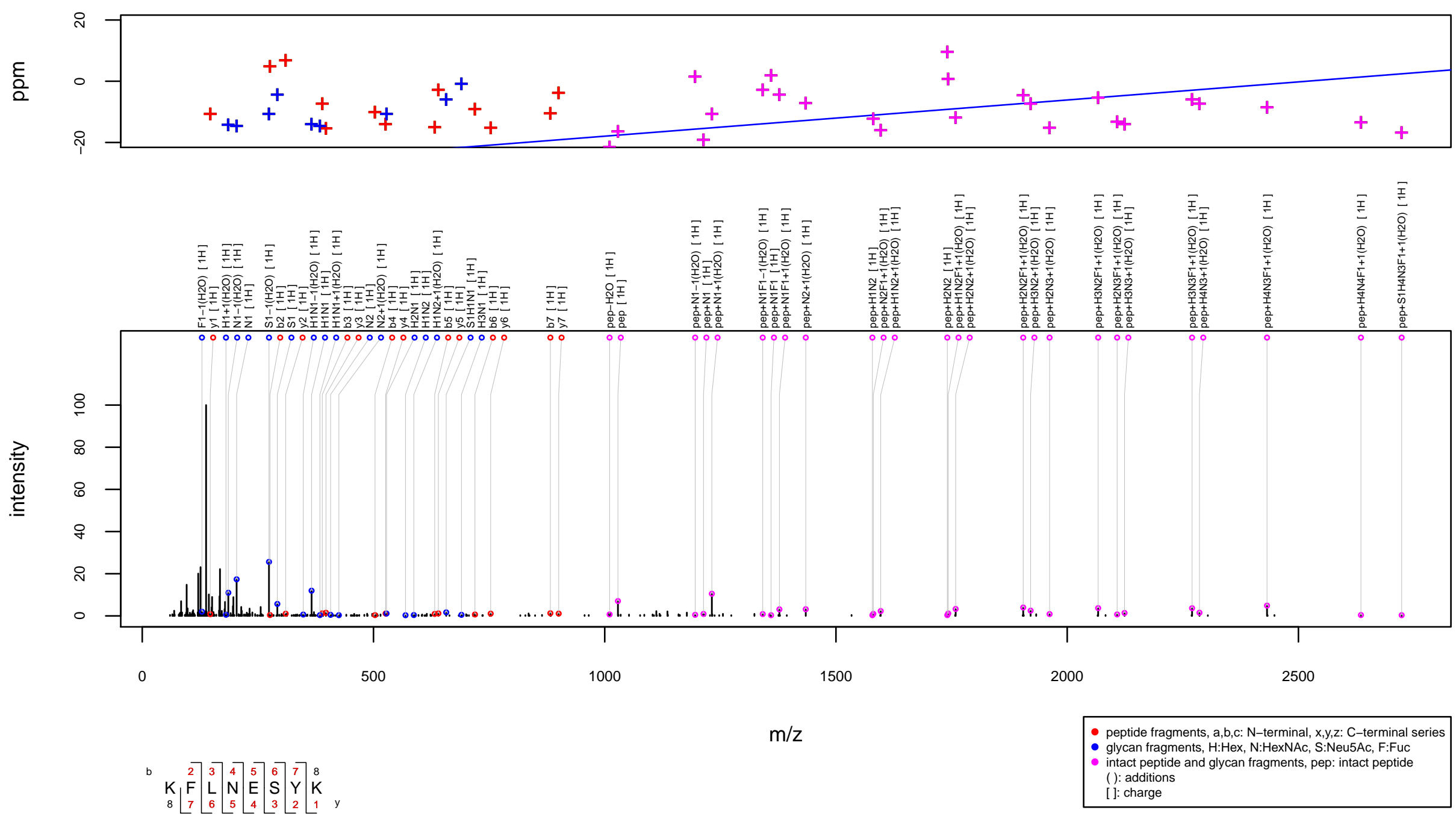
$\mathrm{m} / \mathrm{z} 851.687$ charge 3 scan $0-0$

Score $=66.26$, Hits $=43$, Explained Intensity $=0.27$

Peptide: SEMG2_HUMAN[272,282]:NLSQDQEHGRK

Glycan: $\mathrm{NH}(\mathrm{H}) \mathrm{HN}(\mathrm{F}) \mathrm{N}, \mathrm{H} 3 \mathrm{~N} 3 \mathrm{~F} 1$

Charge: $3 \mathrm{H}$
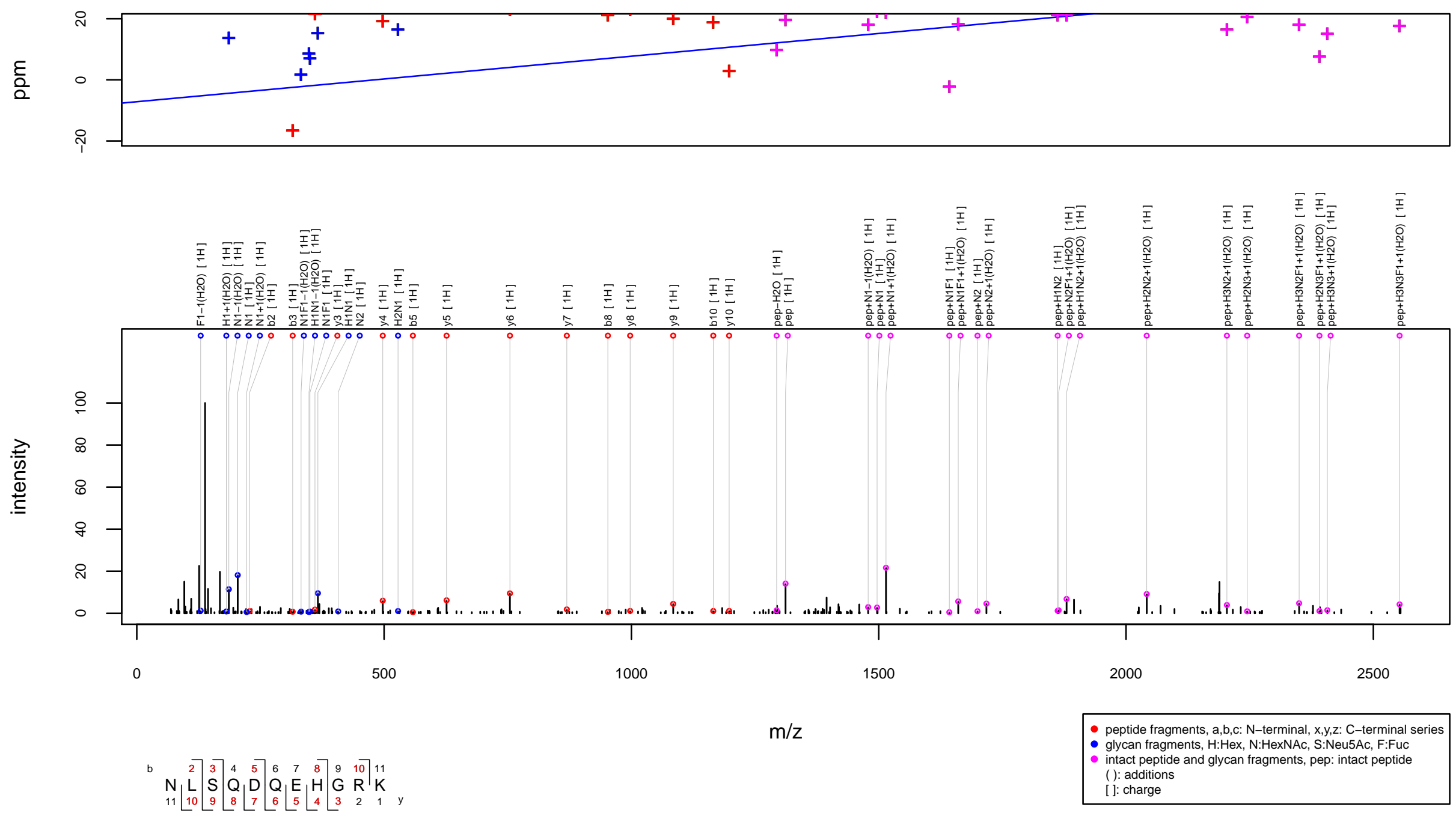
m/z 854.9505 charge 5 scan 0-0

Score $=52.17$, Hits $=55$, Explained Intensity $=0.19$

Peptide: SEMG2_HUMAN[266,281]:NQHQTKNLSQDQEHGR

Glycan: S2H5N4F1

Charge: $5 \mathrm{H}$
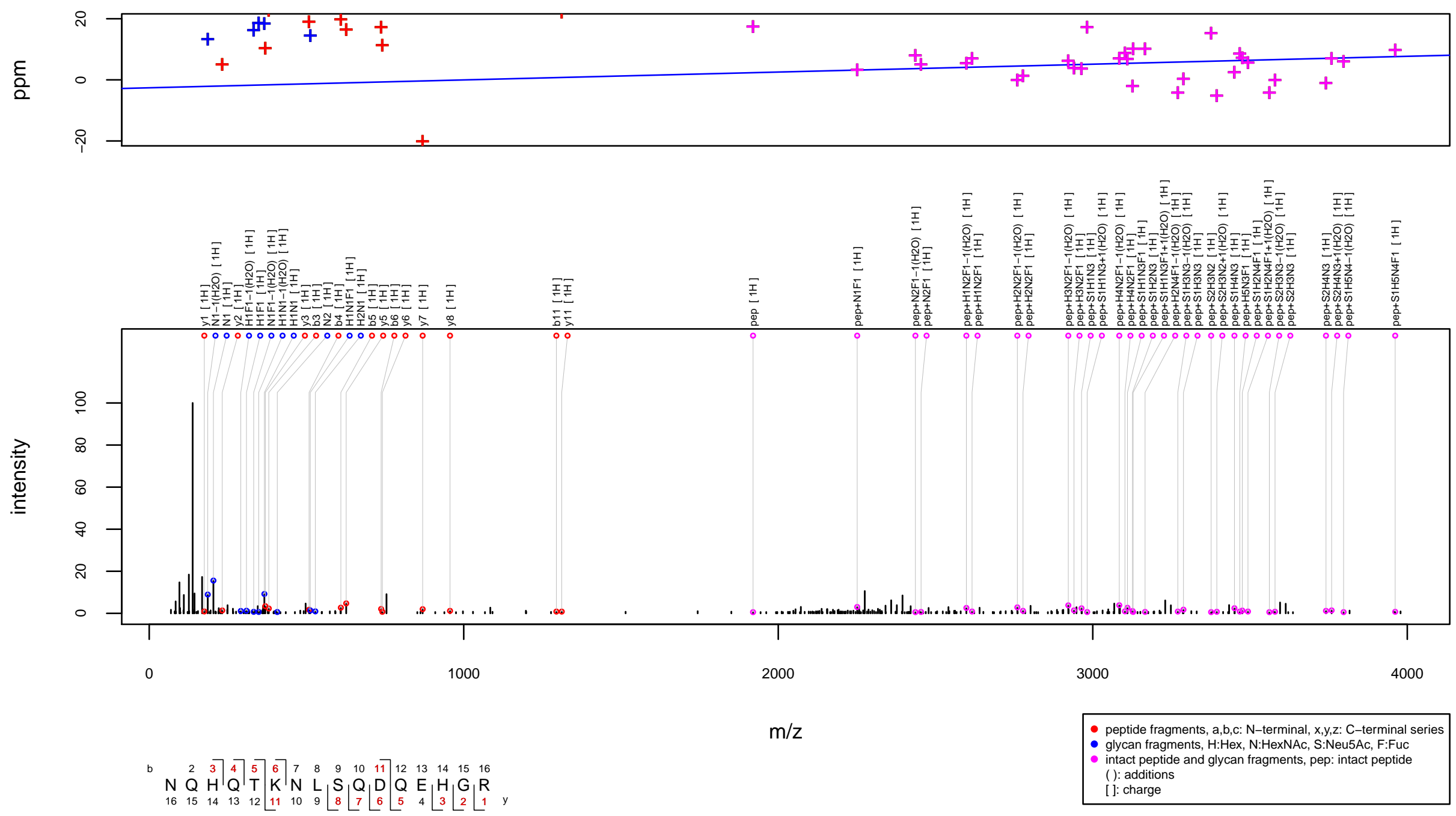
$\mathrm{m} / \mathrm{z} 862.9187$ charge 4 scan $0-0$

Score $=40.95$, Hits $=24$, Explained Intensity $=0.27$

Peptide: FINC_HUMAN[997,1016]:LDAPTNLQFVNETDSTVLVR

Glycan: $\mathrm{H}(\mathrm{H}) \mathrm{H}(\mathrm{H}) \mathrm{HNN}, \mathrm{H} 5 \mathrm{~N} 2$

Charge: $4 \mathrm{H}$
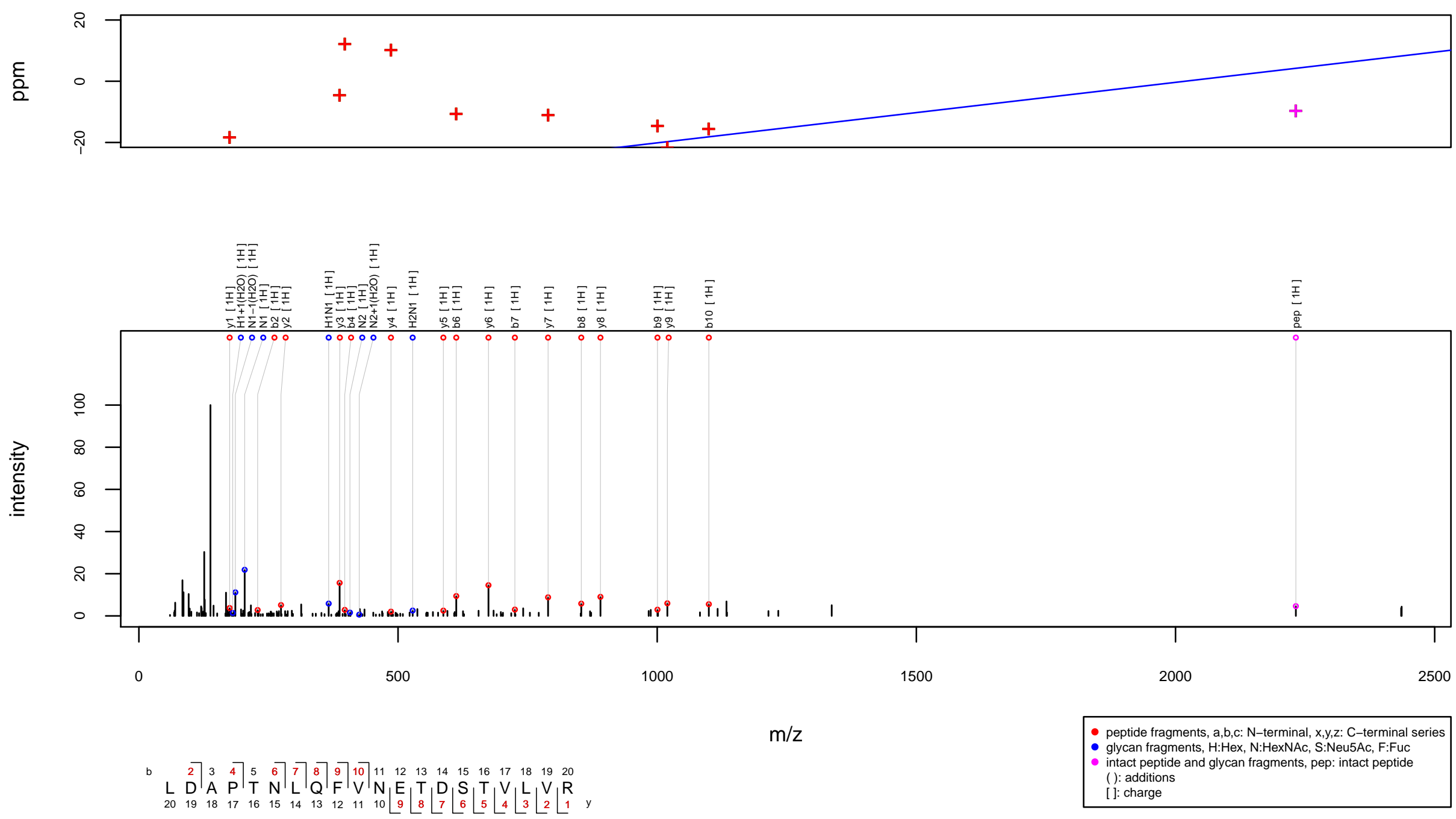
m/z 877.1305 charge 4 scan 0-0

Score $=39.14$, Hits $=26$, Explained Intensity $=0.16$

Peptide: CD38_HUMAN[215,234]:IFDKNSTFGSVEVHNLQPEK

Glycan: $\mathrm{H}(\mathrm{H}) \mathrm{H}(\mathrm{H}) \mathrm{HNN}, \mathrm{H} 5 \mathrm{~N} 2$

Charge: $4 \mathrm{H}$
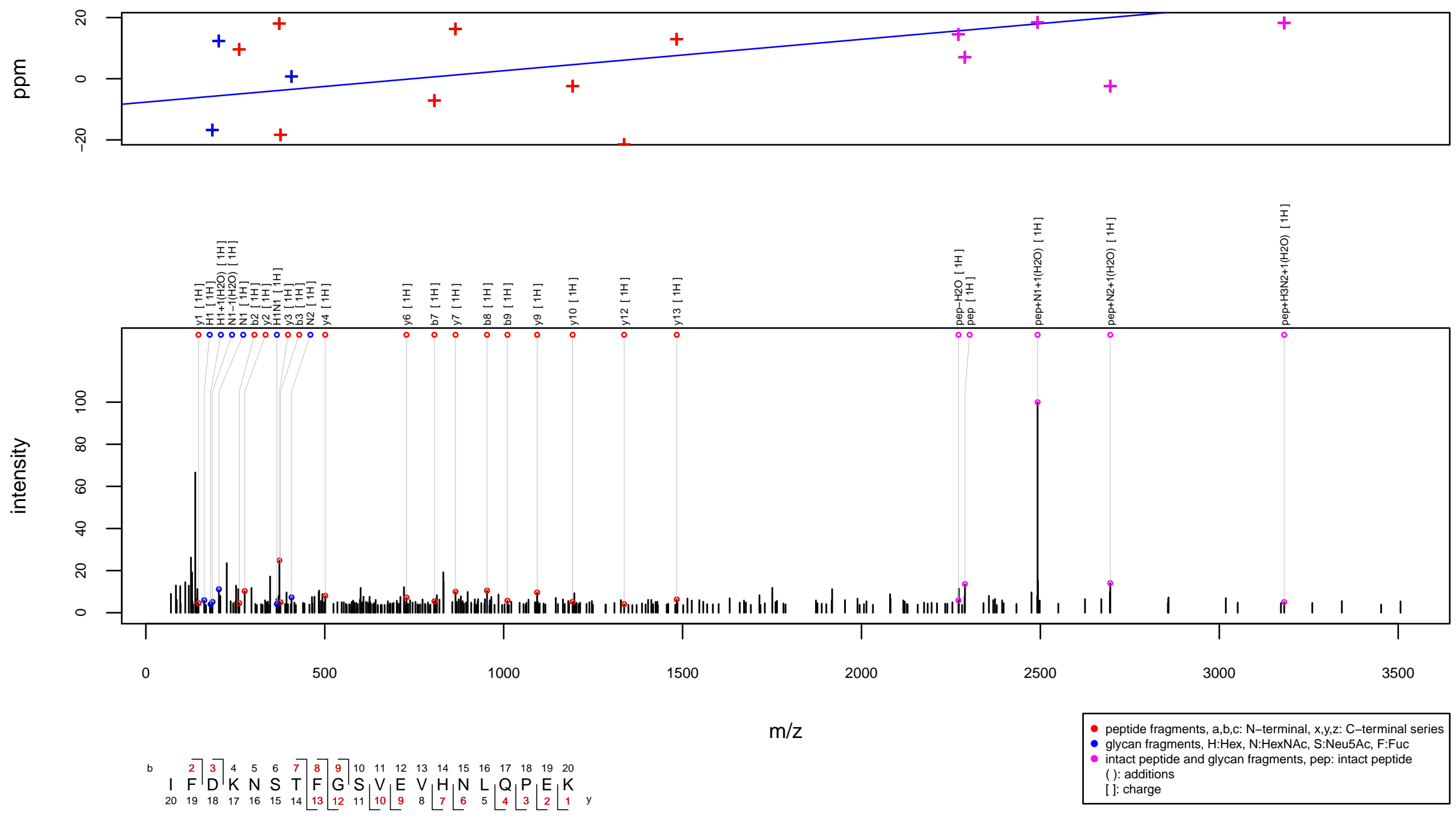
$\mathrm{m} / \mathrm{z} 881.1559$ charge 4 scan $0-0$

Score $=45.76$, Hits $=35$, Explained Intensity $=0.31$

Peptide: TIMP1_HUMAN[46,60]:FVGTPEVNQTTLYQR

Glycan: H5N4F1

Charge: $4 \mathrm{H}$
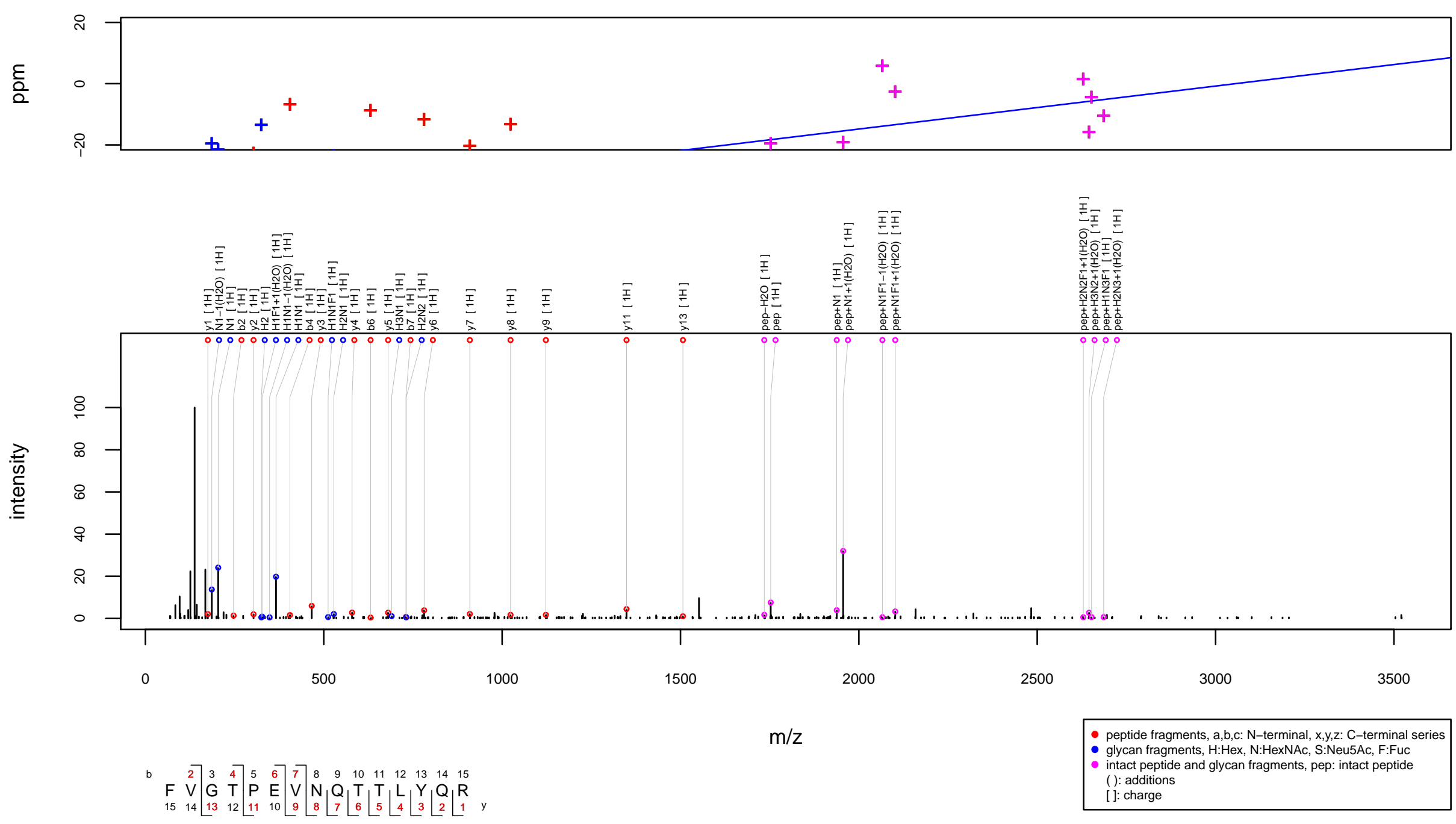
$\mathrm{m} / \mathrm{z} 881.161$ charge 4 scan $0-0$

Score $=44.27$, Hits $=30$, Explained Intensity $=0.2$

Peptide: TIMP1_HUMAN[46,60]:FVGTPEVNQTTLYQR

Glycan: $\mathrm{FHNH}(\mathrm{HNH}) \mathrm{HNN}, \mathrm{H} 5 \mathrm{~N} 4 \mathrm{~F} 1$

Charge: $4 \mathrm{H}$
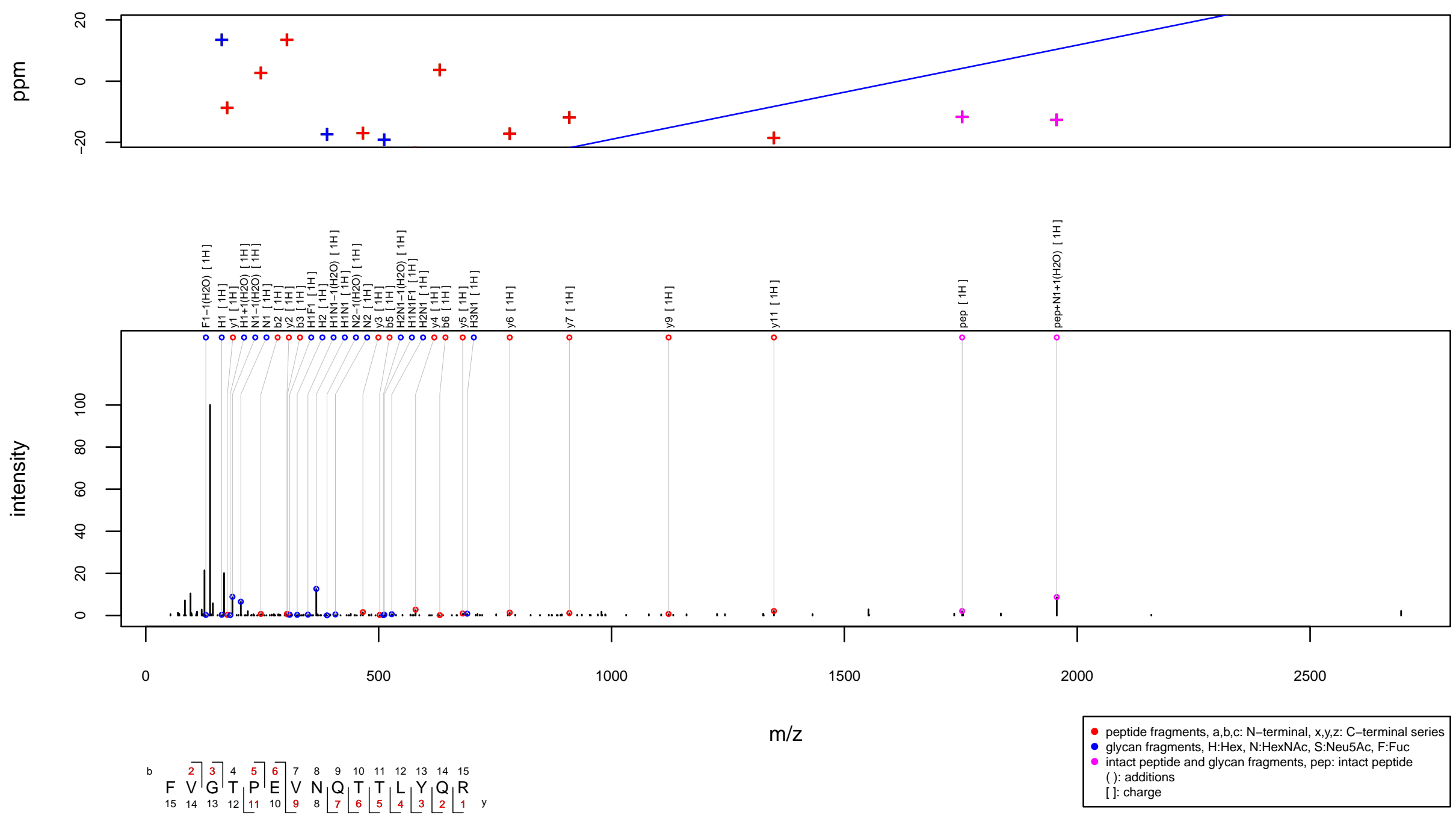
m/z 890.7584 charge 3 scan $0-0$

Score $=48.52$, Hits $=30$, Explained Intensity $=0.43$ Peptide: CBPE_HUMAN[138,150]:GNETIVNLIHSTR Glycan: $\mathrm{H}(\mathrm{H}) \mathrm{H}(\mathrm{H}) \mathrm{HNN}, \mathrm{H} 5 \mathrm{~N} 2$

Charge: $3 \mathrm{H}$
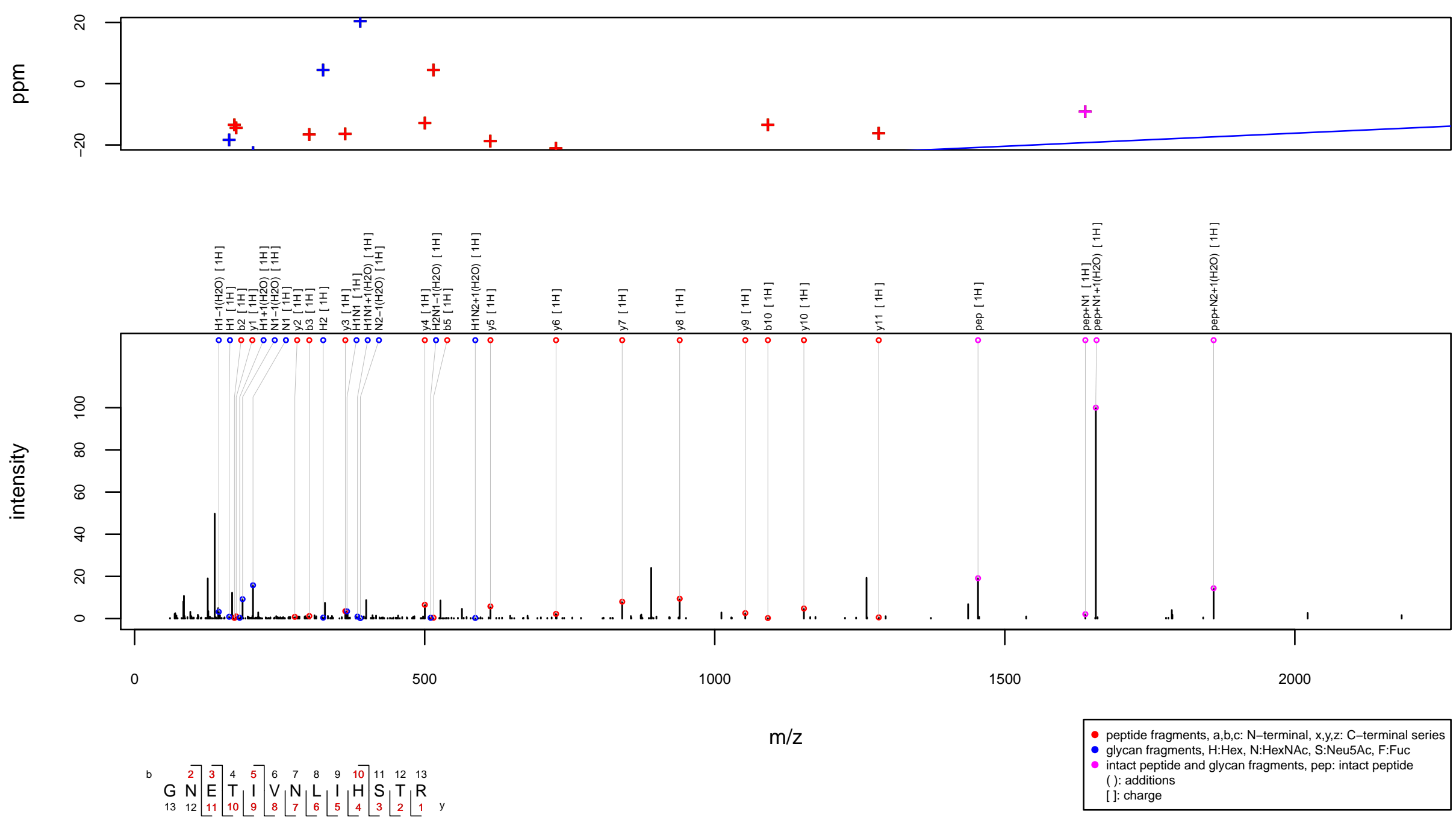
$\mathrm{m} / \mathrm{z} 900.4032$ charge 4 scan $0-0$

Score $=60.37$, Hits $=46$, Explained Intensity $=0.26$ Peptide: CLUS_HUMAN[372,385]:LANLTQGEDQYYLR Glycan: $\mathrm{FHNH}(\mathrm{HNH}) \mathrm{HN}(\mathrm{F}) \mathrm{N}, \mathrm{H} 5 \mathrm{~N} 4 \mathrm{~F} 2$

Charge: $4 \mathrm{H}$
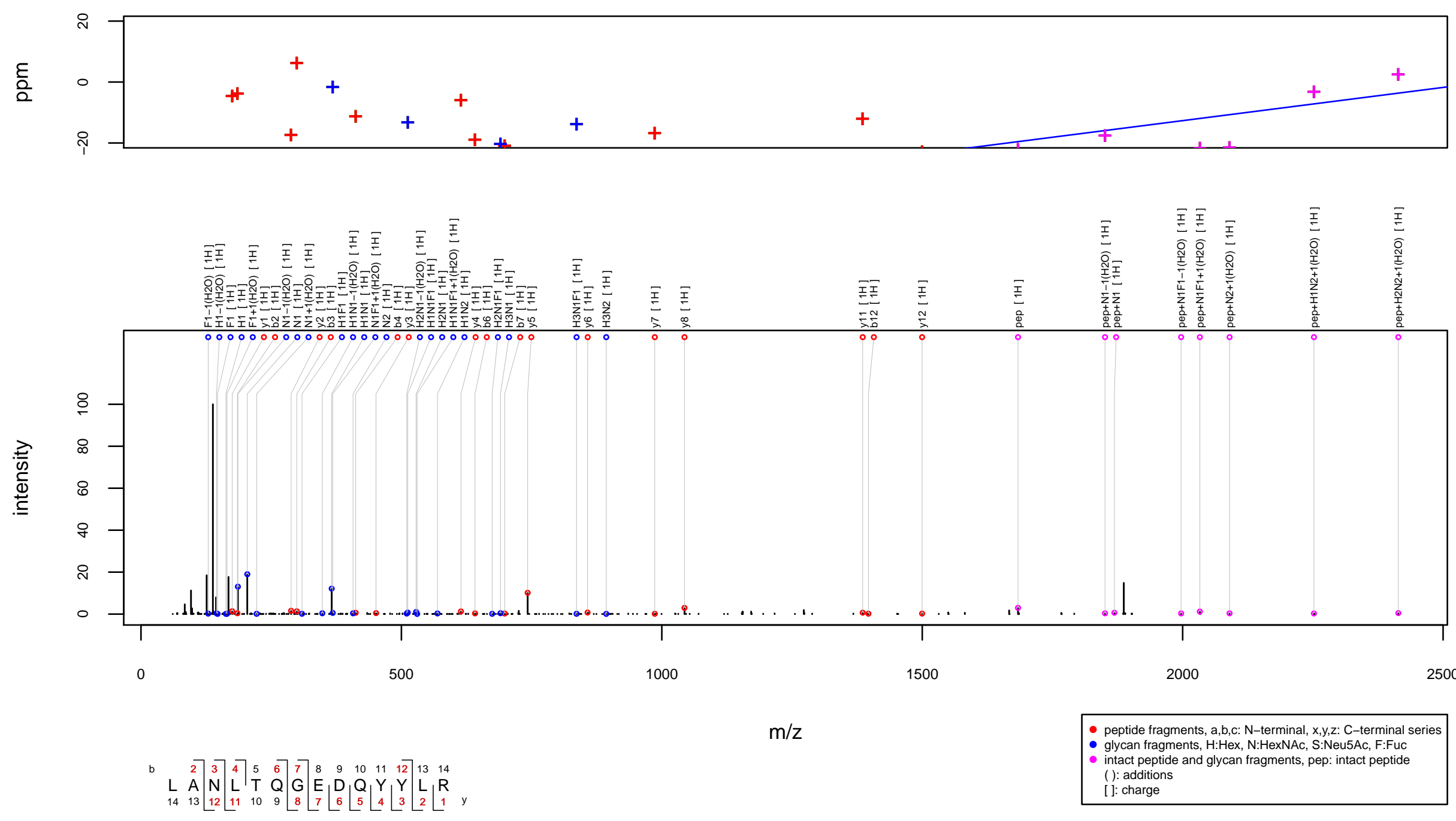
$\mathrm{m} / \mathrm{z} 902.7649$ charge 3 scan $0-0$

Score $=31.66$, Hits $=23$, Explained Intensity $=0.11$ Peptide: TMTC3_HUMAN[856,866]:QTQIVKTSDNK Glycan: H3N4F1

Charge: $3 \mathrm{H}$
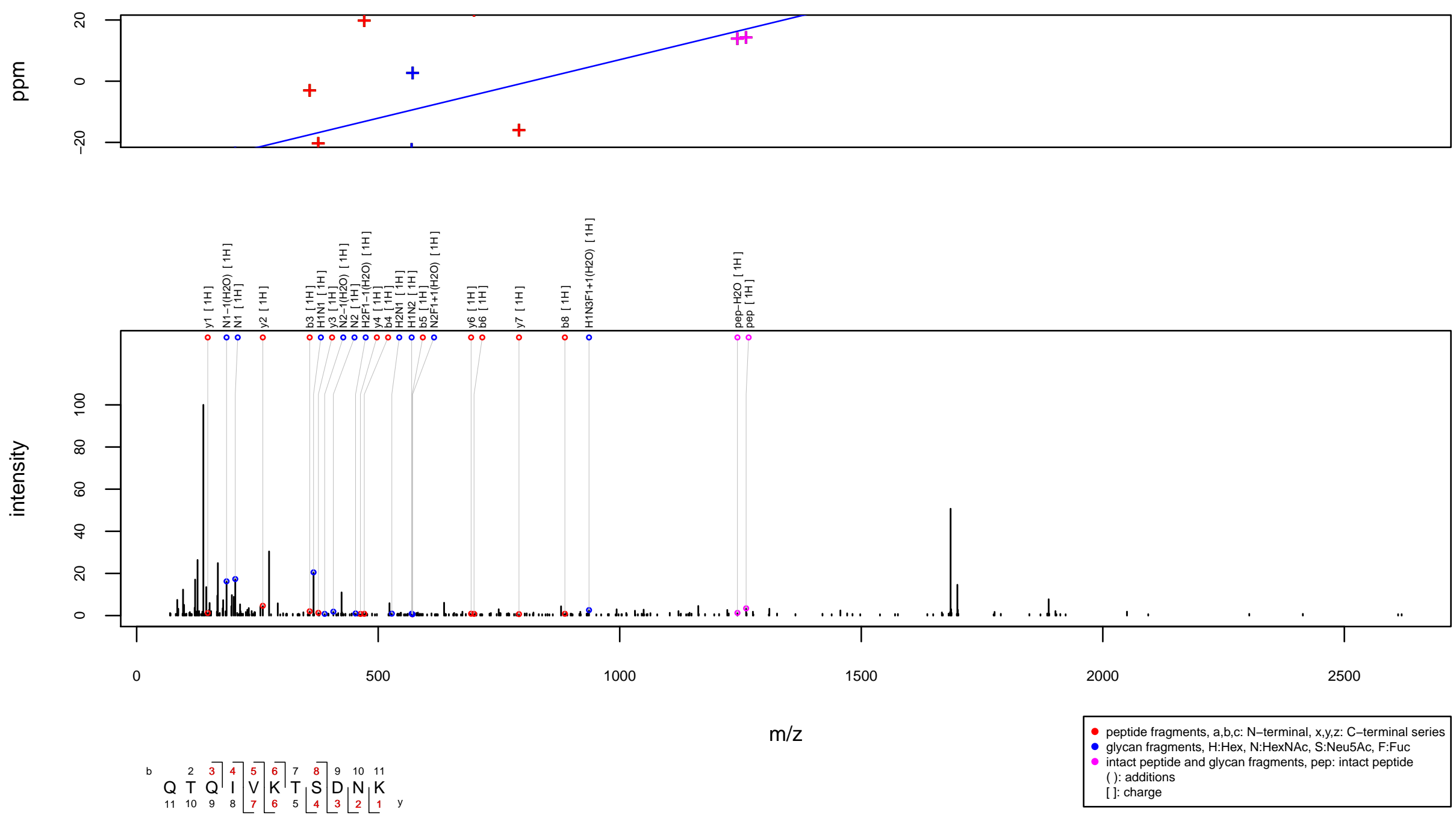
m/z 905.7047 charge 3 scan 0-0

Score $=85.29$, Hits $=57$, Explained Intensity $=0.37$ Peptide: SEMG2_HUMAN[272,282]:NLSQDQEHGRK Glycan: H4N3F1

Charge: $3 \mathrm{H}$
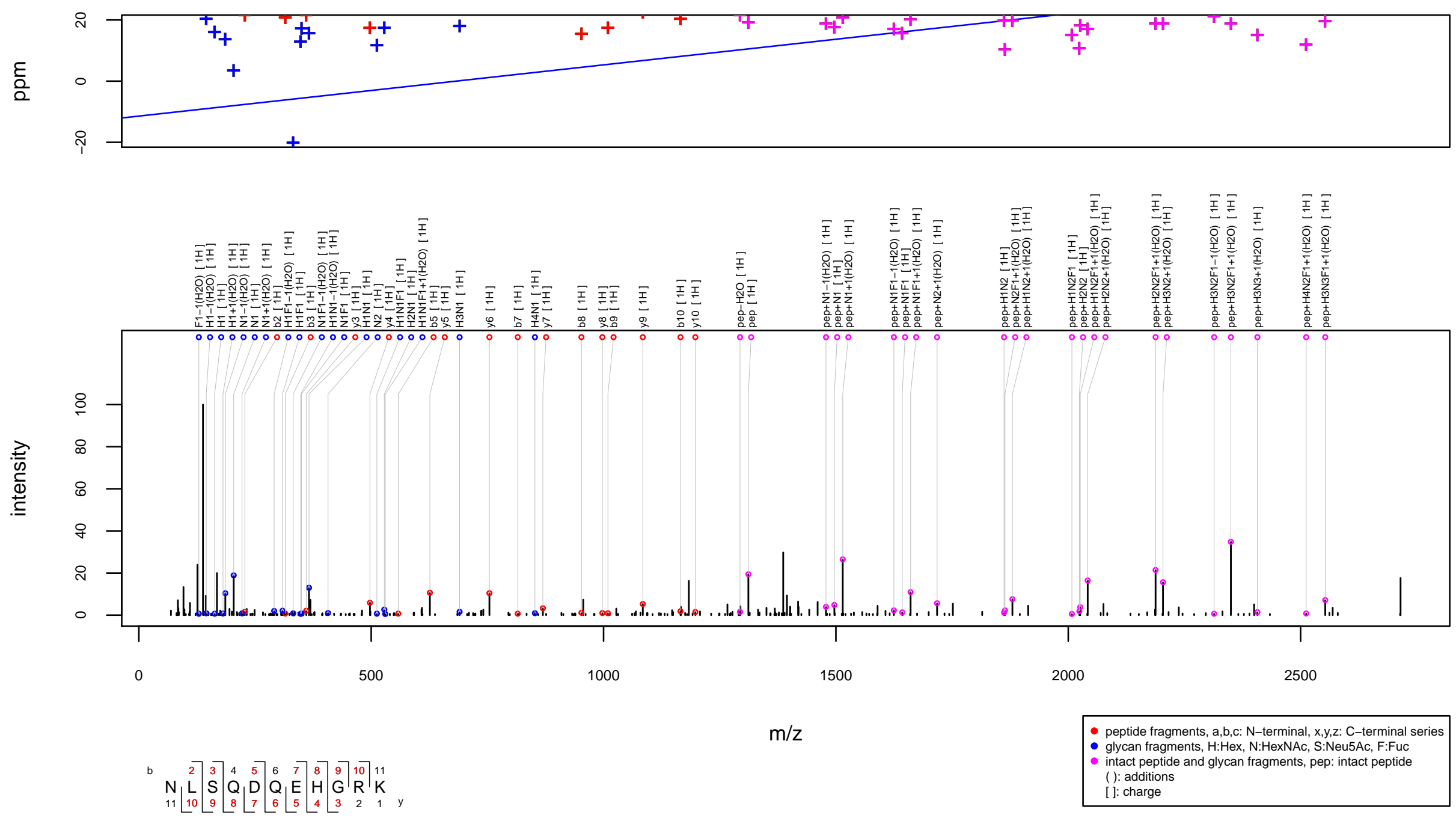
$\mathrm{m} / \mathrm{z} 911.2233$ charge 5 scan $0-0$

Score $=24.95$, Hits $=33$, Explained Intensity $=0.19$

Peptide: SOLH2_HUMAN[183,198]:NGNGLELNASLSEFEK

Glycan: S2H4N5F4

Charge: $5 \mathrm{H}$
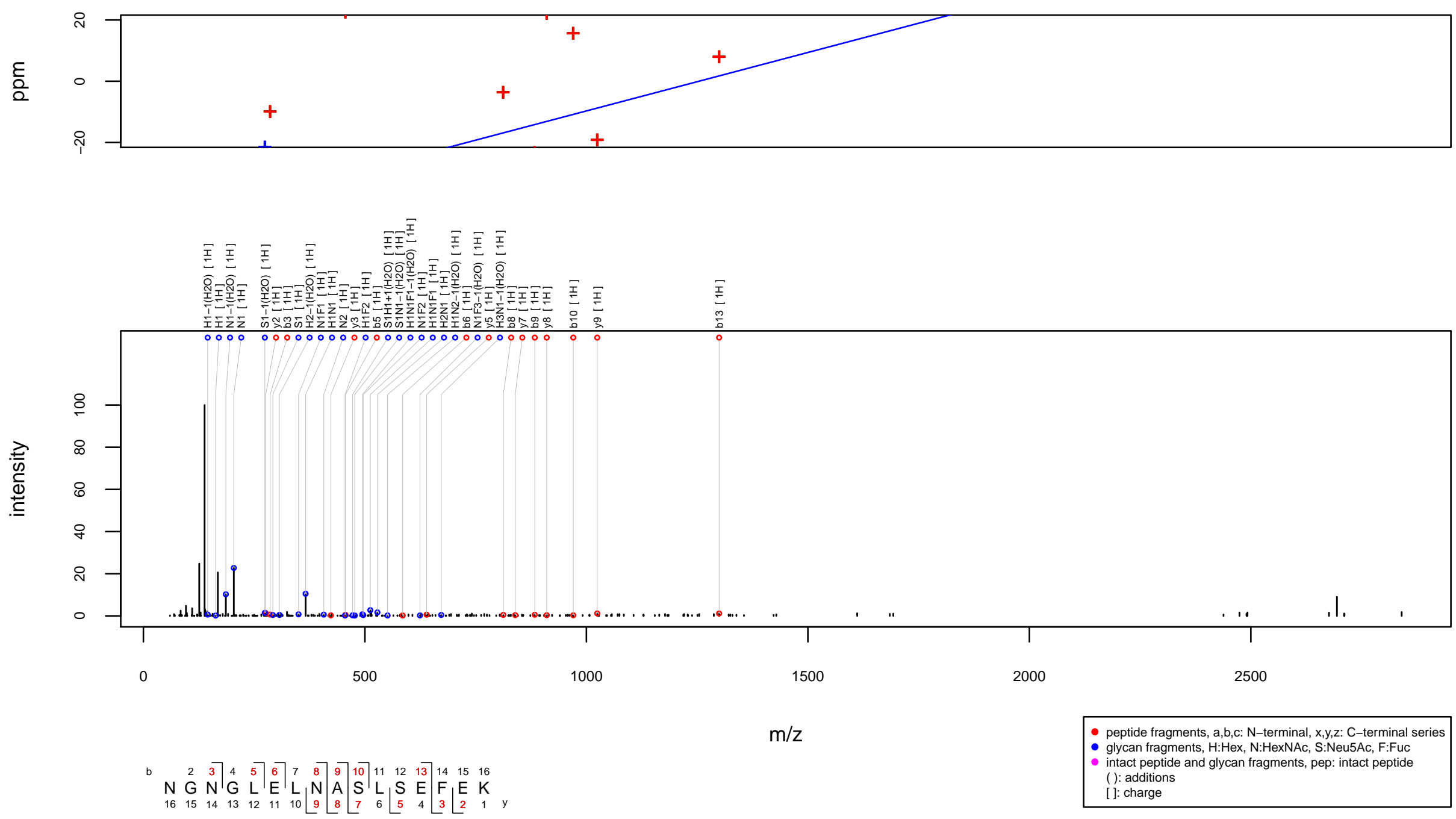
$\mathrm{m} / \mathrm{z} 917.6738$ charge 4 scan $0-0$

Score $=67.5$, Hits $=47$, Explained Intensity $=0.39$

Peptide: TIMP1_HUMAN[46,60]:FVGTPEVNQTTLYQR

Glycan: $\mathrm{F}(\mathrm{H}) \mathrm{HNH}(\mathrm{NH}) \mathrm{HN}(\mathrm{F}) \mathrm{N}, \mathrm{H} 5 \mathrm{~N} 4 \mathrm{~F} 2$

Charge: $4 \mathrm{H}$
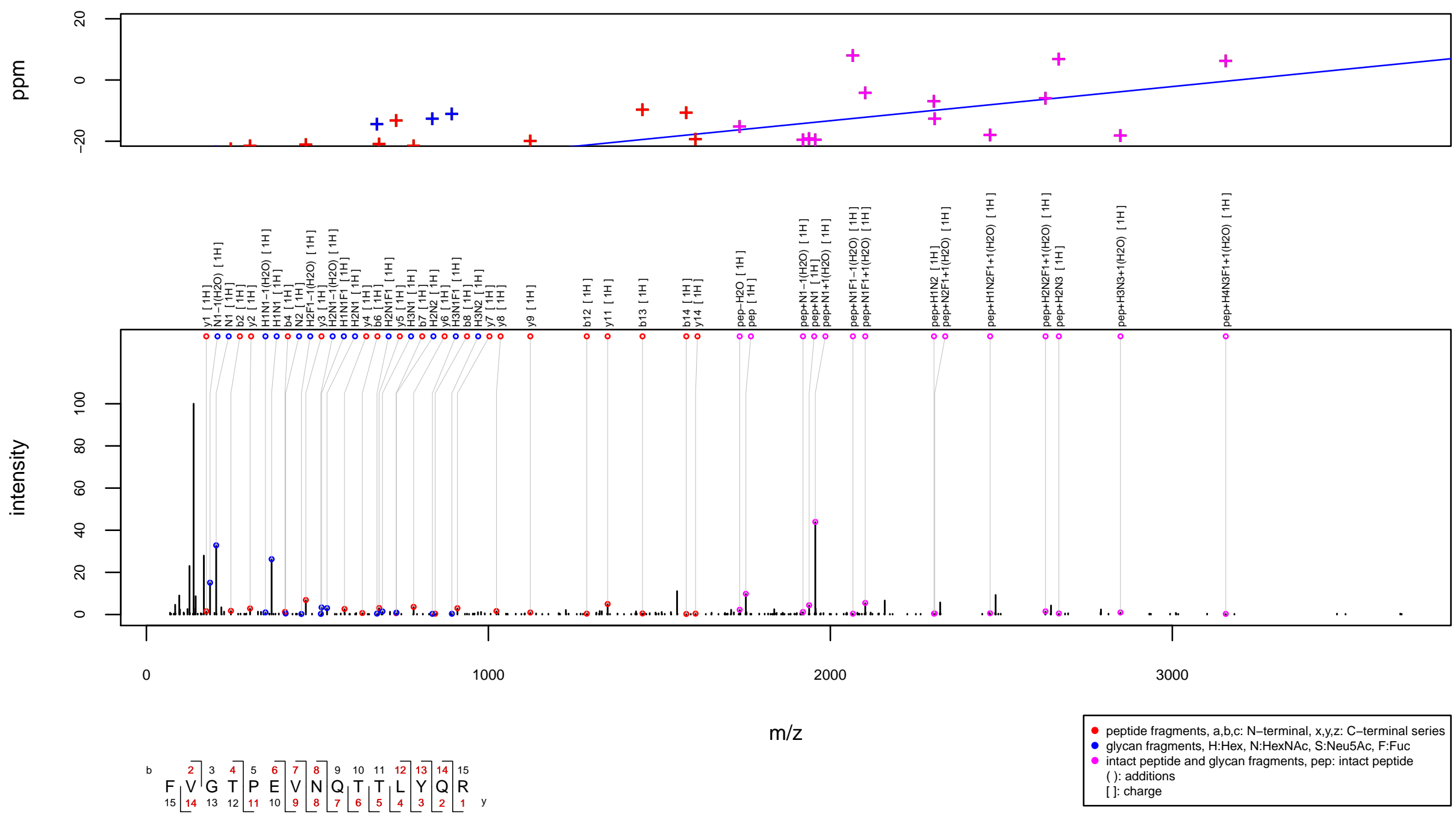
$\mathrm{m} / \mathrm{z} 921.1638$ charge 4 scan $0-0$

Score $=63.9$, Hits $=42$, Explained Intensity $=0.37$

Peptide: TRFE_HUMAN[421,433]:CGLVPVLAENYNK

Glycan: SHNH(SHNH)HNN, S2H5N4

Charge: $4 \mathrm{H}$
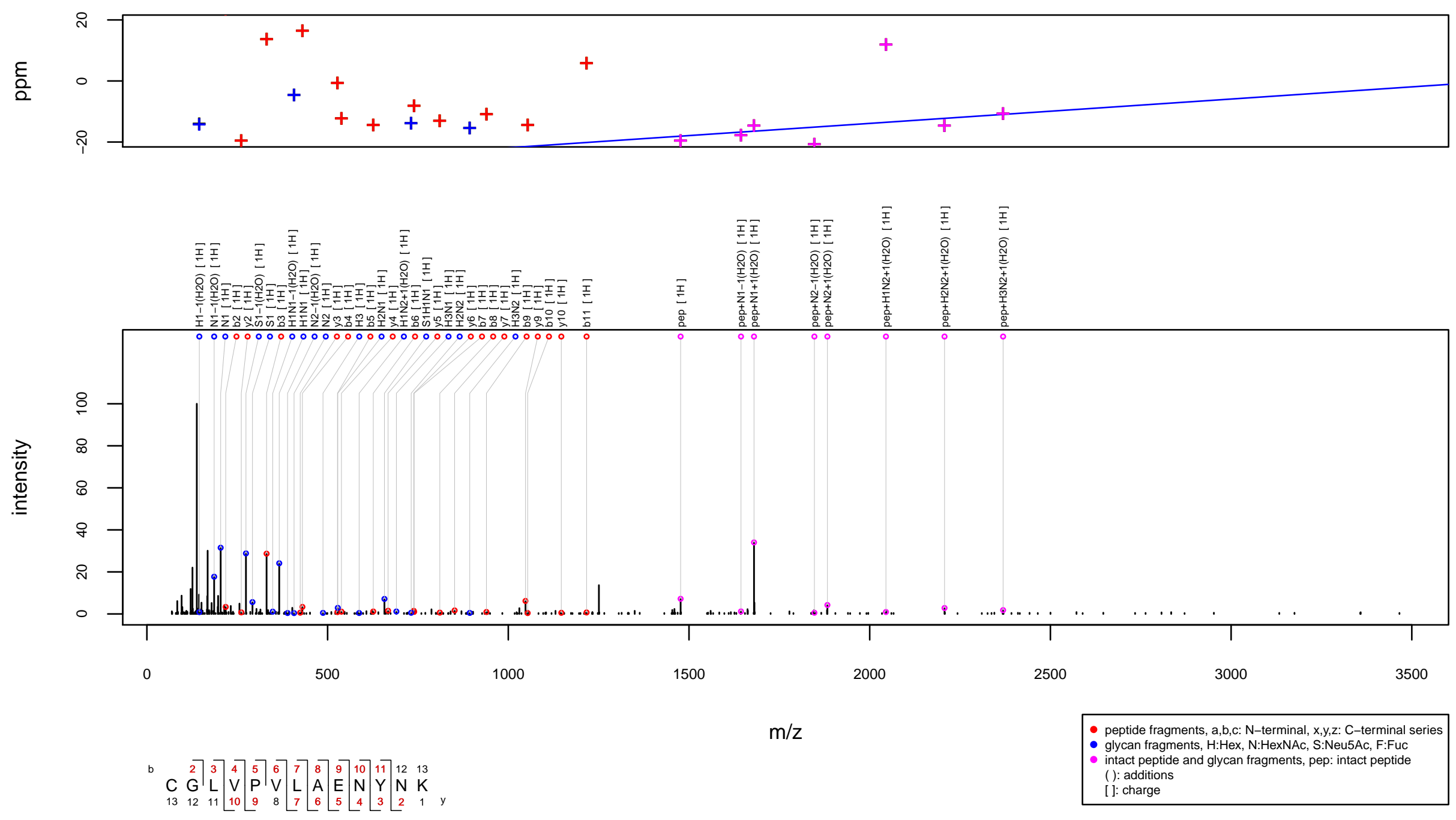
$\mathrm{m} / \mathrm{z} 922.402$ charge 3 scan $0-0$

Score $=49.21$, Hits $=32$, Explained Intensity $=0.26$ Peptide: ACE_HUMAN[706,718]:KFDVNQLQNTTIK Glycan: $\mathrm{H}(\mathrm{H}) \mathrm{H}(\mathrm{H}) \mathrm{HNN}, \mathrm{H} 5 \mathrm{~N} 2$

Charge: $3 \mathrm{H}$
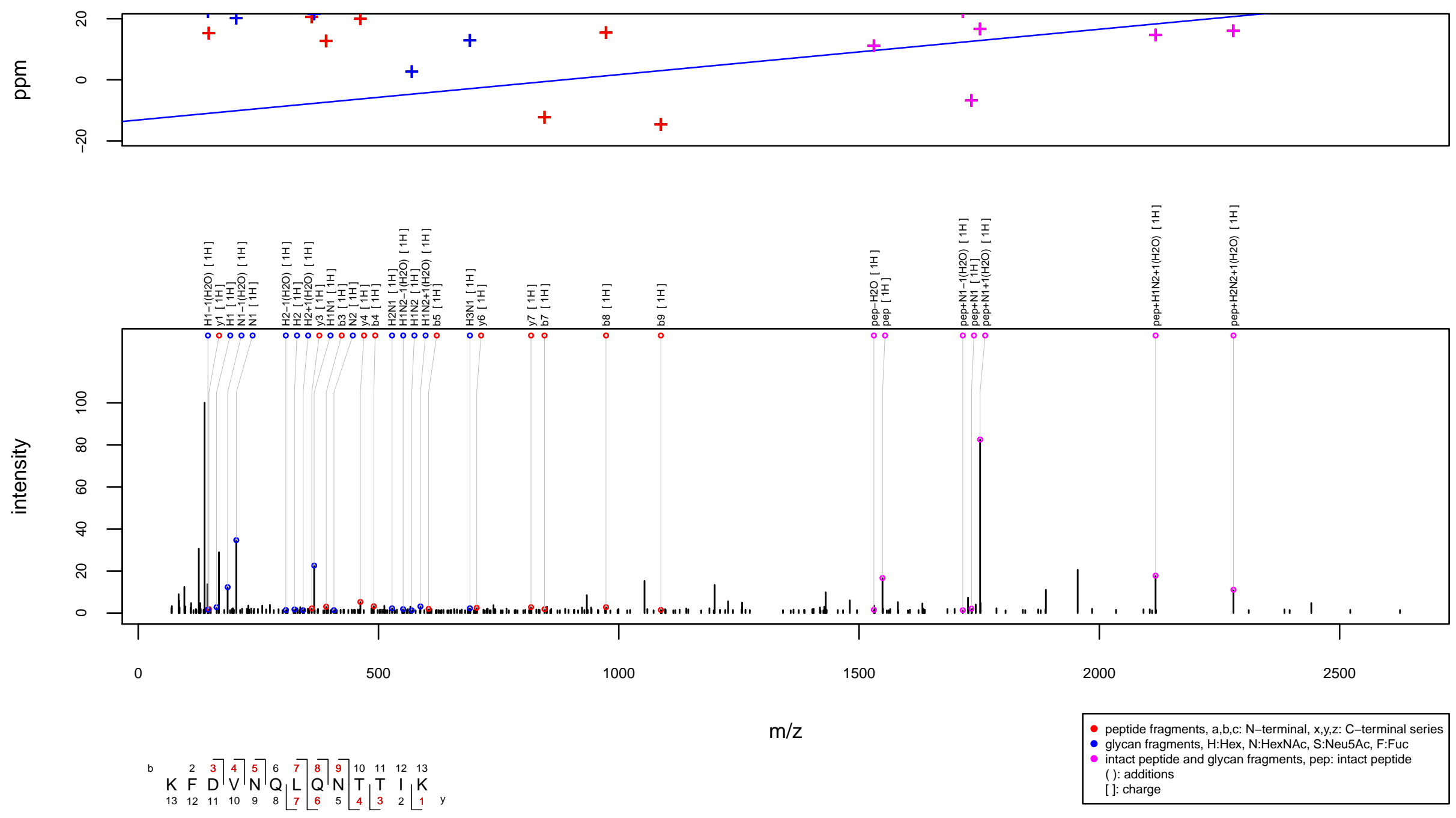
$\mathrm{m} / \mathrm{z} 925.7268$ charge 3 scan $0-0$

Score $=66.63$, Hits $=46$, Explained Intensity $=0.52$ Peptide: MUC6_HUMAN[484,491]:TRNITVFR

Glycan: $\mathrm{HNH}(\mathrm{HNH}) \mathrm{HN}(\mathrm{F}) \mathrm{N}, \mathrm{H} 5 \mathrm{~N} 4 \mathrm{~F} 1$

Charge: $3 \mathrm{H}$
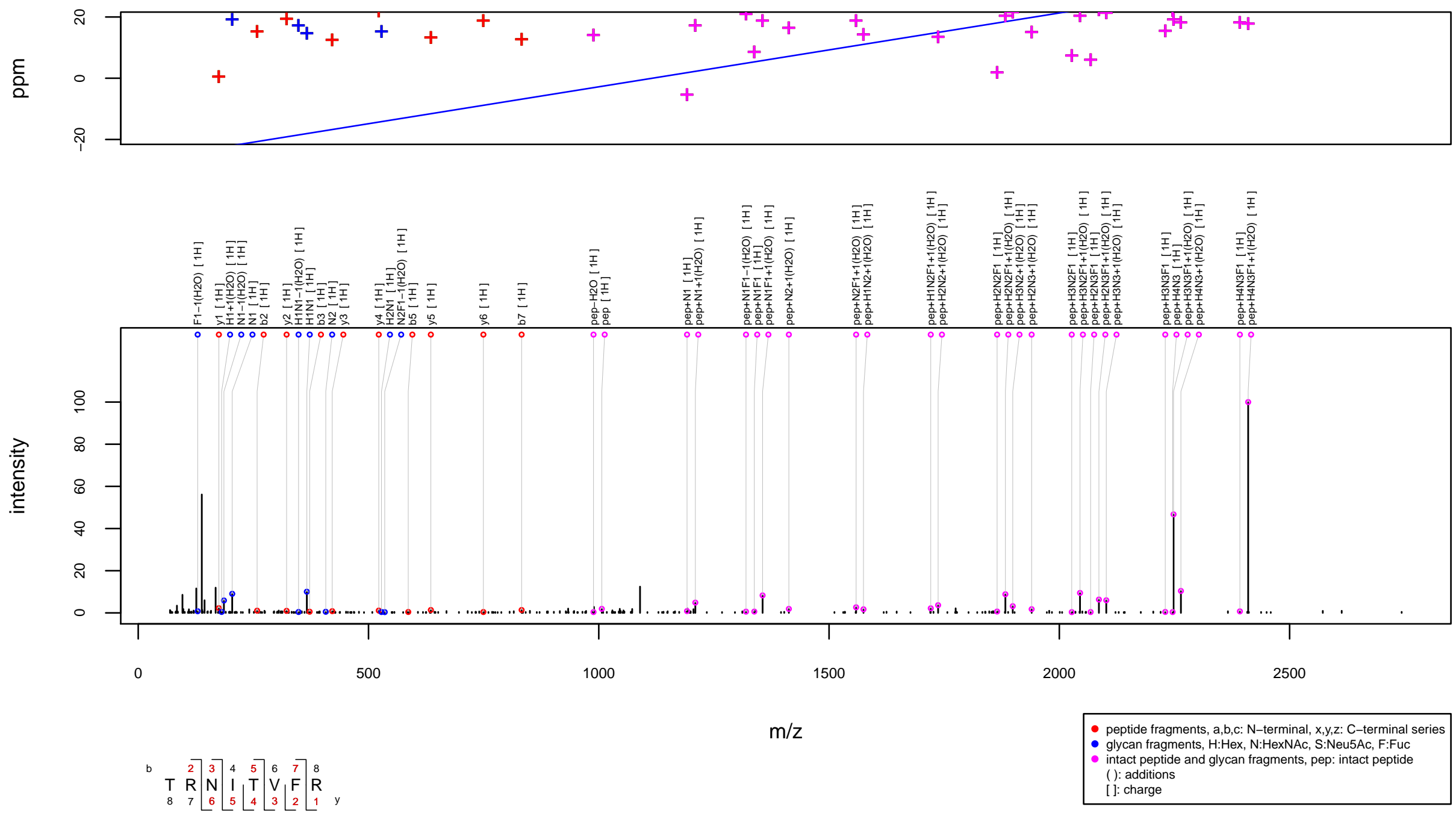
$\mathrm{m} / \mathrm{z} 936.667$ charge 4 scan $0-0$

Score $=46.68$, Hits $=35$, Explained Intensity $=0.32$

Peptide: CLUS_HUMAN[372,385]:LANLTQGEDQYYLR

Glycan: $\mathrm{SHNH}(\mathrm{HNH}) \mathrm{HN}(\mathrm{F}) \mathrm{N}, \mathrm{S} 1 \mathrm{H} 5 \mathrm{~N} 4 \mathrm{~F} 1$

Charge: $4 \mathrm{H}$
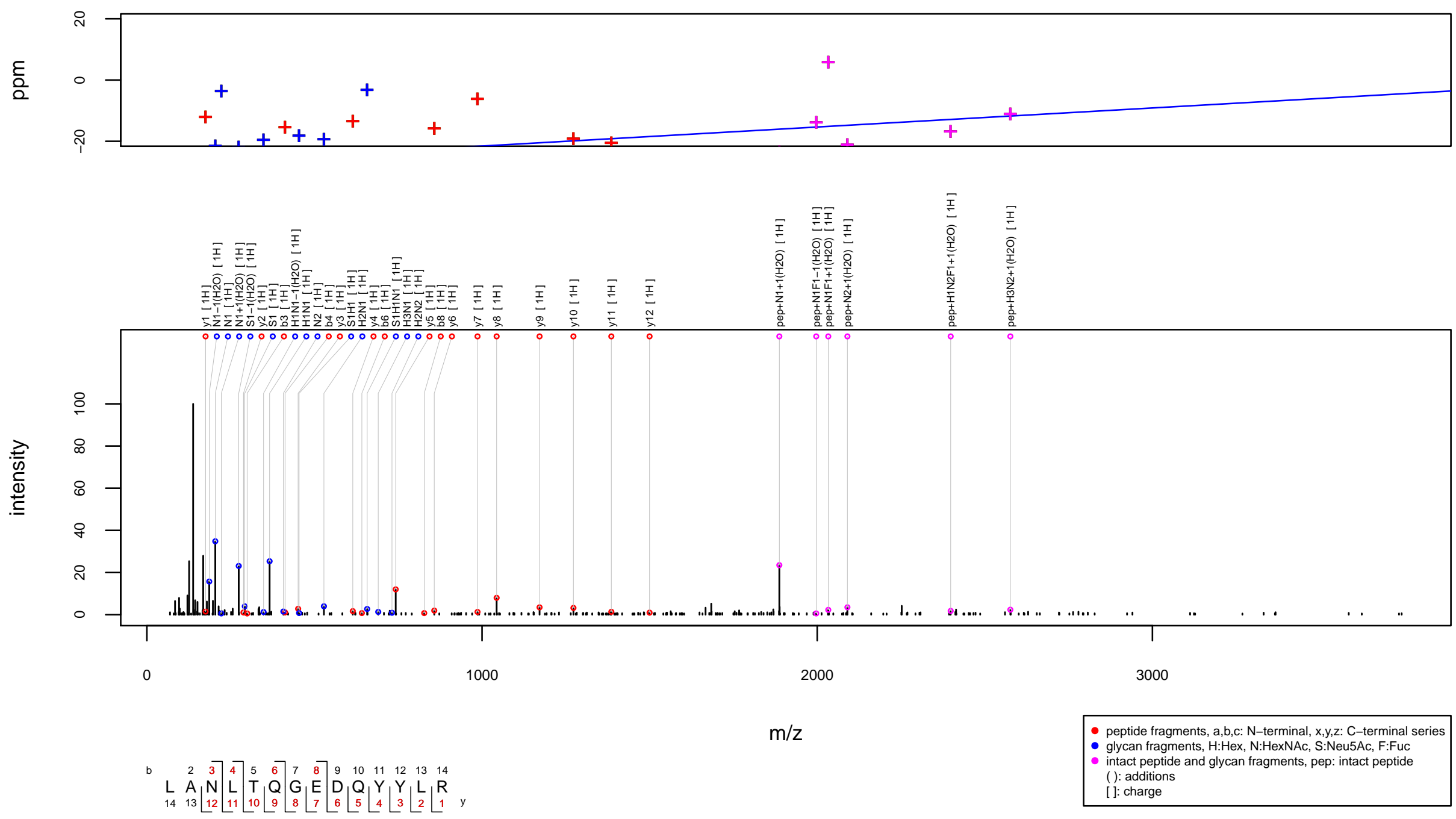
$\mathrm{m} / \mathrm{z} 936.8868$ charge 4 scan $0-0$

Score $=83.27$, Hits $=59$, Explained Intensity $=0.46$

Peptide: CLUS_HUMAN[372,385]:LANLTQGEDQYYLR

Glycan: $\mathrm{FH}(\mathrm{F}) \mathrm{NH}(\mathrm{HNH}) \mathrm{HN}(\mathrm{F}) \mathrm{N}, \mathrm{H} 5 \mathrm{~N} 4 \mathrm{~F} 3$

Charge: $4 \mathrm{H}$
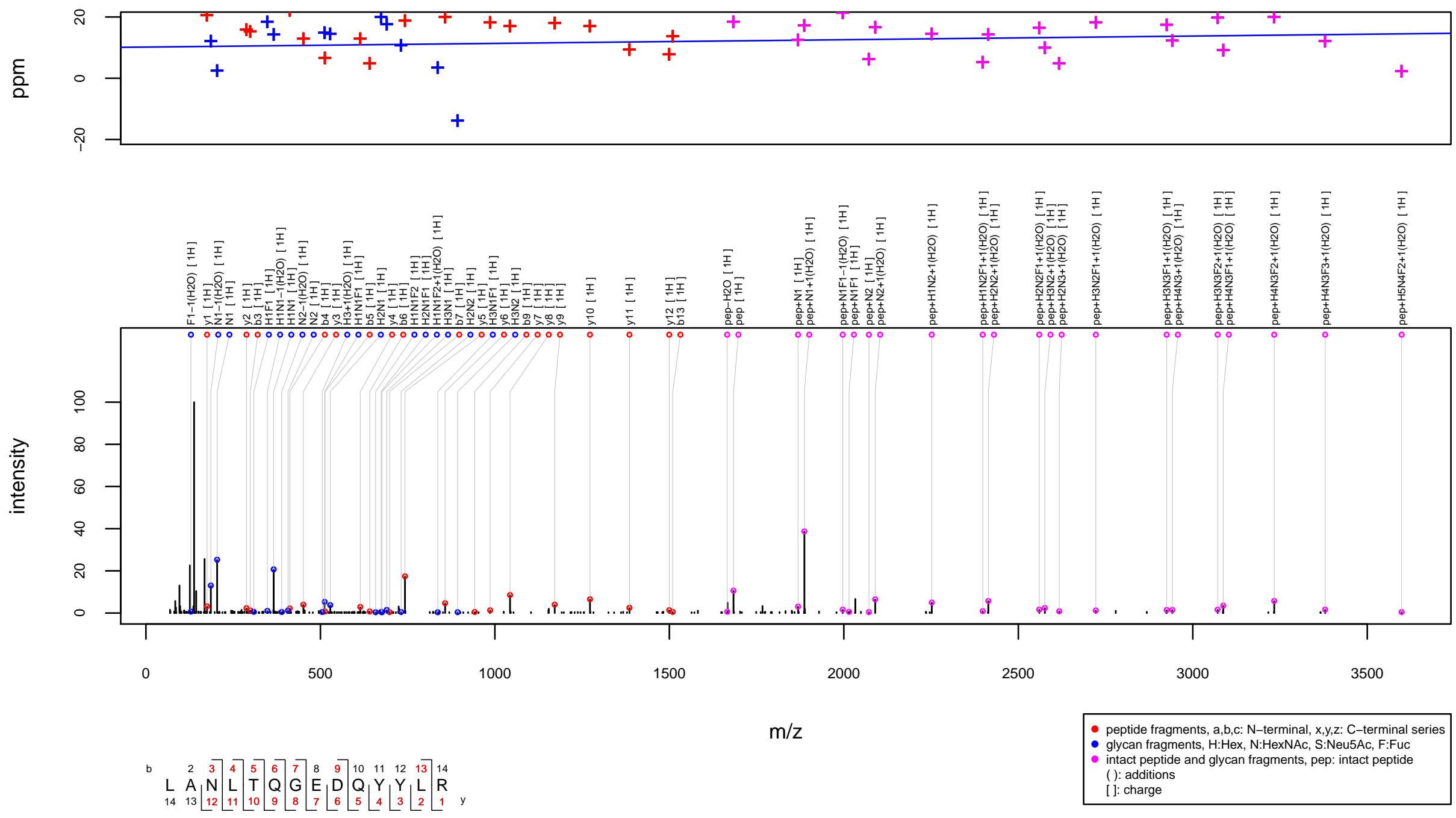
m/z 937.9322 charge 4 scan $0-0$

Score $=53.2$, Hits $=42$, Explained Intensity $=0.22$

Peptide: PIGR_HUMAN[466,479]:VPGNVTAVLGETLK

Glycan: $\mathrm{SHNH}(\mathrm{SHNH}) \mathrm{HN}(\mathrm{F}) \mathrm{N}, \mathrm{S} 2 \mathrm{H} 5 \mathrm{~N} 4 \mathrm{~F} 1$

Charge: $4 \mathrm{H}$
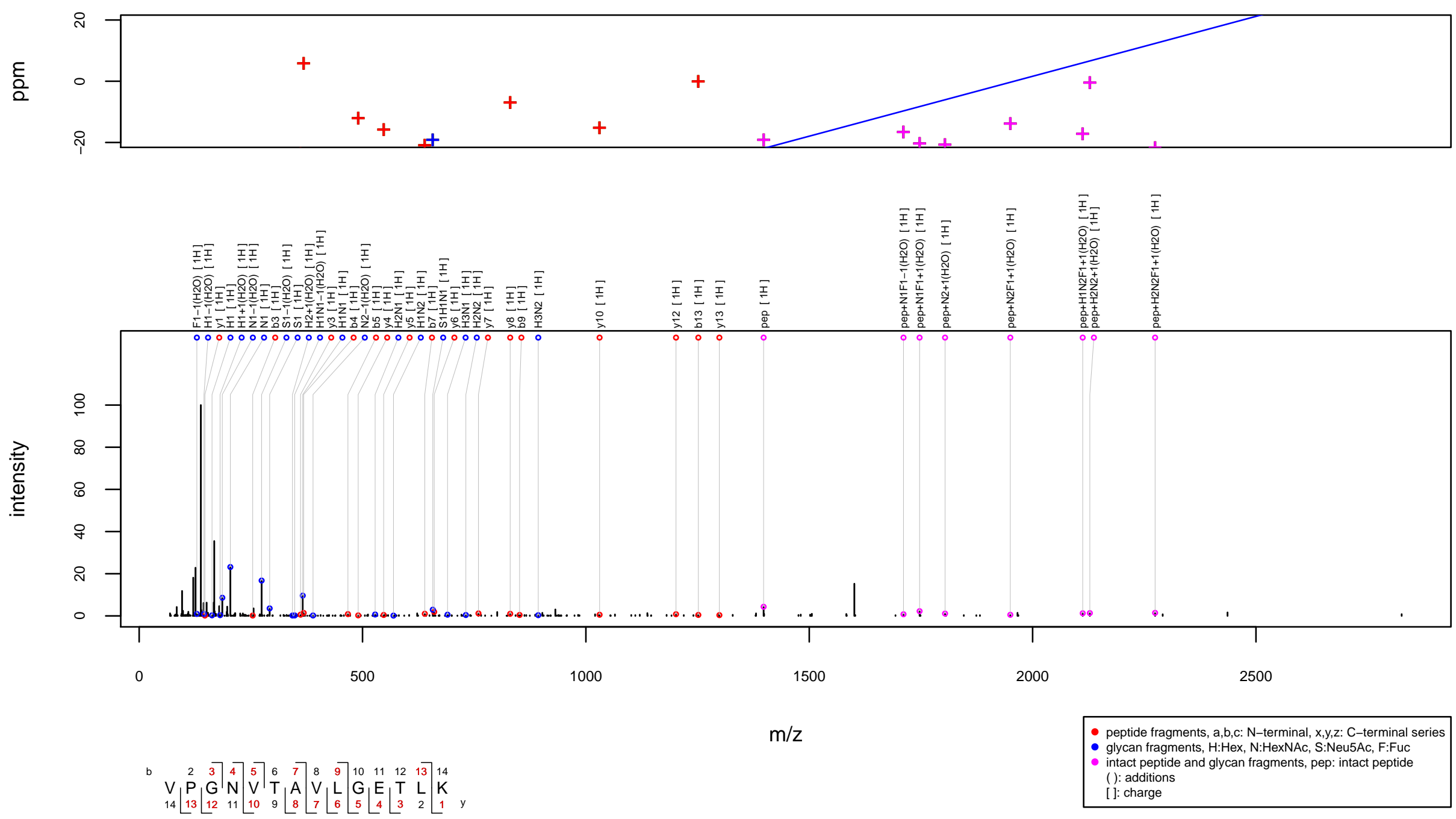
$\mathrm{m} / \mathbf{z} 938.7386$ charge 3 scan $0-0$

Score $=56.02$, Hits $=37$, Explained Intensity $=0.3$

Peptide: PPAP_HUMAN[92,98]:FLNESYK

Glycan: $\mathrm{SHNH}(\mathrm{HNH}) \mathrm{HNN}, \mathrm{S} 1 \mathrm{H} 5 \mathrm{~N} 4$

Charge: $3 \mathrm{H}$
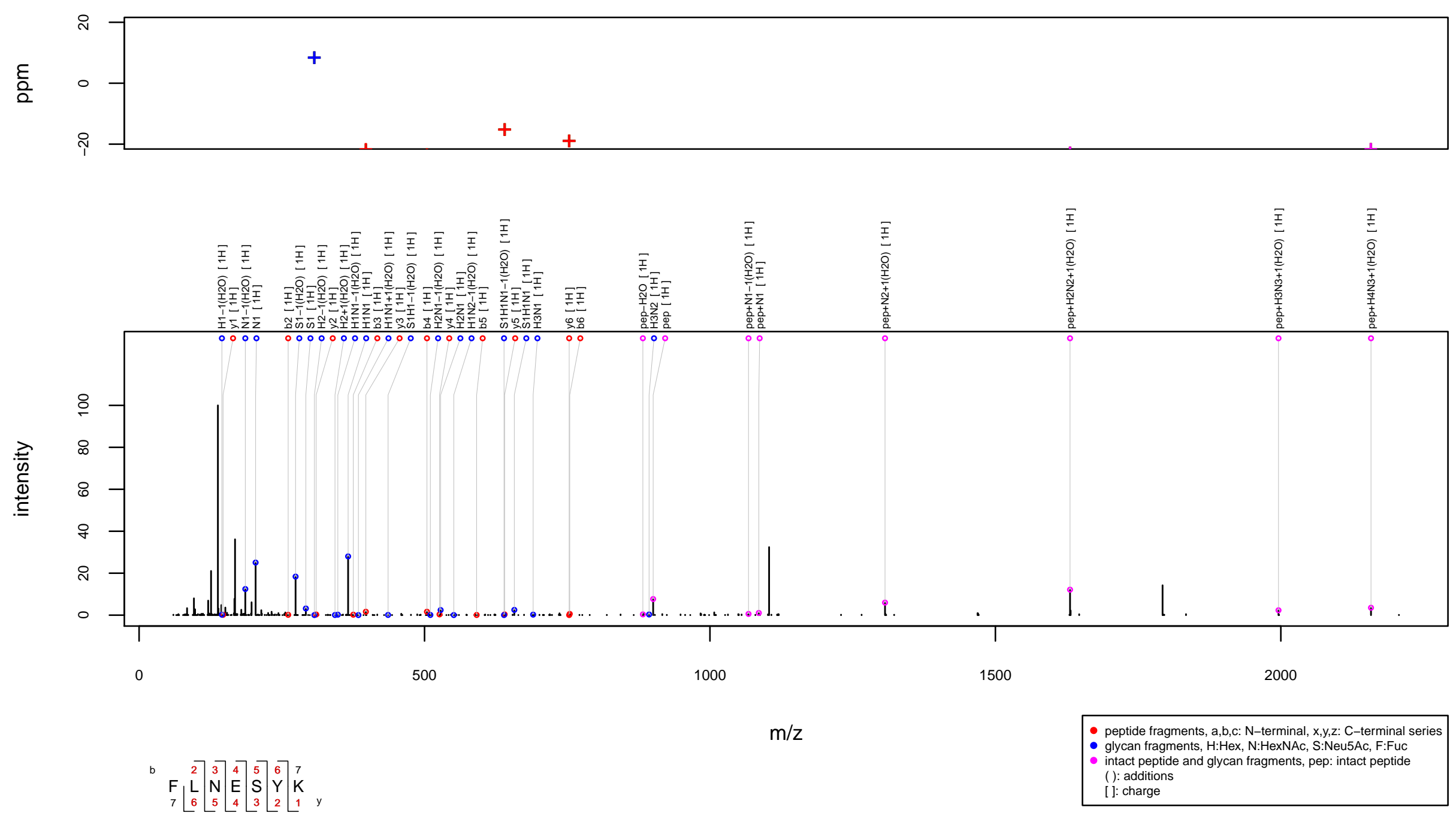
$\mathrm{m} / \mathrm{z} 941.1365$ charge 4 scan $0-0$

Score $=48.02$, Hits $=35$, Explained Intensity $=0.35$

Peptide: PPAP_HUMAN[92,98]:FLNESYK

Glycan: $\mathrm{SHNH}(\mathrm{SHN}(\mathrm{SHN}) \mathrm{H}) \mathrm{HNN}$, S3H6N5

Charge: $4 \mathrm{H}$
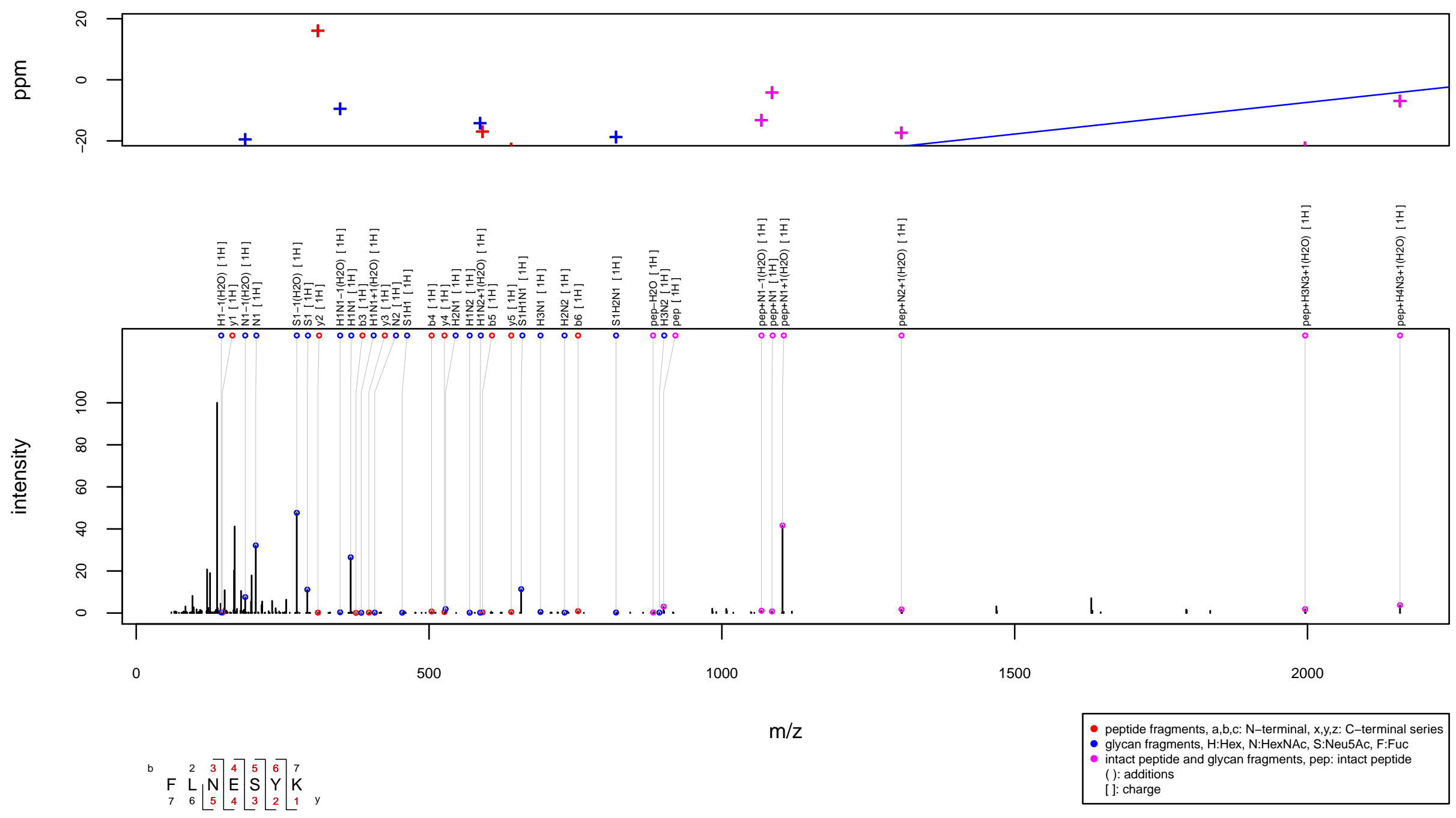
$\mathrm{m} / \mathrm{z} 944.2063$ charge 5 scan $0-0$

Score $=69.53$, Hits $=55$, Explained Intensity $=0.29$

Peptide: KLK11_HUMAN[120,140]:TATESFPHPGFNNSLPNKDHR

Glycan: $\mathrm{SHNH}(\mathrm{SHNH}) \mathrm{HN}(\mathrm{F}) \mathrm{N}, \mathrm{S} 2 \mathrm{H} 5 \mathrm{~N} 4 \mathrm{~F} 1$

Charge: $5 \mathrm{H}$
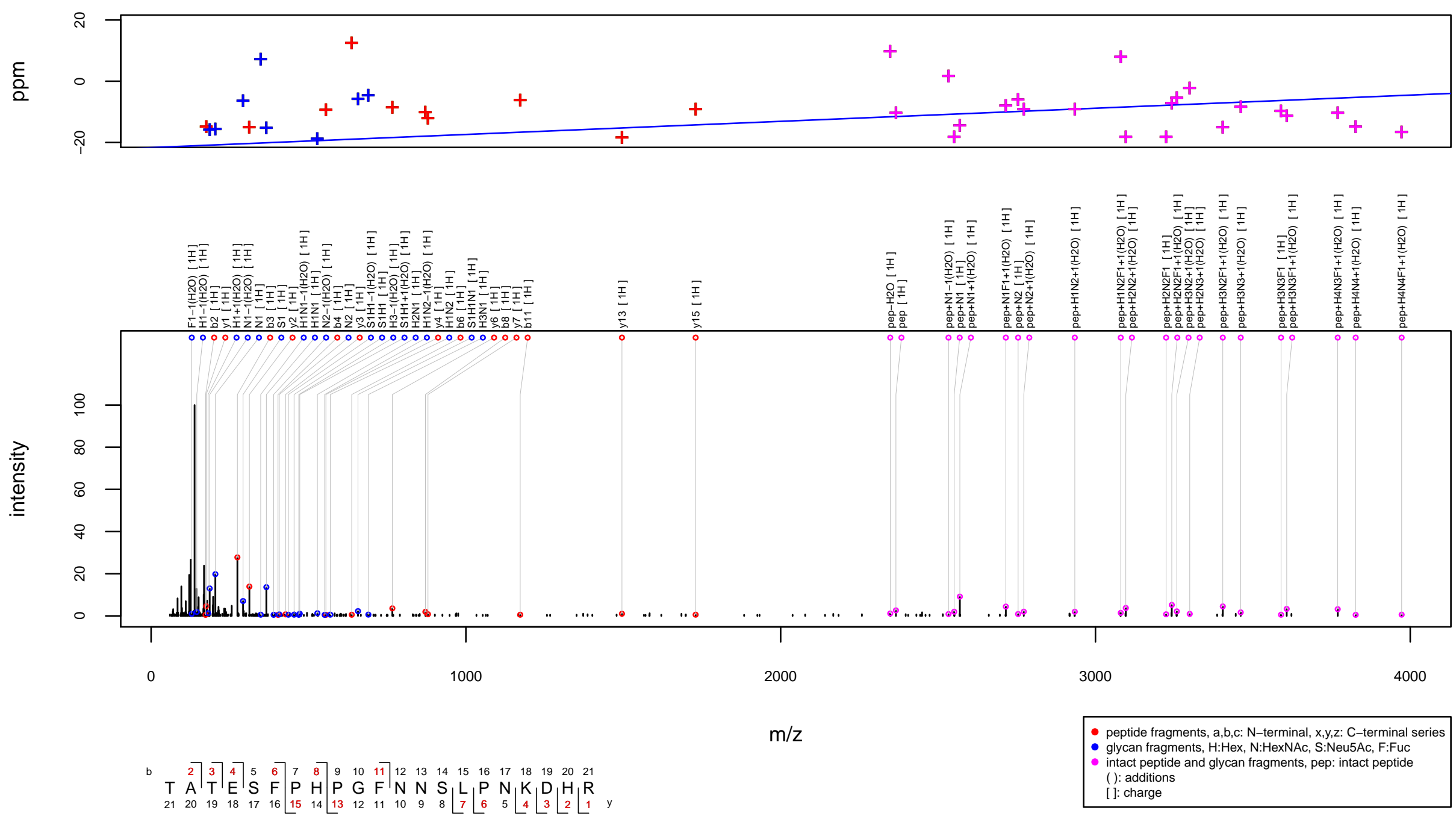
$\mathrm{m} / \mathrm{z} 944.6371$ charge 4 scan $0-0$

Score $=114.31$, Hits $=84$, Explained Intensity $=0.52$

Peptide: CLUS_HUMAN[80,89]:KKEDALNETR

Glycan: $\mathrm{F}(\mathrm{H}) \mathrm{NH}(\mathrm{F}(\mathrm{H}) \mathrm{N}(\mathrm{F}(\mathrm{H}) \mathrm{N}) \mathrm{H}) \mathrm{HN}(\mathrm{F}) \mathrm{N}, \mathrm{H} 6 \mathrm{~N} 5 \mathrm{~F} 4$

Charge: $4 \mathrm{H}$
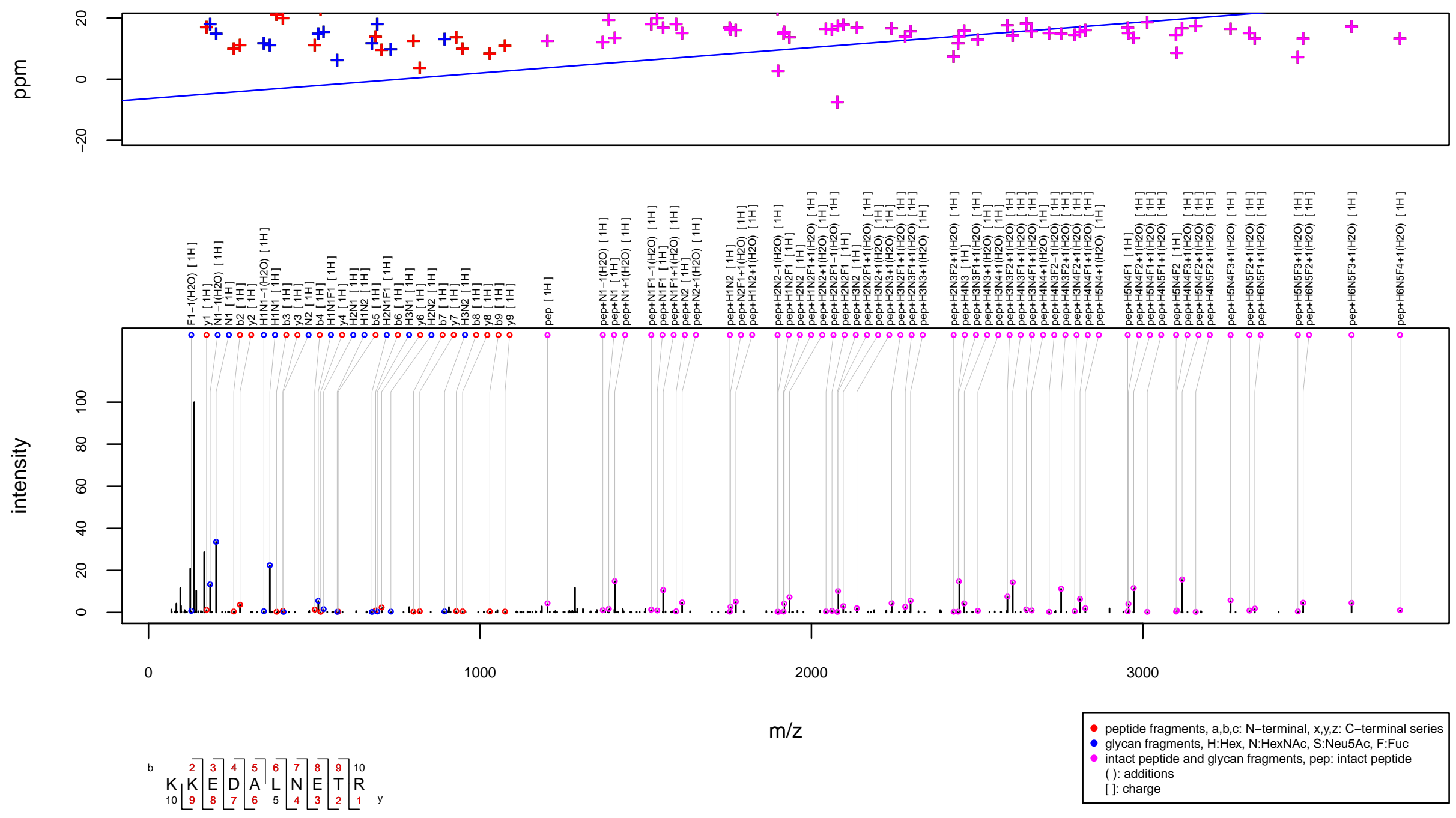
$\mathrm{m} / \mathrm{z} 944.7821$ charge 3 scan $0-0$

Score $=62.04$, Hits $=36$, Explained Intensity $=0.53$ Peptide: CBPE_HUMAN[138,150]:GNETIVNLIHSTR Glycan: $\mathrm{HH}(\mathrm{H}(\mathrm{H}) \mathrm{H}) \mathrm{HNN}, \mathrm{H} 6 \mathrm{~N} 2$

Charge: $3 \mathrm{H}$
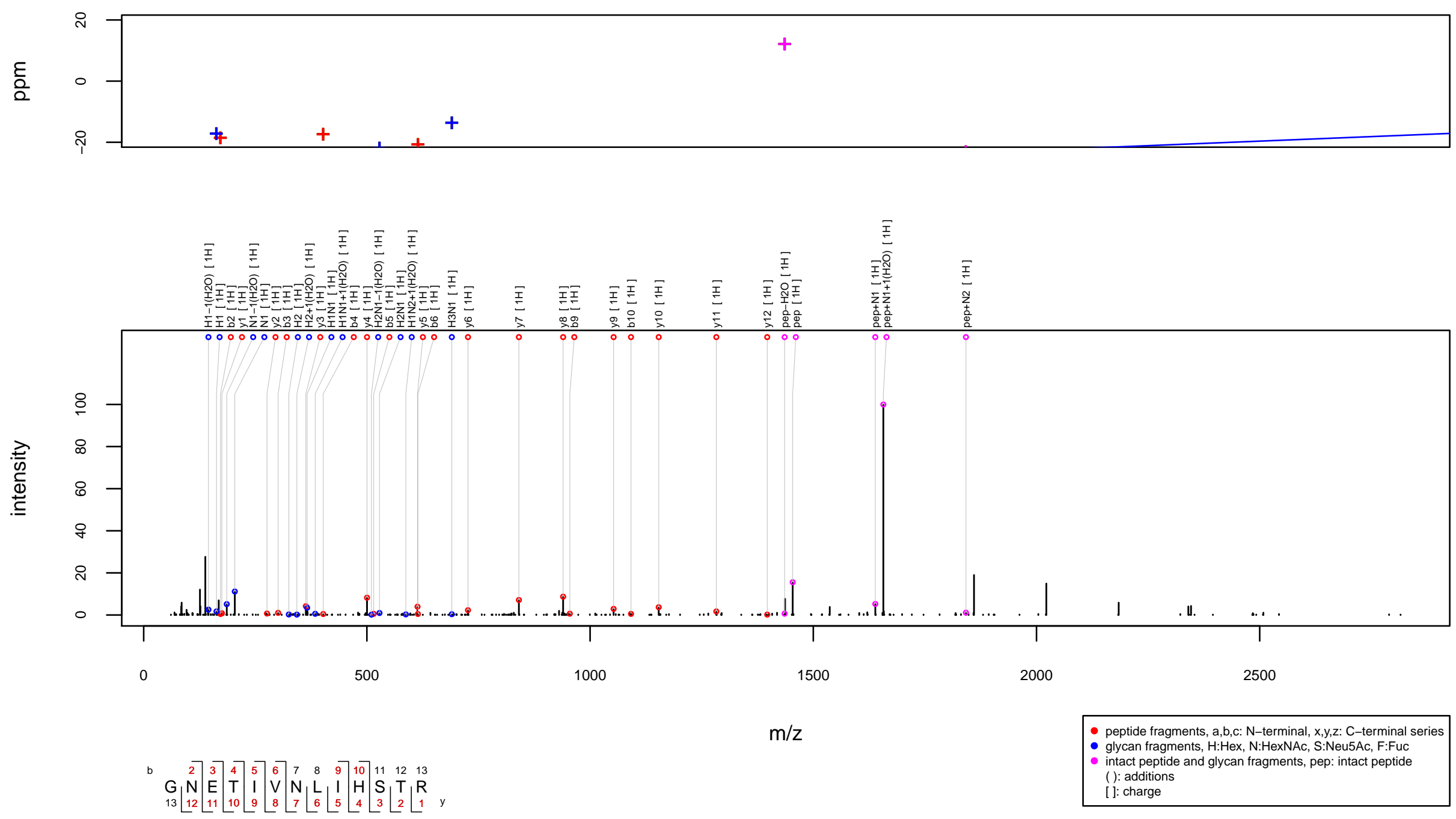
$\mathrm{m} / \mathrm{z} 951.1036$ charge 3 scan $0-0$

Score $=33.45$, Hits $=24$, Explained Intensity $=0.14$ Peptide: PREX2_HUMAN[61,71]:VDKNVTEETVK

Glycan: $\mathrm{SNNH}(\mathrm{H}) \mathrm{HNN}, \mathrm{S} 1 \mathrm{H} 3 \mathrm{~N} 4$

Charge: $3 \mathrm{H}$
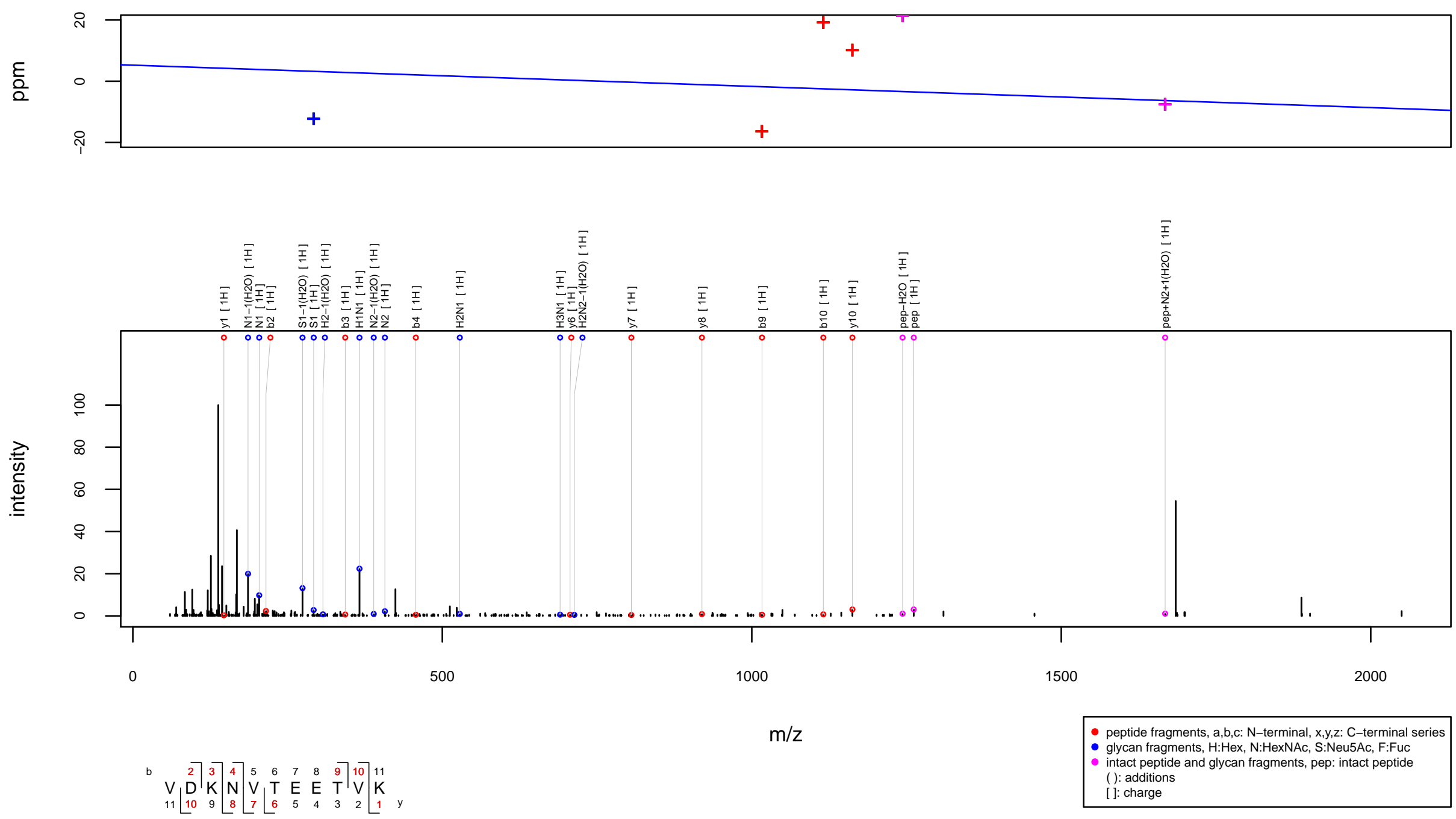
$\mathrm{m} / \mathrm{z} 951.4512$ charge 3 scan $0-0$

Score $=41.96$, Hits $=33$, Explained Intensity $=0.15$ Peptide: TMTC3_HUMAN[856,866]:QTQIVKTSDNK Glycan: H3N4F2

Charge: $3 \mathrm{H}$
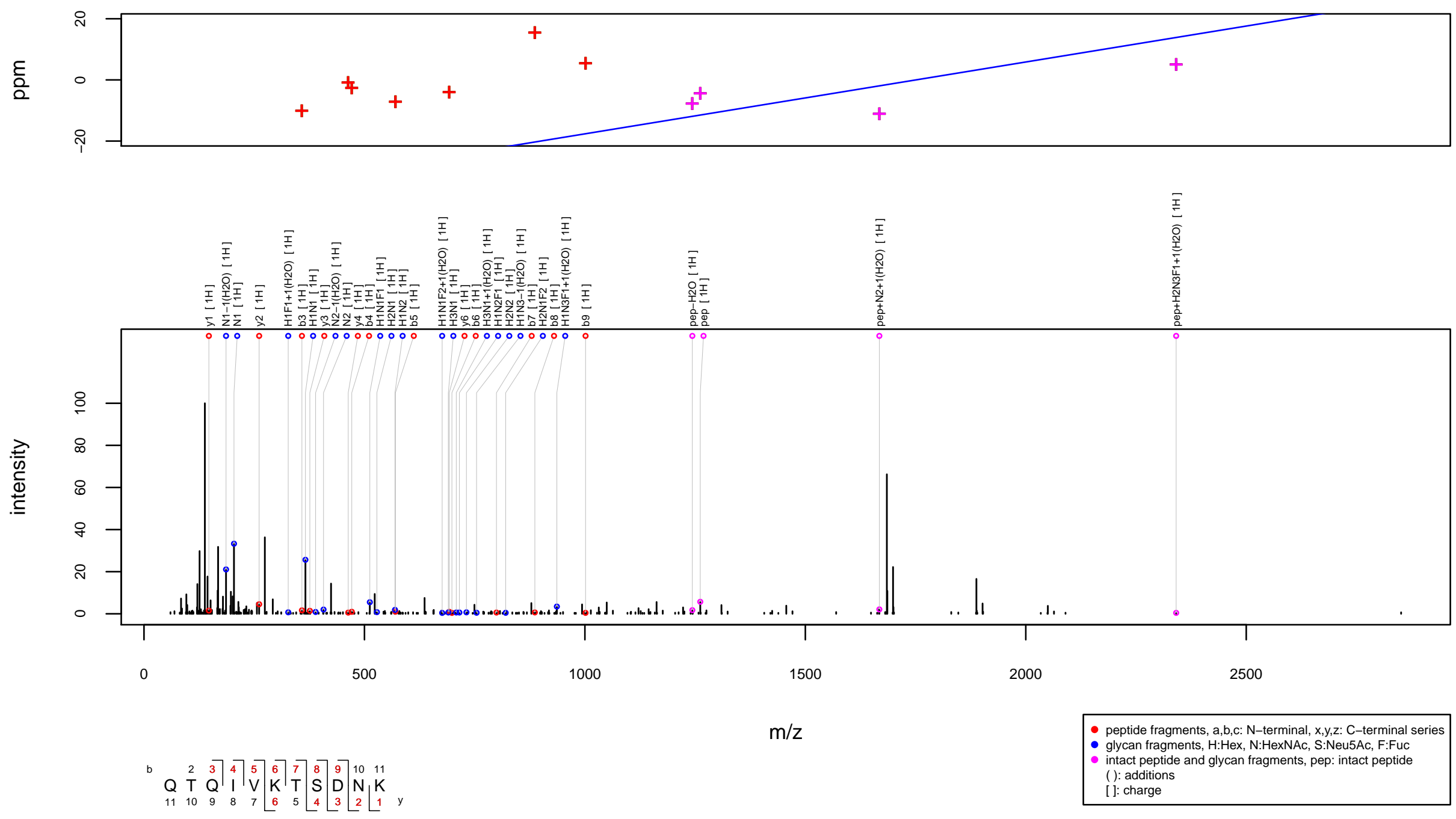
$\mathrm{m} / \mathrm{z} 951.6673$ charge 4 scan $0-0$

Score $=36.63$, Hits $=28$, Explained Intensity $=0.16$

Peptide: FINC_HUMAN[516,533]:DQCIVDDITYNVNDTFHK

Glycan: H4N4F1

Charge: $4 \mathrm{H}$
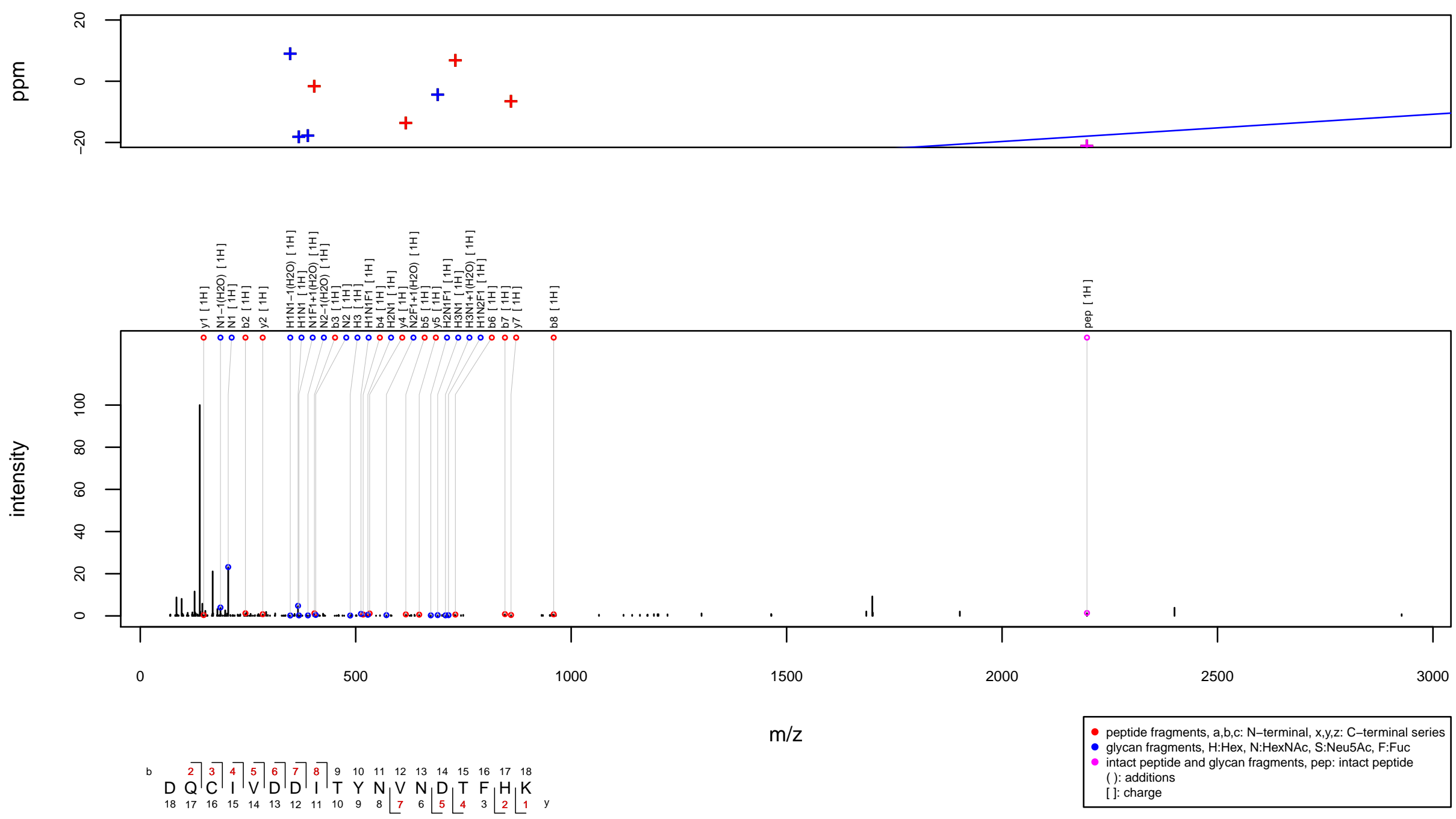
m/z 953.9296 charge 4 scan 0-0

Score $=55.48$, Hits $=42$, Explained Intensity $=0.36$

Peptide: TIMP1_HUMAN[46,60]:FVGTPEVNQTTLYQR

Glycan: $\mathrm{SHNH}(\mathrm{HNH}) \mathrm{HN}(\mathrm{F}) \mathrm{N}, \mathrm{S} 1 \mathrm{H} 5 \mathrm{~N} 4 \mathrm{~F} 1$

Charge: $4 \mathrm{H}$
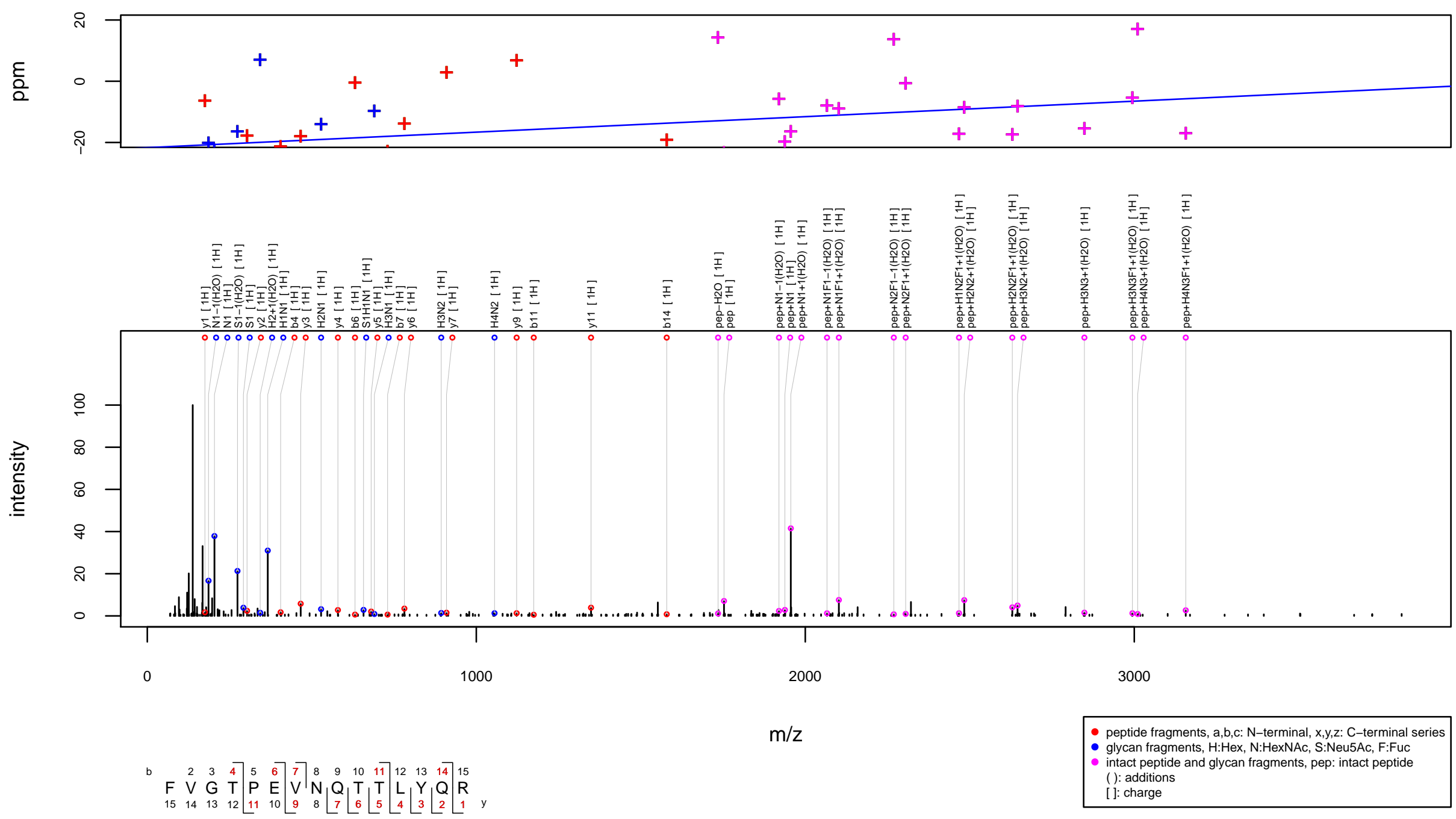
$\mathrm{m} / \mathrm{z} 954.1893$ charge 4 scan $0-0$

Score $=64.56$, Hits $=46$, Explained Intensity $=0.41$

Peptide: TIMP1_HUMAN[46,60]:FVGTPEVNQTTLYQR

Glycan: $\mathrm{FHNH}(\mathrm{FHNH}) \mathrm{HN}(\mathrm{F}) \mathrm{N}, \mathrm{H} 5 \mathrm{~N} 4 \mathrm{~F} 3$

Charge: $4 \mathrm{H}$
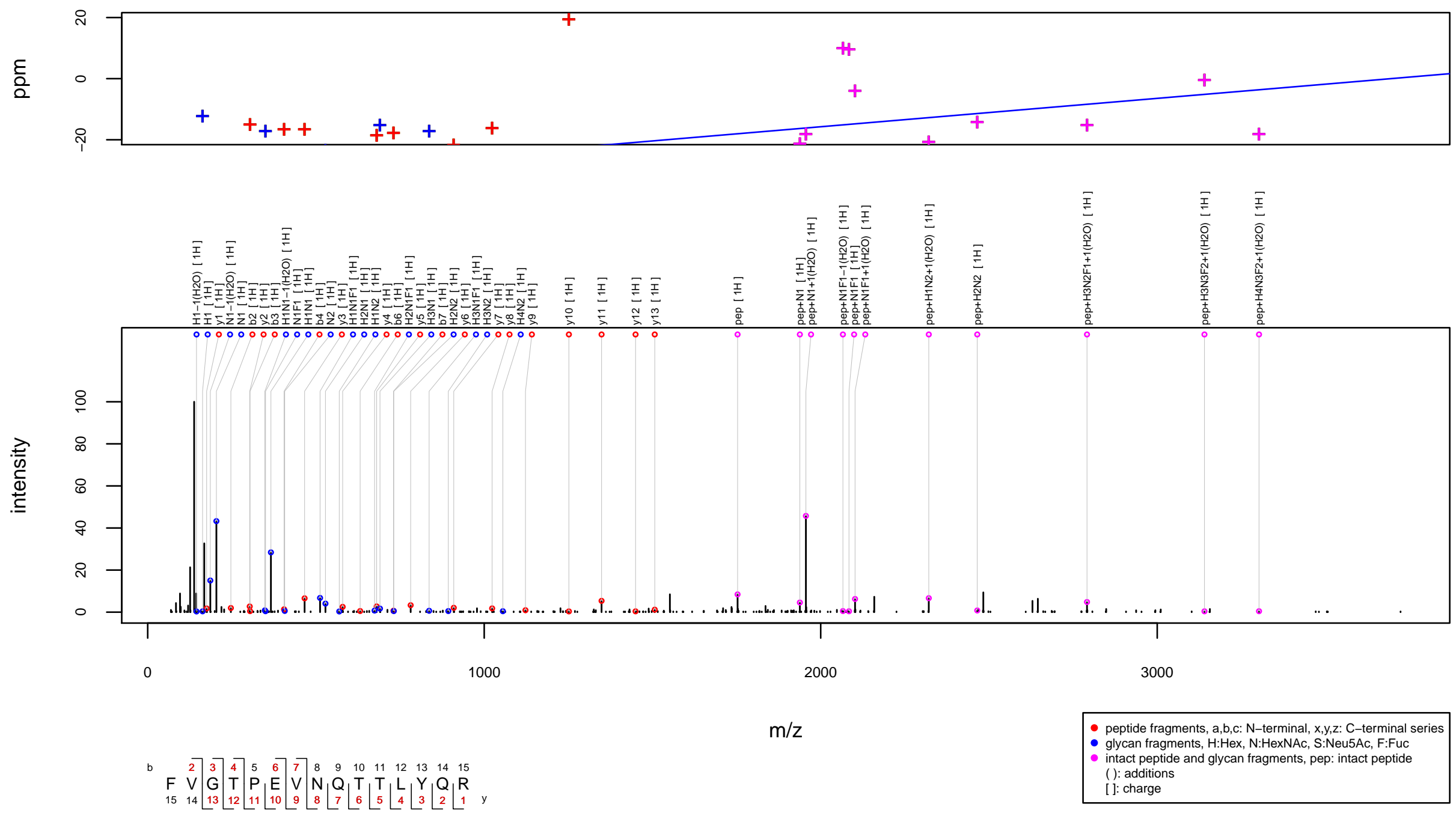
$\mathrm{m} / \mathrm{z} 954.389$ charge 3 scan $0-0$

Score $=91$, Hits $=58$, Explained Intensity $=0.53$

Peptide: SEMG2_HUMAN[272,282]:NLSQDQEHGRK

Glycan: $\mathrm{FHNH}(\mathrm{H}) \mathrm{HN}(\mathrm{F}) \mathrm{N}, \mathrm{H} 4 \mathrm{~N} 3 \mathrm{~F} 2$

Charge: $3 \mathrm{H}$
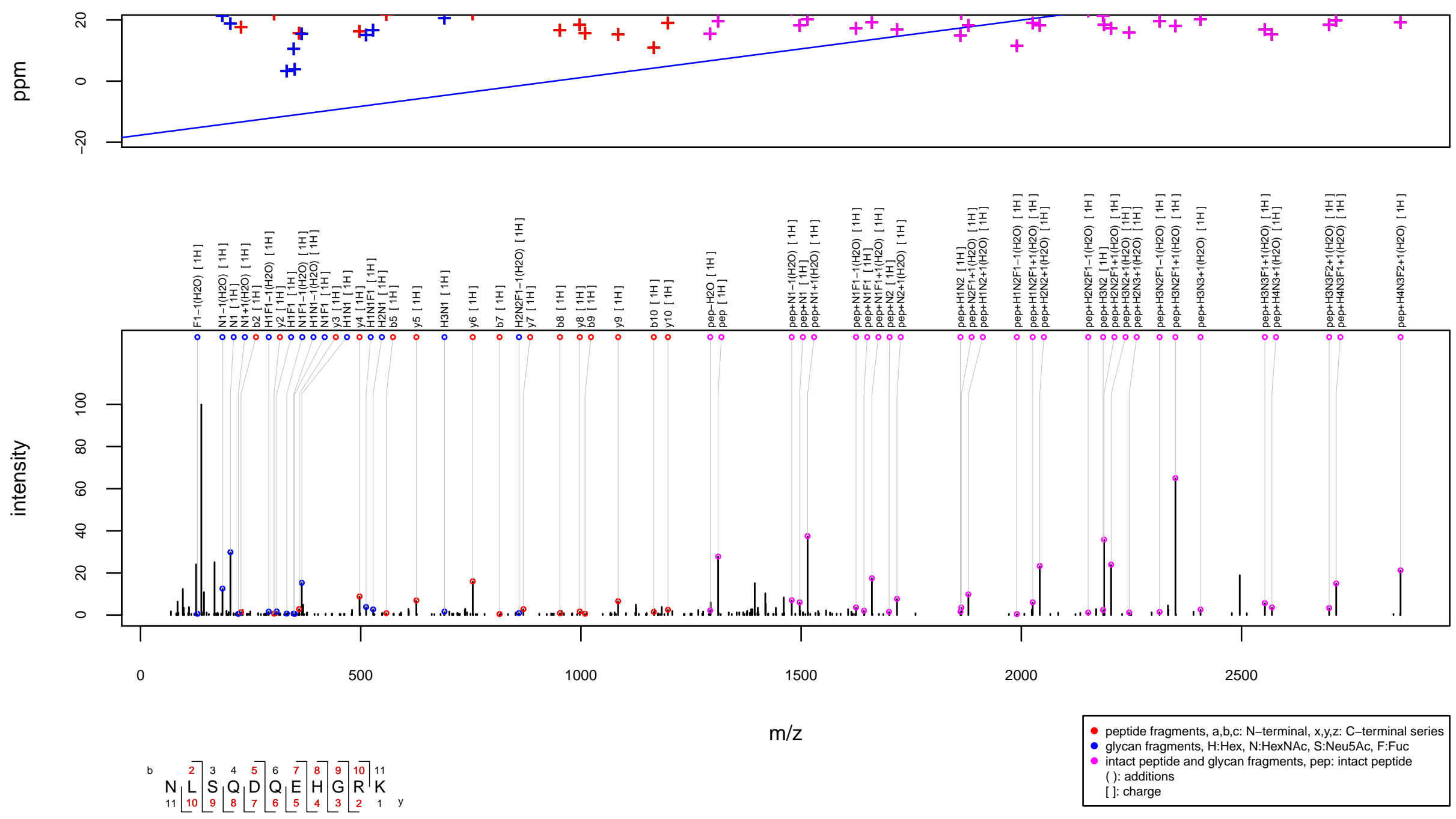
$\mathrm{m} / \mathrm{z} 954.8487$ charge 5 scan $0-0$

Score $=45.87$, Hits $=33$, Explained Intensity $=0.27$

Peptide: CBPE_HUMAN[382,406]:DLQGNPIANATISVEGIDHDVTSAK

Glycan: $\mathrm{SHNH}(\mathrm{SHNH}) \mathrm{HNN}, \mathrm{S} 2 \mathrm{H} 5 \mathrm{~N} 4$

Charge: $5 \mathrm{H}$
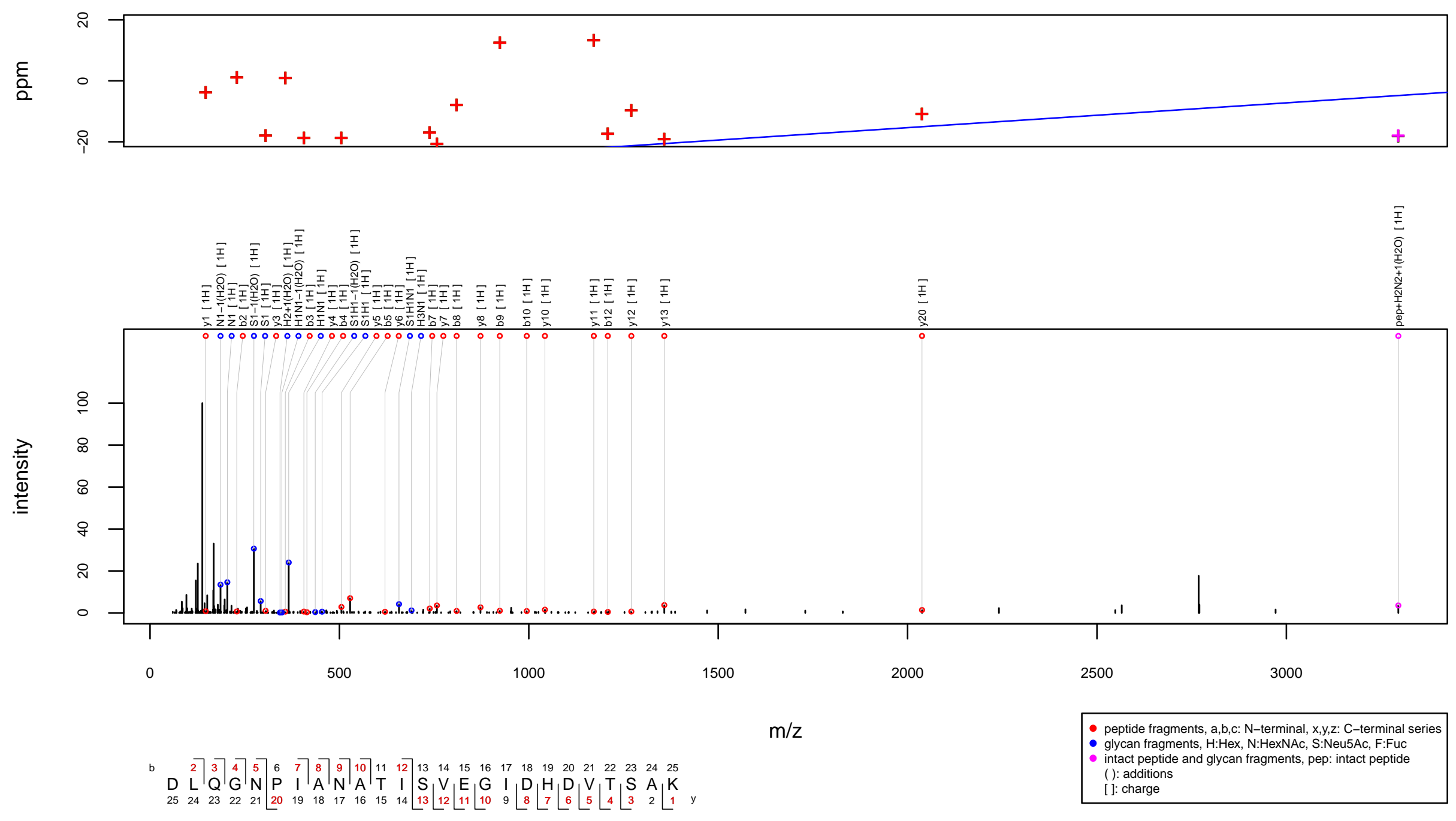
m/z 955.1741 charge 4 scan $0-0$

Score $=52.19$, Hits $=37$, Explained Intensity $=0.28$

Peptide: CLUS_HUMAN[372,385]:LANLTQGEDQYYLR

Glycan: $\mathrm{HNHNH}(\mathrm{F}(\mathrm{H}) \mathrm{NH}) \mathrm{HNN}, \mathrm{H} 6 \mathrm{~N} 5 \mathrm{~F} 1$

Charge: $4 \mathrm{H}$
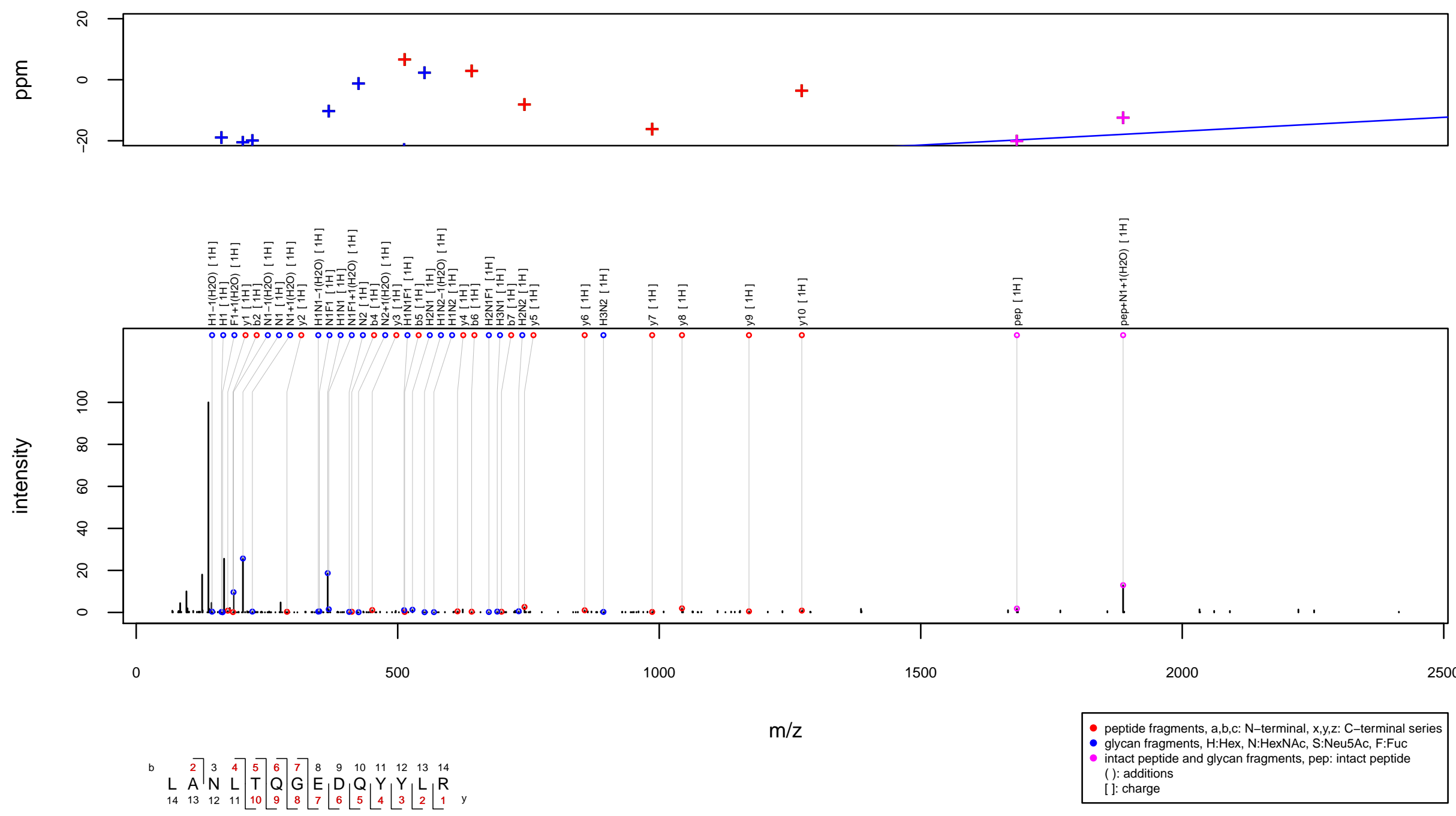
m/z 955.6932 charge 4 scan 0-0

Score $=44.77$, Hits $=32$, Explained Intensity $=0.37$

Peptide: QSOX1_HUMAN[130,147]:NGSGAVFPVAGADVQTLR

Glycan: $\mathrm{FHNH}(\mathrm{FHNH}) \mathrm{HN}(\mathrm{F}) \mathrm{N}, \mathrm{H} 5 \mathrm{~N} 4 \mathrm{~F} 3$

Charge: $4 \mathrm{H}$
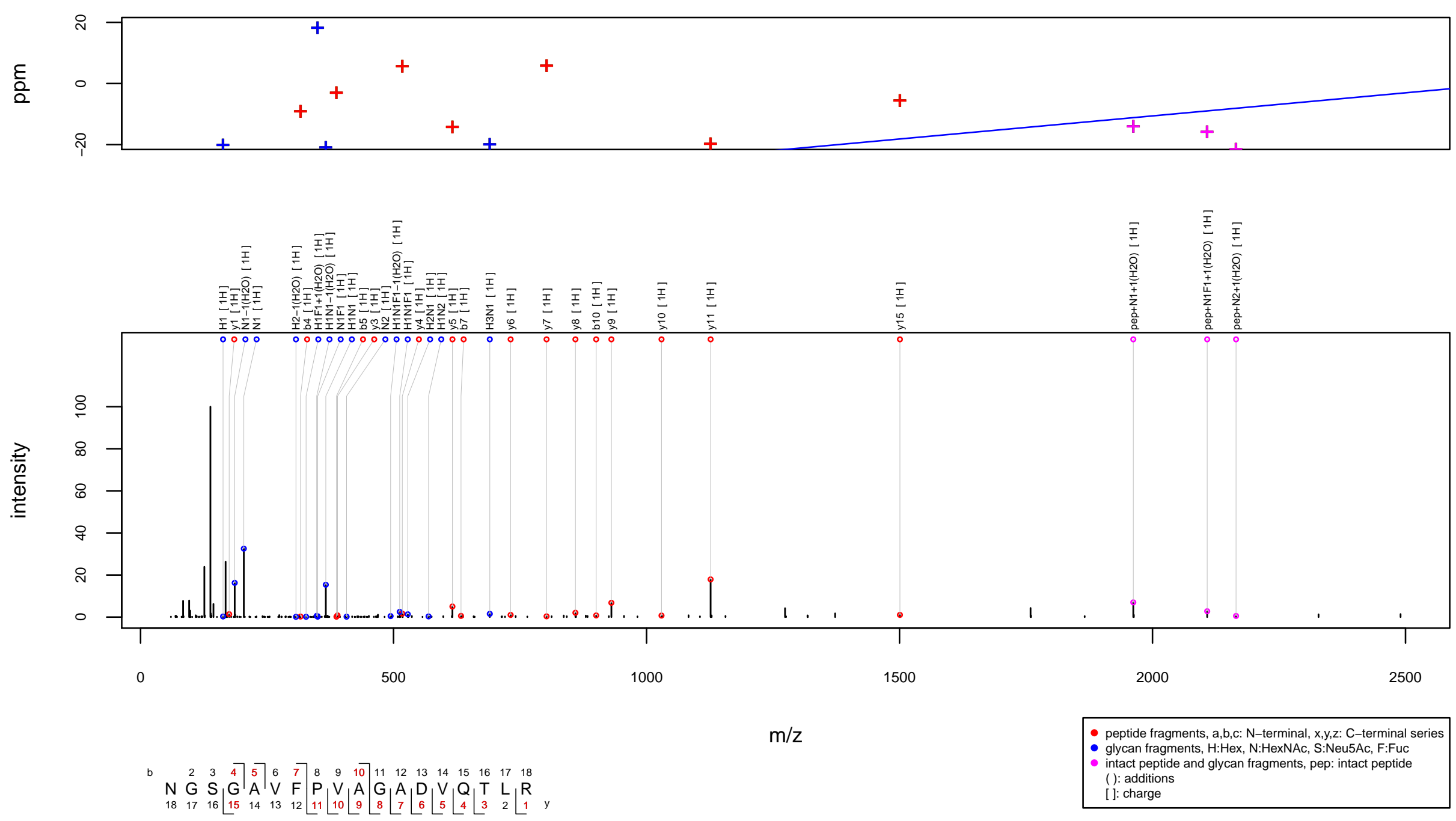
$\mathrm{m} / \mathrm{z} 964.7293$ charge 4 scan $0-0$

Score $=52.34$, Hits $=35$, Explained Intensity $=0.26$

Peptide: AMPN_HUMAN[674,690]:VPVTLALNNTLFLIEER

Glycan: $\mathrm{SHNH}(\mathrm{HNH}) \mathrm{HNN}, \mathrm{S} 1 \mathrm{H} 5 \mathrm{~N} 4$

Charge: $4 \mathrm{H}$
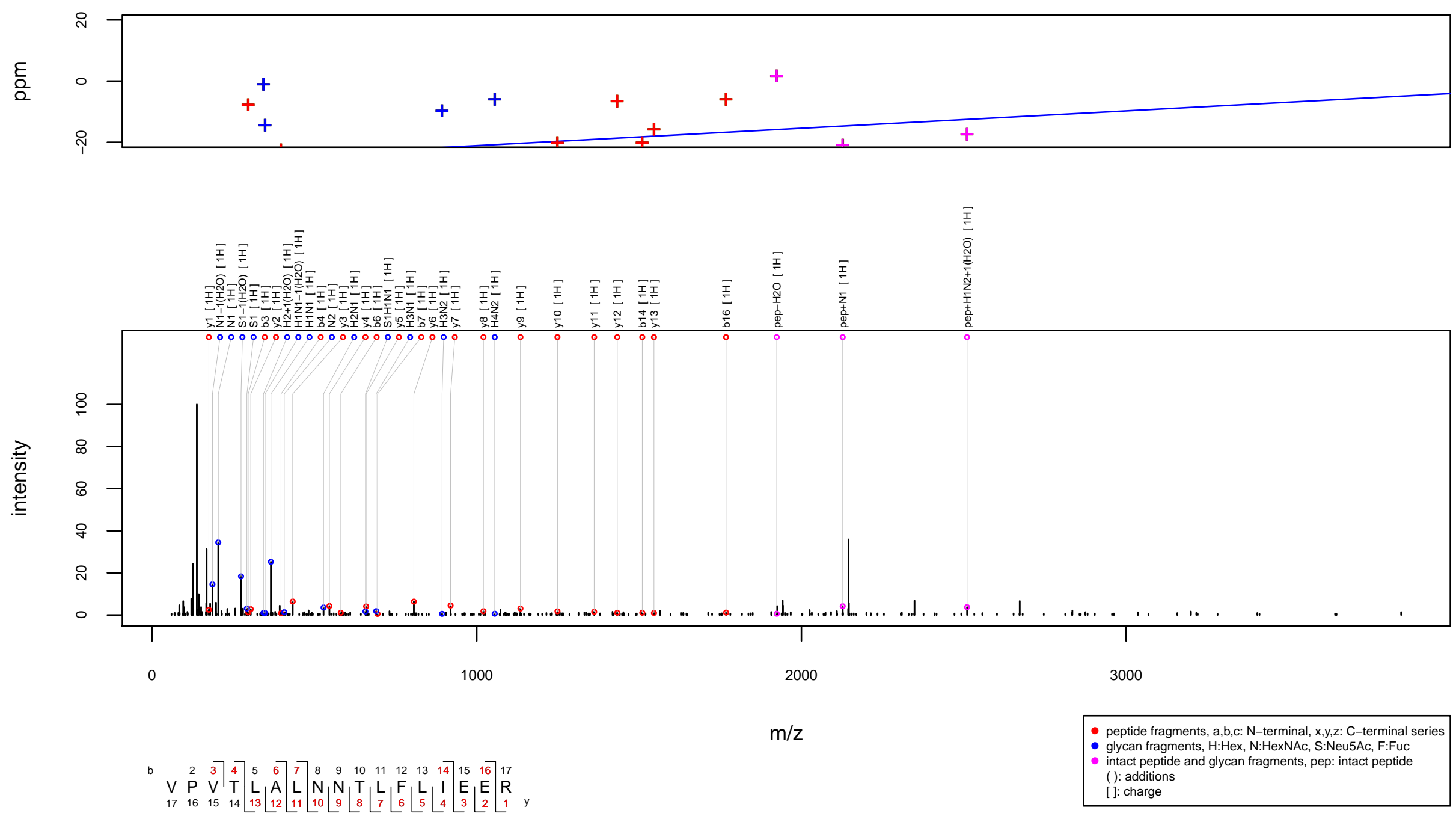
$\mathrm{m} / \mathrm{z} 966.883$ charge 4 scan $0-0$

Score $=71.31$, Hits $=51$, Explained Intensity $=0.35$ Peptide: NBL1_HUMAN[38,51]:NITQIVGHSGCEAK Glycan: $\mathrm{SHNH}(\mathrm{SHNH}) \mathrm{HN}(\mathrm{F}) \mathrm{N}, \mathrm{S} 2 \mathrm{H} 5 \mathrm{~N} 4 \mathrm{~F} 1$

Charge: $4 \mathrm{H}$
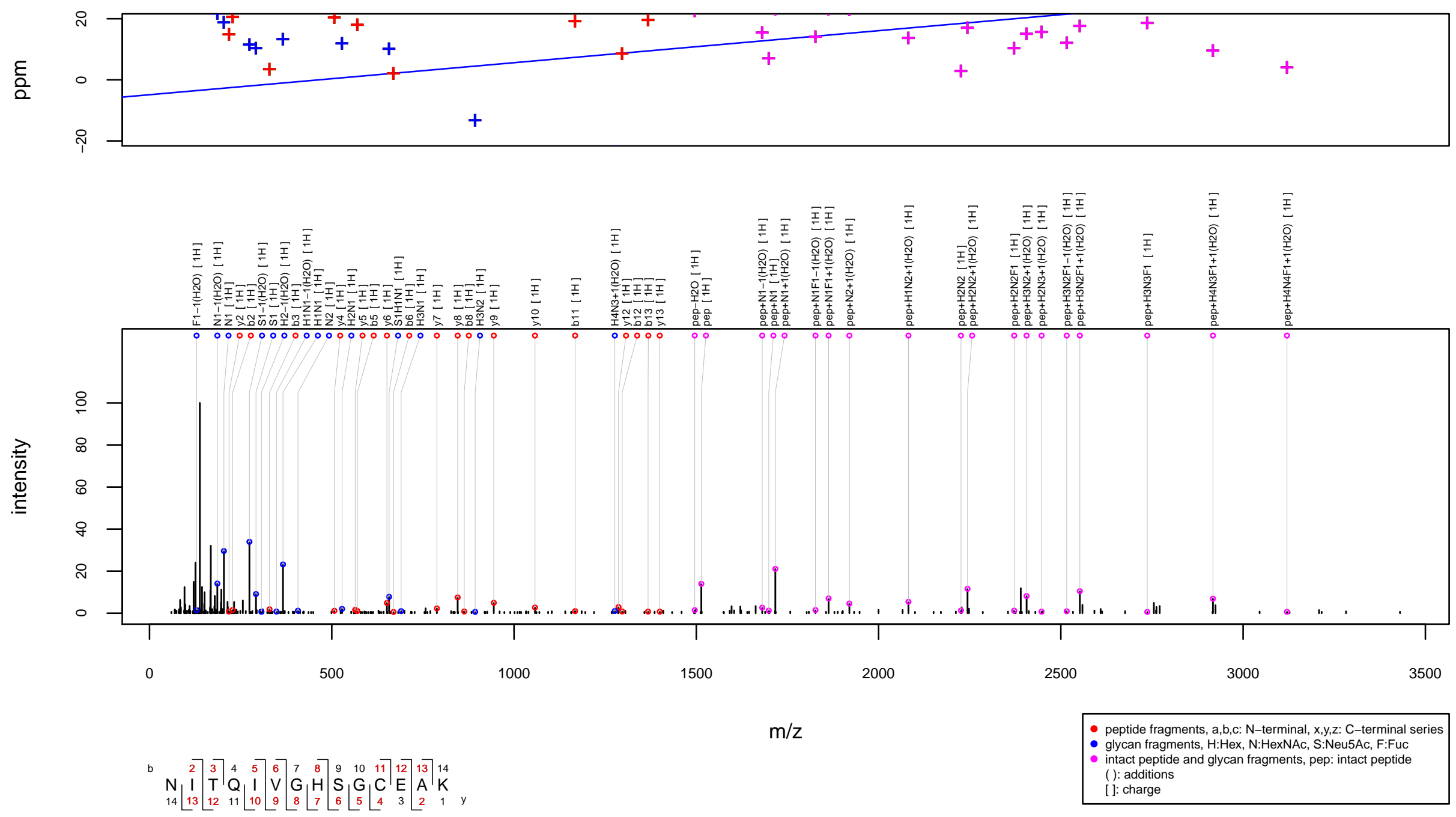
$\mathrm{m} / \mathrm{z} 973.1835$ charge 4 scan $0-0$

Score $=65.12$, Hits $=48$, Explained Intensity $=0.44$ Peptide: CLUS_HUMAN[372,385]:LANLTQGEDQYYLR Glycan: $\mathrm{SHNH}(\mathrm{FHNH}) \mathrm{HN}(\mathrm{F}) \mathrm{N}, \mathrm{S} 1 \mathrm{H} 5 \mathrm{~N} 4 \mathrm{~F} 2$

Charge: $4 \mathrm{H}$
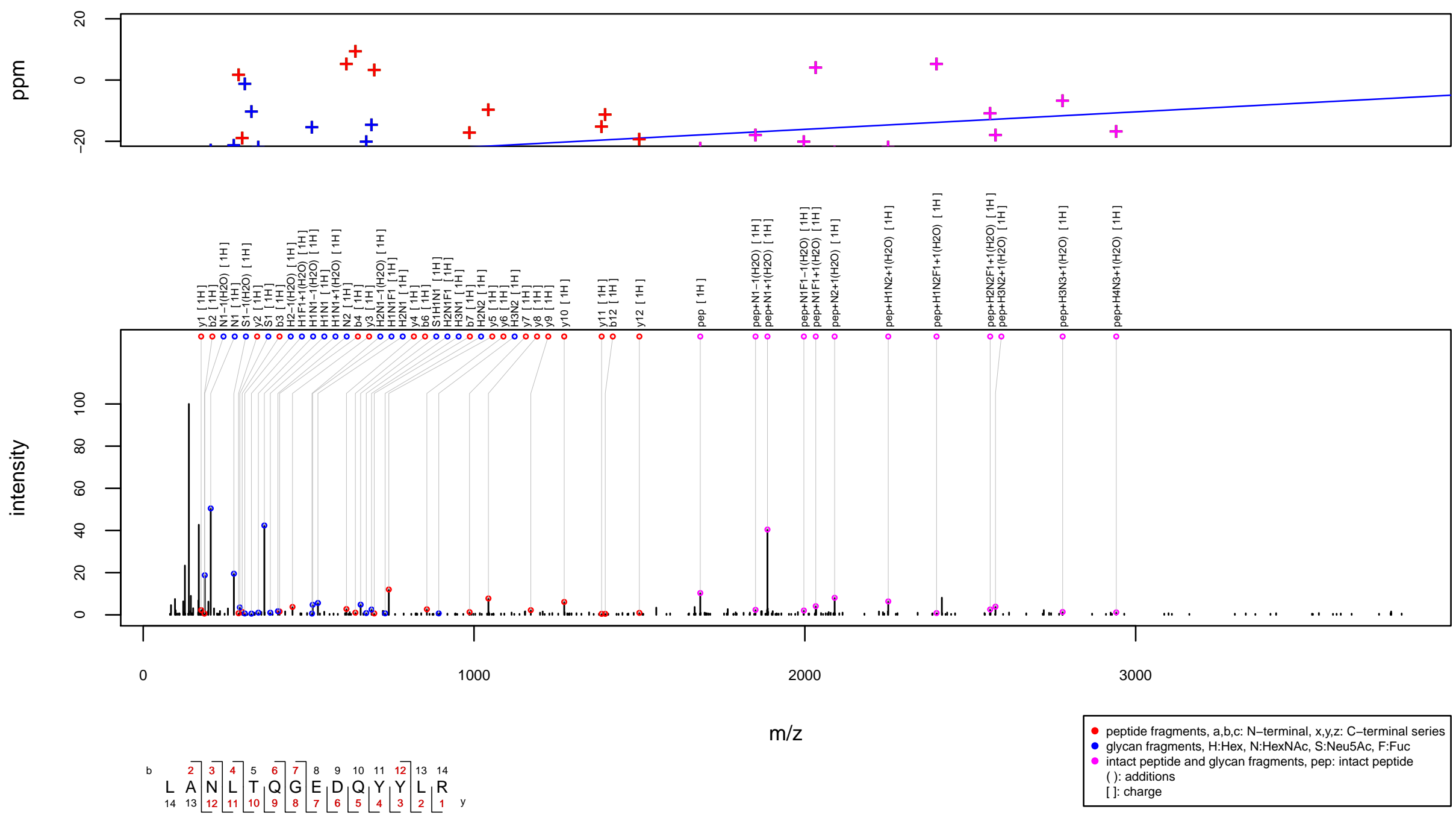
$\mathrm{m} / \mathrm{z} 981.4209$ charge 3 scan $0-0$

Score $=71.53$, Hits $=46$, Explained Intensity $=0.32$ Peptide: PPAP_HUMAN[91,98]:KFLNESYK

Glycan: $\mathrm{SHNH}(\mathrm{HNH}) \mathrm{HNN}, \mathrm{S} 1 \mathrm{H} 5 \mathrm{~N} 4$

Charge: $3 \mathrm{H}$
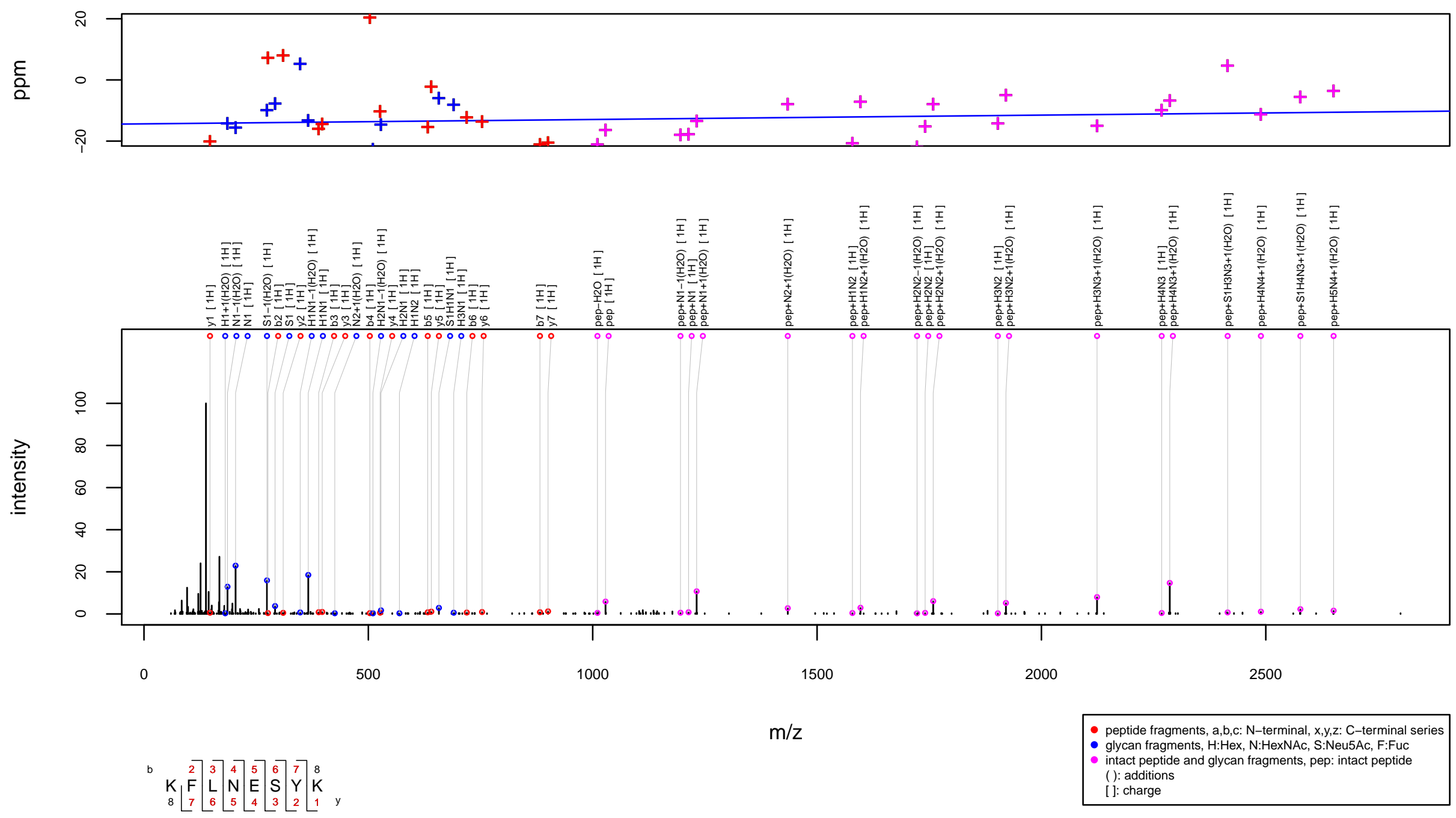
$\mathrm{m} / \mathrm{z} 981.7139$ charge 4 scan $0-0$

Score $=39.5$, Hits $=32$, Explained Intensity $=0.24$

Peptide: SAP_HUMAN[214,227]:TNSTFVQALVEHVK

Glycan: $\mathrm{SHNH}(\mathrm{SHNH}) \mathrm{HN}(\mathrm{F}) \mathrm{N}, \mathrm{S} 2 \mathrm{H} 5 \mathrm{~N} 4 \mathrm{~F} 1$

Charge: $4 \mathrm{H}$
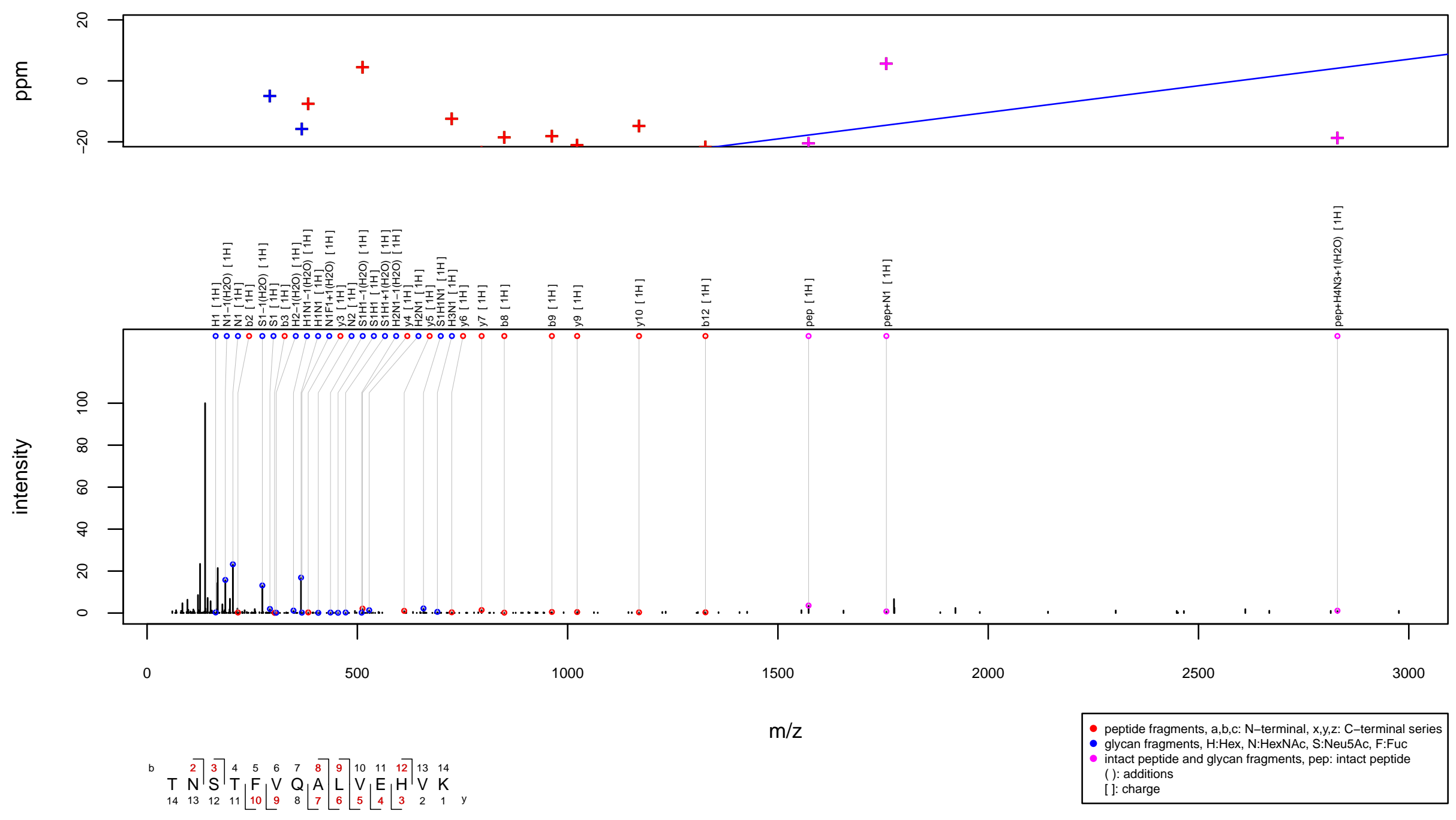
m/z 982.9459 charge 4 scan 0-0

Score $=29.39$, Hits $=32$, Explained Intensity $=0.12$ Peptide: EFNA5_HUMAN[32,42]:YAVYWNSSNPR Glycan: H6N5F4

Charge: $4 \mathrm{H}$
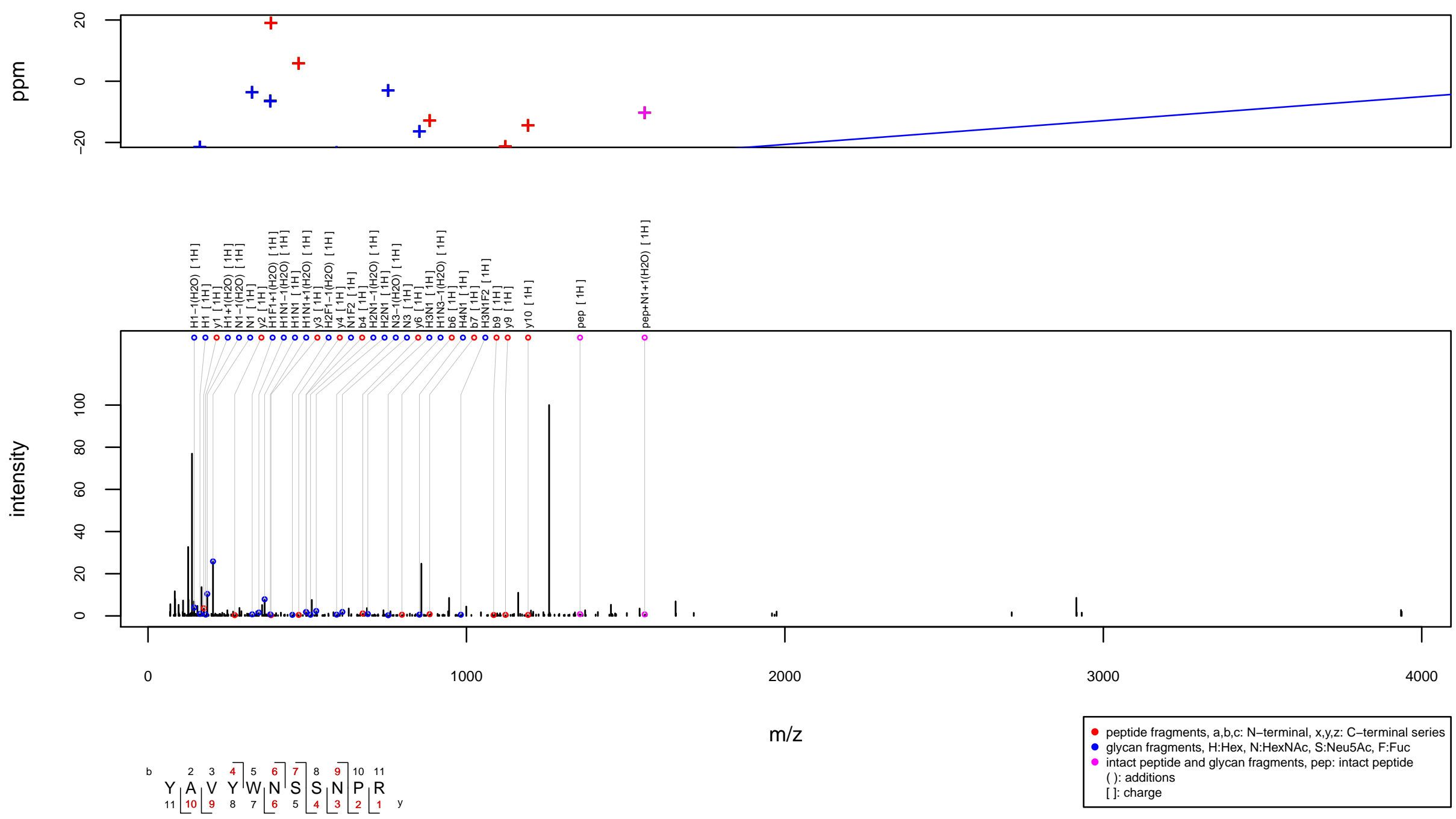
$\mathrm{m} / \mathrm{z} 984.7131$ charge 3 scan $0-0$

Score $=84.35$, Hits $=57$, Explained Intensity $=0.42$ Peptide: SEMG2_HUMAN[272,281]:NLSQDQEHGR Glycan: $\mathrm{HN}(\mathrm{HN}) \mathrm{H}(\mathrm{H}) \mathrm{HN}(\mathrm{F}) \mathrm{N}, \mathrm{H} 5 \mathrm{~N} 4 \mathrm{~F} 1$

Charge: $3 \mathrm{H}$
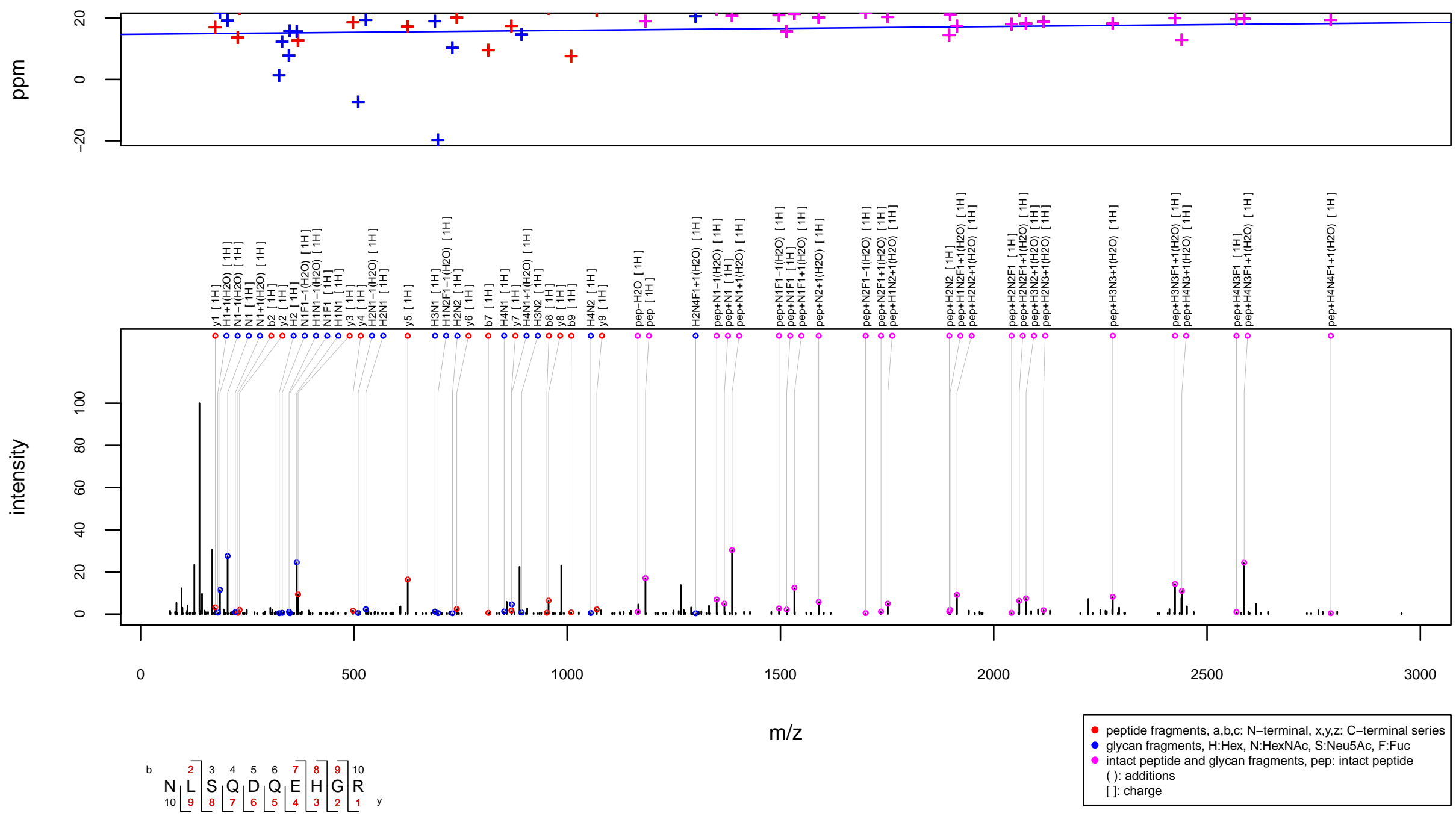
m/z 985.9465 charge 4 scan 0-0

Score $=61.94$, Hits $=40$, Explained Intensity $=0.36$

Peptide: HEMO_HUMAN[447,462]:ALPQPQNVTSLLGCTH

Glycan: $\mathrm{SHNH}(\mathrm{SHNH}) \mathrm{HNN}, \mathrm{S} 2 \mathrm{H} 5 \mathrm{~N} 4$

Charge: $4 \mathrm{H}$
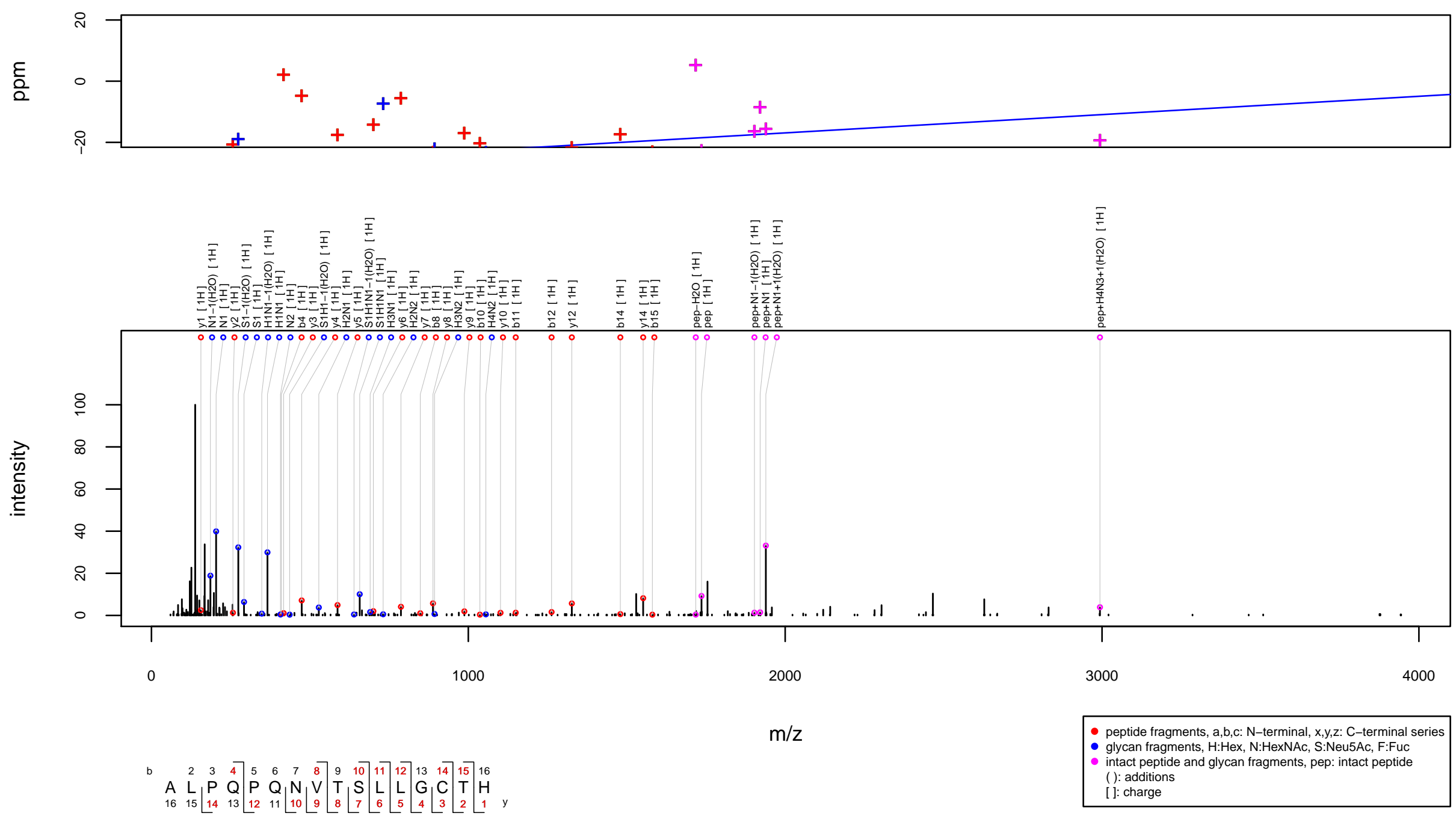
$\mathrm{m} / \mathrm{z} 987.4114$ charge 3 scan $0-0$

Score $=69.76$, Hits $=51$, Explained Intensity $=0.32$

Peptide: PPAP_HUMAN[92,98]:FLNESYK

Glycan: $\mathrm{SHNH}(\mathrm{HNH}) \mathrm{HN}(\mathrm{F}) \mathrm{N}, \mathrm{S} 1 \mathrm{H} 5 \mathrm{~N} 4 \mathrm{~F}$

Charge: $3 \mathrm{H}$
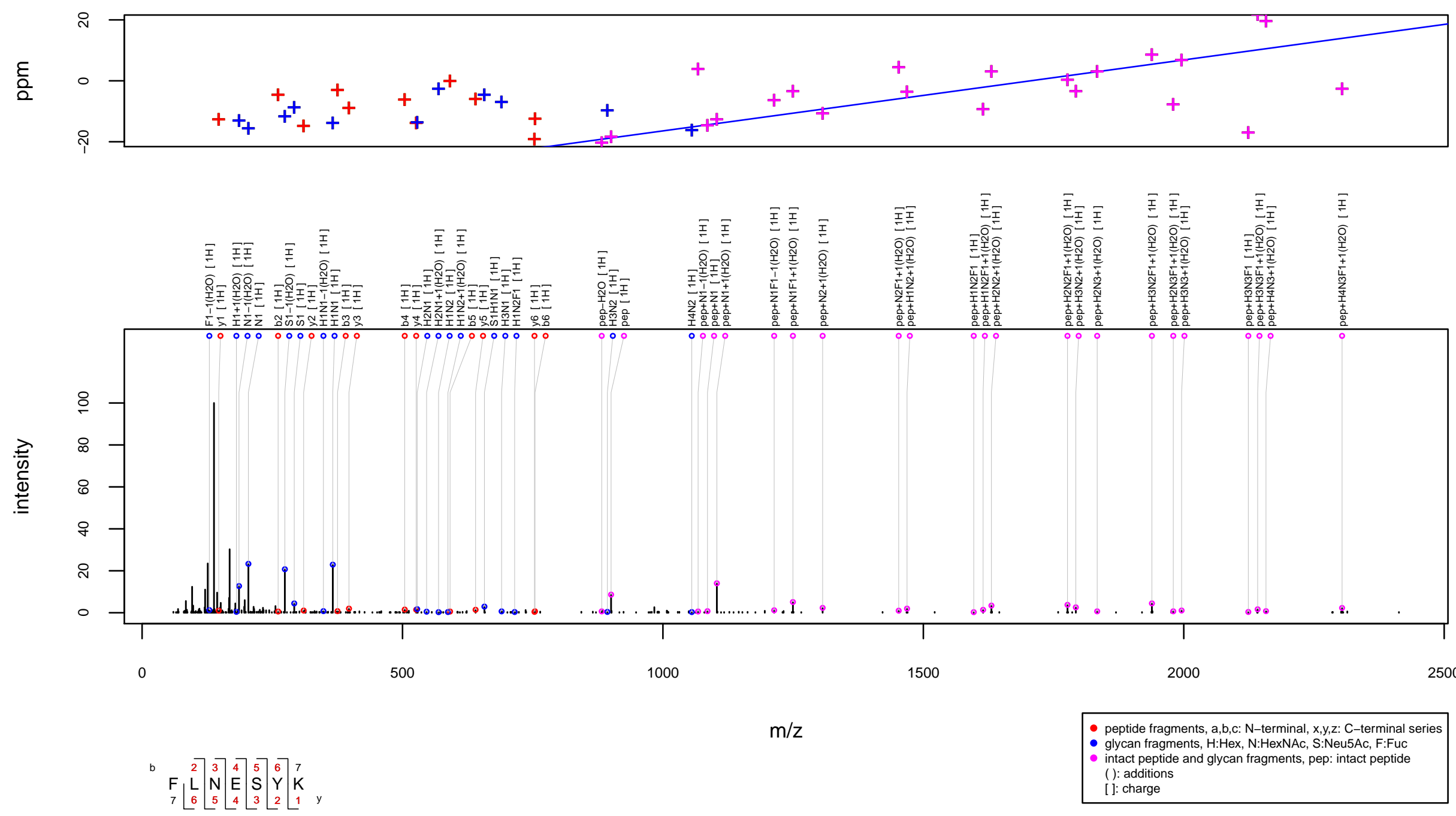
m/z 990.1922 charge 4 scan 0-0

Score $=52.77$, Hits $=36$, Explained Intensity $=0.38$ Peptide: TIMP1_HUMAN[46,60]:FVGTPEVNQTTLYQR Glycan: $\mathrm{SHNH}(\mathrm{SHNH}) \mathrm{HNN}, \mathrm{S} 2 \mathrm{H} 5 \mathrm{~N} 4$

Charge: $4 \mathrm{H}$
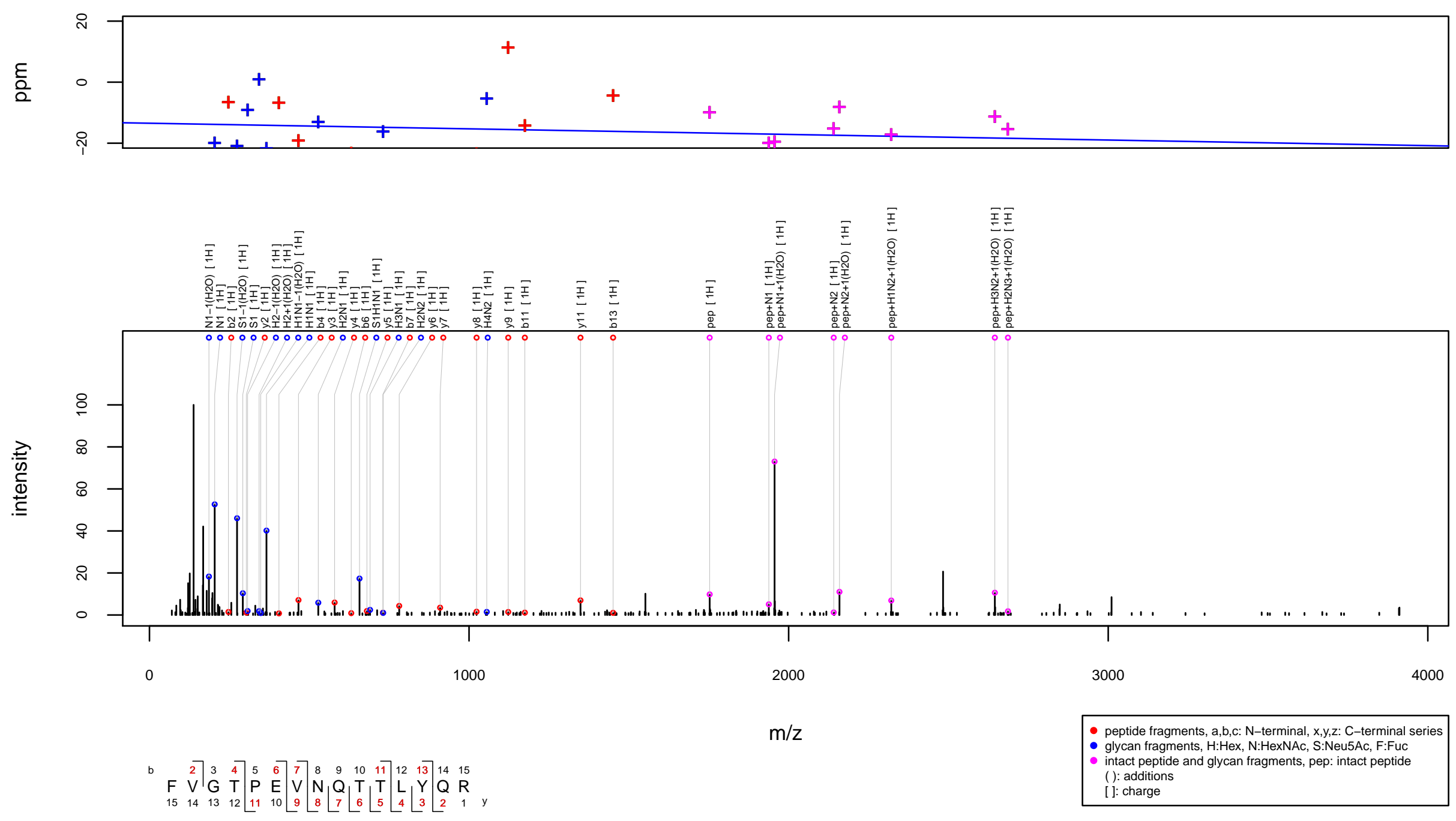
m/z 991.6913 charge 4 scan 0-0

Score $=61.27$, Hits $=45$, Explained Intensity $=0.42$ Peptide: CLUS_HUMAN[372,385]:LANLTQGEDQYYLR Glycan: $\mathrm{F}(\mathrm{H}) \mathrm{NHNH}(\mathrm{HNH}) \mathrm{HN}(\mathrm{F}) \mathrm{N}, \mathrm{H} 6 \mathrm{~N} 5 \mathrm{~F} 2$

Charge: $4 \mathrm{H}$
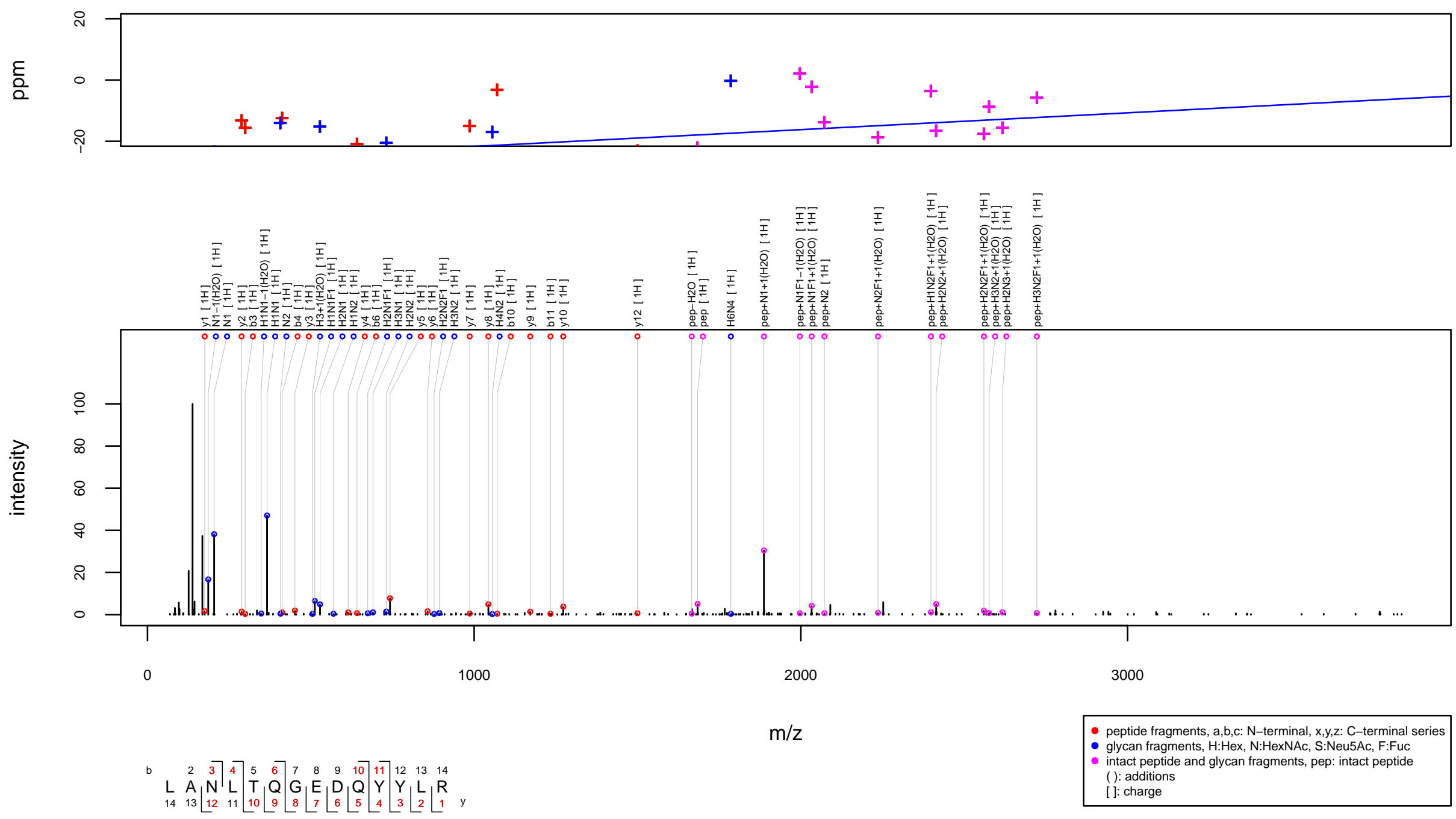
$\mathrm{m} / \mathrm{z} 992.1829$ charge 4 scan $0-0$

Score $=71.18$, Hits $=49$, Explained Intensity $=0.37$

Peptide: FINC_HUMAN[516,533]:DQCIVDDITYNVNDTFHK

Glycan: H5N4F1

Charge: $4 \mathrm{H}$
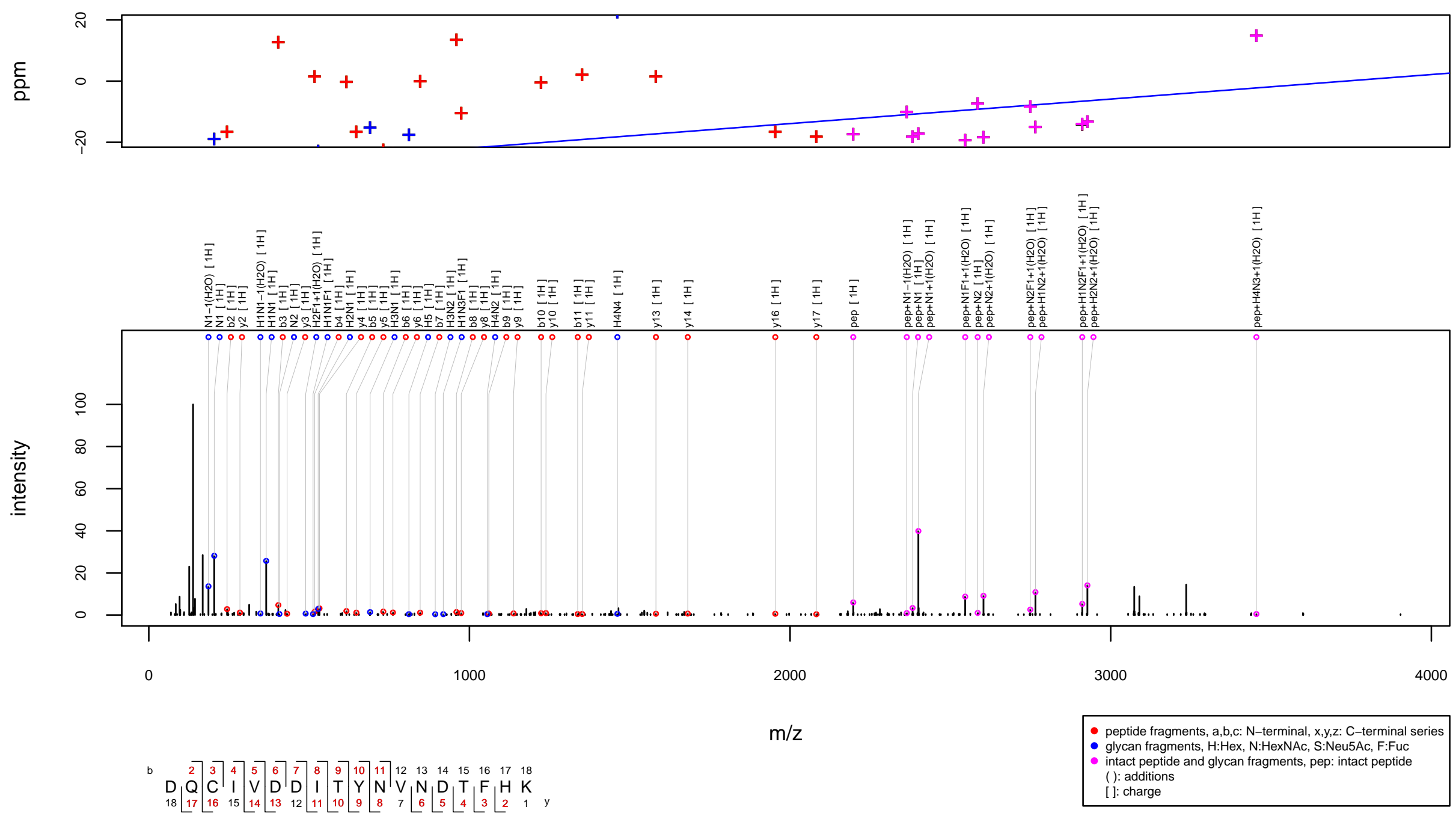
m/z 995.4917 charge 4 scan 0-0

Score $=40.12$, Hits $=31$, Explained Intensity $=0.2$

Peptide: FCGBP_HUMAN[1734,1753]:VVTVAALGTNISIHKDEIGK

Glycan: $\mathrm{SHNH}(\mathrm{HNH}) \mathrm{HNN}, \mathrm{S} 1 \mathrm{H} 5 \mathrm{~N} 4$

Charge: $4 \mathrm{H}$
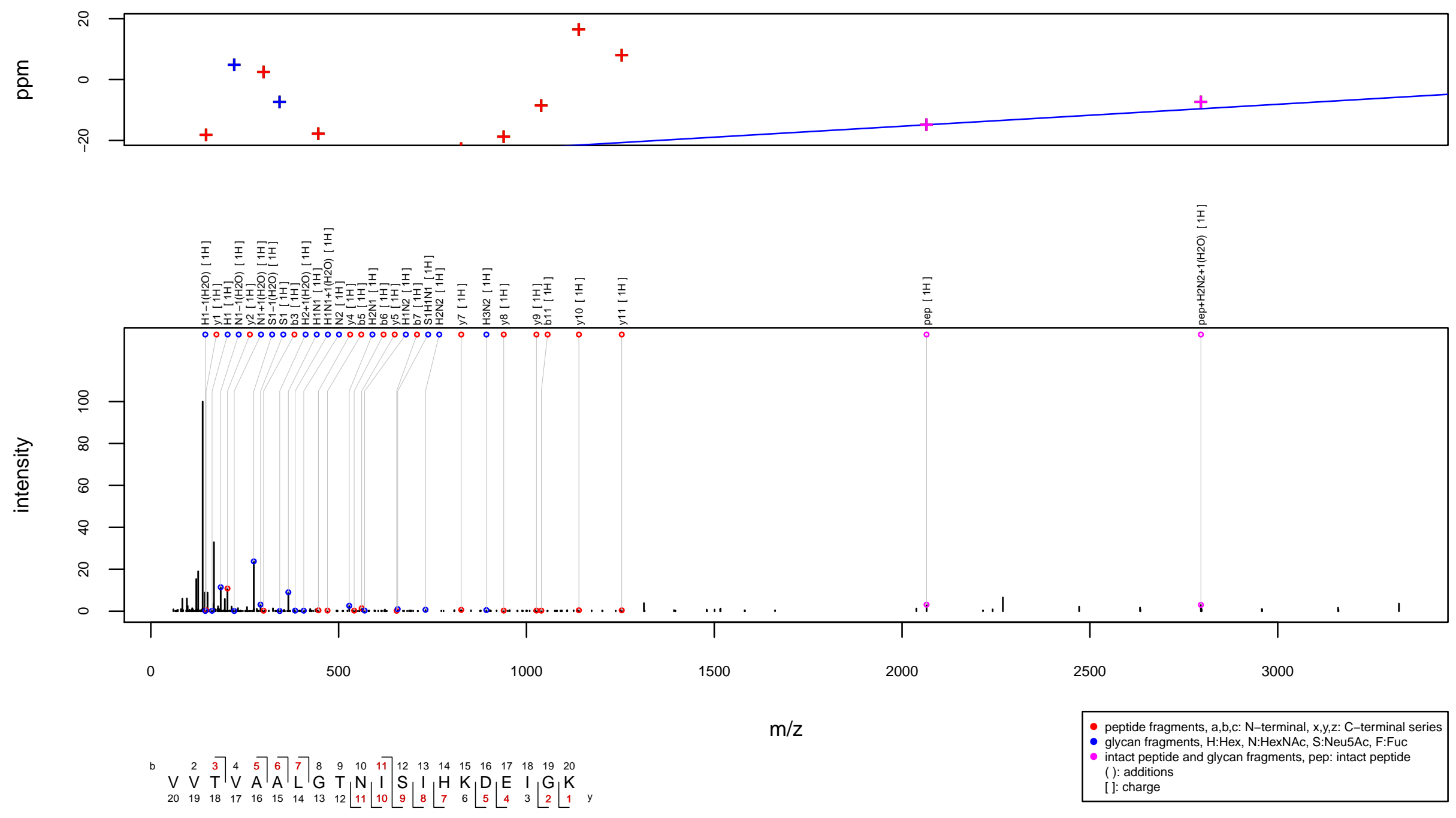
m/z 996.1277 charge 4 scan 0-0

Score $=48.71$, Hits $=63$, Explained Intensity $=0.31$

Peptide: PPAP_HUMAN[92,98]:FLNESYK

Glycan: S2H7N6F1

Charge: $4 \mathrm{H}$
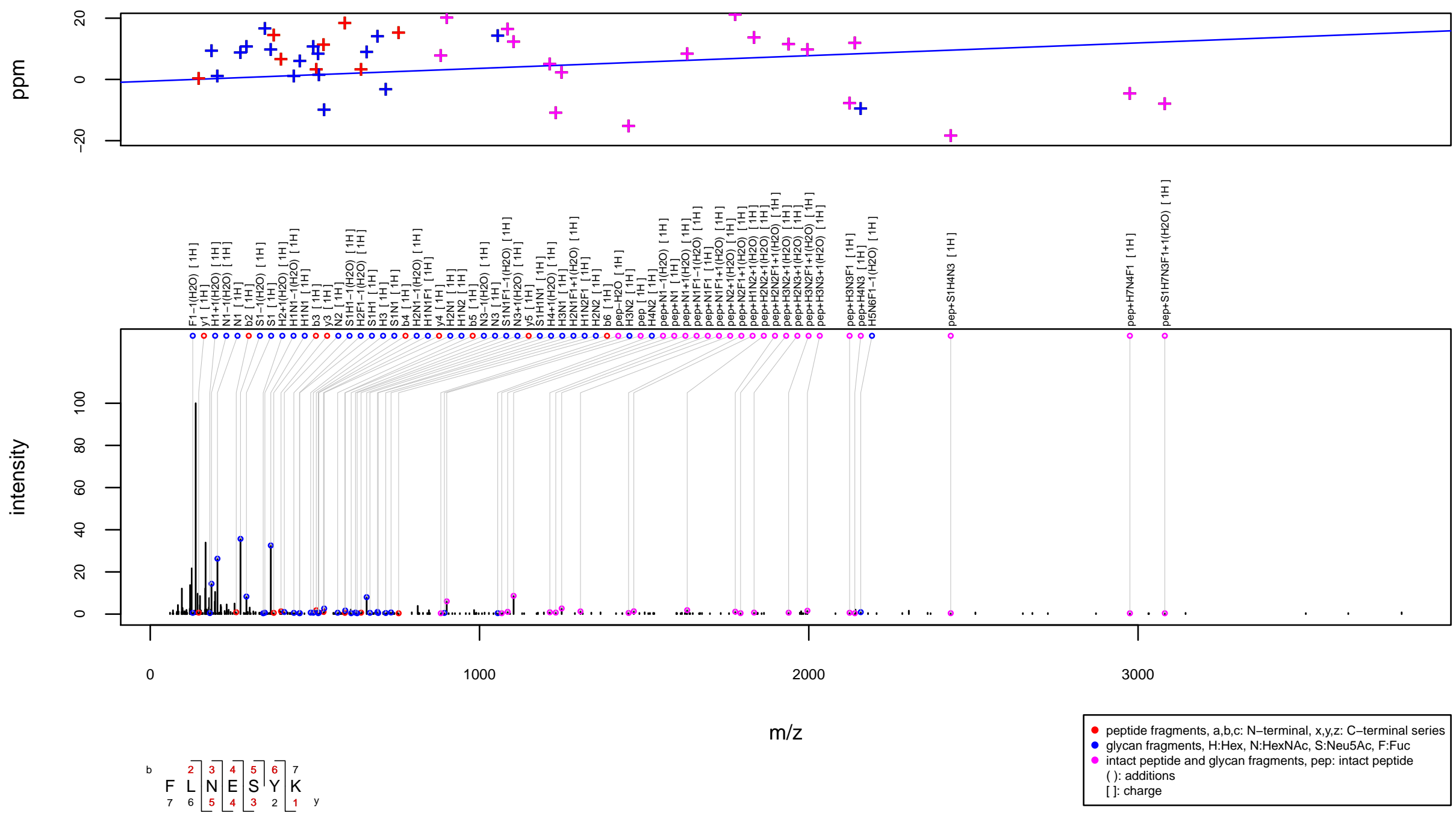
$\mathrm{m} / \mathrm{z} 998.4266$ charge 3 scan $0-0$

Score $=66.29$, Hits $=41$, Explained Intensity $=0.42$ Peptide: AMPN_HUMAN[231,241]:AEFNITLIHPK

Glycan: $\mathrm{SHNH}(\mathrm{HH}) \mathrm{HNN}, \mathrm{S} 1 \mathrm{H} 5 \mathrm{~N} 3$

Charge: $3 \mathrm{H}$
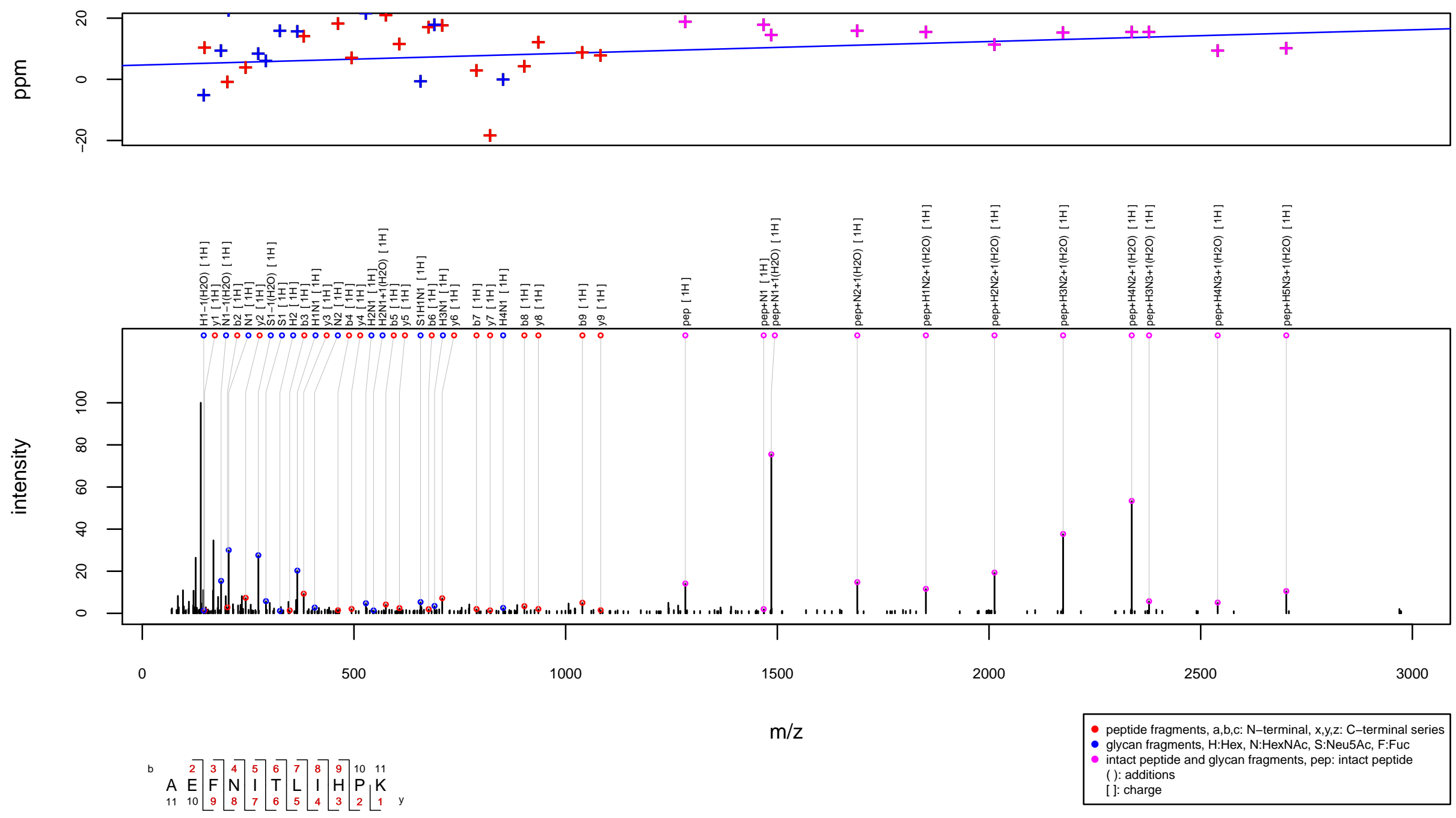
$\mathrm{m} / \mathrm{z} 999.1657$ charge 4 scan $0-0$

Score $=33.73$, Hits $=22$, Explained Intensity $=0.22$

Peptide: FINC_HUMAN[535,550]:HEEGHMLNCTCFGQGR

Glycan: $\mathrm{SHNH}(\mathrm{HNH}) \mathrm{HNN}, \mathrm{S} 1 \mathrm{H} 5 \mathrm{~N} 4$

Charge: $4 \mathrm{H}$
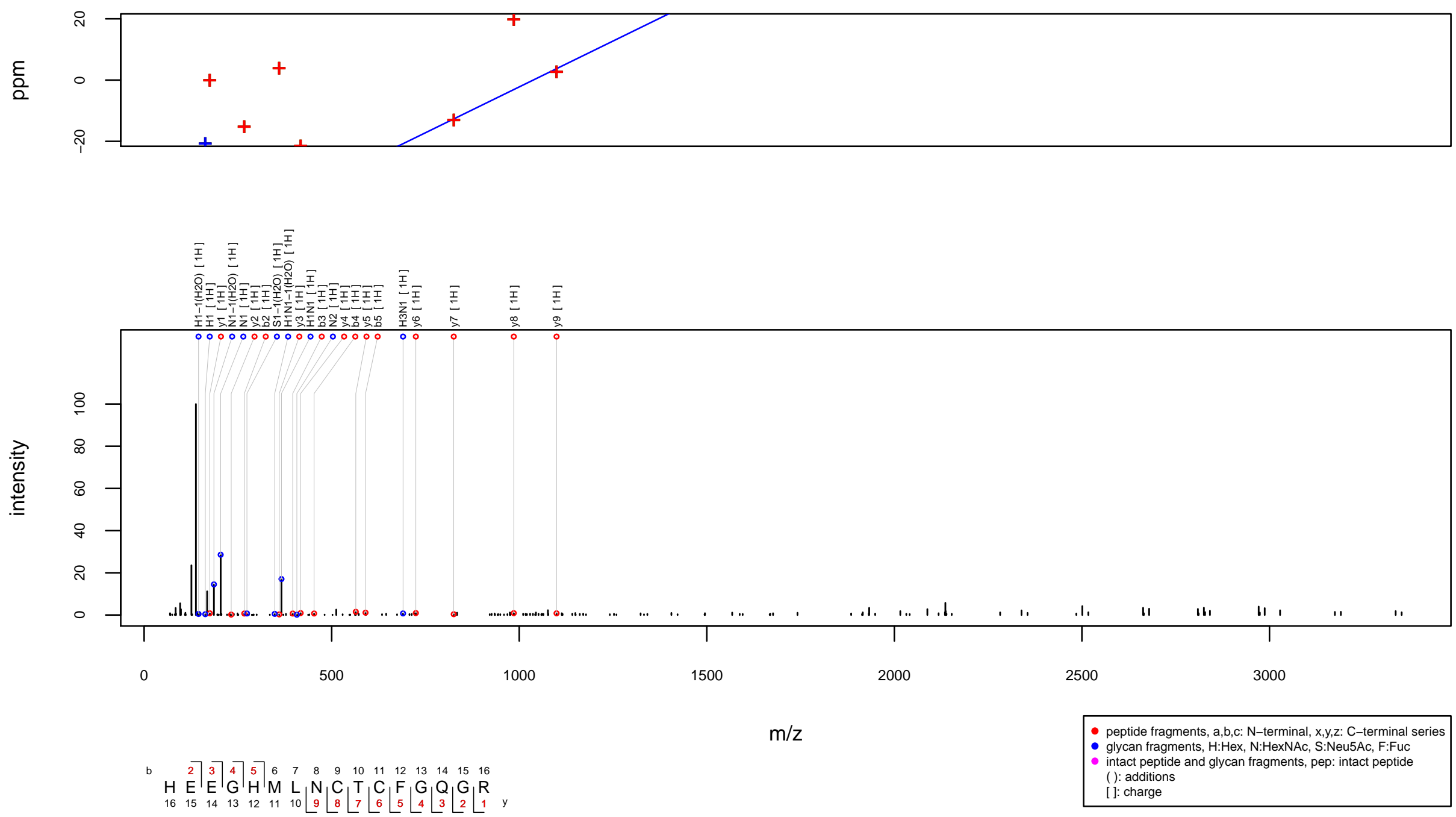